\title{
Combinatorial and Geometric Dualities in Graph Homomorphism Optimization Problems
}

\author{
Nathan Benedetto Proença
}

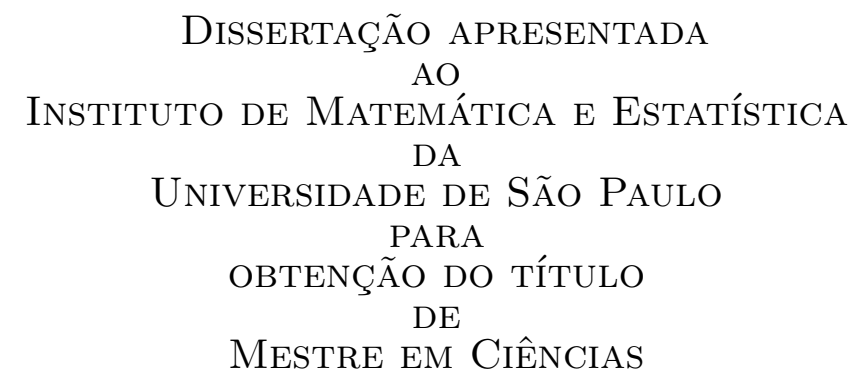

Programa: Ciência da Computação

Orientador: Prof. Dr. Marcel K. de Carli Silva

Durante o desenvolvimento deste trabalho o autor recebeu auxílio financeiro do $\mathrm{CNPq}$

Setembro de 2021 



\title{
Combinatorial and Geometric Dualities in Graph Homomorphism Optimization Problems
}

\author{
Nathan Benedetto Proença
}

Esta versão da dissertação contém as correções e alterações sugeridas pela Comissão Julgadora durante a defesa da versão original do trabalho, realizada em 19/07/2021.

Comissão Julgadora:

- Prof. Dr. Marcel K. de Carli Silva - USP

- Prof. Dr. Levent Tunçel - University of Waterloo

- Prof. Dr. Carlos Hoppen — UFRGS 



\section{Resumo}

Um homomorfismo de grafos é uma função entre os vértices de dois grafos que mapeia pares de vértices adjacentes em pares de vértices adjacentes. Diversos parâmetros de grafos podem ser formulados em termos de encontrar um homomorfismo que maximize ou minimize um certo valor objetivo: o número cromático $\chi$, o número de clique $\omega$ e a função $\vartheta$ de Lovász são exemplos notáveis. Este trabalho estuda otimização de homomorfismos de grafos utilizando otimização convexa e combinatória. Apresentamos um arcabouço, fundamentado na teoria de conjuntos pré-ordenados, que evidencia uma dualidade combinatória entre o número de clique e o número cromático, além de ser capaz de formular diversos parâmetros na literatura. Demonstramos resultados conhecidos sobre cantos convexos e anti-bloqueadores, e então utilizamos esses conceitos geométricos para explicar certas propriedades de alguns dos parâmetros que nos interessam. Em particular, descrevemos uma dualidade geométrica entre limitantes superiores ao número de estabilidade de um grafo e limitantes inferiores ao número cromático fracionário de um grafo. Utilizamos essa dualidade para fornecer um novo entendimento sobre a relação entre dois famosos limitantes espectrais introduzidos por

Hoffman. Aproveitando os conceitos previamente discutidos, abordamos diretamente construções que definem cantos convexos e generalizações de homomorfismos a partir de cones de matrizes simétricas. Relacionamos a representação de Choi de uma transformação linear às formulações cônicas de homomorfismos, obtendo assim uma nova conexão entre ideias presentes na teoria quântica da informação. Diversos resultados e conceitos são apresentados de distintas maneiras no decorrer do texto, estabelecendo através de teoremas e exemplos a coesão entre as perspectivas combinatória e geométrica.

Palavras Chave: homomorfismos de grafos, número cromático fracionário, função teta de Lovász, cantos convexos, programação cônica, teoria quântica da informação. 



\begin{abstract}
A graph homomorphism is a function between the vertex set of two graphs which maps pairs of adjacent vertices to pairs of adjacent vertices. Many graph parameters can be expressed in terms of finding a homomorphism that maximizes or minimizes a certain objective function: the chromatic number $\chi$, the clique number $\omega$, and the Lovász $\vartheta$ function are noteworthy examples. This work studies graph homomorphisms optimization using combinatorial and convex optimization. Grounded on the theory of preordered sets, we develop a framework that captures a combinatorial duality between the clique and coloring numbers, and that formulates several graph parameters in the literature. We demonstrate known results on convex corners and antiblockers, and we use these geometric concepts to explain some properties of the graph parameters of interest. In particular, we present a geometric duality between upper bounds for the stability number of a graph and lower bounds for the fractional chromatic number of a graph. We use this duality to provide a new understanding of the relationship between two famous spectral bounds introduced by Hoffman. Building on the concepts previously presented, we study convex corners and generalizations of graph homomorphisms which use cones of symmetric matrices. We relate the Choi representation of linear transformations with conic formulations of graph homomorphisms, thus uncovering a new connection between ideas from quantum information theory. Several results and concepts are approached in distinct ways throughout the text, establishing with theorems and examples the cohesion between the combinatorial and geometric perspectives.
\end{abstract}

Keywords: graph homomorphisms, fractional chromatic number, Lovász theta function, convex corners, conic programming, quantum information theory. 



\section{Agradecimentos}

Este trabalho só foi possível graças ao auxílio de muitas pessoas. Curiosamente, não existe uma única língua na qual eu possa escrever esse agradecimento e saber que todos os envolvidos podem ler minhas palavras. Por isso, escrevo meus agradecimentos duas vezes, uma em português e outra em inglês.

Agradeço à Amanda por tudo que é Nosso. Agradeço ao Enzo pela companhia e pela consultoria em vocabulário medieval. Luciano, Lourdes, Afonso, Cândida e Magda: o apoio de vocês é tão importante que nada mais justo que eu carregue vocês sempre comigo, inclusive no meu nome. Ao Marcel, agradeço pelos anos me orientando sobre como transformar minha paixão em minha profissão. Agradeço ao Levent e ao Carlos pela coragem de participarem tanto da minha qualificação quanto da minha defesa. Agradeço aos professores Cris, Yoshiko, Coelho e Carlinhos por me fazerem querer seguir o caminho que eu escolhi. Agradeço também ao apoio obtido com meus colegas nessa caminhada: Bruno, Fabrício, Gabriel, Giovanne, Tássio, Victor e Renzo.

\section{Acknowledgments}

This work was only possible through the help of many people. Interestingly, there is no unique language in which I can write my acknowledgments and be sure that everyone involved can read my words. Thus, I write it twice, first in Portuguese and then in English.

I thank Amanda for everything which is ours. I thank Enzo for the company and for the counseling on medieval vocabulary. Luciano, Lourdes, Afonso, Cândida, and Magda: your support is so important that it is only fitting that I carry you with me everywhere, even in my name. I thank Marcel for the years guiding me from my passion to my profession. I thank Levent and Carlos for being so brave as to participate both on my qualifying exam and on my dissertation defense committee. I thank the professors Cris, Yoshiko, Coelho, and Carlinhos for inspiring me to follow the path I have chosen. I also thank the support from the ones walking beside me: Bruno, Fabrício, Gabriel, Giovanne, Tássio, Victor, and Renzo. 


\section{Contents}

1 Introduction $\quad 1$

1.1 Graph Homomorphism Optimization Problems . . . . . . . . . . . . . . . . . . . 1

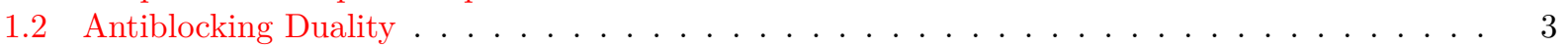

1.3 Methodology and Organization of the Text $\ldots \ldots \ldots \ldots \ldots$

2 Preliminaries and Notation $\quad 7$

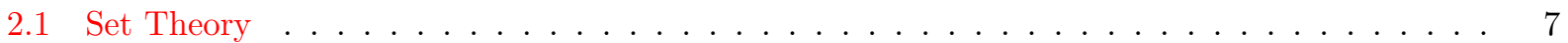

2.2 Linear Algebra . . . . . . . . . . . . . . . . . . . . . . . . . . . . . . 9

2.3 Basic Results on Graphs and Graph Homomorphisms . . . . . . . . . . . . . . . . . . . 11

2.4 Category Theory . . . . . . . . . . . . . . . . . . . . . . . 13

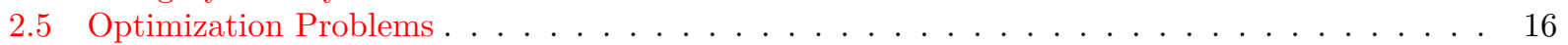

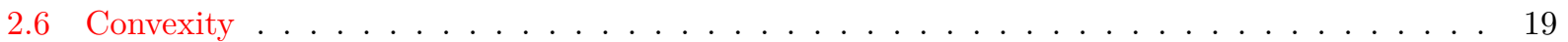

3 Graph Homomorphism Optimization Problems 23

3.1 Order Duality . . . . . . . . . . . . . . . . . . . . . . . . . . . 23

3.2 Graph Universes . . . . . . . . . . . . . . . . . . . . . . . . . . 38

3.3 Generalized Clique and Coloring Numbers . . . . . . . . . . . . . . . . . . . 41

3.4 Known Generalized Clique and Coloring Parameters . . . . . . . . . . . . . . . . . . . 46

3.4.1 The Fractional Chromatic Number . . . . . . . . . . . . . . . . . . . . . . . 48

3.4.2 The Circular Chromatic Number . . . . . . . . . . . . . . . . . . . . . . 52

3.4 .3 The Vector Chromatic Number . . . . . . . . . . . . . . . . . . . . . . 57

3.4 .4 The Quantum Chromatic Number . . . . . . . . . . . . . . . . . . . . . 62

3.4.5 The Orthogonal and Projective Rank . . . . . . . . . . . . . . . . . 65

3.4.6 A Unified Picture of the Presented Parameters . . . . . . . . . . . . . . . . . . . . 69

3.4.7 Relations Defined by the Homomorphic Product . . . . . . . . . . . . . . . . 72

3.4 .8 The Fractional Cut-Covering Number . . . . . . . . . . . . . . . . . 76

4 Antiblocking and Gauge Duality $\quad 81$

4.1 Antiblocking and Gauge Duality . . . . . . . . . . . . . . . . . . . 81

4.1 Basic Definitions . . . . . . . . . . . . . . . . . . . . . . . 81

4.1 .2 Strong Duality for Convex Corners and Gauges . . . . . . . . . . . . . . . . . 87

4.1 .3 Convex Corners from Graph Theory . . . . . . . . . . . . . . . . . . . . . . 94

4.2 Generalized Cauchy-Schwarz and No-Homomorphism Lemma . . . . . . . . . . . . . . . 96

4.3 Geometric Conversion of Bounds . . . . . . . . . . . . . . . . . . . . 98

4.4 Luz's Upper Bound on the Stability Number and the Lovász Theta Function . . . . . . . . 100

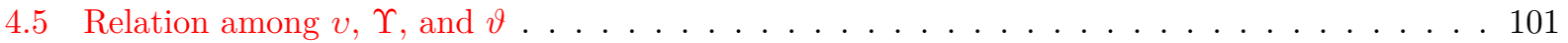

4.6 Unified Chain of Inequalities . . . . . . . . . . . . . . . . . . . . 103

5 Conic Generalizations of Parameters and Homomorphisms 105

5.1 Matrix Cones . . . . . . . . . . . . . . . . . . . . . . . . . . . . 105

5.2 Theta Bodies . . . . . . . . . . . . . . . . . . . . . . . 108

5.3 Dual Conic-programming Bounds for the Maximum Cut and Cut-Covering Problems . . . . 117

5.3 .1 Conic Approximations for the CUT Polytope . . . . . . . . . . . . . . . 117 
5.3.2 An SDP-based Approximation Algorithm for the Fractional Cut-covering Number . 125

5.3 .3 Conic Approximations for the Fractional Cut-covering Number . . . . . . . . . . . 130

5.4 Conic Formulations of Graph Homomorphisms ～. . . . . . . . . . . . . . . . . . 133

5.4 .1 Kronecker Product of Matrices . . . . . . . . . . . . . . . . . . . . . . . . . 134

5.4 .2 Gram Matrices and Composition . . . . . . . . . . . . . . . . . . . . . . 141

5.4 .3 Conic Formulations of Graph Homomorphisms . . . . . . . . . . . . . . . . . . 148

6 Conclusions $\quad 163$

$\begin{array}{ll}\text { Bibliography } & 167\end{array}$ 


\section{Chapter 1}

\section{Introduction}

It is impossible for this introduction to present a cohesive theory upon which this work is built. This predicament arises from the own nature of this text, which attempts to evidence the relationship between two seemingly disparate approaches. Our core contribution is to present each theory and to highlight results which attest to their connection. This method is employed in this dissertation as a whole, and in the following sections in particular.

\subsection{Graph Homomorphism Optimization Problems}

We start by introducing examples of graph homomorphism optimization problems. This section is a miniature of Chapter 3, which surveys the literature for more examples and develops Figure 3.1, a Hasse diagram relating most of the graph parameters studied in this work. As a consequence, we assure the reader that each argument presented here will be covered in depth later in this work. Figure 3.1 is so important that we could not write this introduction without sketching the proof of a small-scaled version of it, equation (1.6). This proof sketch will highlight some of the core ideas of this work and provide a meaningful example of the connection between the approaches presented in this text.

An $n$-coloring of a graph $G$ is a function $f: V(G) \rightarrow[n]:=\{1, \ldots, n\}$ such that $f(i) \neq f(j)$ whenever $i j \in E(G)$. The chromatic number $\chi(G)$ of a graph $G$ is the minimum $n \in \mathbb{N}$ such that there exists an $n$-coloring of $G$. Homomorphisms are a natural generalization of $n$-colorings: a graph homomorphism from a graph $G$ to a graph $H$ is a function $f: V(G) \rightarrow V(H)$ such that $f(i) f(j) \in E(H)$ whenever $i j \in E(G)$. An $n$-coloring of a graph $G$ is a homomorphism from $G$ to $K_{n}$, the complete graph on $n$ vertices. If we denote by $G \rightarrow H$ the existence of a homomorphism from $G$ to $H$, we may express the chromatic number succinctly as

$$
\chi(G)=\inf \left\{n \in \mathbb{N} \mid G \rightarrow K_{n}\right\} .
$$

Equation (1.1) presents the quintessential graph homomorphism optimization problem. In (1.1), the colorings of $G$ determine the feasible region, and the objective function is the number of colors used. If $f$ is a graph homomorphism from $G$ to $H$, we may also say that $f$ is an $H$-coloring of $G$. The similarity between " $H$-coloring" and " $n$-coloring" arises from the interpretation of graph homomorphisms as generalized colorings. It is no surprise then that variations of the chromatic number can be formulated in ways similar to (1.1). Indeed, this is a common point of discussion in introductory texts in graph homomorphisms [52, 57], and we proceed to look at some examples.

Let $a, b \in \mathbb{N}$ be such that $0<b \leq a$. The Kneser graph $\operatorname{KG}(a, b)$ is the graph whose vertices are the subsets of $[a]$ of size $b$, and two vertices are adjacent whenever they are disjoint. A KG $(a, b)$-coloring of a graph $G$ has $a$ available labels and assigns $b$ labels to each vertex of $G$ in such a way that adjacent vertices are mapped to disjoint sets of labels. The fractional chromatic number $\chi_{f}(G)$ of a graph $G$ may be defined as

$$
\chi_{f}(G):=\inf \left\{\frac{a}{b} \mid 0<b \leq a, G \rightarrow \operatorname{KG}(a, b)\right\} .
$$

When working with vectors in $\mathbb{R}^{n}$, we have an inner product $x^{\top} y=\sum_{i=1}^{n} x_{i} y_{i}$ defined for every $x, y \in \mathbb{R}^{n}$; the norm of a vector $x \in \mathbb{R}^{n}$ is given by $\|x\|=\sqrt{x^{\top} x}$ and the unit sphere of $\mathbb{R}^{n}$ is the set of vectors $u \in \mathbb{R}^{n}$ 
such that $\|u\|=1$. Define the graph $\operatorname{Strict}(n, \gamma)$ for every $n \in \mathbb{N}$ and real $\gamma \in[-1,1]$ as the graph whose vertex set is the unit sphere of $\mathbb{R}^{n}$, and two vertices adjacent whenever their inner product is $\gamma$. Karger, Motwani, and Sudan [65] studied strict vector colorings: an assignment of vectors in the unit sphere to the vertices of a graph such that adjacent vertices are mapped to vectors whose inner product is precisely a given value. Just as $K_{n}$-colorings are $n$-colorings, the $\operatorname{Strict}(n, \gamma)$-colorings of a graph $G$ are the $\gamma$-strict vector colorings of $G$. The authors of [65] prove that

$$
\vartheta(\bar{G})=\inf \{1-1 / \gamma \mid n \in \mathbb{N},-1 \leq \gamma<0, G \rightarrow \operatorname{Strict}(n, \gamma)\},
$$

where $\bar{G}$ denotes the complement of the graph $G$, and $\vartheta$ denotes the Lovász theta function [77]. This function has numerous equivalent definitions and interesting applications; we postpone this discussion, as our primary goal for now is to highlight the similarities among (1.1), (1.2), and (1.3). Similarly to (1.1), both (1.2) and (1.3) are graph homomorphism optimization problems. For convenience, for every function $\beta$ on graphs, we write $\bar{\beta}$ for the function defined as $\bar{\beta}(G):=\beta(\bar{G})$ for every graph $G$. Thus, the LHS of (1.3) may be written as $\bar{\vartheta}(G)$.

The focus on colorings skewed our discussion. If $G$ is a subgraph of $H$, then the identity function on $V(G)$ is a homomorphism from $G$ to $H$. Hence, the homomorphic relation captures both the colorings and the subgraphs of a graph. If $G$ is a graph, a set $K \subseteq V(G)$ is a clique if the subgraph of $G$ induced by $K$ is complete. The clique number $\omega(G)$ of a graph $G$ is the size of a largest clique in $G$. A homomorphism from the complete graph $K_{n}$ to $G$ has as its image a complete subgraph of $G$ of size $n$. We may thus write

$$
\omega(G)=\sup \left\{n \in \mathbb{N} \mid K_{n} \rightarrow G\right\} .
$$

Equations (1.1) and (1.4) hint at a certain combinatorial duality between $\omega$ and $\chi$. Recalling (1.2) and (1.3), it is reasonable to consider the counterparts of $\chi_{f}$ and $\bar{\vartheta}$, and study which properties relating $\omega$ and $\chi$ carry over to these other pairs. The literature already has examples of this idea. Zhu [121] defined the circular complete graph $\operatorname{Circ}(a, b)$ for $a, b \in \mathbb{N}$ with $0<b \leq a$, and used these graphs to formulate and study the circular chromatic number $\chi_{c}(G)$, first introduced by Vince [116], and the circular clique number $\omega_{c}(G)$ of a graph $G$.

An effort to dissect the duality between (1.1) and (1.4) can start by looking at the relation ' $\rightarrow$ ' itself. Let $F, G$, and $H$ be graphs. We have that $\rightarrow$ is reflexive, i.e., that $G \rightarrow G$, as the identity function on the vertex set is always a homomorphism from a graph to itself. Furthermore, it is easy to use that graph homomorphisms are closed under composition to prove that

$$
\rightarrow \text { is transitive, }
$$

i.e., that $F \rightarrow G$ and $G \rightarrow H$ imply that $F \rightarrow H$. Therefore, the homomorphic relation $\rightarrow$ is a preorder on graphs. The set $[-\infty,+\infty]$, defined with the usual arithmetic and order relations, is called the extended reals. A function on graphs $\beta$ taking extended real values is homomorphism monotone if $G \rightarrow H$ implies $\beta(G) \leq \beta(H)$. The formulations above make it easy to see that $\omega, \chi, \chi_{f}$, and $\bar{\vartheta}$ are homomorphism monotone. We prove it for $\chi$ : if $G$ and $H$ are graphs such that $G \rightarrow H$, then $H \rightarrow K_{n}$ implies $G \rightarrow K_{n}$ by (1.5). We then conclude that $\chi(G) \leq \chi(H)$ from (1.1),

Most of the aforementioned parameters fit in a single chain of inequalities. In fact,

$$
\omega \leq \bar{\vartheta} \leq \chi_{f} \leq \chi
$$

where the inequality of graph parameters should be taken pointwise. To prove that $\bar{\vartheta} \leq \chi_{f}$, we first show that for every $a, b \in \mathbb{N}$ with $0<b \leq a$,

$$
\mathrm{KG}(a, b) \rightarrow \operatorname{Strict}\left(a, \frac{-b}{a-b}\right) .
$$

The proof of (1.7) follows from mapping each set $S \in V(\operatorname{KG}(a, b))$ to $\mathbb{1}_{S}-\frac{b}{a} \mathbb{1}$, where $\mathbb{1}_{S} \in\{0,1\}^{[a]}$ denotes 
the incidence vector of $S$ and $\mathbb{1}$ denotes the all-ones vector. We then have that for every graph $G$,

$$
\begin{aligned}
G \rightarrow \operatorname{KG}(a, b) & \Longrightarrow G \rightarrow \operatorname{Strict}\left(a, \frac{-b}{a-b}\right) & & \text { by }(1.7) \text { and }(1.5) \\
& \Longrightarrow \bar{\vartheta}(G) \leq 1-\left(\frac{-b}{a-b}\right)^{-1} & & \text { by }(1.3) \\
& \Longrightarrow \bar{\vartheta}(G) \leq \frac{a}{b}, & &
\end{aligned}
$$

so (1.2) implies that $\chi_{f}(G) \leq \bar{\vartheta}(G)$. The inequality $\chi_{f} \leq \chi$ follows similarly from $K_{n} \rightarrow \operatorname{KG}(n, 1),(1.5)$, (1.1), and (1.2). Since $\bar{\vartheta}$ is homomorphism monotone, (1.4) reduces proving $\omega \leq \bar{\vartheta}$ to proving that $\bar{\vartheta}\left(K_{n}\right) \geq n$ for every complete graph, which amounts to a direct computation. After sketching the ideas which prove (1.6), we invite the reader to look back at (1.2) and (1.3) with the above arguments in mind. We have shown how an $n$-coloring of a graph $G$ can be used to define a $\operatorname{KG}(n, 1)$-coloring of $G$, and then a $\operatorname{Strict}(n,-1 /(n-1))$ coloring of $G$. The main tool in this "coloring conversion" procedure is homomorphism composition, which we use whenever we use (1.5). As the objective value of the colorings derived from an $n$-coloring is $n$, the values assigned to the colorings in (1.2) and (1.3) are elucidated - even the obscure $1-1 / \gamma$ for a $\operatorname{Strict}(s, \gamma)$-coloring.

The chain of inequalities in (1.6) not only is helpful in extending the coloring analogy to $\chi_{f}$ and $\bar{\vartheta}$, but also has practical applications. It is NP-hard to approximate $\omega$ [54], $\chi_{f}$ [78], or $\chi$ [78]. Therefore, unless $\mathrm{P}=\mathrm{NP}$, the graph parameter $\bar{\vartheta}$ is the only one in (1.6) whose value can be approximated in polynomial time given any graph and error tolerance as input. Therefore, the function $\bar{\vartheta}$ can be seen as an efficiently computable lower bound for both $\chi_{f}$ and $\chi$, and as an efficiently computable upper bound for $\omega$. In particular, we can compute the chromatic number of any graph $G$ such that $\omega(G)=\chi(G)$ in polynomial time.

\subsection{Antiblocking Duality}

We now shift to the geometric framework of this text. Whereas graph homomorphism optimization problems have a somewhat primitive notion of duality between them, which we can only refer to by means of an example ((1.1) and (1.4)), convex corners are rigorously related by antiblocking duality. We start by introducing some examples of convex corners and discussing the optimization problems they define, and we conclude our exposition by recovering (1.6). This section is a miniature of Chapter 4, so once again, all the results proved here will be covered in much more depth later in this work.

Fulkerson [39, 40] studied what he called antiblocking pairs of polyhedra. Let $V$ be a finite set, and let $\mathbb{R}_{+}^{V}$ denote the set of coordinatewise nonnegative vectors in $\mathbb{R}^{V}$. The antiblocker of a set $\mathcal{C} \subseteq \mathbb{R}_{+}^{V}$ is defined as

$$
\operatorname{abl}(\mathcal{C}):=\left\{y \in \mathbb{R}_{+}^{V} \mid \forall x \in \mathcal{C}, y^{\top} x \leq 1\right\} .
$$

Even though we have defined $\operatorname{abl}(\mathcal{C})$ for any $\mathcal{C} \subseteq \mathbb{R}_{+}^{V}$, it is useful to focus on some special sets. A set $\mathcal{C} \subseteq \mathbb{R}_{+}^{V}$ is lower-comprehensive if $0 \leq x \leq y$ and $y \in \mathcal{C}$ imply $x \in \mathcal{C}$. A convex corner is a compact convex lower-comprehensive set with nonempty interior. We are interested in convex corners because the antiblocking relation defines a duality correspondence on them. More precisely, as we shall show on Theorem 4.25, the function $\operatorname{abl}(\cdot)$ is an involution on the set of convex corners: if $\mathcal{C}$ is a convex corner, then so is abl $(\mathcal{C})$, and furthermore $\mathcal{C}=\operatorname{abl}(\operatorname{abl}(\mathcal{C}))$. We proceed to look at some examples.

Let $G:=(V, E)$ be a graph. A set $S \subseteq V$ is stable if there are no edges between vertices of $S$. Let $\mathcal{S}(G)$ denote the set of stable sets of the graph $G$. Define

$$
\operatorname{STAB}(G):=\operatorname{conv}\left(\left\{\mathbb{1}_{S} \mid S \in \mathcal{S}(G)\right\}\right),
$$

where $\operatorname{conv}(\mathcal{C})$ denotes the convex hull of $\mathcal{C} \subseteq \mathbb{R}^{V}$. It is easy to see that $\operatorname{STAB}(G)$ is compact and convex. Using that every subset of a stable set is stable, one may prove that $\operatorname{STAB}(G)$ is lower-comprehensive. We have that $|V|^{-1} \mathbb{1} \in \operatorname{STAB}(G)$ by convexity and the fact that $\{i\} \in \mathcal{S}(G)$ for every $i \in V(G)$. Using this and the fact that $\operatorname{STAB}(G)$ is lower-comprehensive, we conclude that $\operatorname{STAB}(G)$ has nonempty interior. Hence, $\operatorname{STAB}(G)$ is a convex corner. Define

$$
\operatorname{QSTAB}(G):=\left\{x \in \mathbb{R}_{+}^{V} \mid \forall K \in \mathcal{S}(\bar{G}), \mathbb{1}_{K}^{\top} x \leq 1\right\} .
$$


The set $\operatorname{QSTAB}(G)$ consists of the nonnegative vectors which satisfy the clique inequalities, i.e., the inequalities of the form $\mathbb{1}_{K}^{\top} x \leq 1$ for some clique $K \subseteq V$ in $G$. To conclude that $\operatorname{QSTAB}(G)$ is a convex corner, it suffices to note that

$$
\operatorname{QSTAB}(\bar{G})=\operatorname{abl}(\operatorname{STAB}(G))
$$

and use the fact that $\operatorname{STAB}(G)$ is a convex corner. We now go beyond the work of Fulkerson and consider a convex corner which need not be polyhedral. Let $\mathbb{S}^{V}$ denote the set of symmetric matrices in $\mathbb{R}^{V \times V}$. Define

$$
\operatorname{TH}(G):=\left\{x \in \mathbb{R}_{+}^{V} \mid \exists X \in \mathbb{S}^{V},\left[\begin{array}{cc}
1 & x^{\top} \\
x & X
\end{array}\right] \succeq 0, \begin{array}{l}
\forall i \in V, x_{i}=X_{i i}, \\
\forall i j \in E, X_{i j}=0
\end{array}\right\},
$$

where $A \succeq 0$ denotes the fact that the symmetric matrix $A$ is positive semidefinite - a symmetric matrix is positive semidefinite if all of its eigenvalues are nonnegative. It is not hard to argue that $\operatorname{TH}(G)$ is compact, convex, and lower-comprehensive. To conclude that $\mathrm{TH}(G)$ has nonempty interior one may prove that

$$
\operatorname{STAB}(G) \subseteq \operatorname{TH}(G) .
$$

This follows from $X:=\mathbb{1}_{S} \mathbb{1}_{S}{ }^{\top}$ certifying that $\mathbb{1}_{S} \in \mathrm{TH}(G)$ for every $S \in \mathcal{S}(G)$, and convexity of $\operatorname{TH}(G)$. Although the definition in (1.12) seems convoluted, it works particularly well with antiblocking duality: it is known [49, 67] that

$$
\operatorname{abl}(\operatorname{TH}(G))=\operatorname{TH}(\bar{G}) .
$$

Parallel to what we did with graph parameters in (1.6), we may accommodate the presented convex corners in a single chain of inclusions. Following an argument by Grötschel, Lovász, and Schrijver [49], we claim that for every graph $G$,

$$
\operatorname{STAB}(G) \subseteq \operatorname{TH}(G) \subseteq \operatorname{QSTAB}(G) .
$$

Note that (1.8) implies that if $\mathcal{C}, \mathcal{D} \subseteq \mathbb{R}_{+}^{V(G)}$ are such that $\mathcal{C} \subseteq \mathcal{D}$, then $\operatorname{abl}(\mathcal{D}) \subseteq \operatorname{abl}(\mathcal{C})$. Combining this with (1.11), (1.13), and (1.14), we have that $\operatorname{TH}(\bar{G}) \subseteq \operatorname{QSTAB}(\bar{G})$ for every graph $G$. Hence, (1.15) follows. We further contextualize (1.15) by considering certain optimization problems. Let $G:=(V, E)$ be a graph. For every $w \in \mathbb{R}_{+}^{V}$, define

$$
\alpha(G, w):=\max \left\{w^{\top} x \mid x \in \operatorname{STAB}(G)\right\} .
$$

It is easy to see that $\alpha(G, w)=\max \left\{w^{\top} \mathbb{1}_{S} \mid S \in \mathcal{S}(G)\right\}$. The stability number of $G$, denoted $\alpha(G)$, is the size of a largest stable set of $G$. Clearly, we have that $\alpha(G)=\alpha(G, \mathbb{1})$. Although weights do not arise in the combinatorial discussion involving $\alpha(G)$ or $\omega(G)$ - which equals $\alpha(\bar{G})$ 一, definition (1.16) is natural: instead of comparing stable sets by cardinality, we compare stable sets by the sum of their weights. For every $w \in \mathbb{R}_{+}^{V}$, define

$$
\chi_{f}(G, w):=\max \left\{w^{\top} x \mid x \in \operatorname{QSTAB}(\bar{G})\right\} .
$$

The parameter $\chi_{f}(G, w)$ may be formulated as a linear program. Linear programming optimizes linear functions over sets defined by linear inequalities - e.g., (1.10). Each linear program has an associated dual linear program. The notation $\chi_{f}$ is justified by the well-known fact that $\chi_{f}(G, \mathbb{1})=\chi_{f}(G)[44,56$, 106]. This can be shown by relating the feasible solutions of the linear programming dual of (1.17) to the $\mathrm{KG}(a, b)$-colorings of $G$. Finally, for every $w \in \mathbb{R}_{+}^{V}$, define

$$
\vartheta(G, w):=\max \left\{w^{\top} x \mid x \in \mathrm{TH}(G)\right\} .
$$

The parameter $\vartheta(G, w)$ may be formulated as a semidefinite program. Semidefinite programming optimizes linear functions over sets defined by linear and semidefinite inequalities - e.g., (1.12). Each semidefinite program has an associated dual semidefinite program. One can show that $\vartheta(G, \mathbb{1})=\vartheta(G)$ by relating the feasible solutions of the semidefinite programming dual of (1.18) to the $\operatorname{Strict}(n, \gamma)$-colorings of $\bar{G}$.

By combining (1.15), (1.16), (1.17), and (1.18), we conclude that for every graph $G$ and $w \in \mathbb{R}_{+}^{V(G)}$,

$$
\alpha(G, w) \leq \vartheta(G, w) \leq \chi_{f}(\bar{G}, w) .
$$

The only inequality in (1.6) not implied by considering $\bar{G}$ and $w=\mathbb{1}$ in $(1.19)$ is $\chi_{f}(G) \leq \chi(G)$. However, it is easy to directly prove this inequality using (1.17). In fact, the linear program defined by $\chi_{f}(G, \mathbb{1})$ is usually 
presented as the linear programming relaxation of an integer linear formulation of $\chi(G)$ [106]. Therefore, quite remarkably, we have just sketched a complete proof of (1.6) with no mention to graph homomorphisms.

We invite the reader to take some time to appreciate what just happened. When proving the same statement with two distinct arguments, it is reasonable to assume that both proofs codify the same objects and overcome the same difficulties. The length of this dissertation presents our best effort in grasping how a

chain of convex-corner inclusions (1.15)

reflects upon a

chain of graph-parameter inequalities (1.6).

We study both approaches presented: the combinatorial one, grounded on graph homomorphisms, and the geometric one, grounded on convex optimization. The connection between these frameworks, here exemplified by (1.6), provides the cohesion of the work. We particularly pursue an understanding of the role played by duality in each point of view. As we have already alluded to, the structure of the text resembles the structure of this introduction: Chapter 3 focuses on graph homomorphism optimization problems, Chapter 4 discusses convex corners and antiblocking duality, and Chapter 5 uses matrix cones to define convex corners and generalizations of graph homomorphisms.

\subsection{Methodology and Organization of the Text}

Here we discuss our methodology, and how it shapes the structure of the text in the large and in the small. This text was produced in a very specific, deliberate, and somewhat uncommon way, to the point of a justification becoming appropriate. The very style of work we are about to advocate, as well as many ideas presented throughout these pages, were presented to me by my supervisor, Marcel K. de Carli Silva, both in classes as well as in our work sessions.

There is a distinctive form in the statements and proofs presented throughout the following pages. This form arises from an attempt to employ an inherently human strategy: enhance our capabilities through well-crafted devices. Concretely, we strive for more than theorem proving, we strive for developing a language that aids reasoning. A mathematical proof is a certificate of the validity of a given statement. The whole point of a certificate is that it can be verified. In this manner, reasoning and verification are two fundamental complementary activities of mathematical research. To facilitate reasoning by alleviating verification, we borrow ideas developed for the most inept verifiers: computers. Leslie Lamport [69] advocates for a style with strong similarity to ours, stating that a good process for eliminating errors is to imagine a child pestering him by asking "why?" at each step of a proof. His article shows an example in which the infant's inquiries uncover an implicitly used theorem. When we state that we want to alleviate reasoning by borrowing ideas developed for computer verification, we are just saying that we want to automate Lamport's imaginary child labor. We make an explicit point of saying that, to borrow ideas developed for computer verification is much more about employing useful programming constructs in mathematical writing than actually using proof-checking and proof-assistant technology. A similar point of view is also present in the 1979 ACM Turing award lecture by Kenneth E. Iverson [64], entitled "notation as a tool of thought". Iverson, through his language APL, was highly influential in the development of further programming languages, and [64] may be an early proponent of language-aided reasoning. Note, in particular, that although his talk was tied to a computer science award, its title remarks the role of notation in thinking. In any case, even though we are mostly concerned about mathematical writing, the soundness of our approach stems from the intimate link between logic and computation. Philip Wadler [117] explains a deep result certifying this link: the Curry-Howard isomorphism, that casts propositions as types, and proofs as programs. This isomorphism provides the foundations for modern proof-checking and proof-assistant technology. Granted, these works do not serve as bibliographic evidence for our approach. They do, however, set the tone for what is meant by "language" and emphasize the role of computation and programming in our style. Above all, citing [64, 69, $117]$ is an honest acknowledgment of some influences of this work.

In short, to equip ourselves against the formidable task of research, we strive for developing a language which, beyond capturing our results, excels in what humans do not - namely, rigor — and thus emancipates our reasoning. 
Equations (1.1) and (1.4) exemplify the idea that there is a built-in notion of duality in graph homomorphism optimization problems. Chapter 3 has the language developed to capture this intuition. We define a notion of generalized clique and coloring numbers in terms of order duality, and then survey the literature for graph parameters that fit in these definitions - just as we have done with (1.2) and (1.3). The precise definitions in Chapter 3 demarcate the subject matter of the text, providing objective criteria for deciding what will be studied and what will not. Interestingly, most of the graph parameters presented fit in a single context captured by Figure 3.1, the generalization of (1.6).

Despite the structural importance of demarcation in Chapter 3, Chapter 4 presents the most compelling payoff of demarcation as a byproduct of language development. Chapter 4 delves into convex analysis to define a geometric bound-conversion procedure. In particular, it naturally associates upper bounds for the stability number $\alpha$ of a graph to lower bounds for the fractional chromatic number $\chi_{f}$ of a graph - which itself is a lower bound for the chromatic number $\chi$ by (1.6). The elucidative intuition captured is that a technique used for upper bounding the stability number may be adapted for lower bounding the chromatic number. Although this intuition is a tacit guideline followed by many researchers, it is in the language itself that we find the benefits of demarcation. Picture a graph theorist who, after developing a new technique, only obtained a result for the stability number. Is the absent dual result due to a lack of ingenuity, or is our theorist facing an impossible task? To answer this question is to demarcate the reach of the geometric bound conversion.

To reify something is to make it more concrete. Language aids reasoning when it reifies abstractions. This is true for languages in general, not just in mathematics or programming; the very act of writing reifies ideas and arguments, allowing for revisions and debates. The most direct form of reification is to introduce words to convey an idea. Obviously, it is much harder to ponder about concepts you lack vocabulary to describe. The reified intuitions of Chapters 3 and 4 allow Chapter 5 to address questions which would otherwise be even difficult to formulate. Section 5.2 presents a framework by de Carli Silva and Tunçel [28] that defines convex corners and their antiblockers using matrix cones. In doing so, the authors provide a language that captures how conic programming duality is related to the geometric bound-conversion procedure from Chapter 4 . If the appearance of matrix cones seems surprising, note that even our constrained discussion mentioned the positive semidefinite cone right after (1.12). Section 5.4 presents a framework by Roberson [100] that defines relaxations of graph homomorphisms using matrix cones. In doing so, the author provides a language that geometrically represents graph homomorphisms. In this manner, Sections 5.2 and 5.4 exhibit the vocabulary to tackle the pivotal question of this work: how do the tools from Chapters 4 and 5 apply to the objects of Chapter 3. Section 5.3 hints at a certain robustness of this connection, as it uses convex corners to express new results on the fractional cut-covering number, a graph parameter which in Chapter 3 stood apart from the others. Further exhibiting the value of vocabulary, Section 4.3 naturally exploits antiblocking duality to present new results regarding the well-known Hoffman bounds. Once again, note the value of the language itself: homomorphisms, convex corners, and antiblockers form the vernacular that grounds the reasoning.

We now acknowledge any reader that questions the necessity of referencing programming language design to justify the advantages of carefully crafted definitions or the somewhat straightforward organization of this text. Our point is that the overall structure of this dissertation is grounded on these ideas, even though it is in the routine parts of this work that the influence of proofs as computations is most present. The reader who ventures through these pages will quickly grow accustomed to simple statements being given prominence and, most importantly, a number. This small-scale reification, which we have done already several times, is put to good use in one of the distinctive features of this text: proofs which list a sequence of expressions, annotated with the supporting justification for each step of the derivation. Note an example in this very introduction, right after (1.7): each statement by the mathematician is followed by the answer provided to Lamport's child. We hope that the reader, when going through the pages of a chapter, will get acquainted with the manipulations used, and possibly only reach for the justifying annotation at momentarily obscure steps. We hope that even expressions which are not justified by an annotation still serve to communicate, expressing the triviality of a given step. As computers cannot handle ambiguity nor implicit assumptions, it is reasonable to expect proofs made via computations to mitigate such issues. Surely, there are places in these pages which may leave the reader completely puzzled, just as in any other mathematical work. What may be uncommon is the way in which our style reflects an attempt to lessen these issues. 


\section{Chapter 2}

\section{Preliminaries and Notation}

\subsection{Set Theory}

We adopt the von Neumann-Bernays-Gödel (NBG) set theory. However, any reader familiar with ZermeloFraenkel set theory with Choice (ZFC) will have no trouble reading this text. It is quite reasonable to understand NBG as ZFC with the additional notion of a class: Lévy [76] even says that set theory with classes and set theory with sets "are, essentially, different formulations of the same underlying theory". Although we expect the reader to be familiar with ZFC, we now provide a brief discussion on the core properties of classes which are used in this work. We refer the reader to appropriate sources [76, 111, 114] for precise axiomatizations.

The defining feature of classes is the axiom of predicative comprehension: we may form the class of all sets satisfying a given predicate. For example, if for every set $V$ we denote by $\left(\begin{array}{c}V \\ 2\end{array}\right)$ the set of subsets of $V$ with 2 elements, we may define the class of all simple graphs by

$$
\left.\mathcal{O}(\text { Graph }):=\langle g| \exists v, \exists e, g=(v, e) \text { and } e \subseteq\left(\begin{array}{c}
v \\
2
\end{array}\right)\right\rangle .
$$

Lévy [76] motivates this axiom by saying that classes capture the "extension of pure conditions". Attempting to express the same idea in a way more palatable for computer scientists, I say that classes are syntactic sugar for predicates on sets. Intuitively, the expression $G \in \mathcal{O}(\mathrm{Graph})$ unfolds into the predicate "there exist sets $V$ and $E$ such that $E \subseteq\left(\begin{array}{c}V \\ 2\end{array}\right)$ and $G=(V, E)$ ". As Lévy shows, this intuitive notion makes it clear which operations are possible on classes, and which are not. For example, if $A$ and $B$ are classes, we may define their union by

$$
A \cup B:=\langle x| x \in A \text { or } x \in B\rangle .
$$

Intuitively, since the expressions ' $x \in A$ ' and ' $x \in B$ ' unfold into predicates, we may also unfold ' $x \in A \cup B$ ' into a predicate, so we have a class. When using predicative comprehension, we reserve lower case letter for sets and upper case letters for classes, as the reader can see in (2.1) and (2.2).

The definition of subclasses fits nicely into the predicate analogy: a class $A$ is a subclass of a class $B$ if $x \in A$ implies that $x \in B$; we denote this fact by $A \subseteq B$. The axiom of extensionality for classes state that two classes $A$ and $B$ are equal, which we denote by $A=B$, if $A \subseteq B$ and $B \subseteq A$. There are classes which have the same elements as some set. Let $\mathbb{N}, \mathbb{Z}, \mathbb{Q}, \mathbb{R}$, and $\mathbb{C}$ denote the sets of natural, integers, rationals, reals, and complex numbers, respectively. It is immediate that the set $\mathbb{N}$ has the same elements as the class

$$
\langle x \mid x \in \mathbb{N}\rangle .
$$

In general, every set can be "promoted" into a class in this manner. We implicitly promote sets into classes, so that we can define concepts for classes and obtain the corresponding definition for sets. Moreover, we identify a class and a set which have the same elements. In this manner, we may actually write

$$
\mathbb{N}=\langle x \mid x \in \mathbb{N}\rangle
$$

and use $\langle x \mid x \in \mathbb{N}\rangle$ in every expression that expects a set. The usual paradoxes that motivated the axiomatization of set theory imply that there are classes which are not sets [114, Chapter 2, Theorem 1.1]. We refer to such classes as proper classes. 
The reader may easily see that we can form the Cartesian product $A \times B$ of classes $A$ and $B$. It is natural then to define define relations on classes, and functions on classes. For example, the identity function is defined by

$$
\text { id }:=\langle p \mid \exists x, p=(x, x)\rangle .
$$

As its name implies, the identity function is a function, and in usual notation we write $\operatorname{id}(x)=x$ for every set $x$. If $S$ and $T$ are classes, we write $f: S \rightarrow T$ to say that $f$ is a function with domain $S$ taking values in $T$. If $S$ and $T$ are classes, and $U \subseteq S$, we denote by $F \uparrow_{U}: U \rightarrow S$ the restriction of a function $F: S \rightarrow T$ to the subclass $U$ :

$$
\left.F \uparrow_{U}:=\langle p| \exists x, \exists y, p=(x, y), p \in F, \text { and } x \in U\right\rangle .
$$

We usually work with restrictions of 'id', which receive special notation: for every class $C$, define $\mathrm{id}_{C}:=\mathrm{id} \uparrow_{C}$. Whenever context allows, we may omit the restriction annotation. We remark that the function 'id' is a proper class. It is extremely convenient to define class functions on every set using the $\mapsto$ notation: for example, we have that id $=(x \mapsto x)$. The expression involving ' $\mapsto$ ' should be understood as a shorthand for the axiom of predicative comprehension. As long as what comes to the right of ' $\mapsto$ ' is a set, we have a well-defined class function. We may also restrict the domain of a function defined in this way: for example, for every class $U$, we have that $\operatorname{id}_{U}=(x \in U \mapsto x)$.

For every set $S$, we denote by $\mathcal{P}(S)$ the power set of $S$, that is, the set of all subsets of $S$. Note that we have not defined the class of all subclasses of a class, as this is not possible under NBG. For every finite set $V$, we denote by $|V| \in \mathbb{N}$ its cardinality. For every $n \in \mathbb{N}$, define $[n):=\{0, \ldots, n-1\}$ and $[n]:=\{1, \ldots, n\}$. We shall make use of the Minkowski notation: if $S$ and $T$ are classes and $f: S \rightarrow T$ is a function, we write

$$
f(S):=\langle f(s) \mid s \in S\rangle \subseteq T .
$$

We shall also adopt the Iverson notation: for every predicate $P$, define

$$
[P]:= \begin{cases}1 & \text { if } P \text { holds }, \\ 0 & \text { otherwise }\end{cases}
$$

We adopt the convention that the Iverson notation is strongly zero: if it is part of an expression in which $P$ does not hold, the whole expression is equal to zero, even if parts of it are nonsensical. For example, the expression

$$
[x \neq 0] \frac{1}{x}
$$

is defined for every $x \in \mathbb{R}$.

Let $k \in \mathbb{N}$. Define $\operatorname{Tag}_{k}:=(V \mapsto V \times\{k\})$. The class function $\operatorname{Tag}_{k}$ is such that

$$
\operatorname{Tag}_{k}(V)=V \times\{k\}=\{(v, k) \mid v \in V\}
$$

for every set $V$. Similarly, define $\operatorname{tag}_{k}:=(v \mapsto(v, k))$. For every set $V$, we have that $\operatorname{tag}_{k}: V \rightarrow \operatorname{Tag}_{k}(V)$ is such that $\operatorname{tag}_{k}(v):=(v, k)$ for every $v \in V$. As the notation suggests, the purpose of these definitions is to tag elements with labels, so we can differentiate them.

Let $n \in \mathbb{N}$. For any finite sequence $\left(S_{i}\right)_{i \in[n)}$ of sets, define their disjoint union or coproduct by

$$
S_{0} \sqcup \ldots \sqcup S_{n-1}:=\bigcup_{i \in[n)} \operatorname{Tag}_{i}\left(S_{i}\right) .
$$

For every set $U$ and for every finite sequence of functions $\left(f_{i}\right)_{i \in[n)}$ such that $f_{i}: S_{i} \rightarrow U$ for every $i \in[n)$, define their coproduct

$$
\left(f_{1} \sqcup \ldots \sqcup f_{n-1}\right): S_{0} \sqcup \ldots \sqcup S_{n-1} \rightarrow U
$$

by

$$
\left(f_{0} \sqcup \ldots \sqcup f_{n-1}\right)(x, i):=f_{i}(x) .
$$

Let $V$ be a finite set, and let $i, j \in V$. We shall denote by $i j$ both the ordered pair $(i, j) \in V \times V$ and the set $\{i, j\} \subseteq V \times V$. Usually $i j$ appears in an expression in which only one of $(i, j)$ or $\{i, j\}$ make sense, so confusion does not arise. 


\subsection{Linear Algebra}

Our definitions of vectors and matrices exploit the set-theoretic foundations. The most used kind of vector space throughout this work is $\mathbb{R}^{V}$, with $V$ a finite set. The standard basis of $\mathbb{R}^{V}$ is denoted by $\left\{e_{i} \mid i \in V\right\}$. There are two related functions we shall use a lot in the context of vector and matrix manipulations. Let $S$ be a finite set. Define

$$
\begin{aligned}
\mathbb{1} & :=(x \mapsto 1) \text { and } \\
\mathbb{1}_{S} & :=(x \mapsto[x \in S]) .
\end{aligned}
$$

A restriction of $\mathbb{1}$ to some finite set is the all-ones vector, and a restriction of $\mathbb{1}_{S}$ to some finite set containing $S$ is the incidence vector of $S$. As both functions are only used in vector and matrix manipulations, the appropriate restriction is usually evident. When we need to be explicit about the domain $V$, we may write $\mathbb{1} \in \mathbb{R}^{V}$ or $\mathbb{1}_{S} \in \mathbb{R}^{V}$. For every finite set $V$, define the support of a vector $x \in \mathbb{R}^{V}$ by $\operatorname{supp}(x):=\left\{i \in V \mid x_{i} \neq 0\right\}$.

A matrix is a function whose domain is the Cartesian product of two finite sets. Let $S$ and $T$ be finite sets. This work focuses on real matrices, i.e., elements of $\mathbb{R}^{S \times T}$, although a few places require $\mathbb{Q}^{S \times T}$ or $\mathbb{C}^{S \times T}$. We refer to $S$ as the row set and to $T$ as the column set. Following tradition, we denote by $A_{i j} \in \mathbb{R}$ the application of the function $A \in \mathbb{R}^{S \times T}$ to the pair $(i, j) \in S \times T$. If $A \in \mathbb{R}^{S \times T}$, we denote by $A^{\top} \in \mathbb{R}^{T \times S}$ the function defined by $\left(A^{\top}\right)_{j i}:=A_{i j}$ for every $i \in S$ and $j \in T$. Whenever the matrix is taking values in a commutative ring, we define the product of matrices in the usual way, with the convention that our vectors are column vectors. In particular, if $x \in \mathbb{R}^{S}$ and $y \in \mathbb{R}^{T}$, then

$$
x y^{\top} \in \mathbb{R}^{S \times T} .
$$

Subsection 5.4.1 provides a more precise explanation of what is meant by column vector, as this discussion becomes relevant in Section 5.4. The rank of a matrix $A \in \mathbb{R}^{S \times T}$ is denoted by $\operatorname{rank}(A)$. We denote by $I \in \mathbb{R}^{S \times S}$ the identity matrix.

Let $R, S, T$, and $U$ be finite sets, let $A \in \mathbb{R}^{R \times S}$ and let $B \in \mathbb{R}^{T \times U}$. The Kronecker product is the matrix $A \otimes B \in \mathbb{R}^{(R \times T) \times(S \times U)}$ defined by

$$
(A \otimes B)_{r t, s u}:=A_{r s} B_{t u}
$$

for every $r \in R, s \in S, t \in T$, and $u \in U$. Subsection 5.4.1 is dedicated to the Kronecker product and proves the results we are about to state; see also [61, Chapter 4]. The Mixed Product Property of the Kronecker product states that whenever $A, B, C$, and $D$ are matrices such that $A C$ and $B D$ are defined, it holds that

$$
(A \otimes B)(C \otimes D)=A C \otimes B D .
$$

One may easily prove from (2.6) that

$$
\begin{aligned}
& A \otimes(X+\alpha Y)=A \otimes X+\alpha A \otimes Y \text { and } \\
& (A+\alpha B) \otimes X=A \otimes X+\alpha B \otimes X,
\end{aligned}
$$

and that

$$
(A \otimes X)^{\top}=A^{\top} \otimes X^{\top} .
$$

Finally, the Kronecker product extends naturally to vectors, and it holds that

$$
e_{s} \otimes e_{t}=e_{(s, t)} \in \mathbb{R}^{S \times T}
$$

for every $s \in S$ and $t \in T$.

Our definition of direct sum of vectors is made entirely in terms of the coproduct of functions. In short, we simply define $\oplus:=\sqcup$. In this manner, if $S$ and $T$ are finite sets, we have that

$$
x \oplus y \in \mathbb{R}^{S \sqcup T}
$$

for every $x \in \mathbb{R}^{S}$ and $y \in \mathbb{R}^{T}$. We also automatically have a definition of the direct sum of three or more vectors. 
Let $S$ and $T$ be finite sets, and let $f: S \rightarrow T$. Define the function matrix $P_{f} \in \mathbb{R}^{T \times S}$ by

$$
P_{f}:=\sum_{s \in S} e_{f(s)} e_{s}^{\top}
$$

It is immediate that

$$
P_{f} e_{i}=e_{f(i)}
$$

for every $i \in S$. It is not hard to see that for every $Q \subseteq T$,

$$
P_{f}^{\top} \mathbb{1}_{Q}=\mathbb{1}_{f^{-1}(Q)},
$$

where $f^{-1}(Q)$ denotes the preimage of $Q$ under $f$. A direct consequence of (2.13) is that if $f^{-1}: T \rightarrow S$ is the inverse of $f$, then

$$
P_{f^{-1}}=P_{f}^{\top} .
$$

Another useful consequence of (2.13) is that

$$
P_{f}^{\top} \mathbb{1}=\mathbb{1}
$$

Proposition 2.1. Let $R, S$, and $T$ be finite sets, and let $g: R \rightarrow S$ and $f: S \rightarrow T$. Then

$$
P_{f} P_{g}=P_{f \circ g}
$$

Proof. Both $P_{f} P_{g}$ and $P_{f \circ g}$ are matrices in $\mathbb{R}^{T \times R}$. Since

$$
P_{f} P_{g} e_{i}=P_{f} e_{g(i)}=e_{f(g(i))}=P_{f \circ g} e_{i}
$$

for every $i \in R$, the statement follows.

Let $\mathbb{E}$ be a real vector space. A function $\langle\cdot, \cdot\rangle: \mathbb{E} \times \mathbb{E} \rightarrow \mathbb{R}$ is an inner product if

1. $\langle x, x\rangle \geq 0$ for every $x \in \mathbb{E}$, with equality if and only if $x=0$;

2. $\langle y, x\rangle=\langle x, y\rangle$ for every $x, y \in \mathbb{E}$;

3. for every $x \in \mathbb{E}$, the function $y \mapsto\langle y, x\rangle$ is linear, and for every $y \in \mathbb{E}$ the function $x \mapsto\langle y, x\rangle$ is linear.

An Euclidean space is a pair $(\mathbb{E},\langle\cdot, \cdot\rangle)$ where $\langle\cdot, \cdot\rangle$ is an inner product on $\mathbb{E}$. When the inner product is clear, we may simply say that $\mathbb{E}$ is an Euclidean space. Note that, in this text, an inner product is always defined on a real vector space; in the occasion we work with $\mathbb{C}$, we introduce specific notation. An inner product induces a metric in an Euclidean space; define the norm $\|x\|:=\langle x, x\rangle^{1 / 2}$ for every $x \in \mathbb{E}$. Subsection 4.1.1 has more results on norms, as they play an important role in Chapters 4 and 5 . For every linear transformation $\mathcal{A}: \mathbb{E}_{0} \rightarrow \mathbb{E}_{1}$ between the Euclidean spaces $\mathbb{E}_{0}$ and $\mathbb{E}_{1}$, we denote by $\mathcal{A}^{*}: \mathbb{E}_{1} \rightarrow \mathbb{E}_{0}$ its adjoint, which is the unique linear transformation such that $\langle y, \mathcal{A}(x)\rangle=\left\langle\mathcal{A}^{*}(y), x\right\rangle$ for every $x \in \mathbb{E}_{0}$ and $y \in \mathbb{E}_{1}$.

Let $V$ be a finite set. For every $A \in \mathbb{R}^{V \times V}$, define the trace of $A$ by

$$
\operatorname{Tr}(A):=\sum_{i \in V} A_{i i}
$$

It is immediate that

$$
\operatorname{Tr}(A)=\operatorname{Tr}\left(A^{\top}\right) .
$$

Let $S$ and $T$ be finite sets. For every matrices $A \in \mathbb{R}^{S \times T}$ and $B \in \mathbb{R}^{T \times S}$, it holds that

$$
\operatorname{Tr}(A B)=\operatorname{Tr}(B A) .
$$

One may easily check that the function $\langle\cdot, \cdot\rangle: \mathbb{R}^{S \times T} \times \mathbb{R}^{S \times T} \rightarrow \mathbb{R}$ defined for every $X, Y \in \mathbb{R}^{S \times T}$ by

$$
\langle X, Y\rangle:=\operatorname{Tr}\left(X^{\top} Y\right)
$$

defines an inner product on $\mathbb{R}^{S \times T}$. This inner product is known as the Frobenius inner product or the trace inner product. We shall adopt the latter in this text. 
Let $V$ be a finite set. Define Diag: $\mathbb{R}^{V} \rightarrow \mathbb{R}^{V \times V}$ by

$$
\operatorname{Diag}(x):=\sum_{i \in V} x_{i} e_{i} e_{i}^{\top}
$$

for every $x \in \mathbb{R}^{V}$. Define diag: $\mathbb{R}^{V \times V} \rightarrow \mathbb{R}^{V}$ by

$$
\operatorname{diag}(X)_{i}:=X_{i i}
$$

for every $i \in V$ and for every $X \in \mathbb{R}^{V \times V}$. It is easy to see that

$$
\operatorname{Diag}^{*}=\operatorname{diag}
$$

and that

$$
\operatorname{Diag}(x) e_{i}=x_{i} e_{i}
$$

for every $i \in V$.

Let $V$ be a finite set. We denote by $\mathbb{S}^{V}:=\left\{A \in \mathbb{R}^{V \times V} \mid A^{\top}=A\right\}$ the set of symmetric matrices. We use $\lambda_{\max }(A)$ and $\lambda_{\min }(A)$ to denote the largest and smallest eigenvalue of $A \in \mathbb{S}^{V}$, respectively. Define the set of positive semidefinite matrices by $\mathbb{S}_{+}^{V}:=\left\{A \in \mathbb{S}^{V} \mid \lambda_{\min }(A) \geq 0\right\}$ and the set of positive definite matrices by $\mathbb{S}_{++}^{V}:=\left\{A \in \mathbb{S}^{V} \mid \lambda_{\min }(A)>0\right\}$. We adopt a similar convention regarding real numbers. Define $\mathbb{R}_{+}:=\{x \in \mathbb{R} \mid x \geq 0\}$ and $\mathbb{R}_{++}:=\{x \in \mathbb{R} \mid x>0\}$. A vector in $\mathbb{R}_{+}^{V}:=\left(\mathbb{R}_{+}\right)^{V}$ is nonnegative, and a vector in $\mathbb{R}_{++}^{V}:=\left(\mathbb{R}_{++}\right)^{V}$ is positive.

\subsection{Basic Results on Graphs and Graph Homomorphisms}

Throughout this text, a graph is a pair $(V, E)$, where $V$ is a set and $E \subseteq\left(\begin{array}{l}V \\ 2\end{array}\right)$. In other words, a graph is an element of the class (2.1). Arguably, we are studying symmetric binary relations over sets, as our graphs are always simple and without loops, and we have not imposed any restriction on the size of $V$. Indeed, infinite graphs play an important role in Chapter 3. If $G:=(V, E)$ is a graph, we denote by $V(G):=V$ its vertex set and by $E(G):=E$ its edge set. Two vertices $i, j \in V$ are adjacent if $i j=\{i, j\} \in E$. For every $S \subseteq V(G)$, the induced subgraph $G[S]$ is the graph with vertex set $S$ and vertices adjacent if they are adjacent in $G$. A set $S \subseteq V$ is stable if $E(G[S])=\varnothing$, i.e., if there are no edges between two vertices of $S$. We denote by $\mathcal{S}(G)$ the set of stable sets of $G$. We denote by $\bar{G}$ the complement of $G$, which is the graph with vertex set $V(\bar{G}):=V(G)$ and edge set $E(\bar{G}):=\left(\begin{array}{c}V \\ 2\end{array}\right) \backslash E(G)$.

For every set $S$, define the complete graph on $S$ to be the graph $K_{S}$ such that

$$
\begin{aligned}
& V\left(K_{S}\right):=S \text { and } \\
& E\left(K_{S}\right):=\left(\begin{array}{l}
S \\
2
\end{array}\right) .
\end{aligned}
$$

We usually work with $K_{n}:=K_{[n)}$, defined for every $n \in \mathbb{N}$. Similarly, for every $n \in \mathbb{N}$ with $n>0$, define the cycle graph on $n$ vertices to be the graph $C_{n}$ such that

$$
\begin{aligned}
& V\left(C_{n}\right):=[n) \text { and } \\
& E\left(C_{n}\right):=\{\{i, i+1\} \mid i \in[n-1)\} \cup\{\{0, n-1\}\} .
\end{aligned}
$$

Definition 2.2. Let $G$ and $H$ be graphs. A (graph) homomorphism from $G$ to $H$ is a function $f: V(G) \rightarrow$ $V(H)$ such that $f(i) f(j) \in E(H)$ whenever $i j \in E(G)$. The set of homomorphisms from $G$ to $H$ is denoted by $\operatorname{Hom}(G, H)$.

Proposition 2.3. Let $F, G$, and $H$ be graphs. If $f \in \operatorname{Hom}(F, G)$ and $g \in \operatorname{Hom}(G, H)$, then $g \circ f \in \operatorname{Hom}(F, H)$.

Proof. Let $i j \in E(F)$. Since $f \in \operatorname{Hom}(F, G)$, we have that $f(i) f(j) \in E(G)$. Since $g \in \operatorname{Hom}(G, H)$, we have that

$$
(g \circ f)(i)(g \circ f)(j)=g(f(i)) g(f(j)) \in E(H) .
$$


Let $G$ and $H$ be graphs.

$$
\text { If } \operatorname{Hom}(G, H) \text { is nonempty, we write } G \rightarrow H \text {. }
$$

The relation ' $\rightarrow$ ' may seem odd to a reader who has worked with group homomorphisms, as the homomorphic relation defined by group homomorphisms is trivial. More precisely, the function which maps every element of a group to the identity of another group is always a group homomorphism, regardless of the groups involved. Hence, the relation analogous to (2.26) for groups holds between any pair of groups. On the other hand, as (1.1) and (1.4) show, there is nothing trivial about the homomorphic relation on graphs. If $G$ and $H$ are graphs such that $G \rightarrow H$ and $H \rightarrow G$, we say that $G$ and $H$ are homomorphically equivalent, which is denoted by $G \leftrightarrow H$. For every graph $G$ and for every $S \subseteq V(G)$, one may easily check that

$$
\operatorname{id}_{S} \in \operatorname{Hom}(G[S], G) .
$$

In particular, we have that $\operatorname{id}_{G}:=\operatorname{id}_{V(G)} \in \operatorname{Hom}(G, G)$ for every graph $G$. A homomorphism $f \in \operatorname{Hom}(G, H)$ is a (graph) isomorphism if there exists $g \in \operatorname{Hom}(H, G)$ such that

$$
f \circ g=\operatorname{id}_{H} \text { and } g \circ f=\operatorname{id}_{G} .
$$

If there exists an isomorphism between $G$ and $H$, we say that $G$ and $H$ are isomorphic. If $G$ is a graph, an automorphism of $G$ is an isomorphism in $\operatorname{Hom}(G, G)$. The set of automorphisms of $G$ is a group under composition, and it is denoted by $\operatorname{Aut}(G)$.

By definition, isomorphic graphs are homomorphically equivalent. We now further explain the difference between both relations with a couple of examples.

Example 2.4. The graphs $C_{4}$ and $K_{2}$ are homomorphically equivalent.

Proof. Note that $K_{2}=K_{[2)}=C_{4}[[2)]$, so (2.27) implies that $C_{2} \rightarrow K_{4}$. Define $f:[4) \rightarrow[2)$ by

$$
f(x):=[x \text { is odd }] .
$$

for every $x \in[4)$. Recalling (2.25), if $i j \in E\left(C_{4}\right)$, then precisely one element of $\{i, j\}$ is even, so $f \in$ $\operatorname{Hom}\left(C_{4}, K_{2}\right)$.

Example 2.5. The graphs $\overline{K_{5}}$ and $K_{1}$ are homomorphically equivalent.

Proof. Note that $K_{1}=K_{[1)}=\overline{K_{5}}[[1)]$, so $(2.27)$ implies that $K_{1} \rightarrow \overline{K_{5}}$. Furthermore, as $E\left(\overline{K_{5}}\right)=\varnothing$, it follows that $(x \in[5) \mapsto 0) \in \operatorname{Hom}\left(\overline{K_{5}}, K_{1}\right)$.

Examples 2.4 and 2.5 highlight the fact that homomorphisms can be used to "simplify" a graph. A core of a graph $G$ is a graph that is homomorphically equivalent to $G$ and whose vertex set has minimal cardinality. It is actually the case that $C_{2}$ is a core of $K_{4}$ and that $K_{1}$ is a core of $\overline{K_{5}}$. For more on core of graphs, see [52, Section 2.4] or [44, Section 6.2]. A graph parameter is a function on graphs which is constant on isomorphism classes. One may check that

$$
G \mapsto|V(G)| \text { and } G \mapsto|E(G)|
$$

are graph parameters by using that isomorphisms are bijective functions. On the other hand, as Examples 2.4 and 2.5 show, homomorphically equivalent graphs may not have vertex and edge sets of the same cardinality. As a more interesting example, define the stability number of a graph $G$ by

$$
\alpha(G):=\max \{|S| \mid S \in \mathcal{S}(G)\} .
$$

Since $E\left(\overline{K_{5}}\right)=E\left(K_{1}\right)=\varnothing$, it is easy to see that $\alpha\left(\overline{K_{5}}\right)=5$ and $\alpha\left(K_{1}\right)=1$, so Example 2.5 proves that the stability number of homomorphically equivalent graphs is not necessarily the same. The following useful proposition implies that $\alpha$ is a graph parameter.

Proposition 2.6. Let $G$ and $H$ be graphs. A function $f: V(G) \rightarrow V(H)$ is a homomorphism if and only if

$$
f^{-1}(\mathcal{S}(H)) \subseteq \mathcal{S}(G) .
$$


Proof. Let $S \in \mathcal{S}(H)$ be such that $f^{-1}(S) \notin \mathcal{S}(G)$. Then there exist two vertices $i, j \in f^{-1}(S)$ such that $i j \in E(G)$. Thus $f(i) f(j) \notin E(H)$, as $\{f(i), f(j)\} \subseteq S \in \mathcal{S}(H)$. Hence $f$ is not a homomorphism. For the converse result, assume that $f: V(G) \rightarrow V(H)$ is a function such that the preimage of every stable set is stable, and let $i, j \in V(G)$ be such that $i j \in E(G)$. Then $\{f(i), f(j)\}$ cannot be stable, as its preimage $\{i, j\}$ is not stable. This can only happen if $f(i) f(j) \in E(H)$, so we conclude that $f \in \operatorname{Hom}(G, H)$.

Corollary 2.7. Let $G$ and $H$ be isomorphic graphs. Then $\alpha(G)=\alpha(H)$.

Proof. Let $f \in \operatorname{Hom}(G, H)$ and $g \in \operatorname{Hom}(H, G)$ satisfy (2.28). Then

$$
\begin{aligned}
\mathcal{S}(G) & =(g \circ f)(\mathcal{S}(G)) \\
& =f^{-1}\left(g^{-1}(\mathcal{S}(G))\right) \\
& \subseteq f^{-1}(\mathcal{S}(H)) \\
& \subseteq \mathcal{S}(G) .
\end{aligned}
$$$$
\text { since } f=g^{-1} \text { and } g=f^{-1}
$$

by Proposition 2.6

by Proposition 2.6

Hence $\mathcal{S}(G)=f^{-1}(\mathcal{S}(H))=g(\mathcal{S}(H))$. Since (2.28) also implies that $g$ is bijective, we have that $|g(S)|=|S|$ for every $S \subseteq V(G)$. The statement follows:

$$
\begin{aligned}
\alpha(G) & =\max \{|S| \mid S \in \mathcal{S}(G)\} \\
& =\max \{|g(T)| \mid T \in \mathcal{S}(H)\} \\
& =\max \{|T| \mid T \in \mathcal{S}(H)\} \\
& =\alpha(H) .
\end{aligned}
$$

since $\mathcal{S}(G)=g(\mathcal{S}(H))$

since $g$ is bijective

The adjacency matrix $A_{G} \in \mathbb{S}^{V(G)}$ of a graph $G$, is defined by

$$
A_{G}:=\sum_{i j \in E(G)}\left(e_{i} e_{j}^{\top}+e_{j} e_{i}^{\top}\right) .
$$

\subsection{Category Theory}

Category theory $[1,84,99]$ attempts to unify several results in mathematics by placing homomorphisms at the center of the stage. Riehl [99] tells that the field was born with the publication of [34], which offers a proper definition of the notion of natural transformation. Mac Lane himself, one of the authors of [34], states that the core idea of category theory is already present in our function notation $-f: X \rightarrow Y$, for sets $X$ and $Y$ - and credits Emmy Nöther for being the first one to focus on homomorphisms [84, Notes, Chapter 1]. Hahn and Tardif [52] call some of the early researchers working with graph homomorphisms the Prague School of Category Theory; by the title of the book with many of their results [97], the claim that they work with categories does not seem controversial. Moggi [88] related the categorical idea of monads with the semantics or programming languages; the Haskell programming language then employed monads to provide pure input/output operations [62].

Category theory studies "objects" and "homomorphisms" between them. For example, we may say that a function $f: S \rightarrow T$ between the sets $S$ and $T$ is a (set) isomorphism if there exists $g: T \rightarrow S$ such that

$$
f \circ g=\operatorname{id}_{S} \text { and } g \circ f=\operatorname{id}_{T} .
$$

Set isomorphisms are usually called bijections. It is clear that both (2.28) and (2.30) express the same idea. To obtain a unified definition, one must abstract one's "objects" - graphs in (2.28) and sets in (2.30) — and one's "homomorphisms" - graph homomorphisms in (2.28) and functions in (2.30). We now present this common framework.

Let $\mathcal{O}$ and $\mathcal{H}$ be classes, and let desc: $\mathcal{H} \rightarrow \mathcal{O} \times \mathcal{O}$ and id: $\mathcal{O} \rightarrow \mathcal{H}$ be functions. Define

$$
\mathcal{C}:=\langle(f, g) \in \mathcal{H} \times \mathcal{H}| \exists a \in \mathcal{O}, \exists b \in \mathcal{O}, \exists c \in \mathcal{O},(c, b)=\operatorname{desc}(f) \text { and }(b, a)=\operatorname{desc}(g)\rangle .
$$

Let $\circ: \mathcal{C} \rightarrow \mathcal{H}$ be a function. The classes $\mathcal{O}$ and $\mathcal{H}$ and the functions desc, id, and $\circ$ define a category when they satisfy the following: 
1. for every $(f, g) \in \mathcal{C}$ it holds that $\operatorname{desc}(f \circ g)=(c, a)$, where $(c, b):=\operatorname{desc}(f)$ and $(b, a):=\operatorname{desc}(g)$;

2. for every $a \in \mathcal{O}$ it holds that $\operatorname{desc}(\operatorname{id}(a))=(a, a)$;

3. for every $a \in \mathcal{O}$ and for every $f \in \mathcal{H}$ such that $\left(f, \operatorname{id}_{a}\right) \in \mathcal{C}$, it holds that $f \circ \operatorname{id}_{a}=f$;

4. for every $a \in \mathcal{O}$ and for every $f \in \mathcal{H}$ such that $\left(\operatorname{id}_{a}, f\right) \in \mathcal{C}$, it holds that $\operatorname{id}_{a} \circ f=f$.

5. for every $f, g, h \in \mathcal{H}$ such that $(f, g) \in \mathcal{C}$ and $(g, h) \in \mathcal{C}$, it holds that $(f \circ g) \circ h=f \circ(g \circ h)$;

We refer to the elements of $\mathcal{O}$ as objects, and to the elements of $\mathcal{H}$ as homomorphisms. Some authors refer to the elements of $\mathcal{H}$ as arrows or morphisms. For every $f \in \mathcal{H}$, if $(b, a):=\operatorname{desc}(f)$ then $a$ is the domain of $f$, and $b$ is the codomain of $f$. We refer to $\operatorname{id}_{a}:=\operatorname{id}(a)$ as the identity homomorphism on $a$ for every $a \in \mathcal{O}$. The function $\circ$ is the composition of homomorphisms. Our definition of category is distinct from most of the definitions in this text, as the formal definition of a category lies outside of set theory: the core issue is that (under NBG) we cannot form an ordered tuple $(\mathcal{O}, \mathcal{H}$, desc, id, o) whenever these objects are proper classes. Mac Lane [84] and Shulman [111] write something similar to what we have written above as axioms, and say that a category is any model of those axioms within set theory.

We now present the category Set of sets. The objects of Set are defined by

$$
\mathcal{O}(\text { Set }):=\langle v \mid v=v\rangle,
$$

which represents the class of all sets. The homomorphisms of Set are the set functions, together with their domains and codomains:

$$
\left.\mathcal{H}(\text { Set }):=\langle(f, s, t)| \exists s, t \in \mathcal{O}(\text { Set }), f \in t^{s}\right\rangle .
$$

We define descset $:=((f, s, t) \in \mathcal{H}(\mathrm{Set}) \mapsto(t, s))$, and id $\mathrm{Set}_{\mathrm{Set}}:=\left(v \mapsto \mathrm{id}_{v}\right)$, where the 'id' in the right hand side the the identity function defined in Section 2.1. Composition is given by usual function composition. Similar to how we defined Hom in Definition 2.2, we may define for every sets $s$ and $t$,

$$
\left.\operatorname{Hom}_{\text {Set }}(s, t):=\langle f|(f, s, t) \in \mathcal{H}(\text { Set })\right\rangle=t^{s} .
$$

A couple of remarks are in place. Note that the class $\mathcal{H}($ Set $)$ carries the domain and codomain of a function. This is necessary to define descset, and highlights the fact that the codomain of a function is not defined in set theory. For example, the domain and image of the function $(x \in\{0\} \mapsto x)$ is $\{0\}$, and it is completely reasonable to write both

$$
(x \in\{0\} \mapsto x) \in \operatorname{Hom}_{\text {Set }}(\{0\}, \mathbb{N}) \text { and }(x \in\{0\} \mapsto x) \in \operatorname{Hom}_{\text {Set }}(\{0\}, \mathbb{Z}) .
$$

This is why we "pack" this extra information when defining $\mathcal{H}($ Set $)$ and "unpack" it when using Homset , as in the latter case the codomain is clear. Given the foundational role of set theory, this technical nuisance carries over to several other examples of categories.

The category Graph of graphs is the most important in this text. The class in (2.1) contains all of its objects - i.e., graphs — and its homomorphisms are graph homomorphisms:

$$
\mathcal{H}(\text { Graph }):=\langle(\phi, g, h)| \exists g, h \in \mathcal{O}(\text { Graph }), \phi \in \operatorname{Hom}(g, h)\rangle .
$$

Note that we are using the definition of Hom we presented in Section 2.3. Define descGraph $:=((\phi, g, h) \in$ $\mathcal{H}(\mathrm{Graph}) \mapsto(h, g))$ and $\operatorname{id}_{\mathrm{Graph}}:=\left((v, e) \mapsto \mathrm{id}_{v}\right)$, with composition given by usual function composition. We may define for every graphs $g$ and $h$,

$$
\operatorname{Hom}_{\text {Graph }}(g, h):=\langle f \mid(f, g, h) \in \mathcal{H}(\operatorname{Graph})\rangle=\operatorname{Hom}(g, h) .
$$

One may easily check (using Proposition 2.3) that Graph is indeed a category. Define the class of all finite sets by

$$
\mathcal{O}(\text { FinSet }):=\langle s|\exists n \in \mathbb{N},| s \mid=n\rangle .
$$

We denote by FinSet the category whose objects are finite sets, whose homomorphisms are functions, and composition is given by usual function composition. The category FinSet is a subcategory of the category Set: 
its objects are a subclass of $\mathcal{O}$ (Set), and FinSet inherits the categorical structure of Set. Yet another category is the category Matrix $\mathbb{R}_{\mathbb{R}}$ of real matrices. Define its objects by

$$
\mathcal{O}\left(\text { Matrix }_{\mathbb{R}}\right):=\mathcal{O}(\text { FinSet })
$$

and its homomorphisms by

$$
\left.\mathcal{H}\left(\text { Matrix }_{\mathbb{R}}\right):=\langle(a, s, t)| \exists s, t \in \mathcal{O}(\text { Matrix }), a \in \mathbb{R}^{t \times s}\right\rangle .
$$

Further define $\operatorname{desc}_{\text {Matrix }_{\mathbb{R}}}:=\left((a, s, t) \in \mathcal{H}\left(\right.\right.$ Matrix $\left.\left._{\mathbb{R}}\right) \mapsto(t, s)\right)$ and $\operatorname{id}_{\text {Matrix } \mathbb{R}}:=\left(s \mapsto I \in \mathbb{R}^{s \times s}\right)$, where the $I$ after ' $\mapsto$ ' denotes the identity matrix. Composition in Matrix $\mathbb{R}_{\mathbb{R}}$ is defined by matrix multiplication. For every finite sets $s$ and $t$, define

$$
\left.\operatorname{Hom}_{\text {Matrix }}(s, t):=\langle f|(f, s, t) \in \mathcal{H}\left(\text { Matrix }_{\mathbb{R}}\right)\right\rangle=\mathbb{R}^{t \times s} .
$$

Another simple example of a category will play an important role in our work. Let $S$ be a set. A preorder $R$ on $S$ is a relation $R \subseteq S \times S$ on $S$ which is

1. reflexive, i.e., for every $p \in S$ we have that $(p, p) \in R$, and

2. transitive, i.e., if $a, b, c \in S$ are such that $(a, b) \in R$ and $(b, c) \in R$, then $(a, c) \in R$.

The ordered pair $P:=(S, R)$ is a preordered set. It is traditional to present a relation in infix position, writing $a R b$ whenever $(a, b) \in R$. Section 3.1 studies preordered sets in depth; for now we define the category $\widehat{P}$ defined by $P$. Define $\mathcal{O}(\widehat{P}):=S$ and $\mathcal{H}(\widehat{P}):=R$. Set $\operatorname{desc}((p, q))=(q, p)$ for every $(p, q) \in R=\mathcal{H}(\widehat{P})$ and $\operatorname{id}(p):=(p, p)$ for every $p \in S$. Finally, define

$$
(a, b) \circ(b, c):=(a, c)
$$

for every $(a, b) \in R$ and $(b, c) \in R$. The close relationship between categories and preorders motivates our study of order theory in Chapter 3.

There is also a notion of "homomorphism between categories". Consider the functions

$$
\begin{aligned}
V_{\mathcal{O}} & :=(g \in \mathcal{O}(\text { Graph }) \mapsto V(g)) \text { and } \\
V_{\mathcal{H}} & :=\left((f, g, h) \in \mathcal{H}(\text { Graph }) \mapsto\left(f, V_{\mathcal{O}}(g), V_{\mathcal{O}}(h)\right)\right) .
\end{aligned}
$$

Note that $V_{\mathcal{O}}$ maps the objects of the category Graph to the objects of the category Set, whereas $V_{\mathcal{H}}$ maps the homomorphisms of Graph to the homomorphisms of Set. Let $F, G$, and $H$ be graphs. Then

$$
\text { for every } f \in \operatorname{Hom}(F, G) \text {, we have that } V_{\mathcal{H}}(f) \in \operatorname{Hom}_{\text {Set }}\left(V_{\mathcal{O}}(F), V_{\mathcal{O}}(G)\right) \text {. }
$$

Moreover, for every $f \in \operatorname{Hom}(F, G)$ and for every $g \in \operatorname{Hom}(G, H)$, we have that

$$
V_{\mathcal{H}}(g) \circ V_{\mathcal{H}}(f)=V_{\mathcal{H}}(g \circ f) .
$$

Finally, for every $G \in \mathcal{O}$ (Graph), we have that

$$
V_{\mathcal{H}}\left(\operatorname{id}_{\operatorname{Graph}}(G)\right)=\operatorname{id}_{\text {Set }}\left(V_{\mathcal{O}}(G)\right) .
$$

Two functions which satisfy (2.32), (2.33), and (2.34) for a given pair of categories are called a functor. We refer to $V_{\mathcal{O}}$ as the object function and to $V_{\mathcal{H}}$ the homomorphism function, and adopt similar nomenclature for any functor. As it is the case with categories, we cannot formalize the notion of a functor in set theory, even though it is easy to find models, i.e., examples. We write $V:$ Graph $\rightarrow$ Set to say that $V$ is a functor from the category Graph to the category Set, and simply use $V(G)$ and $V(f)$ to denote the image of the object function on $G \in \mathcal{O}$ (Graph) and the image of the homomorphism function on $f \in \mathcal{H}(\mathrm{Graph})$, respectively. We adopt a similar convention for other functors.

There are a couple of functors that appear throughout this work. The function matrices from Section 2.2 define a functor $P$ : FinSet $\rightarrow$ Matrix $_{\mathbb{R}}$, where the function between objects is $(v \in \mathcal{O}$ (FinSet) $\mapsto v)$ and the function between homomorphisms is $\left((f, s, t) \in \mathcal{H}(\right.$ FinSet $) \mapsto\left(P_{f}, s, t\right) \in \mathcal{H}\left(\right.$ Matrix $\left.\left._{\mathbb{R}}\right)\right)$. Proposition 2.1 precisely states that this functor satisfies (2.33). 
Definition 2.8. Let $P:=(S, R)$ be a preordered set, and let $\widehat{P}$ be the category defined by $P$. Assume $\mathrm{C}$ is a category whose class of objects is $\mathcal{O}(\mathrm{C})$. A function $\beta: \mathcal{O}(\mathrm{C}) \rightarrow S$ is homomorphism monotone when it is the object function of a functor $\mathrm{C} \rightarrow \widehat{S}$.

Let $P:=(S, \leq)$ be a preordered set, and let $\widehat{P}$ be the category defined by $P$. Since there is at most one homomorphism in $\widehat{P}$ between any two objects, it is easy to see that a homomorphism monotone function completely defines a functor to $\widehat{P}$. We are particularly interested in homomorphism monotone functions $\beta: \mathcal{O}$ (Graph) $\rightarrow S$, i.e., functions such that $G \rightarrow H$ implies $\beta(G) \leq \beta(H)$, for every graphs $G$ and $H$. Chapter 3 studies in great detail several examples of homomorphism monotone functions taking extended real values. By extended reals $[-\infty,+\infty]$ we mean the set of real numbers $\mathbb{R}$ equipped with two extra elements: $-\infty$ and $+\infty$. We equip $[-\infty,+\infty]$ with the usual arithmetic and order; in particular, it holds that $\sup \varnothing=-\infty$ and inf $\varnothing=+\infty$. Furthermore, the expressions $0 \cdot( \pm \infty),( \pm \infty) /( \pm \infty)$, and $\pm \infty+\mp \infty$ are undefined. Using that the preorder on $[-\infty,+\infty]$ is antisymmetric, i.e., such that $a \leq b$ and $b \leq a$ imply that $a=b$ for every $a, b \in[-\infty,+\infty]$, one can see that every homomorphism monotone function $\beta: \mathcal{O}($ Graph $) \rightarrow[-\infty,+\infty]$ is a graph parameter.

\subsection{Optimization Problems}

This section presents the basic vernacular of optimization adopted in this the text. We have two goals: to fix a common language and to show that some key categorical notions we have presented in Section 2.3 play an important role when working with optimization problems.

An optimization problem is a triplet $(t, X, f)$ where $X$ is a set, $f: X \rightarrow[-\infty,+\infty]$, and $t \in\{ \pm 1\}$. A minimization problem is an optimization problem of the form $(-1, X, f)$, and a maximization problem is an optimization problem of the form $(1, X, f)$. Let $(t, X, f)$ be an optimization problem. We refer to $X$ as the feasible region and to $f$ as the objective function. An element $x \in X$ is called a feasible solution and we refer to $f(x)$ as its objective value. Define

$$
-(t, X, f):=(-t, X,-f)
$$

where $-f: X \rightarrow[-\infty,+\infty]$ is defined by $(-f)(x):=-f(x)$ for every $x \in X$. This definition is useful in going from minimization to maximization problems. It is clear from (2.35) that

$$
-(-(t, X, f))=(t, X, f) \text {. }
$$

An optimization problem is feasible whenever $X \neq \varnothing$. Interestingly, under our definitions, there are only two optimization problems that are infeasible, i.e., not feasible. Those optimization problems are

$$
(-1, \varnothing, \varnothing) \text { and }(1, \varnothing, \varnothing) \text {. }
$$

Let $X$ be a set, and let $f: X \rightarrow[-\infty,+\infty]$. We may call the pair $(X, f)$ a minimization problem or a maximization problem; the former refers to $(-1, X, f)$, and the latter refers to $(1, X, f)$. A minimization problem $(X, f)$ is usually presented as

$$
\begin{aligned}
\text { Minimize } & f(x) \\
\text { s.t. } & x \in X
\end{aligned}
$$

and a maximization problem $(X, f)$ is usually presented as

$$
\begin{aligned}
\text { Maximize } & f(x) \\
\text { s.t. } & x \in X .
\end{aligned}
$$

Let $X$ be a set and let $f: X \rightarrow[-\infty,+\infty]$. The optimal value of the minimization problem $(X, f)$ is defined by

$$
\inf \{f(x) \mid x \in X\} \text {. }
$$


A minimization problem $(X, f)$ is solvable when there exists $s \in X$ such that $f(s)=\inf \{f(x) \mid x \in X\}$. A minimization problem is bounded when its optimal value is not $-\infty$. The optimal value of a maximization problem $(X, f)$ is defined by

$$
\sup \{f(x) \mid x \in X\} .
$$

For every optimization problem $(t, X, f)$,

if $\alpha \in[-\infty,+\infty]$ is the optimal value of $(t, X, f)$,

then $-\alpha \in[-\infty,+\infty]$ is the optimal value of $-(t, X, f)$.

since

$$
\begin{aligned}
\inf \{-f(x) \mid x \in X\} & =-\sup \{f(x) \mid x \in X\}, \text { and } \\
\sup \{-f(x) \mid x \in X\} & =-\inf \{f(x) \mid x \in X\} .
\end{aligned}
$$

A maximization problem $(1, X, f)$ is solvable or bounded when its $-(1, X, f)$ is solvable or bounded, respectively. By definition, a solvable optimization problem is always feasible. Moreover, a solvable optimization problem whose objective function is finite for every feasible solution is bounded.

Let $(X, f)$ and $(Y, g)$ be minimization problems. A homomorphism of minimization problems from $(X, f)$ to $(Y, g)$ is a function $\psi: X \rightarrow Y$ such that

$$
g(\psi(x)) \leq f(x) \text { for every } x \in X .
$$

It is clear that homomorphisms of minimization problems compose, and that $\operatorname{id}_{X}: X \rightarrow X$ is a homomorphism of minimization problems for every minimization problem $(X, f)$. Therefore, we may talk about the category MinProb of minimization problems: we use $\mathcal{O}$ (MinProb) and $\mathcal{H}$ (MinProb) to denote its classes of objects and homomorphisms, respectively.

Let $(X, f)$ and $(Y, g)$ be maximization problems. A homomorphism of maximization problems from $(X, f)$ to $(Y, g)$ is a homomorphism of minimization problems from $-(1, X, f)$ to $-(1, Y, g)$. Note that $\psi: X \rightarrow Y$ is a homomorphism of maximization problems if and only if

$$
g(\psi(x)) \geq f(x) \text { for every } x \in X .
$$

Once again, we may talk about the category MaxProb of maximization problems: we use $\mathcal{O}$ (MaxProb) and $\mathcal{H}$ (MaxProb) to denote its classes of objects and homomorphisms, respectively. The best way to remember whether to use (2.40) or (2.41) is to note that

a homomorphism of optimization problems is a mapping between feasible regions

that improves each solution's objective value.

Homomorphisms of optimization problems are too broad of a notion to preserve some important concepts in optimization. Define $\psi:(1,2] \rightarrow\{1\}$ by $\psi(x):=1$ for every $x \in(1,2]$. We may represent several minimization problems and some homomorphisms between them in the following diagram:

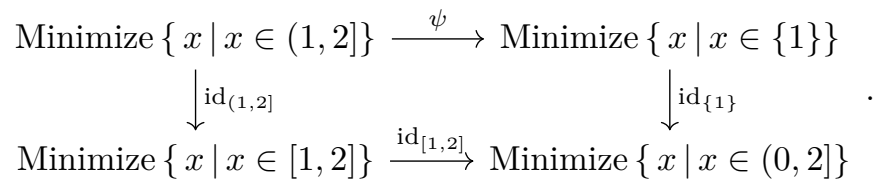

Inspecting the diagram above, the reader will find an example of:

1. a homomorphism between minimization problems with distinct optimal values;

2. a homomorphism from a solvable minimization problem to a minimization problem which is not solvable; and

3. a homomorphism from a minimization problem which is not solvable to a solvable minimization problem. 
On the other hand, the notion of isomorphism of optimization problems is too restrictive. For every minimization problems $(X, f)$ and $(Y, g)$, a homomorphism $\phi: X \rightarrow Y$ from $(X, f)$ to $(Y, g)$ is a (minimizationproblem) isomorphism from $(X, f)$ to $(Y, g)$ if there exists a homomorphism of minimization problems $\phi: Y \rightarrow X$ from $(Y, g)$ to $(X, f)$ such that

$$
\phi \circ \psi=\operatorname{id}_{X} \text { and } \psi \circ \phi=\operatorname{id}_{Y} .
$$

A homomorphism between the maximization problems $(1, X, f)$ and $(1, Y, g)$ is a (maximization-problem) isomorphism if it is an isomorphism between the optimization problems $-(1, X, f)$ and $-(1, Y, g)$. Two minimization problems are isomorphic if there exists an isomorphism between them, and two maximization problems are isomorphic if there exists an isomorphism between them. It is easy to see that two isomorphic optimization problems have feasible regions with the same cardinality. Hence, the optimization problems

$$
\text { Minimize }\{x \mid x \in\{0,1\}\} \text { and Minimize }\{x \mid x \in[0,1]\}
$$

are not isomorphic, even though it is clearly desirable to understand these problems as "similar".

This motivates our interest in a notion broader than that of isomorphisms of optimization problems. Two minimization problems $(X, f)$ and $(Y, g)$ are homomorphically equivalent if there exist homomorphisms of minimization problems from $(X, f)$ to $(Y, g)$ and from $(Y, g)$ to $(X, f)$. Two maximization problems $(1, X, f)$ and $(1, Y, g)$ are homomorphically equivalent if $-(1, X, f)$ and $-(1, Y, g)$ are homomorphically equivalent. Since every optimization problem is either a maximization or a minimization problem, we have just defined homomorphically equivalent optimization problems.

Proposition 2.9. For every minimization problems $(X, f)$ and $(Y, g)$, if there exists a homomorphism of minimization problems $\psi: X \rightarrow Y$ from $(X, f)$ to $(Y, g)$, then

$$
\inf \{f(x) \mid x \in X\} \geq \inf \{g(y) \mid y \in Y\} .
$$

For every maximization problems $(X, f)$ and $(Y, g)$, if there exists a homomorphism of maximization problems $\psi: X \rightarrow Y$ from $(X, f)$ to $(Y, g)$, then

$$
\sup \{f(x) \mid x \in X\} \leq \sup \{g(y) \mid y \in Y\} .
$$

Proof. Let $(X, f)$ and $(Y, g)$ be minimization problems, and let $\psi$ be an homomorphism from $(X, f)$ to $(Y, g)$. Then

$$
\begin{aligned}
\inf \{f(x) \mid x \in X\} & \geq \inf \{g(\psi(x)) \mid x \in X\} & & \text { by }(2.40) \\
& \geq \inf \{g(y) \mid y \in Y\} . & & \text { since } \psi(X) \subseteq Y
\end{aligned}
$$

Hence (2.42) holds

Let $(X, f)$ and $(Y, g)$ be maximization problems. Let $\alpha$ denote the optimal value of $(1, X, f)$ and let $\beta$ denote the optimal value of $(1, Y, g)$. Let $\psi: X \rightarrow Y$ be a homomorphism of maximization problems. By definition, we have that $\psi$ is a homomorphism between the minimization problems $-(1, X, f)$ and $-(1, Y, g)$. Both (2.42) and (2.39) imply that $-\alpha \geq-\beta$, so (2.39) again implies (2.43).

Proposition 2.9 proves that the function sup: $\mathcal{O}(\mathrm{MaxProb}) \rightarrow[-\infty,+\infty]$ mapping a maximization problem to its optimal value is homomorphism monotone, as well as the analogous fact for minimization problems. One may define the opposite category of a given category, and then formulate inf: $\mathcal{O}$ (MinProb) $\rightarrow[-\infty,+\infty]$ as a homomorphism monotone function as well - see [99, Section 1.2].

Corollary 2.10. Let $(t, X, f)$ and $(u, Y, g)$ be homomorphically equivalent optimization problems. Then

1. both problems have the same optimal value;

2. either both are feasible, or both are infeasible;

3. either both are bounded, or both are unbounded;

4. either both are solvable, or both are not solvable. 
Proof. Item 1 follows directly from Proposition 2.9.

Item 2: it suffices to prove that if $(t, X, f)$ is feasible, then $(u, Y, g)$ is feasible. Let $\psi: X \rightarrow Y$ be a homomorphism of optimization problems. Since $(t, X, f)$ is feasible, there exists $x \in X$. Hence $\psi(x) \in Y$, so $(u, Y, g)$ is feasible.

Item 3: let $(X, f)$ and $(Y, g)$ be homomorphically equivalent minimization problems. Assume $(X, f)$ is bounded. Item 1 implies that $(Y, g)$ is bounded, which directly implies that item 3 holds for minimization problems.

Now let $(X, f)$ and $(Y, g)$ be homomorphically equivalent maximization problems. By definition, the problems $-(1, X, f)$ and $-(1, Y, g)$ are homomorphically equivalent, so our previous argument implies that either both are bounded or both are unbounded. Since $(1, X, f)$ and $(1, Y, g)$ are bounded if and only if $-(1, X, f)$ and $-(1, Y, g)$ are bounded, we conclude Item 3.

Item 4: let $(X, f)$ and $(Y, g)$ be homomorphically equivalent minimization problems. Assume $(X, f)$ is solvable, so there exists $s \in X$ such that $f(s)=\inf \{f(x) \mid x \in X\}$. Let $\psi: X \rightarrow Y$ and $\phi: Y \rightarrow X$ be homomorphisms of minimization problems. To prove that

$$
g(\psi(s))=\inf \{g(y) \mid y \in Y\},
$$

note that for every $y \in Y$,

$$
\begin{aligned}
g(y) & \geq f(\phi(y)) \\
& \geq \inf \{f(x) \mid x \in X\} \\
& =f(s) \\
& \geq g(\psi(s)) .
\end{aligned}
$$

since $\phi(y) \in X$

hypothesis on $s \in X$

by $(2.40)$

Therefore item 4 holds for minimization problems.

Now let $(X, f)$ and $(Y, g)$ be homomorphically equivalent maximization problems. By definition, the problems $-(1, X, f)$ and $-(1, Y, g)$ are homomorphically equivalent, so our previous argument implies that either both are solvable or both are not solvable. Since $(1, X, f)$ and $(1, Y, g)$ are solvable if and only if $-(1, X, f)$ and $-(1, Y, g)$ are solvable, we conclude item 4 .

\subsection{Convexity}

Convex analysis plays an important role in this text. Our notation and terminology mostly follows [102]. Chapter 4 in general, and Section 4.1 in particular, will further develop some concepts presented here. Using the language from Section 2.5, we may state that convexity is a convenient source of solvable optimization problems.

Let $\mathbb{E}$ be an Euclidean space. A set $C \subseteq \mathbb{E}$ is convex if for every $\lambda \in(0,1)$ and $x, y \in C$ we have that $\lambda x+(1-\lambda) y \in C$. A function $f: \mathbb{E} \rightarrow[-\infty,+\infty]$ is convex if

$$
f(\lambda x+(1-\lambda) y)<\lambda \alpha+(1-\lambda) \beta
$$

whenever $\lambda \in(0,1)$ and $\alpha, \beta \in[-\infty,+\infty]$ are such that $f(x)<\alpha$ and $f(y)<\beta$. If $C \subseteq \mathbb{E}$ is a convex set, a function $g: C \rightarrow \mathbb{R}$ such that

$$
g(\lambda x+(1-\lambda) y) \leq \lambda g(x)+(1-\lambda) g(y)
$$

for every $x, y \in C$ and $\lambda \in(0,1)$ can be encoded as a convex function $f: \mathbb{E} \rightarrow[-\infty,+\infty]$ by defining it to be $+\infty$ outside of $C$. In short, the extended reals allow us to uniformly work with convex functions defined over distinct domains. Our definition of convexity is slightly unusual only because it works for functions taking extended-real values. A set $K \subseteq \mathbb{E}$ is a cone if $\alpha x \in K$ for every $\alpha>0$ and $x \in K$.

Let $\mathbb{E}$ be an Euclidean space, and let $K \subseteq \mathbb{E}$ be a convex cone. Convexity implies that $\frac{1}{2}(K+K) \subseteq K$, so we may use that $K$ is a cone to conclude that

$$
K+K \subseteq K
$$


We denote by $\preceq_{K}$ the relation on $\mathbb{E}$ such that $x \preceq_{K} y$ if $y-x \in K$, for every $x, y \in \mathbb{E}$. Note that (2.44) implies that $\preceq_{K}$ is transitive. Therefore, if $0 \in K$, we have that $\preceq_{K}$ is reflexive, so it defines a preorder on $\mathbb{E}$. We also write $x \succeq_{K} y$ when $y \preceq_{K} x$ for $x, y \in \mathbb{E}$. Let $V$ be a finite set. We denote by $\preceq_{=} \preceq_{\mathbb{S}_{+}^{V}}$ and $\succeq:=\succeq_{\mathbb{S}_{+}^{V}}$ the Löwner order on $\mathbb{S}^{V}$, and by $\leq:=\preceq_{\mathbb{R}_{+}^{V}}$ and $\geq:=\succeq_{\mathbb{R}_{+}^{V}}$ the pointwise order on $\mathbb{R}^{V}$.

Let $\mathbb{E}$ be an Euclidean space, and let $C \subseteq \mathbb{E}$. The convex hull conv $C$ of $C$ is the intersection of every convex set in $\mathbb{E}$ containing $C$. One may easily check that for every sets $C, D \subseteq \mathbb{E}$, we have that

$$
\begin{gathered}
C \subseteq \operatorname{conv} C, \\
C \subseteq D \Longrightarrow \operatorname{conv} C \subseteq \operatorname{conv} D, \\
\operatorname{conv}(\operatorname{conv} C)=\operatorname{conv} C .
\end{gathered}
$$

Recalling that the inner product defines a norm on $\mathbb{E}$, we have that $\mathbb{E}$ is a metric space and is naturally equipped with a topology. The closure $\operatorname{cl} C$ of $C$ is the intersection of every closed set in $\mathbb{E}$ containing $C$. For every sets $C, D \subseteq \mathbb{E}$, we have that

$$
\begin{gathered}
C \subseteq \operatorname{cl} C, \\
C \subseteq D \Longrightarrow \operatorname{cl} C \subseteq \operatorname{cl} D, \\
\operatorname{cl}(\operatorname{cl} C)=\operatorname{cl} C .
\end{gathered}
$$

The interior int $C$ of $C$ is the union of every open set contained in $C$. One may easily check that

$$
\begin{gathered}
\operatorname{int} C \subseteq C, \\
C \subseteq D \Longrightarrow \operatorname{int} C \subseteq \operatorname{int} D, \\
\operatorname{int}(\operatorname{int} C)=C
\end{gathered}
$$

for every $C, D \subseteq \mathbb{E}$.

Let $\mathbb{E}$ be an Euclidean space, and let $C \subseteq \mathbb{E}$ be a set. Define the support function $\delta^{*}(\cdot \mid C): \mathbb{E} \rightarrow[-\infty,+\infty]$ for every $x \in \mathbb{E}$ by

$$
\delta^{*}(x \mid C):=\sup \{\langle x, z\rangle \mid z \in C\} .
$$

This notation comes from [102], and is used extensively throughout this work. It is easy to see that $\delta^{*}(\cdot \mid C)$ is a convex function. Furthermore, it holds that

$$
\delta^{*}(x \mid C)=\delta^{*}(x \mid \operatorname{cl} C),
$$

that

$$
\delta^{*}(x \mid C)=\delta^{*}(x \mid \operatorname{conv} C)
$$

and that for every $D \subseteq \mathbb{E}$,

$$
\text { if } C \subseteq D \text { then } \delta^{*}(x \mid C) \leq \delta^{*}(x \mid D) .
$$

Let $\mathbb{E}_{0}$ and $\mathbb{E}_{1}$ be Euclidean spaces, let $\mathcal{A}: \mathbb{E}_{0} \rightarrow \mathbb{E}_{1}$ be a linear transformation, and let $C \subseteq \mathbb{E}_{0}$. It holds that

$$
\operatorname{conv}(\mathcal{A} C)=\mathcal{A}(\operatorname{conv} C) .
$$

Furthermore, for every $x \in \mathbb{E}_{1}$,

$$
\delta^{*}(\mathcal{A}(x) \mid C)=\delta^{*}\left(x \mid \mathcal{A}^{*}(C)\right) .
$$

Let $\mathbb{E}$ be an Euclidean spaces, and let $C \subseteq \mathbb{E}$. The polar $C^{\circ}$ of $C$ is defined as

$$
C^{\circ}:=\{y \in \mathbb{E} \mid \forall x \in C,\langle y, x\rangle \leq 1\} .
$$

The dual $C^{*}$ of $C$ is defined as

$$
C^{*}:=\{y \in \mathbb{E} \mid \forall x \in C,\langle y, x\rangle \geq 0\} .
$$

One may trivially check that both $C^{\circ}$ and $C^{*}$ are closed convex sets, and that $C^{*}$ is a cone. For every closed convex cone $K \subseteq \mathbb{E}$, it holds that

$$
K^{* *}=K
$$


Let $\mathbb{E}$ be an Euclidean space. A cone $K \subseteq \mathbb{E}$ is regular if $K$ is a closed convex cone such that int $K \neq \varnothing$ and $\operatorname{int}\left(K^{*}\right) \neq \varnothing$. Using (2.53) we see that a closed convex cone is regular if and only if its dual is regular. Equivalently, the cone $K \subseteq \mathbb{E}$ is regular if $K$ is a closed convex cone with nonempty interior and such that $K \cap(-K)=\{0\}$. A hyperplane is a set of the form $\{y \in \mathbb{E} \mid\langle y, x\rangle \leq \alpha\}$ for some nonzero $x \in \mathbb{E}$ and $\alpha \in \mathbb{R}$. A set is polyhedral if it is a finite intersection of hyperplanes. For every finite set $V$, the positive semidefinite matrices $\mathbb{S}_{+}^{V}$ form a regular cone, and the nonnegative orthant $\mathbb{R}_{+}^{V}$ is a polyhedral cone.

Theorem 2.11 ([93, Theorem 7.12] Conic Programming Strong Duality). Let $U$ and $V$ be finite sets. Let $\mathcal{A}: \mathbb{S}^{V} \rightarrow \mathbb{R}^{U}$ be a linear transformation, let $b \in \mathbb{R}^{U}$, and let $C \in \mathbb{S}^{V}$. Let $K \subseteq \mathbb{R}^{U}$ be a polyhedral cone, and let $L \subseteq \mathbb{S}^{V}$ be a regular cone. Consider the optimization problems

$$
\begin{gathered}
\inf \left\{\langle C, X\rangle \mid \mathcal{A}(X) \succeq_{K} b, X \in L^{*}\right\}, \\
\sup \left\{b^{\top} y \mid y \in K^{*}, \mathcal{A}^{*}(y) \preceq_{L} C\right\} .
\end{gathered}
$$

Then

1. for every $y \in K^{*}$ such that $\mathcal{A}^{*}(y) \preceq_{L} C$ and for every $X \in L^{*}$ such that $\mathcal{A}(x) \succeq_{K} b$,

$$
b^{\top} y \leq\langle C, X\rangle
$$

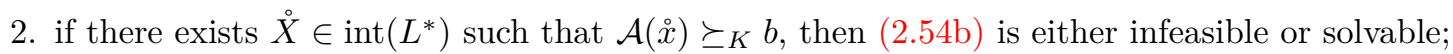

3. if there exits $\stackrel{\circ}{y} \in K^{*}$ such that $C-\mathcal{A}^{*}(\stackrel{\circ}{y}) \in \operatorname{int}(L)$, then $(2.54 a)$ is either infeasible or solvable.

Theorem 2.11 can be readily understood as a generalization of Linear Programming Strong Duality — and this is how it is presented in [93, Section 7.1]. We assume the reader is familiar with basic results about linear programming - i.e., some of the material in [107] or [24]. In particular, we will make use of the following result.

Theorem 2.12. Let $U$ and $V$ be finite sets. Let $A \in \mathbb{Q}^{U \times V}$, let $b \in \mathbb{Q}^{U}$, and let $c \in \mathbb{R}^{V}$. If the linear program

$$
\max \left\{c^{\top} x \mid x \in \mathbb{R}^{U}, A x=b\right\}
$$

has an optimal solution, then it has an optimal solution in $\mathbb{Q}^{U}$. In particular, if $c \in \mathbb{Q}^{V}$, then the optimal value of $(2.55)$ belongs to $\mathbb{Q}$.

Theorem 2.12 is a natural consequence of a couple of well-known results from linear programming. In particular, it follows from the correctness of the simplex algorithm, which only looks for optimal solutions among the basic feasible solutions; solutions whose coordinates can be found by solving a linear system of equations, and thus, must reside on the same field as the matrix defining the LP. 


\section{Chapter 3}

\section{Graph Homomorphism Optimization Problems}

\section{$3.1 \quad$ Order Duality}

This section introduces the results from order theory which are crucial to the remaining of the chapter. Order theory arises as the common language necessary for going beyond the graph homomorphic relation into some of its generalizations - see Subsection 3.4.7. Order duality lies at the core of the theory presented here, and special care was taken to provide such results in a useful manner: most of all, we avoided proving results by simply stating them to be "dual" to some other result. Only the exposition here is new.

Let $S$ be a set. A preorder $\preceq$ on $S$ is a relation on $S$ which is

1. reflexive, i.e., for every $p \in S$ we have that $p \preceq p$, and

2. transitive, i.e., if $a, b, c \in S$ are such that $a \preceq b$ and $b \preceq c$, then $a \preceq c$.

The ordered pair $P:=(S, \preceq)$ is a preordered set. We will use a notation similar to graph-theoretic notation: the ground set $S$ of $P$ may be denoted by $V(P)$, and $\preceq$ may be denoted by $\preceq_{P}$. We also sometimes omit the $P$ in $\preceq_{P}$ and simply write $\preceq$ when $P$ can be deduced from context. The dual $P^{*}$ of the preordered set $P$ is the preordered set $(S, \succeq)$, where $a \succeq b$ holds if $b \preceq a$, for every $a, b \in S$. By definition

$$
V(P)=V\left(P^{*}\right)
$$

and, for every $a, b \in S$,

$$
a \preceq^{*} b \text { if and only if } b \preceq_{P} a \text {. }
$$

To the reader uncomfortable with the use of $V$ to denote both the ground set of a preordered set and the vertex set of a graph, we offer a theoretical justification. A simple digraph is an ordered pair $(V, A)$ where $V$ is a set and $A \subseteq V \times V$. If we focus on symmetric relations on sets, we are studying objects equivalent to simple graphs, whereas if we focus on reflexive and transitive relations we recover preordered sets. In short, both examples may be easily framed as special cases of simple digraphs. In particular, we may consider $V(D):=V$ to be defined for every simple digraph $D=(V, A)$, so that we are specializing, and not overloading, the notation. Although we will bring them up once more to enlighten the discussion, simple digraphs will not be studied in this work.

Theorem 3.1. If $P$ is a preordered set, then so is $P^{*}$.

Proof. Let $S$ be the ground set of $P$. Reflexivity of $\preceq_{P^{*}}$ follows from (3.2) and from reflexivity of $\preceq_{P}$. If $a, b, c \in S$ are such that $a \preceq_{P^{*}} b$ and $b \preceq_{P^{*}} c$, then $c \preceq_{P} b$ and $b \preceq_{P} a$ by (3.2). Transitivity of $\preceq_{P}$ implies that $c \preceq_{P} a$, so $a \preceq^{*} c$. 
Let $P$ be a preordered set. Theorem 3.1 states that $P^{*}$ is a preordered set. It therefore makes sense to consider what $\left(P^{*}\right)^{*}$ is. Both (3.1) and (3.2) imply that

$$
\left(P^{*}\right)^{*}=P \text {. }
$$

Theorem 3.1 is stated as a theorem because it establishes the basic construction which we shall exploit. After relating the dual of a preordered set to other constructions in order theory, we unify statements which tend to be vaguely mentioned as "duals".

Let $S$ be a set. A partial order on $S$ is a preorder $\preceq$ on $S$ that is antisymmetric, i.e., such that $a \preceq b$ and $b \preceq a$ imply $a=b$, for every $a, b \in S$. A partially ordered set is a pair $(S, \preceq)$ where $\preceq$ is a partial order on $S$. Since every partially ordered set $P$ is a preordered set, the dual $P^{*}$ is defined.

Proposition 3.2. Let $P$ be a partially ordered set. Then $P^{*}$ is a partially ordered set.

Proof. Theorem 3.1 implies $P^{*}$ is a preordered set. Set $(S, \preceq):=P$. Let $a, b \in S$ be such that $a \preceq P^{*} b$ and $b \preceq_{P^{*}} a$. Then $b \preceq_{P} a$ and $a \preceq_{P} b$ by (3.2). The relation $\preceq_{P}$ is antisymmetric since $P$ is partially ordered, so $a=b$ follows.

Let $P:=(S, \preceq)$ be a preordered set. Define $[\cdot]: S \rightarrow \mathcal{P}(S)$ by

$$
[s]:=\{x \in S \mid s \preceq x \text { and } x \preceq s\}
$$

for every $s \in S$. Define $[P]:=([S], \leq)$, where $[S]$ is simply the image of $S$ under $[\cdot]$, and

$$
A \leq B \text { if there exist } a, b \in S \text { such that } a \preceq_{P} b, A=[a] \text {, and } B=[b],
$$

for every $A, B \in[S]$. We call $[P]$ the partially ordered set defined by $P$, or the quotient of $P$ - the next proposition shows that $[P]$ is indeed a partially ordered set. By definition, we have that

$$
V([P])=[V(P)] .
$$

Our current goal is to prove that for every $a, b \in S$,

$$
[a] \preceq_{[P]}[b] \text { if and only if } a \preceq_{P} b .
$$

To prove (3.7), we first prove that for every $a, b \in S$,

$$
[a]=[b] \text { if and only if } a \in[b] .
$$

Let $a, b \in S$ be such that $[a]=[b]$. Then

$$
\begin{aligned}
a & \in[a] & & \text { by reflexivity } \\
& =[b] . & & \text { by hypothesis }
\end{aligned}
$$

Now let $a, b \in S$ be such that $a \in[b]$. For every $s \in[a]$, we have that

$$
\begin{aligned}
s & \preceq a & & \text { by } s \in[a] \text { and }(3.4) \\
& \preceq b & & \text { by } a \in[b] \text { and }(3.4) \\
& \text { } a & & \text { by } a \in[b] \text { and }(3.4) \\
& \text { s. } & & \text { by } s \in[a] \text { and }(3.4)
\end{aligned}
$$

Therefore, transitivity of ' $\preceq$ ' implies that $s \preceq b$ and that $b \preceq s$, so we conclude that $s \in[b]$ by (3.4). We have thus proved that

$$
a \in[b] \text { implies that }[a] \subseteq[b] .
$$

Now note that $a \in[b]$ and (3.4) imply that $a \preceq b$ and $b \preceq a$, so $b \in[a]$ by (3.4). Thus (3.9) implies (3.8). We now prove (3.7). Let $a, b \in S$ be such that $a \preceq b$. Then $[a] \leq[b]$ by (3.5). Conversely, assume that $[a] \leq[b]$ for $a, b \in S$. By (3.5) and (3.8), there exist $a^{\prime}, b^{\prime} \in S$ such that

$$
\begin{aligned}
a^{\prime} & \preceq b^{\prime}, \\
a^{\prime} & \in[a], \text { and } \\
b^{\prime} & \in[b] .
\end{aligned}
$$


Therefore

$$
\begin{aligned}
a & \preceq a^{\prime} & & \text { by }(3.4) \text { and }(3.10 \mathrm{~b}) \\
& \preceq b^{\prime} & & \text { by }(3.10 \mathrm{a}) \\
& \preceq b . & & \text { by }(3.4) \text { and }(3.10 \mathrm{c})
\end{aligned}
$$

Transitivity of $\preceq$ implies that $a \preceq b$. This proves (3.7).

Proposition 3.3. Let $P$ be a preordered set. Then $[P]$ is a partially ordered set.

Proof. Set $(S, \preceq):=P$. We prove that $[P]$ is a partially ordered set. By construction, we have that every element of $V([P])=[S]$ is of the form $[s]$ for $s \in S$. Hence, it is immediate from (3.7) that $\preceq_{[P]}$ is reflexive and transitive. Let $a, b \in S$ be such that $[a] \leq[b]$ and $[b] \leq[a]$. Using (3.7) twice we conclude that

$$
a \preceq b \text { and } b \preceq a \text {. }
$$

Both (3.4) and (3.11) imply that $a \in[b]$, so (3.8) implies that $[a]=[b]$.

Let $P$ be a preordered set. If we denote by $[\cdot]^{*}: V\left(P^{*}\right) \rightarrow V\left(\left[P^{*}\right]\right)$ the function defined in (3.4) for $P^{*}$ and by $[\cdot]: V(P) \rightarrow V([P])$ the function defined in (3.4) for $P$, we have that

$$
[\cdot]=[\cdot]^{*}
$$

since

$$
\begin{aligned}
{[s] } & =\left\{x \in V(P) \mid s \preceq_{P} x \text { and } x \preceq_{P} s\right\} & & \text { by }(3.4) \\
& =\left\{x \in V(P) \mid s \preceq_{P^{*}} x \text { and } x \preceq_{P^{*}} s\right\} & & \text { by }(3.2) \text { (twice) } \\
& =\left\{x \in V\left(P^{*}\right) \mid s \preceq_{P^{*}} x \text { and } x \preceq_{P^{*}} s\right\} & & \text { by }(3.1) \\
& =[s]^{*} . & & \text { by }(3.4)
\end{aligned}
$$

We may then prove that

$$
[P]^{*}=\left[P^{*}\right] .
$$

First note that both partially ordered sets in (3.13) have the same ground sets, as

$$
\begin{aligned}
V\left([P]^{*}\right) & =V([P]) & & \text { by }(3.1) \\
& =[V(P)] & & \text { by }(3.6) \\
& =[V(P)]^{*} & & \text { by }(3.12) \\
& =\left[V\left(P^{*}\right)\right]^{*} & & \text { by }(3.1) \\
& =V\left(\left[P^{*}\right]\right) . & & \text { by }(3.6)
\end{aligned}
$$

For every $a, b \in V(P)$, we have that

$$
\begin{aligned}
{[a] \preceq_{[P]^{*}}[b] } & \Longleftrightarrow[b] \preceq_{[P]}[a] & & \text { by }(3.2) \\
& \Longleftrightarrow b \preceq_{P} a & & \text { by }(3.7) \\
& \Longleftrightarrow a \bigcap_{P^{*}} b & & \text { by }(3.2) \\
& \Longleftrightarrow[a] \preceq_{\left[P^{*}\right]}[b], & & \text { by }(3.7)
\end{aligned}
$$

so (3.13) holds.

We now introduce some notation for working with inequalities in preordered sets. Let $P$ be a preordered set, and let $S \subseteq V(P)$. Define

$$
\operatorname{lb}_{P} S:=\left\{b \in V(P) \mid \forall s \in S, b \preceq_{P} s\right\} .
$$

An element of $\operatorname{lb}_{P} S$ is a lower bound for $S$ with respect to (w.r.t.) $P$. We may omit $P$ whenever no ambiguity arises. We do so both in notation and in text: we may simply say that and element of $\mathrm{lb} S$ is a lower bound 
of $S$. Of course, a similar convention applies to the similar definitions we are about to present. A minimum of $S$ w.r.t. $P$ is a lower bound for $S$ which belongs to $S$, i.e., an element of

$$
\min _{P} S:=S \cap \operatorname{lb}_{P} S .
$$

We may also refer to elements of $\min _{P} S$ using the adjective least: for example, every element of $\min _{P} S$ is a least element of $S$. Define

$$
\mathrm{ub}_{P} S:=\left\{b \in V(P) \mid \forall s \in S, s \preceq_{P} b\right\} .
$$

An element of $\mathrm{ub}_{P} S$ is an upper bound for $S$ w.r.t. $P$. A maximum of $S$ w.r.t. $P$ is an upper bound for $S$ which belongs to $S$, i.e., an element of

$$
\max _{P} S:=S \cap \mathrm{ub}_{P} S .
$$

We may refer to elements of $\max _{P} S$ using the adjective greatest. An infimum of $S$ w.r.t. $P$ is a greatest lower bound of $S$, i.e., an element of

$$
\inf _{P} S:=\max _{P}\left(\operatorname{lb}_{P} S\right),
$$

and a supremum of $S$ w.r.t. $P$ is a least upper bound of $S$, i.e., an element of

$$
\sup _{P} S:=\min _{P}\left(\operatorname{ub}_{P} S\right) .
$$

We may expand the definition (3.19) of supremum using (3.15) to see that

$$
\sup _{P} S=\min _{P}\left(\operatorname{ub}_{P} S\right)=\left(\operatorname{ub}_{P} S\right) \cap \operatorname{lb}_{P}\left(\operatorname{ub}_{P} S\right),
$$

so

$$
\begin{aligned}
& \sup _{P} S \subseteq \mathrm{ub}_{P} S, \text { and } \\
& \sup _{P} S \subseteq \operatorname{lb}_{P}\left(\mathrm{ub}_{P} S\right) .
\end{aligned}
$$

Dually, using (3.18) and (3.17) we see that

$$
\inf _{P} S=\max _{P}\left(\operatorname{lb}_{P} S\right)=\left(\operatorname{lb}_{P} S\right) \cap \mathrm{ub}_{P}\left(\operatorname{lb}_{P} S\right),
$$

so

$$
\begin{aligned}
& \inf _{P} S \subseteq \operatorname{lb}_{P} S \text {, and } \\
& \inf _{P} S \subseteq \operatorname{ub}_{P}\left(\operatorname{lb}_{P} S\right) .
\end{aligned}
$$

In words, we have that every infimum of $S$ is a lower bound of $S$, and also an upper bound for the lower bounds of $S$.

Let $P$ be a preordered set, and let $S \subseteq V(P)$. We prove that

$$
\begin{aligned}
\mathrm{ub}_{P^{*}} S & =\operatorname{lb}_{P} S, \text { and } \\
\operatorname{lb}_{P^{*}} S & =\mathrm{ub}_{P} S .
\end{aligned}
$$

Note the implicit use of Theorem 3.1 when assuming that $P^{*}$ is a preordered set, so the expressions $\mathrm{ub}_{P^{*}} S$ and $\mathrm{lb}_{P^{*}} S$ are defined. We first prove (3.24a):

$$
\begin{aligned}
\operatorname{ub}_{P^{*}} S & =\left\{b \in V(P) \mid \forall s \in S, s \preceq_{P^{*}} b\right\} & & \text { by }(3.16) \\
& =\left\{b \in V(P) \mid \forall s \in S, b \preceq_{P} s\right\} & & \text { by }(3.2) \\
& =\operatorname{lb}_{P} S . & & \text { by }(3.14)
\end{aligned}
$$

Then (3.24b) follows, as

$$
\begin{aligned}
\operatorname{lb}_{P^{*}} S & =\operatorname{ub}_{\left(P^{*}\right)^{*}} S & & \text { by }(3.24 \mathrm{a}) \\
& =\mathrm{ub}_{P} S . & & \text { by }(3.3)
\end{aligned}
$$


Concerning max and min, we have that

$$
\begin{aligned}
\max _{P^{*}} S & =\min _{P} S, \text { and } \\
\min _{P^{*}} S & =\max _{P} S .
\end{aligned}
$$

The proof of (3.25a) basically follows from (3.24a), as

$$
\begin{aligned}
\max _{P^{*}} S & =S \cap\left(\operatorname{ub}_{P^{*}} S\right) & & \text { by }(3.17) \\
& =S \cap\left(\operatorname{lb}_{P} S\right) & & \text { by }(3.24 \mathrm{a}) \\
& =\min _{P} S . & & \text { by }(3.15)
\end{aligned}
$$

It is then easy to conclude (3.25b):

$$
\begin{aligned}
\min _{P^{*}} S & =\max _{\left(P^{*}\right)^{*}} S & & \text { by }(3.25 \mathrm{a}) \\
& =\max _{P} S . & & \text { by }(3.3)
\end{aligned}
$$

Furthermore,

$$
\begin{aligned}
\sup _{P^{*}} S & =\inf _{P} S, \text { and } \\
\inf _{P^{*}} S & =\sup _{P} S .
\end{aligned}
$$

We first prove (3.26a):

$$
\begin{aligned}
\sup _{P^{*}} S & =\min _{P^{*}}\left(\operatorname{ub}_{P^{*}} S\right) \\
& =\max _{P}\left(\mathrm{ub}_{P^{*}} S\right) \\
& =\max _{P}\left(\operatorname{lb}_{P} S\right) \\
& =\inf _{P} S .
\end{aligned}
$$

by $(3.18)$

Finally, we conclude (3.26b):

$$
\begin{aligned}
\inf _{P^{*}} S & =\sup _{\left(P^{*}\right)^{*}} S & & \text { by }(3.26 \mathrm{a}) \\
& =\sup _{P} S . & & \text { by }(3.3)
\end{aligned}
$$

Results (3.24), (3.25), and (3.26) provide a precise language to compute dual results.

As our first application of our duality results, we establish the basic facts regarding the quotient of a preordered set. Let $P$ be a preordered set, and let $S \subseteq V(P)$. We claim that

$$
\begin{aligned}
\mathrm{ub}_{[P]}[S] & =\left[\mathrm{ub}_{P} S\right] \text {, and } \\
\operatorname{lb}_{[P]}[S] & =\left[\operatorname{lb}_{P} S\right] .
\end{aligned}
$$

To prove $(3.27 \mathrm{a})$, note that

$$
\begin{aligned}
\mathrm{ub}_{[P]}[S] & =\left\{b \in V([P]) \mid \forall s \in[S], s \preceq_{[P]} b\right\} & & \text { by }(3.16) \\
& =\left\{b \in[V(P)] \mid \forall s \in[S], s \preceq_{[P]} b\right\} & & \text { by }(3.6) \\
& =\left\{[b] \mid b \in V(P), \forall s \in[S], s \preceq_{[P]}[b]\right\} & & \text { by }(2.3) \\
& =\left\{[b] \mid b \in V(P), \forall s \in S,[s] \preceq_{[P]}[b]\right\} & & \text { by }(2.3) \\
& =\left\{[b] \mid b \in V(P), \forall s \in S, s \preceq_{P} b\right\} & & \text { by }(3.7) \\
& =\left[\left\{b \in V(P) \mid \forall s \in S, s \preceq_{P} b\right\}\right] & & \text { by }(2.3) \\
& =\left[\operatorname{ub}_{P} S\right] & & \text { by }(3.16)
\end{aligned}
$$

This proves (3.27a). It is easy then to conclude (3.27b):

$$
\begin{aligned}
\operatorname{lb}_{[P]}[S] & =\mathrm{ub}_{[P]^{*}}[S] & & \text { by }(3.24 \mathrm{a}) \\
& =\mathrm{ub}_{\left[P^{*}\right]}[S] & & \text { by }(3.13) \\
& =\left[\mathrm{ub}_{P^{*}} S\right] & & \text { by }(3.27 \mathrm{a}) \\
& =\left[\operatorname{lb}_{P} S\right] . & & \text { by }(3.24 \mathrm{a})
\end{aligned}
$$


Moreover, we have that

$$
\begin{aligned}
\max _{[P]}[S] & =\left[\max _{P} S\right], \text { and } \\
\min _{[P]}[S] & =\left[\min _{P} S\right] .
\end{aligned}
$$

It is immediate from (3.16) and (3.17) that

$$
\max _{P} S=\left\{b \in S \mid \forall s \in S, s \preceq_{P} b\right\} .
$$

Therefore (3.28a) holds, as

$$
\begin{aligned}
\max _{[P]}[S] & =\left\{b \in[S] \mid \forall s \in[S], s \preceq_{[P]} b\right\} \\
& =\left\{[b] \mid b \in S, \forall s \in[S], s \preceq_{[P]}[b]\right\} \\
& =\left\{[b] \mid b \in S, \forall s \in S,[s] \preceq_{[P]}[b]\right\} \\
& =\left\{[b] \mid b \in S, \forall s \in S, s \preceq_{P} b\right\} \\
& =\left[\left\{b \in S \mid \forall s \in S, s \preceq_{P} b\right\}\right] \\
& =\left[\max _{P} S\right] .
\end{aligned}
$$

We conclude (3.28b):

$$
\begin{aligned}
\min _{[P]}[S] & =\max _{[P]^{*}}[S] & & \text { by }(3.25 \mathrm{a}) \\
& =\max _{\left[P^{*}\right]}[S] & & \text { by }(3.13) \\
& =\left[\max _{P^{*}} S\right] & & \text { by }(3.28 \mathrm{a}) \\
& =\left[\min _{P} S\right] . & & \text { by }(3.25 \mathrm{a})
\end{aligned}
$$

Finally, we have that

$$
\begin{aligned}
\sup _{[P]}[S] & =\left[\sup _{P} S\right], \text { and } \\
\inf _{[P]}[S] & =\left[\inf _{P} S\right] .
\end{aligned}
$$

Equations (3.30) basically follow from (3.27) and (3.28). We first prove (3.30a):

$$
\begin{aligned}
\sup _{[P]}[S] & =\min _{[P]}\left(\operatorname{ub}_{[P]}[S]\right) & & \text { by }(3.19) \\
& =\min _{[P]}\left[\operatorname{ub}_{P} S\right] & & \text { by }(3.27 \mathrm{a}) \\
& =\left[\min _{P}\left(\mathrm{ub}_{P} S\right)\right] & & \text { by }(3.28 \mathrm{~b}) \\
& =\left[\sup _{P} S\right] . & & \text { by }(3.19)
\end{aligned}
$$

Thus (3.30b) follows, as

$$
\begin{aligned}
\inf _{[P]}[S] & =\sup _{[P]^{*}}[S] & & \text { by }(3.26 \mathrm{a}) \\
& =\sup _{\left[P^{*}\right]}[S] & & \text { by }(3.13) \\
& =\left[\sup _{P^{*}} S\right] & & \text { by }(3.30 \mathrm{a}) \\
& =\left[\inf _{P} S\right] . & & \text { by }(3.26 \mathrm{a})
\end{aligned}
$$

Let $P$ be a preordered set, and let $A, B \subseteq V(P)$. It is immediate from (3.14) and (3.16) that

$A \subseteq B$ implies $\operatorname{ub}_{P} B \subseteq \mathrm{ub}_{P} A$, and

$A \subseteq B$ implies $\mathrm{lb}_{P} B \subseteq \mathrm{lb}_{P} A$.

Furthermore, we claim that for every $S \subseteq V(P)$,

$$
\begin{aligned}
& S \subseteq \operatorname{lb}_{P}\left(\operatorname{ub}_{P} S\right), \text { and } \\
& S \subseteq \operatorname{ub}_{P}\left(\operatorname{lb}_{P} S\right) .
\end{aligned}
$$


We prove (3.32a) by noting that for every $S \subseteq V(P)$, for every $s \in S$, and for every $b \in \operatorname{ub}_{P} S$,

$$
s \preceq_{P} b,
$$

so $s \in \operatorname{lb}_{P}\left(\operatorname{ub}_{P} S\right)$. The result dual to (3.32a) is (3.32b), and it follows from Theorem 3.1 and

$$
\begin{aligned}
S & \subseteq \operatorname{lb}_{P^{*}}\left(\operatorname{ub}_{P^{*}} S\right) & & \text { by }(3.32 \mathrm{a}) \\
& =\operatorname{ub}_{P}\left(\operatorname{ub}_{P^{*}} S\right) & & \text { by }(3.24 \mathrm{~b}) \\
& =\operatorname{ub}_{P}\left(\operatorname{lb}_{P} S\right) . & & \text { by }(3.24 \mathrm{a})
\end{aligned}
$$

We may then prove that every minimum is an infimum, i.e., that for every $S \subseteq V(P)$,

$$
\min _{P} S \subseteq \inf _{P} S
$$

Indeed,

$$
\begin{aligned}
\min _{P} S & =S \cap\left(\operatorname{lb}_{P} S\right) & & \text { by }(3.15) \\
& \subseteq\left(\operatorname{ub}_{P}\left(\operatorname{lb}_{P} S\right)\right) \cap\left(\operatorname{lb}_{P} S\right) & & \text { by }(3.32 \mathrm{~b}) \\
& =\max _{P}\left(\operatorname{lb}_{P} S\right) & & \text { by }(3.17) \\
& =\inf _{P} S . & & \text { by }(3.18)
\end{aligned}
$$

Moreover, we have that

$$
\inf _{P}\left(\operatorname{ub}_{P} S\right)=\min _{P}\left(\operatorname{ub}_{P} S\right) .
$$

It is clear that (3.33) implies ' $\supseteq$ ' in (3.34). Equality holds in (3.34) because

$$
\begin{aligned}
\inf _{P}\left(\operatorname{ub}_{P} S\right) & =\max _{P}\left(\operatorname{lb}_{P}\left(\operatorname{ub}_{P} S\right)\right) & & \text { by }(3.18) \\
& =\left(\operatorname{lb}_{P}\left(\operatorname{ub}_{P} S\right)\right) \cap\left(\operatorname{ub}_{P}\left(\operatorname{lb}_{P}\left(\mathrm{ub}_{P} S\right)\right)\right) & & \text { by }(3.17) \\
& \subseteq\left(\operatorname{lb}_{P}\left(\mathrm{ub}_{P} S\right)\right) \cap\left(\mathrm{ub}_{P} S\right) & & \text { by }(3.32 \mathrm{a}) \text { and }(3.31 \mathrm{a}) \\
& =\min _{P}\left(\mathrm{ub}_{P} S\right) . & & \text { by }(3.15)
\end{aligned}
$$

We therefore conclude that

$$
\begin{aligned}
\sup _{P} S & =\inf _{P}\left(\operatorname{ub}_{P} S\right), \text { and } \\
\inf _{P} S & =\sup _{P}\left(\operatorname{lb}_{P} S\right) .
\end{aligned}
$$

These hold because

$$
\begin{aligned}
\sup _{P} S & =\min _{P}\left(\operatorname{ub}_{P} S\right) \\
& =\inf _{P}\left(\operatorname{ub}_{P} S\right)
\end{aligned}
$$

and

$$
\begin{aligned}
\inf _{P} S & =\sup _{P^{*}} S & & \text { by }(3.26 \mathrm{a}) \\
& =\inf _{P^{*}}\left(\operatorname{ub}_{P^{*}} S\right) & & \text { by }(3.35 \mathrm{a}) \\
& =\sup _{P}\left(\operatorname{ub}_{P^{*}} S\right) & & \text { by }(3.26 \mathrm{~b}) \\
& =\sup _{P}\left(\operatorname{lb}_{P} S\right) . & & \text { by }(3.24 \mathrm{a})
\end{aligned}
$$

We have defined infimum and supremum as sets of elements. When working with preordered sets, it may indeed be the case that a subset of the ground set has more than one supremum and infimum: consider the preordered set $P:=(C, \preceq)$, where $C:=\{0,1\}$ and $\preceq:=C \times C$. Then

$$
\min _{P} C=C=\{0,1\} .
$$


Our interest in graphs extends to functions on graphs. We will exploit a simple construction which defines a preorder on functions taking values on a preordered set. Let $P$ be a preordered set, and let $X$ be a set. We denote by $\operatorname{Fun}(X, P)$ the preordered set with ground set

$$
V(\operatorname{Fun}(X, P)):=V(P)^{X}
$$

and relation $\preceq_{\text {Fun }(X, P)}$ defined by the pointwise order, i.e., such that for every $f, g: X \rightarrow V(P)$,

$$
f \preceq_{\text {Fun }(X, P)} g \quad \text { if } f(x) \preceq_{P} g(x) \text { for every } x \in X .
$$

We first prove that $\operatorname{Fun}(X, P)$ is indeed a preordered set, and then argue that the order-theoretic properties of $P$ propagate to $\operatorname{Fun}(X, P)$.

Proposition 3.4. Let $P$ be a preordered set, and let $X$ be a set. Then $\operatorname{Fun}(X, P)$ is a preordered set.

Proof. Let $(S, \preceq):=P$. For every $f \in S^{X}$ and $x \in X$, reflexivity of $\preceq$ implies that $f(x) \preceq f(x)$. Hence $f \preceq_{\operatorname{Fun}(X, P)} f$ for every $f \in S^{X}$. Let $f, g, h \in S^{X}$ be such that $f \preceq_{\operatorname{Fun}(X, P)} g$ and $g \preceq_{\text {Fun }(X, P)} h$, and let $x \in X$. Then $f(x) \preceq g(x)$ and $g(x) \preceq h(x)$ by (3.37). Therefore $f(x) \preceq h(x)$, as $\preceq$ is transitive. Since $x \in X$ was arbitrary, we conclude that $f \preceq$ Fun $(X, P) h$.

Proposition 3.5. Let $P$ be a partially ordered set, and let $X$ be a set. Then $\operatorname{Fun}(X, P)$ is a partially ordered set.

Proof. Proposition 3.4 states that $\operatorname{Fun}(X, P)$ is a preordered set. Let $f, g \in V(\operatorname{Fun}(X, P))$ be such that

$$
\begin{aligned}
& f \preceq_{\operatorname{Fun}(X, P)} g \text { and } \\
& g \preceq_{\operatorname{Fun}(X, P)} f .
\end{aligned}
$$

Let $x \in X$. Then (3.37), (3.38), and (3.39) imply that $f(x) \preceq_{P} g(x)$ and $g(x) \preceq_{P} f(x)$. Therefore $f(x)=g(x)$, as $\preceq_{P}$ is antisymmetric. As this holds for every $x \in X$, we conclude that $f=g$.

The next result relates the two core constructions presented: the duality of preordered sets and the preordered sets defined by Fun.

Proposition 3.6. Let $P$ be a preordered set, and let $X$ be a set. Then $\operatorname{Fun}(X, P)^{*}=\operatorname{Fun}\left(X, P^{*}\right)$.

Proof. First note that both preordered sets have the same ground set, as

$$
\begin{aligned}
V\left(\operatorname{Fun}(X, P)^{*}\right) & =V(\operatorname{Fun}(X, P)) & & \text { by }(3.1) \\
& =V(P)^{X} & & \text { by }(3.36) \\
& =V\left(P^{*}\right)^{X} & & \text { by }(3.1) \\
& =V\left(\operatorname{Fun}\left(X, P^{*}\right)\right) . & & \text { by }(3.36)
\end{aligned}
$$

Thus, for every $f, g: X \rightarrow V(P)$,

$$
\begin{aligned}
f \preceq_{\text {Fun }(X, P)^{*}} g & \Longleftrightarrow g \preceq_{\text {Fun }(X, P)} f & & \text { by }(3.2) \\
& \Longleftrightarrow\left(\forall x \in X, g(x) \preceq_{P} f(x)\right) & & \text { by }(3.37) \\
& \Longleftrightarrow\left(\forall x \in X, f(x) \preceq_{P^{*}} g(x)\right) & & \text { by }(3.2) \\
& \Longleftrightarrow f \preceq_{\text {Fun }\left(X, P^{*}\right)} g . & & \text { by }(3.37)
\end{aligned}
$$

Let $P$ be a preordered set, and let $S \subseteq V(P)$. We denote by $P[S]$ the preordered set with ground set

$$
V(P[S]):=S
$$

and with relation $\preceq_{P[S]}$ such that

$$
a \preceq_{P[S]} b \text { if and only if } a \preceq_{P} b
$$

for every $a, b \in S$. We first prove that $P[S]$ is indeed a preordered set. 
Proposition 3.7. Let $P$ be a preordered set, and let $S \subseteq V(P)$. Then $P[S]$ is a preordered set.

Proof. Let $s \in S$. Reflexivity of $\preceq_{P}$ implies that $s \preceq_{P} s$, so (3.41) implies that $s \preceq_{P[S]} s$. As this holds for every $s \in S$, we have proved reflexivity of $\preceq_{P[S]}$. Let $a, b, c \in S$ be such that $a \preceq_{P[S]} b$ and $b \preceq_{P[S]} c$. Then (3.41) (twice) implies that $a \preceq_{P}$ and $b \preceq_{P} c$. Transitivity of $\preceq_{P}$ implies that $a \preceq_{P} c$, and since $a, b \in S$, we use (3.41) to conclude that $a \preceq_{P[S]} c$.

Proposition 3.8. Let $P$ be a preordered set, and let $S \subseteq V(P)$. Then

$$
P[S]^{*}=P^{*}[S] .
$$

Proof. We have that $P[S]$ is a preordered set by Proposition 3.7, and both Theorem 3.1 and Proposition 3.7 imply that $P^{*}[S]$ is a preordered set. Both preordered sets have the same ground set:

$$
\begin{aligned}
V\left(P[S]^{*}\right) & =V(P[S]) & & \text { by }(3.1) \\
& =S & & \text { by }(3.40) \\
& =V\left(P^{*}[S]\right) . & & \text { by }(3.40)
\end{aligned}
$$

Moreover, for every $a, b \in S$,

$$
\begin{aligned}
a \preceq_{P[S]^{*}} b & \Longleftrightarrow b \preceq_{P[S]} a & & \text { by }(3.2) \\
& \Longleftrightarrow b \preceq_{P} a & & \text { by }(3.41), a \in S, \text { and } b \in S \\
& \Longleftrightarrow a \preceq_{P^{*}} b & & \text { by }(3.2) \\
& \Longleftrightarrow a \preceq_{P^{*}[S]} b . & & \text { by }(3.41), a \in S, \text { and } b \in S
\end{aligned}
$$

Let $P$ and $Q$ be preordered sets. A function $f: V(P) \rightarrow V(Q)$ is $(P, Q)$-monotone if

$$
a \preceq_{P} b \text { implies } f(a) \preceq_{Q} f(b)
$$

for every $a, b \in V(P)$. Whenever $P$ and $Q$ are clear from context, we may omit them. The similarity between the definition of monotone function and graph homomorphism is connected to the relation among graphs, preordered sets, and simple digraphs. We may define a homomorphism of simple digraphs to be a function which maps the vertex sets of one digraph to the other while preserving the relations involved. This notion of homomorphism, when specialized to simple graphs, is equivalent to graph homomorphisms; when specialized to preordered sets, is equivalent to monotone functions.

Let $P$ and $Q$ be preordered sets, and let $X$ and $Y$ be sets. We first show that for every monotone $f: V(P) \rightarrow V(Q)$ and for every $g, h \in V(\operatorname{Fun}(X, P))$,

$$
\text { if } g \preceq_{\operatorname{Fun}(X, P)} h \text {, then } f \circ g \preceq_{\operatorname{Fun}(X, Q)} f \circ h \text {. }
$$

Let $f: V(P) \rightarrow V(Q)$ be monotone, and let $g, h \in \operatorname{Fun}(X, P)$. Then

$$
\begin{aligned}
g \preceq_{\operatorname{Fun}(X, P)} h & \Longrightarrow\left(\forall x \in X, g(x) \preceq_{P} h(x)\right) \\
& \Longrightarrow\left(\forall x \in X, f(g(x)) \preceq_{Q} f(h(x))\right) \\
& \Longrightarrow f \circ g \preceq_{\operatorname{Fun}(X, Q)} f \circ h .
\end{aligned}
$$

We now show that, for every $f: X \rightarrow Y$ and for every $g, h \in V(\operatorname{Fun}(Y, P))$,

$$
\text { if } g \preceq_{\text {Fun }(Y, P)} h \text {, then } g \circ f \preceq_{\operatorname{Fun}(X, P)} h \circ f \text {. }
$$

Let $f: X \rightarrow Y$, and let $g, h \in \operatorname{Fun}(Y, P)$. Then

$$
\begin{aligned}
g \preceq_{\text {Fun }(Y, P)} h & \Longrightarrow\left(\forall y \in Y, g(y) \preceq{ }_{P} h(y)\right) & & \text { by }(3.37) \\
& \Longrightarrow\left(\forall x \in X, g(f(x)) \preceq{ }_{P} h(f(x))\right) & & \text { since } f: X \rightarrow Y \\
& \Longrightarrow g \circ f \preceq \text { Fun }(X, P) h \circ f . & & \text { by }(3.37)
\end{aligned}
$$


Proposition 3.9. Let $P$ and $Q$ be preordered sets. Then $f: V(P) \rightarrow V(Q)$ is monotone if and only if

$$
\operatorname{lb}_{P} S \subseteq f^{-1}\left(\operatorname{lb}_{Q}(f(S))\right)
$$

for every $S \subseteq V(P)$.

Proof. Let $f: V(P) \rightarrow V(Q)$ be monotone. Let $S \subseteq V(P)$, and let $b \in \operatorname{lb}_{P} S$. Then

$$
\begin{aligned}
b \in \operatorname{lb}_{P} S & \Longrightarrow\left(\forall s \in S, b \preceq_{P} s\right) & & \text { by }(3.14) \\
& \Longrightarrow\left(\forall s \in S, f(b) \preceq_{Q} f(s)\right) & & \text { by }(3.42) \\
& \Longrightarrow f(b) \in \operatorname{lb}_{Q}(\{f(s) \mid s \in S\}) & & \text { by }(3.14) \\
& \Longrightarrow b \in f^{-1}\left(\operatorname{lb}_{Q}(f(S))\right) . & &
\end{aligned}
$$

As this holds for every $b \in \operatorname{lb}_{P} S$, the necessity part of the proof is done. Assume that $f: V(P) \rightarrow V(Q)$ is not monotone. Then there exist $a, b \in V(P)$ such that $a \preceq_{P} b$ and $f(a) \swarrow_{Q} f(b)$. Therefore $a \in \operatorname{lb}_{P}\{b\}$, yet $a \notin f^{-1}\left(\mathrm{lb}_{Q} f(\{b\})\right)$.

Let $P$ be a preordered set, let $S \subseteq V(P)$, and let $R \subseteq S$. For a first application of monotone functions and Proposition 3.9, we prove that

$$
\begin{aligned}
\operatorname{lb}_{P[S]} R & =S \cap \operatorname{lb}_{P} R \text { and } \\
\operatorname{ub}_{P[S]} R & =S \cap \mathrm{ub}_{P} R .
\end{aligned}
$$

Note that (3.41) implies that

$$
\iota_{S}: V(P[S]) \rightarrow V(P) \text {, defined by } \iota_{S}(s):=q \text { for every } s \in S, \text { is monotone. }
$$

Proposition 3.9 implies that

$$
\mathrm{lb}_{P[S]} R \subseteq \iota_{S}^{-1}\left(\operatorname{lb}_{P}\left(\iota_{S}(R)\right)\right)=\iota_{S}^{-1}\left(\mathrm{lb}_{P}(R)\right)=S \cap\left(\mathrm{lb}_{P} R\right) .
$$

Now let $a \in S \cap \operatorname{lb}_{P} R$. For every $r \in R$, we have that $a \preceq_{P} r$. Then (3.41) implies that $a \preceq_{P[S]} r$ for every $r \in R$, as $a \in S$ and $R \subseteq S$. In short, we have that $S \cap\left(\operatorname{lb}_{P} R\right) \subseteq \operatorname{lb}_{P[S]} R$, concluding the proof of (3.45a). Duality then implies (3.45b), as

$$
\begin{aligned}
\mathrm{ub}_{P[S]} R & =\mathrm{lb}_{P[S]^{*}} R & & \text { by }(3.24 \mathrm{~b}) \\
& =\mathrm{lb}_{P^{*}[S]} R & & \text { by Proposition } 3.8 \\
& =S \cap \mathrm{lb}_{P^{*}} R & & \text { by }(3.45 \mathrm{a}) \\
& =S \cap \mathrm{ub}_{P} R . & & \text { by }(3.24 \mathrm{~b})
\end{aligned}
$$

Proposition 3.10. Let $P$ be a partially ordered set, and let $S \subseteq V(P)$. Then $P[S]$ is a partially ordered set.

Proof. Proposition 3.7 implies that $P[S]$ is a preordered set. Let $a, b \in S$ be such that $a \preceq_{P[S]} b$ and $b \preceq_{P[S]} a$. Then (3.41) implies that $a \preceq_{P} b$ and $b \preceq_{P} a$. Since $\preceq_{P}$ is antisymmetric, we conclude that $a=b$.

Let $P$ and $Q$ be preordered sets. Define

$$
\operatorname{Mon}(P, Q):=\operatorname{Fun}(V(P), Q)[M]
$$

where

$$
M:=\{f: V(P) \rightarrow V(Q) \mid f \text { is }(P, Q) \text {-monotone }\},
$$

Recalling (3.40) we have that

$$
V(\operatorname{Mon}(P, Q))=\{f: V(P) \rightarrow V(Q) \mid f \text { is }(P, Q) \text {-monotone }\}
$$

and recalling (3.41), we have that for every monotone $f, g: V(P) \rightarrow V(Q)$,

$$
f \preceq_{\operatorname{Mon}(P, Q)} g \text { if and only if } f \preceq_{\text {Fun }(V(P), Q)} g \text {. }
$$


Comparing Mon with Fun, Proposition 3.7 implies a result analogous to Proposition 3.4: if $Q$ is preordered, then

$$
\operatorname{Mon}(P, Q) \text { is preordered, }
$$

as

$$
\begin{aligned}
Q \text { is preordered } & \Longrightarrow \operatorname{Fun}(V(P), Q) \text { is preordered } & & \text { by Proposition } 3.4 \\
& \Longrightarrow \operatorname{Fun}(V(P), Q)[M] \text { is preordered } & & \text { by Proposition } 3.7 \\
& \Longrightarrow \operatorname{Mon}(Q, P) \text { is preordered. } & & \text { by }(3.47)
\end{aligned}
$$

Similarly, Proposition 3.10 implies a result analogous to Proposition 3.5: if $P$ is partially ordered, then

$$
\operatorname{Mon}(Q, P) \text { is partially ordered, }
$$

as

$$
\begin{aligned}
Q \text { is partially ordered } & \Longrightarrow \operatorname{Fun}(V(P), Q) \text { is partially ordered } & \text { by Proposition } 3.5 \\
& \Longrightarrow \operatorname{Fun}(V(P), Q)[M] \text { is partially ordered } & \text { by Proposition } 3.10 \\
& \Longrightarrow \operatorname{Mon}(P, Q) \text { is partially ordered. } & \text { by }(3.47)
\end{aligned}
$$

In short, we may argue that $\operatorname{Mon}(P, Q)$ inherits the order-theoretic properties of $Q$. The following result is arguably a corollary of Proposition 3.6: it is a similar result focused on monotone functions.

Proposition 3.11. Let $P$ and $Q$ be preordered sets. Then

$$
\operatorname{Mon}(P, Q)^{*}=\operatorname{Mon}\left(P^{*}, Q^{*}\right) .
$$

Proof. First note that both preordered sets have the same ground set, as

$$
\begin{aligned}
& V\left(\operatorname{Mon}\left(P^{*}, Q^{*}\right)\right) \\
& =\left\{f: V\left(P^{*}\right) \rightarrow V\left(Q^{*}\right) \mid \forall a, b \in V\left(P^{*}\right), a \preceq_{P^{*}} b \Longrightarrow f(a) \preceq_{Q^{*}} f(b)\right\} \quad \text { by (3.48) } \\
& =\left\{f: V(P) \rightarrow V(Q) \mid \forall a, b \in V(P), a \preceq_{P^{*}} b \Longrightarrow f(a) \preceq_{Q^{*}} f(b)\right\} \quad \text { by (3.1) } \\
& =\left\{f: V(P) \rightarrow V(Q) \mid \forall a, b \in V(P), b \preceq_{P} a \Longrightarrow f(b) \preceq_{Q} f(a)\right\} \quad \text { by (3.2) } \\
& =V(\operatorname{Mon}(P, Q)) \quad \text { by (3.48) } \\
& =V\left(\operatorname{Mon}(P, Q)^{*}\right) . \quad \text { by }(3.1)
\end{aligned}
$$

Proposition 3.6 finishes the proof, as for every $f, g \in V\left(\operatorname{Mon}\left(P^{*}, Q^{*}\right)\right)=V\left(\operatorname{Mon}(P, Q)^{*}\right)$,

$$
\begin{aligned}
f \preceq_{\operatorname{Mon}\left(P^{*}, Q^{*}\right)} g & \Longleftrightarrow f \preceq_{\operatorname{Fun}\left(V\left(P^{*}\right), Q^{*}\right)} g & & \text { by }(3.49) \\
& \Longleftrightarrow f \preceq_{\operatorname{Fun}\left(V(P), Q^{*}\right)} g & & \text { by }(3.1) \\
& \Longleftrightarrow f \preceq_{\text {Fun }(V(P), Q)^{*}} g & & \text { by Proposition } 3.6 \\
& \Longleftrightarrow g \preceq_{\text {Fun }(V(P), Q)} f & & \text { by }(3.2) \\
& \Longleftrightarrow g \preceq_{\operatorname{Mon}(P, Q)} f & & \text { by }(3.49) \\
& \Longleftrightarrow f \preceq_{\operatorname{Mon}(P, Q)^{*}} g & & \text { by }(3.2) .
\end{aligned}
$$

Proposition 3.12. Let $P$ be a preordered set, let $X$ be a set, let $F \subseteq V(\operatorname{Fun}(X, P))$, and let $g \in$ $V(\operatorname{Fun}(X, P))$.

$$
\begin{aligned}
& \text { If } g(x) \in \sup _{P}\{f(x) \mid f \in F\} \text { for every } x \in X \text {, then } g \in \sup _{\operatorname{Fun}(X, P)} F \text {, and } \\
& \text { if } g(x) \in \inf _{P}\{f(x) \mid f \in F\} \text { for every } x \in X \text {, then } g \in \inf _{\operatorname{Fun}(X, P)} F \text {. }
\end{aligned}
$$

Proof. By Proposition 3.4, we have that Fun $(X, P)$ is a preordered set. Let $g: X \rightarrow V(P)$ be such that for every $x \in X$,

$$
g(x) \in \sup _{P}\{f(x) \mid f \in F\} .
$$


Then

$$
\begin{aligned}
\left(\forall x \in X, g(x) \in \operatorname{ub}_{P}\{f(x) \mid f \in F\}\right) & \\
& \Longleftrightarrow\left(\forall x \in X, \forall f \in F, f(x) \preceq_{P} g(x)\right) \\
& \Longleftrightarrow\left(\forall f \in F, f \preceq_{\mathrm{Fun}(X, P)} g\right) \\
& \Longleftrightarrow g \in \operatorname{ub}_{\operatorname{Fun}(X, P)} F .
\end{aligned}
$$

Let $h \in \mathrm{ub}_{\mathrm{Fun}(X, P)} F$. We claim that for every $x \in X$,

$$
h(x) \in \operatorname{ub}_{P}\{f(x) \mid f \in F\} .
$$

This holds because

$$
\begin{aligned}
h \in \operatorname{ub}_{\mathrm{Fun}(X, P)} F & \Longleftrightarrow\left(\forall f \in F, f \preceq_{\mathrm{Fun}(X, P)} h\right) \\
& \Longleftrightarrow\left(\forall f \in F, \forall x \in X, f(x) \preceq_{P} h(x)\right) \\
& \Longleftrightarrow\left(\forall x \in X, h(x) \in \mathrm{ub}_{P}\{f(x) \mid f \in F\}\right) .
\end{aligned}
$$

Note that (3.55) implies that $g(x) \preceq_{P} h(x)$ for every $x \in X$, since

$$
\begin{aligned}
g(x) & \in \sup _{P}\{f(x) \mid f \in F\} & & \text { by }(3.54) \\
& \subseteq \operatorname{lb}_{P}\left(\operatorname{ub}_{P}\{f(x) \mid f \in F\}\right) . & & \text { by (3.21b) }
\end{aligned}
$$

As this is true for every $x \in X$, we conclude that $g \preceq_{\mathrm{Fun}(X, P)} h$. As this is true for every $h \in \operatorname{ub}_{\mathrm{Fun}(X, P)} F$, we conclude that $g \in \sup _{\operatorname{Fun}(X, P)} F$. Therefore (3.52) holds.

To prove (3.53), assume $g(x) \in \inf _{P}\{f(x) \mid f \in F\}$ for every $x \in X$. Then

$$
\begin{aligned}
\left(\forall x \in X, g(x) \in \inf _{P}\{f(x) \mid f \in F\}\right) & \Longrightarrow\left(\forall x \in X, g(x) \in \sup _{P^{*}}\{f(x) \mid f \in F\}\right) & & \text { by (3.26a) } \\
& \Longrightarrow g \in \sup _{\operatorname{Fun}\left(X, P^{*}\right)} F & & \text { by (3.52) } \\
& \Longrightarrow g \in \sup _{\operatorname{Fun}(X, P)^{*}} F & & \text { by Proposition 3.6 } \\
& \Longrightarrow g \in \inf _{\mathrm{Fun}(X, P)} F . & & \text { by (3.26a) }
\end{aligned}
$$

A lattice is a partially ordered set $L$ such that for every nonempty finite subset $S \subseteq V(L)$,

$$
\begin{aligned}
& \sup _{L} S \neq \varnothing \text { and } \\
& \inf _{L} S \neq \varnothing .
\end{aligned}
$$

Let $(C, \preceq):=L$. The axiom of choice implies there exist binary operations $\vee, \wedge: C \times C \rightarrow C$ such that

$$
a \vee b \in \sup _{L}\{a, b\} \text { and } a \wedge b \in \inf _{L}\{a, b\}
$$

for every $a, b \in C$. The operation $\vee$ is commonly called the meet of the lattice $L$, and $\wedge$ is commonly called the join of the lattice $L$.

Proposition 3.13. Let $L$ be a lattice. Then $L^{*}$ is also a lattice.

Proof. The statement follows from Proposition 3.2, (3.26), and (3.56).

Proposition 3.14. Let $P$ be a preordered set. Assume that for every $a, b \in V(P)$,

$$
\begin{aligned}
& \sup _{P}\{a, b\} \neq \varnothing \text { and } \\
& \inf _{P}\{a, b\} \neq \varnothing .
\end{aligned}
$$

Then $[P]$ is a lattice. 
Proof. We prove that for every preordered set $P$,

$$
\text { if } \sup _{P}\{a, b\} \neq \varnothing \text { for every } a, b \in V(P),
$$

then $\sup _{P} S \neq \varnothing$ for every nonempty finite $S \subseteq V(P)$.

To prove (3.59), we prove that for every finite set $S$ such that $|S|>1$, for every $a \in S$, and for every $b \in \sup _{P}(S \backslash\{a\})$, we have that

$$
\sup _{P} S \supseteq \sup _{P}\{a, b\} .
$$

Let $x \in \sup _{P}\{a, b\}$. For every $s \in S \backslash\{a\}$,

$$
\begin{array}{ll}
s \preceq_{P} b & \text { since } b \in \sup _{P}(S \backslash\{a\}) \\
\preceq_{P} x, & \text { since } x \in \sup _{P}\{a, b\}
\end{array}
$$

so transitivity of $\preceq_{P}$ implies that $x \in \mathrm{ub}_{P}(S \backslash\{a\})$. As $a \in\{a, b\}$, we conclude that $x \in \mathrm{ub}_{P} S$. Now let $y \in \operatorname{ub}_{P} S$. Using $S \backslash\{a\} \subseteq S$ and (3.31b) we conclude that $y \in \operatorname{ub}_{P}(S \backslash\{a\})$. But then (3.21b) implies that $b \preceq_{P} y$. Furthermore, as $a \in S$, we have that $a \preceq_{P} y$, so we conclude that $y \in \mathrm{ub}_{P}\{a, b\}$. Using (3.21b) again we conclude that $x \preceq_{P} y$. In short, we have that $x \in \operatorname{lb}_{P}\left(\mathrm{ub}_{P} S\right)$, concluding the proof of (3.60) by (3.20).

We now prove (3.59). Let $P$ be a preordered set $\operatorname{such}_{\text {that }} \sup _{P}\{a, b\} \neq \varnothing$ for every $a, b \in V(P)$. Assume $T \subseteq V(P)$ is a nonempty finite set such that $\sup _{P} T=\varnothing$, and further assume that $T$ has minimal cardinality among such sets. Our hypothesis implies that $|T| \geq 3$. Let $a \in T$. By minimality of $T$, there exists $b \in \sup _{P}(T \backslash\{a\})$. But then (3.60) implies that $\sup _{P}\{a, b\}=\varnothing$, as

$$
\varnothing=\sup _{P} T \supseteq \sup _{P}\{a, b\} .
$$

But this contradicts the choice of $T$, as $|T| \geq 3>2 \geq|\{a, b\}|$. We have thus proved (3.59).

Now let $S \subseteq V(P)$ be a nonempty finite set. Then

$$
\begin{aligned}
\sup _{[P]}[S] & =\left[\sup _{P} S\right] & & \text { by }(3.30 \mathrm{a}) \\
& \neq \varnothing . & & \text { by }(3.58 \mathrm{a}) \text { and }(3.59)
\end{aligned}
$$

Moreover,

$$
\begin{aligned}
\inf _{[P]}[S] & =\left[\inf _{P} S\right] & & \text { by }(3.30 \mathrm{~b}) \\
& =\left[\sup _{P^{*}} S\right] & & \text { by }(3.26 \mathrm{a}) \\
& \neq \varnothing . & & \text { by }(3.26 \mathrm{a}),(3.58 \mathrm{~b}), \text { and }(3.59)
\end{aligned}
$$

Proposition 3.3 implies that $[P]$ is a partially ordered class. Since $[\cdot]: V(P) \rightarrow V([P])$ is surjective, we have just proved that $[P]$ satisfies $(3.56)$.

A complete lattice is a lattice $L$ such that for every $S \subseteq V(L)$,

$$
\begin{aligned}
& \sup _{L} S \neq \varnothing, \text { and } \\
& \inf _{L} S \neq \varnothing .
\end{aligned}
$$

As the empty set has an infimum and a supremum, one may prove that complete lattices have a maximum element, i.e., that $\max _{L} V(L)$ is nonempty, and similarly that $\min _{L} V(L)$ is nonempty. Define $\overline{\mathbb{R}}:=([-\infty,+\infty], \leq)$, where $[-\infty,+\infty]$ are the extended reals and $\leq$ is the usual order on it. It is well known that $\overline{\mathbb{R}}$ is a complete lattice, and it is the example the reader should keep in mind throughout this text.

Proposition 3.15. Let $L$ be a complete lattice. Then so is $L^{*}$.

Proof. The statement follows from Proposition 3.13 and (3.26).

Proposition 3.16. Let $L$ be a lattice, and let $X$ be a set. Then $\operatorname{Fun}(X, L)$ is a lattice. 
Proof. Let $F \subseteq V(\operatorname{Fun}(X, L))$ be nonempty and finite. Since $L$ is a lattice, we can use (3.56) and the axiom of choice to conclude that there exist $g, h: X \rightarrow V(L)$ such that

$$
g(x) \in \sup _{L}\{f(x) \mid f \in F\} \text { and } h(x) \in \inf _{L}\{f(x) \mid f \in F\}
$$

for every $x \in X$. Proposition 3.12 finishes the proof.

Proposition 3.17. Let $L$ be a complete lattice, and let $X$ be a set. Then $\operatorname{Fun}(X, L)$ is a complete lattice.

Proof. Let $F \subseteq V(\operatorname{Fun}(X, L))$. Since $L$ is a complete lattice, we can use (3.61) and the axiom of choice to conclude that there exist $g, h: X \rightarrow V(L)$ such that

$$
g(x) \in \sup _{L}\{f(x) \mid f \in F\} \text { and } h(x) \in \inf _{L}\{f(x) \mid f \in F\}
$$

for every $x \in X$. Proposition 3.12 finishes the proof.

Proposition 3.18. Let $L$ be a complete lattice, and let $M \subseteq V(L)$ be such that

$$
M \cap \inf _{L} S \neq \varnothing \text { and } M \cap \sup _{L} S \neq \varnothing
$$

for every $S \subseteq M$. Then $L[M]$ is a complete lattice.

Proof. We prove that for every $S \subseteq M$,

$$
\begin{aligned}
M \cap \inf _{L} S \subseteq \inf _{L[M]} S, \text { and } \\
M \cap \sup _{L} S \subseteq \sup _{L[M]} S .
\end{aligned}
$$

By (3.62), this implies the statement. Note that

$$
\begin{aligned}
\mathrm{lb}_{L[M]} S \subseteq \mathrm{lb}_{L} S & & & \text { by (3.45a) } \\
& \Longrightarrow \mathrm{ub}_{L}\left(\mathrm{lb}_{L} S\right) \subseteq \mathrm{ub}_{L}\left(\mathrm{lb}_{L[M]} S\right) & & \text { by (3.31a) } \\
& \Longrightarrow M \cap \mathrm{ub}_{L}\left(\mathrm{lb}_{L} S\right) \subseteq M \cap \mathrm{ub}_{L}\left(\mathrm{lb}_{L[M]} S\right) & & \\
& \Longrightarrow M \cap \mathrm{ub}_{L}\left(\mathrm{lb}_{L} S\right) \subseteq \mathrm{ub}_{L[M]}\left(\operatorname{lb}_{L[M]} S\right) . & & \text { by (3.45b) }
\end{aligned}
$$

Combining with (3.23b), we conclude that

$$
M \cap \inf _{L} S \subseteq \mathrm{ub}_{L[M]}\left(\mathrm{lb}_{L[M]} S\right) .
$$

Moreover

$$
M \cap \inf _{L} S \subseteq \operatorname{lb}_{L[M]} S,
$$

as

$$
\begin{array}{rlr}
\inf _{L} S \subseteq \operatorname{lb}_{L} S & & \text { by (3.23a) } \\
& \Longrightarrow M \cap \inf _{L} S \subseteq M \cap \mathrm{lb}_{L} S & \\
& \Longrightarrow M \cap \inf _{L} S \subseteq \mathrm{lb}_{L[M]} S . &
\end{array}
$$

Combining (3.63) and (3.64), we conclude that

$$
\begin{aligned}
M \cap \inf _{L} S & \subseteq\left(\operatorname{lb}_{L[M]} S\right) \cap\left(\operatorname{ub}_{L[M]}\left(\operatorname{lb}_{L[M]} S\right)\right) \\
& =\max _{L[M]}\left(\operatorname{lb}_{L[M]} S\right) \\
& =\inf _{L[M]} S .
\end{aligned}
$$

Then (3.62b) follows:

$$
\begin{aligned}
M \cap \sup _{L} S & =M \cap \inf _{L^{*}} S & & \text { by }(3.26 \mathrm{~b}) \\
& \subseteq \inf _{L^{*}[M]} S & & \text { by }(3.62 \mathrm{a}) \\
& =\inf _{L[M]^{*}} S & & \text { by Proposition } 3.8 \\
& =\sup _{L[M]} S . & & \text { by }(3.26 \mathrm{~b})
\end{aligned}
$$


Proposition 3.19. Let $P$ be a preordered set, and let $L$ be a complete lattice. Then $\operatorname{Mon}(P, L)$ is a complete lattice.

Proof. Set $M:=\{f: V(P) \rightarrow V(L) \mid f$ is monotone $\}$ and let $F \subseteq M$.

We first prove that

$$
g \in M \cap \inf _{\operatorname{Fun}(V(P), L)} F
$$

for every $g: V(P) \rightarrow V(L)$ such that

$$
g(x) \in \inf _{L}\{f(x) \mid f \in F\}
$$

for every $x \in V(P)$. Let $g: V(P) \rightarrow L$ satisfy (3.66). Let $x, y \in V(P)$ be such that $x \preceq_{P} y$. For every $f \in F$,

$$
\begin{array}{rlrl}
g(x) & \preceq_{L} f(x) & & \text { by }(3.66) \text { and (3.23a) } \\
\preceq_{L} f(y) . & & \text { as } f \text { is monotone }
\end{array}
$$

Since this holds for every $f \in F$, we have that $g(x) \in \mathrm{lb}_{L}\{f(y) \mid f \in F\}$. This implies that $g(x) \preceq_{L} g(y)$, since

$$
\begin{aligned}
g(y) & \in \inf _{L}\{f(y) \mid f \in F\} & & \text { by }(3.66) \\
& \subseteq \operatorname{ub}_{L}\left(\operatorname{lb}_{L}\{f(y) \mid f \in F\}\right) . & & \text { by }(3.23 \mathrm{~b})
\end{aligned}
$$

Hence $g \in M$. Using (3.66) and (3.53) we conclude that $g \in \inf _{\mathrm{Fun}(V(P), L)} F$, so (3.65) follows.

We now prove that

$$
h \in M \cap \sup _{\operatorname{Fun}(V(P), L)} F
$$

for every $h: V(P) \rightarrow V(L)$ such that

$$
h(x) \in \sup _{L}\{f(x) \mid f \in F\}
$$

for every $x \in V(P)$. This follows from (3.65), since

$$
\begin{array}{rlrl}
\left(\forall x \in V(P), h(x) \in \sup _{L}\{f(x) \mid f \in F\}\right) & & & \text { by (3.68) } \\
& \Longrightarrow\left(\forall x \in V(P), h(x) \in \inf _{L^{*}}\{f(x) \mid f \in F\}\right) & \text { by (3.26b) } \\
& \Longrightarrow h \in M \cap \inf _{\operatorname{Fun}\left(V(P), L^{*}\right)} F & & \text { by (3.65) } \\
& \Longrightarrow h \in M \cap \inf _{\operatorname{Fun}(V(P), L)^{*}} F & & \text { by Proposition 3.6 } \\
& \Longrightarrow h \in M \cap \sup _{\operatorname{Fun}(V(P), L)} F . & \text { by (3.26b) }
\end{array}
$$

We have that $\operatorname{Fun}(V(P), L)$ is a complete lattice by Proposition 3.17. Using (3.61) and the axiom of choice, we have that for every $F \subseteq M$ there exist $g, h: V(P) \rightarrow V(L)$ in $\operatorname{Fun}(V(P), L)$ such that (3.66) and (3.68) hold. Therefore, for every $F \subseteq M$, the sets in the RHS of (3.65) and (3.67) are nonempty, so Proposition 3.18 and (3.47) finish the proof.

Proposition 3.19 presents the core object we shall study on Section 3.3. Before going on to the next section, let us prove two small results to make our exposition simpler.

Proposition 3.20. Let $P$ be a partially ordered set, and let $S \subseteq V(P)$. Then $\left|\min _{P} S\right| \leq 1$ and $\left|\max _{P} S\right| \leq 1$.

Proof. Let $a, b \in \min _{P} S$. By (3.14) and (3.15) we have that $a \preceq b$, since $a \in \operatorname{lb}_{P} S$ and $b \in S$. Using (3.14) and (3.15) again, we see that $b \preceq a$, since $b \in \operatorname{lb}_{P} S$ and $a \in S$. As $P$ is partially ordered, we conclude that $a=b$. Theorem 3.1 and (3.25b) finish the proof:

$$
\left|\max _{P} S\right|=\left|\min _{P^{*}} S\right| \leq 1 .
$$

Corollary 3.21. Let $P$ be a partially ordered set, and let $S \subseteq V(P)$. Then $\left|\inf _{P} S\right| \leq 1$ and $\left|\sup _{P} S\right| \leq 1$. 
Proof. Proposition 3.20 and (3.18) imply that

$$
\left|\inf _{P} S\right|=\left|\max _{P}\left(\operatorname{lb}_{P} S\right)\right| \leq 1 .
$$

Hence (3.26b) finishes the proof:

$$
\left|\sup _{P} S\right|=\left|\inf _{P^{*}} S\right| \leq 1
$$

Let $L$ be a complete lattice. Both (3.61) and Corollary 3.21 imply that, for every $S \subseteq V(L)$, there exist $x, y \in V(L)$ such that

$$
\inf _{L} S=\{x\} \text { and } \sup _{L} S=\{y\} .
$$

Let $\overline{\inf }: \mathcal{P}(V(L)) \rightarrow V(L)$ and $\overline{\sup }: \mathcal{P}(V(L)) \rightarrow V(L)$ be the unique functions such that

$$
\overline{\inf }(S) \in \inf _{L} S \text { and } \overline{\sup }(S) \in \sup _{L} S
$$

for every $S \in \mathcal{P}(V(L))$. The functions $\overline{\text { inf }}$ and $\overline{\text { sup }}$ capture the usual notion of infimum and supremum as taking values in the complete lattice itself, and not as subsets of the lattice. From now on, we actually refer to $\overline{\inf }(S)$ and $\overline{\sup }(S)$ using $\inf _{L} S$ and $\sup _{L} S$.

\subsection{Graph Universes}

A homomorphism monotone function is a function on graphs. Since $\mathcal{O}(\mathrm{Graph})$ is a proper class, every homomorphism monotone function is a proper class. As homomorphism monotone functions are classes, NBG does not allow us to talk about the "collection" of all homomorphism monotone functions. Therefore, we seem to be in a sorry state of affairs, in which a theory developed around a motivating example does not apply to the motivating example. This brief subsection addresses this issue.

We start by showing what is trivial to do in NBG.

Proposition 3.22. Let $\mathrm{C}$ be a category. Denote by $\mathcal{O}(\mathrm{C})$ the class of objects of $\mathrm{C}$, and denote by $\operatorname{Hom}_{\mathrm{C}}(A, B)$ the set of homomorphisms with domain $A$ and codomain $B$, for every $A, B \in \mathcal{O}(\mathrm{C})$. The class

$$
\rightarrow^{\mathrm{C}}:=\left\langle(a, b) \mid \exists a, b \in \mathcal{O}(\mathrm{C}), \operatorname{Hom}_{\mathrm{C}}(a, b) \neq \varnothing\right\rangle
$$

is a reflexive and transitive relation with domain and codomain $\mathcal{O}(\mathrm{C})$.

Proof. The identity homomorphisms certify that $A \rightarrow^{\mathrm{C}} A$ for every object $A \in \mathcal{O}(\mathrm{C})$. If $f \in \operatorname{Hom}_{\mathrm{C}}(A, B)$ and $g \in \operatorname{Hom}_{\mathrm{C}}(B, C)$ for objects $A, B, C \in \mathcal{O}(\mathrm{C})$, then $g \circ f \in \operatorname{Hom}_{\mathrm{C}}(A, C)$. Hence, the relation $\rightarrow^{\mathrm{C}}$ is transitive.

Similar to our situation with categories, a definition of preordered class lies outside of set theory. Proposition 3.22 shows that it is not so unreasonable to think about graphs as a preordered set. Although we could define a preordered class in a manner similar to how we defined a category, this would not get us much further than Proposition 3.22. Let $P$ be a preordered set, and let $X$ be a set. Recalling (3.36), we see that the ground set of $\operatorname{Fun}(X, P)$ is the set of all functions from $X$ to $V(P)$. This construction is only possible under NBG if $X$ is a set.

Let $S$ be a set. Recall the definition of the complete graph (2.24). Define the $S$-graph universe by

$$
\mathcal{U}_{S}:=\left\{H \mid H \text { is a subgraph of } K_{S}\right\} .
$$

The most important feature of a graph universe is that it is a set. Consequently, we can work with

$$
\operatorname{Fun}\left(\mathcal{U}_{S}, \overline{\mathbb{R}}\right)
$$

which contains all the functions from graphs in $\mathcal{U}_{S}$. Furthermore, we may equip $\mathcal{U}_{S}$ with the restriction of the homomorphic relation on graphs, obtaining a preordered set. Formally, define

$$
P:=\left(\mathcal{U}_{S},\left\{\{G, H\} \mid G, H \in \mathcal{U}_{S}, G \rightarrow H\right\}\right) .
$$


We now obtain a set which contains all homomorphism monotone functions we care about:

$$
\operatorname{Mon}(P, \overline{\mathbb{R}}) .
$$

This shows that if we develop our graph theory inside of the universe $\mathcal{U}_{S}$, we may readily apply our developments from Section 3.1. As the reader shall see, all of our graphs fit inside of $\mathcal{U}_{\mathbb{R}}$. Any reader convinced by the construction above that we do not risk facing paradoxes is free to advance to Section 3.3. However, I am not satisfied with this situation: our graph theory was not developed inside of $\mathcal{U}_{\mathbb{R}}$.

We start by being explicit about our problem with "developing graph theory inside $\mathcal{U}_{\mathbb{R}}$ ". Imagine for a moment that we desire to state and prove some result showing that graphs form a lattice. In this task, two natural definitions arise. Let $G$ and $H$ be graphs. Recall from (2.4) the definition of the disjoint union of sets. Define the disjoint union of $G$ and $H$ as the graph $G \sqcup H$ such that

$$
\begin{aligned}
V(G \sqcup H) & :=V(G) \sqcup V(H), \\
E(G \sqcup H) & :=\left\{\left\{\operatorname{tag}_{0}(i), \operatorname{tag}_{0}(j)\right\} \mid i j \in E(G)\right\} \\
& \cup\left\{\left\{\operatorname{tag}_{1}(i), \operatorname{tag}_{1}(j)\right\} \mid i j \in E(H)\right\} .
\end{aligned}
$$

Define the categorical product of $G$ and $H$ as the graph $G \times H$ such that

$$
\begin{aligned}
& V(G \times H):=V(G) \times V(H), \\
& E(G \times H):=\{\{(i, k),(j, \ell)\} \mid i j \in E(G), k \ell \in E(H)\} .
\end{aligned}
$$

These definitions define the meet and join of our desired lattice on graphs. Since we need a lattice to be a set, consider for a moment that we are working with graphs in $\mathcal{U}_{\mathbb{R}}$. The vertex set of every graph in $\mathcal{U}_{\mathbb{R}}$ is a set of real numbers. Let $G, H \in \mathcal{U}_{\mathbb{R}}$. The categorical product $G \times H$ is not and element of $\mathcal{U}_{\mathbb{R}}$, since

$$
V(G \times H)=V(G) \times V(H) \subseteq \mathbb{R} \times \mathbb{R} \nsubseteq \mathbb{R}
$$

by (3.73). A similar problem happens to the coproduct defined in (3.72), and to almost any other basic graph-theoretic construction beyond taking subgraphs. On the other hand, it is clear $G \times H$ is $i s o m o r p h i c$ to some graph in $\mathcal{U}_{\mathbb{R}}$, so that one could simply append our definition of cartesian product with some phrase along the lines of "and take a graph in $\mathcal{U}_{\mathbb{R}}$ isomorphic to $G \times H$ ". The following argument is my best attempt at implementing this idea in NBG.

Let $S$ be a set. We now introduce a new category. Define the class

$$
\left.\mathcal{O}\left(\text { Graph }_{\leq S}\right):=\langle G \in \mathcal{O}(\text { Graph })| \text { there exists } f: V(G) \rightarrow S \text { injective }\right\rangle .
$$

Just as FinSet was defined as a subcategory of Set, we now simply define Graph $<S$ as a subcategory of Graph: the homomorphisms, composition, and identity are the ones from Graph restricted to the objects in $\mathcal{O}\left(\mathrm{Graph}_{\leq S}\right)$. The axiom of global choice [76, Section 4] of NBG states that there exists a class function 'choice' whose domain is every nonempty set and such that choice $(S) \in S$ for every set $S \neq \varnothing$. Define

$$
\ell_{\leq S}:=\text { choice } \circ\left(G \in \mathcal{O}\left(\text { Graph }_{\leq S}\right) \mapsto\{f: V(G) \rightarrow S \mid f \text { injective }\}\right)
$$

Note how (3.74) implies that we can indeed use the choice function, as there are no empty sets in the image of the class function to the right of o. Therefore, for every $G \in \mathcal{O}\left(\operatorname{Graph}_{\leq S}\right)$, we have that $\ell_{\leq S}(G): V(G) \rightarrow S$ is an injective function. This is a labeling of the vertices of a graph that we can use to obtain a graph in our universe. We then obtain a class function which chooses for every graph in $\mathcal{O}\left(\mathrm{Graph}_{\leq S}\right)$ an isomorphic graph in $\mathcal{U}_{S}$ : define

$$
\mathcal{L}_{\leq S}:=\left(G:=(V, E) \in \mathcal{O}\left(\operatorname{Graph}_{\leq S}\right) \mapsto(\ell(V),\{\ell(i) \ell(j) \mid i j \in E\}) \text { where } \ell:=\ell_{\leq S}(G)\right) .
$$

Let $S$ be a set. For every graph $G \in \mathcal{O}\left(\mathrm{Graph}_{\leq S}\right)$, we have that

$$
\begin{gathered}
\mathcal{L}_{\leq S}(G) \in \mathcal{U}_{S} \text { and } \\
\ell_{\leq S}(G) \in \operatorname{Hom}\left(G, \mathcal{L}_{\leq S}(G)\right) \text { is an isomorphism. }
\end{gathered}
$$


Our plan is to develop our graph theory inside of $\mathrm{Graph}_{\leq S}$. Note that we may keep our definitions untouched: the cartesian product of two graphs in $\mathrm{Graph}_{\leq \mathbb{R}}$ is another graph in $\mathrm{Graph}_{\leq \mathbb{R}}$ because there exists an injective function $\mathbb{R} \times \mathbb{R} \rightarrow \mathbb{R}$. However, this very convenience implies that $\mathcal{O}\left(\mathrm{Graph}_{\leq S}\right)$ is a proper class, so we still cannot consider all the homomorphism monotone functions on $\mathcal{O}\left(\mathrm{Graph}_{\leq S}\right)$. But we are finally in a position to circumvent this issue.

Let $S$ be a set. Let $\beta: \mathcal{O}($ Graph $) \rightarrow[-\infty,+\infty]$ be a homomorphism monotone function. Define $\tilde{\beta}:=\beta \uparrow_{\mathcal{U}_{S}}$. We claim that

$$
\beta \uparrow_{\mathcal{O}\left(\mathrm{Graph}_{\leq S}\right)}=\tilde{\beta} \circ \mathcal{L}_{\leq S} .
$$

This is true, as for every graph $G \in \mathrm{Graph}_{\leq S}$,

$$
\begin{aligned}
\beta(G) & =\beta\left(\mathcal{L}_{\leq S}(G)\right) & & \text { by }(3.78) \text { and because } \beta \text { is homomorphism monotone } \\
& =\tilde{\beta}\left(\mathcal{L}_{\leq S}(G)\right) . & & \text { by }(3.77) \text { and the definition of restriction }
\end{aligned}
$$

What is important for our discussion is that $\tilde{\beta}$ is a set function, as its domain is the set $\mathcal{U}_{S}$. If we define $P$ as in (3.70), we have that

$$
\tilde{\beta} \in \operatorname{Mon}(P, \overline{\mathbb{R}}),
$$

so we can safely apply our theory from Section 3.1 to the preordered set $\operatorname{Mon}(P, \overline{\mathbb{R}})$, and use (3.79) to be sure that we indeed have the objects we desire.

Henceforth we define that every graph we work with is an element of $\mathcal{O}\left(\mathrm{Graph}_{\leq \mathbb{R}}\right)$. Let $P$ be defined as in (3.70). We will refer to $P$ as the preordered set of graphs under the homomorphic relation. A function is homomorphism monotone if it is an element of $\operatorname{Mon}(P, \overline{\mathbb{R}})$. We may implicitly promote a homomorphism monotone function to a class function: if $\beta \in \operatorname{Mon}(P, \overline{\mathbb{R}})$, we write $\beta: \mathcal{O}\left(\operatorname{Graph}_{\leq \mathbb{R}}\right) \rightarrow[-\infty,+\infty]$ to refer to the class function $\beta \circ \mathcal{L}_{\leq \mathbb{R}}$. In short, we keep our definitions, our theory, and our language, and NBG shields us from potential paradoxes.

Theorem 3.23. Let $P$ denote the preordered set of graphs under the homomorphic relation. Then $[P]$ is a lattice.

Proof. Let $G$ and $H$ be graphs in $\mathcal{U}_{\mathbb{R}}$. Set $G \vee H:=\mathcal{L}_{\leq \mathbb{R}}(G \sqcup H) \in \mathcal{U}_{\mathbb{R}}$. We first prove that

$$
G \vee H \in \sup _{P}\{G, H\} .
$$

Both (3.72b) and (3.78) imply that $\left(\ell_{\leq \mathbb{R}}(G \sqcup H) \circ \operatorname{tag}_{0}\right): V(G) \rightarrow V(G \vee H)$ belongs to $\operatorname{Hom}(G, G \vee H)$. Both (3.72c) and (3.78) imply that $\left(\ell_{\leq \mathbb{R}}(G \sqcup H) \circ \operatorname{tag}_{1}\right): V(H) \rightarrow V(G \vee H)$ belongs to Hom $(H, G \vee H)$. Therefore $G \preceq_{P}(G \vee H)$ and $H \preceq_{P}(G \vee H)$, so

$$
G \vee H \in \mathrm{ub}_{P}\{G, H\} .
$$

Let $K \in \operatorname{ub}_{P}\{G, H\}$, let $f \in \operatorname{Hom}(G, K)$, and let $g \in \operatorname{Hom}(H, K)$. We claim that

$$
f \sqcup g \in \operatorname{Hom}(G \sqcup H, K) .
$$

We now prove (3.81). Let $k \ell \in E(G \sqcup H)$. By (3.72), either $k \ell=\{(i, 0),(j, 0)\}$ for some $i j \in E(G)$, or $k \ell=\{(i, 1),(j, 1)\}$ for some $i j \in E(H)$. In the first case, we have that

$$
\begin{aligned}
\{(f \sqcup g)(k),(f \sqcup g)(\ell)\} & =\{(f \sqcup g)(i, 0),(f \sqcup g)(j, 0)\} \\
& =\{f(i), f(j)\} \\
& \in E(K) .
\end{aligned}
$$$$
\text { since } f \in \operatorname{Hom}(G, K) \text { and } i j \in E(G)
$$

In the second case, we have that

$$
\begin{aligned}
\{(f \sqcup g)(k),(f \sqcup g)(\ell)\} & =\{(f \sqcup g)(i, 1),(f \sqcup g)(j, 1)\} \\
& =\{g(i), g(j)\} \\
& \in E(K) .
\end{aligned}
$$
since $g \in \operatorname{Hom}(H, K)$ and $i j \in E(H)$ 
This concludes the proof of (3.81). By combining (3.78) and (3.81) we conclude that

$$
G \vee H \preceq_{P} K \text {. }
$$

As $K \in \mathrm{ub}_{P}\{G, H\}$ was arbitrary, we have that $G \vee H \in \operatorname{lb}_{P}\left(\mathrm{ub}_{P}\{G, H\}\right)$, thus proving (3.80) by (3.20).

Set $G \wedge H:=\mathcal{L}_{\leq \mathbb{R}}(G \times H)$. We claim that

$$
G \wedge H \in \inf _{P}\{G, H\}
$$

Define $\lambda: V(G \times H) \rightarrow V(G)$ by $\lambda(i, j):=i$ for every $(i, j) \in V(G \times H)$. Define $\rho: V(G \times H) \rightarrow V(H)$ by $\rho(i, j):=j$ for every $(i, j) \in V(G \times H)$. Note that $\lambda \in \operatorname{Hom}(G \times H, G)$, as for every $\{(i, k),(j, \ell)\} \in E(G \times H)$,

$$
\begin{aligned}
\{\lambda(i, k), \lambda(j, \ell)\} & =\{i, j\} & \\
& \in E(G) . & \text { by }(3.73 \mathrm{~b})
\end{aligned}
$$

Similarly, we have that $\rho \in \operatorname{Hom}(G \times H, H)$, as for every $\{(i, k),(j, \ell)\} \in E(G \times H)$,

$$
\begin{aligned}
\{\rho(i, k), \rho(j, \ell)\} & =\{k, \ell\} & \\
& \in E(H) . & \text { by }(3.73 \mathrm{~b})
\end{aligned}
$$

Using (3.78) we conclude that both $G \wedge H \preceq_{P} G$ and $G \wedge H \preceq_{P} H$, so

$$
G \wedge H \in \operatorname{lb}_{P}\{G, H\} .
$$

Let $K \in \operatorname{lb}_{P}\{G, H\}$, let $f \in \operatorname{Hom}(K, G)$, and let $g \in \operatorname{Hom}(K, H)$. Define $f \times g: V(K) \rightarrow V(G \times H)$ by $(f \times g)(k):=(f(k), g(k))$ for every $k \in V(K)$. We claim that

$$
f \times g \in \operatorname{Hom}(K, G \times H) .
$$

Let $i j \in E(K)$. We have that $f(i) f(j) \in E(G)$ and $g(i) g(j) \in E(H)$, since $f \in \operatorname{Hom}(K, G)$ and $g \in$ $\operatorname{Hom}(K, H)$. Therefore

$$
\{(f \times g)(i),(f \times g)(j)\}=\{(f(i), g(i)),(f(j), g(j))\}
$$$$
\in E(G \times H) \quad \text { by }(3.73 b) \text {. }
$$

Thus (3.83) holds. Combining (3.83) and (3.78) we conclude that

$$
K \preceq_{P} G \wedge H .
$$

As $K \in \operatorname{lb}_{P}\{G, H\}$ was arbitrary, we conclude that $G \wedge H \in \mathrm{ub}_{P}\left(\operatorname{lb}_{P}\{G, H\}\right)$, thus proving (3.82) by (3.22). Proposition 3.14, (3.80), and (3.82) finish the proof.

\subsection{Generalized Clique and Coloring Numbers}

The definition of graph homomorphism is commonly motivated as a generalization of graph colorings. Unsurprisingly, there are several "coloring-like" graph parameters which can be formulated using graph homomorphisms. This chapter as a whole studies the similarities among such formulations, and relates them with their "clique-like" counterparts. This section in particular utilizes the results from Section 3.1 to formulate a common framework for the parameters we wish to study, formally capturing the duality between them.

Let $G$ be a graph, and let $n \in \mathbb{N}$. Recall that a homomorphism from $K_{n}$ to $G$ exists if and only if there exists a clique of size $n$ in $G$, whereas a homomorphism from $G$ to $K_{n}$ exists if and only if there exists a coloring of $G$ using $n$ colors. Hence

$$
\begin{aligned}
& \omega(G)=\sup \left\{n \in \mathbb{N} \mid K_{n} \rightarrow G\right\}, \\
& \chi(G)=\inf \left\{n \in \mathbb{N} \mid G \rightarrow K_{n}\right\} .
\end{aligned}
$$


Both statements may be taken as definitions of the corresponding graph parameters (as we have done in (1.4) and (1.1), respectively). Equations (3.84) are the hub of this chapter: we wish to study variations of the clique and coloring numbers which can be formulated in similar terms. Two introductory texts in graph homomorphisms already present a similar perspective - see [57, Chapter 6] and [52, Section 4]. However, both works are skewed towards (3.84b), presenting only variations of graph coloring and thus making no attempt to uniformly work with clique and coloring numbers.

Recalling Chapter 1, we can discuss the role of (3.84) in a language developed for reasoning. We start by stating the obvious: it is standard practice among researchers to adapt techniques developed for the chromatic number to the clique number, and vice versa. There is a real, well defined duality expressed in (3.84), and it is not hard to find examples of works exploiting it. We therefore categorize "graph homomorphism optimization duality", which is exemplified by (3.84), as an useful intuition yet to be captured by formalization. A framework to express the basic properties of "graph homomorphism optimization duality" becomes even more important given the several similar examples throughout the literature we survey in Section 3.4. Having developed a solid order-theoretic foundation in Section 3.1, we now attempt to dissect (3.84) into its constituents, to break it into smaller dualities, so as to make clear what comes naturally, and what must be earned. In short, we now attempt to condensate "graph homomorphism optimization duality" from an ad hoc research guideline into a cohesive theory.

Definition 3.24. Let $P$ be a preordered set, let $Q \subseteq V(P)$, and let $L$ be a complete lattice. The generalized clique number is the function $\omega: V(\operatorname{Fun}(Q, L)) \rightarrow V(\operatorname{Mon}(P, L))$ defined by

$$
\omega_{g}:=\inf _{\operatorname{Mon}(P, L)}\left\{f \mid g \preceq_{\operatorname{Fun}(Q, L)} f \circ \iota_{Q}\right\}
$$

for every $g \in V(\operatorname{Fun}(Q, L))$.

Definition 3.25. Let $P$ be a preordered set, let $Q \subseteq V(P)$, and let $L$ be a complete lattice. The generalized coloring number is the function $\chi: V(\operatorname{Fun}(Q, L)) \rightarrow V(\operatorname{Mon}(P, L))$ defined by

$$
\chi_{g}:=\sup _{\operatorname{Mon}(P, L)}\left\{f \mid f \circ \iota_{Q} \preceq_{\mathrm{Fun}(Q, L)} g\right\}
$$

for every $g \in V(\operatorname{Fun}(Q, L))$.

We make a small remark on the notation used. Let $P$ be a preordered set, let $Q \subseteq V(P)$, let $L$ be a complete lattice, and let $g \in V(P) \rightarrow V(L)$. When we write

$$
\inf _{\operatorname{Mon}(P, L)}\left\{f \mid g \preceq_{\operatorname{Fun}(P, L)} f \circ \iota_{Q}\right\}
$$

what is actually meant is

$$
\inf _{\operatorname{Mon}(P, L)}\left\{f \in V(\operatorname{Mon}(P, L)) \mid g \preceq_{\operatorname{Fun}(Q, L)} f \circ \iota_{Q}\right\} .
$$

The repetition of $\operatorname{Mon}(P, L)$ is unavoidable if one wants the set and the infimum to be well defined expressions by themselves. We must annotate the infimum with the lattice to apply results like (3.26), and we must have " $f \in V(\operatorname{Mon}(P, L))$ " to avoid unbounded set comprehensions. However, as it is possible to infer the set comprehension given the expressions in Definitions 3.24 and 3.25, we adopt this simpler notation. Note that we are not using the axiom of predicative comprehension: we are working exclusively with sets.

Let $P$ be a preordered set, let $Q \subseteq V(P)$, and let $g: Q \rightarrow \overline{\mathbb{R}}$. Note that the function $f \circ \iota_{Q}$ is the restriction of $f$ to $Q$. Unpacking the preorder on $\operatorname{Fun}(Q, \overline{\mathbb{R}})$, we conclude that $\omega_{g}$ is the infimum over every monotone function $f: V(P) \rightarrow V(\overline{\mathbb{R}})$ such that $g(q) \preceq_{\mathbb{R}} f(q)$ for every $q \in Q$. A similar argument casts $\chi_{g}$ as the supremum over every monotone function with domain $V(P)$ and codomain $V(\overline{\mathbb{R}})$ which is pointwise at most $g$ on $Q$. Since the functions $\omega_{g}$ and $\chi_{g}$ are an infimum and a supremum of functions in $\operatorname{Mon}(P, \overline{\mathbb{R}})$, Proposition 3.19 implies that

$$
\omega_{g} \text { and } \chi_{g} \text { are }(P, \overline{\mathbb{R}}) \text {-monotone. }
$$

Therefore, both $\omega_{g}$ and $\chi_{g}$ can be seen as ways of "extending" the domain of a function $g$ from $Q$ to $V(P)$ and obtain a monotone function. Note that the definitions of $\omega_{g}$ and $\chi_{g}$ do not guarantee that these functions are actually extensions of $g$; in other words,

$$
\text { it may not hold that } \omega_{g} \circ \iota_{Q}=\chi_{g} \circ \iota_{Q}=g \text {. }
$$


Our upcoming developments in this subsection will provide a better understanding of this issue.

We now formally relate Definitions 3.24 and 3.25. Let $P$ be a preordered set, let $Q \subseteq V(P)$, and let $L$ be a complete lattice. By (3.1) we have that $Q \subseteq V\left(P^{*}\right)$. Denote by $\iota_{Q}^{*}: V\left(P^{*}[Q]\right) \rightarrow V\left(P^{*}\right)$ the function defined by $\iota_{Q}^{*}(q)=q$ for every $q \in Q$. Proposition 3.8 and (3.1) imply

$$
V\left(P^{*}[Q]\right)=V\left(P[Q]^{*}\right)=V(P[Q]) .
$$

Hence, both $\iota_{Q}$ and $\iota_{Q}^{*}$ have the same domain. It is then obvious that

$$
\iota_{Q}=\iota_{Q}^{*} .
$$

By Proposition 3.15 we have that $L^{*}$ is a complete lattice. Let $\omega^{*}$ denote the generalized clique number defined by $P^{*}, Q$, and $L^{*}$, i.e., define

$$
\omega_{g}^{*}:=\inf _{\operatorname{Mon}\left(P^{*}, L^{*}\right)}\left\{f \mid g \preceq_{\text {Fun }\left(Q, L^{*}\right)} f \circ \iota_{Q}^{*}\right\}
$$

for every $g \in V\left(\operatorname{Fun}\left(Q, L^{*}\right)\right)$. Similarly, let $\chi^{*}$ denote the generalized coloring number defined by $P^{*}, Q$, and $L^{*}$, i.e., define

$$
\chi_{g}^{*}:=\sup _{\operatorname{Mon}\left(P^{*}, L^{*}\right)}\left\{f \mid f \circ \iota_{Q}^{*} \preceq_{\mathrm{Fun}\left(Q, L^{*}\right)} g\right\}
$$

for every $g \in V\left(\operatorname{Fun}\left(Q, L^{*}\right)\right)$. Note that both $\omega^{*}$ and $\chi^{*}$ are functions with domain $V\left(\operatorname{Fun}\left(Q, L^{*}\right)\right)$ and codomain $V\left(\operatorname{Mon}\left(P^{*}, L^{*}\right)\right)$. Proposition 3.6 and (3.1) imply

$$
V\left(\operatorname{Fun}\left(Q, L^{*}\right)\right)=V\left(\operatorname{Fun}(Q, L)^{*}\right)=V(\operatorname{Fun}(Q, L)) .
$$

Similarly, Proposition 3.11 and (3.1) imply

$$
V\left(\operatorname{Mon}\left(P^{*}, L^{*}\right)\right)=V\left(\operatorname{Mon}(P, L)^{*}\right)=V(\operatorname{Mon}(P, L)) .
$$

Hence, we may write

$$
\omega^{*}, \chi^{*}: V(\operatorname{Fun}(Q, L)) \rightarrow V(\operatorname{Mon}(P, L)) .
$$

Since $\omega^{*}, \chi^{*}, \omega$, and $\chi$ have the same domain, we may check if such functions are equal. For every $g \in V(\operatorname{Fun}(Q, L))$,

$$
\begin{aligned}
\omega_{g}^{*} & =\inf _{\operatorname{Mon}\left(P^{*}, L^{*}\right)}\left\{f \mid g \preceq_{\operatorname{Fun}\left(Q, L^{*}\right)} f \circ \iota_{Q}^{*}\right\} & & \text { by }(3.88 \mathrm{a}) \\
& =\inf _{\operatorname{Mon}(P, L)^{*}}\left\{f \mid g \preceq_{\operatorname{Fun}\left(Q, L^{*}\right)} f \circ \iota_{Q}^{*}\right\} & & \text { by Proposition } 3.11 \\
& =\sup _{\operatorname{Mon}(P, L)}\left\{f \mid g \preceq_{\text {Fun }\left(Q, L^{*}\right)} f \circ \iota_{Q}^{*}\right\} & & \text { by }(3.26 \mathrm{~b}) \\
& =\sup _{\operatorname{Mon}(P, L)}\left\{f \mid g \preceq_{\operatorname{Fun}(Q, L)^{*}} f \circ \iota_{Q}^{*}\right\} & & \text { by Proposition } 3.6 \\
& =\sup _{\operatorname{Mon}(P, L)}\left\{f \mid f \circ \iota_{Q}^{*} \preceq_{\operatorname{Fun}(Q, L)} g\right\} & & \text { by }(3.2) \\
& =\sup _{\operatorname{Mon}(P, L)}\left\{f \mid f \circ \iota_{Q} \preceq_{\operatorname{Fun}(Q, L)} g\right\} & & \text { by }(3.87) \\
& =\chi_{g} . & & \text { by Definition } 3.25
\end{aligned}
$$

Dually, for every $g \in V(\operatorname{Fun}(Q, L))$,

$$
\begin{aligned}
\chi_{g}^{*} & =\sup _{\operatorname{Mon}\left(P^{*}, L^{*}\right)}\left\{f \mid f \circ \iota_{Q}^{*} \preceq_{\mathrm{Fun}\left(Q, L^{*}\right)} g\right\} & & \text { by }(3.88 \mathrm{~b}) \\
& =\sup _{\operatorname{Mon}(P, L)^{*}}\left\{f \mid f \circ \iota_{Q}^{*} \preceq_{\mathrm{Fun}\left(Q, L^{*}\right)} g\right\} & & \text { by Proposition } 3.11 \\
& =\inf _{\operatorname{Mon}(P, L)}\left\{f \mid f \circ \iota_{Q}^{*} \preceq_{\mathrm{Fun}\left(Q, L^{*}\right)} g\right\} & & \text { by }(3.26 \mathrm{a}) \\
& =\inf _{\operatorname{Mon}(P, L)}\left\{f \mid f \circ \iota_{Q}^{*} \preceq_{\operatorname{Fun}(Q, L)^{*}} g\right\} & & \text { by Proposition } 3.6 \\
& =\inf _{\operatorname{Mon}(P, L)}\left\{f \mid g \preceq_{\operatorname{Fun}(Q, L)} f \circ \iota_{Q}^{*}\right\} & & \text { by }(3.2) \\
& =\inf _{\operatorname{Mon}(P, L)}\left\{f \mid g \preceq_{\operatorname{Fun}(Q, L)} f \circ \iota_{Q}\right\} & & \text { by }(3.87) \\
& =\omega_{g} . & & \text { by Definition } 3.24
\end{aligned}
$$


In short, we have a precise duality statement relating the generalized clique number and the generalized coloring number: for every $g \in V(\operatorname{Fun}(Q, L))$,

$$
\begin{aligned}
& \omega_{g}^{*}=\chi_{g}, \\
& \chi_{g}^{*}=\omega_{g} .
\end{aligned}
$$

One may also check that

$$
\begin{aligned}
& g \preceq_{\operatorname{Fun}(Q, L)} \omega_{g} \circ \iota_{Q}, \\
& \chi_{g} \circ \iota_{Q} \preceq_{\operatorname{Fun}(Q, L)} g .
\end{aligned}
$$

It is easy to see that (3.91a) follows from Definition 3.24 and (3.85), and that (3.91b) follows from Definition 3.25 and (3.85). One can also prove one of the statements from the other using Proposition 3.6 and (3.90) although the direct proof in this case is actually shorter. Note that we have expressed the symmetry between $\omega_{g}$ and $\chi_{g}$ noticeable in Definitions 3.24 and 3.25 using order duality.

Two obstacles stand in our way of recovering our motivating example (3.84) from our Definitions 3.24 and 3.25. The first is the obvious one: to correctly choose the objects $P$ and $Q$. The second one is that, although (3.84a) defines the clique number of a graph as a supremum, Definition 3.24 defines the generalized clique number as an infimum. We are already equipped to solve the first issue, and the second will be solved by the upcoming Proposition 3.26. Let $P$ denote the preordered set of graphs under the homomorphic relation. It is easy to see that $V(\operatorname{Mon}(P, \overline{\mathbb{R}}))$ is the set of homomorphism monotone functions taking extended-real values. Let $\beta$ be a homomorphism monotone function such that $\beta\left(K_{n}\right) \geq n$ for every $n \in \mathbb{N}$. We may conclude that the clique number $\omega$ is pointwise at most $\beta$, since $K_{\omega(G)} \rightarrow G$ implies that

$$
\omega(G) \leq \beta\left(K_{\omega(G)}\right) \leq \beta(G)
$$

for every graph $G$. Similarly, if $\beta$ is homomorphism monotone such that $\beta\left(K_{n}\right) \leq n$ for every $n \in \mathbb{N}$, we conclude from $G \rightarrow K_{\chi(G)}$ that the coloring number $\chi$ is at least $\beta$, as

$$
\beta(G) \leq \beta\left(K_{\chi(G)}\right) \leq \chi(G)
$$

for every graph $G$. Recalling Definitions 3.24 and 3.25, it seems reasonable to look at the generalized clique and coloring numbers defined by $P$ and $\overline{\mathbb{R}}$ as above, and with $Q:=\left\{K_{n} \mid n \in \mathbb{N}\right\}$ and $g:=\left(K_{n} \in Q \mapsto n\right)$.

Proposition 3.26. Let $P$ be a preordered set, and let $Q \subseteq V(P)$. For every $g \in V(\operatorname{Fun}(Q, \overline{\mathbb{R}}))$ and $p \in V(P)$,

$$
\begin{aligned}
& \omega_{g}(p)=\sup _{\overline{\mathbb{R}}}\left\{g(q) \mid q \in Q, q \preceq_{P} p\right\}, \text { and } \\
& \chi_{g}(p)=\inf _{\overline{\mathbb{R}}}\left\{g(q) \mid q \in Q, p \preceq_{P} q\right\} .
\end{aligned}
$$

Proof. Let $(C, \preceq):=P$. Set $\hat{g}:=\left(p \in C \mapsto \sup _{\overline{\mathbb{R}}}\left\{g(q) \mid q \in Q, q \preceq_{P} p\right\}\right)$. We first prove that

$$
\omega_{g} \preceq_{\operatorname{Fun}(C, \overline{\mathbb{R}})} \hat{g} .
$$

Let $a, b \in C$ be such that $a \preceq_{P} b$. Transitivity of $\preceq_{P}$ implies that $\left\{q \in Q \mid q \preceq_{P} a\right\} \subseteq\left\{q \in Q \mid q \preceq_{P} b\right\}$. Hence $\hat{g}(a) \preceq_{\overline{\mathbb{R}}} \hat{g}(b)$, so $\hat{g} \in V(\operatorname{Mon}(P, \overline{\mathbb{R}}))$. Furthermore, as $q \preceq_{P} q$ for every $q \in Q$, we have that

$$
g(q) \preceq_{\overline{\mathbb{R}}} \sup _{\overline{\mathbb{R}}}\left\{g(x) \mid x \in Q, x \preceq_{P} q\right\}=\hat{g}(q) .
$$

Definition 3.24 implies (3.95), as we have just proved that $\hat{g} \in\left\{f \in V(\operatorname{Mon}(P, \overline{\mathbb{R}})) \mid g \preceq_{\text {Fun }(Q, \overline{\mathbb{R}})} f \circ \iota_{Q}\right\}$.

We now prove

$$
\hat{g} \preceq_{\mathrm{Fun}(C, \overline{\mathbb{R}})} \omega_{g} .
$$

Let $p \in C$, and let $q \in Q$ be such that $q \preceq_{P} p$. Inequality (3.91a) and (3.85) imply that

$$
g(q) \preceq_{\overline{\mathbb{R}}} \omega_{g}(q) \preceq_{\overline{\mathbb{R}}} \omega_{g}(p) .
$$


Hence $\omega_{g}(p)$ is an upper bound for $\left\{g(q) \mid q \in Q, q \preceq_{P} p\right\}$, which implies that $\hat{g}(p) \preceq_{\overline{\mathbb{R}}} \omega_{g}(p)$. Then (3.96) follows, which combined with with (3.95), implies (3.94a).

Finally, define $\omega^{*}$ as in (3.88a). For every $p \in C$,

$$
\begin{aligned}
\chi_{g}(p) & =\omega_{g}^{*}(p) & & \text { by (3.90a) } \\
& =\sup _{\overline{\mathbb{R}}^{*}}\left\{g(q) \mid q \in Q, \preceq_{P^{*}} p\right\} & & \text { by }(3.94 \mathrm{a}) \\
& =\inf _{\overline{\mathbb{R}}}\left\{g(q) \mid q \in Q, q \preceq_{P^{*}} p\right\} & & \text { by }(3.26) \\
& =\inf _{\overline{\mathbb{R}}}\left\{g(q) \mid q \in Q, p \preceq_{P} q\right\} . & & \text { by }(3.2)
\end{aligned}
$$

We may finally argue that "generalized clique number" and "generalized coloring number" are suitable names for the definitions proposed. Let $P$ be the preordered set of graphs under the homomorphic relation. Define

$$
\begin{aligned}
\mathcal{Q}_{K}:= & \left\{K_{n} \mid n \in \mathbb{N}, n>0\right\}, \\
g_{K}: & \mathcal{Q}_{K} \rightarrow[-\infty,+\infty], \\
& g_{K}\left(K_{n}\right):=n
\end{aligned}
$$

Then for every graph $G$,

$$
\omega(G)=\omega_{g_{K}}(G),
$$

since

$$
\begin{aligned}
\omega_{g_{K}}(G) & =\sup \left\{g_{K}(F) \mid F \in \mathcal{Q}_{K}, F \rightarrow G\right\} \\
& =\sup \left\{n \mid n \in \mathbb{N}, K_{n} \rightarrow G\right\} \\
& =\omega(G) .
\end{aligned}
$$

by Proposition 3.26

by $(3.97)$

by $(3.84 \mathrm{a})$

Moreover, for every graph $G$,

$$
\chi(G)=\chi_{g_{K}}(G)
$$

as

$$
\begin{array}{rlrl}
\chi_{g_{K}}(G) & =\inf \left\{g_{K}(F) \mid F \in \mathcal{Q}_{K}, G \rightarrow F\right\} & \\
& =\inf \left\{n \mid n \in \mathbb{N}, G \rightarrow K_{n}\right\} & & \text { by Propositio } \\
& =\chi(G) . & \text { by (3.97) } \\
& & \text { by }(3.84 \mathrm{~b})
\end{array}
$$

by Proposition 3.26

Let $P$ be a preordered set, let $Q \subseteq V(P)$, and let $g: Q \rightarrow[-\infty,+\infty]$. After recovering our motivating example in (3.97), it is natural to gauge the framework presented by comparing it with results regarding the clique number $\omega$ and the coloring number $\chi$. For example, we know that $\omega \leq \chi$ : is it true that $\omega_{g} \leq \chi_{g}$ ? Similarly, for every $n \in \mathbb{N}$ we have that $\omega\left(K_{n}\right)=\chi\left(K_{n}\right)=n$, whereas (3.86) remarks that the obvious generalization may not always hold. For a related question, note that (3.92) and (3.93) imply that

if $\beta: \mathcal{O}\left(\mathrm{Graph}_{\leq \mathbb{R}}\right) \rightarrow[-\infty,+\infty]$ is homomorphism monotone and $\beta\left(K_{n}\right)=n$ for every $n \in \mathbb{N}$,

$$
\text { then } \omega \leq \beta \leq \chi \text {. }
$$

Note that if either $\omega_{g}$ or $\chi_{g}$ is a extension of $g$, then both serve as a suitable $\beta$, and further that the existence of such $\beta$ implies $\omega_{g} \leq \chi_{g}$. Does a suitable $\beta$ exist whenever $\omega_{g} \leq \chi_{g}$ ? Note also that $g_{K}$ is monotone if considered as a function with domain $\mathcal{Q}_{K}$. Does $g \in V(\operatorname{Mon}(P[Q], \overline{\mathbb{R}}))$ imply some property of $\omega_{g}$ or $\chi_{g}$ ? Our next theorem relates all of these questions.

Theorem 3.27. Let $P$ be a preordered set, let $Q \subseteq V(P)$, and let $g \in V(\operatorname{Fun}(Q, \overline{\mathbb{R}}))$. The following are equivalent:

1. it holds that $\omega_{g} \preceq_{\operatorname{Mon}(P, \overline{\mathbb{R}})} \chi_{g}$;

2. both $\omega_{g}$ and $\chi_{g}$ are extensions of $g$, i.e., $g=\omega_{g} \circ \iota_{Q}=\chi_{g} \circ \iota_{Q}$; 
3. there exists $f \in V(\operatorname{Mon}(P, \overline{\mathbb{R}}))$ such that $g=f \circ \iota_{Q}$;

4. it holds that $g \in V(\operatorname{Mon}(P[Q], \overline{\mathbb{R}}))$.

Proof. Item 1 implies item 2: note that

$$
\begin{aligned}
g & \preceq_{\mathrm{Fun}(Q, \overline{\mathbb{R}})} \omega_{g} \circ \iota_{Q} & & \text { by }(3.91 \mathrm{a}) \\
& \preceq_{\mathrm{Fun}(Q, \overline{\mathbb{R}})} \chi_{g} \circ \iota_{Q} & & \text { by item } 1,(3.44), \text { and }(3.49) \\
& \preceq_{\text {Fun }(Q, \overline{\mathbb{R}})} g . & & \text { by }(3.91 \mathrm{~b})
\end{aligned}
$$

As $\overline{\mathbb{R}}$ is partially ordered, Fun $(Q, \overline{\mathbb{R}})$ is partially ordered by Proposition 3.5. Item 2 then follows.

Item 2 implies item 3: Immediate from (3.85).

Item 3 implies item 4: trivial.

Item 4 implies item 1: let $p \in V(P)$, and let $x, y \in Q$ be such that $x \preceq_{P} p$ and $p \preceq_{P} y$. Transitivity implies that $x \preceq_{P} y$, so $g(x) \preceq_{\mathbb{R}} g(y)$. Proposition 3.26 finishes the proof:

$$
\omega_{g}(p)=\sup _{\overline{\mathbb{R}}}\left\{g(x) \mid x \in Q, x \preceq_{P} p\right\} \preceq_{\overline{\mathbb{R}}} \inf _{\overline{\mathbb{R}}}\left\{g(y) \mid y \in Q, p \preceq_{P} y\right\}=\chi_{g}(p) .
$$

We motivated Theorem 3.27 with the known inequality $\omega \leq \chi$; we now prove it using that theorem. Let $n \in \mathbb{N}$ and let $G$ be a graph. Every homomorphism in $\operatorname{Hom}\left(K_{n}, G\right)$ must be injective, otherwise there exists an edge in $K_{n}$ both of whose ends are mapped to the same vertex. Therefore $g_{K}$ is monotone, as $K_{n} \rightarrow K_{m}$ implies that

$$
g_{K}\left(K_{n}\right)=\left|V\left(K_{n}\right)\right| \leq\left|V\left(K_{m}\right)\right|=g_{K}\left(K_{m}\right)
$$

for every $n, m \in \mathbb{N}$. Recalling (3.97), this statement is precisely item 4 of Theorem 3.27, so item 1 states that

$$
\omega \leq \chi
$$

\subsection{Known Generalized Clique and Coloring Parameters}

Recall that for every $n \in \mathbb{N}$ we denote by $[n)$ the set $\{m \in \mathbb{N} \mid m<n\}=\{0,1, \ldots, n-1\}$. For every finite set $V$ and $b \in \mathbb{N}$, the Kneser graph $\operatorname{KG}(V, b)$ is the graph with vertex set $\left(\begin{array}{l}V \\ b\end{array}\right)$ and two vertices are adjacent if disjoint. For every $a, b \in \mathbb{N}$, set $\operatorname{KG}(a, b):=\operatorname{KG}([a), b)$. The fractional chromatic number $\chi_{f}(G)$ of a graph $G$ is

$$
\chi_{f}(G):=\inf \left\{\frac{a}{b} \mid a, b \in \mathbb{N}, 0<b \leq a, G \rightarrow \operatorname{KG}(a, b)\right\} .
$$

Our current goal is to describe a way of relating homomorphism optimization problems, so we introduced $\chi_{f}$ before its own subsection (Subsection 3.4.1) simply to prove that $\chi_{f} \leq \chi$. Let $n \in \mathbb{N}$ be such that $G \rightarrow K_{n}$. The function $f:[n) \rightarrow\left(\begin{array}{c}{[n)} \\ 1\end{array}\right)$ given by $f(i):=\{i\}$ for every $i \in[n)$ certifies that $K_{n} \rightarrow \operatorname{KG}(n, 1)$. Consequently, transitivity of $\rightarrow$ implies that $G \rightarrow \mathrm{KG}(n, 1)$. As this holds for every $n \in \mathbb{N}$ such that $G \rightarrow K_{n}$, both (3.84b) and (3.102) imply that $\chi_{f}(G) \leq \chi(G)$. The following result, which will be our main tool for relating graph parameters in this section, captures the proof we have just sketched, and places it in the context presented in the preceding sections.

Theorem 3.28. Let $P$ be a preordered set. Let $Q, S \subseteq V(P)$, let $g: Q \rightarrow[-\infty,+\infty]$, and let $h: S \rightarrow$ $[-\infty,+\infty]$. Then:

1. if $\psi: Q \rightarrow S$ is such that $\psi(q) \preceq_{P} q$ and $g(q) \preceq_{\overline{\mathbb{R}}} h(\psi(q))$ for every $q \in Q$, then $\omega_{g} \preceq_{\operatorname{Mon}(P, \overline{\mathbb{R}})} \omega_{h}$.

2. if $\phi: S \rightarrow Q$ is such that $s \preceq_{P} \phi(s)$ and $g(\phi(s)) \preceq_{\overline{\mathbb{R}}} h(s)$ for every $s \in S$, then $\chi_{g} \preceq_{\operatorname{Mon}(P, \overline{\mathbb{R}})} \chi_{h}$.

3. if $\psi: Q \rightarrow S$ is surjective and for every $q \in Q$ we have that $\psi(q) \preceq_{P} q$ and $g(q) \preceq_{\overline{\mathbb{R}}} h(\psi(q))$, then $\omega_{g} \preceq_{\operatorname{Mon}(P, \overline{\mathbb{R}})} \omega_{h}$ and $\chi_{g} \preceq_{\operatorname{Mon}(P, \overline{\mathbb{R}})} \chi_{h}$. 
Proof. Item 1: Let $\iota_{Q}: Q \rightarrow V(P)$ and $\iota_{S}: S \rightarrow V(P)$ be defined as in (3.46). From (3.91a) we have that

$$
h \preceq_{\mathrm{Fun}(S, \overline{\mathbb{R}})} \omega_{h} \circ \iota_{S} .
$$

The condition that $\psi(q) \preceq_{P} q$ for every $q \in Q$ may be written as

$$
\iota_{S} \circ \psi \preceq_{\mathrm{Fun}(Q, P)} \iota_{Q} .
$$

Similarly, note that

$$
g \preceq_{\text {Fun }(Q, \overline{\mathbb{R}})} h \circ \psi
$$

as for every $q \in Q$ we have that $g(q) \preceq_{\overline{\mathbb{R}}} h(\psi(q))$. Therefore

$$
\begin{aligned}
& g \preceq_{\operatorname{Fun}(Q, \overline{\mathbb{R}})} h \circ \psi \\
& \text { by (3.105) } \\
& \preceq_{\text {Fun }(Q, \overline{\mathbb{R}})} \omega_{h} \circ \iota_{S} \circ \psi \\
& \text { by (3.44) and (3.103) } \\
& \preceq_{\operatorname{Fun}(Q, \overline{\mathbb{R}})} \omega_{h} \circ \iota_{Q} .
\end{aligned}
$$

Since $\omega_{h}$ is monotone by $(3.85)$, we have that $\omega_{g} \preceq_{\operatorname{Mon}(P, \overline{\mathbb{R}})} \omega_{h}$ by Definition 3.24.

Item 2: Define $\omega^{*}$ as in (3.88a). Using (3.2) twice we conclude that $\phi(s) \preceq_{P^{*}} s$ and that $h(s) \preceq_{\overline{\mathbb{R}}^{*}} g(\phi(s))$ for every $s \in S$. Hence

$$
\begin{aligned}
& \omega_{h}^{*} \preceq_{\operatorname{Mon}\left(P^{*}, \overline{\mathbb{R}}^{*}\right)} \omega_{g}^{*} \\
& \Longrightarrow \chi_{h} \preceq_{\operatorname{Mon}\left(P^{*}, \overline{\mathbb{R}}^{*}\right)} \omega_{g}^{*} \quad \text { by (3.90a) } \\
& \Longrightarrow \chi_{h} \preceq_{\operatorname{Mon}(P, \overline{\mathbb{R}})^{*}} \omega_{g}^{*} \quad \text { by Proposition } 3.11 \\
& \Longrightarrow \omega_{g}^{*} \preceq_{\operatorname{Mon}(P, \overline{\mathbb{R}})} \chi_{h} \quad \text { by }(3.2) \\
& \Longrightarrow \chi_{g} \preceq_{\operatorname{Mon}(P, \overline{\mathbb{R}})} \chi_{h} . \quad \text { by (3.90a) }
\end{aligned}
$$

Item 3: item 1 implies that $\omega_{g} \preceq_{\operatorname{Mon}(P, \overline{\mathbb{R}})} \omega_{h}$. Note also that both (3.104) and (3.105) hold. Since $\psi$ is surjective, there exists a right inverse $\phi: S \rightarrow Q$ of $\psi$. Then

$$
\begin{aligned}
\iota_{S} & =\iota_{S} \circ \psi \circ \phi & & \text { since } \psi \circ \phi=\operatorname{id}_{S} \\
& \preceq_{\text {Fun }(S, P)} \iota_{Q} \circ \phi . & & \text { by }(3.44) \text { and }(3.104)
\end{aligned}
$$

In other words, for every $s \in S$ we have that $s \preceq_{P} \phi(s)$. Moreover,

$$
\begin{aligned}
g \circ \phi & \preceq_{\operatorname{Fun}(S, \overline{\mathbb{R}})} h \circ \psi \circ \phi & & \text { by }(3.44) \text { and }(3.105) \\
& =h . & & \text { since } \psi \circ \phi=\operatorname{id}_{S}
\end{aligned}
$$

In other words, for every $s \in S$ we have that $g(\phi(s)) \preceq_{\overline{\mathbb{R}}} h(s)$. Item 2 implies $\chi_{g} \preceq_{\text {Mon }(P, \overline{\mathbb{R}})} \chi_{h}$, thus finishing the proof.

We are about to shift our perspective, from considering the generalized clique and coloring numbers in the context of order theory, to discussing examples in graph theory. This motivates a shift in language: we are about to present several graph parameters as instances of Definitions 3.24 and 3.25, so it is helpful to have a shorthand for this process. Let $P$ be a preordered set, let $L$ be a complete lattice, let $Q \subseteq V(P)$, and let $g: Q \rightarrow L$. We will refer to $\omega_{g}$ as the least $(P, L)$-monotone function which is at least $g$ on $Q$, and to $\chi_{g}$ as the greatest $(P, L)$-monotone function which is at most $g$ on $Q$. Note that we are exploiting the consequences of our exposition, rather than actually introducing new vocabulary: see (3.85) and (3.91). Interestingly, (3.90a) may be restated as

the least $\left(P^{*}, L^{*}\right)$-monotone function which is at least $g$ on $Q$ is equal to

the greatest $(P, L)$-monotone function which is at most $g$ on $Q$, which although more verbose than (3.90a), precisely displays the objects involved. 


\subsubsection{The Fractional Chromatic Number}

We now present results from the literature regarding the fractional chromatic number $\chi_{f}$, as defined in (3.102), which is the first variant of the chromatic number we shall study. It is also arguably the most important, for a couple of reasons. First, the fractional chromatic number and the Lovász theta function provide the clearest connection between the combinatorial framework presented in this chapter and the geometric framework presented in Chapter 4. Second, there is significant interest from the graph theory community on $\chi_{f}$, as there are conjectures which are either open or false when stated in terms of $\chi$, but are known to be true when stated in terms of $\chi_{f}$ (see $[89,90,110,122]$ ). We make no attempt at a comprehensive survey on the fractional chromatic number: we have simply selected results relevant to the themes of this text.

We start by discussing the combinatorial problem encoded by $\chi_{f}$. Let $G$ be a graph, and let $a, b \in \mathbb{N}$ be such that $0<b \leq a$. A homomorphism in $\operatorname{Hom}(G, \operatorname{KG}(a, b))$ is an assignment of exactly $b$ distinct colors from $[a)$ to each vertex of $G$ such that adjacent vertices have disjoint sets of colors. Define $\chi_{b}$ for every nonzero $b \in \mathbb{N}$ by

$$
\chi_{b}(G):=\inf \{a \mid a \in \mathbb{N}, b \leq a, G \rightarrow \operatorname{KG}(a, b)\} .
$$

Note that if $f \in \operatorname{Hom}(G, \operatorname{KG}(a, b))$, then for each $c \in[a)$, the set $\{i \in V(G) \mid c \in f(i)\}$ is stable - otherwise there is a pair of adjacent vertices of $G$ mapped to an intersecting pair of sets of colors. In other words, $f$ describes a covering of the vertices of $G$, using $a$ stable sets, such that each vertex is assigned to precisely $b$ stable sets. It is easy to see that

$$
\chi_{f}(G)=\inf \left\{\frac{\chi_{b}(G)}{b} \mid b \in \mathbb{N}, b>0\right\} .
$$

These remarks capture the vague idea that $\chi_{f}$ is a "normalization" of $\chi$ and provide another proof that $\chi_{f} \leq \chi_{1}=\chi$. Although we will not do so, note that for every $b \in \mathbb{N}$ the graph parameter $\chi_{b}$ may be formulated using Definition 3.25. We also point the reader to Scheinerman and Ullman [106, Chapter 1] for an interesting reasoning related to (3.107).

Let $P$ denote the preordered set of graphs under the homomorphic relation. Define

$$
\begin{gathered}
\mathcal{Q}_{\mathrm{KG}}:=\left\{K_{1}\right\} \cup\{\operatorname{KG}(a, b) \mid a, b \in \mathbb{N}, 0<2 b \leq a\}, \\
g_{\mathrm{KG}}: \mathcal{Q}_{\mathrm{KG}} \rightarrow[-\infty,+\infty], \\
g_{\mathrm{KG}}\left(K_{1}\right):=1, \\
g_{\mathrm{KG}}(\mathrm{KG}(a, b)):=a / b .
\end{gathered}
$$

Denote by $\omega_{\mathrm{KG}}:=\omega_{g_{\mathrm{KG}}}$ the least $(P, \overline{\mathbb{R}})$-monotone function which is at least $g_{\mathrm{KG}}$ on $\mathcal{Q}_{\mathrm{KG}}$, and by $\chi_{\mathrm{KG}}:=\chi_{g_{\mathrm{KG}}}$ the greatest $(P, \overline{\mathbb{R}})$-monotone function which is at most $g_{\mathrm{KG}}$ on $\mathcal{Q}_{\mathrm{KG}}$. Our immediate goal is to motivate why $\mathcal{Q}_{\mathrm{KG}}$ is not defined by $\{\operatorname{KG}(a, b) \mid a, b \in \mathbb{N}, 0<b \leq a\}$. Note that, if $a, b \in \mathbb{N}$ are such that $0<b \leq a$ and $2 b>a$, then $E(\operatorname{KG}(a, b))=\varnothing$, as every pair of sets in $\left(\begin{array}{c}{[a)} \\ b\end{array}\right)$ must have an element in common by the pigeonhole principle. Note also that every graph $G$ such that $V(G) \neq \varnothing$ and $E(G)=\varnothing$ is homomorphically equivalent to $K_{1}$. Item 4 of Theorem 3.27 explains why we wish to avoid a set of homomorphically equivalent graphs associated with all of the values in $\{a / b \mid a, b \in \mathbb{N}, 0<b \leq a, a<2 b\}=\mathbb{Q} \cap[1,2)$. In other words, since we want $\omega_{\mathrm{KG}} \leq \chi_{\mathrm{KG}}$ to hold, we had to adapt our definition of $\mathcal{Q}_{\mathrm{KG}}$, as

$$
\sup \left\{a / b \mid a, b \in \mathbb{N}, 0<b \leq a, \operatorname{KG}(a, b) \rightarrow K_{1}\right\}=2>1=\inf \left\{a / b \mid a, b \in \mathbb{N}, 0<b \leq a, K_{1} \rightarrow \operatorname{KG}(a, b)\right\} .
$$

We later prove that $\omega_{\mathrm{KG}} \leq \chi_{\mathrm{KG}}$ holds. Proposition 3.26 and a case analysis on $E(G)=\varnothing$ and $E(G) \neq \varnothing$ imply that for every graph $G$,

$$
\begin{aligned}
\omega_{\mathrm{KG}}(G) & =\sup \left\{g_{\mathrm{KG}}(F) \mid F \in \mathcal{Q}_{\mathrm{KG}}, F \rightarrow G\right\} \\
& = \begin{cases}1, & \text { if } E(G)=\varnothing, \\
\sup \{a / b \mid a, b \in \mathbb{N}, 0<b \leq a, \operatorname{KG}(a, b) \rightarrow G\}, & \text { otherwise. }\end{cases}
\end{aligned}
$$

Similarly, Proposition 3.26 and a case analysis on $E(G)=\varnothing$ and $E(G) \neq \varnothing$ imply that for every graph $G$,

$$
\begin{aligned}
\chi_{\mathrm{KG}}(G) & =\inf \left\{g_{\mathrm{KG}}(F) \mid F \in \mathcal{Q}_{\mathrm{KG}}, G \rightarrow F\right\} \\
& =\inf \{a / b \mid a, b \in \mathbb{N}, 0<b \leq a, G \rightarrow \operatorname{KG}(a, b)\} \\
& =\chi_{f}(G) .
\end{aligned}
$$


Proposition 3.29. It holds that

$$
\omega \leq \omega_{\mathrm{KG}} .
$$

Proof. Let $P$ denote the preordered set of graphs under the homomorphic relation. Define $\psi: \mathcal{Q}_{K} \rightarrow \mathcal{Q}_{\mathrm{KG}}$ by

$$
\psi\left(K_{n}\right):=\left\{\begin{array}{l}
K_{1}, \text { if } n=1 \\
\operatorname{KG}(n, 1), \text { otherwise. }
\end{array}\right.
$$

Let $n \in \mathbb{N}$ be greater than 1. Recall from $(2.24)$ that $V\left(K_{n}\right)=[n)$, so $\psi\left(K_{n}\right) \preceq_{P} K_{n}$, as

$$
(\{x\} \mapsto x) \in \operatorname{Hom}\left(\operatorname{KG}(n, 1), K_{n}\right) .
$$

Moreover,

$$
g_{K}\left(K_{n}\right)=n=g_{\mathrm{KG}}(\mathrm{KG}(n, 1))=g_{\mathrm{KG}}\left(\psi\left(K_{n}\right)\right) .
$$

It is immediate $\psi\left(K_{1}\right)=K_{1} \preceq_{P} K_{1}$ and that $g_{K}\left(K_{1}\right)=g_{\mathrm{KG}}\left(\psi\left(K_{1}\right)\right)$. Theorem 3.28 item 1 and (3.98) finish the proof.

The fractional chromatic number is usually presented as the optimal value of a linear program. As a warm-up for Chapter 4, we present a weighted version of said linear program. Recall that $\mathcal{S}(G)$ denotes the set of stable sets of a graph $G$. For every graph $G$ and $w \in \mathbb{R}_{+}^{V(G)}$, define

$$
\chi_{f}(G, w):=\min \left\{\mathbb{1}^{\top} y \mid y \in \mathbb{R}_{+}^{\mathcal{S}(G)}, \sum_{S \in \mathcal{S}(G)} y_{S} \mathbb{1}_{S} \geq w\right\} .
$$

We have that

$$
\chi_{f}(G, w)=\max \left\{w^{\top} x \mid x \in \mathbb{R}_{+}^{V(G)}, \forall S \in \mathcal{S}(G), \mathbb{1}_{S}^{\top} x \leq 1\right\},
$$

since the linear programs in (3.109) and (3.110) are LP duals to each other, and they have $\stackrel{\circ}{y}:=\left(\max _{i \in V} w_{i}\right) \mathbb{1}$ and $\stackrel{\circ}{x}:=0$ as feasible solutions. It is also useful to define

$$
\operatorname{QSTAB}(G):=\left\{x \in \mathbb{R}_{+}^{V(G)} \mid \forall S \in \mathcal{S}(\bar{G}),\left\langle\mathbb{1}_{S}, x\right\rangle \leq 1\right\},
$$

so we can restate $(3.110)$ as

$$
\chi_{f}(G, w)=\max \left\{w^{\top} x \mid x \in \operatorname{QSTAB}(\bar{G})\right\} .
$$

The proof we are about to show that $\chi_{f}(G)$ may be formulated using (3.109) appears in several texts [44, 57, 106]. However, the emphasis on homomorphism monotonicity and the following (geometric) proposition is, to the best of our knowledge, ours.

Proposition 3.30. Let $G$ and $H$ be graphs, and let $f \in \operatorname{Hom}(G, H)$. Then

$$
P_{f} \operatorname{QSTAB}(\bar{G}) \subseteq \operatorname{QSTAB}(\bar{H}) \text {. }
$$

Proof. Let $x \in \operatorname{QSTAB}(\bar{G})$, and let $S \in \mathcal{S}(H)$. Proposition 2.6 implies that $T:=f^{-1}(S) \in \mathcal{S}(G)$. Then

$$
\begin{aligned}
\mathbb{1}_{S}^{\top}\left(P_{f} x\right) & =\left(P_{f}^{\top} \mathbb{1}_{S}\right)^{\top} x & & \\
& =\mathbb{1}_{f-1}^{\top}(S) & & \text { by }(2.13) \\
& =\mathbb{1}_{T}^{\top} x & & \\
& \leq 1 . & & \text { since } x \in \operatorname{QSTAB}(\bar{G}) \text { and } T \in \mathcal{S}(G)
\end{aligned}
$$

Corollary 3.31. The function $G \mapsto \chi_{f}(G, \mathbb{1})$ defined for every graph $G$ is homomorphism monotone.

Proof. Let $G$ and $H$ be graphs such that $G \rightarrow H$, and let $f \in \operatorname{Hom}(G, H)$. Then

$$
\begin{aligned}
\chi_{f}(G, \mathbb{1}) & =\max \left\{\mathbb{1}^{\top} x \mid x \in \operatorname{QSTAB}(\bar{G})\right\} & & \text { by }(3.112) \\
& \left.=\max \left\{\left(P_{f}^{\top} \mathbb{1}\right)^{\top} x \mid x \in \operatorname{QSTAB}(\bar{G})\right)\right\} & & \text { by }(2.15) \\
& =\max \left\{\mathbb{1}^{\top} P_{f} x \mid x \in \operatorname{QSTAB}(\bar{G})\right\} & & \\
& \leq \max \left\{\mathbb{1}^{\top} z \mid z \in \operatorname{QSTAB}(\bar{H})\right\} & & \text { by Proposition } 3.30 \\
& =\chi_{f}(H, \mathbb{1}) . & & \text { by }(3.112)
\end{aligned}
$$


Proposition 3.32. For every graph $G$, there exists a nonzero $\rho \in \mathbb{N}$ such that $\rho \chi_{f}(G, \mathbb{1}) \in \mathbb{N}$ and $G \rightarrow \operatorname{KG}\left(\rho \chi_{f}(G, \mathbb{1}), \rho\right)$.

Proof. Set $B:=\sum_{S \in \mathcal{S}(G)} \mathbb{1}_{S} e_{S}^{\top} \in \mathbb{R}^{V(G) \times \mathcal{S}(G)}$. The linear program

$$
\inf \left\{\mathbb{1}^{\top} B y \mid y \in \mathbb{R}_{+}^{\mathcal{S}(G)}, \mathbb{1}^{\top} y=\chi_{f}(G), B y \geq \mathbb{1}\right\}
$$

is bounded, as $B y \geq \mathbb{1}$ implies $\mathbb{1}^{\top} B y \geq \mathbb{1}^{\top} \mathbb{1}=|V(G)|$. Moreover, any optimal solution of (3.109) is feasible in (3.113), which implies that (3.113) is feasible. Therefore (3.113) has an optimal solution by LP Strong Duality. Since the constraints in (3.109) only have rational numbers, Theorem 2.12 implies $\chi_{f}(G)=\chi_{f}(G, \mathbb{1})$ is rational. Consequently, the constraints in (3.113) only have rational numbers, so Theorem 2.12 implies (3.113) has an optimal solution $\bar{y} \in \mathbb{Q}_{+}^{\mathcal{S}(G)}$. We claim that

$$
B \bar{y}=\mathbb{1} .
$$

Indeed, assume $i \in V(G)$ is such that $e_{i}^{\top} B \bar{y}>1$. Then there exists $S \in \mathcal{S}(G)$ such that $\bar{y}_{S}>0$ and $i \in S$. Set $\alpha:=\min \left\{\bar{y}_{S}, e_{i}^{\top} B \bar{y}-1\right\}>0$ and $z:=\bar{y}-\alpha\left(e_{S}-e_{S \backslash\{i\}}\right) \geq 0$. Note that $z$ is feasible in (3.113), as

$$
\mathbb{1}^{\top} z=\mathbb{1}^{\top} \bar{y}-\alpha \mathbb{1}^{\top}\left(e_{S}-e_{S \backslash\{i\}}\right)=\mathbb{1}^{\top} \bar{y}=\chi_{f}(G)
$$

and

$$
\begin{aligned}
B z & =B \bar{y}-\alpha B\left(e_{S}-e_{S \backslash\{i\}}\right) & & \\
& =B \bar{y}-\alpha\left(\mathbb{1}_{S}-\mathbb{1}_{S \backslash\{i\}}\right) & & \text { as } B e_{T}=\mathbb{1}_{T} \text { for every } T \in \mathcal{S}(G) \\
& =B \bar{y}-\alpha e_{i} & & \\
& \geq B \bar{y}-\left(e_{i}^{\top} B \bar{y}-1\right) e_{i} & & \text { by the definition of } \alpha \\
& \geq \mathbb{1} . & & \text { since } B \bar{y} \geq \mathbb{1}
\end{aligned}
$$

Moreover the objective value of $z$ in (3.113) is better than that of $\bar{y}$ :

$$
\begin{array}{rlrl}
\mathbb{1}^{\top} B z & =\mathbb{1}^{\top} B \bar{y}-\alpha \mathbb{1}^{\top} B\left(e_{S}-e_{S \backslash\{i\}}\right) & \\
& =\mathbb{1}^{\top} g B \bar{y}-\alpha \mathbb{1}^{\top}\left(\mathbb{1}_{S}-\mathbb{1}_{S \backslash\{i\}}\right) & & \text { as } B e_{T}=\mathbb{1}_{T} \text { for every } T \in \mathcal{S}(G) \\
& =\mathbb{1}^{\top} B \bar{y}-\alpha \mathbb{1}^{\top} e_{i} & & \\
& =\mathbb{1}^{\top} B \bar{y}-\alpha & & \text { since } \alpha>0 \\
& <\mathbb{1}^{\top} B \bar{y} . &
\end{array}
$$

This contradiction implies (3.114).

As $\bar{y} \in \mathbb{Q}_{+}^{\mathcal{S}(G)}$, there exists a nonzero $\rho \in \mathbb{N}$ such that $\rho \bar{y} \in \mathbb{N}^{\mathcal{S}(G)}$. We now use $\rho \bar{y}$ to define a homomorphism that certifies that

$$
G \rightarrow \operatorname{KG}\left(\rho \chi_{f}(G, \mathbb{1}), \rho\right) .
$$

Define

Note that $|U|=\rho \mathbb{1}^{\top} \bar{y}$, as

$$
U:=\left\{(S, k) \mid S \in \mathcal{S}(G), k \in\left[\rho \bar{y}_{S}\right)\right\} .
$$

$$
\begin{array}{rlrl}
|U| & =\sum_{S \in \mathcal{S}(G)}|\{k \in \mathbb{N} \mid(S, k) \in U\}| & & \text { since } U \subseteq \mathcal{S}(G) \times \mathbb{N} \\
& =\sum_{S \in \mathcal{S}(G)} \rho \bar{y}_{S} & & \text { by }(3.116) \\
& =\rho \mathbb{1}^{\top} \bar{y} . &
\end{array}
$$

For every $i \in V(G)$, define

$$
f(i):=\{(S, k) \in U \mid i \in S\} .
$$


We have that $|f(i)|=\rho$ for every $i \in V(G)$, since

$$
\begin{aligned}
|f(i)| & =\sum_{S \in \mathcal{S}(G)}|\{(S, k) \in U \mid i \in S\}| & & \text { by }(3.117) \\
& =\sum_{S \in \mathcal{S}(G)}\left|\left\{k \in \mathbb{N} \mid i \in S, k \in\left[\rho \bar{y}_{S}\right)\right\}\right| & & \text { by }(3.116) \\
& =\sum_{S \in \mathcal{S}(G)}[i \in S] \rho \bar{y}_{S} & & \\
& =\rho e_{i}^{\top} B \bar{y} & & \text { by definition of } B \\
& =\rho . & & \text { by }(3.114)
\end{aligned}
$$

Thus $f: V(G) \rightarrow\left(\begin{array}{c}U \\ \rho\end{array}\right)$. We claim that

$$
f \in \operatorname{Hom}(G, \operatorname{KG}(U, \rho)) .
$$

Let $i j \in E(G)$, let $(S, n) \in f(i)$, and let $(T, m) \in f(j)$. It suffices to show that $S \neq T$, as this implies that $f(i) \cap f(j)=\varnothing$, thus proving (3.118). By (3.117), we have that $i \in S$. Since $S \in \mathcal{S}(G)$ is stable and $i j \in E(G)$, we conclude that $j \notin S$. But then $j \in T \backslash S$, as $j \in T$ by (3.117). Thus $S \neq T$.

Set $a:=|U|=\rho \chi_{f}(G)$ and $b:=\rho$. Let $\ell: U \rightarrow[a)$ be any bijective function. The function $g:\left(\begin{array}{c}U \\ \rho\end{array}\right) \rightarrow\left(\begin{array}{c}{[a)} \\ b\end{array}\right)$ defined by

$$
g(S):=\ell(S)=\{\ell(i) \mid i \in S\}
$$

belongs to $\operatorname{Hom}(\operatorname{KG}(U, \rho), \operatorname{KG}([a), b))$ because $\ell$ is injective. Then $g \circ f \in \operatorname{Hom}(G, \operatorname{KG}(a, b))$ by (3.118), so (3.115) holds.

Theorem 3.33. For every graph $G$ we have that $\chi_{f}(G, \mathbb{1})=\chi_{\mathrm{KG}}(G)$.

Proof. Proposition 3.32 and (3.102) imply $\chi_{\mathrm{KG}}(G) \leq \chi_{f}(G, \mathbb{1})$. As $G \mapsto \chi_{f}(G, \mathbb{1})$ is homomorphism monotone by Corollary 3.31, our plan is to show that $\chi_{f}(F, \mathbb{1}) \leq g_{\mathrm{KG}}(F)$ for every $F \in \mathcal{Q}_{\mathrm{KG}}$, so we can conclude $\chi_{f}(G, \mathbb{1}) \leq \chi_{\mathrm{KG}}(G)$ from Definition 3.25. As it is clear from (3.110) that $\chi_{f}\left(K_{1}, \mathbb{1}\right)=1$, it suffices to prove that

$$
\text { for every } a, b \in \mathbb{N} \text { such that } 0<b \leq a \text {, we have that } \chi_{f}(\operatorname{KG}(a, b), \mathbb{1}) \leq \frac{a}{b} .
$$

Let $a, b \in \mathbb{N}$ be such that $0<b \leq a$. We now exhibit a feasible solution for (3.109) with $G:=\operatorname{KG}(a, b)$ and with objective value $a / b$. Define $\phi:[a) \rightarrow \mathcal{S}(\operatorname{KG}(a, b))$ by $\phi(i):=\{S \in V(\operatorname{KG}(a, b)) \mid i \in S\}$ and set $\bar{y}:=P_{\phi} \mathbb{1}$. Then, for every $T \in V(\operatorname{KG}(a, b))$,

$$
\begin{array}{rlrl}
e_{T}^{\top}\left(\sum_{S \in \mathcal{S}(\mathrm{KG}(a, b))} \bar{y}_{S} \mathbb{1}_{S}\right) & =\sum_{S \in \mathcal{S}(\mathrm{KG}(a, b))}[T \in S] \bar{y}_{S} & \\
& =\sum_{S \in \mathcal{S}(\mathrm{KG}(a, b))}[T \in S] e_{S}^{\top} P_{\phi} \mathbb{1} & & \text { by the definition of } \bar{y} \\
& =\sum_{S \in \mathcal{S}(\mathrm{KG}(a, b))}[T \in S] e_{S}^{\top}\left(\sum_{i \in[a)} e_{\phi(i)}\right) & & \text { by }(2.12) \\
& =\sum_{i \in[a)} \sum_{S \in \mathcal{S}(\mathrm{KG}(a, b))}[T \in S][S=\phi(i)] & \\
& =\sum_{i \in[a)}[T \in \phi(i)] & & \text { since } \phi \text { is a function } \\
& \geq b . & & \text { since } T \in \phi(i) \text { for every } i \in T
\end{array}
$$

Moreover (2.15) implies

$$
\mathbb{1}^{\top} \bar{y}=\mathbb{1}^{\top} P_{\phi} \mathbb{1}=\left(P_{\phi}^{\top} \mathbb{1}\right)^{\top} \mathbb{1}=\mathbb{1}^{\top} \mathbb{1}=a .
$$

Hence $(1 / b) \bar{y}$ is a feasible solution with objective value $a / b$, so (3.119) holds. 
Let $P$ be a preordered set, let $Q \subseteq V(P)$, and let $g: Q \rightarrow \overline{\mathbb{R}}$. Our developments do not guarantee that the 'sup' in (3.94a) or the 'inf' in (3.94b) are attained: although the completeness of $\overline{\mathbb{R}}$ implies $\left\{g(q) \mid q \in Q, p \preceq_{P} q\right\}$ has an infimum for every $p \in P$, there may not exist a function $\bar{q}: P \rightarrow Q$ such that $\chi_{g}(p)=g(\bar{q}(p))$ for every $p \in P$. Such a function clearly exists for $\omega$ and $\chi$, as for every graph $G$ we only need to consider finitely many complete graphs to decide its clique and coloring number. Corollary 3.34 shows this is also the case for $\chi_{f}$.

Corollary 3.34. Let $G$ be graph. Then

$$
\chi_{f}(G)=\min \left\{\frac{a}{b} \mid a, b \in \mathbb{N}, 0<b \leq a, G \rightarrow \operatorname{KG}(a, b)\right\} .
$$

Proof. Proposition 3.32 states the existence of a nonzero $\rho \in \mathbb{N}$ such that $G \rightarrow \operatorname{KG}\left(\rho \chi_{f}(G, \mathbb{1}), \rho\right)$. Note that

$$
g_{\mathrm{KG}}\left(\operatorname{KG}\left(\rho \chi_{f}(G, \mathbb{1}), \rho\right)\right)=\frac{\rho \chi_{f}(G, \mathbb{1})}{\rho}=\chi_{f}(G, \mathbb{1}) .
$$

Theorem 3.33 then implies the optimality of $\operatorname{KG}\left(\rho \chi_{f}(G, \mathbb{1}), \rho\right)$ in (3.102).

Corollary 3.35. We have that $\chi_{f}(G) \in \mathbb{Q}$ for every graph $G$.

Proof. Let $G$ be a graph. Corollary 3.34 implies there exist $a, b \in \mathbb{N}$ with $0<b \leq a$ such that $\chi_{f}(G)=a / b$.

Theorem 3.33 and its consequences showcase the power of linear programming strong duality. We now compare it with the duality between Definitions 3.24 and 3.25. Let $P$ be a preordered set, let $Q \subseteq V(P)$, and let $g: Q \rightarrow V(\overline{\mathbb{R}})$. Assume that $\omega_{g} \leq \chi_{g}$. Proposition 3.26 implies that, for every $p \in V(P)$,

if there exists $q \in Q$ such that $q \preceq_{P} p$, then $g(q) \leq \omega_{g}(p)$, and if there exists $q \in Q$ such that $p \preceq_{P} q$, then $\chi_{g}(p) \leq g(q)$.

This provides a straightforward way of producing lower bounds for $\omega_{g}$ and upper bounds for $\chi_{g}$. Furthermore, as $\omega_{g} \leq \chi_{g}$, lower bounds for $\omega_{g}$ are also lower bounds for $\chi_{g}$, and upper bounds for $\chi_{g}$ are also upper bounds for $\omega_{g}$. In this sense, the situation with $\omega_{g}$ and $\chi_{g}$ is quite similar to linear primal and dual programs: see how this idea is used with the linear programming formulations in (3.119) or Proposition 3.30. However, one crucial difference concerns the existence of optimal solutions. For every graph $G$ and $w \in \mathbb{R}_{+}^{V(G)}$, if $\chi_{f}(G, w) \geq \rho$ for some $\rho \in \mathbb{R}$, then there exists $\bar{x} \in \operatorname{QSTAB}(\bar{G})$ such that $w^{\top} \bar{x} \geq \rho$. Things may be more complicated when working with generalized clique and coloring numbers. Note, for example, that $\omega\left(C_{5}\right)=2$, so it is impossible to exhibit a clique which certifies that $\chi\left(C_{5}\right) \geq 3$, which we know to be true. In other words, if we consider Definitions 3.24 and 3.25 as defining a pair of primal and dual problems whenever $g$ is monotone, our theory must embrace a duality gap, as shown by the our motivating example: $\omega$ and $\chi$.

\subsubsection{The Circular Chromatic Number}

Let $a, b \in \mathbb{N}$ be such that $0<b \leq a$. Define the circular complete graph $\operatorname{Circ}(a, b)$ to be the graph such that

$$
\begin{aligned}
& V(\operatorname{Circ}(a, b)):=[a), \\
& E(\operatorname{Circ}(a, b)):=\left\{\{i, j\} \in\left(\begin{array}{c}
{[a)} \\
2
\end{array}\right)|b \leq| i-j \mid \leq a-b\right\} .
\end{aligned}
$$

Let $P$ be the preordered set of graphs under the homomorphic relation. Define

$$
\begin{gathered}
\mathcal{Q}_{\text {Circ }}:=\left\{K_{1}\right\} \cup\{\operatorname{Circ}(a, b) \mid a, b \in \mathbb{N}, 0<2 b \leq a\}, \\
g_{\text {Circ }}: \mathcal{Q}_{\text {Circ }} \rightarrow[-\infty,+\infty], \\
g_{\text {Circ }}\left(K_{1}\right):=1, \\
g_{\text {Circ }}(\operatorname{Circ}(a, b)):=a / b .
\end{gathered}
$$


Note that if $2 b>a$, then $E(\operatorname{Circ}(a, b))=\varnothing$, so our remarks about the definition of $\mathcal{Q}_{\mathrm{KG}}$ apply to the definition of $\mathcal{Q}_{\text {Circ }}$. In particular, $\mathcal{Q}_{\text {Circ }}$ is defined so that its clique number is at most its chromatic number, and we shall prove this fact shortly. We denote by

$$
\omega_{\text {Circ }}:=\omega_{g_{\text {Circ }}} \text { the least }(P, \overline{\mathbb{R}}) \text {-monotone function which is at least } g_{\text {Circ }} \text { on } \mathcal{Q}_{\text {Circ }} \text {, }
$$

and by

$$
\chi_{\text {Circ }}:=\chi_{g_{\text {Circ }}} \text { the greatest }(P, \overline{\mathbb{R}}) \text {-monotone function which is at most } g_{\text {Circ }} \text { on } \mathcal{Q}_{\text {Circ }} \text {. }
$$

The parameter $\chi_{\text {Circ }}$ is commonly denoted by $\chi_{c}$ and called the circular chromatic number, although it was introduced by Vince [116] as the "star chromatic number". Although the paper [116] defines circular complete graphs and even computes the circular chromatic number of such graphs, it does not formulate $\chi_{c}$ as a graph homomorphism optimization problem. For results on $\chi_{c}$, see the survey [120]. Zhu [121] introduced $\omega_{\text {Circ }}$ as the circular clique number, denoting it by $\omega_{c}$; the paper introduces $\omega_{c}$ using the same graph homomorphism optimization problem we are about to present. Proposition 3.26 and a case analysis on $E(G)=\varnothing$ and $E(G) \neq \varnothing$ imply that for every graph $G$,

$$
\omega_{c}(G)= \begin{cases}1, & \text { if } E(G)=\varnothing, \\ \sup \{a / b \mid a, b \in \mathbb{N}, 0<b \leq a, \operatorname{Circ}(a, b) \rightarrow G\}, & \text { otherwise. }\end{cases}
$$

Similarly, Proposition 3.26 and a case analysis on $E(G)=\varnothing$ and $E(G) \neq \varnothing$ imply that for every graph $G$,

$$
\chi_{c}(G)=\inf \left\{\frac{a}{b} \mid a, b \in \mathbb{N}, 0<b \leq a, G \rightarrow \operatorname{Circ}(a, b)\right\} .
$$

Proposition 3.36. Let $a, b \in \mathbb{N}$ be such that $0<b \leq a$. Then

$$
\begin{aligned}
K_{\lfloor a / b\rfloor} & \rightarrow \operatorname{Circ}(a, b), \text { and } \\
\operatorname{Circ}(a, b) & \rightarrow K_{\lceil a / b\rceil} .
\end{aligned}
$$

Proof. Define $f:[\lfloor a / b\rfloor) \rightarrow[a)$ by $f(i):=i b$. Let $i, j \in[\lfloor a / b\rfloor)$ be distinct, and assume without loss of generality that $i<j$. Then

$$
\begin{aligned}
b & \leq b(j-i) \\
& \leq b(\lfloor a / b\rfloor-1-i) \\
& \leq b(\lfloor a / b\rfloor-1) \\
& \leq a-b .
\end{aligned}
$$

$$
\begin{aligned}
& \text { since } i<j \\
& \text { since } j \leq\lfloor a / b\rfloor-1 \\
& \text { since } i \geq 0 \\
& \text { since } b\lfloor a / b\rfloor \leq a
\end{aligned}
$$

As $b(j-i)=|f(j)-f(i)|$, it follows that $f \in \operatorname{Hom}\left(K_{|a / b|}, \operatorname{Circ}(a, b)\right)$, thus proving (3.125).

Define $g:[a) \rightarrow[\lceil a / b\rceil)$ by $g(i):=\lfloor i / b\rfloor$. Let $i j \in E(\operatorname{Circ}(a, b))$, and assume without loss of generality that $i<j$. Then $b \leq|j-i|=j-i$ implies that $i \leq j-b$, so

$$
\begin{aligned}
g(i) & =\lfloor i / b\rfloor & & \text { by the definition of } g \\
& \leq\left\lfloor\frac{j-b}{b}\right\rfloor & & \text { since } i \leq j-b \text { and }\lfloor\cdot\rfloor \text { is monotone } \\
& =\left\lfloor\frac{j}{b}-1\right\rfloor & & \\
& =\lfloor j / b\rfloor-1 & & \text { since }\lfloor x+k\rfloor=\lfloor x\rfloor+k \text { for every } x \in \mathbb{R} \text { and } k \in \mathbb{Z} \\
& <\lfloor j / b\rfloor & & \\
& =g(j) . & & \text { by the definition of } g
\end{aligned}
$$

Hence $g \in \operatorname{Hom}\left(\operatorname{Circ}(a, b), K_{\lceil a / b\rceil}\right)$, so (3.126) holds.

Corollary 3.37. Let $a, b \in \mathbb{N}$ be such that $0<b \leq a$. If $b$ divides $a$, then

$$
\operatorname{Circ}(a, b) \leftrightarrow K_{a / b}
$$


Proof. We conclude that $K_{a / b} \rightarrow \operatorname{Circ}(a, b)$ from (3.125), and that $\operatorname{Circ}(a, b) \rightarrow K_{a / b}$ from (3.126).

Corollary 3.38. It holds that

$$
\omega \leq \omega_{\mathrm{Circ}} \text { and } \chi_{\mathrm{Circ}} \leq \chi
$$

Proof. Let $P$ denote the preordered set of graphs under the homomorphic relation. Define $\psi: \mathcal{Q}_{K} \rightarrow \mathcal{Q}_{\text {Circ }}$ by

$$
\psi\left(K_{n}\right):=\left\{\begin{array}{l}
K_{1}, \text { if } n=1 \\
\operatorname{Circ}(n, 1), \text { otherwise. }
\end{array}\right.
$$

Corollary 3.37 and $K_{1} \preceq_{P} K_{1}$ imply that

$$
\begin{gathered}
\psi\left(K_{n}\right) \preceq_{P} K_{n} \text { and } \\
K_{n} \preceq_{P} \psi\left(K_{n}\right)
\end{gathered}
$$

for every nonzero $n \in \mathbb{N}$. Furthermore

$$
g_{K}\left(K_{n}\right)=n=g_{\text {Circ }}\left(\psi\left(K_{n}\right)\right)
$$

for every nonzero $n \in \mathbb{N}$. Theorem 3.28 finishes the proof: item 1, (3.127a), and (3.128) imply that $\omega \leq \omega_{\text {Circ }}$, whereas item $2,(3.127 \mathrm{~b})$, and (3.128) imply that $\chi_{\text {Circ }} \leq \chi$.

As it was the case with the fractional chromatic number, we have that the supremum and infimum in (3.124) are attained.

Proposition 3.39 ([17]). For every graph $G$,

$$
\chi_{c}(G)=\min \left\{\frac{a}{b} \mid a, b \in \mathbb{N}, 0<b \leq a, G \rightarrow \operatorname{Circ}(a, b)\right\} .
$$

Proposition 3.40 ([121]). For every graph $G$ with $E(G) \neq \varnothing$,

$$
\omega_{c}(G)=\max \left\{\frac{a}{b} \mid a, b \in \mathbb{N}, 0<b \leq a, \operatorname{Circ}(a, b) \rightarrow G\right\} .
$$

Bondy and Hell [17] provide a combinatorial argument showing that

$$
\chi_{c}(G)=\inf \left\{\frac{a}{b}|a, b \in \mathbb{N}, 0<b \leq a \leq| V(G) \mid, G \rightarrow \operatorname{Circ}(a, b)\right\} .
$$

As the set $\{a, b \in \mathbb{N}|0<b \leq a<| V(G) \mid\}$ is finite, Proposition 3.39 follows. Zhu [121] provides a combinatorial argument showing that if $a, b \in \mathbb{N}$ are coprime and such that $a / b=\omega_{c}(G)$, then $G$ has an induced subgraph isomorphic to $\operatorname{Circ}(a, b)$. As a graph has finitely many induced subgraphs, Proposition 3.40 follows.

Corollary 3.41. It holds that

$$
\left\lfloor\omega_{\text {Circ }}\right\rfloor \leq \omega \text { and } \chi \leq\left\lceil\chi_{\text {Circ }}\right\rceil .
$$

Proof. Let $P$ denote the preordered set of graphs under the homomorphic relation. Define

$$
\begin{gathered}
\mathcal{Q}_{\lfloor\text {Circ }\rfloor}:=\mathcal{Q}_{\text {Circ }}, \\
g_{\lfloor\text {Circ }\rfloor}: \mathcal{Q}_{\lfloor\text {Circ }\rfloor} \rightarrow[-\infty,+\infty], \\
g_{\lfloor\text {Circ }\rfloor}(H):=\left\lfloor g_{\text {Circ }}(H)\right\rfloor \text { for every } H \in \mathcal{Q}_{\text {Circ }} .
\end{gathered}
$$

Let $\omega_{g_{\lfloor\text {Circ }\rfloor}}$ be the least $(P, \overline{\mathbb{R}})$-monotone function which is at least $g_{\lfloor\text {Circ }\rfloor}$ on $\mathcal{Q}_{\lfloor\text {Circ }\rfloor}$. We claim that

$$
\omega_{\lfloor\text {Circ }\rfloor}(G)=\left\lfloor\omega_{\text {Circ }}(G)\right\rfloor
$$

for every graph $G$. If $G$ is a graph such that $E(G)=\varnothing$, then $K_{1}$ is the only graph $H \in \mathcal{Q}_{\lfloor\text {[irc }\rfloor}=\mathcal{Q}_{\text {Circ }}$ such that $H \rightarrow G$. Hence

$$
\omega_{\lfloor\text {Circ }\rfloor}(G)=1=\omega_{\text {Circ }}(G)=\left\lfloor\omega_{\text {Circ }}(G)\right\rfloor .
$$


If $G$ is such that $E(G) \neq \varnothing$, then

$$
\begin{aligned}
\left\lfloor\omega_{\operatorname{Circ}}(G)\right\rfloor & =\left\lfloor\max \left\{\frac{a}{b} \mid a, b \in \mathbb{N}, 0<b \leq a, \operatorname{Circ}(a, b) \rightarrow G\right\}\right\rfloor \\
& =\max \left\{\left\lfloor\frac{a}{b}\right\rfloor \mid a, b \in \mathbb{N}, 0<b \leq a, \operatorname{Circ}(a, b) \rightarrow G\right\} \\
& =\sup \left\{g_{\lfloor\operatorname{Circ}\rfloor}(H) \mid H \in \mathcal{Q}_{\lfloor\text {Circ }\rfloor}, H \preceq_{P} G\right\} \\
& =\omega_{\lfloor\operatorname{Circ}\rfloor}(G) .
\end{aligned}
$$

by Proposition 3.40

by monotonicity of $\lfloor\cdot\rfloor$

by $(3.129)$ and $E(G) \neq \varnothing$

by $(3.94 a)$

Thus, we conclude (3.130).

Define $\psi: \mathcal{Q}_{\lfloor\text {Circ }\rfloor} \rightarrow \mathcal{Q}_{K}$ by

$$
\begin{aligned}
\psi\left(K_{1}\right) & :=K_{1}, \\
\psi(\operatorname{Circ}(a, b)) & :=K_{\lfloor a / b\rfloor} \text { for every } \operatorname{Circ}(a, b) \in \mathcal{Q}_{\lfloor\operatorname{Circ}\rfloor} .
\end{aligned}
$$

Note that

$$
g_{\lfloor\text {Circ }\rfloor}\left(K_{1}\right)=1=g_{K}\left(K_{1}\right)=g_{K}\left(\psi\left(K_{1}\right)\right)
$$

and that

$$
g_{\lfloor\operatorname{Circ}\rfloor}(\operatorname{Circ}(a, b))=\left\lfloor\frac{a}{b}\right\rfloor=g_{K}\left(K_{\lfloor a / b\rfloor}\right)=g_{K}(\psi(\operatorname{Circ}(a, b)))
$$

for every $a, b \in \mathbb{N}$ with $0<2 b \leq a$. Both $K_{1} \preceq_{P} K_{1}$ and (3.125) imply that $\psi(H) \preceq_{P} H$ for every $H \in \mathcal{Q}_{\lfloor\text {Circ }\rfloor}$. Thus, by Theorem 3.28 item 1 ,

$$
\left\lfloor\omega_{\text {Circ }}\right\rfloor=\omega_{\lfloor\text {Circ }\rfloor} \leq \omega
$$

Define

$$
\begin{gathered}
\mathcal{Q}_{\lceil\mathrm{Circ}\rceil}:=\mathcal{Q}_{\text {Circ }}, \\
g_{\lceil\mathrm{Circ}\rceil}: \mathcal{Q}_{\text {Circ }} \rightarrow[-\infty,+\infty], \\
g_{\lceil\mathrm{Circ}\rceil}:=\left\lceil g_{\text {Circ }}(H)\right\rceil \text { for every } H \in \mathcal{Q}_{\lceil\mathrm{Circ}\rceil} .
\end{gathered}
$$

Let $\chi_{g_{\lceil\text {Circ }\rceil}}$ be the greatest $(P, \overline{\mathbb{R}})$-monotone function which is at most $g_{\lceil\text {Circ }\rceil}$ on $\mathcal{Q}_{\lceil\text {Circ }\rceil}$. We claim that for every graph $G$,

$$
\chi_{\lceil\operatorname{Circ}\rceil}(G)=\left\lceil\chi_{\text {Circ }}(G)\right\rceil .
$$

Indeed, for every graph $G$,

$$
\begin{aligned}
\left\lceil\chi_{\operatorname{Circ}}(G)\right\rceil & =\left\lceil\min \left\{\frac{a}{b} \mid a, b \in \mathbb{N}, 0<b \leq a, G \rightarrow \operatorname{Circ}(a, b)\right\}\right\rceil \\
& =\min \left\{\left\lceil\frac{a}{b}\right\rceil \mid a, b \in \mathbb{N}, 0<b \leq a, G \rightarrow \operatorname{Circ}(a, b)\right\} \\
& =\inf \left\{g_{\lceil\operatorname{Circ}\rceil}(H) \mid H \in \mathcal{Q}_{\lceil\operatorname{Circ}\rceil}, G \preceq_{P} H\right\} \\
& =\chi_{\lceil\operatorname{Circ}\rceil}(G) .
\end{aligned}
$$

by Proposition 3.39

by monotonicity of $\lceil\cdot\rceil$

by $(3.131)$

by $(3.94 b)$

Define $\phi: \mathcal{Q}_{\lceil\mathrm{Circ}\rceil} \rightarrow \mathcal{Q}_{K}$ by

$$
\begin{aligned}
\phi\left(K_{1}\right) & :=K_{1}, \\
\phi(\operatorname{Circ}(a, b)) & :=K_{\lceil a / b\rceil} \text { for every } \operatorname{Circ}(a, b) \in \mathcal{Q}_{\lceil\operatorname{Circ}\rceil} .
\end{aligned}
$$

Note that

$$
g_{\lceil\operatorname{Circ}\rceil}\left(K_{1}\right)=1=g_{K}\left(K_{1}\right)=g_{K}\left(\phi\left(K_{1}\right)\right)
$$

and that

$$
g_{\lceil\operatorname{Circ}\rceil}(\operatorname{Circ}(a, b))=\left\lceil\frac{a}{b}\right\rceil=g_{K}\left(K_{\lceil a / b\rceil}\right)=g_{K}(\phi(\operatorname{Circ}(a, b)))
$$

for every $a, b \in \mathbb{N}$ such that $0<2 b \leq a$. Both $K_{1} \preceq_{P} K_{1}$ and and (3.126) imply that $H \preceq_{P} \phi(H)$ for every $H \in \mathcal{Q}_{\lceil\text {Circ }\rceil}$. Thus, by Theorem 3.28 item 2,

$$
\chi \leq \chi_{\lceil\operatorname{Circ}\rceil}=\left\lceil\chi_{\text {Circ }}\right\rceil .
$$


Using that $\omega$ and $\chi$ are always integers and Corollaries 3.38 and 3.41, we conclude that

$$
\left\lfloor\omega_{\text {Circ }}\right\rfloor=\omega \leq \omega_{\text {Circ }} \text { and } \chi_{\text {Circ }} \leq \chi=\left\lceil\chi_{\text {Circ }}\right\rceil .
$$

These results are as old as the parameters they relate: [116] proves that $\left\lceil\chi_{c}\right\rceil=\chi$, and [121] proves that $\left\lfloor\omega_{c}\right\rfloor=\omega$.

Proposition 3.42. Let $a, b \in \mathbb{N}$ be such that $0<b \leq a$. Then

$$
\operatorname{Circ}(a, b) \rightarrow \operatorname{KG}(a, b) .
$$

Proof. Define $f:[a) \rightarrow\left(\begin{array}{c}{[a)} \\ b\end{array}\right)$ by

$$
f(i):=\{k \bmod a \mid k \in \mathbb{N}, i \leq k \leq i+b-1\},
$$

where $k \bmod a$ denotes the remainder of the division of $k$ by $a$. Let $i j \in E(\operatorname{Circ}(a, b))$ and assume without loss of generality that $i<j$. Note that $b \leq j-i \leq a-b$ implies that $b-1<j-i<a-b+1$, which in turn implies that

$$
\begin{aligned}
& i+b-1<j \text { and } \\
& j+b-1<a+i .
\end{aligned}
$$

Note that (3.134) implies that $f(i)=\{i, i+1, \ldots, i+b-1\}$, since $j<a$. Let $k \in \mathbb{N}$ be such that

$$
j \leq k \leq j+b-1 \text {. }
$$

If $k<a$, then

$$
\begin{aligned}
k & \geq j \\
& >i+b-1,
\end{aligned}
$$

which implies that $(k \bmod a)=k \notin f(i)$. If $k \geq a$, we have that

$$
\begin{aligned}
a & \leq k \\
& \leq j+b-1 \\
& <a+i \\
& <2 a,
\end{aligned}
$$

since $i \in[a)$

which implies that $(k \bmod a)<i$, so $(k \bmod a) \notin f(i)$. Recalling the definition of $f$, we have just proved that $f(i) \cap f(j)=\varnothing$, which implies that $f \in \operatorname{Hom}(\operatorname{Circ}(a, b), \operatorname{KG}(a, b))$.

Corollary 3.43. It holds that

$$
\omega_{\mathrm{KG}} \leq \omega_{\mathrm{Circ}} \text { and } \chi_{\mathrm{KG}} \leq \chi_{\mathrm{Circ}} .
$$

Proof. Let $P$ denote the preordered set of graphs under the homomorphic relation. Define $\psi: \mathcal{Q}_{\mathrm{KG}} \rightarrow \mathcal{Q}_{\text {Circ }}$ by

$$
\begin{aligned}
\psi\left(K_{1}\right) & :=K_{1}, \\
\psi(\operatorname{KG}(a, b)) & :=\operatorname{Circ}(a, b) \text { for every } \operatorname{KG}(a, b) \in \mathcal{Q}_{\mathrm{KG}}
\end{aligned}
$$

Both (3.108) and (3.121) imply that $\psi$ is surjective. Proposition 3.42 and $K_{1} \preceq_{P} K_{1}$ imply that $\psi(H){ }_{P} H$ for every $H \in \mathcal{Q}_{\mathrm{KG}}$. Finally,

$$
g_{\mathrm{KG}}(\operatorname{KG}(a, b))=\frac{a}{b}=g_{\mathrm{Circ}}(\operatorname{Circ}(a, b))=g_{\mathrm{Circ}}(\psi(\operatorname{KG}(a, b))) .
$$

Theorem 3.28 item 3 finishes the proof.

We conclude this subsection by proving that $\omega_{c} \leq \chi_{c}$ and that $\omega_{\mathrm{KG}} \leq \chi_{f}$. It is remarkable how the geometric formulation (3.112) of the fractional chromatic number proves Proposition 3.44, which has a "purely combinatorial" statement. 
Proposition 3.44. Let $a, b \in \mathbb{N}$ be such that $0<2 b \leq a$. Then

$$
\chi_{\mathrm{KG}}(\operatorname{Circ}(a, b))=\chi_{\mathrm{KG}}(\operatorname{KG}(a, b))=\frac{a}{b} .
$$

Proof. Any subset of $[a)$ with at least $b+1$ elements must have an edge in $\operatorname{Circ}(a, b)$ : in the best scenario they are consecutive elements in the cyclic order, and the first vertex must be adjacent to the last vertex. Therefore $\alpha(\operatorname{Circ}(a, b)) \leq b$. Hence $\bar{x}:=(1 / b) \mathbb{1} \in \operatorname{QSTAB}(\overline{\operatorname{Circ}(a, b)}) \subseteq \mathbb{R}^{[a)}$, as for every stable set $S \in \mathcal{S}(\operatorname{Circ}(a, b))$ we have that $\mathbb{1}_{S}^{\top} \bar{x}=|S| / b \leq 1$. Thus

$$
\begin{aligned}
\frac{a}{b} & =\mathbb{1}^{\top} \bar{x} & & \\
& \leq \max \left\{\mathbb{1}^{\top} x \mid x \in \operatorname{QSTAB}(\overline{\operatorname{Circ}(a, b)})\right\} & & \text { since } \bar{x} \in \operatorname{QSTAB}(\overline{\operatorname{Circ}(a, b)}) \\
& =\chi_{f}(\operatorname{Circ}(a, b), \mathbb{1}) & & \text { by }(3.112) \\
& =\chi_{\mathrm{KG}}(\operatorname{Circ}(a, b)) & & \text { by Theorem } 3.33 \\
& \leq \chi_{\mathrm{KG}}(\operatorname{KG}(a, b)) & & \text { by Proposition } 3.42 \text { and }(3.85) \\
& \leq \frac{a}{b} . & & \text { by }(3.91 \mathrm{~b})
\end{aligned}
$$

Corollary 3.45. It holds that

$$
\omega_{\mathrm{Circ}} \leq \chi_{\mathrm{KG}}
$$

Proof. We have that $\chi_{\mathrm{KG}}$ is a homomorphism monotone function (by $(3.85)$ ) such that $\chi_{\mathrm{KG}}(G) \geq g_{\mathrm{Circ}}(G)$ for every $G \in \mathcal{Q}_{\text {Circ }}$ (by Theorem 3.33 and Proposition 3.44). Recalling Definition 3.24, the statement follows.

Note how Corollaries 3.43 and 3.45 imply that

$$
\omega_{\mathrm{Circ}} \leq \chi_{\mathrm{Circ}} \text { and } \omega_{\mathrm{KG}} \leq \chi_{\mathrm{KG}} .
$$

That these inequalities follow from Proposition 3.44 can be seen directly from Theorem 3.27, as Proposition 3.44 shows that the homomorphism monotone function $\chi_{f}$ agrees with $g_{\text {Circ }}$ on $\mathcal{Q}_{\text {Circ }}$ and agrees with $g_{\mathrm{KG}}$ on $\mathcal{Q}_{\mathrm{KG}}$.

\subsubsection{The Vector Chromatic Number}

Karger, Motwani, and Sudan [65] defined the vector chromatic number of a graph. The authors of [65] reformulate this parameter as the optimal value of a semidefinite programming problem, and they use this SDP to provide an approximation algorithm for the chromatic number of a graph. Although the vector chromatic number was defined in [65] as a step towards an approximation algorithm, it was already known in other contexts. Lovász [77] introduced the graph parameter $\vartheta$, which became known as the Lovász $\vartheta$ function, to solve a problem in information theory. While [65] already noted the relation between the vector chromatic number and the $\vartheta$ function, Szegedy [115] stated the relation precisely, observing that the vector chromatic number of a graph $G$ is equal to $\vartheta^{\prime}(\bar{G})$, where $\vartheta^{\prime}$ is a variant of $\vartheta$ introduced independently by McEliece, Rodemich, and Rumsey [87] and Schrijver [108]. The paper [115] also introduces $\vartheta^{+}$, another variation of the Lovász $\vartheta$ function, which may also be formulated as a coloring problem. The names for the variants of the vector chromatic number in this subsection come from [101, Section 6.7]. For recent papers on the vector chromatic number see [41, 42,43]. The history of the vector chromatic number just sketched provides a glimpse of the rich relations around the Lovász $\vartheta$ function, its variants, and their applications. The introductions of $[6,28]$ point to further results regarding the parameter. To complement the references just mentioned, we remark that [47, 72] provide a context for $\vartheta$ in algebraic geometry, whereas [21, 98] use the $\vartheta$ function to compare the power of classical and quantum computational models.

Define

$$
\text { Ball : }=\left\{\mathcal{B} \mid n \in \mathbb{N}, \mathcal{B} \subseteq \mathbb{R}^{n}, \forall x \in \mathcal{B}, x^{\top} x=1\right\} .
$$

Let $\mathcal{B} \in$ Ball and let $\gamma \in \mathbb{R}$. Define $\operatorname{Vect}(\mathcal{B}, \gamma)$ to be the graph such that

$$
\begin{aligned}
& V(\operatorname{Vect}(\mathcal{B}, \gamma)):=\mathcal{B}, \\
& E(\operatorname{Vect}(\mathcal{B}, \gamma)):=\left\{\{x, y\} \in\left(\begin{array}{c}
\mathcal{B} \\
2
\end{array}\right) \mid x^{\top} y \leq \gamma\right\} .
\end{aligned}
$$


Similarly, define $\operatorname{Strict}(\mathcal{B}, \gamma)$ to be the graph such that

$$
\begin{aligned}
& V(\operatorname{Strict}(\mathcal{B}, \gamma)):=\mathcal{B}, \\
& E(\operatorname{Strict}(\mathcal{B}, \gamma)):=\left\{\{x, y\} \in\left(\begin{array}{c}
\mathcal{B} \\
2
\end{array}\right) \mid x^{\top} y=\gamma\right\}
\end{aligned}
$$

Intuitively, we may think that $\operatorname{Vect}(\mathcal{B}, \gamma)$ has edges between vertices which are "far enough" apart, where the notion of distance comes from the inner product. If the set $\mathcal{B}$ is such that $x^{\top} y \geq \gamma$ for every $x, y \in \mathcal{B}$, we say that $\mathcal{B}$ is $\gamma$-rigid.

Let $P$ be the preordered set of graphs under the homomorphic relation. Define

$$
\begin{gathered}
\mathcal{Q}_{\text {Vect }}:=\left\{K_{1}\right\} \cup\left\{\operatorname{Vect}(\mathcal{B}, \gamma) \mid \gamma \in-\mathbb{R}_{++}, \mathcal{B} \in \text { Ball }\right\}, \\
g_{\text {Vect }}: \mathcal{Q}_{\text {Vect }} \rightarrow[-\infty,+\infty], \\
g_{\text {Vect }}\left(K_{1}\right):=1, \\
g_{\text {Vect }}(\operatorname{Vect}(\mathcal{B}, \gamma)):=1-\frac{1}{\gamma} .
\end{gathered}
$$

We denote by $\chi_{\text {Vect }}:=\chi_{g_{\text {Vect }}}$ the greatest $(P, \overline{\mathbb{R}})$-monotone function which is at most $g_{\text {Vect }}$ on $\mathcal{Q}_{\text {Vect }}$. Moreover, define

$$
\begin{gathered}
\mathcal{Q}_{\text {Strict }}:=\left\{K_{1}\right\} \cup\left\{\operatorname{Strict}(\mathcal{B}, \gamma) \mid \gamma \in-\mathbb{R}_{++}, \mathcal{B} \in \text { Ball }\right\}, \\
g_{\text {Strict }}: \mathcal{Q}_{\text {Strict }} \rightarrow[-\infty,+\infty], \\
g_{\text {Strict }}\left(K_{1}\right):=1, \\
g_{\text {Strict }}(\operatorname{Strict}(\mathcal{B}, \gamma)):=1-\frac{1}{\gamma} .
\end{gathered}
$$

We denote by $\chi_{\text {Strict }}:=\chi_{g_{\text {Strict }}}$ the greatest $(P, \overline{\mathbb{R}})$-monotone function which is at most $g_{\text {Strict }}$ on $\mathcal{Q}_{\text {Strict }}$. Finally, define

$$
\begin{aligned}
\mathcal{Q}_{\text {Rigid }}:=\left\{K_{1}\right\} \cup\left\{\operatorname{Strict}(\mathcal{B}, \gamma) \mid \gamma \in-\mathbb{R}_{++}, \mathcal{B} \in \text { Ball, } \mathcal{B} \text { is } \gamma \text {-rigid }\right\}, \\
g_{\text {Rigid }}: \mathcal{Q}_{\text {Rigid }} \rightarrow[-\infty,+\infty], \\
g_{\text {Rigid }}\left(K_{1}\right):=1, \\
g_{\text {Rigid }}(\operatorname{Strict}(\mathcal{B}, \gamma)):=1-\frac{1}{\gamma} .
\end{aligned}
$$

We denote by $\chi_{\text {Rigid }}:=\chi_{g_{\text {Rigid }}}$ the greatest $(P, \overline{\mathbb{R}})$-monotone function which is at most $g_{\text {Rigid }}$ on $\mathcal{Q}_{\text {Rigid }}$.

The colorings just defined revolve around representing a graph as points in the unit sphere, with certain constraints on the inner products among them. Hence the name vector coloring. An example may elucidate the discussion. Let $\gamma \in \mathbb{R}$ be a negative number, let $\mathcal{B} \in$ Ball be a $\gamma$-rigid set, and let $G$ be a graph. A homomorphism $f \in \operatorname{Hom}(G, \operatorname{Strict}(\mathcal{B}, \gamma))$ is a function $f: V(G) \rightarrow \mathcal{B}$ such that

$$
\begin{gathered}
\|f(i)\|=1 \text { for every } i \in V(G), \\
f(i)^{\top} f(j) \geq \gamma \text { for every } i, j \in V(G), \text { and } \\
f(i)^{\top} f(j)=\gamma \text { for every } i j \in E(G) .
\end{gathered}
$$

Proposition 3.46. For every $\mathcal{B} \in$ Ball and for every $\gamma \in-\mathbb{R}_{++}$,

$$
\operatorname{Strict}(\mathcal{B}, \gamma) \rightarrow \operatorname{Vect}(\mathcal{B}, \gamma) \text {. }
$$

Proof. As $\operatorname{Strict}(\mathcal{B}, \gamma)$ is a subgraph of $\operatorname{Vect}(\mathcal{B}, \gamma)$, it follows that $\operatorname{id}_{\mathcal{B}}$ is the desired homomorphism.

Corollary 3.47. It holds that

$$
\chi_{\text {Vect }} \leq \chi_{\text {Strict }} .
$$


Proof. Define $\phi: \mathcal{Q}_{\text {Strict }} \rightarrow \mathcal{Q}_{\text {Vect }}$ by

$$
\begin{aligned}
\phi\left(K_{1}\right) & :=K_{1} \\
\phi(\operatorname{Strict}(\mathcal{B}, \gamma)) & :=\operatorname{Vect}(\mathcal{B}, \gamma) \text { for every } \operatorname{Strict}(\mathcal{B}, \gamma) \in \mathcal{Q}_{\text {Strict }}
\end{aligned}
$$

Since

$$
g_{\text {Vect }}(\phi(\operatorname{Strict}(\mathcal{B}, \gamma)))=1-\frac{1}{\gamma}=g_{\text {Strict }}(\operatorname{Strict}(\mathcal{B}, \gamma))
$$

for every $\gamma \in-\mathbb{R}_{++}$and for every $\mathcal{B} \in$ Ball, we have that Proposition 3.46, $K_{1} \preceq_{P} K_{1}$, and Theorem 3.28 item 2 imply the statement.

Proposition 3.48. It holds that

$$
\chi_{\text {Strict }} \leq \chi_{\text {Rigid }} .
$$

Proof. Note that $\mathcal{Q}_{\text {Rigid }} \subseteq \mathcal{Q}_{\text {Strict }}$, so we may define $\psi: \mathcal{Q}_{\text {Rigid }} \rightarrow \mathcal{Q}_{\text {Strict }}$ by $\psi:=\iota_{\mathcal{Q}_{\text {Rigid }}}$. As

$$
g_{\text {Strict }} \circ \psi=g_{\text {Strict }} \circ \iota_{\mathcal{Q}_{\text {Rigid }}}=g_{\text {Rigid }},
$$

Theorem 3.28 item 2 implies the statement.

We now relate the vector chromatic numbers with the Lovász $\vartheta$ function and its variants. Whereas $\bar{\vartheta}=\chi_{\text {Strict }}$ is proved in [65], the equality $\overline{\vartheta^{\prime}}=\chi_{\text {Vect }}$ is simply mentioned in [115]. This is part of a common practice of relegating proofs about $\vartheta^{\prime}$ and $\vartheta^{+}$to parenthetical remarks around a proof about $\vartheta$. The next argument, which will treat the vector colorings in a unified manner, can be seen as a warm-up for Section 5.2, where we discuss a framework from [28] which, among other things, properly handles the three parameters presented in a unified manner.

Let $V$ be a finite set, and let $E^{+}, E^{-} \subseteq\left(\begin{array}{c}V \\ 2\end{array}\right)$. Define

$$
\mathbb{A}_{E^{+}, E^{-}}:=\left\{A \in \mathbb{S}^{V} \mid \forall i j \in E^{+}, A_{i j} \geq 0, \forall i j \in E^{-}, A_{i j} \leq 0\right\} .
$$

One may easily check that $\mathbb{A}_{E^{+}, E^{-}}$is a cone, i.e., that

$$
\text { for every } \alpha \in \mathbb{R}_{++}, \alpha \mathbb{A}_{E^{+}, E^{-}} \subseteq \mathbb{A}_{E^{+}, E^{-}} .
$$

Let $w \in \mathbb{R}_{+}^{V}$. Adapting the notation from [28], define

$$
\vartheta_{2}\left(\mathbb{A}_{E^{+}, E^{-}}, w\right):=\inf \left\{\rho \mid \rho \in \mathbb{R}, \sqrt{w} \sqrt{w}^{\top}-Y \preceq \rho I, \operatorname{diag}(Y)=0, Y \in \mathbb{A}_{\overline{E^{+}}, \overline{E^{-}}}\right\},
$$

where $\overline{E^{+}}$and $\overline{E^{-}}$denote the complement of the respective sets in $\left(\begin{array}{c}V \\ 2\end{array}\right)$. We take (3.144) as the bridge between the vector colorings and the bibliography on the Lovász $\vartheta$ function and its variants. More specifically, one may check that [77, Theorem 3] proves that

$$
\vartheta(G)=\vartheta_{2}\left(\mathbb{A}_{E, E}, \mathbb{1}\right)
$$

whereas the formulations of $\vartheta^{\prime}$ and $\vartheta^{+}$in $[115$, Section 2] state that

$$
\vartheta^{\prime}(G)=\vartheta_{2}\left(\mathbb{A}_{E \cup \bar{E}, E}, \mathbb{1}\right)
$$

and

$$
\vartheta^{+}(G)=\vartheta_{2}\left(\mathbb{A}_{\varnothing, E}, \mathbb{1}\right)
$$

for every graph $G:=(V, E)$.

Proposition 3.49. Let $V$ be a finite set. Then for every $E^{+}, E^{-} \subseteq\left(\begin{array}{c}V \\ 2\end{array}\right)$,

$$
\vartheta_{2}\left(\mathbb{A}_{E^{+}, E^{-}}, \mathbb{1}\right)=\inf \left\{1-\frac{1}{\gamma} \mid \gamma \in-\mathbb{R}_{++}, C \in \mathbb{S}_{+}^{V}, \operatorname{diag}(C)=\mathbb{1}, C-\gamma \mathbb{1} \mathbb{1}^{\top} \in \mathbb{A}_{\overline{E^{+}}, \overline{E^{-}}}\right\} .
$$


Proof. Set $\overline{\mathbb{A}}:=\mathbb{A}_{\overline{E^{+}}, \overline{E^{-}}}$. Let $(\gamma, C)$ be a feasible solution in (3.146). Set $\rho:=1-\frac{1}{\gamma}$, and define $Y:=$ $(\rho-1) C+\mathbb{1}^{\top}-\rho I$. Since $C \in \mathbb{S}_{+}^{V}$ and $\rho \geq 1$,

$$
\mathbb{1}^{\top}-Y=\rho I-(\rho-1) C \preceq \rho I .
$$

Moreover

$$
\operatorname{diag}(Y)=(\rho-1) \mathbb{1}+\mathbb{1}-\rho \mathbb{1}=0 .
$$

As $\gamma<0$, it follows that $\rho-1>0$, so (3.143) implies that

$$
Y+\rho I=(\rho-1) C+\mathbb{1}^{\top}=(\rho-1)\left(C-\gamma \mathbb{1}^{\top}\right) \in \overline{\mathbb{A}} .
$$

Recalling (3.142), we conclude that

$$
Y \in \overline{\mathbb{A}} \text {. }
$$

By combining (3.147), (3.148), and (3.149) we conclude that $(\rho, Y)$ is feasible in (3.144), and has objective value $\rho=1-\frac{1}{\gamma}$.

Let $(\rho, Y)$ be feasible in (3.144). Note that

$$
\rho \geq \lambda_{\max }\left(\mathbb{1} \mathbb{1}^{\top}-Y\right) \geq \max \left\{\left(\mathbb{1} \mathbb{1}^{\top}-Y\right)_{i i} \mid i \in V\right\}=1 .
$$

Let $\varepsilon>0$ and define

$$
\begin{aligned}
\gamma_{\varepsilon} & :=-\frac{1}{\rho+\varepsilon-1}, \\
C_{\varepsilon} & :=-\gamma_{\varepsilon}\left(Y-\mathbb{1}^{\top}+(\rho+\varepsilon) I\right) .
\end{aligned}
$$

We first prove that

$$
C_{\varepsilon} \in \mathbb{S}_{+}^{V}
$$

Note that

$$
\begin{aligned}
& -\frac{1}{\gamma_{\varepsilon}} C_{\varepsilon}=Y-\mathbb{1}^{\top}+(\rho+\varepsilon) I \quad \text { by }(3.151) \\
& =-\left(\mathbb{1} \mathbb{1}^{\top}-Y\right)+(\rho+\varepsilon) I \\
& \succeq-\rho I+(\rho+\varepsilon) I \quad \text { since }(\rho, Y) \text { is feasible in (3.144) } \\
& =\varepsilon I \\
& \in \mathbb{S}_{+}^{V}
\end{aligned}
$$

Since $\mathbb{S}_{+}^{V}$ is a cone and $-1 / \gamma_{\varepsilon}>0$ (as $\left.\rho \geq 1\right)$, we conclude (3.152). Furthermore,

$$
\operatorname{diag}\left(C_{\varepsilon}\right)=\mathbb{1},
$$

since

$$
\begin{aligned}
\operatorname{diag}\left(C_{\varepsilon}\right) & =\operatorname{diag}\left(-\gamma_{\varepsilon}\left(Y-\mathbb{1}^{\top}+(\rho+\varepsilon) I\right)\right) & & \text { by }(3.151) \\
& =-\gamma_{\varepsilon}(\operatorname{diag}(Y)-\mathbb{1}+(\rho+\varepsilon) \mathbb{1}) & & \\
& =-\gamma_{\varepsilon}(\rho+\varepsilon-1) \mathbb{1} & & \text { since }(\rho, Y) \\
& =\mathbb{1} . & & \text { by }(3.150)
\end{aligned}
$$

Finally, we have that

$$
C_{\varepsilon}-\gamma_{\varepsilon} \mathbb{1}^{\top} \in \overline{\mathbb{A}}
$$

since

$$
\begin{aligned}
C_{\varepsilon}-\gamma_{\varepsilon} \mathbb{1} \mathbb{1}^{\top} & =-\gamma_{\varepsilon}\left(Y-\mathbb{1}^{\top}+(\rho+\varepsilon) I\right)-\gamma_{\varepsilon} \mathbb{1}^{\top} & & \text { by }(3.151) \\
& =-\gamma_{\varepsilon}(Y+(\rho+\varepsilon) I) & & \\
& \in \overline{\mathbb{A}} . & & \text { by }(3.143) \text { and since }(\rho, Y) \text { is feasible in }(3.144)
\end{aligned}
$$


By combining (3.152), (3.153), and (3.154), we conclude that $\left(\gamma_{\varepsilon}, C_{\varepsilon}\right)$ is feasible in (3.146) and has objective value

$$
1-\frac{1}{\gamma_{\varepsilon}}=1+(\rho+\varepsilon-1)=\rho+\varepsilon
$$

As this holds for every $\varepsilon>0$, the proof is done.

Corollary 3.50. Let $V$ be a finite set. Then for every $E^{+}, E^{-} \subseteq\left(\begin{array}{c}V \\ 2\end{array}\right)$,

$$
\vartheta_{2}\left(\mathbb{A}_{\overline{E^{+}}, \overline{E^{-}}}, \mathbb{1}\right)=\inf \left\{1-\frac{1}{\gamma} \mid \begin{array}{c}
\gamma \in-\mathbb{R}_{++}, \exists n \in \mathbb{N}, \exists f: V \rightarrow \mathbb{R}^{n}, \\
\forall i \in V,\|f(i)\|=1, \\
\forall i j \in E^{+},\langle f(i), f(j)\rangle \geq \gamma, \forall i j \in E^{-},\langle f(i), f(j)\rangle \leq \gamma
\end{array}\right\} .
$$

Proof. Set $\mathbb{A}:=\mathbb{A}_{E^{+}, E^{-}}$. Proposition 3.49 implies that

$$
\vartheta_{2}\left(\mathbb{A}_{\overline{E^{+}}, \overline{E^{-}}}, \mathbb{1}\right)=\inf \left\{1-\frac{1}{\gamma} \mid \gamma \in-\mathbb{R}_{++}, C \in \mathbb{S}_{+}^{V}, \operatorname{diag}(C)=\mathbb{1}, C-\gamma \mathbb{1} \mathbb{1}^{\top} \in \mathbb{A}\right\} .
$$

Let $f: V \rightarrow \mathbb{R}^{n}$ and $\gamma \in-\mathbb{R}_{++}$be feasible in (3.155). Let $C \in \mathbb{S}_{+}^{V}$ be defined by $C_{i j}:=\langle f(i), f(j)\rangle$ for every $i, j \in V$. We have that $\operatorname{diag}(C)=\mathbb{1}$ because $\|f(i)\|=1$ for every $i \in V$, and that

$$
C-\gamma \mathbb{1} \mathbb{1}^{\top} \in \mathbb{A} \text {. }
$$

Hence $(\gamma, C)$ is feasible in $(3.156)$ and with objective value $1-\frac{1}{\gamma}$. Thus ' $\leq$ ' holds in the statement.

Let $(\gamma, C)$ be feasible in (3.156). Then there exist $n \in \mathbb{N}$ and $B \in \mathbb{R}^{[n) \times V}$ such that $C=B^{\top} B$. Define $f: V \rightarrow \mathbb{R}^{n}$ by $f(i):=B e_{i}$ for every $i \in V$. For every $i, j \in V$

$$
\begin{aligned}
\langle f(i), f(j)\rangle & =e_{i}^{\top} B^{\top} B e_{j} \\
& =e_{i}^{\top} C e_{j} \\
& =C_{i j} .
\end{aligned}
$$

Since $\operatorname{diag}(C)=\mathbb{1}$ we have that $\|f(i)\|=1$ for every $i \in V$. Moreover, as $C-\gamma \mathbb{1} \mathbb{1}^{\top} \in \mathbb{A}$, we conclude that $\langle f(i), f(j)\rangle \geq \gamma$ for every $i j \in E^{+}$, since

$$
0 \leq C_{i j}-\gamma=\langle f(i), f(j)\rangle-\gamma .
$$

Similarly, we have that $\langle f(i), f(j)\rangle \leq \gamma$ for every $i j \in E^{-}$. Thus $(\gamma, f)$ is feasible in (3.155).

To show that the Lovász theta function and its variants are equal to the vector colorings presented, it is convenient to show that the graph $K_{1}$ may be safely discarded when computing the vector chromatic numbers. Proposition 3.51 is the crucial step in this argument, and can be interpreted as stating that " $K_{1}$ is in the closure of the vector colorings". An analogous argument explains why there is no mention to $K_{1}$ in (3.102) or $(3.124 b)$.

Proposition 3.51. There exist $\gamma \in-\mathbb{R}_{++}^{\mathbb{N}}$ and $\mathcal{B} \in$ Ball such that for every $n \in \mathbb{N}$,

$$
K_{1} \rightarrow \operatorname{Strict}\left(\mathcal{B}, \gamma_{n}\right)
$$

and

$$
\mathcal{B} \text { is } \gamma_{n} \text {-rigid. }
$$

Moreover, it holds that

$$
\lim _{n \rightarrow \infty} 1-\frac{1}{\gamma_{n}}=1 \text {. }
$$

Proof. Set $\mathcal{B}:=\{1\} \subseteq \mathbb{R}$, and set $\gamma_{n}:=-(n+1)$ for every $n \in \mathbb{N}$. It is immediate that (3.159) holds. Now let $n \in \mathbb{N}$. As $E\left(K_{1}\right)=\varnothing$, we have that

$$
\left(x \in V\left(K_{1}\right) \mapsto 1\right) \in \operatorname{Hom}\left(K_{1}, \operatorname{Strict}\left(\mathcal{B}, \gamma_{n}\right)\right),
$$

so (3.157) holds. And so does (3.158), as for every $x, y \in \mathcal{B}$,

$$
\begin{array}{rlrl}
x^{\top} y & =1 & & \text { since } \mathcal{B}=\{1\} \\
>\gamma_{n} . & & \text { since } \gamma_{n} \in-\mathbb{R}_{++}
\end{array}
$$


Corollary 3.52. Let $G$ be a graph. Then

$$
\begin{aligned}
\chi_{\text {Vect }}(G) & =\inf \left\{1-\frac{1}{\gamma} \mid \gamma \in-\mathbb{R}_{++}, \mathcal{B} \in \operatorname{Ball}, G \rightarrow \operatorname{Vect}(\mathcal{B}, \gamma)\right\}, \\
\chi_{\text {Strict }}(G) & =\inf \left\{1-\frac{1}{\gamma} \mid \gamma \in-\mathbb{R}_{++}, \mathcal{B} \in \operatorname{Ball}, G \rightarrow \operatorname{Strict}(\mathcal{B}, \gamma)\right\}, \\
\chi_{\text {Rigid }}(G) & =\inf \left\{1-\frac{1}{\gamma} \mid \gamma \in-\mathbb{R}_{++}, \mathcal{B} \in \operatorname{Ball}, \mathcal{B} \text { is } \gamma \text {-rigid, } G \rightarrow \operatorname{Strict}(\mathcal{B}, \gamma)\right\} .
\end{aligned}
$$

Proof. It is immediate from (3.94b) and the definitions in (3.139), (3.140), and (3.141) that ' $\leq$ ' holds in each equality of the statement. On the other hand, if $G \rightarrow K_{1}$, then Proposition 3.51 and transitivity of ' $\rightarrow$ ' imply that there exists a sequence of vector colorings ensuring that ' $\geq$ ' holds in each equality of the statement.

Let $G:=(V, E)$ be a graph. We wish to prove that

$$
\chi_{\text {Vect }}=\overline{\vartheta^{\prime}}, \chi_{\text {Strict }}=\bar{\vartheta} \text {, and } \chi_{\text {Rigid }}=\overline{\vartheta^{+}} .
$$

Note how (3.137) implies that for every $\gamma \in-\mathbb{R}_{++}$,

$$
\text { there exist } \mathcal{B} \in \text { Ball and } f \in \operatorname{Vect}(\mathcal{B}, \gamma)
$$

if and only if

$$
\text { there exists }(\gamma, n, f) \text { feasible in }(3.155) \text { with }\left(E^{+}, E^{-}\right):=(\varnothing, E) .
$$

Using (3.145b) and Corollaries 3.50 and 3.52 we conclude that $\overline{\vartheta^{\prime}}=\chi_{\text {vect }}$. Moreover, we have that (3.138) implies that for every $\gamma \in-\mathbb{R}_{++}$,

$$
\text { there exist } \mathcal{B} \in \text { Ball and } f \in \operatorname{Strict}(\mathcal{B}, \gamma)
$$

if and only if

$$
\text { there exists }(\gamma, n, f) \text { feasible in }(3.155) \text { with }\left(E^{+}, E^{-}\right):=(E, E) .
$$

Using (3.145a) and Corollaries 3.50 and 3.52 we conclude that $\bar{\vartheta}=\chi_{\text {Strict }}$. Finally, the definition of $\gamma$-rigid and (3.138) imply that for every $\gamma \in-\mathbb{R}_{++}$,

$$
\text { there exist } \gamma \text {-rigid } \mathcal{B} \in \text { Ball and } f \in \operatorname{Strict}(\mathcal{B}, \gamma)
$$

if and only if

$$
\text { there exists }(\gamma, n, f) \text { feasible in }(3.155) \text { with }\left(E^{+}, E^{-}\right):=(E \cup \bar{E}, E) \text {. }
$$

Using (3.145c) and Corollaries 3.50 and 3.52 we conclude that $\overline{\vartheta^{+}}=\chi_{\text {Rigid }}$. We have thus proved (3.160).

\subsubsection{The Quantum Chromatic Number}

Cameron, Montanaro, Newman, Severini, and Winter [22] consider the coloring game, in which two players attempt to convince a referee that $G \rightarrow K_{n}$ for a given graph $G$ and $n \in \mathbb{N}$. Mančinska and Roberson [85] generalize this setting into the homomorphism game, in which both players attempt to convince a referee that $G \rightarrow H$ for a pair of graphs $G$ and $H$. Formulations of decision problems as a "game" in which provers attempt to convince a verifier that a given input has a given property were previously employed by the theoretical computer science community in the study of interactive proof protocols - see, e.g., [11]. Feige and Lovász [36] accidentally studied a special case of the homomorphism game using semidefinite programming decades before [85]. We say "accidentally" because, as it is pointed out in [25], much of this early work by the TCS community implicitly forbids the players from using quantum strategies, whereas the definitions in $[22,85]$ are suitable for understanding what can be done in a quantum model of computation. The coloring and homomorphism games are examples of nonlocal games, and the study of nonlocal games is an active area of research [48, 112, 113].

In this setting, the quantum chromatic number of a graph captures the best strategy possible for players sharing quantum information, as opposed to the chromatic number, which captures the best strategy possible for players sharing classical information. Although introduced in [22], the idea of a quantum chromatic 
number is mentioned in a previous work by Avis, Hasegawa, Kikuchi, and Sasaki [5]. Both papers, however, cite even earlier results which make assertions about the quantum chromatic number of certain graphs, such as [18]. Aside from the discussion in [22], we also point the reader to [73, Section 2.3] and [25]. For recent results on the quantum chromatic number, check [96, 119].

We have so far presented no results on complex numbers. This is a deliberate choice in exposition, as the only places in this text which actually work with the complex field are this subsection and Subsection 3.4.5. This kind of choice has an obvious cost: there are simple results which we lack the language to demonstrate. We now briefly pay this toll by introducing some definitions and stating without proof some results. The diligent reader could look into [118, Chapter 1]. Let $V$ be a finite set. Define the trace $\operatorname{Tr}_{\mathbb{C}}: \mathbb{C}^{V \times V} \rightarrow \mathbb{C}$ by

$$
\operatorname{Tr}_{\mathbb{C}}(X):=\sum_{i \in V} X_{i i}
$$

for every $X \in \mathbb{C}^{V \times V}$. It is easy to see that

$$
\operatorname{Tr}_{\mathbb{C}}(X+\alpha Y)=\operatorname{Tr}_{\mathbb{C}}(X)+\alpha \operatorname{Tr}_{\mathbb{C}}(Y)
$$

for every $X, Y \in \mathbb{C}^{V \times V}$ and $\alpha \in \mathbb{C}$. Furthermore, one may also prove that for every finite sets $S$ and $T$, and for every matrices $A \in \mathbb{C}^{S \times T}$ and $B \in \mathbb{C}^{T \times S}$, it holds that

$$
\operatorname{Tr}_{\mathbb{C}}(A B)=\operatorname{Tr}_{\mathbb{C}}(B A)
$$

Let $S$ and $T$ be finite sets. We denote by $\bar{x} \in \mathbb{C}^{S}$ the componentwise complex conjugate of $x \in \mathbb{C}^{S}$. Similarly, we denote by $\bar{X} \in \mathbb{C}^{S \times T}$ the componentwise complex conjugate of $X \in \mathbb{C}^{S \times T}$. For every $X \in \mathbb{C}^{S \times T}$, we denote by $X^{\top} \in \mathbb{C}^{T \times S}$ the matrix defined by $\left(X^{\top}\right)_{j i}:=X_{i j}$ for every $i \in S$ and $j \in T$.

Let $V$ be a finite set. Define the set

$$
\mathcal{H}_{V}:=\left\{A \in \mathbb{C}^{V \times V} \mid \overline{A^{\top}}=A\right\}
$$

of Hermitian matrices. As usual, define $\mathcal{H}_{a}:=\mathcal{H}_{[a}$ for every $a \in \mathbb{N}$. Surprisingly, Hermitian matrices form a real vector space, and the real vector spaces $\mathbb{R}^{V \times V}$ and $\mathcal{H}_{V}$ are linearly isomorphic. One such isomorphism is given by the following function:

$$
\left[\begin{array}{ll}
\alpha & \beta \\
\gamma & \delta
\end{array}\right] \in \mathbb{R}^{2 \times 2} \mapsto\left[\begin{array}{cc}
\alpha & \beta+i \gamma \\
\beta-i \gamma & \delta
\end{array}\right] \in \mathcal{H}_{2}
$$

Our use of this correspondence is encapsulated by the following proposition.

Proposition 3.53 ([118, Equation (1.100)]). Let $V$ be a finite set. There exists a bijective real linear transformation $\Phi: \mathcal{H}_{V} \rightarrow \mathbb{R}^{V \times V}$ such that

$$
\langle\Phi(A), \Phi(B)\rangle=\operatorname{Tr}_{\mathbb{C}}(A B)
$$

for every $A, B \in \mathcal{H}_{V}$.

We now reach the last relevant result needed on complex matrices. Let $V$ be a finite set, and let $P, Q \in \mathcal{H}_{V}$. If $P^{2}=P$ and $Q^{2}=Q$, then

$$
\operatorname{Tr}_{\mathbb{C}}(P Q) \geq 0 .
$$

This result follows directly from extending the notion of positive semidefinite matrices to complex matrices. Similar to the real case, one may use a complex version of the spectral theorem [118, Theorem 1.3] to show that if $X \in \mathcal{H}_{V}$ is such that $X^{2}=X$, then $X$ is a positive semidefinite complex matrix, so a standard argument implies (3.163). The discussion following [118, Theorem 1.2] presents several equivalent formulations of positive semidefinite complex matrices.

We now formulate the quantum chromatic number as a generalized coloring number. Let $G:=(V, E)$ be a graph, and let $d \in \mathbb{N}$. Following [85], define the measurement graph $\mathcal{M}(G, d)$ as the graph such that

$$
\begin{aligned}
& V(\mathcal{M}(G, d)):=\left\{P \in\left(\mathbb{C}^{d \times d}\right)^{V} \mid P_{i} \in \mathcal{H}_{d} \text { is such that } P_{i}^{2}=P_{i} \text { for every } i \in V, \sum_{i \in V} P_{i}=I\right\}, \\
& E(\mathcal{M}(G, d)):=\left\{\{P, Q\} \in\left(\begin{array}{c}
V(\mathcal{M}(G, d)) \\
2
\end{array}\right) \mid P_{i} Q_{j}=0 \text { for every } i, j \in V \text { such that } i j \notin E\right\} .
\end{aligned}
$$


Definition 3.54. Let $G$ and $H$ be graphs. A quantum homomorphism from $G$ to $H$ is an homomorphism in $\operatorname{Hom}(G, \mathcal{M}(H, d))$ for some $d \in \mathbb{N}$. We write $G \rightarrow^{q} H$ if there exists $d \in \mathbb{N}$ such that $\operatorname{Hom}(G, \mathcal{M}(H, d)) \neq \varnothing$.

Let $P$ denote the preordered set of graphs under the homomorphic relation. Define

$$
\begin{gathered}
\mathcal{Q}_{q}:=\left\{\mathcal{M}\left(K_{n}, d\right) \mid n \in \mathbb{N}, n>0, d \in \mathbb{N}\right\}, \\
g_{q}: \mathcal{Q}_{q} \rightarrow[-\infty,+\infty], \\
g_{q}\left(\mathcal{M}\left(K_{n}, d\right)\right):=n
\end{gathered}
$$

We denote by $\chi_{q}:=\chi_{g_{q}}$ the greatest $(P, \overline{\mathbb{R}})$-monotone function which is at most $g_{q}$ on $\mathcal{Q}_{q}$. Note that the vertices of the measurement graph are functions taking values in a certain space of complex matrices. This explains the clumsy function in the following important result. Recall our notation on function matrices (2.12). The reader familiar with functional programming may interpret the next result as using currying to translate a function matrix in $\mathbb{C}^{V(H) \times V(G)}$ into an object in

$$
V(G) \rightarrow\left(V(H) \rightarrow \mathbb{C}^{1 \times 1}\right)
$$

for $G$ and $H$ graphs. We also point out that nowhere in the following proof, we employ the useful Iverson notation: to avoid confusion, all of the uses of square brackets represent matrices.

Proposition 3.55. Let $G$ and $H$ be graphs, and let $f \in \operatorname{Hom}(G, H)$. For every $i \in V(G)$, define $F(i): V(H) \rightarrow \mathbb{C}^{1 \times 1}$ by

$$
F(i):=\left(k \in V(H) \mapsto\left[\left(P_{f}\right)_{k i}\right] \in \mathbb{C}^{1 \times 1}\right) .
$$

Then $F \in \operatorname{Hom}(G, \mathcal{M}(H, 1))$.

Proof. Let $i \in V(G)$. Set $P:=F(i)$. Note that $P: V(H) \rightarrow \mathbb{C}^{1 \times 1}$. We first prove that

$$
P \in V(\mathcal{M}(H, 1)) .
$$

Since for every $k \in V(H)$ we have that $\left(P_{f}\right)_{k i} \in\{0,1\}$, it follows that

$$
P_{k}^{2}=\left[\left(P_{f}\right)_{k i}\right]^{2}=\left[\left(P_{f}\right)_{k i}^{2}\right]=\left[\left(P_{f}\right)_{k i}\right]=P_{k} .
$$

Similarly, we conclude that $\overline{P_{k}^{\top}}=P_{k}$. Moreover, by $(2.15)$,

$$
\sum_{k \in V(H)} P_{k}=\sum_{k \in V(H)}\left[\left(P_{f}\right)_{k i}\right]=[1]
$$

which is the identity in $\mathbb{C}^{1 \times 1}$. Using (3.164a) we conclude (3.166).

Now let $i j \in E(G)$, set $P:=F(i)$, and set $Q:=F(j)$. Then for every $k, \ell \in V(H)$,

$$
P_{k} Q_{l}=\left[\left(P_{f}\right)_{k i}\right]\left[\left(P_{f}\right)_{\ell j}\right]=\left[\left(P_{f}\right)_{k i}\left(P_{f}\right)_{\ell j}\right] .
$$

The product in the rightmost matrix in (3.167) is one if and only if $f(i)=k$ and $f(j)=\ell$. Since $f \in \operatorname{Hom}(G, H)$, we have that $\{f(i), f(j)\} \in E(H)$. Therefore, for every $k, \ell \in V(H)$ such that $k \ell \notin E(H)$, the product must be zero, so $\{F(i), F(j)\} \in E(\mathcal{M}(H, 1))$ by (3.164b).

Corollary 3.56. For every graph $G$,

$$
G \rightarrow \mathcal{M}(G, 1) .
$$

Proof. Proposition 3.55 and $\operatorname{id}_{V(G)} \in \operatorname{Hom}(G, G)$ imply the statement.

Corollary 3.57. It holds that

$$
\chi_{q} \leq \chi
$$


Proof. Let $P$ denote the preordered set of graphs under the homomorphic relation. Define $\phi: \mathcal{Q}_{K} \rightarrow \mathcal{Q}_{q}$ by $\phi\left(K_{n}\right):=\mathcal{M}\left(K_{n}, 1\right)$ for every nonzero $n \in \mathbb{N}$. Corollary 3.56 implies $H \preceq_{P} \phi(H)$ for every $H \in \mathcal{Q}_{K}$, and we have that

$$
g_{q}\left(\phi\left(K_{n}\right)\right)=g_{q}\left(\mathcal{M}\left(K_{n}, 1\right)\right)=n=g_{K}\left(K_{n}\right)
$$

for every nonzero $n \in \mathbb{N}$. Theorem 3.28 item 2 implies the statement.

Let $G$ and $H$ be graphs. Section 5.4 presents a generalization of quantum homomorphisms. For the sake of clarity, we remark that there is a one to one correspondence [100] between

$$
\bigcup\{\operatorname{Hom}(G, \mathcal{M}(H, d)) \mid d \in \mathbb{N}\} \text { and } \operatorname{Hom}_{\mathbb{C S}_{+}}(G, H),
$$

where $\operatorname{Hom}_{\mathbb{C S}}(G, H)$ is one of the main subjects of Section 5.4. Theorem 5.73 actually restates (3.168). Using Theorems 5.72 and 5.73 and Proposition 3.22, we obtain the quantum homomorphic relation. The restriction of this relation to our universe $\mathcal{U}_{\mathbb{R}}$ defines the preordered set $P_{q}$ of graphs under the quantum homomorphic relation. We may then define a quantum analogue of the clique and coloring numbers for each parameter presented in this section. For example, the "quantum circular chromatic number" is the greatest $\left(P_{q}, \overline{\mathbb{R}}\right)$ monotone function which is at most $g_{\text {Circ }}$ on $\mathcal{Q}_{\text {Circ }}$. In this manner, graph parameters formulated using Definitions 3.24 and 3.25 have a built-in classical-to-quantum conversion. This is in tune with the literature: although using other language, [85] defines the quantum clique number $\omega_{q}$ as the greatest $\left(P_{q}, \overline{\mathbb{R}}\right)$-monotone function which is at most $g_{K}$ on $\mathcal{Q}_{K}$.

\subsubsection{The Orthogonal and Projective Rank}

For every $d \in \mathbb{N}$, define $\Omega(d)$ as the graph such that

$$
\begin{aligned}
& V(\Omega(d)):=\left\{x \in \mathbb{C}^{d} \mid \bar{x}^{\top} x=1\right\}, \text { and } \\
& E(\Omega(d)):=\left\{\{x, y\} \in\left(\begin{array}{c}
V(\Omega(d)) \\
2
\end{array}\right) \mid \bar{x}^{\top} y=0\right\} .
\end{aligned}
$$

Note the use of the componentwise conjugate. Let $P$ denote the preordered set of graphs under the homomorphic relation. Define

$$
\begin{gathered}
\mathcal{Q}_{\xi}:=\{\Omega(d) \mid d \in \mathbb{N}, d>0\}, \\
g_{\xi}: \mathcal{Q}_{\xi} \rightarrow[-\infty,+\infty], \\
g_{\xi}(\Omega(d)):=d .
\end{gathered}
$$

We denote by $\xi:=\chi_{g_{\xi}}$ the greatest $(P, \overline{\mathbb{R}})$-monotone function which is at most $g_{\xi}$ on $\mathcal{Q}_{\xi}$. The parameter $\xi$ is called the orthogonal rank of a graph. Lovász [77] considered orthonormal representations of a graph $G$, which are precisely the elements of $\operatorname{Hom}(\bar{G}, \Omega(d))$ for a given $d \in \mathbb{N}$, and proved that $\xi \geq \bar{\vartheta}$. The graph parameter $\bar{\xi}$ has been studied as the minimum positive semidefinite rank of a graph [10,35]. Briët and Zuiddam [19] bound $\xi$ on Cayley graphs and use those bounds to prove a conjecture on quantum interactive protocols.

Proposition 3.58. For every $n \in \mathbb{N}$,

$$
K_{n} \rightarrow \Omega(n) .
$$

Proof. Let $e:[n) \rightarrow \mathbb{C}^{[n)}$ denote the standard basis. In other words, let $e$ be such that

$$
e_{i}(j):=[i=j]
$$

for every $i, j \in[n)$. Note that for every $i, j \in[n)$,

$$
{\overline{e_{i}}}^{\top} e_{j}=e_{i}^{\top} e_{j}=[i=j] \text {. }
$$

Thus $e \in \operatorname{Hom}\left(K_{n}, \Omega(n)\right)$.

Corollary 3.59. It holds that

$$
\xi \leq \chi
$$


Proof. Let $P$ denote the preordered set of graphs under the homomorphic relation. Define $\phi: \mathcal{Q}_{K} \rightarrow \mathcal{Q}_{\xi}$ by $\phi\left(K_{n}\right):=\Omega(n)$ for every nonzero $n \in \mathbb{N}$. Proposition 3.58 implies that $H \preceq_{P} \phi(H)$ for every $H \in \mathcal{Q}_{K}$, and we have that

$$
g_{\xi}\left(\phi\left(K_{n}\right)\right)=g_{\xi}(\Omega(n))=n=g_{K}\left(K_{n}\right)
$$

for every nonzero $n \in \mathbb{N}$. Theorem 3.28 item 2 finishes the proof.

For every $a, b \in \mathbb{N}$ such that $0<b \leq a$, define the graph $\Omega(a, b)$ as the graph such that

$$
\begin{aligned}
& V(\Omega(a, b)):=\left\{P \in \mathbb{C}^{a \times a} \mid P \in \mathcal{H}_{a}, P^{2}=P, \operatorname{Tr}_{\mathbb{C}}(P)=b\right\}, \\
& E(\Omega(a, b)):=\left\{\{P, Q\} \in\left(\begin{array}{c}
V(\Omega(a, b)) \\
2
\end{array}\right) \mid \operatorname{Tr}_{\mathbb{C}}(P Q)=0\right\} .
\end{aligned}
$$

Let $P$ denote the preordered set of graphs under the homomorphic relation. Define

$$
\begin{gathered}
\mathcal{Q}_{\xi f}:=\left\{K_{1}\right\} \cup\{\Omega(a, b) \mid a, b \in \mathbb{N}, 0<2 b \leq a\}, \\
g_{\xi f}: \mathcal{Q}_{\xi f} \rightarrow[-\infty,+\infty], \\
g_{\xi f}\left(K_{1}\right):=1, \\
g_{\xi f}(\Omega(a, b)):=\frac{a}{b} .
\end{gathered}
$$

Let $\xi_{f}:=\chi_{g_{\xi f}}$ denote the largest $(P, \overline{\mathbb{R}})$-monotone function which is at most $g_{\xi f}$ on $\mathcal{Q}_{\xi f}$. Mančinska and Roberson [85] introduced $\xi_{f}$ as the projective rank of a graph. Some of the similarities between the pairs $\left(\chi, \chi_{f}\right)$ and $\left(\xi, \xi_{f}\right)$ will become apparent after our upcoming propositions; they are also discussed in [59, 85].

Proposition 3.60 ([85, Section 6.1]). For every $a, b \in \mathbb{N}$ such that $0<b \leq a$,

$$
\mathrm{KG}(a, b) \rightarrow \Omega(a, b) .
$$

Proof. Define $P: V(\operatorname{KG}(a, b)) \rightarrow \Omega(a, b)$ for every $S \in V(\operatorname{KG}(a, b))$ by

$$
P_{S}:=\sum_{i \in S} e_{i} e_{i}^{\top}
$$

For every $S, T \in V(\operatorname{KG}(a, b))$ we have that

$$
P_{S} P_{T}=\sum_{i \in S \cap T} e_{i} e_{i}^{\top}
$$

since

$$
P_{S} P_{T}=\sum_{i \in S} \sum_{j \in T} e_{i} e_{i}^{\top} e_{j} e_{j}^{\top}=\sum_{i \in S} \sum_{j \in T}[i=j] e_{i} e_{j}^{\top}=\sum_{i \in S}[i \in T] e_{i} e_{i}^{\top}=\sum_{i \in S \cap T} e_{i} e_{i}^{\top} .
$$

Since for every set $S$, the matrix $P_{S}$ has only real entries, we conclude that $\overline{P_{S}}=P_{S}$, so (3.173) implies that $P_{S}^{2}=\overline{P_{S}^{\top}}=P_{S}$. Furthermore,

$$
\begin{aligned}
\operatorname{Tr}_{\mathbb{C}}\left(P_{S}\right) & =\sum_{i \in S} \operatorname{Tr}_{\mathbb{C}}\left(e_{i} e_{i}^{\top}\right) & & \text { by }(3.162 \mathrm{a}) \\
& =\sum_{i \in S} 1 & & \text { by }(3.161) \\
& =b . & & \text { since } S \in V(\operatorname{KG}(a, b))
\end{aligned}
$$

Finally,

$$
\begin{aligned}
\operatorname{Tr}_{\mathbb{C}}\left(P_{S} P_{T}\right) & =\operatorname{Tr}_{\mathbb{C}}\left(\sum_{i \in S \cap T} e_{i} e_{i}^{\top}\right) & & \text { by }(3.173) \\
& =\operatorname{Tr}_{\mathbb{C}}(0) & & \text { since } S \cap T=\varnothing \\
& =0 . & & \text { by }(3.162 \mathrm{a})
\end{aligned}
$$

Hence $P \in \operatorname{Hom}(\operatorname{KG}(a, b), \Omega(a, b))$. 
Corollary 3.61. It holds that

$$
\xi_{f} \leq \chi_{f} .
$$

Proof. Let $P$ denote the preordered set of graphs under the homomorphic relation. Define $\phi: \mathcal{Q}_{\mathrm{KG}} \rightarrow \mathcal{Q}_{\xi f}$ by

$$
\begin{aligned}
\phi\left(K_{1}\right) & :=K_{1}, \\
\phi(\operatorname{KG}(a, b)) & :=\Omega(a, b) \text { for every } \operatorname{KG}(a, b) \in \mathcal{Q}_{\mathrm{KG}} .
\end{aligned}
$$

Proposition 3.60 and $K_{1} \preceq_{P} K_{1}$ imply that $H \preceq_{P} \phi(H)$ for every $H \in \mathcal{Q}_{K}$. Moreover, we have that $g_{\mathrm{KG}}\left(K_{1}\right)=g_{\xi f}\left(K_{1}\right)$ and that

$$
g_{\mathrm{KG}}(\operatorname{KG}(a, b))=\frac{a}{b}=g_{\xi f}(\Omega(a, b))=g_{\xi f}(\phi(\operatorname{KG}(a, b)))
$$

for every $\operatorname{KG}(a, b) \in \mathcal{Q}_{\mathrm{KG}}$. Theorem 3.28 item 2 finishes the proof.

Proposition 3.62. For every $d \in \mathbb{N}$,

$$
\Omega(d) \rightarrow \Omega(d, 1) .
$$

Proof. Define $P: V(\Omega(d)) \rightarrow \mathbb{C}^{d \times d}$ by

$$
P_{x}:=x \bar{x}^{\top}
$$

for every $x \in V(\Omega(d))$. It is then clear that

$$
P_{x} P_{y}=\left(\bar{x}^{\top} y\right) x \bar{y}^{\top}
$$

for every $x, y \in V(\Omega(d))$.

Let $x \in V(\Omega(d))$. It is immediate that $\overline{P_{x}^{\top}}=P_{x}$. Furthermore,

$$
\begin{aligned}
P_{x}^{2} & =\left(\bar{x}^{\top} x\right) x \bar{x}^{\top} & & \text { by }(3.175) \\
& =x \bar{x}^{\top} & & \text { since } x \in V(\Omega(d)) \text { and (3.169a) } \\
& =P_{x} . & & \text { by }(3.174)
\end{aligned}
$$

We conclude that

$$
\begin{aligned}
\operatorname{Tr}_{\mathbb{C}}\left(P_{x}\right) & =\operatorname{Tr}_{\mathbb{C}}\left(x \bar{x}^{\top}\right) & & \text { by }(3.174) \\
& =\operatorname{Tr}_{\mathbb{C}}\left(\bar{x}^{\top} x\right) & & \text { by }(3.162 \mathrm{~b}) \\
& =1, & & \text { since } x \in V(\Omega(d)) \text { and }(3.169 \mathrm{a})
\end{aligned}
$$

so $P_{x} \in V(\Omega(d, 1))$.

To show that $P \in \operatorname{Hom}(\Omega(d), \Omega(d, 1))$, let $\{x, y\} \in E(\Omega(d))$. Then

$$
\begin{aligned}
\operatorname{Tr}_{\mathbb{C}}\left(P_{x} P_{y}\right) & =\operatorname{Tr}_{\mathbb{C}}\left(\left(\bar{x}^{\top} y\right) x \bar{y}^{\top}\right) & & \text { by }(3.175) \\
& =\operatorname{Tr}_{\mathbb{C}}(0) & & \text { by }(3.169 \mathrm{~b}) \\
& =0 . & &
\end{aligned}
$$

Hence $\left\{P_{x}, P_{y}\right\} \in E(\Omega(d, 1))$ by $(3.171 b)$.

Corollary 3.63. It holds that

$$
\xi_{f} \leq \xi .
$$

Proof. Let $P$ denote the preordered set of graphs under the homomorphic relation. Define $\phi: \mathcal{Q}_{\xi} \rightarrow \mathcal{Q}_{\xi f}$ by $\phi(\Omega(d)):=\Omega(d, 1)$ for every nonzero $d \in \mathbb{N}$. Proposition 3.62 states that $H \preceq_{P} \phi(H)$ for every $H \in \mathcal{Q}_{\xi}$, and we have that

$$
g_{\xi}(\Omega(d))=d=g_{\xi f}(\Omega(d, 1))=g_{\xi f}(\phi(\Omega(d)))
$$

for every $\Omega(d) \in \mathcal{Q}_{\xi}$. Theorem 3.28 item 2 finishes the proof. 
While we are not going to develop the theory around Proposition 3.53, the following similar proposition, which we also state and use without proof, refers to an object that is one of the main topics of the upcoming Subsection 5.4.1.

Proposition 3.64. Let $n \in \mathbb{N}$. There exists a linear transformation vec: $\mathbb{R}^{[n) \times[n)} \rightarrow \mathbb{R}^{[n \times n)}$ such that

$$
\langle A, B\rangle=\operatorname{vec}(A)^{\top} \operatorname{vec}(B)
$$

for every $A, B \in \mathbb{R}^{V \times V}$.

Let $n \in \mathbb{N}$. All the function vec: $\mathbb{R}^{[n) \times[n)} \rightarrow \mathbb{R}^{[n \times n)}$ does is to transform matrices in vectors.

Proposition 3.65. Let $a, b \in \mathbb{N}$ be such that $0<b<a$. Set $\gamma:=-b /(a-b)$. There exists a $\gamma$-rigid $\mathcal{B} \in$ Ball such that

$$
\Omega(a, b) \rightarrow \operatorname{Strict}(\mathcal{B}, \gamma) .
$$

Proof. Let $\Phi$ denote the transformation mentioned in Proposition 3.53. Define $f: V(\Omega(a, b)) \rightarrow \mathbb{R}^{a \times a}$ for every $P \in V(\Omega(a, b))$ by

$$
f(P):=\left(\frac{a}{b(a-b)}\right)^{1 / 2}\left(\Phi(P)-\frac{b}{a} \Phi(I)\right) .
$$

Let $P, Q \in V(\Omega(a, b))$. We first prove that

$$
\langle\Phi(I), \Phi(P)\rangle=b .
$$

This follows from

$$
\begin{aligned}
\langle\Phi(P), \Phi(I)\rangle & =\operatorname{Tr}_{\mathbb{C}}(P I) & & \text { by Proposition } 3.53 \\
& =b . & & \text { by }(3.171 \mathrm{a})
\end{aligned}
$$

We have that

$$
\langle f(P), f(Q)\rangle=\frac{a \operatorname{Tr}_{\mathbb{C}}(P Q)}{b(a-b)}-\frac{b}{a-b},
$$

since

$$
\begin{aligned}
& \langle f(P), f(Q)\rangle \\
& =\frac{a}{b(a-b)}\left\langle\Phi(P)-\frac{b}{a} \Phi(I), \Phi(Q)-\frac{b}{a} \Phi(I)\right\rangle \\
& =\frac{a}{b(a-b)}\left(\langle\Phi(P), \Phi(Q)\rangle-\frac{b}{a}\langle\Phi(P), \Phi(I)\rangle-\frac{b}{a}\langle\Phi(I), \Phi(Q)\rangle+\frac{b^{2}}{a^{2}}\langle\Phi(I), \Phi(I)\rangle\right) \\
& =\frac{a}{b(a-b)}\left(\langle\Phi(P), \Phi(Q)\rangle-2 \frac{b^{2}}{a}+\frac{b^{2}}{a^{2}}\langle\Phi(I), \Phi(I)\rangle\right) \\
& =\frac{a}{b(a-b)}\left(\langle\Phi(P), \Phi(Q)\rangle-2 \frac{b^{2}}{a}+\frac{b^{2}}{a^{2}} \operatorname{Tr}_{\mathbb{C}}(I I)\right) \\
& =\frac{a}{b(a-b)}\left(\langle\Phi(P), \Phi(Q)\rangle-\frac{b^{2}}{a}\right) \\
& =\frac{a}{b(a-b)}\left(\operatorname{Tr}_{\mathbb{C}}(P Q)-\frac{b^{2}}{a}\right) \\
& =\frac{a \operatorname{Tr}_{\mathbb{C}}(P Q)}{b(a-b)}-\frac{b}{a-b} .
\end{aligned}
$$

Using (3.171a) and (3.178) we conclude that $\|f(P)\|=1$ for every $P \in V(\Omega(a, b))$. Furthermore, the first fraction in the RHS of (3.178) is always nonnegative by (3.163) and (3.171a). Thus, if $\{P, Q\} \in E(\Omega(a, b))$, then (3.171b) and (3.178) imply that $\langle f(P), f(Q)\rangle=\gamma$.

Define $\mathcal{B}:=(\operatorname{vec} \circ f \circ V)(\Omega(a, b))$. Proposition 3.64 implies that

$$
(\operatorname{vec} \circ f) \in \operatorname{Hom}(\Omega(a, b), \operatorname{Strict}(\mathcal{B}, \gamma)) .
$$




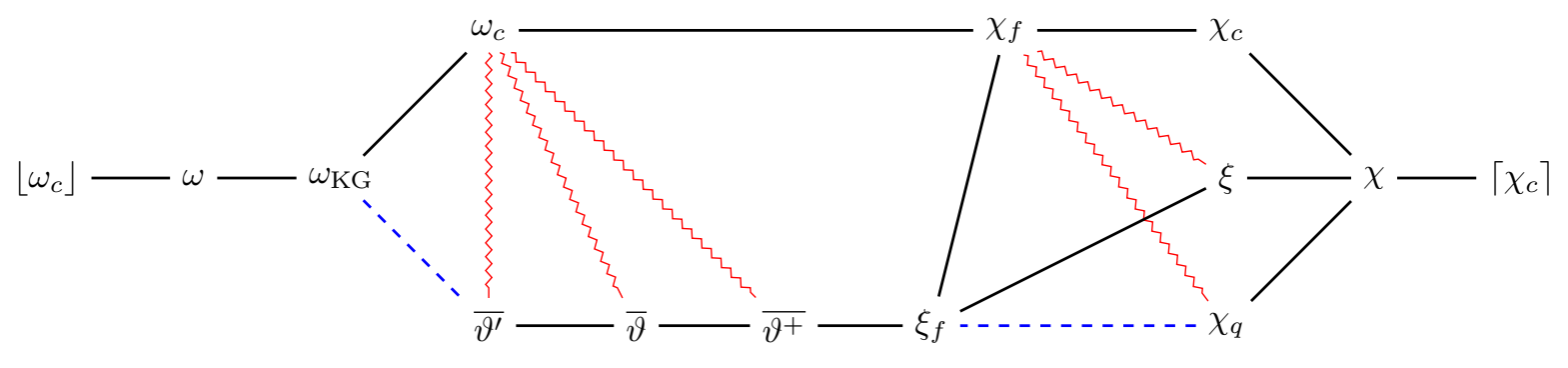

Figure 3.1: Hasse diagram of parameters. A straight (black) or dashed (blue) line indicates an inequality between parameters, with the smaller one to the left. The squiggly (red) lines indicate parameters known to be incomparable. The straight (black) lines are reserved for the results already proved.

Corollary 3.66. It holds that

$$
\overline{\vartheta^{+}} \leq \xi_{f}
$$

Proof. Let $P$ denote the preordered set of graphs under the homomorphic relation. Define $\phi: \mathcal{Q}_{\xi f} \rightarrow \mathcal{Q}_{\text {Rigid }}$ by

$$
\begin{aligned}
\phi\left(K_{1}\right) & :=K_{1}, \\
\phi(\Omega(a, b)) & :=\operatorname{Strict}\left(\mathcal{B}_{a, b},-\frac{b}{a-b}\right) \text { for every } \Omega(a, b) \in \mathcal{Q}_{\xi f},
\end{aligned}
$$

where $\mathcal{B}_{a, b}$ is the $\gamma$-rigid set given by Proposition 3.65, with $\gamma:=-b /(a-b)$. Proposition 3.65 implies that $H \preceq_{P} \phi(H)$ for every $H \in \mathcal{Q}_{\xi f}$. It is clear that $g_{\xi f}\left(K_{1}\right)=g_{\text {Rigid }}\left(\phi\left(K_{1}\right)\right)$. Furthermore,

$$
g_{\xi f}(\Omega(a, b))=\frac{a}{b}=1+\frac{a-b}{b}=g_{\text {Rigid }}\left(\operatorname{Strict}\left(\mathcal{B}_{a, b},-\frac{b}{a-b}\right)\right)=g_{\text {Rigid }}(\phi(\Omega(a, b)))
$$

for every $\Omega(a, b) \in \mathcal{Q}_{\xi f}$. Theorem 3.28 item 2 finishes the proof.

\subsubsection{A Unified Picture of the Presented Parameters}

Both Portuguese and English have adages expressing the idea that "a picture is worth a thousand words". Confusion may arise in this relationship, as one may have the picture and lack the words, or have the words and lack the picture. From this point of view, we find ourselves in a privileged position: we have both a picture and a couple of thousand words. Figure 3.1 restates Corollaries 3.38, 3.41, 3.43, 3.45, 3.47, 3.57, 3.59, 3.61, 3.63, and 3.66, and Propositions 3.29 and 3.48. Figure 3.2 annotates each inequality in Figure 3.1 with a reference to its proof. The Hasse diagram in Figure 3.1 captures the inequalities known among the parameters presented, and explains our criteria for deciding which inequalities to prove when presenting a parameter in the previous subsections. Figure 3.1 showcases the power of Theorem 3.28 and captures the idea that the parameters presented belong to a single context, directly related to $\omega$ and $\chi$.

There are four results in Figure 3.1 regarding $\overline{\vartheta^{\prime}}, \bar{\vartheta}$ and $\overline{\vartheta^{+}}$which are missing so far: that each of these parameters is incomparable with $\omega_{c}$, and that $\omega_{\mathrm{KG}} \leq \overline{\vartheta^{\prime}}$. Propositions 4.40 and 4.41 prove these results. Chapter 4 presents ways of computing the value of $\vartheta$ and its variants for specific families of graphs, and this is precisely what will be used in the proof of the two upcoming propositions. For the statements that $\chi_{f}$ is incomparable with both $\xi$ and $\chi_{q}$, as well as the inequality $\xi_{f} \leq \chi_{q}$, we refer the reader to [85].

Figure 3.1 also contains the celebrated sandwich theorem [67], first proved in [77]: the statement that

$$
\omega \leq \bar{\vartheta} \leq \chi .
$$

As we are missing one of the inequalities necessary for its proof, we add a simple argument to show that $\bar{\vartheta} \geq \omega$. 


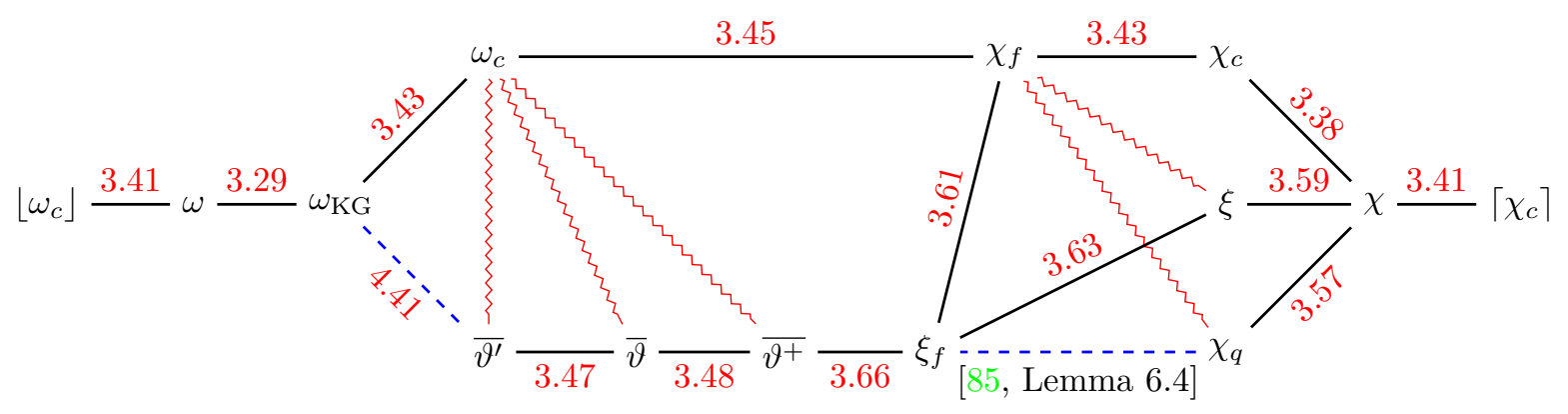

Figure 3.2: Figure 3.1 with the inequalities annotated by a reference to its proof.

Proposition 3.67. Let $n \in \mathbb{N}$. Let $\mathcal{B} \in$ Ball, and let $\gamma \in[-1,0)$. If $K_{n} \rightarrow \operatorname{Strict}(\mathcal{B}, \gamma)$, then

$$
n \leq 1-\frac{1}{\gamma} \text {. }
$$

Proof. Let $f \in \operatorname{Hom}\left(K_{n}, \operatorname{Strict}(\mathcal{B}, \gamma)\right)$. Define the matrix $C \in \mathbb{S}_{+}^{[n)}$ by

$$
C_{i j}=\langle f(i), f(j)\rangle
$$

for every $i, j \in[n)$. Since $f \in \operatorname{Hom}\left(K_{n}, \operatorname{Strict}(\mathcal{B}, \gamma)\right)$, we have that $C=I+\gamma\left(\mathbb{1}^{\top}-I\right)$. Hence

$$
C \mathbb{1}=(1+\gamma(n-1)) \mathbb{1},
$$

so $1+\gamma(n-1)$ is an eigenvalue of $C$. As $C$ is positive semidefinite, this eigenvalue must be nonnegative. If we rearrange the terms keeping in mind that $\gamma<0$, we conclude that $n \leq 1-\frac{1}{\gamma}$.

Corollary 3.68. It holds that

$$
\omega \leq \bar{\vartheta}
$$

Proof. We have that $\bar{\vartheta}$ is homomorphism monotone by (3.160) and (3.85). Proposition 3.67 implies $\bar{\vartheta}$ is at least $n$ for every $K_{n} \in \mathcal{Q}_{K}$. Definition 3.24 finishes the proof.

A real number is computable in polynomial time if its value can be approximated in polynomial time given any error bound as input. The sandwich theorem implies that for every graph $G$ such that $\omega(G)=\chi(G)$, then both $\omega(G)$ and $\chi(G)$ are computable in polynomial time, as $\bar{\vartheta}(G)$ is computable in polynomial time. One may see Figure 3.1 as a generalization of this result for several other parameters: for every graph $G$ with $\omega(G)=\chi(G)$, every parameter in Figure 3.1 is computable in polynomial time, as all of them are equal to $\bar{\vartheta}(G)$. This includes the important family of perfect graphs: a graph is perfect if all of its induced subgraphs $H$ are such that $\omega(H)=\chi(H)$. We thus have a nontrivial infinite family of graphs for which we know the values of some complicated parameters, such as $\xi$ or $\omega_{c}$. This is a special case of a pragmatic use of Figure 3.1: if for a given graph we can compute (or perhaps simply bound) any parameter in the figure, we can at a glimpse understand what other parameters are bounded by this value for this particular graph. An argument by Bachoc, Pêcher, and Thiéry [7] is also relevant to our discussion. In [7], the authors prove that for every graph $G$ such that $\omega_{\text {Circ }}(G)=\chi_{\text {Circ }}(G)$, both parameters can be computed in polynomial time. They exploit Propositions 3.39 and 3.40 to conclude that, when $\omega_{c}(G)=\chi_{c}(G)$, it holds that $G \leftrightarrow \operatorname{Circ}(a, b)$ for some $a, b \in \mathbb{N}$ such that $0<b \leq a$. By showing how to efficiently recover both $a$ and $b$ from $\bar{\vartheta}(\operatorname{Circ}(a, b))$, the authors prove that one may compute $a / b=\omega_{c}(G)=\chi_{c}(G)$ in polynomial time. A question lingering in this discussion is how Definitions 3.24 and 3.25 may guide a generalization of the notion of perfect graphs. Zhu [121], and consequently [7], defines a graph to be circular perfect if all of its induced subgraphs are homomorphically equivalent to some circular complete graph. This clearly can be cast in a more general setting using our framework. 
We also point out that there are generalized clique and coloring numbers which do not fit in Figure 3.1. Whereas this highlights the expressiveness of Definitions 3.24 and 3.25, it also serves as criticism of the naming we adopted. One such parameter is the fractional cut-covering number, which is the main topic of Subsection 3.4.8. Another generalized coloring number that does not fit in Figure 3.1 is the odd girth of a graph. For every graph $G$, define the odd girth $\operatorname{og}(G)$ of $G$ by

$$
\operatorname{og}(G):=\inf \left\{2 n+1 \mid n \in \mathbb{N}, C_{2 n+1} \rightarrow G\right\} .
$$

Let $P$ denote the preordered set of graphs under the homomorphic relation. Define

$$
\begin{gathered}
\mathcal{Q}_{\mathrm{og}}:=\left\{C_{2 n+1} \mid n \in \mathbb{N}\right\}, \\
g_{\mathrm{og}}: \mathcal{Q}_{\mathrm{og}} \rightarrow[-\infty,+\infty], \\
g_{\mathrm{og}}\left(C_{2 n+1}\right):=2 n+1 .
\end{gathered}
$$

Let $\chi_{g_{\mathrm{og}}}$ denote the greatest $\left(P^{*}, \overline{\mathbb{R}}\right)$-monotone function which is at most $g_{\text {og }}$ on $\mathcal{Q}_{\text {og }}$. For every graph $G$,

$$
\begin{aligned}
\chi_{g_{\mathrm{og}}}(G) & =\inf \left\{2 n+1 \mid n \in \mathbb{N}, G \preceq_{P^{*}} C_{2 n+1}\right\} & & \text { by }(3.94 \mathrm{~b}) \\
& =\inf \left\{2 n+1 \mid n \in \mathbb{N}, C_{2 n+1} \preceq_{P} G\right\} & & \text { by }(3.2) \\
& =\inf \left\{2 n+1 \mid n \in \mathbb{N}, C_{2 n+1} \rightarrow G\right\} & & \\
& =\operatorname{og}(G) . & & \text { by }(3.179)
\end{aligned}
$$

The developments in Section 3.3 then apply to the odd girth. For example, we conclude that

$$
G \rightarrow H \Longrightarrow H \preceq_{P^{*}} G \Longrightarrow \operatorname{og}(H) \leq \mathrm{og}(G)
$$

for every pair of graphs $G, H$.

There are also alternative formulations of some parameters in Figure 3.1 as generalized clique or generalized coloring numbers. For every $r \in \mathbb{R}_{++}$, define $I(r)$ to be the graph such that

$$
\begin{aligned}
& V(I(r)):=[0, r), \\
& E(I(r)):=\left\{\{i, j\} \in\left(\begin{array}{c}
{[0, r)} \\
2
\end{array}\right)|1 \leq| i-j \mid\right\} .
\end{aligned}
$$

We briefly show that these graphs give us nothing new, as

$$
I(r) \leftrightarrow K_{\lceil r\rceil}
$$

for every $r \in \mathbb{R}_{++}$. It is clear that $(x \mapsto x) \in \operatorname{Hom}\left(K_{\lceil r\rceil}, I(r)\right)$, since $V\left(K_{\lceil r\rceil}\right)=[\lceil r\rceil)=\{0, \ldots,\lceil r\rceil-1\}$. On the other hand, as $1 \leq|x-y|$ implies $\lfloor x\rfloor \neq\lfloor y\rfloor$ for every $x, y \in \mathbb{R}$, we have that $(x \mapsto\lfloor x\rfloor) \in \operatorname{Hom}\left(I(r), K_{\lceil r\rceil}\right)$. Theorem 3.28 and (3.182) imply that extending the function $I(r) \mapsto r$ to every graph via a generalized clique would define $\omega$, and extending it to every graph via a generalized coloring would define $\chi$. Zhu [120] states that it is well known that the graphs $I(r)$ define the same chromatic number as $\chi$, and presents a similar construction for the circular chromatic number. For every $r \in \mathbb{R}_{++}$, define $S(r)$ as the graph such that

$$
\begin{aligned}
& V(S(r)):=[0, r), \\
& E(S(r)):=\left\{\{i, j\} \in\left(\begin{array}{c}
{[0, r)} \\
2
\end{array}\right)|1 \leq| i-j \mid \leq r-1\right\} .
\end{aligned}
$$

Zhu [120] proves that

$$
S(a / b) \leftrightarrow \operatorname{Circ}(a, b)
$$

for every $a, b \in \mathbb{N}$ such that $0<b \leq a$. Both (3.182) and (3.184) showcase how homomorphisms may aid the study of infinite graphs. For example, we know that $\beta(I(r))=\beta\left(K_{\lceil r\rceil}\right)$ for every homomorphism monotone function $\beta$ and for every $r \in \mathbb{R}_{++}$. For another related example, define the graph $M(r)$ by

$$
\begin{aligned}
& V(M(r)):=\{S \subseteq[0,1] \mid S \text { is a measurable set with measure } 1 / r\}, \\
& E(M(r)):=\left\{\{S, T\} \in\left(\begin{array}{c}
V(M(r)) \\
2
\end{array}\right) \mid S \cap T=\varnothing\right\} .
\end{aligned}
$$


Deuber and Zhu [32] prove that

$$
\chi_{f}(G)=\inf \{r \mid r \in \mathbb{R}, r \geq 1, G \rightarrow M(r)\} .
$$

The previous discussion approaches an important issue we have not yet directly addressed. Let $P$ be the preordered set of graphs under the homomorphic relation. If we choose $Q \subseteq V(P)$ and $g: V(P) \rightarrow[-\infty,+\infty]$, then Definitions 3.24 and 3.25 provide a pair of dual homomorphism monotone functions. However, we are usually not interested in the pair $(Q, g)$, and rather in a given homomorphism monotone function $\beta: V(P) \rightarrow[-\infty,+\infty]$. It is always possible to find a pair whose generalized coloring number is equal to the original homomorphism monotone function: for every homomorphism monotone $\beta$, we may simply take $Q:=V(P)$ and $g:=\beta$. Hence, the examples throughout this chapter show that there may be more than one possible formulation. Whereas it is hard to have any qualms with (3.97), we felt inclined to justify $\mathcal{Q}_{\mathrm{KG}}$ right after its definition in (3.108). Even more pertinent, the motivation for our definition of $\mathcal{Q}_{\mathrm{KG}}$ provides an example in which an alternative pair $(Q, g)$ would change the generalized clique number obtained, without changing the generalized coloring number. Note that (3.187) provides yet another way of formulating $\chi_{f}$ as a generalized coloring number. We then arrive at the following question:

for a given $(P, \overline{\mathbb{R}})$-monotone function $\beta$,

what is the "best" $Q \subseteq V(P)$ and $g: Q \rightarrow[-\infty,+\infty]$ such that $\chi_{g}=\beta$ ?

Of course, there is a parallel question regarding $\omega_{g}$. It may well be the case that competing answers to the question posed in (3.188) have complementary qualities. Even in such a scenario, there may be reasonable "regularity" conditions which we can always impose when studying such problems. Theorem 3.27 hints at requiring $g$ to be monotone on $Q$, for example. At a high level, we are dealing with a situation that permeates the field of optimization. In general, there is more than one way of formulating an optimization problem, and the choice of formulation has technical ramifications. The main difference is that in, say, linear programming, we already have good technical assumptions and conditions to look out for when formulating the optimization problem.

\subsubsection{Relations Defined by the Homomorphic Product}

We developed our theory in terms of an arbitrary preordered set in Section 3.1. However, except for a comment in Subsection 3.4.4 regarding the quantum homomorphic relation, we have so far only worked with the preordered set of graphs under the homomorphic relation. This choice is justified by our interest in the graph parameters in Figure 3.1. In this subsection we exhibit a construction that defines a relation on graphs and is known to define a preorder in some cases.

Recall from (2.6) that $\otimes$ denotes the Kronecker product of two matrices. Our current interest in the Kronecker product is for its convenience in defining graph products. Let $G$ and $H$ be graphs. One may easily check that

$$
A_{G \times H}=A_{G} \otimes A_{H},
$$

where $A_{F} \in \mathbb{S}^{V(F)}$ denotes the adjacency matrix of a graph $F$, as defined in (2.29). It is easy to see that the equality above could be used as the definition of the graph $G \times H$ : the rows and columns of the adjacency matrix determine the vertex set of the graph, and the entries of the adjacency matrix determine the edge set of the graph. We define the Cartesian product $G \square H$ as the graph with vertex set $V(G) \times V(H)$ whose adjacency matrix is given by

$$
A_{G \square H}=A_{G} \otimes I+I \otimes A_{H} .
$$

The categorical and Cartesian products of graphs are examples of graph products. There is a whole book on graph products [53], and some recent work on them [42, 43, 122]. Notable among recent papers is a counterexample by Shitov [110] to a conjecture posed by Hedetniemi [55] in 1966, regarding the chromatic number of the categorical product of two graphs. We are interested in another graph product. The homomorphic product $G \ltimes H$ of $G$ and $H$ is the graph with vertex set $V(G) \times V(H)$ whose adjacency matrix is given by

$$
A_{G \ltimes H}=I \otimes\left(\mathbb{1}^{\top}-I\right)+A_{G} \otimes\left(\mathbb{1} \mathbb{1}^{\top}-A_{H}\right) .
$$

Although Hell [56] worked with this definition in 1979, Chvátal [23] worked with $G \ltimes K_{n}$ for $n \in \mathbb{N}$ in 1973. 
To prove our main result about the homomorphic product, it is convenient to introduce special notation for relations on sets. In particular, we generalize our notion of function matrix from (2.12) using that

$$
f: A \rightarrow B \text { is a subset of } A \times B \text { for every sets } A \text { and } B .
$$

Let $S$ and $T$ be finite sets, and let $\sim \subseteq S \times T$. Following tradition, we denote by $s \sim t$ the fact that $(s, t) \in \sim$. Define the matrix $P \sim \in \mathbb{R}^{T \times S}$ by

$$
P_{\sim}:=\sum_{i \in T} \sum_{j \in S}[j \sim i] e_{i} e_{j}^{\top} .
$$

It is immediate from (2.11), (3.191), and (3.192) that we are indeed generalizing function matrices. If we consider the equality relation $=\subseteq S \times S$ and the inequality relation $\neq \subseteq S \times S$, we have that

$$
\begin{aligned}
& P_{=}=I, \\
& P_{\neq}=\mathbb{1} \mathbb{1}^{\top}-I .
\end{aligned}
$$

Let $G$ be a graph. We have two relations of interest on $V(G) \times V(G)$ : the adjacency relation $i \sim_{G} j$ holds whenever $i j \in E(G)$ for $i, j \in V(G)$, and the non-adjacency relation $i \chi_{G} j$ holds whenever $i j \notin E(G)$ for $i, j \in V(G)$. It is clear that

$$
\begin{aligned}
& P_{\sim_{G}}=A_{G}, \\
& P_{\varkappa_{G}}=\mathbb{1}^{\top}-A_{G} .
\end{aligned}
$$

Proposition 3.69. Let $S$ and $T$ be finite sets. Let $\sim_{S} \subseteq S \times S$, let $\sim_{T} \subseteq T \times T$, and let $F \subseteq T \times S$. Then

$$
\mathbb{1}_{F}^{\top}\left(P \sim_{T} \otimes P_{\sim_{S}}\right) \mathbb{1}_{F}=\sum_{i, j \in S} \sum_{k, \ell \in T}\left[j \sim_{S} i\right]\left[\ell \sim_{T} k\right][(k, i) \in F][(\ell, j) \in F] .
$$

Proof. The statement follows from a direct computation:

$$
\begin{array}{rlrl}
\mathbb{1}_{F}^{\top}\left(P_{\sim_{T}} \otimes P_{\sim_{S}}\right) \mathbb{1}_{F} & =\sum_{i, j \in S}\left[j \sim_{S} i\right] \mathbb{1}_{F}^{\top}\left(P_{\sim_{T}} \otimes e_{i} e_{j}^{\top}\right) \mathbb{1}_{F} & & \text { by }(2.8) \text { and (3.192) } \\
& =\sum_{i, j \in S} \sum_{k, \ell \in T}\left[j \sim_{S} i\right]\left[\ell \sim_{T} k\right] \mathbb{1}_{F}^{\top}\left(e_{k} e_{\ell}^{\top} \otimes e_{i} e_{j}^{\top}\right) \mathbb{1}_{F} & & \text { by }(2.8) \text { and (3.192) } \\
& =\sum_{i, j \in S} \sum_{k, \ell \in T}\left[j \sim_{S} i\right]\left[\ell \sim_{T} k\right] \mathbb{1}_{F}^{\top}\left(\left(e_{k} \otimes e_{i}\right)\left(e_{\ell}^{\top} \otimes e_{j}^{\top}\right)\right) \mathbb{1}_{F} & & \text { by }(2.7) \\
& =\sum_{i, j \in S} \sum_{k, \ell \in T}\left[j \sim_{S} i\right]\left[\ell \sim_{T} k\right] \mathbb{1}_{F}^{\top}\left(\left(e_{k} \otimes e_{i}\right)\left(e_{\ell} \otimes e_{j}\right)^{\top}\right) \mathbb{1}_{F} & & \text { by }(2.9) \\
& =\sum_{i, j \in S} \sum_{k, \ell \in T}\left[j \sim_{S} i\right]\left[\ell \sim_{T} k\right] \mathbb{1}_{F}^{\top} e_{(k, i)} e_{(\ell, j)}^{\top} \mathbb{1}_{F} & & \text { by (2.10) } \\
& =\sum_{i, j \in S} \sum_{k, \ell \in T}\left[j \sim_{S} i\right]\left[\ell \sim_{T} k\right][(k, i) \in F][(\ell, j) \in F] . &
\end{array}
$$

Recall from Section 2.3 that if $S \subseteq V(G)$, we denote by $G[S]$ the subgraph induced by $S$. We now have the core result regarding the homomorphic product of two graphs.

Proposition 3.70 ([56]). Let $G$ and $H$ be graphs. Then

$$
\mathcal{S}(G \ltimes H)=\bigcup_{T \subseteq V(G)} \operatorname{Hom}(G[T], H) .
$$

Proof. Note that for every graph $K$,

$$
S \in \mathcal{S}(K) \text { if and only if } \mathbb{1}_{S}^{\top} A_{K} \mathbb{1}_{S}=0 .
$$


Let $U$ and $V$ be finite sets. Let $S \subseteq U \times V$. We claim that

$$
\mathbb{1}_{S}^{\top}\left(I \otimes\left(\mathbb{1}^{\top}-I\right)\right) \mathbb{1}_{S}=0 \text { if and only if } S \text { is a function. }
$$

Note that

$$
\begin{array}{rlrl}
\mathbb{1}_{S}^{\top}\left(I \otimes\left(\mathbb{1}^{\top}-I\right)\right) \mathbb{1}_{S} & =\mathbb{1}_{S}^{\top}\left(P=\otimes\left(\mathbb{1} \mathbb{1}^{\top}-I\right)\right) \mathbb{1}_{S} & & \text { by }(3.193) \\
& =\mathbb{1}_{S}^{\top}(P=\otimes P \neq) \mathbb{1}_{S} & & \text { by }(3.194) \\
& =\sum_{i, j \in V} \sum_{k, \ell \in U}[i \neq j][k=\ell][(k, i) \in S][(\ell, j) \in S] & & \text { by Proposition 3.69 } \\
& =\sum_{k \in U} \sum_{i, j \in V}[i \neq j][(k, i) \in S][(k, j) \in S] & & \\
& =|\{(k,(i, j)) \in U \times(V \times V) \mid i \neq j,(k, i) \in S,(k, j) \in S\}| .
\end{array}
$$

This implies (3.198).

We then claim that for every $S \subseteq V(G)$ and $f: S \rightarrow V(H)$,

$$
\mathbb{1}_{f}^{\top}\left(A_{G} \otimes\left(\mathbb{1}^{\top}-A_{H}\right)\right) \mathbb{1}_{f}=0 \text { if and only if } f \in \operatorname{Hom}(G[S], H) .
$$

Indeed, note that

$$
\begin{aligned}
& \mathbb{1}_{f}^{\top}\left(A_{G} \otimes\left(\mathbb{1}^{\top}-A_{H}\right)\right) \mathbb{1}_{f} \\
& =\mathbb{1}_{f}^{\top}\left(P_{\sim_{G}} \otimes\left(\mathbb{1}^{\top}-A_{H}\right)\right) \mathbb{1}_{f} \quad \text { by }(3.195) \\
& =\mathbb{1}_{f}^{\top}\left(P_{\sim_{G}} \otimes P_{\not_{H}}\right) \mathbb{1}_{f} \quad \text { by }(3.196) \\
& =\sum_{i, j \in V(H)} \sum_{k, \ell \in V(G)}\left[j \nsim_{H} i\right]\left[\ell \sim_{G} k\right][(k, i) \in f][(\ell, j) \in f] \quad \text { by Proposition } 3.69 \\
& =\sum_{i, j \in V(H)} \sum_{k, \ell \in V(G)}\left[j \nsim_{H} i\right]\left[\ell \sim_{G} k\right][k \in S][f(k)=i][\ell \in S][f(\ell)=j] \\
& =\sum_{k, \ell \in V(G)}[f(\ell) f(k) \notin E(H)][\ell k \in E(G)][k \in S][\ell \in S] \\
& =2|\{k \ell \in E(G[S]) \mid f(k) f(\ell) \notin E(H)\}| .
\end{aligned}
$$

This implies (3.199).

Results (3.190), (3.197), (3.198), and (3.199) imply the statement.

Corollary 3.71 ([56]). For every graphs $G$ and $H$, we have that

$$
G \rightarrow H \text { if and only if } \alpha(G \ltimes H)=|V(G)| \text {. }
$$

Proof. If $f \in \operatorname{Hom}(G, H)$, then $f \in \mathcal{S}(G \ltimes H)$ by Proposition 3.70, and $|f|=|V(G)|$. Conversely, let $S \in \mathcal{S}(G \ltimes H)$ be such that $|S|=|V(G)|$. Proposition 3.70 implies that there exists $T \subseteq V(G)$ such that $S=f$ for some $f: T \rightarrow V(H)$; we conclude that $T=V(G)$ from $|S|=|V(G)|$. Hence $f \in \operatorname{Hom}(G, H)$.

Corollary 3.72 ([23]). For every graph $G$,

$$
\chi(G)=\min \left\{n \in \mathbb{N}\left|\alpha\left(G \ltimes K_{n}\right)=\right| V(G) \mid\right\} .
$$

Proof. Note that

$$
\begin{aligned}
\chi(G) & =\min \left\{n \in \mathbb{N} \mid G \rightarrow K_{n}\right\} & & \text { by }(3.84 \mathrm{~b}) \\
& =\min \left\{n \in \mathbb{N}\left|\alpha\left(G \ltimes K_{n}\right)=\right| V(G) \mid\right\} . & & \text { by Corollary } 3.71
\end{aligned}
$$


For every $n \in \mathbb{N}$, we have that

$$
G \square K_{n}=G \ltimes K_{n} .
$$

This follows from (3.189), (3.190), and the fact that $A_{K_{n}}=\mathbb{1}^{\top}-I$. For this reason, the reader may find results in the literature about the Cartesian product which are actually statements about the homomorphic product. A particular example is [44, Section 7.14], which states that the Cartesian product "can be used to provide information about the size of $r$-colorable induced subgraphs of a graph" right before proving the special case of Proposition 3.70 in which $H$ is a complete graph. The maximum $k$-colorable subgraph problem asks for the size of the largest induced $k$-colorable subgraph, for $k \in \mathbb{N}$. In other words, given a graph $G$ and $n \in \mathbb{N}$, what is the value of $\alpha\left(G \ltimes K_{n}\right)$ ? There are both old [92] and new [68] results on efficient bounds for this problem.

We may use the homomorphic product to turn upper bounds on the stability number into relaxations of the homomorphic relation on graphs. Let $\beta$ be a graph parameter such that $\beta \geq \alpha$. Define the relation $\rightarrow^{\beta}$ by

$$
G \rightarrow^{\beta} H \text { if and only if } \beta(G \ltimes H) \geq|V(G)|
$$

for every pair of graphs $G$ and $H$. Every relation $\rightarrow^{\beta}$ is a relaxation of the homomorphic relation, as

$$
\begin{aligned}
G \rightarrow H & \Longrightarrow \alpha(G \ltimes H)=|V(G)| & & \text { by Corollary } 3.71 \\
& \Longrightarrow \beta(G \ltimes H) \geq|V(G)| & & \text { since } \alpha \leq \beta \\
& \Longrightarrow G \rightarrow^{\beta} H . & & \text { by }(3.200)
\end{aligned}
$$

The complement of any parameter in Figure 3.1 defines a relation in this manner, so we may actually interpret Figure 3.1 as a Hasse diagram of relations on graphs. For example, note that $\overline{\vartheta^{+}} \leq \xi_{f}$ implies that $\rightarrow \overline{\xi_{f}}$ is a relaxation of $\rightarrow^{\vartheta^{+}}$.

We now outline an argument from Bačík that proves that $\rightarrow \overline{\overline{\chi f}_{f}}$ fails to be transitive. Bačík [8, Chapter 3, Theorem 27] proves that

$$
G \rightarrow^{\overline{\chi_{f}}} H \text { if and only if } \omega(G) \leq \chi_{f}(H)
$$

for every graphs $G$ and $H$. The Mycielski [91] transformation of a graph $G$ is the graph $\mu(G)$ with vertex set $\{0\} \sqcup V \sqcup V$ and adjacency matrix defined by

$$
A_{\mu(G)}=\left[\begin{array}{ccc}
0 & 0^{\top} & \mathbb{1}^{\top} \\
0 & P_{1} A_{G} P_{1}^{\top} & P_{1} A_{G} P_{2}^{\top} \\
\mathbb{1} & P_{2} A_{G} P_{1}^{\top} & 0
\end{array}\right],
$$

where $P_{i}:=P_{\operatorname{tag}_{i}} \in \mathbb{R}^{(V \times \mathbb{N}) \times V}$ is the function matrix defined by the tag functions, for $i \in\{1,2\}$. It is not hard to prove that

$$
\omega(\mu(G))=\omega(G)
$$

for every graph $G$. Larsen, Propp, and Ullman [70] prove that for every graph $G$,

$$
\chi_{f}(\mu(G))=\chi_{f}(G)+\frac{1}{\chi_{f}(G)} .
$$

Set $H:=\mu\left(\mu\left(\mu\left(K_{2}\right)\right)\right)$. Combining (3.201) and (3.202), we conclude that

$$
K_{3} \rightarrow^{\overline{\chi_{f}}} H
$$

since $\omega\left(K_{3}\right)=3<941 / 290=\chi_{f}(H)$. We use (3.201) again to conclude that

$$
H \rightarrow \overline{\chi_{f}} K_{2}
$$

since $\omega(H)=2=\chi_{f}\left(K_{2}\right)$ by (3.202a). On the other hand, we have from (3.201) that

$$
K_{3} \operatorname{t}^{\overline{\chi_{f}}} K_{2}
$$


Hence (3.203), (3.204), and (3.205) certify that $\rightarrow \overline{\chi_{f}}$ is not transitive. Bačík's PhD. thesis [8, Chapter 3] has results on $\rightarrow^{\vartheta^{\prime}}, \rightarrow^{\vartheta}$, and $\rightarrow^{\overline{\chi_{f}}}$. See also [9].

The $\rightarrow \overline{\overline{x f}_{f}}$ relation shows that we may not always be able to apply the results from Sections 3.1 and 3.3 to a relation given by (3.200). Given a graph parameter $\beta$ such that $\beta \geq \alpha$, we must first show $\rightarrow^{\beta}$ to be transitive. Hence, the following theorems show that $\rightarrow^{\vartheta}$ is special among the relations we have defined. We remark that Theorem 5.75 implies Theorem 3.73.

Theorem 3.73 ([8, 36]). The relation $\rightarrow^{\vartheta}$ is a preorder on graphs.

Theorem 3.74 ([51]). Let $P$ be the preordered set of graphs under the $\rightarrow^{\vartheta}$ relation. Then $\lceil\bar{\vartheta}\rceil$ is the largest $(P, \overline{\mathbb{R}})$-monotone function which is at most $n$ on $K_{n}$, for every $n \in \mathbb{N}$.

The work by Gvozdenović and Laurent [51] is not at first sight related to homomorphism monotone and generalized colorings. Let $\beta$ be any graph parameter which is at least $\alpha$ and at most $\bar{\chi}$. Gvozdenović and Laurent [51] defined

$$
\Psi_{\beta}(G):=\min \left\{t \in \mathbb{N}\left|\beta\left(G \ltimes K_{t}\right)=\right| V(G) \mid\right\},
$$

for every graph $G$. Corollary 3.72 states that $\Psi_{\alpha}=\chi$. Furthermore, as $\chi\left(\overline{G \ltimes K_{t}}\right) \leq t$ for every $t \in \mathbb{N}$, it is easy to see that $\alpha \leq \beta \leq \bar{\chi}$ implies that $\Psi_{\beta}$ is well-defined for every graph $G$. There is also a natural "order reversal" when applying $\Psi$, so that $\alpha \leq \beta$ implies that $\Psi_{\beta} \leq \Psi_{\alpha}=\chi$. In short, $\Psi$ converts upper bounds on $\alpha$ to lower bounds on $\chi$. The only reason we cannot say that (3.206) is computing the generalized coloring number defined by the relation $\rightarrow^{\beta}$ is that $\rightarrow^{\beta}$ may fail to define a preorder. The authors of [51] compute $\Psi_{\beta}$ for several graph parameters. In particular, they prove that for every graph $G$,

$$
\Psi_{\vartheta}(G)=\lceil\vartheta(\bar{G})\rceil, \Psi_{\vartheta^{\prime}}(G)=\left\lceil\vartheta^{+}(\bar{G})\right\rceil, \text { and } \Psi_{\overline{\chi_{f}}}(G)=\omega(G) .
$$

Hence [51] combinatorially relates graph parameters which we geometrically relate in Chapter 4 . We remark that [51] is written using the cartesian product.

\subsubsection{The Fractional Cut-Covering Number}

Section 3.2 provides a standard way of obtaining a preordered set from a category on graphs. There are other categories whose objects are graphs which have been studied by the graph theory community [103, 104, 105]. As DeVos, Nešetřil, and Raspaud [33] put it, "[s]ometimes strange looking definitions are far from arbitrary as they reflect the experience gained with dealing with concrete problems"; although they are talking about cycle- and flow-continuous functions, the statement applies just as well to quantum homomorphisms. In this context, Śámal [105, Chapters 4 and 5] worked with the category of cut-continuous functions, and introduced the fractional cut-covering number, which is the subject of this subsection. See also [103].

Let $G:=(V, E)$ be a graph. For every $S \subseteq V$, define

$$
\delta_{G}(S):=\{i j \in E||\{i, j\} \cap S \mid=1\} .
$$

Whenever $G$ is clear from context, we may simply write $\delta(S)$. A cut is a set of edges of the form $\delta_{G}(S)$ for some $S \subseteq V$. A cut-cover of $G$ is a set of cuts of $G$ whose union is $E$. Note that $\{\delta(\{i\}) \mid i \in V(G)\}$ is always a cut-cover of $G$, as every edge is incident to some vertex. Neto and Ben-Ameur [94] remark that it is well known that the minimum number of cuts necessary to cover the edges of a graph $G$ is

$$
\lceil\lg \chi(G)\rceil .
$$

We now proceed to formulate a relaxation of this problem as a generalized coloring number.

For every $a, b \in \mathbb{N}$ such that $0<b \leq a$, define the graph $\operatorname{Ham}(a, b)$ as the graph such that

$$
\begin{aligned}
& V(\operatorname{Ham}(a, b)):=\{0,1\}^{[a)}, \\
& E(\operatorname{Ham}(a, b)):=\left\{\{x, y\} \in\left(\begin{array}{c}
V(\operatorname{Ham}(a, b)) \\
2
\end{array}\right) \mid d_{H}(x, y) \geq b\right\},
\end{aligned}
$$

where $d_{H}:\{0,1\}^{[a)} \times\{0,1\}^{[a)} \rightarrow[a+1)$ is the Hamming distance, which is defined by

$$
d_{H}(x, y):=\left|\left\{i \in[a) \mid x_{i} \neq y_{i}\right\}\right|
$$


for every $x, y \in\{0,1\}^{[a)}$. For $b \in \mathbb{N}$, a b-cover by cuts of $G$ is a function $X: S \rightarrow \mathcal{P}(V(G))$, with $S$ a finite set, such that for each edge $e \in E(G)$ it holds that

$$
\left|\left\{i \in S \mid e \in \delta\left(X_{i}\right)\right\}\right| \geq b .
$$

We refer to $|S|$ as the size of the $b$-cover.

Proposition 3.75 ([103]). Let $a, b \in \mathbb{N}$ be such that $0<b \leq a$, and let $G$ be a graph. Then $G$ has a $b$-cover by cuts of size $a$ if and only if $G \rightarrow \operatorname{Ham}(a, b)$.

Proof. Set $(V, E):=G$. Let $X: S \rightarrow \mathcal{P}(V)$ be a $b$-cover by cuts of $G$, and set $a:=|S|$. We may assume that $S=[a)$; if not, take any bijective function $g:[a) \rightarrow S$ and note that $(X \circ g):[a) \rightarrow \mathcal{P}(V)$ is a $b$-cover of size $a$. Define the function $f: V \rightarrow\{0,1\}^{[a)}$ by

$$
f(v)_{i}:=\left[v \in X_{i}\right]
$$

for every $v \in V$ and for every $i \in[a)$. Then for every $u v \in E$,

$$
\begin{aligned}
d_{H}(f(u), f(v)) & =\left|\left\{i \in[a) \mid f(u)_{i} \neq f(v)_{i}\right\}\right| & & \text { by (3.209) } \\
& =\left|\left\{i \in[a) \mid\left[u \in X_{i}\right] \neq\left[v \in X_{i}\right]\right\}\right| & & \text { by (3.211) } \\
& =\left|\left\{i \in[a) \mid u v \in \delta\left(X_{i}\right)\right\}\right| & & \\
& \geq b . & & \text { by (3.210) }
\end{aligned}
$$

Recalling (3.208), we conclude that $f \in \operatorname{Hom}(G, \operatorname{Ham}(a, b))$.

Assume then that $f \in \operatorname{Hom}(G, \operatorname{Ham}(a, b))$. Define $X:[a) \rightarrow \mathcal{P}(V)$ by

$$
X_{i}:=\left\{v \in V \mid f(v)_{i}=1\right\} .
$$

We have that $X$ is a $b$-cover by cuts of $G$ with size $|[a)|=a$, since for every $u v \in E$,

$$
\begin{aligned}
\left|\left\{i \in[a) \mid u v \in \delta\left(X_{i}\right)\right\}\right| & =\left|\left\{i \in[a) \mid\left[u \in X_{i}\right] \neq\left[v \in X_{i}\right]\right\}\right| & & \\
& =\left|\left\{i \in[a) \mid f(u)_{i} \neq f(v)_{i}\right\}\right| & & \text { by }(3.212) \\
& =d_{H}(f(u), f(v)) & & \text { by }(3.209) \\
& \geq b . & & \text { since } f \in \operatorname{Hom}(G, \operatorname{Ham}(a, b))
\end{aligned}
$$

Let $a \in \mathbb{N}$ be nonzero. It is not hard to define an isomorphism from $\operatorname{Ham}(a, 1)$ to $K_{2^{a}}$. The fact that $\operatorname{Ham}(a, 1)$ and $K_{2^{a}}$ are isomorphic and Proposition 3.75 directly imply that $\lceil\lg \chi(G)\rceil$ is the minimum number of cuts necessary to cover the edges of a graph $G$, as we remarked on (3.207).

Let $P$ be the preordered set of graphs under the homomorphic relation. Define

$$
\begin{gathered}
\mathcal{Q}_{x}:=\{\operatorname{Ham}(a, b) \mid a, b \in \mathbb{N}, 0<b \leq a\} \\
g_{x}: \mathcal{Q}_{x} \rightarrow[-\infty,+\infty], \\
g_{x}(\operatorname{Ham}(a, b)):=\frac{a}{b} .
\end{gathered}
$$

We denote by $x:=\chi_{g_{x}}$ the largest $(P, \overline{\mathbb{R}})$-monotone function which is at most $g_{x}$ on $\mathcal{Q}_{x}$. The parameter $x$ is called the fractional cut-covering number of a graph, and was introduced in Šámal's PhD. thesis [105, Chapter 5], and further explored in [103]. For every graph $G$,

$$
\begin{aligned}
x(G) & =\inf \left\{\frac{a}{b} \mid a, b \in \mathbb{N}, 0<b \leq a, G \rightarrow \operatorname{Ham}(a, b)\right\} & & \text { by }(3.94 \mathrm{~b}) \\
& =\inf \left\{\frac{a}{b} \mid a, b \in \mathbb{N}, 0<b \leq a, G \text { has a } b \text {-cover by cuts of size } a\right\} & & \text { by Proposition } 3.75
\end{aligned}
$$

Recalling Subsection 3.4.1, the name "fractional cut-covering number" becomes clear. Proposition 3.75 formulates cut-covers using homomorphisms, and thus allows the fractional cut-covering number to be formulated as a generalized coloring number. The analogy with $\chi_{f}$ goes further: Subsection 4.1.3 proves that $x$ is also the optimal value of a linear program (so the infimum in its definition is attained and $x$ is a rational valued). 
Corollary 3.76. Let $n \in \mathbb{N}$. Then

$$
K_{2 n} \rightarrow \operatorname{Ham}\left(\left(\begin{array}{c}
2 n \\
n
\end{array}\right), 2\left(\begin{array}{c}
2 n-2 \\
n-1
\end{array}\right)\right) .
$$

Proof. Define $X:\left(\begin{array}{c}{[2 n)} \\ n\end{array}\right) \rightarrow 2^{V\left(K_{2 n}\right)}$ by

$$
X_{S}:=S .
$$

To see that $X$ is a $b$-cover by cuts with $b:=2\left(\begin{array}{c}2 n-2 \\ n-1\end{array}\right)$, note that for every $i j \in E\left(K_{2 n}\right)$,

$$
\left\{S \in\left(\begin{array}{c}
{[2 n)} \\
n
\end{array}\right) \mid i j \in \delta(S)\right\}=\bigcup\left\{\{S \cup\{i\}, S \cup\{j\}\} \mid S \in\left(\begin{array}{c}
{[2 n) \backslash\{i, j\}} \\
n-1
\end{array}\right)\right\} .
$$

Proposition 3.75 finishes the proof.

Corollary 3.77. Let $n \in \mathbb{N}$. Then

$$
x\left(K_{2 n}\right) \leq 2-\frac{1}{n} .
$$

Proof. Let $H_{n}$ denote the graph in the RHS of Corollary 3.76. Using (3.213c) we have that

$$
g_{x}\left(H_{n}\right)=\frac{\left(\begin{array}{c}
2 n \\
n
\end{array}\right)}{2\left(\begin{array}{c}
2 n-2 \\
n-1
\end{array}\right)}=\frac{(2 n) !}{n ! n !} \frac{(n-1) !(n-1) !}{2(2 n-2) !}=\frac{2 n(2 n-1)}{2 n^{2}}=2-\frac{1}{n} .
$$

The statement follows:

$$
\begin{aligned}
x\left(K_{2 n}\right) & \leq x\left(H_{n}\right) & & \text { by }(3.85) \\
& \leq g_{x}\left(H_{n}\right) & & \text { by }(3.91 \mathrm{~b} \\
& =2-\frac{1}{n} . & &
\end{aligned}
$$

Corollary 3.78. For every graph $G$,

$$
x(G)=\inf \left\{\frac{a}{b} \mid a, b \in \mathbb{N}, 0<b \leq a<2 b, G \rightarrow \operatorname{Ham}(a, b)\right\} .
$$

In particular, $x(G)<2$.

Proof. Let $G$ be a graph. Set

$$
S:=\left\{\frac{a}{b} \mid a, b \in \mathbb{N}, 0<b \leq a, G \rightarrow \operatorname{Ham}(a, b)\right\} \text { and } T:=\{s \in S \mid s<2\} .
$$

The statement amounts to proving that $\inf S=\inf T$, which follows from

$$
\mathrm{lb}_{\overline{\mathbb{R}}} S=\mathrm{lb}_{\overline{\mathbb{R}}} T
$$

by (3.18).

We have that

$$
\inf S<2
$$

since

$$
\begin{aligned}
G \rightarrow K_{2|V(G)|} & \Longrightarrow x(G) \leq x\left(K_{2|V(G)|}\right) & & \text { by }(3.85) \\
& \Longrightarrow x(G) \leq 2-\frac{1}{|V(G)|} & & \text { by Corollary } 3.77 \\
& \Longrightarrow x(G)<2 & & \\
& \Longrightarrow \inf S<2 . & & \text { by }(3.94 \mathrm{~b})
\end{aligned}
$$


We now prove (3.214). Since $T \subseteq S$, we have that ' $\subseteq$ ' holds in (3.214) by (3.31a). By (3.215), there exists $\stackrel{\circ}{s} \in S$ such that $\stackrel{\circ}{s}<2$. Let $b \in \mathrm{lb}_{\overline{\mathbb{R}}} T$. By definition of $T$, for every $s \in S$ we have that either $s \in T$ or $s \geq 2$. If $s \in T$, then $s \geq b$. If $s \geq 2$, then

$$
\begin{aligned}
& s \geq 2 \\
& >\stackrel{\circ}{ } \text { by hypothesis on } \stackrel{s}{\circ} \\
& \geq b . \quad \text { since } b \in \operatorname{lb}_{\overline{\mathbb{R}}} T \text { and } \stackrel{s}{\quad \in T}
\end{aligned}
$$

Transitivity implies that $s \geq b$. In short, we have just prove that for every $b \in \operatorname{lb}_{\overline{\mathbb{R}}} T$ and for every $s \in S$, we have that $b \leq s$. This implies that $\operatorname{lb}_{\overline{\mathbb{R}}} T \subseteq \mathrm{lb}_{\overline{\mathbb{R}}} S$, and concludes the proof of (3.214).

Proposition 3.79. Let $a, b \in \mathbb{N}$ be such that $0<b \leq a<2 b$. Define $\gamma:=1-2 b / a$. Then there exists $\mathcal{B} \in$ Ball such that

$$
\operatorname{Ham}(a, b) \rightarrow \operatorname{Vect}(\mathcal{B}, \gamma)
$$

Proof. Define $f:\{0,1\}^{[a)} \rightarrow \mathbb{R}^{a}$ by

$$
f(x)_{i}:=a^{-1 / 2}(2 x-\mathbb{1})
$$

for every $x \in\{0,1\}^{[a)}$. Then for every $x, y \in\{0,1\}^{[a)}$,

$$
\begin{array}{rlrl}
\langle f(x), f(y)\rangle & =a^{-1}\left(\sum_{i \in[a)}\left(2 x_{i}-1\right)\left(2 y_{i}-1\right)\right) & \text { by }(3.216) \\
& =a^{-1}\left(\sum_{i \in[a)}(-1)^{\left[x_{i} \neq y_{i}\right]}\right) & \\
& =\frac{\left|\left\{i \in[a) \mid x_{i}=y_{i}\right\}\right|-\left|\left\{i \in[a) \mid x_{i} \neq y_{i}\right\}\right|}{a} & & \text { by (3.209) } \\
& =\frac{a-d_{H}(x, y)-d_{H}(x, y)}{a} & \\
& =1-\frac{2 d_{H}(x, y)}{a} . &
\end{array}
$$

It follows that $\|f(x)\|=1$ for every $x \in V(\operatorname{Ham}(a, b))$. Moreover, if $x y \in E(\operatorname{Ham}(a, b))$, then $d_{H}(x, y) \geq b$, so $\langle f(x), f(y)\rangle \leq 1-2 b / a$. Set $\mathcal{B}:=\left\{x \in \mathbb{R}^{a} \mid x^{\top} x=1\right\}$, so we conclude that $f \in \operatorname{Hom}(\operatorname{Ham}(a, b)$, $\operatorname{Vect}(\mathcal{B}, \gamma))$.

Corollary 3.80. For every graph $G$,

$$
2\left(1-\frac{1}{\overline{\vartheta^{\prime}}(G)}\right) \leq x(G)
$$

Proof. Let $a, b \in \mathbb{N}$ be such that $0<b \leq a<2 b$ and such that $G \rightarrow \operatorname{Ham}(a, b)$. Define $\gamma:=1-2 b / a$, so $\gamma<0$ follows from $a<2 b$. Further note that

$$
1-\frac{1}{\gamma}=1-\frac{1}{1-2 b / a}=1-\frac{a}{a-2 b}=\frac{a-2 b-a}{a-2 b}=\frac{2 b}{2 b-a} .
$$

Proposition 3.79 and transitivity of ' $\rightarrow$ ' imply there exists $H \in \mathcal{Q}_{\text {vect }}$ such that $G \rightarrow H$ and

$$
g_{\text {Vect }}(H)=2 b /(2 b-a) \text {. }
$$

Then (3.160) implies that

$$
\overline{\vartheta^{\prime}}(G) \leq \frac{2 b}{2 b-a}
$$

since

$$
\begin{aligned}
\overline{\vartheta^{\prime}}(G) & \leq \overline{\vartheta^{\prime}}(H) & & \text { by }(3.85) \text { and }(3.160) \\
& \leq g_{\text {Vect }}(H) & & \text { by }(3.91 \mathrm{~b}) \\
& =\frac{2 b}{2 b-a} . & & \text { by }(3.217)
\end{aligned}
$$


Therefore

$$
\begin{aligned}
2\left(1-\frac{1}{\overline{\vartheta^{\prime}}(G)}\right) & \leq 2\left(1-\frac{2 b-a}{2 b}\right) \\
& =\frac{a}{b} .
\end{aligned}
$$

Corollary 3.78 finishes the proof.

Since we want to obtain a minimum cut-cover of a graph, it is natural to be interested in large cuts, i.e., cuts with many edges. For example, note how Corollary 3.76 obtains, from large cuts, a good cut-cover of complete graphs of even size, and how this yields an upper bound in Corollary 3.77. Therefore, it is natural to exploit results developed for the maximum cut problem, which asks for the cardinality of the largest cut in a graph, to obtain results on the fractional cut-covering number. Goemans and Williamson [46] showed that the optimal vale of an SDP is an $\alpha_{\mathrm{GW}}$ approximation to the size of the largest cut of a graph, where

$$
\alpha_{\mathrm{GW}}:=\max _{0 \leq \theta \leq \pi} \frac{2}{\pi} \frac{\theta}{1-\cos \theta}>0.87856 .
$$

They first show that this semidefinite program is a relaxation of the problem of finding a maximum cut, and then show that they can obtain a cut from the optimal solution by sampling a uniform vector in the unit sphere. This approximation result has been adapted to the fractional cut-covering number.

Proposition 3.81 ([94, 103]). Let $G$ be a graph with nonempty edge set. Then

$$
x(G) \leq \frac{\pi}{\arccos \left(\left(1-\vartheta^{\prime}(\bar{G})\right)^{-1}\right)} \leq \frac{1}{\alpha_{\mathrm{GW}}} x(G),
$$

where $\alpha_{\mathrm{GW}}$ is defined as in (3.219).

Lemma 3.82 ([46]). Let $n \in \mathbb{N}$ and let $u, v \in \mathbb{R}^{n}$ be such that $u^{\top} u=v^{\top} v=1$. Let $r \in\left\{x \in \mathbb{R}^{n} \mid x^{\top} x=1\right\}$ be uniformly sampled. Then

$$
\mathbb{P}\left(\left(r^{\top} u\right)\left(r^{\top} v\right)<0\right)=\frac{\arccos u^{\top} v}{\pi} .
$$

Let $G$ be a graph with nonempty edge set. Let $a \in \mathbb{N}$ and $\gamma \in[-1,0)$ be such that $G \rightarrow \operatorname{Vect}(\mathcal{B}, \gamma)$, where $\mathcal{B}:=\left\{x \in \mathbb{R}^{a} \mid x^{\top} x=1\right\}$. Let $f \in \operatorname{Hom}(G$, $\operatorname{Vect}(\mathcal{B}, \gamma))$. A vector $r \in \mathcal{B}$ defines a cut once one defines

$$
S:=\left\{i \in V \mid f(i)^{\top} r>0\right\} .
$$

By Lemma 3.82, the probability of an edge belonging to this cut is $p:=\pi / \arccos \gamma$. Hence, after sampling $n$ independent cuts, the expected value of cuts an edge belongs to is $n / p$. Intuitively, this provides a cover by cuts with objective value $1 / p$, as we have used $n$ cuts to obtain a cut cover where each edge belongs to $n / p$ cuts. Of course, there may be edges which belong to fewer than $n / p$ cuts; the proof in [103] overcomes this issue by exploiting a concentration of measure result. It is easy to see that Proposition 3.81 implies that

$$
x(G) \leq \frac{\pi}{\arccos \gamma} .
$$

Corollary 3.80 exploits the representation of a graph in the unit sphere given by $f \in \operatorname{Hom}(G, \operatorname{Vect}(\mathcal{B}, \gamma))$, and also that the objective function in Corollary 3.52 is "compatible" with the goal of minimizing cut covers: when one maximizes the angle between a pair of vertices, one improves their chances of being separated by a random hyperplane, and thus minimize the amounts of cuts used. Note that (3.220) captures such idea: since arccos is a monotone nonincreasing function, the smaller the value of $\gamma$ the better the bound produced. However, this reasoning does not really explain the quality of the bound produced, i.e., the inequality in Corollary 3.80 missing from (3.220). In Section 5.3 we come back to cut-covers, and Subsection 5.3.2 has a new proof of Corollary 3.80 . 


\section{Chapter 4}

\section{Antiblocking and Gauge Duality}

\subsection{Antiblocking and Gauge Duality}

This section introduces the theory of convex corners, antiblocking duality, and real-valued positive definite monotone gauges. Terminology and notation used mostly follows [102], which has a much deeper treatment of the concepts presented here. We also remark that the optimization community has recent publications [3, 38] about dual gauge programs, which were introduced by Freund [37].

\subsubsection{Basic Definitions}

This subsection introduces some basic results regarding convex corners. In particular, it introduces the notion of a lower-comprehensive set. After establishing the basic facts regarding lower-comprehensive sets, we then establish a correspondence between real-valued positive definite monotone gauges and convex corners.

Let $\mathbb{E}$ be an Euclidean space. A set $\mathcal{C} \subseteq \mathbb{E}$ is convex if for every $\lambda \in[0,1]$ and $x, y \in \mathcal{C}$ we have that $\lambda x+(1-\lambda) y \in \mathcal{C}$. A function $\beta: \mathcal{C} \rightarrow[-\infty,+\infty]$ is convex if

$$
\mathcal{C} \text { is convex and } \beta(\lambda x+(1-\lambda) y) \leq \lambda \beta(x)+(1-\lambda) \beta(y)
$$

for every $x, y \in \mathcal{C}$ and $\lambda \in[0,1]$. A set $\mathcal{K} \subseteq \mathbb{E}$ is a cone if $\alpha x \in \mathcal{K}$ for every positive $\alpha \in \mathbb{R}$ and $x \in \mathcal{K}$. A function $\beta: \mathcal{K} \rightarrow[-\infty,+\infty]$ is positively homogeneous if

$$
\mathcal{K} \text { is a cone and } \beta(\alpha x)=\alpha x
$$

for every positive $\alpha \in \mathbb{R}$ and $x \in \mathcal{K}$. A set $\mathcal{C} \subseteq \mathbb{E}$ is closed under addition if $\mathcal{C}+\mathcal{C} \subseteq \mathcal{C}$. A function $\beta: \mathcal{C} \rightarrow[-\infty,+\infty]$ is sublinear if

$$
\mathcal{C} \text { is closed under addition and } \beta(x+y) \leq \beta(x)+\beta(y)
$$

for every $x, y \in \mathcal{K}$. It is easy to see that a cone is convex if and only if it is also closed under addition, and then conclude that a positively homogeneous function $\beta: \mathcal{K} \rightarrow(-\infty,+\infty]$ is convex if and only if it is sublinear. A function $\beta: \mathcal{C} \rightarrow[-\infty,+\infty]$ is positive semidefinite if

$$
\text { it is nonnegative, } 0 \in \mathcal{C} \text {, and } \beta(0)=0 \text {. }
$$

A positive semidefinite function $\beta$ is positive definite if $\beta(x)=0$ implies $x=0$. A gauge is a positive semidefinite, positively homogeneous convex function. Note that the domain of a gauge is a convex cone of $\mathbb{E}$ containing the origin.

Let $V$ be a finite set. The nonnegative orthant $\mathbb{R}_{+}^{V}$ is a convex cone containing the origin in the Euclidean space $\mathbb{R}^{V}$. A function $\beta: \mathbb{R}_{+}^{V} \rightarrow[-\infty,+\infty]$ is monotone if

$$
x \leq y \text { implies } \beta(x) \leq \beta(y)
$$


for every $x, y \in \mathbb{R}_{+}^{V}$. It is easy to see that the sets of convex, positive semidefinite, positive definite, positively homogeneous, and sublinear functions defined on the same domain are closed under pointwise supremum; we shall use this fact repeatedly. In particular, we state that

monotone gauges are closed under pointwise supremum.

We now introduce some useful examples. Let $V$ be a finite set. For $p \in[1,+\infty)$, define the $p$-norm on $\mathbb{R}^{V}$ by $\|x\|_{p}:=\left(\sum_{i \in V}\left|x_{i}\right|^{p}\right)^{1 / p}$. Similarly, define the $\infty$-norm on $\mathbb{R}^{V}$ as $\|x\|_{\infty}:=\max _{i \in V}\left|x_{i}\right|$. When restricted to the nonnegative orthant $\mathbb{R}_{+}^{V}$, the $\infty$-norm and every $p$-norm for $p \in[1,+\infty)$ define real-valued positive definite monotone gauges. It is easy to check that for every $y \in \mathbb{R}_{+}^{V}$ the function

$$
x \in \mathbb{R}_{+}^{V} \mapsto y^{\top} x \text { is a monotone gauge. }
$$

We may use such simple monotone gauges to define more interesting ones. For every graph $G:=(V, E)$ and for every $w \in \mathbb{R}_{+}^{V}$, define the weighted stability number $\alpha(G, w)$ as

$$
\alpha(G, w):=\max \left\{w^{\top} \mathbb{1}_{S} \mid S \in \mathcal{S}(G)\right\} .
$$

The function $\alpha(G, \cdot)$ is a monotone gauge by (4.5) and (4.6). Furthermore, it is real-valued since $\mathcal{S}(G)$ is finite, and it is positive definite since $\{i\} \in \mathcal{S}(G)$ for every $i \in V(G)$ implies that $\|w\|_{\infty} \leq \alpha(G, w)$. In short,

$$
\alpha(G, \cdot) \text { is a real-valued positive definite monotone gauge. }
$$

Let $V$ be a finite set. We now present an example of a positive definite gauge whose domain is not $\mathbb{R}_{+}^{V}$. Recall that $\mathbb{S}^{V}$ denotes the set of symmetric matrices in $\mathbb{R}^{V \times V}$. The subset of $\mathbb{S}^{V}$ of positive semidefinite matrices is denoted by $\mathbb{S}_{+}^{V}$. We write $A \preceq B$ if $B-A \in \mathbb{S}_{+}^{V}$. Moreover, we use $\lambda_{\max }(A)$ and $\lambda_{\min }(A)$ to denote the largest and smallest eigenvalues of $A \in \mathbb{S}^{V}$, respectively. The set of positive semidefinite matrices $\mathbb{S}_{+}^{V}$ is a convex cone containing the origin. It is easy to see that for every $x \in \mathbb{R}^{V}$, the function on $\mathbb{S}_{+}^{V}$ defined by

$$
A \in \mathbb{S}_{+}^{V} \mapsto x^{\top} A x \text { is a gauge. }
$$

Using the spectral theorem, we see that $\lambda_{\max }(A)=0$ implies $A=0$ for $A \succeq 0$. Consequently,

$$
\lambda_{\max }: \mathbb{S}_{+}^{V} \rightarrow \mathbb{R} \text { is a positive definite gauge }
$$

follows from (4.8), (4.5), and the well known fact that $\lambda_{\max }(A)=\max \left\{x^{\top} A x \mid\|x\|=1\right\}$.

Let $V$ be a finite set. For every positively homogeneous function $\beta: \mathbb{R}_{+}^{V} \rightarrow[-\infty,+\infty]$, define the unit convex corner of $\beta$ by

$$
\operatorname{unit}(\beta):=\left\{x \in \mathbb{R}_{+}^{V} \mid \beta(x) \leq 1\right\} .
$$

For $\mathcal{C} \subseteq \mathbb{R}_{+}^{V}$, the Minkowski functional $\gamma(\cdot \mid \mathcal{C}): \mathbb{R}_{+}^{V} \rightarrow[-\infty,+\infty]$ is the function defined by

$$
\gamma(x \mid \mathcal{C}):=\inf \left\{\mu \in \mathbb{R}_{++} \mid x \in \mu \mathcal{C}\right\}
$$

for every $x \in \mathbb{R}_{+}^{V}$.

Let $V$ be a finite set. A subset $\mathcal{C} \subseteq \mathbb{R}_{+}^{V}$ is lower comprehensive if

$$
0 \leq x \leq y \text { for some } y \in \mathcal{C} \text { implies that } x \in \mathcal{C} .
$$

It is easy to see that $\mathbb{R}_{+}^{V}$ is lower comprehensive, and that the intersection of lower-comprehensive subsets of $\mathbb{R}_{+}^{V}$ is lower comprehensive. Denote by $\operatorname{lc}(\mathcal{C})$ the lower-comprehensive hull of $\mathcal{C} \subseteq \mathbb{R}_{+}^{V}$, i.e., the intersection of every lower-comprehensive set in $\mathbb{R}_{+}^{V}$ which contains $\mathcal{C}$. It is clear that

$$
\text { if } \mathcal{C} \subseteq \mathcal{D} \text { then } \operatorname{lc}(\mathcal{C}) \subseteq \operatorname{lc}(\mathcal{D})
$$

Proposition 4.1. Let $V$ be a finite set, and let $\mathcal{C} \subseteq \mathbb{R}_{+}^{V}$. Then

$$
\operatorname{lc}(\mathcal{C})=\bigcup\left\{\left\{x \in \mathbb{R}_{+}^{V} \mid x \leq c\right\} \mid c \in \mathcal{C}\right\} .
$$


Proof. For every $c \in \mathcal{C}$, define $L_{c}:=\left\{x \in \mathbb{R}_{+}^{V} \mid x \leq c\right\}$. Further define $L:=\bigcup_{c \in \mathcal{C}} L_{c}$. We wish to prove that $L=\operatorname{lc}(\mathcal{C})$.

Let $\mathcal{D} \subseteq \mathbb{R}_{+}^{V}$ be a lower-comprehensive set such that $\mathcal{C} \subseteq \mathcal{D}$. If $x \in L$, then there exists $c \in \mathcal{C}$ such that $x \in L_{c}$. Since $\mathcal{C} \subseteq \mathcal{D}$, we have that $x \in \mathcal{D}$, as $\mathcal{D}$ is lower comprehensive and $0 \leq x \leq c \in \mathcal{D}$. As $x \in L$ was arbitrary, we proved that $L \subseteq \mathcal{D}$. Taking $\mathcal{D}:=\operatorname{lc}(\mathcal{C})$, we conclude that $L \subseteq \operatorname{lc}(\mathcal{C})$.

Since $c \in L_{c}$ for every $c \in \mathcal{C}$, we have that $\mathcal{C} \subseteq L$. If we prove that $L$ is lower comprehensive, we conclude that $\operatorname{lc}(\mathcal{C}) \subseteq L$ and finish the proof. Let $x \in \mathbb{R}_{+}^{V}$ and $y \in L$ be such that $x \leq y$. By the definition of $L$, there exists $c \in C$ such that $y \in L_{c}$. Consequently, it holds that $x \leq c$, as $x \leq y \leq c$. As $x \in \mathbb{R}_{+}^{V}$, we conclude that $x \in L_{c} \subseteq L$. Thus $L$ is lower comprehensive, and the proof is done.

Proposition 4.2. Let $V$ be a finite set, and let $\mathcal{C} \subseteq \mathbb{R}_{+}^{V}$. Assume $\mathcal{C}$ is such that, for every $i \in V$,

$$
\text { if } x \in \mathcal{C} \text { then } x-x_{i} e_{i} \in \mathcal{C} \text {. }
$$

Then $\operatorname{conv}(\mathcal{C})$ is lower-comprehensive.

Proof. We first show that, for every $i \in V$,

$$
\text { if } \tilde{x} \in \operatorname{conv}(\mathcal{C}) \text { then } \tilde{x}-\tilde{x}_{i} e_{i} \in \operatorname{conv}(\mathcal{C}) \text {. }
$$

Let $\tilde{x} \in \operatorname{conv}(\mathcal{C})$. Then there exists a finite set $\mathcal{D} \subseteq \mathcal{C}$ and $\lambda \in \mathbb{R}_{++}^{\mathcal{D}}$ such that $\lambda^{\top} \mathbb{1}=1$ and $\tilde{x}=\sum_{x \in \mathcal{D}} \lambda_{x} x$. Then (4.14) implies that

$$
\tilde{x}-\tilde{x}_{i} e_{i}=\sum_{x \in \mathcal{D}} \lambda_{x}\left(x-x_{i} e_{i}\right) \in \operatorname{conv}(\mathcal{C})
$$

Hence (4.15) holds.

Let $y \in \mathbb{R}_{+}^{V}$ be such that $0 \leq y \leq x$ for some $x \in \operatorname{conv}(\mathcal{C})$. We prove that $y \in \operatorname{conv}(\mathcal{C})$. Let $z \in \mathbb{R}_{+}^{V}$ be an element of

$$
\{x \in \operatorname{conv}(\mathcal{C}) \mid y \leq x\}
$$

which maximizes $\left|\left\{i \in V \mid x_{i}=y_{i}\right\}\right|$. If $y=z$, we are done. Otherwise, there exists $i \in V$ such that $y_{i}<z_{i}$. In particular, we have that $z_{i}>0$. Set $\lambda:=\left(z_{i}-y_{i}\right) / z_{i} \in(0,1)$. Then

$$
\begin{array}{rlrl}
z-z_{i} e_{i}+y_{i} e_{i} & =z-\left(z_{i}-y_{i}\right) e_{i} & \\
& =z-\lambda z_{i} e_{i} & & \text { by definition of } \lambda \\
& =\lambda\left(z-z_{i} e_{i}\right)+(1-\lambda) z & & \\
& \in \operatorname{conv}(\mathcal{C}) . & & \text { by }(4.15) \text { and } z \in \operatorname{conv}(\mathcal{C})
\end{array}
$$

Hence $z-z_{i} e_{i}+y_{i} e_{i}$ is an element of $\operatorname{conv}(\mathcal{C})$ which has more coordinates in common with $y$ than $z$. By the definition of $z$ this is impossible, so the proof is done.

Corollary 4.3. Let $V$ be a finite set. If $\mathcal{C} \subseteq \mathbb{R}_{+}^{V}$ is lower comprehensive, then $\operatorname{conv}(\mathcal{C})$ is lower comprehensive.

Proof. Immediate from Proposition 4.2.

Corollary 4.4. Let $V$ be a finite set. Let $\mathcal{C} \subseteq \mathbb{R}_{+}^{V}$. Then

$$
\operatorname{lc}(\operatorname{conv}(\operatorname{lc} \mathcal{C}))=\operatorname{conv}(\operatorname{lc} \mathcal{C})
$$

Proof. Since $\operatorname{lc}(\mathcal{C})$ is lower comprehensive, Corollary 4.3 implies that $\operatorname{conv}(\operatorname{lc} \mathcal{C})$ is lower comprehensive. Thus $\operatorname{lc}(\operatorname{conv}(\operatorname{lc} \mathcal{C}))=\operatorname{conv}(\operatorname{lc} \mathcal{C})$.

Proposition 4.5. Let $V$ be a finite set. Let $\mathcal{C} \subseteq \mathbb{R}_{+}^{V}$ be a convex set. Then $\operatorname{lc}(\mathcal{C})$ is convex. 
Proof. Let $n \in \mathbb{N}$, let $x \in(\operatorname{lc}(\mathcal{C}))^{[n)}$, and let $\lambda \in \mathbb{R}_{+}^{[n)}$ be such that $\lambda^{\top} \mathbb{1}=1$. Set $\bar{x}:=\sum_{i \in[n)} \lambda_{i} x_{i}$. We wish to prove that $\bar{x} \in \operatorname{lc}(\mathcal{C})$. Since $\mathbb{R}_{+}^{V}$ is convex, we have that $\bar{x} \in \mathbb{R}_{+}^{V}$. Proposition 4.1 implies that there exists $c \in \mathcal{C}^{[n)}$ such that $x_{i} \leq y_{i}$ for every $i \in[n)$. Therefore

$$
\begin{aligned}
\bar{x} & =\sum_{i \in[n)} \lambda_{i} x_{i} & & \\
& \leq \sum_{i \in[n)} \lambda_{i} c_{i} & & \text { since } x_{i} \leq c_{i} \text { for every } i \in[n) \\
& \in \mathcal{C} & & \text { since } \mathcal{C} \text { is convex } \\
& \in \operatorname{lc}(\mathcal{C}) . & & \text { by }(4.13)
\end{aligned}
$$

Using that $\operatorname{lc}(\mathcal{C})$ is lower comprehensive, we conclude that $\bar{x} \in \operatorname{lc}(\mathcal{C})$.

Corollary 4.6. Let $V$ be a finite set. Let $\mathcal{C} \subseteq \mathbb{R}_{+}^{V}$. Then

$$
\operatorname{conv}(\operatorname{lc}(\operatorname{conv} \mathcal{C}))=\operatorname{lc}(\operatorname{conv} \mathcal{C})
$$

Proof. Since $\operatorname{conv}(\mathcal{C})$ is convex, Proposition 4.5 implies that $\operatorname{lc}(\operatorname{conv} \mathcal{C})$ is convex. Thus $\operatorname{conv}(\operatorname{lc}(\operatorname{conv} \mathcal{C}))=$ $\operatorname{lc}(\operatorname{conv} \mathcal{C})$.

Proposition 4.7. Let $V$ be a finite set. Let $\mathcal{C} \subseteq \mathbb{R}_{+}^{V}$. If $\mathcal{C}$ is compact, then lc $(\mathcal{C})$ is compact.

Proof. Since $\mathcal{C}$ is bounded, there exists $M \in \mathbb{R}$ such that

$$
\mathcal{C} \subseteq\left\{x \in \mathbb{R}_{+}^{V} \mid\|x\| \leq M\right\} .
$$

Since the set in the RHS of (4.16) is lower comprehensive, we conclude that $l c(\mathcal{C})$ is bounded.

Let $y: \mathbb{N} \rightarrow \operatorname{lc}(\mathcal{C})$ be a sequence, and let $\bar{y} \in \mathbb{R}_{+}^{V}$ be such that $\bar{y}=\lim _{n \rightarrow \infty} y_{n}$. Proposition 4.1 implies that there exists $x: \mathbb{N} \rightarrow \mathcal{C}$ such that

$$
y_{n} \leq x_{n}
$$

for every $n \in \mathbb{N}$. Since $\mathcal{C}$ is compact, we may assume (perhaps after taking a subsequence) that there exists $\bar{x} \in \mathcal{C}$ such that $\bar{x}=\lim _{n \rightarrow \infty} x_{n}$. We have that $\bar{y} \leq \bar{x}$, as

$$
\begin{aligned}
\bar{x}-\bar{y} & =\lim _{n \rightarrow \infty}\left(x_{n}-y_{n}\right) & \\
& \in \mathbb{R}_{+}^{V} . & \text { by }(4.17) \text { and since } \mathbb{R}_{+}^{V} \text { is closed }
\end{aligned}
$$

As $\bar{x} \in \mathcal{C}$, Proposition 4.1 implies that $\bar{y} \in \operatorname{lc}(\mathcal{C})$, so we conclude that $\operatorname{lc}(\mathcal{C})$ is closed, and thus compact.

A convex corner is a lower-comprehensive compact convex subset of the nonnegative orthant with nonempty interior.

Proposition 4.8. Let $V$ be a finite set, and let $\mathcal{C} \subseteq \mathbb{R}_{+}^{V}$. If $\mathcal{C}$ is lower comprehensive, then

$$
\mu \mathcal{C} \text { is lower comprehensive }
$$

for every $\mu \in \mathbb{R}_{++}$.

Proof. Let $\mu \in \mathbb{R}_{++}$and let $x, y \in \mathbb{R}_{+}^{V}$ be such that $x \leq y$ and $y \in \mu \mathcal{C}$. We have that

$$
\begin{aligned}
& \frac{1}{\mu} x \leq \frac{1}{\mu} y \quad \text { since } x \leq y \text { and } \mu>0 \\
& \Longrightarrow \frac{1}{\mu} x \in \mathcal{C} \quad \text { since } \frac{1}{\mu} y \in \mathcal{C} \text { and } \mathcal{C} \text { is lower comprehensive } \\
& \Longrightarrow x \in \mu \mathcal{C} \text {. }
\end{aligned}
$$

Proposition 4.9. Let $V$ be a finite set. If $\mathcal{C} \subseteq \mathbb{R}_{+}^{V}$ is a convex corner, then so is $\mu \mathcal{C}$ for every $\mu \in \mathbb{R}_{++}$. 
Proof. Let $\mathcal{C} \subseteq \mathbb{R}_{+}^{V}$ be a convex corner, let $\mu \in \mathbb{R}_{++}$, and define $f: \mathbb{R}_{+}^{V} \rightarrow \mathbb{R}_{+}^{V}$ for every $x \in \mathbb{R}_{+}^{V}$ by $f(x):=\mu x$. Since $f$ is a continuous function, we have that $\mu \mathcal{C}=f(\mathcal{C})$ is compact. Since $f$ is a linear function, we have that $\mu \mathcal{C}=f(\mathcal{C})$ is convex. Since $f$ has a continuous inverse, we have that $f(\operatorname{int}(\mathcal{C}))$ is an open subset of $f(\mathcal{C})$, so that

$$
\operatorname{int}(\mu \mathcal{C})=\operatorname{int}(f(\mathcal{C})) \supseteq f(\operatorname{int}(\mathcal{C})) \neq \varnothing .
$$

Proposition 4.8 finishes the proof.

The main result of this subsection is that there is a one-to-one correspondence between real-valued positive definite monotone gauges and convex corners, where unit $(\cdot)$ and $\mathcal{C} \mapsto \gamma(\cdot \mid \mathcal{C})$ are the functions defining the pairing. In the proof of this correspondence, we will use the following simple observation:

$$
\text { for every } x \in \mathbb{R}_{+}^{V} \text { it holds that } x \leq\|x\|_{\infty} \mathbb{1} \text {. }
$$

Proposition 4.10. Let $V$ be a finite set, and let $\beta: \mathbb{R}_{+}^{V} \rightarrow \mathbb{R}$ be a positive definite monotone gauge. Then $\operatorname{unit}(\beta)$ is a convex corner and $\gamma(\cdot \mid \operatorname{unit}(\beta))=\beta$.

Proof. Note that unit $(\beta)$ is a subset of $\mathbb{R}_{+}^{V}$ by $(4.10)$.

Let $x, y \in \operatorname{unit}(\beta)$ and let $\lambda \in[0,1]$. Then unit $(\beta)$ is convex, since

$$
\begin{aligned}
\beta(\lambda x+(1-\lambda) y) & \leq \lambda \beta(x)+(1-\lambda) \beta(y) & & \text { by }(4.1) \\
& \leq \lambda+(1-\lambda) & & \text { by }(4.10) \text { since } x, y \in \text { unit }(\beta) \text { and } \lambda \in[0,1] \\
& =1 . & &
\end{aligned}
$$

To prove that unit $(\beta)$ is lower comprehensive, let $x \in \mathbb{R}_{+}^{V}$ be such that $x \leq y$ for $y \in \operatorname{unit}(\beta)$. Then

$$
\begin{aligned}
\beta(x) & \leq \beta(y) & & \text { by }(4.4) \\
& \leq 1 . & & \text { by }(4.10) \text { since } y \in \operatorname{unit}(\beta)
\end{aligned}
$$

Hence $x \in \operatorname{unit}(\beta)$ by (4.10).

We claim that

$$
\left\{x \in \mathbb{R}_{+}^{V} \mid\|x\|_{\infty} \leq \beta(\mathbb{1})^{-1}\right\} \subseteq \operatorname{unit}(\beta) .
$$

Since the set on the LHS of (4.19) has nonempty interior, this implies that unit $(\beta)$ has nonempty interior. We are using that $\beta$ is positive definite to be sure that $\beta(\mathbb{1})>0$ in (4.19). To see that (4.19) holds, let $x \in \mathbb{R}_{+}^{V}$ be such that $\|x\|_{\infty} \leq \beta(\mathbb{1})^{-1}$. Then

$$
\begin{aligned}
\beta(x) & \leq \beta\left(\|x\|_{\infty} \mathbb{1}\right) & & \text { by }(4.4) \text { and }(4.18) \\
& =\|x\|_{\infty} \beta(\mathbb{1}) & & \text { by }(4.2) \\
& \leq 1 . & & \text { since }\|x\|_{\infty} \leq \beta(\mathbb{1})^{-1}
\end{aligned}
$$

Hence (4.19) follows.

Let $\left(x_{n}\right)_{n \in \mathbb{N}} \subseteq \mathbb{R}_{+}^{V}$ be a converging sequence, and set $\bar{x}:=\lim _{n \rightarrow+\infty} x_{n}$. We now argue that $\beta$ is lower semi-continuous, i.e., that

$$
\beta(\bar{x}) \leq \liminf _{n \rightarrow \infty} \beta\left(x_{n}\right) .
$$

Let $n \in \mathbb{N}$. If we denote by $|z|$ the componentwise absolute value of $z \in \mathbb{R}^{V}$, it is immediate that

$$
\bar{x} \leq\left|\bar{x}-x_{n}\right|+x_{n} .
$$

Then

$$
\begin{aligned}
\beta(\bar{x}) & \leq \beta\left(\left|\bar{x}-x_{n}\right|+x_{n}\right) & & \text { by }(4.4) \text { and }(4.21) \\
& \leq \beta\left(\left|\bar{x}-x_{n}\right|\right)+\beta\left(x_{n}\right) & & \text { by }(4.3) \\
& \leq \beta\left(\left\|\bar{x}-x_{n}\right\|_{\infty} \mathbb{1}\right)+\beta\left(x_{n}\right) & & \text { by }(4.18) \text { and }(4.4) \\
& =\left\|\bar{x}-x_{n}\right\|_{\infty} \beta(\mathbb{1})+\beta\left(x_{n}\right) . & & \text { by }(4.2)
\end{aligned}
$$


Since $\lim _{n \rightarrow+\infty}\left\|\bar{x}-x_{n}\right\|_{\infty}=0$ and $\beta(\mathbb{1})<+\infty$, we have that (4.20) holds. It follows from (4.20) and (4.10) that $\operatorname{unit}(\beta)$ is closed.

Set $\mathcal{B}:=\left\{x \in \mathbb{R}_{+}^{V} \mid\|x\|=1\right\}$ and set $M:=\inf \{\beta(x) \mid x \in \mathcal{B}\}$. Let $x: \mathbb{N} \rightarrow \mathcal{B}$ be a sequence such that $\lim _{n \rightarrow \infty} \beta\left(x_{n}\right)=M$. Since $\mathcal{B}$ is compact, we may assume (perhaps after taking a subsequence) that there exists $\bar{x} \in \mathcal{B}$ such that $\lim _{n \rightarrow \infty} x_{n}=\bar{x}$. But then $\beta(\bar{x})=M$, as

$$
\begin{aligned}
M & =\lim _{n \rightarrow \infty} \beta\left(x_{n}\right) & & \text { by choice of }\left(x_{n}\right)_{n \in \mathbb{N}} \\
& \geq \beta(\bar{x}) & & \text { by }(4.20) \\
& \geq M . & & \text { since } \bar{x} \in \mathcal{B} \text { and } M=\inf \{\beta(x) \mid x \in \mathcal{B}\}
\end{aligned}
$$

We know that $0 \notin \mathcal{B}$, since $\|\cdot\|$ is positive definite. Therefore $\bar{x} \neq 0$, so $M=\beta(\bar{x})>0$, since $\beta$ is positive definite. We now claim that

$$
\beta(\bar{x})\|x\| \leq \beta(x)
$$

for every $x \in \mathbb{R}_{+}^{V}$. If $x=0$, then (4.22) clearly holds. On the other hand, if $x \neq 0$, then $\|x\| \neq 0$ since $\|\cdot\|$ is positive definite, so $\frac{1}{\|x\|} x \in \mathcal{B}$ because $\|\cdot\|$ is positively homogeneous. Hence

$$
\begin{aligned}
\beta(\bar{x}) & \leq \beta\left(\frac{1}{\|x\|} x\right) & & \text { since } \frac{1}{\|x\|} x \in \mathcal{B} \text { and } \beta(\bar{x})=\inf \{\beta(y) \mid y \in \mathcal{B}\} \\
& =\frac{1}{\|x\|} \beta(x) . & & \text { by }(4.2)
\end{aligned}
$$

Therefore (4.22) follows. It is then clear that

$$
\operatorname{unit}(\beta)=\left\{x \in \mathbb{R}_{+}^{V} \mid \beta(x) \leq 1\right\} \subseteq\left\{x \in \mathbb{R}_{+}^{V} \mid\|x\| \leq \beta(\bar{x})^{-1}\right\},
$$

so we conclude that unit $(\beta)$ is bounded.

We now show that $\beta \leq \gamma(\cdot \mid \operatorname{unit}(\beta))$. Let $x \in \mathbb{R}_{+}^{V}$ and let $\mu>0$ be such that $x \in \mu$ unit $(\beta)$. Then

$$
\begin{aligned}
x \in \mu \operatorname{unit}(\beta) & \Longrightarrow \frac{1}{\mu} x \in \operatorname{unit}(\beta) & & \text { since } \mu>0 \\
& \Longrightarrow \beta\left(\frac{1}{\mu} x\right) \leq 1 & & \text { by }(4.10) \\
& \Longrightarrow \frac{1}{\mu} \beta(x) \leq 1 & & \text { by }(4.2) \\
& \Longrightarrow \beta(x) \leq \mu . & & \text { since } \mu>0
\end{aligned}
$$

Using (4.11), we conclude $\beta \leq \gamma(\cdot \mid \operatorname{unit}(\beta))$.

Using that $\beta(0)=0$ and (4.10) we conclude that $0 \in \operatorname{unit}(\beta)$, so (4.11) implies that $\gamma(0 \mid$ unit $(\beta))=0=$ $\beta(0)$. To show that $\gamma(\cdot \mid$ unit $(\beta)) \leq \beta$, let $x \in \mathbb{R}_{+}^{V}$ be nonzero. We claim that

$$
x \in \beta(x) \operatorname{unit}(\beta) .
$$

Since $\beta$ is positive definite, we have that $\beta(x)>0$. Then (4.2) implies that

$$
\beta\left(\frac{1}{\beta(x)} x\right)=\frac{1}{\beta(x)} \beta(x)=1 .
$$

Recalling (4.10), we conclude that $\frac{1}{\beta(x)} x \in$ unit $(\beta)$, which readily implies (4.23). Thus

$$
\begin{aligned}
\gamma(x \mid \operatorname{unit}(\beta)) & =\inf \left\{\mu \in \mathbb{R}_{++} \mid x \in \mu \operatorname{unit}(\beta)\right\} & & \text { by }(4.11) \\
& \leq \beta(x) . & & \text { by }(4.23) \text { and } \beta(x)>0
\end{aligned}
$$

Thus $\gamma(\cdot \mid \operatorname{unit}(\beta)) \leq \beta$.

Proposition 4.11. Let $V$ be a finite set, and let $\mathcal{C} \subseteq \mathbb{R}_{+}^{V}$ be a convex corner. Then $\gamma(\cdot \mid \mathcal{C})$ is a real-valued positive definite monotone gauge, and $\operatorname{unit}(\gamma(\cdot \mid \mathcal{C}))=\mathcal{C}$. 
Proof. We first prove that $\gamma(\cdot \mid \mathcal{C})$ is a real-valued positive definite monotone gauge. The function $\gamma(\cdot \mid \mathcal{C})$ is nonnegative and positively homogeneous by definition. Consequently, to prove that $\gamma(\cdot \mid \mathcal{C})$ is convex, it suffices to prove that $\gamma(\cdot \mid \mathcal{C})$ is sublinear. Let $x, y \in \mathbb{R}_{+}^{V}$ and let $\mu_{x}, \mu_{y} \in \mathbb{R}_{++}$be such that $x \in \mu_{x} \mathcal{C}$ and $y \in \mu_{y} \mathcal{C}$. Then $\mu_{x}^{-1} x$ and $\mu_{y}^{-1} y$ are in $\mathcal{C}$, which together with convexity of $\mathcal{C}$ implies that

$$
\frac{x+y}{\mu_{x}+\mu_{y}}=\frac{\mu_{x}}{\mu_{x}+\mu_{y}} \frac{1}{\mu_{x}} x+\frac{\mu_{y}}{\mu_{x}+\mu_{y}} \frac{1}{\mu_{y}} y \in \mathcal{C} .
$$

In other words, we have that $(x+y) \in\left(\mu_{x}+\mu_{y}\right) \mathcal{C}$, so $(4.11)$ implies that $\gamma(x+y \mid \mathcal{C}) \leq \gamma(x \mid \mathcal{C})+\gamma(y \mid \mathcal{C})$.

As $\mathcal{C}$ is bounded, there exists $M>0$ such that $\mathcal{C} \subseteq\left\{x \in \mathbb{R}_{+}^{V} \mid\|x\| \leq M\right\}$. Therefore, for every $x \in \mathbb{R}_{+}^{V}$ and for every $\mu \in \mathbb{R}_{++}$we have that

$$
\begin{aligned}
x \in \mu \mathcal{C} & \Longrightarrow x \in \mu\left\{x \in \mathbb{R}_{+}^{V} \mid\|x\| \leq M\right\} \\
& \Longrightarrow\|x\| \leq \mu M \\
& \Longrightarrow M^{-1}\|x\| \leq \mu .
\end{aligned}
$$

Therefore, $M^{-1}\|x\| \leq \gamma(x \mid \mathcal{C})$ for every $x \in \mathbb{R}_{+}^{V}$. Hence $\gamma(\cdot \mid \mathcal{C})$ is positive definite.

To see that $\gamma(\cdot \mid \overline{\mathcal{C}})$ is monotone, note that for $x, y \in \mathbb{R}_{+}^{V}$ such that $x \leq y$, we have that

$$
\begin{array}{rlrl} 
& \left\{\mu \in \mathbb{R}_{++} \mid y \in \mu \mathcal{C}\right\} \subseteq\left\{\mu \in \mathbb{R}_{++} \mid x \in \mu \mathcal{C}\right\} & & \text { by Proposition } 4.8 \text { since } x \leq y \\
\Longrightarrow & \inf \left\{\mu \in \mathbb{R}_{++} \mid x \in \mu \mathcal{C}\right\} \leq \inf \left\{\mu \in \mathbb{R}_{++} \mid y \in \mu \mathcal{C}\right\} & \\
\Longrightarrow & \gamma(x \mid \mathcal{C}) \leq \gamma(y \mid \mathcal{C}) . & & \text { by }(4.11)
\end{array}
$$

To see that $\gamma(\cdot \mid \mathcal{C})$ is real valued, let $\bar{x}$ be in the interior of $\mathcal{C}$. Since $\mathcal{C} \subseteq \mathbb{R}_{+}^{V}$, we have that $\bar{x}>0$. Set $\mu:=\min \left\{\bar{x}_{i} \mid i \in V\right\}$. We have that

$$
\mathbb{1} \leq \mu^{-1} \bar{x}
$$

Therefore, for every $x \in \mathbb{R}_{+}^{V}$,

$$
\begin{aligned}
\gamma(x \mid \mathcal{C}) & \leq \gamma\left(\|x\|_{\infty} \mathbb{1} \mid \mathcal{C}\right) \\
& =\|x\|_{\infty} \gamma(\mathbb{1} \mid \mathcal{C}) \\
& \leq\|x\|_{\infty} \gamma\left(\mu^{-1} \bar{x} \mid \mathcal{C}\right) \\
& =\|x\|_{\infty} \mu^{-1} \gamma(\bar{x} \mid \mathcal{C}) \\
& \leq\|x\|_{\infty} \mu^{-1} .
\end{aligned}
$$

by $(4.18)$ since $\gamma(\cdot \mid \mathcal{C})$ is monotone

since $\gamma(\cdot \mid \mathcal{C})$ is positively homogeneous

by (4.24) since $\gamma(\cdot \mid \mathcal{C})$ monotone

since $\gamma(\cdot \mid \mathcal{C})$ is positively homogeneous

by (4.11) since $\bar{x} \in \mathcal{C}$

Since $\|x\|_{\infty}$ is real valued, we conclude that $\gamma(\cdot \mid \mathcal{C})$ is real valued.

The fact that $\mathcal{C} \subseteq \operatorname{unit}(\gamma(\cdot \mid \mathcal{C}))$ is trivial. If $x \in \operatorname{unit}(\gamma(\cdot \mid \mathcal{C}))$, then there exists a sequence of positive real numbers $\left(\mu_{n}\right)_{n \in \mathbb{N}}$ such that $\lim _{n \rightarrow+\infty} \mu_{n}=\mu \leq 1$ and $x \in \mu_{n} \mathcal{C}$ for every $n \in \mathbb{N}$. If $\mu=0$, then as $\mathcal{C}$ is bounded we conclude that $x=0$, so that $x \in\{0\}=\mu \mathcal{C}$. If $\mu \neq 0$, we have that $\lim _{n \rightarrow+\infty} \mu_{n}^{-1}=\mu^{-1}$. Define $x_{n}:=\mu_{n}^{-1} x$ for every $n \in \mathbb{N}$. By construction, we have that $x_{n} \in \mathcal{C}$ for every $n \in \mathbb{N}$, and that $\lim _{n \rightarrow \infty} x_{n}=\mu^{-1} x$. Since $\mathcal{C}$ is closed, we conclude that $x \in \mu \mathcal{C}$. Using that $\mathcal{C}$ is a convex set containing the origin and that $\mu \leq 1$ we conclude that $\mu \mathcal{C} \subseteq \mathcal{C}$, which finishes proof.

\subsubsection{Strong Duality for Convex Corners and Gauges}

Propositions 4.10 and 4.11 have established a one-to-one correspondence between convex corners and realvalued positive definite monotone gauges. There is, however, another way to define a real-valued positive definite monotone gauge from a convex corner. Let $V$ be a finite set, and let $\mathcal{C} \subseteq \mathbb{R}_{+}^{V}$ be a convex corner. Define

$$
\beta(w):=\max \left\{w^{\top} x \mid x \in \mathcal{C}\right\},
$$

for every $w \in \mathbb{R}_{+}^{V}$. The function $\beta$ is a monotone gauge, as it is the pointwise supremum of monotone gauges. It is positive definite because $\mathcal{C}$ has nonempty interior. Using Cauchy-Schwarz and the fact that $\mathcal{C}$ is bounded we conclude that $\beta$ is real-valued. Therefore, Proposition 4.10 implies that unit $(\beta)$ is a convex corner, so that 
we have implicitly defined a mapping among convex corners. Making this mapping explicit will allow us to formulate $\beta$ as the Minkowski functional of a suitable convex corner.

Following the terminology from Fulkerson [39,40], define the antiblocker abl $(\mathcal{C})$ of a set $\mathcal{C} \subseteq \mathbb{R}_{+}^{V}$ as

$$
\operatorname{abl}(\mathcal{C}):=\left\{y \in \mathbb{R}_{+}^{V} \mid \forall x \in \mathcal{C}, y^{\top} x \leq 1\right\}
$$

Note that, by definition,

$$
\mathcal{C} \subseteq \mathcal{D} \Longrightarrow \operatorname{abl}(\mathcal{D}) \subseteq \operatorname{abl}(\mathcal{C})
$$

It is also evident that

$$
\operatorname{abl}(\mathcal{C})=\left\{x \in \mathbb{R}_{+}^{V} \mid \delta^{*}(x \mid \mathcal{C}) \leq 1\right\}
$$

In particular, we conclude that

$$
\operatorname{abl}(\operatorname{cl} \mathcal{C})=\operatorname{abl}(\mathcal{C})
$$

since

$$
\begin{aligned}
\operatorname{abl}(\operatorname{cl} \mathcal{C}) & =\left\{x \in \mathbb{R}_{+}^{V} \mid \delta^{*}(x \mid \operatorname{cl} \mathcal{C}) \leq 1\right\} & & \text { by }(4.27) \\
& =\left\{x \in \mathbb{R}_{+}^{V} \mid \delta^{*}(x \mid \mathcal{C}) \leq 1\right\} & & \text { by }(2.46) \\
& =\operatorname{abl}(\mathcal{C}) & & \text { by }(4.27)
\end{aligned}
$$

Further note that (4.25) implies that

$$
\mathcal{C} \subseteq \operatorname{abl}(\operatorname{abl} \mathcal{C})
$$

as $y^{\top} x \leq 1$ for every $y \in \mathcal{C}$ and $x \in \operatorname{abl}(\mathcal{C})$.

As the reader shall see, the antiblocking operator defines an involution on convex corners. This is extremely useful, as it provides us with a concrete object - convex corners - and a way to transform them into similar objects. However, this subsection must both establish that the antiblocking operator is an involution on convex corners and provide facilities for obtaining convex corners. For this reason, in the following pages, we will focus on a definition that is less stringent than convex corners, which will describe the objects that form the basic blocks for reasoning about convex corners. Let $V$ be a finite set. For every $w \in \mathbb{R}^{V}$ and $\alpha \in \mathbb{R}$ define

$$
\mathcal{B}(w, \alpha):=\left\{x \in \mathbb{R}_{+}^{V} \mid w^{\top} x \leq \alpha\right\} .
$$

A set of the form $\mathcal{B}(w, \alpha)$ with $w \in \mathbb{R}_{+}^{V}$ and $\alpha \in \mathbb{R}$ is a basic corner. For every $\mathcal{C} \subseteq \mathbb{R}_{+}^{V}$ and $w \in \mathbb{R}^{V}$, define

$$
\mathcal{B}(w \mid \mathcal{C}):=\mathcal{B}\left(w, \delta^{*}(w \mid \mathcal{C})\right)
$$

A set of the form $\mathcal{B}(w \mid \mathcal{C})$ with $\mathcal{C} \subseteq \mathbb{R}_{+}^{V}$ and $w \in \mathbb{R}^{V}$ is a supporting basic corner. The reader who is familiar with convexity will realize that a basic corner is simply the intersection of a closed half-space with the nonnegative orthant. Basically, all of our following results are standard results regarding closed half-spaces adapted to basic corners. Note that

$$
\operatorname{abl}(\mathcal{C})=\bigcap\{\mathcal{B}(z, 1) \mid z \in \mathcal{C}\}
$$

for every $\mathcal{C} \subseteq \mathbb{R}_{+}^{V}$, since

$$
\begin{aligned}
\operatorname{abl}(\mathcal{C}) & =\left\{y \in \mathbb{R}_{+}^{V} \mid \forall z \in \mathcal{C}, y^{\top} z \leq 1\right\} \\
& =\bigcap\left\{\left\{y \in \mathbb{R}_{+}^{V} \mid y^{\top} z \leq 1\right\} \mid z \in \mathcal{C}\right\} \\
& =\bigcap\{\mathcal{B}(z, 1) \mid z \in \mathcal{C}\} .
\end{aligned}
$$

Proposition 4.12. Let $V$ be a finite set. For every $w \in \mathbb{R}_{+}^{V}$ and for every $\alpha \in \mathbb{R}$, the set $\mathcal{B}(w, \alpha)$ is a lower-comprehensive closed convex subset of $\mathbb{R}_{+}^{V}$. 
Proof. Let $w \in \mathbb{R}_{+}^{V}$. The set $\mathcal{B}(w, \alpha)$ is closed and convex because it is the intersection of $|V|+1$ closed and convex sets. It is a subset of $\mathbb{R}_{+}^{V}$ by definition. We show that $\mathcal{B}(w, \alpha)$ is lower comprehensive. Let $x, y \in \mathbb{R}_{+}^{V}$ be such that $0 \leq x \leq y$ and $y \in \mathcal{B}(w, \alpha)$. Then

$$
\begin{aligned}
w^{\top} x & \leq w^{\top} y & & \text { since } 0 \leq w \text { and } x \leq y \\
& \leq \alpha . & & \text { since } y \in \mathcal{B}(w, \alpha) \text { and }(4.30)
\end{aligned}
$$

Using that $x \in \mathbb{R}_{+}^{V}$, we conclude from (4.30) that $x \in \mathcal{B}(w, \alpha)$.

Corollary 4.13. Let $V$ be a finite set, and let $\mathcal{C} \subseteq \mathbb{R}_{+}^{V}$. Then $\operatorname{abl}(\mathcal{C})$ is a lower-comprehensive closed convex set.

Proof. As lower-comprehensive closed convex sets are closed under intersection, Proposition 4.12 and (4.32) finish the proof.

Proposition 4.14. Let $V$ be a finite set. If $w \in \mathbb{R}_{++}^{V}$, then $\mathcal{B}(w, \alpha)$ is bounded for every $\alpha \in \mathbb{R}$.

Proof. Let $w \in \mathbb{R}_{++}^{V}$ and $\alpha \in \mathbb{R}$. Set $\beta:=\min _{i \in V} w_{i}>0$. It is clear that

$$
\beta \mathbb{1} \leq w .
$$

If $x \in \mathcal{B}(w, \alpha)$, then

$$
\begin{aligned}
\beta\|x\|_{1} & =\beta \mathbb{1}^{\top} x \\
& \leq w^{\top} x \quad \text { by (4.33) and } x \geq 0
\end{aligned}
$$$$
\leq \alpha . \quad \text { by } x \in \mathcal{B}(w, \alpha) \text { and }(4.30)
$$

Therefore $\|x\|_{1} \leq \beta^{-1} \alpha$. Since $x \in \mathcal{B}(w, \alpha)$ was arbitrary, the proof is done.

Proposition 4.15. Let $V$ be a finite set, and let $\mathcal{C} \subseteq \mathbb{R}_{+}^{V}$ be a lower-comprehensive set. For every $\bar{x} \in \mathbb{R}_{+}^{V} \backslash \operatorname{cl}(\operatorname{conv} \mathcal{C})$ there exists $y \in \mathbb{R}_{+}^{V}$ such that

$$
\delta^{*}(y \mid \mathcal{C})<y^{\top} \bar{x}
$$

Proof. Let $\bar{x} \in \mathbb{R}_{+}^{V} \backslash \operatorname{cl}(\operatorname{conv} \mathcal{C})$. Since $\operatorname{cl}(\operatorname{conv} \mathcal{C})$ is a closed convex set and $\bar{x} \notin \operatorname{cl}(\operatorname{conv} \mathcal{C})$, it is a standard result in convex analysis that there exists $y \in \mathbb{R}^{V}$ such that the inequality in the statement holds. See, for example, [102, Section 11]. We only have to prove that there exists a nonnegative vector with this property. Set

$$
\mathcal{B}:=\left\{y \in \mathbb{R}^{V} \mid \delta^{*}(y \mid \mathcal{C})<y^{\top} \bar{x}\right\} .
$$

Let $y \in \mathcal{B}$ be an element which maximizes

$$
\left|\left\{i \in V \mid y_{i} \geq 0\right\}\right| .
$$

If $\left|\left\{i \in V \mid y_{i} \geq 0\right\}\right|=|V|$, the proof is done. Otherwise, there exists $i \in V$ be such that $y_{i}<0$. Using that $\mathcal{C}$ is lower comprehensive and that $\bar{x} \geq 0$, we have that

$$
\begin{aligned}
\delta^{*}\left(y-y_{i} e_{i} \mid \mathcal{C}\right) & =\sup \left\{\left(y-y_{i} e_{i}\right)^{\top} x \mid x \in \mathcal{C}\right\} & & \text { by }(2.45) \\
& =\sup \left\{y^{\top}\left(x-x_{i} e_{i}\right) \mid x \in \mathcal{C}\right\} & & \\
& \leq \sup \left\{y^{\top} x \mid x \in \mathcal{C}\right\} & & \text { by }(4.12) \text { and } 0 \leq x-x_{i} e_{i} \leq x \\
& =\delta^{*}(y \mid \mathcal{C}) & & \text { by }(2.45) \\
& <y^{\top} \bar{x} & & \text { by hypothesis } \\
& \leq\left(y-y_{i} e_{i}\right)^{\top} \bar{x} . & & \text { since } y_{i}<0
\end{aligned}
$$

Therefore (4.34) implies that if $y \in \mathcal{B}$ then $y-y_{i} e_{i} \in \mathcal{B}$. But $y-y_{i} e_{i}$ has one nonnegative entry more than $y$, contradicting the definition of $y$. 
Corollary 4.16. Let $V$ be a finite set, and let $\mathcal{C}_{0}, \mathcal{C}_{1} \subseteq \mathbb{R}_{+}^{V}$ be convex corners. Then $\mathcal{C}_{0} \subseteq \mathcal{C}_{1}$ if and only if

$$
\delta^{*}\left(w \mid \mathcal{C}_{0}\right) \leq \delta^{*}\left(w \mid \mathcal{C}_{1}\right)
$$

for every $w \in \mathbb{R}_{+}^{V}$. In particular, $\mathcal{C}_{0}=\mathcal{C}_{1}$ if and only if $\delta^{*}\left(w \mid \mathcal{C}_{0}\right)=\delta^{*}\left(w \mid \mathcal{C}_{1}\right)$ for every $w \in \mathbb{R}_{+}^{V}$.

Proof. If $\mathcal{C}_{0} \subseteq \mathcal{C}_{1}$, it is obvious that $\delta^{*}\left(w \mid \mathcal{C}_{0}\right) \leq \delta^{*}\left(w \mid \mathcal{C}_{1}\right)$ for every $w \in \mathbb{R}_{+}^{V}$. Conversely, assume that there exists $\bar{x} \in \mathcal{C}_{0} \backslash \mathcal{C}_{1}$. Proposition 4.15 implies that there exists $w \in \mathbb{R}_{+}^{V}$ such that $\delta^{*}\left(w \mid \mathcal{C}_{1}\right)<w^{\top} \bar{x}$. Thus $\delta^{*}\left(w \mid \mathcal{C}_{0}\right) \geq w^{\top} \bar{x}>\delta^{*}\left(w \mid \mathcal{C}_{1}\right)$.

The second part of the statement follows trivially from the first.

Although Theorems 4.25 and 4.26 are the theorems most used outside of this chapter, it is Theorem 4.17 that better captures strong duality in our context. It also explains our nomenclature, as it implies that a convex corner is the intersection of its supporting basic corners. Even more, it shows how the lower-comprehensive condition appears naturally from our focus on the nonnegative orthant. The many corollaries of Theorem 4.17 provide auxiliary results regarding convex corner which we shall use in the remainder of the text.

Theorem 4.17. Let $V$ be a finite set, and $\mathcal{C} \subseteq \mathbb{R}_{+}^{V}$ be a set. Then

$$
\operatorname{cl}(\operatorname{conv}(\operatorname{lc} \mathcal{C}))=\bigcap\left\{\mathcal{B}(w \mid \mathcal{C}) \mid w \in \mathbb{R}_{+}^{V}\right\} .
$$

Proof. Set

$$
\mathcal{B}:=\bigcap\left\{\mathcal{B}(w \mid \mathcal{C}) \mid w \in \mathbb{R}_{+}^{V}\right\} .
$$

We now prove that $\operatorname{cl}(\operatorname{conv}(\operatorname{lc} \mathcal{C}))=\mathcal{B}$.

We first show that for every $w \in \mathbb{R}_{+}^{V}$,

$$
\mathcal{C} \subseteq \mathcal{B}\left(w, \delta^{*}(w \mid \mathcal{C})\right)
$$

Let $w \in \mathbb{R}_{+}^{V}$. Then

$$
\begin{aligned}
\mathcal{C} & \subseteq\left\{x \in \mathbb{R}_{+}^{V} \mid w^{\top} x \leq \delta^{*}(w \mid \mathcal{C})\right\} & & \text { by }(2.45) \\
& =\mathcal{B}\left(w, \delta^{*}(w \mid \mathcal{C})\right) & & \text { by }(4.30)
\end{aligned}
$$

We thus have that for every $w \in \mathbb{R}_{+}^{V}$,

$$
\operatorname{cl}(\operatorname{conv}(\operatorname{lc} \mathcal{C})) \subseteq \mathcal{B}(w \mid \mathcal{C})
$$

since

$$
\begin{aligned}
\mathcal{C} \subseteq \mathcal{B}\left(w, \delta^{*}(w \mid \mathcal{C})\right) & & & \text { by }(4.36) \\
& \Longrightarrow \operatorname{lc}(\mathcal{C}) \subseteq \mathcal{B}\left(w, \delta^{*}(w \mid \mathcal{C})\right) & & \text { by Proposition } 4.12 \\
& \Longrightarrow \operatorname{conv}(\operatorname{lc} \mathcal{C}) \subseteq \mathcal{B}\left(w, \delta^{*}(w \mid \mathcal{C})\right) & & \text { by Proposition } 4.12 \\
& \Longrightarrow \operatorname{cl}(\operatorname{conv}(\operatorname{lc} \mathcal{C})) \subseteq \mathcal{B}\left(w, \delta^{*}(w \mid \mathcal{C})\right) & & \text { by Proposition } 4.12 \\
& \Longrightarrow \operatorname{cl}(\operatorname{conv}(\operatorname{lc} \mathcal{C})) \subseteq \mathcal{B}(w \mid \mathcal{C}) . & & \text { by }(4.31)
\end{aligned}
$$

It is immediate from (4.35) and (4.37) that

$$
\operatorname{cl}(\operatorname{conv}(\operatorname{lc} \mathcal{C})) \subseteq \bigcap\left\{\mathcal{B}(w \mid \mathcal{C}) \mid w \in \mathbb{R}_{+}^{V}\right\}=\mathcal{B} .
$$

Assume that there exists $x \in \mathcal{B} \backslash \operatorname{cl}(\operatorname{conv}(\operatorname{lc} \mathcal{C}))$. Then there exists $y \in \mathbb{R}_{+}^{V}$ such that

$$
\begin{aligned}
\delta^{*}(y \mid \operatorname{lc} \mathcal{C}) & <y^{\top} x & & \text { by Proposition } 4.15 \\
& \leq \delta^{*}(y \mid \mathcal{C}) & & \text { by }(4.31) \text { and } x \in \mathcal{B} \subseteq \mathcal{B}(y \mid \mathcal{C}) \\
& \leq \delta^{*}(y \mid \operatorname{lc} \mathcal{C}) . & & \text { by }(2.48) \text { and } \mathcal{C} \subseteq \text { lc } \mathcal{C}
\end{aligned}
$$

As this is a contradiction, we conclude that $\mathcal{B} \subseteq \operatorname{cl}(\operatorname{conv}(\operatorname{lc} \mathcal{C}))$. 
Corollary 4.18. Let $V$ be a finite set, and let $\mathcal{C} \subseteq \mathbb{R}_{+}^{V}$. Then $\operatorname{cl}(\operatorname{conv}(\operatorname{lc} \mathcal{C}))$ is a lower-comprehensive closed convex set.

Proof. Theorem 4.17 and (4.31) imply that

$$
\operatorname{cl}(\operatorname{conv}(\operatorname{lc} \mathcal{C}))=\bigcap\left\{\mathcal{B}(w \mid \mathcal{C}) \mid w \in \mathbb{R}_{+}^{V}\right\}=\bigcap\left\{\mathcal{B}\left(w, \delta^{*}(w \mid \mathcal{C})\right) \mid w \in \mathbb{R}_{+}^{V}\right\} .
$$

As lower-comprehensive closed convex sets are closed under intersection, Proposition 4.12 finishes the proof.

Corollary 4.19. Let $V$ be a finite set. Let $\mathcal{C} \subseteq \mathbb{R}_{+}^{V}$ be a bounded set such that $\mathcal{C} \cap \mathbb{R}_{++}^{V}$ is nonempty. Then $\operatorname{cl}(\operatorname{conv}(\operatorname{lc} \mathcal{C}))$ is a convex corner.

Proof. Since $\mathcal{C}$ is bounded, there exists $M \in \mathbb{R}_{++}$such that

$$
\mathcal{C} \subseteq \mathcal{B}
$$

where $\mathcal{B}:=\left\{x \in \mathbb{R}_{+}^{V} \mid\|x\| \leq M\right\}$. The set $\mathcal{B}$ is lower comprehensive, since for every $x, y \in \mathbb{R}_{+}^{V}$ such that $y \leq x$ we have that $\|y\| \leq\|x\|$. Furthermore, $\mathcal{B}$ is clearly closed and convex. Thus

$$
\begin{aligned}
\mathcal{C} \subseteq \mathcal{B} & \Longrightarrow \operatorname{lc}(\mathcal{C}) \subseteq \mathcal{B} & & \text { since } \mathcal{B} \text { is lower comprehensive } \\
& \Longrightarrow \operatorname{conv}(\operatorname{lc} \mathcal{C}) \subseteq \mathcal{B} & & \text { since } \mathcal{B} \text { is convex } \\
& \Longrightarrow \operatorname{cl}(\operatorname{conv}(\operatorname{lc} \mathcal{C})) \subseteq \mathcal{B} . & & \text { since } \mathcal{B} \text { is closed }
\end{aligned}
$$

In short,

$$
\operatorname{cl}(\operatorname{conv}(\operatorname{lc} \mathcal{C})) \text { is bounded. }
$$

Let $\stackrel{\circ}{x} \in \mathcal{C} \cap \mathbb{R}_{++}^{V}$. Set $\beta:=\min \left\{\stackrel{\circ}{x}_{i} \mid i \in V\right\}>0$. Let $x \in \mathbb{R}_{+}^{V}$ be such that $\|x\|_{\infty} \leq \beta$. Then

$$
\begin{aligned}
x & \leq\|x\|_{\infty} \mathbb{1} & & \text { by }(4.18) \\
& \leq \beta \mathbb{1} & & \\
& \leq \stackrel{x}{x} . & & \text { by choice of } \beta
\end{aligned}
$$

Since $\operatorname{cl}(\operatorname{conv}(\operatorname{lc} \mathcal{C}))$ is lower comprehensive by Corollary 4.18 and $\stackrel{\circ}{x} \in \mathcal{C} \subseteq \operatorname{cl}(\operatorname{conv}(\operatorname{lc} \mathcal{C}))$, we conclude that $x \in \operatorname{cl}(\operatorname{conv}(\operatorname{lc} \mathcal{C}))$. Therefore

$$
\left\{x \in \mathbb{R}_{+}^{V} \mid\|x\|_{\infty} \leq \beta\right\} \subseteq \operatorname{cl}(\operatorname{conv}(\operatorname{lc} \mathcal{C})),
$$

which implies that $\operatorname{cl}(\operatorname{conv}(\operatorname{lc} \mathcal{C}))$ has nonempty interior.

Corollary 4.20. Let $V$ be a finite set. Let $\mathcal{C} \subseteq \mathbb{R}_{+}^{V}$ be a compact convex set such that $\mathcal{C} \cap \mathbb{R}_{++}^{V}$ is nonempty. Then $\operatorname{lc}(\mathcal{C})$ is a convex corner.

Proof. Note that

$$
\operatorname{lc}(\mathcal{C})=\operatorname{cl}(\operatorname{conv}(\operatorname{lc} \mathcal{C}))
$$

since

$$
\begin{aligned}
\operatorname{lc}(\mathcal{C}) & =\operatorname{cl}(\operatorname{lc} \mathcal{C}) & & \text { by Proposition } 4.7 \text { and since } \mathcal{C} \text { compact } \\
& =\operatorname{cl}(\operatorname{lc}(\operatorname{conv} \mathcal{C})) & & \text { since } \mathcal{C} \text { convex } \\
& =\operatorname{cl}(\operatorname{conv}(\operatorname{lc}(\operatorname{conv} \mathcal{C}))) & & \text { by Corollary } 4.6 \\
& =\operatorname{cl}(\operatorname{conv}(\operatorname{lc} \mathcal{C})) . & & \text { since } \mathcal{C} \text { convex }
\end{aligned}
$$

Corollary 4.19 and (4.39) finish the proof.

Corollary 4.21. Let $V$ be a finite set, and let $\mathcal{C} \subseteq \mathbb{R}_{+}^{V}$ be a set. Then for every $w \in \mathbb{R}_{+}^{V}$,

$$
\delta^{*}(w \mid \operatorname{cl}(\operatorname{conv}(\operatorname{lc} \mathcal{C})))=\delta^{*}(w \mid \mathcal{C}) .
$$


Proof. Let $w \in \mathbb{R}_{+}^{V}$. Then

$$
\begin{aligned}
x \in \operatorname{cl}(\operatorname{conv}(\operatorname{lc} \mathcal{C})) & \Longrightarrow x \in \mathcal{B}(w \mid \mathcal{C}) & & \text { by Theorem } 4.17 \\
& \Longrightarrow x \in \mathcal{B}\left(w, \delta^{*}(w \mid \mathcal{C})\right) & & \text { by }(4.31) \\
& \Longrightarrow w^{\top} x \leq \delta^{*}(w \mid \mathcal{C}) . & & \text { by }(4.30)
\end{aligned}
$$

As this holds for every $x \in \operatorname{cl}(\operatorname{conv}(\operatorname{lc} \mathcal{C}))$, we have that

$$
\delta^{*}(w \mid \operatorname{cl}(\operatorname{conv}(\operatorname{lc} \mathcal{C}))) \leq \delta^{*}(w \mid \mathcal{C}) .
$$

Therefore

$$
\begin{aligned}
\delta^{*}(w \mid \mathcal{C}) & \leq \delta^{*}(w \mid \operatorname{cl}(\operatorname{conv}(\operatorname{lc} \mathcal{C}))) & & \text { by }(2.48) \text { and } \mathcal{C} \subseteq \operatorname{cl}(\operatorname{conv}(\operatorname{lc} \mathcal{C})) \\
& \leq \delta^{*}(w \mid \mathcal{C}) . & & \text { by }(4.40)
\end{aligned}
$$

Corollary 4.22. Let $V$ be a finite set, and let $\mathcal{C} \subseteq \mathbb{R}_{+}^{V}$. Then for every $w \in \mathbb{R}_{+}^{V}$,

$$
\delta^{*}(w \mid \operatorname{lc} \mathcal{C})=\delta^{*}(w \mid \mathcal{C}) .
$$

Proof. Combine Corollary 4.21 with (2.46) and (2.47).

Corollary 4.23. Let $V$ be a finite set, and let $\mathcal{C} \subseteq \mathbb{R}_{+}^{V}$. Then

$$
\operatorname{abl}(\mathcal{C})=\operatorname{abl}(\operatorname{lc}(\mathcal{C}))
$$

Proof. Note that

$$
\begin{aligned}
y \in \operatorname{abl}(\mathcal{C}) & \Longleftrightarrow y \in \mathbb{R}_{+}^{V} \text { and } \delta^{*}(y \mid \mathcal{C}) \leq 1 & & \text { by }(4.27) \\
& \Longleftrightarrow y \in \mathbb{R}_{+}^{V} \text { and } \delta^{*}(y \mid \operatorname{lc} \mathcal{C}) \leq 1 & & \text { by Corollary } 4.22 \\
& \Longleftrightarrow y \in \operatorname{abl}(\operatorname{lc} \mathcal{C}) . & & \text { by }(4.27)
\end{aligned}
$$

Corollary 4.24. Let $V$ be a finite set. Let $\mathcal{C} \subseteq \mathbb{R}_{+}^{V}$ be such that $\mathcal{C} \cap \mathbb{R}_{++}^{V} \neq \varnothing$. Then

$$
\operatorname{abl}(\operatorname{abl} \mathcal{C})=\operatorname{cl}(\operatorname{conv}(\operatorname{lc} \mathcal{C}))
$$

Proof. We prove that

$$
\text { for every } w \in \mathbb{R}_{+}^{V} \backslash\{0\} \text { there exists } z \in \operatorname{abl} \mathcal{C} \text { such that } \mathcal{B}(w \mid \mathcal{C})=\mathcal{B}(z, 1) \text {. }
$$

Let $w \in \mathbb{R}_{+}^{V} \backslash\{0\}$. Since $\mathcal{C} \cap \mathbb{R}_{++}^{V}$ is nonempty, it follows that $\delta^{*}(w \mid \mathcal{C})>0$. Define $z:=\delta^{*}(w \mid \mathcal{C})^{-1} w$. By (4.27), it is clear that $z \in \operatorname{abl} \mathcal{C}$. Furthermore

$$
\begin{aligned}
\mathcal{B}(w \mid \mathcal{C}) & =\mathcal{B}\left(w, \delta^{*}(w \mid \mathcal{C})\right) & & \text { by }(4.31) \\
& =\left\{x \in \mathbb{R}_{+}^{V} \mid w^{\top} x \leq \delta^{*}(w \mid \mathcal{C})\right\} & & \text { by }(4.30) \\
& =\left\{x \in \mathbb{R}_{+}^{V} \mid\left(\delta^{*}(w \mid \mathcal{C}) z\right)^{\top} x \leq \delta^{*}(w \mid \mathcal{C})\right\} & & \text { by definition of } z \\
& =\left\{x \in \mathbb{R}_{+}^{V} \mid z^{\top} x \leq 1\right\} & & \text { since } \delta^{*}(w \mid \mathcal{C})>0 \\
& =\mathcal{B}(z, 1) . & & \text { by }(4.30)
\end{aligned}
$$

We thus conclude (4.41).

Then

$$
\begin{aligned}
\operatorname{cl}(\operatorname{conv}(\operatorname{lc} \mathcal{C})) & \subseteq \operatorname{cl}(\operatorname{conv}(\operatorname{lc}(\operatorname{abl}(\operatorname{abl} \mathcal{C})))) & & \text { by }(4.29) \text { and monotonicity of cl, conv, and lc } \\
& =\operatorname{abl}(\operatorname{abl} \mathcal{C}) & & \text { by Corollary } 4.13 \\
& =\bigcap\{\mathcal{B}(z, 1) \mid z \in \operatorname{abl} \mathcal{C}\} & & \text { by }(4.32) \\
& \subseteq \bigcap\left\{\mathcal{B}(w \mid \mathcal{C}) \mid w \in \mathbb{R}_{+}^{V} \backslash\{0\}\right\} & & \text { by }(4.41) \\
& =\bigcap\left\{\mathcal{B}(w \mid \mathcal{C}) \mid w \in \mathbb{R}_{+}^{V}\right\} & & \text { since } \mathcal{B}(0 \mid \mathcal{C})=\mathbb{R}_{+}^{V} \\
& =\operatorname{cl}(\operatorname{conv}(\operatorname{lc} \mathcal{C})) & & \text { by Theorem } 4.17
\end{aligned}
$$


Theorem 4.25. Let $V$ be a finite set, and let $\mathcal{C} \subseteq \mathbb{R}_{+}^{V}$ be a convex corner. Then

1. the set $\operatorname{abl}(\mathcal{C})$ is a convex corner,

2. for every $y \in \mathbb{R}_{+}^{V}$ we have that $\delta^{*}(y \mid \mathcal{C})=\gamma(y \mid \operatorname{abl}(\mathcal{C}))$, and

3. it holds that $\operatorname{abl}(\operatorname{abl}(\mathcal{C}))=\mathcal{C}$.

Proof. Corollary 4.13 states that $\operatorname{abl}(\mathcal{C})$ is a lower-comprehensive closed convex set. Let $\stackrel{x}{x}>0$ be in the interior of $\mathcal{C}$. Then abl $\mathcal{C} \subseteq \mathcal{B}(\stackrel{\circ}{x}, 1)$ by $(4.32)$. Proposition 4.14 implies that $\mathcal{B}(\stackrel{\circ}{x}, 1)$ is bounded, so abl $(\mathcal{C})$ is bounded. As $\mathcal{C}$ is bounded, there exists $M>0$ such that $\|x\| \leq M$ for every $x \in \mathcal{C}$. Let $x \in \mathcal{C}$. Then

$$
\left\{y \in \mathbb{R}_{+}^{V} \mid\|y\| \leq M^{-1}\right\} \subseteq \mathcal{B}(x, 1),
$$

since for every $y \in \mathbb{R}_{+}^{V}$ with $\|y\| \leq M^{-1}$,

$$
y^{\top} x \leq\|y\|\|x\| \leq M^{-1} M=1 .
$$

Using (4.32), we conclude that $\left\{y \in \mathbb{R}_{+}^{V} \mid\|y\| \leq M^{-1}\right\} \subseteq \operatorname{abl}(\mathcal{C})$. Thus, $\operatorname{abl}(\mathcal{C})$ has nonempty interior, and item 1 holds.

Let $y \in \mathbb{R}_{+}^{V}$. Then $\mu>0$ is an upper bound for $\left\{y^{\top} x \mid x \in \mathcal{C}\right\}$ if and only if $y \in \mu \operatorname{abl}(\mathcal{C})$. As the supremum of a set is the infimum of its upper bounds (recall (3.35a)), we have that $\sup \left\{y^{\top} x \mid x \in \mathcal{C}\right\}=\gamma(y \mid \operatorname{abl}(\mathcal{C})$ ). Item 2 follows.

To prove Item 3, note that

$$
\begin{aligned}
\mathcal{C} & =\operatorname{cl}(\operatorname{conv}(\operatorname{lc} \mathcal{C})) & & \text { since } \mathcal{C} \text { is a convex corner } \\
& =\operatorname{abl}(\operatorname{abl} \mathcal{C}) . & & \text { since } \mathcal{C} \text { has nonempty interior, and by Corollary } 4.24
\end{aligned}
$$

Theorem 4.25 describes an involution on convex corners. We now define an involution on gauges. Let $V$ be a finite set, and let $\beta: \mathbb{R}_{+}^{V} \rightarrow \mathbb{R}$ be a positive definite monotone gauge. Define its gauge dual $\beta^{\circ}: \mathbb{R}_{+}^{V} \rightarrow$ $[-\infty,+\infty]$ as

$$
\beta^{\circ}(y):=\max \left\{y^{\top} x \mid x \in \operatorname{unit}(\beta)\right\}
$$

for every $y \in \mathbb{R}_{+}^{V}$. We point out the simple fact that, if $\mathcal{C} \subseteq \mathbb{R}_{+}^{V}$ is a convex corner, then

$$
\delta^{*}(y \mid \mathcal{C})=\max \left\{y^{\top} x \mid x \in \mathcal{C}\right\},
$$

since $\mathcal{C}$ is compact.

Theorem 4.26. Let $\beta: \mathbb{R}_{+}^{V} \rightarrow \mathbb{R}$ be a positive definite monotone gauge. Then

1. the function $\beta^{\circ}$ is a real-valued positive definite monotone gauge;

2. we have that $\operatorname{unit}\left(\beta^{\circ}\right)=\operatorname{abl}(\operatorname{unit}(\beta))$;

3. we have that $\beta^{\circ \circ}=\beta$.

Proof. For every $w \in \mathbb{R}_{+}^{V}$,

$$
\beta^{\circ}(w)=\gamma(w \mid \operatorname{abl}(\operatorname{unit}(\beta))),
$$

since

$$
\begin{aligned}
\beta^{\circ}(w) & =\max \left\{w^{\top} x \mid x \in \operatorname{unit}(\beta)\right\} & & \text { by }(4.42) \\
& =\delta^{*}(w \mid \operatorname{unit}(\beta)) & & \text { by }(4.43) \text { and Proposition } 4.10 \\
& =\gamma(w \mid \operatorname{abl}(\operatorname{unit}(\beta))) . & & \text { by item } 2 \text { of Theorem } 4.25 \text { and Proposition } 4.10
\end{aligned}
$$

Proposition 4.10 and item 1 of Theorem 4.25 imply that

$$
\operatorname{abl}(\operatorname{unit}(\beta)) \text { is a convex corner, }
$$


so that item 1 holds by Proposition 4.11 and (4.44).

Item 2 then follows:

$$
\begin{aligned}
\operatorname{unit}\left(\beta^{\circ}\right) & =\operatorname{unit}(\gamma(\cdot \mid \operatorname{abl}(\operatorname{unit}(\beta)))) & & \text { by }(4.44) \\
& =\operatorname{abl}(\operatorname{unit}(\beta)) . & & \text { by Proposition } 4.11 \text { and }(4.45)
\end{aligned}
$$

For item 3, note that

$$
\begin{aligned}
\beta^{\circ \circ} & =\gamma\left(\cdot \mid \operatorname{abl}\left(\operatorname{unit}\left(\beta^{\circ}\right)\right)\right) & & \text { by item } 1 \text { and }(4.44) \\
& =\gamma(\cdot \mid \operatorname{abl}(\operatorname{abl}(\operatorname{unit}(\beta)))) & & \text { by item } 2 \\
& =\gamma(\cdot \mid \operatorname{unit}(\beta)) & & \text { by item } 3 \text { of Theorem } 4.25 \text { and Proposition } 4.10 \\
& =\beta . & & \text { by Proposition } 4.10
\end{aligned}
$$

\subsubsection{Convex Corners from Graph Theory}

Let $G$ be a graph. Recall (1.9) and (1.10) that

$$
\begin{aligned}
\operatorname{STAB}(G) & =\operatorname{conv}\left(\left\{\mathbb{1}_{S} \mid S \in \mathcal{S}(G)\right\}\right), \\
\operatorname{QSTAB}(G) & =\left\{x \in \mathbb{R}_{+}^{V(G)} \mid \forall K \in \mathcal{S}(\bar{G}), \mathbb{1}_{K}^{\top} x \leq 1\right\} .
\end{aligned}
$$

It is easy to see that $\operatorname{QSTAB}(\bar{G})=\operatorname{unit}(\alpha(G, \cdot))$. Using this and $(3.112)$ we have that for every $w \in \mathbb{R}_{+}^{V}$,

$$
\chi_{f}(G, w)=\max \left\{w^{\top} x \mid x \in \operatorname{QSTAB}(\bar{G})\right\}=\max \left\{w^{\top} x \mid x \in \operatorname{unit}(\alpha(G, \cdot))\right\} .
$$

Since $\alpha(G, \cdot)$ is a real-valued positive definite monotone gauge by (4.7), we have from (4.42) and Theorem 4.26 that

$$
\alpha(G, \cdot) \text { and } \chi_{f}(G, \cdot) \text { are real-valued positive definite monotone gauges, dual to each other. }
$$

Proposition 4.27. Let $G$ be a graph. Then $\operatorname{STAB}(G)$ and $\operatorname{QSTAB}(G)$ are convex corners, and

$$
\operatorname{abl}(\operatorname{STAB}(G))=\operatorname{QSTAB}(\bar{G}) .
$$

Proof. Set $(V, E):=G$. Note that

$$
\operatorname{QSTAB}(\bar{G})=\left\{x \in \mathbb{R}_{+}^{V} \mid \forall S \in \mathcal{S}(G), \mathbb{1}_{S}^{\top} x \leq 1\right\}=\operatorname{abl}(\operatorname{STAB}(G)) .
$$

By Theorem 4.25, the statement follows from proving that $\operatorname{STAB}(G)$ is a convex corner. The set $\operatorname{STAB}(G)$ is convex and compact by construction. We have that $\operatorname{STAB}(G) \cap \mathbb{R}_{++}^{V}$ is nonempty, as

$$
\frac{1}{|V|} \mathbb{1}=\sum_{i \in V} \frac{1}{|V|} \mathbb{1}_{\{i\}} \in \operatorname{STAB}(G)
$$

Corollary 4.20 implies that $\operatorname{lc}(\operatorname{STAB}(G))$ is a convex corner. To finish the proof, it suffices to prove that $\operatorname{STAB}(G)$ is lower comprehensive. Proposition 4.2 then reduces our statement to proving that for every $i \in V$,

$$
\text { if } y \in \operatorname{STAB}(G) \text {, then } y-y_{i} e_{i} \in \operatorname{STAB}(G) \text {. }
$$

Fix $i \in V$, and let $\lambda \in \mathbb{R}_{++}^{\mathcal{S}(G)}$ be such that $\lambda^{\top} \mathbb{1}=1$. If $y=\sum_{S \in \mathcal{S}(G)} \lambda_{S} \mathbb{1}_{S}$, then

$$
y-y_{i} e_{i}=\sum_{S \in \mathcal{T}} \lambda_{S} \mathbb{1}_{S \backslash\{i\}}+\sum_{S \in \mathcal{S}(G) \backslash \mathcal{T}} \lambda_{S} \mathbb{1}_{S},
$$

where $\mathcal{T}:=\{S \in \mathcal{S}(G) \mid i \in S\}$. The element on the RHS is clearly in $\operatorname{STAB}(G)$, which implies (4.47). 
Let $G:=(V, E)$ be a graph. Define

$$
\operatorname{CUT}(G):=\operatorname{conv}\left(\left\{\mathbb{1}_{\delta(S)} \mid S \subseteq V\right\}\right) .
$$

There are many results about $\delta^{*}(w \mid \operatorname{CUT}(G))$ for $w \in \mathbb{R}_{+}^{W}$, as computing this value is the well-known maximum cut problem - see, e.g., [31, 46, 75]. There is another relevant graph parameter related to cuts introduced by [103] — see also [105]. The fractional cut-covering number is defined by

$$
x(G, z):=\inf \left\{\mathbb{1}^{\top} y \mid y \in \mathbb{R}_{+}^{\mathcal{P}(V)}, \sum_{S \subseteq V} y_{s} \mathbb{1}_{S} \geq z\right\}
$$

for every $z \in \mathbb{R}_{+}^{E}$. For every $z \in \mathbb{R}_{+}^{E}$, we have that

$$
\begin{aligned}
x(G, z) & =\min \left\{\mathbb{1}^{\top} y \mid y \in \mathbb{R}_{+}^{\mathcal{P}(V)}, \sum_{S \subseteq V} y_{s} \mathbb{1}_{S} \geq z\right\} \\
& =\max \left\{z^{\top} w \mid w \in \mathbb{R}_{+}^{E}, \forall S \subseteq V, \mathbb{1}_{\delta(S)}^{\top} w \leq 1\right\},
\end{aligned}
$$

as the linear programs in (4.50) are LP duals to each other, and they have $\stackrel{y}{y}:=\|z\|_{\infty} \mathbb{1}$ and $\stackrel{\circ}{x}:=0$ as feasible solutions. The fractional cut-covering number will be studied in depth in Section 5.3. For now, we simply show it to be a real-valued positive definite monotone gauge.

One may want to prove that (4.49) is a real-valued positive definite monotone gauge by showing that $\operatorname{CUT}(G)$ is a convex corner for every graph $G$. However, this is not the case. We now argue that

$$
\mathrm{CUT}\left(K_{3}\right) \text { is not lower-comprehensive. }
$$

Since $K_{3}$ has 3 vertices, and a set and its complement induce the same cut, we have 4 distinct cuts to consider. These cuts are the empty cut, and the cuts defined by each vertex. Therefore

$$
\begin{aligned}
\operatorname{CUT}\left(K_{3}\right) & =\operatorname{conv}\left(\left\{0, \mathbb{1}_{\delta(\{0\})}, \mathbb{1}_{\delta(\{1\})}, \mathbb{1}_{\delta(\{2\})}\right\}\right) \\
& =\operatorname{conv}\left(\left\{0, e_{01}+e_{02}, e_{01}+e_{12}, e_{02}+e_{12}\right\}\right) .
\end{aligned}
$$

It is then clear that $e_{01} \notin \mathrm{CUT}\left(K_{3}\right)$, although $e_{01} \leq e_{01}+e_{02} \in \mathrm{CUT}(G)$, so (4.51) follows.

Proposition 4.28. Let $G$ be a graph with nonempty edge set. Then $\operatorname{lc}(\mathrm{CUT}(G))$ and $\operatorname{abl}(\mathrm{CUT}(G))$ are convex corners, and

$$
\operatorname{abl}(\operatorname{lc}(\operatorname{CUT}(G)))=\operatorname{abl}(\operatorname{CUT}(G)) .
$$

Proof. The set $\operatorname{CUT}(G)$ is compact and convex by construction. We also have that $\operatorname{CUT}(G) \cap \mathbb{R}_{++}^{E}$ is nonempty, as

$$
\frac{2}{|V|} \mathbb{1}=\sum_{i \in V} \frac{1}{|V|} \mathbb{1}_{\delta(\{i\})} \in \operatorname{CUT}(G) .
$$

Corollary 4.20 implies that $\operatorname{lc}(\operatorname{CUT}(G))$ is a convex corner. Corollary 4.23 proves the equality in the statement.

Theorem 4.29. Let $G=(V, E)$ be a graph with nonemtpy edge set. Then for every $z \in \mathbb{R}_{+}^{E}$,

$$
x(G, z)=\delta^{*}(z \mid \operatorname{abl}(\mathrm{CUT}(G))) .
$$

Proof. Note that

$$
\begin{aligned}
\delta^{*}(z \mid \operatorname{abl}(\operatorname{CUT}(G))) & =\max \left\{z^{\top} x \mid x \in \operatorname{abl}(\operatorname{CUT}(G))\right\} & & \text { by (4.43) } \\
& =\max \left\{z^{\top} x \mid x \in \mathbb{R}_{+}^{E}, \forall y \in \operatorname{CUT}(G), y^{\top} x \leq 1\right\} & & \text { by (4.25) } \\
& =\max \left\{z^{\top} x \mid x \in \mathbb{R}_{+}^{E}, \forall S \subseteq V, \mathbb{1}_{\delta(S)}^{\top} x \leq 1\right\} & & \text { by (4.48) } \\
& =x(G, z) . & & \text { by (4.50) }
\end{aligned}
$$




\subsection{Generalized Cauchy-Schwarz and No-Homomorphism Lemma}

This subsection will generalize the Cauchy-Schwarz inequality for gauge dual pairs. Although the proof is quite short, we will relate this geometrical result with the No-Homomorphism Lemma, which has received some attention in the literature. Given graphs $G$ and $H$, what are the approaches for establishing that there is no homomorphism from $G$ to $H$ ? A first idea is to show that there exists a homomorphism monotone function $\beta$ such that $\beta(H)<\beta(G)$. Albertson and Collins [2] found an approach which, at first sight, seems different.

For every graph $G$ define the automorphism group $\operatorname{Aut}(G)$ of $G$ as the set of isomorphisms from $G$ to itself. A graph $G$ is vertex-transitive if for every $i, j \in V(G)$ there exists $f \in \operatorname{Aut}(G)$ such that $f(i)=j$.

Lemma 4.30 (No-Homomorphism lemma). Let $H$ be a vertex-transitive graph. If $G \rightarrow H$ then

$$
\frac{|V(G)|}{\alpha(G)} \leq \frac{|V(H)|}{\alpha(H)} .
$$

Lemma 4.30, which is proved in [2] with a counting argument over the automorphisms of $H$, has a bunch of interesting consequences. The name of the lemma comes from its contrapositive: if $H$ is a vertex-transitive graph and $|V(G)| / \alpha(G)>|V(H)| / \alpha(H)$ for some graph $G$, then $G \not \rightarrow H$. The authors of [2] use it to show that there exists an infinite sequence of graphs, all of which have chromatic number 3, which is strictly increasing in the homomorphism order. By construction, every term of the sequence has a homomorphism to the next, and the lemma is used to show that there are no consecutive terms that are homomorphically equivalent. Hahn and Tardif [52] present the sequence of odd cycles $\left(C_{2 n+3}\right)_{n \in \mathbb{N}}$ as a similar example. The sequence is homomorphically decreasing, as $C_{2 n+3} \rightarrow C_{2(n-1)+3}$ for every $n \in \mathbb{N}$ with $n>0$. Since $\alpha\left(C_{2 n+3}\right)=n+1$, Lemma 4.30 implies that

$$
C_{2 n+3} \rightarrow C_{2 m+3} \Longrightarrow \frac{2 n+3}{n+1} \leq \frac{2 m+3}{m+1} \Longrightarrow n \geq m .
$$

Hence, there are no homomorphically equivalent consecutive terms. Mančinska and Roberson [85] provide a counterexample to the quantum analog of Lemma 4.30, where one replaces $\alpha$ with $\alpha_{q}$. We are about to prove Lemma 4.30 using an argument which appears as exercise 14 of [57, Chapter 6]. As a final hint of the connection we are about to present, we point out that Hell and Nešetřil [57] use Lemma 4.30 to show that if $a, b, c, d \in \mathbb{N}$ are such that $0<b \leq 2 a$, then $\operatorname{KG}(a, b) \rightarrow \operatorname{KG}(c, d)$ implies $a / b \leq c / d$. Recalling Theorem 3.27, this argument can be used to prove that $\chi_{f}$ is homomorphism monotone.

Proposition 4.31 (Generalized Cauchy-Schwarz). Let $V$ be a finite set, and let $\beta: \mathbb{R}_{+}^{V} \rightarrow \mathbb{R}$ be a positive definite monotone gauge. Then for every $y, x \in \mathbb{R}_{+}^{V}$,

$$
y^{\top} x \leq \beta^{\circ}(y) \beta(x) .
$$

Proof. If either $y$ or $x$ is zero, the statement clearly holds. Assume otherwise, and set $\bar{y}:=\beta^{\circ}(y)^{-1} y$ and $\bar{x}:=\beta(x)^{-1} x$. Then $\bar{x} \in$ unit $(\beta)$, and Theorem 4.26 implies that $\bar{y} \in \operatorname{unit}\left(\beta^{\circ}\right)=\operatorname{abl}(\operatorname{unit}(\beta))$. Hence

$$
y^{\top} x=\left(\beta^{\circ}(y) \bar{y}\right)^{\top}(\beta(x) \bar{x})=\beta^{\circ}(y) \beta(x) \bar{y}^{\top} \bar{x} \leq \beta^{\circ}(y) \beta(x) .
$$

Let $G$ be a graph. Proposition 4.31 and (4.46) imply that

$$
|V(G)| \leq \alpha(G, \mathbb{1}) \chi_{f}(G, \mathbb{1})=\alpha(G) \chi_{f}(G) .
$$

Our next proposition shows that, whenever $G$ is vertex-transitive, equality holds.

Proposition 4.32. Let $G$ be a vertex-transitive graph. Then $|V(G)|=\alpha(G) \chi_{f}(G)$.

Proof. Equation (4.52) proves ' $\leq$ '. Consider the optimization problem

$$
\chi_{f}(G, \mathbb{1})=\max \left\{\mathbb{1}^{\top} x \mid x \in \operatorname{QSTAB}(\bar{G})\right\} .
$$


Let $g \in \operatorname{Hom}(G, G)$. Proposition 3.30 implies that $P_{g} x \in \operatorname{QSTAB}(\bar{G})$ for every $x \in \operatorname{QSTAB}(\bar{G})$. Since $P_{g}^{\top} \mathbb{1}=\mathbb{1}$ by $(2.15)$, we conclude that the matrix $P_{g}$ maps elements of $\operatorname{QSTAB}(\bar{G})$ to elements of $\operatorname{QSTAB}(\bar{G})$ with the same objective value. Thus, for every $g \in \operatorname{Aut}(G)$,

$$
P_{g} \text { is a homomorphism of optimization problems from (4.53) to (4.53). }
$$

Let $\bar{x} \in \operatorname{QSTAB}(\bar{G})$ be such that $\chi_{f}(G, \mathbb{1})=\mathbb{1}^{\top} \bar{x}$. We claim that there exists $\mu \in \mathbb{R}_{+}$such that

$$
\mu \mathbb{1}=\frac{1}{|\operatorname{Aut}(G)|} \sum_{g \in \operatorname{Aut}(G)} P_{g} \bar{x} \quad \text { and } \quad \chi_{f}(G, \mathbb{1})=\mathbb{1}^{\top}(\mu \mathbb{1}) .
$$

We remark that (4.55) is a standard use of the Reynolds operator. Let $i, j \in V(G)$, and let $f \in \operatorname{Aut}(G)$ be such that $f(i)=j$. Since $(g \in \operatorname{Aut}(G) \mapsto f \circ g)$ is the inverse of $\left(g \in \operatorname{Aut}(G) \mapsto f^{-1} \circ g\right)$, we have that

$$
g \mapsto f^{-1} \circ g \text { is a bijection on } \operatorname{Aut}(G) .
$$

Then

$$
\begin{array}{rlrl}
e_{i}^{\top}\left(\frac{1}{|\operatorname{Aut}(G)|} \sum_{g \in \operatorname{Aut}(G)} P_{g} \bar{x}\right) & =\frac{1}{|\operatorname{Aut}(G)|} \sum_{g \in \operatorname{Aut}(G)} e_{i}^{\top} P_{g} \bar{x} & \\
& =\frac{1}{|\operatorname{Aut}(G)|} \sum_{g \in \operatorname{Aut}(G)}\left(P_{f} e_{j}\right)^{\top} P_{g} \bar{x} & & \text { since } f(j)=i \text { and by }(2.12) \\
& =\frac{1}{|\operatorname{Aut}(G)|} \sum_{g \in \operatorname{Aut}(G)} e_{j}^{\top} P_{f-1} P_{g} \bar{x} & & \text { by }(2.14) \\
& =\frac{1}{|\operatorname{Aut}(G)|} \sum_{g \in \operatorname{Aut}(G)} e_{j}^{\top} P_{f^{-1} \circ g} \bar{x} & & \text { by Proposition } 2.1 \\
& =\frac{1}{|\operatorname{Aut}(G)|} \sum_{h \in \operatorname{Aut}(G)} e_{j}^{\top} P_{h} \bar{x} & & \text { by }(4.56) \\
& =e_{j}^{\top}\left(\frac{1}{|\operatorname{Aut}(G)|} \sum_{g \in \operatorname{Aut}(G)} P_{g} \bar{x}\right) &
\end{array}
$$

As $i, j \in V(G)$ were arbitrary, there exists $\mu \in \mathbb{R}$ such that $\frac{1}{|\operatorname{Aut}(G)|} \sum_{g \in \operatorname{Aut}(G)} P_{g} \bar{x}=\mu \mathbb{1}$. Using (4.54) we see that $\mu \mathbb{1}$ is a convex combination of optimal solutions to (4.53), which implies (4.55).

Combining (4.54) and (4.55) we conclude that $\mu \mathbb{1} \in \operatorname{QSTAB}(\bar{G})$, so Proposition 4.27 implies that $\mu \mathbb{1} \in \operatorname{abl}(\operatorname{STAB}(G))$. Therefore $\mu \leq \alpha(G)^{-1}$, as for every $S \in \mathcal{S}(G)$ we have that $\mu|S|=(\mu \mathbb{1})^{\top} \mathbb{1}_{S} \leq 1$. The proof is finished:

$$
\chi_{f}(G, \mathbb{1})=\mathbb{1}^{\top}(\mu \mathbb{1}) \leq \frac{|V(G)|}{\alpha(G)} .
$$

A couple of remarks are in place. Proposition 4.32 can be generalized to other functions defined for every pair $(G, w)$ where $G$ is a graph and $w \in \mathbb{R}_{+}^{V(G)}$. However, to be able to exploit the automorphisms of a graph, we need such function to be a weighted graph parameter, i.e., a function $\beta$ such that if $G$ and $H$ are graphs and $f \in \operatorname{Hom}(G, H)$ is an isomorphism, we have that $\beta(G, w)=\beta\left(H, P_{f} w\right)$ for every $w \in \mathbb{R}_{+}^{V(G)}$. See [13] for more details.

Let $G$ and $H$ be graphs, with $H$ vertex-transitive. If $G \rightarrow H$, then

$$
\begin{aligned}
\frac{|V(G)|}{\alpha(G)} & \leq \chi_{f}(G) & & \text { by }(4.52) \\
& \leq \chi_{f}(H) & & \text { by }(3.85) \\
& =\frac{|V(H)|}{\alpha(H)} . & & \text { by Proposition } 4.32
\end{aligned}
$$


Hence, Lemma 4.30 holds. Conversely, if $|V(G)| / \alpha(G)>|V(H)| / \alpha(H)$, then

$$
\begin{aligned}
\chi_{f}(G) & \geq \frac{|V(G)|}{\alpha(G)} \\
& >\frac{|V(H)|}{\alpha(H)} \\
& =\chi_{f}(H) .
\end{aligned}
$$

by Proposition 4.32

As $\chi_{f}$ is homomorphism monotone, $G \not \rightarrow H$ follows. Therefore, we may prove each result mentioned in the beginning of the subsection using that $\chi_{f}$ is homomorphism monotone and Proposition 4.32 - with no mention to Lemma 4.30. Furthermore, we have a more complete criterion, since the condition $\chi_{f}(G) \leq \chi_{f}(H)$ is necessary for $G \rightarrow H$ whether $H$ is vertex-transitive or not. If $H$ is vertex-transitive, Proposition 4.32 computes $\chi_{f}(H)$ from $\alpha(H)$ and recovers the ratio which appears in Lemma 4.30.

\subsection{Geometric Conversion of Bounds}

In this subsection, we lay out the core idea in [13], which is a paper I coauthored with Marcel K. de Carli Silva and Gabriel Coutinho. The starting observation is that, if one can upper bound $\alpha(G, \cdot)$ by a gauge, its gauge dual is a lower bound on $\chi_{f}(G, \cdot)$, and vice versa. We used this procedure to obtain a new graph parameter which upper bounds the stability number, and to explain the relationship among some well-known bounds introduced by Hoffman [58].

Proposition 4.33. Let $V$ be a finite set, and let $\beta, \eta: \mathbb{R}_{+}^{V} \rightarrow \mathbb{R}$ be positive definite monotone gauges. The following are equivalent:

1. for every $w \in \mathbb{R}_{+}^{V}$, it holds that $\eta(w) \leq \beta(w)$,

2. for every $z \in \mathbb{R}_{+}^{V}$, it holds that $\beta^{\circ}(z) \leq \eta^{\circ}(z)$.

Proof. We claim that for every positive definite monotone gauges $\beta, \eta: \mathbb{R}_{+}^{V} \rightarrow \mathbb{R}$,

$$
\eta(w) \leq \beta(w) \text { for every } w \in \mathbb{R}_{+}^{V} \text { if and only if } \operatorname{unit}(\beta) \subseteq \operatorname{unit}(\eta) .
$$

Indeed, if $\eta(w) \leq \beta(w)$ for every $w \in \mathbb{R}_{+}^{V}$, we have that

$$
\operatorname{unit}(\beta)=\left\{w \in \mathbb{R}_{+}^{V} \mid \beta(w) \leq 1\right\} \subseteq\left\{w \in \mathbb{R}_{+}^{V} \mid \eta(w) \leq 1\right\}=\operatorname{unit}(\eta) .
$$

Conversely, if unit $(\beta) \subseteq$ unit $(\eta)$, we have that

$$
\begin{aligned}
\beta(w) & =\gamma(w \mid \operatorname{unit}(\beta)) & & \text { by Proposition } 4.10 \\
& =\inf \left\{\mu \in \mathbb{R}_{++} \mid w \in \mu \operatorname{unit}(\beta)\right\} & & \text { by }(4.11) \\
& \geq \inf \left\{\mu \in \mathbb{R}_{++} \mid w \in \mu \operatorname{unit}(\eta)\right\} & & \text { since unit }(\beta) \subseteq \operatorname{unit}(\eta) \\
& =\gamma(w \mid \operatorname{unit}(\eta)) & & \text { by }(4.11) \\
& =\eta(w) . & & \text { by Proposition } 4.10
\end{aligned}
$$

Thus (4.57) holds.

Furthermore,

$$
\operatorname{unit}(\beta) \subseteq \operatorname{unit}(\eta) \text { if and only if } \operatorname{unit}\left(\eta^{\circ}\right) \subseteq \operatorname{unit}\left(\beta^{\circ}\right),
$$

as

$$
\begin{aligned}
\operatorname{unit}(\beta) \subseteq \operatorname{unit}(\eta) & \Longrightarrow \operatorname{abl}(\operatorname{unit}(\eta)) \subseteq \operatorname{abl}(\operatorname{unit}(\beta)) \\
& \Longrightarrow \operatorname{unit}\left(\eta^{\circ} \subseteq \subseteq \operatorname{unit}\left(\beta^{\circ}\right)\right. \\
& \Longrightarrow \operatorname{abl}\left(\operatorname{unit}\left(\beta^{\circ}\right) \subseteq \operatorname{abl}\left(\operatorname{unit}\left(\beta^{\circ}\right)\right)\right. \\
& \Longrightarrow \operatorname{unit}\left(\beta^{\circ \circ} \subseteq \subseteq \operatorname{unit}\left(\beta^{\circ \circ}\right)\right. \\
& \Longrightarrow \operatorname{unit}(\beta) \subseteq \operatorname{unit}(\beta) .
\end{aligned}
$$

by item 2 of Theorem 4.26

by $(4.26)$

by item 2 of Theorem 4.26

by item 3 of Theorem 4.26

The statement follows from combining (4.57) and (4.58). 
Corollary 4.34. Let $G$ be a graph, and $\beta: \mathbb{R}_{+}^{V(G)} \rightarrow \mathbb{R}$ be a positive definite monotone gauge. The following are equivalent:

1. for every $w \in \mathbb{R}_{+}^{V}$, it holds that $\alpha(G, w) \leq \beta(w)$;

2. for every $z \in \mathbb{R}_{+}^{V}$, it holds that $\beta^{\circ}(z) \leq \chi_{f}(G, z)$.

Proof. Set $\eta(w):=\alpha(G, w)$, so $\eta^{\circ}(z)=\chi_{f}(G, z)$ by (4.46). Apply Proposition 4.33.

We start with a trivial example. Let $G$ be a graph. For every $w \in \mathbb{R}_{+}^{V(G)}$ we have that $\alpha(G, w) \leq\|w\|_{1}$. Note that $\|\cdot\|_{1}$ and $\|\cdot\|_{\infty}$, when restricted to the nonnegative orthant, are real-valued positive definite monotone gauges which are gauge dual to each other. Corollary 4.34 implies that $\|z\|_{\infty} \leq \chi_{f}(G, z)$ for every $z \in \mathbb{R}_{+}^{V(G)}$.

To introduce a more interesting example, we will need some notation. Let $V$ be a finite set. If $A \in \mathbb{S}_{+}^{V}$, we denote by $A^{1 / 2}$ the unique positive semidefinite square root of $A$. For every $A \in \mathbb{S}^{V} \operatorname{such}$ that $\operatorname{diag}(A)=0$, define $\tilde{A} \in \mathbb{S}^{V}$ as

$$
\tilde{A}:=\left\{\begin{array}{lc}
0 & \text { if } A=0, \\
-\lambda_{\min }(A)^{-1} A & \text { otherwise. }
\end{array}\right.
$$

Note that $\operatorname{diag}(A)=0$ implies that the sum of eigenvalues of $A$ is zero. Consequently, if $A \neq 0$, then $\lambda_{\min }(A)<0$, so that the expression above is well defined. Moreover, note that we always have that $I+\tilde{A} \succeq 0$, and that when $A \neq 0$, the nullspace of $I+\tilde{A}$ is precisely the eigenspace of $A$ associated with $\lambda_{\min }(A)$.

Let $V$ be a finite set, and define $H(A, w)$ for every $A \in \mathbb{S}^{V}$ such that $\operatorname{diag}(A)=0$ and $w \in \mathbb{R}_{+}^{V}$ as

$$
H(A, w):=\lambda_{\max }\left(\operatorname{Diag}(w)^{1 / 2}(I+\tilde{A}) \operatorname{Diag}(w)^{1 / 2}\right) .
$$

The fact that $\lambda_{\max }(B C)=\lambda_{\max }(C B)$ for every $B, C \in \mathbb{S}^{V}$ (see [60, Theorem 1.3.20]) implies that

$$
H(A, w)=\lambda_{\max }\left((I+\tilde{A})^{1 / 2} \operatorname{Diag}(w)(I+\tilde{A})^{1 / 2}\right) .
$$

Proposition 4.35. Let $V$ be a finite set and let $A \in \mathbb{S}^{V}$ be such that $\operatorname{diag}(A)=0$. Then $H(A, \cdot)$ is a real-valued positive definite monotone gauge.

Proof. Using (4.60), we have that $H(A, \cdot)$ is a positive definite function:

$$
H(A, w) \geq \max \left\{e_{i}^{\top} \operatorname{Diag}(w)^{1 / 2}(I+\tilde{A}) \operatorname{Diag}(w)^{1 / 2} e_{i} \mid i \in V\right\}=\|w\|_{\infty} .
$$

Define $L: \mathbb{R}_{+}^{V} \rightarrow \mathbb{S}_{+}^{V}$ as $L(w):=(I+\tilde{A})^{1 / 2} \operatorname{Diag}(w)(I+\tilde{A})^{1 / 2}$. Then $(4.61)$ states that $H(A, \cdot)=\lambda_{\max } \circ L$. Using this, (4.9), and linearity of $L$, one proves that $H(A, \cdot)$ is a real-valued positive definite gauge. To see that it is monotone, let $w, z \in \mathbb{R}_{+}^{V}$ be such that $w \leq z$. Then $\operatorname{Diag}(w) \preceq \operatorname{Diag}(z)$, which implies that

$$
H(A, w)=\lambda_{\max }\left((I+\tilde{A})^{1 / 2} \operatorname{Diag}(w)(I+\tilde{A})^{1 / 2}\right) \leq \lambda_{\max }\left((I+\tilde{A})^{1 / 2} \operatorname{Diag}(z)(I+\tilde{A})^{1 / 2}\right)=H(A, z) .
$$

Let $G$ be a graph. The set of generalized adjacency matrices $\mathcal{A}_{G}$ of $G$ is defined as

$$
\mathcal{A}_{G}:=\left\{A \in \mathbb{S}^{V(G)} \mid \operatorname{diag}(A)=0, \forall i j \in E(\bar{G}), A_{i j}=0\right\} .
$$

The adjacency matrix $A_{G}$ of $G$ is the matrix $A_{G} \in \mathbb{S}^{V(G)}$ such that $\left(A_{G}\right)_{i j}=[i j \in E(G)]$. Note that $A_{G} \in \mathcal{A}_{G}$.

Proposition 4.36. Let $G$ be a graph and let $A \in \mathcal{A}_{G}$. Then $\operatorname{STAB}(G) \subseteq \operatorname{unit}(H(A, \cdot))$.

Proof. Let $S \in \mathcal{S}(G)$. Note that $A \in \mathcal{A}_{G}$ and $S \in \mathcal{S}(G)$ imply that

$$
\operatorname{Diag}\left(\mathbb{1}_{S}\right)^{1 / 2}(I+\tilde{A}) \operatorname{Diag}\left(\mathbb{1}_{S}\right)^{1 / 2}=\operatorname{Diag}\left(\mathbb{1}_{S}\right)(I+\tilde{A}) \operatorname{Diag}\left(\mathbb{1}_{S}\right)=\operatorname{Diag}\left(\mathbb{1}_{S}\right) \preceq I .
$$

Therefore, $H\left(A, \mathbb{1}_{S}\right) \leq 1$ by (4.60). Thus

$\left\{\mathbb{1}_{S} \mid S \in \mathcal{S}(G)\right\} \subseteq \operatorname{unit}(H(A, \cdot))$

$\Longrightarrow \operatorname{conv}\left(\left\{\mathbb{1}_{S} \mid S \in \mathcal{S}(G)\right\}\right) \subseteq \operatorname{unit}(H(A, \cdot)) \quad$ since unit $(H)$ is convex by Propositions 4.35 and 4.10

$\Longrightarrow \operatorname{STAB}(G) \subseteq \operatorname{unit}(H(A, \cdot))$. by $(1.9)$ 
Let $V$ be a finite set, and define $\Upsilon(A, z)$ for every $A \in \mathbb{S}^{V}$ such that $\operatorname{diag}(A)=0$ and $z \in \mathbb{R}_{+}^{V}$ as

$$
\Upsilon(A, z):=\max \left\{z^{\top} x \mid x \in \mathbb{R}_{+}^{V},(I+\tilde{A})^{1 / 2} \operatorname{Diag}(x)(I+\tilde{A})^{1 / 2} \preceq I\right\} .
$$

Note that (4.61) implies that $\Upsilon(A, z)=\max \left\{z^{\top} w \mid w \in \operatorname{unit}(H(A, \cdot))\right\}$. Hence Proposition 4.35, (4.42), and Theorem 4.26 imply that, for every $A \in \mathbb{S}^{V}$ with $\operatorname{diag}(A)=0$,

$\Upsilon(A, \cdot)$ and $H(A, \cdot)$ are real-valued positive definite monotone gauges, dual to each other.

Proposition 4.36, (4.63), and Corollary 4.34 imply that for every graph $G$ and $A \in \mathcal{A}_{G}$,

$$
\begin{aligned}
\alpha(G, \cdot) & \leq \Upsilon(A, \cdot), \\
H(A, \cdot) & \leq \chi_{f}(G, \cdot) .
\end{aligned}
$$

Inequality (4.65) is folklore. It is a consequence of a couple of known results we are yet to cover, namely (4.68) and Proposition 5.8. If $G$ is a regular graph, one can compute $\Upsilon\left(A_{G}, \mathbb{1}\right)$ using SDP Weak Duality (see [13]) and conclude that

$$
\Upsilon\left(A_{G}, \mathbb{1}\right)=\frac{|V(G)|}{1-\lambda_{\max }\left(A_{G}\right) / \lambda_{\min }\left(A_{G}\right)} .
$$

Using (4.64), we have that

$$
\alpha(G)=\alpha(G, \mathbb{1}) \leq \Upsilon\left(A_{G}, \mathbb{1}\right)=\frac{|V(G)|}{1-\lambda_{\max }\left(A_{G}\right) / \lambda_{\min }\left(A_{G}\right)}
$$

for every regular graph $G$. Using (4.65), we have that

$$
1-\frac{\lambda_{\max }\left(A_{G}\right)}{\lambda_{\min }\left(A_{G}\right)}=H\left(A_{G}, \mathbb{1}\right) \leq \chi_{f}(G, \mathbb{1}) \leq \chi(G)
$$

for every graph with at least one edge. Hoffman [58] proved (4.66) and (4.67), and both bounds are commonlypresented applications of spectral techniques to graph theory: [44, Lemma 9.6.2], [15, Theorem 8.8]. Newman states that (4.66) is the central tool of his Ph.D. thesis [95], which provides further discussion and applications of this particular bound.

It is easy to see that the bounds (4.66) and (4.67) are somehow related without mentioning gauge duality. Yet, the required hypotheses hint that there is something missing: why is regularity only assumed in (4.66), and not in (4.67)? Inequalities (4.64) and (4.65) explain the relation between the bounds in a symmetric way, and show that the specific assumptions mentioned serve merely to compute $\Upsilon\left(A_{G}, \mathbb{1}\right)$ and $H\left(A_{G}, \mathbb{1}\right)$ for the graph $G$ under scrutiny.

\subsection{Luz's Upper Bound on the Stability Number and the Lovász Theta Function}

This brief subsection presents results which were known before [13]. Such results further contextualize Section 4.3, and most importantly, introduce two weighted graph parameters which will be studied in Section 4.5 .

Let $G$ be a graph. It is possible to use [67, Theorem 33] to prove that for every $w \in \mathbb{R}_{+}^{V(G)}$,

$$
\vartheta(\bar{G}, w)=\max \left\{H(A, w) \mid A \in \mathcal{A}_{G}\right\} .
$$

This is a weighted generalization of $\vartheta(\bar{G})=\max \left\{H(A, \mathbb{1}) \mid A \in \mathcal{A}_{G}\right\}$, which already appeared in [77]. Proposition 4.35 and $(4.68)$ imply that $\vartheta(\bar{G}, \cdot)$ is a positive definite monotone gauge, as $\vartheta(\bar{G}, \cdot)$ is a pointwise supremum of positive definite monotone gauges. By combining (4.65) and (4.68), we get the well-known result that

$$
\vartheta(\bar{G}, \cdot) \leq \chi_{f}(G, \cdot) .
$$


Since $\chi_{f}(G, \cdot)$ is real-valued, we conclude that $\vartheta(\bar{G}, \cdot)$ is a real-valued positive definite monotone gauge. This implies that $\vartheta(G, \cdot)$ is a real-valued positive definite monotone gauge for every graph $G$.

Define $\operatorname{TH}(G):=\operatorname{abl}(\operatorname{unit}(\vartheta(G, \cdot)))$ for every graph $G$. Then for every $w \in \mathbb{R}_{+}^{V(G)}$,

$$
\begin{aligned}
\vartheta(G, w) & =\gamma(w \mid \operatorname{unit}(\vartheta(G, \cdot))) & & \text { by Proposition 4.10 } \\
& =\gamma(w \mid \operatorname{abl}(\operatorname{abl}(\operatorname{unit}(\vartheta(G, \cdot))))) & & \text { by item } 3 \text { of Theorem } 4.25 \\
& =\delta^{*}(w \mid \operatorname{abl}(\operatorname{unit}(\vartheta(G, \cdot)))) & & \text { by item } 2 \text { of Theorem } 4.26 \\
& =\max \left\{w^{\top} x \mid x \in \operatorname{abl}(\operatorname{unit}(\vartheta(G, \cdot)))\right\} & & \text { by }(4.43) \\
& =\max \left\{w^{\top} x \mid x \in \operatorname{TH}(G)\right\} . & &
\end{aligned}
$$

Grötschel, Lovász, and Schrijver [49] already noted that $\vartheta(G, \cdot)$ is a real-valued positive definite monotone gauge, and proved that

$$
\operatorname{abl}(\operatorname{TH}(G))=\operatorname{TH}(\bar{G}) .
$$

Theorem 4.25 implies that $\operatorname{TH}(\bar{G})=\operatorname{abl}(\operatorname{TH}(G))=\operatorname{abl}(\operatorname{abl}(\operatorname{unit}(\vartheta(G, \cdot))))=\operatorname{unit}(\vartheta(G, \cdot))$. Hence

$$
\vartheta(\bar{G}, w)=\max \left\{w^{\top} x \mid x \in \mathrm{TH}(\bar{G})\right\}=\max \left\{w^{\top} x \mid x \in \operatorname{unit}(\vartheta(G, \cdot))\right\} .
$$

By using that $\vartheta(G, \cdot)$ is a gauge and $(4.42)$, we conclude that

$\vartheta(G, \cdot)$ and $\vartheta(\bar{G}, \cdot)$ are real-valued positive definite monotone gauges, dual to each other.

Corollary 4.34, (4.69), and (4.71) imply that

$$
\alpha(G, \cdot) \leq \vartheta(G, \cdot)
$$

which is another well-known result.

Luz [82] introduced another graph parameter, which was later generalized to a weighted version by Luz and Cardoso [83]. For convenience, we will use the weighted generalization from [28]. Let $V$ be a finite set. Define

$$
v(A, w):=\sup \left\{2 w^{\top} x-x^{\top} \operatorname{Diag}(w)^{1 / 2}(I+\tilde{A}) \operatorname{Diag}(w)^{1 / 2} x \mid x \in \mathbb{R}_{+}^{V}\right\}
$$

for every $A \in \mathbb{S}^{V}$ with $\operatorname{diag}(A)=0$, and for every $w \in \mathbb{R}_{+}^{V}$. Refining the work of Luz and Schrijver [80], Luz [81] proved that for every graph $G$ and $w \in \mathbb{R}_{+}^{V(G)}$,

$$
\vartheta(G, w)=\min \left\{v(A, w) \mid A \in \mathcal{A}_{G}\right\} .
$$

By combining (4.72) and (4.74), we get that $\alpha(G, \cdot) \leq v(A, \cdot)$ for every graph $G$ and $A \in \mathcal{A}_{G}$. Luz [82] also proved that whenever $G$ is a regular graph,

$$
v\left(A_{G}, \mathbb{1}\right)=\frac{|V(G)|}{1-\lambda_{\max }\left(A_{G}\right) / \lambda_{\min }\left(A_{G}\right)},
$$

which implies that $v$ also provides the upper bound on $\alpha$ described in (4.66).

\subsection{Relation among $v, \Upsilon$, and $\vartheta$}

We invite the reader to look back to Sections 4.3 and 4.4, focusing on the graph parameters presented. For every graph $G$ and $A \in \mathcal{A}_{G}$, we have that both $v(A, \cdot)$ and $\Upsilon(A, \cdot)$ upper bound $\alpha(G, \cdot)$. Furthermore, $v\left(A_{G}, \mathbb{1}\right)=\Upsilon\left(A_{G}, \mathbb{1}\right)$ whenever $G$ is regular. The matrix in (4.60) appears in a quadratic form in (4.73). Moreover, compare (4.68) to (4.74). Taking these facts as motivation, this subsection presents one of my main contributions to [13], which was to show that $v(A, \cdot)=\Upsilon(A, \cdot)$ whenever all the entries of $A$ are nonnegative.

We first reformulate $v$. Let $\operatorname{supp}(x):=\left\{i \in V \mid x_{i} \neq 0\right\}$ denote the support of $x \in \mathbb{R}^{V}$, and define the orthogonal projector $P_{\operatorname{supp}(x)}:=\sum_{i \in \operatorname{supp}(x)} e_{i} e_{i}^{\top} \in \mathbb{S}^{V}$. We will use that

$$
v(A, w)=\sup \left\{2 \sqrt{w}^{\top} x-x^{\top}(I+\tilde{A}) x \mid x \in \mathbb{R}_{+}^{V}, P_{\operatorname{supp}(w)} x=x\right\},
$$


where $\sqrt{w}$ denotes the componentwise square root of the vector $w$. Define $w^{\dagger} \in \mathbb{R}_{+}^{V}$ such that $w_{i}^{\dagger}=\left[w_{i} \neq 0\right] w_{i}^{-1}$ for every $i \in V$. The changes of variable described by $x \mapsto \operatorname{Diag}(w)^{1 / 2} x$ and $x \mapsto \operatorname{Diag}\left(w^{\dagger}\right)^{1 / 2} x$ establish the equivalence between (4.73) and (4.75).

Let $V$ be a finite set, and $A \in \mathbb{S}^{V}$ be such that $\operatorname{diag}(A)=0$. We first show that $v(A, \cdot)$ may not be real-valued, which already hinders the attempt to prove that $v(A, \cdot)$ is a real-valued positive definite monotone gauge. Let $G$ be a $k$-regular graph, and set $A:=-A_{G} \in \mathcal{A}_{G}$. Then $\lambda_{\min }(A)=-\lambda_{\max }\left(A_{G}\right)=-k$, which implies that $\mathbb{1}$ is an eigenvector associated with the smallest eigenvalue of $A$. Hence $(I+\tilde{A}) \mathbb{1}=0$. Therefore (4.75) implies that for every $w>0$,

$$
v(A, w)=\sup \left\{2 \sqrt{w}^{\top} x-x^{\top}(I+\tilde{A}) x \mid x \in \mathbb{R}_{+}^{V}\right\} \geq \sup \left\{2 \rho \sqrt{w}^{\top} \mathbb{1} \mid \rho \geq 0\right\}=+\infty .
$$

To relate $v$ and $\Upsilon$, we will look at their Lagrangean duals. Let $V$ be a finite set, let $A \in \mathbb{S}^{V}$ be such that $\operatorname{diag}(A)=0$, and let $w \in \mathbb{R}_{+}^{V}$. Since $I+\tilde{A} \succeq 0$, the problem in (4.73) is convex. We have that

$$
\text { if } v(A, w)<+\infty \text {, then } v(A, w)=\min \left\{\|y\|_{2}^{2} \mid \sqrt{w} \leq P_{\operatorname{supp}(w)}(I+\tilde{A})^{1 / 2} y\right\} .
$$

This holds because the optimization problem in (4.76) is an equivalent formulation of the Lagrangean dual of (4.75), so that Strong Duality (see, for example, [12]) and feasibility of (4.75) imply (4.76). Regarding $\Upsilon$, we have that

$$
\Upsilon(A, w)=\min \left\{\operatorname{Tr}(Y) \mid w \leq \operatorname{diag}\left((I+\tilde{A})^{1 / 2} Y(I+\tilde{A})^{1 / 2}\right), Y \succeq 0\right\} .
$$

Here we have used Theorem 2.11, that (4.77) is the SDP dual of (4.62), that (4.62) has a strictly feasible solution, and that $\Upsilon(A, w)$ is real-valued. If $y$ is feasible in (4.76), then $y y^{\top}$ is feasible in (4.77), and has the same objective value. Therefore,

$$
\Upsilon(A, w) \leq v(A, w) .
$$

Hence $\Upsilon(A, w)$ may be seen as a SDP strengthening of $v(A, w)$, which justifies our notation: $\Upsilon$ is the upper case Greek letter for $v$. The inequality in (4.78) may be strict, since $v(A, w)$ may be $+\infty$, and $\Upsilon(A, \cdot)$ is real valued. We now look for conditions on $A$ which guarantee equality. A matrix is nonnegative whenever all of its entries are nonnegative.

Proposition 4.37. Let $V$ be a finite set, and let $A \in \mathbb{S}^{V}$ be a nonnegative matrix such that $\operatorname{diag}(A)=0$. Then $v(A, \cdot)$ is a real-valued positive definite monotone gauge.

Proof. Note that $v(A, \cdot)$ is positive definite by (4.78), and positively homogeneous by (4.73). We will make use of the following observation: For every $x \in \mathbb{R}^{V}$,

$$
\text { if } y \in \mathbb{R}_{+}^{V} \text {, then } P_{\operatorname{supp}(x)} y \in \mathbb{R}_{+}^{V} \text {. }
$$

Let $w, z \in \mathbb{R}_{+}^{V}$ be such that $w \leq \underset{\sim}{ }$. Then $\operatorname{supp}(w) \subseteq \operatorname{supp}(z)$, which implies that $P_{\operatorname{supp}(w)}=P_{\operatorname{supp}(w)} P_{\operatorname{supp}(z)}$. Let $y$ be such that $P_{\operatorname{supp}(z)}(I+\tilde{A}) y \geq \sqrt{z}$. Using (4.79) twice,

$$
P_{\operatorname{supp}(w)}(I+\tilde{A}) y=P_{\operatorname{supp}(w)} P_{\operatorname{supp}(z)}(I+\tilde{A}) y \geq P_{\operatorname{supp}(w)} \sqrt{z} \geq P_{\operatorname{supp}(w)} \sqrt{w}=\sqrt{w} .
$$

Hence, if $y$ is feasible in (4.76) for $z$, then $y$ is also feasible in (4.76) for $w$. Therefore $v(A, \cdot)$ is monotone. We now prove that

$$
w \mapsto \sqrt{w}^{\top} X \sqrt{w} \text { is concave for every nonnegative } X \in \mathbb{S}^{V},
$$

as a function with domain $\mathbb{R}_{+}^{V}$. Let $X \in \mathbb{S}^{V}$ be nonnegative. The function in (4.80) is clearly positively homogeneous and nonnegative. It suffices to prove that it is superlinear. Let $w, z \in \mathbb{R}_{+}^{V}$ and $i, j \in V$. The inequality of arithmetic and geometric means implies that

$$
\left(w_{i}+z_{i}\right)\left(w_{j}+z_{j}\right)=w_{i} w_{j}+z_{i} z_{j}+w_{i} z_{j}+w_{j} z_{i} \geq w_{i} w_{j}+z_{i} z_{j}+2 \sqrt{w_{i} z_{j} w_{j} z_{i}}=\left(\sqrt{w_{i} w_{j}}+\sqrt{z_{i} z_{j}}\right)^{2} .
$$

Hence $\sqrt{w_{i}+z_{i}} \sqrt{w_{j}+z_{j}} \geq \sqrt{w_{i} w_{j}}+\sqrt{z_{i} z_{j}}$. Multiplying both sides by $X_{i j} \geq 0$, and summing over every $i, j \in V$, we have that

$$
\sqrt{w+z}^{\top} X \sqrt{w+z} \geq \sqrt{w}^{\top} X \sqrt{w}+\sqrt{z}^{\top} X \sqrt{z},
$$


which proves (4.80). Using (4.73) and the fact that $\operatorname{Diag}(x) y=\operatorname{Diag}(y) x$ for every $x, y \in \mathbb{R}^{V}$,

$$
v(A, w)=\sup \left\{2 w^{\top} x-\sqrt{w}^{\top} \operatorname{Diag}(x)(I+\tilde{A}) \operatorname{Diag}(x) \sqrt{w} \mid x \in \mathbb{R}_{+}^{V}, P_{\operatorname{supp}(w)} x=x\right\} .
$$

By (4.80), we have that $v(A, \cdot)$ is the pointwise supremum of convex functions, and, as such, convex. Since $A$ is nonnegative, for every $x \in \mathbb{R}_{+}^{V}$ we have that $\|x\|^{2}=x^{\top} x \leq x^{\top}(I+\tilde{A}) x$. Hence

$$
2 \sqrt{w}^{\top} x-x^{\top}(I+\tilde{A}) x \leq 2\|\sqrt{w}\|\|x\|-\|x\|^{2} \leq\|\sqrt{w}\|^{2}=\|w\|_{1}
$$

for every $w \in \mathbb{R}_{+}^{V}$. Consequently, $v(A, \cdot)$ is real-valued, since $v(A, \cdot) \leq\|\cdot\|_{1}$.

Theorem 4.38. Let $V$ be a finite set, and $A \in \mathbb{S}^{V}$ be nonnegative and such that $\operatorname{diag}(A)=0$. Then $\Upsilon(A, \cdot)=v(A, \cdot)$.

We refer the reader to [13], as we will not prove Theorem 4.38, but simply outline its arguments. Let $V$ be a finite set, and let $A \in \mathbb{S}^{V}$ be nonnegative and such that $\operatorname{diag}(A)=0$. Proposition 4.37 reduces Theorem 4.38 to proving that $v(A, \cdot)$ and $H(A, \cdot)$ are gauge dual to each other. Proposition 4.33, (4.63), and (4.78) imply that $v^{\circ}(A, \cdot) \leq H(A, \cdot)$. To prove that $v^{\circ}(A, \cdot) \geq H(A, \cdot)$, we show that for every $z \in \mathbb{R}_{+}^{V}$ there exists $\bar{w} \in \operatorname{unit}(v(A, \cdot))$ such that $z^{\top} \bar{w}=H(A, z)$. We again crucially rely on $A$ being nonnegative when we define $\bar{w}$ using the Perron-Frobenius Theorem [60, Theorem 8.3.1].

Theorem 4.39. Let $G$ be a graph. For every $w \in \mathbb{R}_{+}^{V(G)}$,

$$
\vartheta(G, w)=\min \left\{\Upsilon(A, w) \mid A \in \mathcal{A}_{G}\right\} .
$$

Proof. Let $A \in \mathcal{A}_{G}$. Proposition 4.33, (4.63), (4.68), and (4.71) imply that $\vartheta(G, \cdot) \leq \Upsilon(A, \cdot)$. Therefore $\vartheta(G, \cdot) \leq \inf \left\{\Upsilon(A, \cdot) \mid A \in \mathcal{A}_{G}\right\}$, and both (4.74) and (4.78) finish the proof.

Let $G$ be a graph. In [13] we prove that

$$
\vartheta^{+}(G, w)=\min \left\{\Upsilon(A, w) \mid A \in \mathcal{A}_{G}, A \geq 0\right\}
$$

using that

$$
\vartheta^{\prime}(\bar{G}, w)=\max \left\{H(A, w) \mid A \in \mathcal{A}_{G}, A \geq 0\right\},
$$

which is a well-known fact that can be deduced from [67, Theorem 33]. These definitions recover the weighted versions of the parameters defined in Subsection 3.4.3: for every graph $G$ we have that $\chi_{\text {vect }}(G)=\vartheta^{\prime}(\bar{G}, \mathbb{1})$, that $\chi_{\text {Strict }}(G)=\vartheta(\bar{G}, \mathbb{1})$, and that $\chi_{\text {Rigid }}(G)=\vartheta^{+}(\bar{G}, \mathbb{1})$. One can use $(4.82)$ to prove that $\bar{\vartheta}^{\prime}$ is a real-valued positive definite monotone gauge. Define $\mathrm{TH}^{\prime}(G):=\operatorname{abl}\left(\operatorname{unit}\left(\vartheta^{\prime}(G, \cdot)\right)\right)$ and $\mathrm{TH}^{+}(G):=\operatorname{abl}\left(\operatorname{unit}\left(\vartheta^{+}(G, \cdot)\right)\right)$. Similar to (4.70), we have that

$$
\operatorname{abl}\left(\mathrm{TH}^{\prime}(G)\right)=\mathrm{TH}^{+}(\bar{G}),
$$

which can be used to prove that

$$
\vartheta^{\prime}(G, \cdot) \text { and } \vartheta^{+}(\bar{G}, \cdot) \text { are real-valued positive definite monotone gauges, dual to each other. }
$$

\subsection{Unified Chain of Inequalities}

We now conclude the proof of the inequalities mentioned in Figure 3.1, back in Subsection 3.4.6.

For every $k$-regular graph $G$, one can use (4.66) to reduce the computation of $\Upsilon\left(A_{G}, \mathbb{1}\right)$ to the computation of $\lambda_{\min }\left(A_{G}\right)$, as $\lambda_{\max }\left(A_{G}\right)=k$. For some graphs, these values are known: if $a, b \in \mathbb{N}$ are such that $0<2 b \leq a$, then

$$
\Upsilon\left(A_{C_{a}}, \mathbb{1}\right)=\frac{a \cos (\pi / a)}{1+\cos (\pi / a)}, \quad \Upsilon\left(A_{\mathrm{KG}(a, b)}, \mathbb{1}\right)=\left(\begin{array}{c}
a-1 \\
b-1
\end{array}\right) .
$$

Lovász [77] used (4.85) to compute $\vartheta$ for cycles and Kneser graphs. The value of $\Upsilon\left(A_{\mathrm{KG}(a, b)}, \mathbb{1}\right)$ is particularly special as it implies the Erdős-Ko-Rado Theorem (see, e.g., [45]).

Proposition 4.40. The parameter $\omega_{c}$ is incomparable with each of $\overline{\vartheta^{\prime}}, \bar{\vartheta}$, and $\overline{\vartheta^{+}}$. 
Proof. Since $\overline{\vartheta^{\prime}} \leq \bar{\vartheta} \leq \overline{\vartheta^{+}}$, it is enough to argue that there exist graphs $G$ and $H$ such that

$$
\begin{aligned}
& \overline{\vartheta^{+}}(G)<\omega_{c}(G), \text { and } \\
& \omega_{c}(H)<\overline{\vartheta^{\prime}}(H) .
\end{aligned}
$$

Let $a \in \mathbb{N}$ be at least 4 , and define $G:=\operatorname{Circ}(a, 2)$. Note that $\bar{G}=\overline{\operatorname{Circ}(a, 2)}=C_{a}$. Then

$$
\begin{array}{rlrl}
\overline{\vartheta^{+}}(G) & =\vartheta^{+}\left(C_{a}\right) & \\
& \leq \Upsilon\left(A_{C_{a}}, \mathbb{1}\right) & & \text { by }(4.81) \\
& =\frac{a \cos (\pi / a)}{1+\cos (\pi / a)} & & \text { by }(4.85) \\
& =a\left(1-\frac{1}{1+\cos (\pi / a)}\right) & & \\
& <a\left(1-\frac{1}{2}\right) & & \text { since } \cos (\pi / a)<1 \\
& =\frac{a}{2} & & \\
& \leq \omega_{c}(\operatorname{Circ}(a, 2)) & & \text { by }(3.91 \mathrm{a}) \\
& =\omega_{c}(G) . &
\end{array}
$$

Hence (4.86a) holds. On the other hand, on the Erdős-Rényi model $G(n, p)$ of random graphs, the gap between $\vartheta^{\prime}$ and $\alpha$ can be as large as as $n^{1 / 2-\varepsilon}$ [26]. Since $\omega_{c}<\omega+1$, there exists a graph $H$ such that $\omega_{c}(H)<\overline{\vartheta^{\prime}}(H)$, thus proving (4.86b).

Proposition 4.41. It holds that

$$
\omega_{\mathrm{KG}} \leq \overline{\vartheta^{\prime}}
$$

Proof. Since $\overline{\vartheta^{\prime}}$ is homomorphism monotone by (3.85) and (3.160), Definition 3.24 and (3.108) imply that it suffices to prove that $\overline{\vartheta^{\prime}}\left(K_{1}\right) \geq 1$ and $\overline{\vartheta^{\prime}}(\operatorname{KG}(a, b)) \geq a / b$ for every $a, b \in \mathbb{N}$ such that $0<2 b \leq a$. From Corollary 3.52 and (3.160) it is immediate that $\overline{\vartheta^{\prime}}\left(K_{1}\right) \geq 1$.

Let $a, b \in \mathbb{N}$ be such that $0<2 b \leq a$. Then

$$
\begin{aligned}
\left(\begin{array}{l}
a \\
b
\end{array}\right) & \leq \overline{\vartheta^{\prime}}(\operatorname{KG}(a, b)) \vartheta^{+}(\operatorname{KG}(a, b)) & & \text { by Proposition } 4.31 \text { and }(4.84) \\
& \leq \overline{\vartheta^{\prime}}(\operatorname{KG}(a, b)) \Upsilon\left(A_{\mathrm{KG}(a, b), \mathbb{1})}\right. & & \text { by }(4.81) \\
& =\overline{\vartheta^{\prime}}(\operatorname{KG}(a, b))\left(\begin{array}{l}
a-1 \\
b-1
\end{array}\right) . & & \text { by }(4.85)
\end{aligned}
$$

The inequality $\overline{\vartheta^{\prime}}(\operatorname{KG}(a, b)) \geq a / b$ follows. 


\section{Chapter 5}

\section{Conic Generalizations of Parameters and Homomorphisms}

\subsection{Matrix Cones}

Recall that a cone is a set $K$ in a vector space such that $\alpha K \subseteq K$ for every $\alpha \in \mathbb{R}_{++}$. Cones geometrically represent the intuitive notion of "directions", and as such permeate optimization theory. The ubiquity of cones in optimization reverberates in adjacent areas, and we will look at cones arising in combinatorial optimization in general, and in graph parameters and homomorphisms in particular. Our focus is even narrower, as we will work with cones which are subsets of $\mathbb{S}^{V}$ for some finite set $V$. As we shall see, such cones encode surprising and meaningful information, and offer insight into several objects we have already faced in this text.

Let $S$ and $T$ be finite sets. We will work with the set of matrices $\mathbb{R}^{S \times T}$ equipped with the trace inner product, which is given by

$$
\langle A, B\rangle=\operatorname{Tr}\left(A^{\top} B\right)
$$

for every $A, B \in \mathbb{R}^{S \times T}$. Whereas we have already used this definition, it gains bigger importance in the current context, as it is key in defining the dual of a convex cone. Let $\mathbb{E} \subseteq \mathbb{R}^{S \times T}$ be a linear subspace. The restriction of the trace inner product to $\mathbb{E} \times \mathbb{E}$ defines an inner product on $\mathbb{E}$, turning $\mathbb{E}$ into an Euclidean space. Define for every $C \subseteq \mathbb{E}$,

$$
C^{*}:=\{Y \in \mathbb{E} \mid \forall X \in C,\langle Y, X\rangle \geq 0\} .
$$

One may trivially check that $C^{*}$ is a closed convex cone for every $C \subseteq \mathbb{E}$. It is immediate from (5.2) and (2.45) that

$$
C^{*}=\left\{Y \in \mathbb{E} \mid \delta^{*}(-Y \mid C) \leq 0\right\}
$$

for every $C \subseteq \mathbb{E}$. We conclude from (5.3) that

$$
\text { if } C \subseteq D \text { then } D^{*} \subseteq C^{*}
$$

for every $C, D \subseteq \mathbb{E}$, since

$$
\begin{aligned}
D^{*} & =\left\{Y \in \mathbb{E} \mid \delta^{*}(-Y \mid D) \leq 0\right\} & & \text { by }(5.3) \\
& =\left\{Y \in \mathbb{E} \mid \delta^{*}(-Y \mid C) \leq 0\right\} & & \text { by }(2.48) \text { and } C \subseteq D \\
& =C^{*} . & & \text { by }(5.3)
\end{aligned}
$$

We also prove that for every set $C \subseteq \mathbb{E}$,

$$
C^{*}=(\operatorname{cl} C)^{*},
$$

since

$$
\begin{aligned}
C^{*} & =\left\{Y \in \mathbb{E} \mid \delta^{*}(-Y \mid C) \leq 0\right\} & & \text { by }(5.3) \\
& =\left\{Y \in \mathbb{E} \mid \delta^{*}(-Y \mid \operatorname{cl} C) \leq 0\right\} & & \text { by }(2.46) \\
& =\operatorname{cl}(C)^{*} . & & \text { by }(5.3)
\end{aligned}
$$


For every convex cone $K \subseteq \mathbb{E}$, it holds that

$$
\text { cl } K \text { is a closed convex cone, }
$$

and that

$$
\text { if } K \text { is closed, then } K^{* *}=K \text {. }
$$

We will not prove neither (5.6) or (5.7); we remark that (5.6) is a straightforward check, whereas (5.7) follows from a suitable hyperplane separation result — see, for example, [102, Chapter 14]. One may easily refine (5.7) by proving that

$$
K^{* *}=\operatorname{cl}(K)
$$

for every convex cone $K \subseteq \mathbb{E}$. This is true because

$$
\begin{aligned}
K^{* *} & =(\operatorname{cl} K)^{* *} & & \text { by }(5.5) \\
& =\operatorname{cl} K . & & \text { by }(5.6) \text { and }(5.7)
\end{aligned}
$$

Proposition 5.1. Let $S$ and $T$ be finite sets, let $\mathbb{K}_{S} \subseteq \mathbb{S}^{S}$ and $\mathbb{K}_{T} \subseteq \mathbb{S}^{T}$ be convex cones. Then for every linear transformation $\Phi: \mathbb{S}^{S} \rightarrow \mathbb{S}^{T}$,

$$
\Phi\left(\mathbb{K}_{S}\right) \subseteq \operatorname{cl}\left(\mathbb{K}_{T}\right) \text { if and only if } \Phi^{*}\left(\mathbb{K}_{T}^{*}\right) \subseteq \mathbb{K}_{S}^{*} .
$$

Proof. We first prove that

$$
\Phi\left(\mathbb{K}_{S}\right) \subseteq \operatorname{cl}\left(\mathbb{K}_{T}\right) \text { implies } \Phi^{*}\left(\mathbb{K}_{T}^{*}\right) \subseteq \mathbb{K}_{S}^{*}
$$

Let $Y \in \mathbb{K}_{T}^{*}$. Then

$$
\begin{aligned}
\delta^{*}\left(-\Phi^{*}(Y) \mid \mathbb{K}_{S}\right) & =\delta^{*}\left(-Y \mid \Phi\left(\mathbb{K}_{S}\right)\right) & & \text { by }(2.50) \\
& \leq \delta^{*}\left(-Y \mid \operatorname{cl}\left(\mathbb{K}_{T}\right)\right) & & \text { by }(2.48) \text { and } \Phi\left(\mathbb{K}_{S}\right) \subseteq \operatorname{cl}\left(\mathbb{K}_{T}\right) \\
& =\delta^{*}\left(-Y \mid \mathbb{K}_{T}\right) & & \text { by }(2.46) \\
& \leq 0 . & & \text { by }(5.3) \text { and } Y \in \mathbb{K}_{T}^{*}
\end{aligned}
$$

Using (5.3), we conclude that $\Phi^{*}(Y) \in \mathbb{K}_{S}^{*}$, so (5.9) holds.

Assume $\Phi^{*}\left(\mathbb{K}_{T}^{*}\right) \subseteq \mathbb{K}_{S}^{*}$. Then $\Phi^{*}\left(\mathbb{K}_{T}^{*}\right) \subseteq \operatorname{cl}\left(\mathbb{K}_{S}^{*}\right)$. Duality finishes the proof:

$$
\begin{aligned}
\Phi\left(\mathbb{K}_{S}\right) & \subseteq \Phi\left(\operatorname{cl}\left(\mathbb{K}_{S}\right)\right) & & \\
& =\Phi\left(\mathbb{K}_{S}^{* *}\right) & & \text { by }(5.8) \\
& \subseteq \mathbb{K}_{T}^{* *} & & \text { by }(5.9) \text { and } \Phi^{*}\left(\mathbb{K}_{T}^{*}\right) \subseteq \operatorname{cl}\left(\mathbb{K}_{S}^{*}\right) \\
& =\operatorname{cl}\left(\mathbb{K}_{T}\right) . & & \text { by }(5.8)
\end{aligned}
$$

Let $S, T$, and $V$ be finite sets. Define Gram: $\left(\mathbb{R}^{S \times T}\right)^{V} \rightarrow \mathbb{S}^{V}$ by

$$
\operatorname{Gram}(f)_{i j}:=\langle f(i), f(j)\rangle
$$

for every $f: V \rightarrow \mathbb{R}^{S \times T}$ and $i, j \in V$. A matrix of the form $\operatorname{Gram}(f)$ for some $f: V \rightarrow \mathbb{R}^{S \times T}$ is called a Gram matrix. The main cones we wish to work with are defined in terms of Gram matrices.

Let $V$ be a finite set. Define the completely positive cone

$$
\mathbb{C P}^{V}:=\left\{\operatorname{Gram}(f) \mid f: V \rightarrow \mathbb{R}_{+}^{T} \text { for some finite set } T\right\},
$$

and the completely positive semidefinite cone

$$
\mathbb{C S}_{+}^{V}:=\left\{\operatorname{Gram}(f) \mid f: V \rightarrow \mathbb{S}_{+}^{T} \text { for some finite set } T\right\} .
$$

Moreover, it is well known that

$$
\mathbb{S}_{+}^{V}=\left\{\operatorname{Gram}(f) \mid f: V \rightarrow \mathbb{R}^{T} \text { for some finite set } T\right\} .
$$

Before discussing the two cones just introduced, we prove two helpful propositions regarding Gram matrices. 
Proposition 5.2. Let $S, T$, and $U$ be finite sets. Then for every $f: U \rightarrow \mathbb{R}^{S \times T}$ and $V \subseteq U$,

$$
\operatorname{Gram}(f)[V]=\operatorname{Gram}\left(f \uparrow_{V}\right) .
$$

Proof. Immediate consequence of the definition of the restriction $f \uparrow_{V}: V \rightarrow \mathbb{R}^{S \times T}$.

Proposition 5.3. Let $S, T, U$, and $V$ be finite sets. For every $g: U \rightarrow \mathbb{R}^{S \times T}$ and $A \in \mathbb{R}^{V \times U}$,

$$
A \operatorname{Gram}(g) A^{\top}=\operatorname{Gram}(h)
$$

where $h: V \rightarrow \mathbb{R}^{S \times T}$ is defined by

$$
h(v):=\sum_{u \in U} A_{v u} g(u)
$$

for every $v \in V$.

Proof. For every $i, j \in V$,

$$
\begin{array}{rlrl}
\left(A \operatorname{Gram}(g) A^{\top}\right)_{i j} & =e_{i}^{\top} A \operatorname{Gram}(g) A^{\top} e_{j} & \\
& =\sum_{u, u^{\prime} \in U} A_{i u} A_{u^{\prime} j} \operatorname{Gram}(g)_{u u^{\prime}} & \\
& =\sum_{u, u^{\prime} \in U} A_{i u} A_{u^{\prime} j}\left\langle g(u), g\left(u^{\prime}\right)\right\rangle & & \text { by }(5.10) \\
& =\left\langle\sum_{u \in U} A_{i u} g(u), \sum_{u^{\prime} \in U} A_{j u^{\prime}} g\left(u^{\prime}\right)\right\rangle & & \text { since }\langle\cdot, \cdot\rangle \text { bilinear } \\
& =\langle h(i), h(j)\rangle & & \\
& =\operatorname{Gram}(h)_{i j} . & & \text { by }(5.10)
\end{array}
$$

Let $V$ be a finite set, and let $A \in \mathbb{S}^{V}$. The completely positive cone and its dual arise naturally when optimizing a quadratic function $x \in \mathbb{R}_{+}^{V} \mapsto x^{\top} A x-$ see, for example, $[16,20]$. The dual $\left(\mathbb{C P}^{V}\right)^{*}$ of the completely positive cone is commonly referred to as the copositive cone. It holds that

$$
\left(\mathbb{C P}^{V}\right)^{*}=\left\{A \in \mathbb{S}^{V} \mid x^{\top} A x \geq 0 \text { for every } x \in \mathbb{R}_{+}^{V}\right\} .
$$

Proposition 5.4. Let $V$ be a finite set. The following are equivalent:

1. $X \in \mathbb{C P}^{V}$;

2. there exists a finite set $S$ and $B \in \mathbb{R}_{+}^{S \times V}$ such that $X=B^{\top} B$;

3. $X=\sum_{s \in S} x_{s} x_{s}^{\top}$ for some finite set $S$ and $x: S \rightarrow \mathbb{R}_{+}^{V}$ such that $x_{s} \neq 0$ for every $s \in S$.

Proof. Let $S$ be a finite set, let $f: V \rightarrow \mathbb{R}^{S}$, and let $B \in \mathbb{R}^{S \times V}$. We first prove that

$$
\text { if } B e_{v}=f(v) \text { for every } v \in V \text {, then } \operatorname{Gram}(f)=B^{\top} B \text {. }
$$

This is true, as for every $i, j \in V$,

$$
\begin{aligned}
\left(B^{\top} B\right)_{i j} & =e_{i}^{\top} B^{\top} B e_{j} \\
& =(f(i))^{\top} f(j) \\
& =\operatorname{Gram}(f)_{i j} .
\end{aligned}
$$

Item 1 implies item 2: Let $X \in \mathbb{C P}^{V}$. By (5.11a), there exists a finite set $S$ and $f: V \rightarrow \mathbb{R}_{+}^{S}$ such that $X=\operatorname{Gram}(f)$. Define the matrix $B \in \mathbb{R}^{S \times V}$ by $B e_{v}:=f(v)$ for every $v \in V$. Then $B \geq 0$ and $X=B^{\top} B$ by (5.13).

Item 2 implies item 1 Let $S$ be a finite set and $B \in \mathbb{R}^{S \times V}$ be such that $B \geq 0$ and $X=B^{\top} B$. Define the function $f(v):=B e_{v}$ for every $v \in V$. Then $B^{\top} B=\operatorname{Gram}(f)$ by (5.13). 
Item 3 implies item 2: Let $S$ be a finite set and let $x: S \rightarrow \mathbb{R}_{+}^{V}$ be such that $x_{s} \neq 0$ for every $s \in S$. Define $B \in \mathbb{R}^{S \times V}$ by $B^{\top} e_{s}:=x_{s}$ for every $s \in S$. Then

$$
X=\sum_{s \in S} x_{s} x_{s}^{\top}=\sum_{s \in S} B^{\top} e_{s} e_{s}^{\top} B=B^{\top}\left(\sum_{s \in S} e_{s} e_{s}^{\top}\right) B=B^{\top} B
$$

Item 2 implies item 3: Let $S$ be a finite set, and $B \in \mathbb{R}^{S \times V}$ be such that $B \geq 0$ and $X=B^{\top} B$. Define $x: S \rightarrow \mathbb{R}_{+}^{V}$ by $x_{s}:=B^{\top} e_{s}$ for every $s \in S$. Define $T:=\left\{s \in S \mid x_{s} \neq 0\right\}$. Then $T$ and $\left.x\right|_{T}$ certify that item 3 holds, as

$$
X=B^{\top} B=\sum_{s \in S} B^{\top} e_{s} e_{s}^{\top} B=\sum_{s \in S}\left(B^{\top} e_{s}\right)\left(B^{\top} e_{s}\right)^{\top}=\sum_{s \in S} x_{s} x_{s}^{\top}=\sum_{t \in T} x_{t} x_{t}^{\top} .
$$

Let $V$ be a finite set. We claim that

$$
\mathbb{C P}^{V} \text { is a convex cone with nonempty interior. }
$$

Item 3 of Proposition 5.4 immediately implies that $\mathbb{C P}^{V}$ is a convex cone. Moreover, every subspace of $\mathbb{S}^{V}$ which contains $\mathbb{C P}^{V}$ must also contain

$$
e_{i} e_{j}^{\top}+e_{j} e_{i}^{\top}=\left(e_{i}+e_{j}\right)\left(e_{i}+e_{j}\right)^{\top}-e_{i} e_{i}^{\top}-e_{j} e_{j}^{\top},
$$

for every $i, j \in V$. Thus, the only subspace containing $\mathbb{C P}^{V}$ is $\mathbb{S}^{V}$ it self, so we conclude that $\mathbb{C P}^{V}$ has nonempty interior. One may also easily use Item 3 of Proposition 5.4 to prove (5.12). We refer the reader to [14] for further discussion on the completely positive cone; in particular, [14, Theorem 2.2] uses Carathéodory's theorem to prove that

$$
\mathbb{C P}^{V} \text { is closed. }
$$

Let $V$ be a finite set. Laurent and Piovesan [73] formulated the quantum chromatic number $\chi_{q}$ and the quantum stability number $\alpha_{q}$ as optimization problems over $\mathbb{C S}_{+}^{V}$. They also proved that

$$
\mathbb{C S}_{+}^{V} \text { is a convex cone with nonempty interior, }
$$

and that

$$
\mathbb{C P}^{V} \subseteq \mathbb{C S}_{+}^{V} \subseteq \mathbb{S}_{+}^{V} \subseteq \mathbb{S}^{V}
$$

The completely positive semidefinite cone is the main tool for reasoning about quantum phenomena in this work. Furthermore, it serves as a special example. Whereas $\mathbb{C P}^{V}$ and $\mathbb{S}_{+}^{V}$ share many similarities, like the ones mentioned in Proposition 5.4, the completely positive semidefinite cone is much harder to work with. For example, it took years since the introduction of $\mathbb{C S}_{+}^{V}$ in [73] until Slofstra [113] proved that $\mathbb{C S}_{+}^{V}$ is not closed.

\subsection{Theta Bodies}

This whole chapter is dedicated to relating matrix cones presented in Section 5.1 with Chapters 3 and 4 . We start this effort by presenting a framework by de Carli Silva and Tunçel [28] which converts matrix cones into convex corners, thus relating such cones to Chapter 4 .

Throughout this chapter,

we assume that 0 is not an element of $V$

for every finite set $V$. This becomes important when we expect $\mathbb{S}\{0\} \cup V$ be a higher dimensional object than $\mathbb{S}^{V}$. It is evident that we may always assume without loss of generality that $0 \notin V$; our remark is that we will do this implicitly throughout the text. This convention also applies to to graphs and their vertex sets.

Let $V$ be a finite set, and let $E^{+}, E^{-} \subset\left(\begin{array}{c}V \\ 2\end{array}\right)$. Recall from (3.142) that

$$
\mathbb{A}_{E^{+}, E^{-}}=\left\{A \in \mathbb{S}^{V} \mid \forall i j \in E^{+}, A_{i j} \geq 0, \forall i j \in E^{-}, A_{i j} \leq 0\right\} .
$$

Moreover, we will use the fact (see, e.g., [60, Chapter 7]) that for every $X \in \mathbb{S}_{+}^{V}$ and $S \subseteq V$,

$$
\operatorname{det}(X[S]) \geq 0 .
$$


Consequently, for every $\alpha \in \mathbb{R}$,

$$
\text { for every } X \in \mathbb{S}_{+}^{V} \text {, if } \operatorname{diag}(X) \leq \alpha \mathbb{1} \text { then }-\alpha \mathbb{1} \mathbb{1}^{\top} \leq X \leq \alpha \mathbb{1} \mathbb{1}^{\top} \text {, }
$$

since

$$
0 \leq \operatorname{det}(X[\{i, j\}])=X_{i i} X_{j j}-X_{i j}^{2} \leq \alpha^{2}-X_{i j}^{2}
$$

for every $X \in \mathbb{S}_{+}^{V}$ such that $\operatorname{diag}(X) \leq \alpha \mathbb{1}$.

Definition 5.5. Let $V$ be a finite set. Let $\mathbb{A} \subseteq \mathbb{S}^{V}$ and $\widehat{\mathbb{K}} \subseteq \mathbb{S}\{0\} \cup V$. The lifted theta body $\widehat{\mathrm{TH}}(\mathbb{A}, \widehat{\mathbb{K}})$ is defined by

$$
\widehat{\mathrm{TH}}(\mathbb{A}, \widehat{\mathbb{K}}):=\left\{\left[\begin{array}{cc}
1 & x^{\top} \\
x & X
\end{array}\right] \in \widehat{\mathbb{K}} \mid X \in \mathbb{A}, \operatorname{diag}(X)=x\right\} .
$$

Let $V$ be a finite set. The lifted theta body can be used as a matrix relaxation of binary constraints on variables. Similar construction appear in lift-and-project methods to approximate polytopes with binary variables [71], in formulations of quadratic programming problems with certain variables required to be binary [20], and in a notion of total dual integrality for SDPs [27]. Two simple results illustrate the connection between the lifted theta body and binary constraints. Direct computation shows that for every $\mathbb{A} \subseteq \mathbb{S}^{V}$ and for every $S \subseteq V$,

$$
\left[\begin{array}{cc}
1 & \mathbb{1}_{S}^{\top} \\
\mathbb{1}_{S} & \mathbb{1}_{S} \mathbb{1}_{S}^{\top}
\end{array}\right] \in \widehat{\mathrm{TH}}\left(\operatorname{Diag}\left(\mathbb{R}^{V}\right), \mathbb{C P}^{\{0\} \cup V}\right) \text { if } \mathbb{1}_{S} \mathbb{1}_{S}^{\top} \in \mathbb{A}
$$

Moreover, note that for every $x \in \mathbb{R}^{V}$ and $X \in \mathbb{S}^{V}$,

$$
\text { if }\left[\begin{array}{cc}
1 & x^{\top} \\
x & X
\end{array}\right] \in \widehat{\mathrm{TH}}\left(\mathbb{S}^{V}, \mathbb{S}_{+}^{\{0\} \cup V}\right) \text {, then } 0 \leq x \leq \mathbb{1}
$$

since

$$
x_{i}-x_{i}^{2}=\operatorname{det}(X[\{0, i\}]) \geq 0
$$

for every $i \in V$ by (5.19).

Let $V$ be a finite set. Definition 5.5 requires a cone in $\mathbb{S}\{0\} \cup V$. For our purposes, this cone will be obtained by lifting lower dimensional cones. We now present two liftings, and state without proof their basic properties - we hope the reader agrees that the assertions in this paragraph are either simple to prove or well known. For every $\mathbb{K} \subseteq \mathbb{S}^{V}$, define the $P S D$ lifting of $\mathbb{K}$ as

$$
\operatorname{Psd}(\mathbb{K}):=\left\{\widehat{X} \in \mathbb{S}_{+}^{\{0\} \cup V} \mid \widehat{X}[V] \in \mathbb{K}\right\}
$$

The function $\operatorname{Psd}(\cdot)$ lifts subsets of $\mathbb{S}^{V}$ to subsets of $\mathbb{S}^{\{0\} \cup V}$, as desired. It easy to see that if $\mathbb{K}$ is a cone, then so is $\operatorname{Psd}(\mathbb{K})$. One may easily prove that

$$
\text { if } \mathbb{K} \text { is closed, then } \operatorname{Psd}(\mathbb{K}) \text { is closed. }
$$

and that

$$
\operatorname{Psd}\left(\mathbb{S}_{+}^{V}\right)=\mathbb{S}_{+}^{\{0\} \cup V}
$$

Equation (5.25) is special; for another example, one may easily check that

$$
\mathbb{C P}^{\{0\} \cup V} \subseteq \operatorname{Psd}\left(\mathbb{C P}^{V}\right)
$$

We are also interested in another lifting, which is in a sense dual to $\operatorname{Psd}(\cdot)$. The Schur lifting of $\mathbb{K}$ is defined by

$$
\operatorname{Schur}(\mathbb{K}):=\left\{\left[\begin{array}{cc}
x_{0} & x^{\top} \\
x & X
\end{array}\right] \in \mathbb{S}^{\{0\} \cup V} \mid x_{0} \in \mathbb{R}_{+}, X \in \mathbb{K}, x_{0} X \succeq_{\mathbb{K}} x x^{\top}\right\} .
$$


The function $\operatorname{Schur}(\cdot)$ lifts subsets of $\mathbb{S}^{V}$ to subsets of $\mathbb{S}\{0\} \cup V$, as desired. It easy to see that if $\mathbb{K}$ is a cone, then so is $\operatorname{Schur}(\mathbb{K})$. Whenever

$$
\left[\begin{array}{cc}
x_{0} & x^{\top} \\
x & X
\end{array}\right]:=\widehat{X} \in \mathbb{S}^{\{0\} \cup V}
$$

is such that $x_{0} \neq 0$ the expression $X-x_{0}^{-1} x x^{\top}$ is commonly called the Schur complement of $x_{0}$ in $\widehat{X}$, which motivates the notation. The definition of $\operatorname{Schur}(\cdot)$ is related to the well-known facts that

$$
\mathbb{S}_{+}^{\{0\} \cup V}=\left\{\left[\begin{array}{cc}
x_{0} & x^{\top} \\
x & X
\end{array}\right] \in \mathbb{S}^{\{0\} \cup V} \mid x_{0} \in \mathbb{R}_{+}, X \in \mathbb{S}_{+}^{V}, x_{0} X \succeq x x^{\top}\right\}
$$

and

$$
\mathbb{S}_{++}^{\{0\} \cup V}=\left\{\left[\begin{array}{cc}
x_{0} & x^{\top} \\
x & X
\end{array}\right] \in \mathbb{S}^{\{0\} \cup V} \mid x_{0} \in \mathbb{R}_{++}, X \in \mathbb{S}_{++}^{V}, x_{0} X \succ x x^{\top}\right\} .
$$

See, for example, [60, Theorem 7.7.6]. We conclude that

$$
\operatorname{Schur}\left(\mathbb{S}_{+}^{V}\right)=\mathbb{S}_{+}^{\{0\} \cup V}
$$

directly from (5.28a). When manipulating cones using these liftings, it will be extremely helpful to exploit their monotonicity. Precisely, note that for every $\mathbb{K}_{0}, \mathbb{K}_{1} \subseteq \mathbb{S}^{V}$,

$$
\begin{aligned}
\text { if } \mathbb{K}_{0} \subseteq \mathbb{K}_{1} \text {, then } \operatorname{Psd}\left(\mathbb{K}_{0}\right) \subseteq \operatorname{Psd}\left(\mathbb{K}_{1}\right), \\
\text { if } \mathbb{K}_{0} \subseteq \mathbb{K}_{1} \text {, then } \operatorname{Schur}\left(\mathbb{K}_{0}\right) \subseteq \operatorname{Schur}\left(\mathbb{K}_{1}\right) \text {. }
\end{aligned}
$$

Furthermore, for every $\mathbb{A}_{0}, \mathbb{A}_{1} \subseteq \mathbb{S}^{V}$ and $\widehat{\mathbb{K}}_{0}, \widehat{\mathbb{K}}_{1} \subseteq \mathbb{S}\{0\} \cup V$,

$$
\text { if } \mathbb{A}_{0} \subseteq \mathbb{A}_{1} \text { and } \widehat{\mathbb{K}}_{0} \subseteq \widehat{\mathbb{K}}_{1} \text {, then } \widehat{\mathrm{TH}}\left(\mathbb{A}_{0}, \widehat{\mathbb{K}}_{0}\right) \subseteq \widehat{\mathrm{TH}}\left(\mathbb{A}_{1}, \widehat{\mathbb{K}}_{1}\right) \text {. }
$$

One may easily see that

$$
\widehat{\mathrm{TH}}(\mathbb{A}, \widehat{\mathbb{K}})=\left\{\widehat{X} \in \widehat{\mathbb{K}} \mid \mathcal{B}_{V}(\widehat{X})=1 \oplus 0, \widehat{X}[V] \in \mathbb{A}\right\}
$$

for every $\mathbb{A} \subseteq \mathbb{S}^{V}$ and $\widehat{\mathbb{K}} \subseteq \mathbb{S}^{\{0\} \cup V}$, where $\mathcal{B}_{V}: \mathbb{S}^{\{0\} \cup V} \rightarrow \mathbb{R}^{\{0\} \cup V}$ is defined by

$$
\begin{aligned}
\mathcal{B}_{V}(\widehat{X})_{0} & :=\widehat{X}_{00}, \\
\mathcal{B}_{V}(\widehat{X})_{i} & :=\widehat{X}_{0 i}+\widehat{X}_{i 0}-2 \widehat{X}_{i i} \text { for every } i \in V
\end{aligned}
$$

for every $\widehat{X} \in \mathbb{S}\{0\} \cup V$.

Proposition 5.6. Let $V$ be a finite set. Let $\mathbb{K} \subseteq \mathbb{S}_{+}^{V}$ be a closed convex cone, and let $E^{+}, E^{-} \subseteq\left(\begin{array}{c}V \\ 2\end{array}\right)$. Then

$$
\widehat{\mathrm{TH}}\left(\mathbb{A}_{E^{+}, E^{-}}, \operatorname{Psd}(\mathbb{K})\right) \text { is compact. }
$$

Proof. Set $\mathbb{A}:=\mathbb{A}_{E^{+}, E^{-}}$. We claim that

$$
\widehat{\mathrm{TH}}(\mathbb{A}, \operatorname{Psd}(\mathbb{K})) \subseteq \widehat{\mathrm{TH}}\left(\mathbb{S}^{V}, \mathbb{S}_{+}^{\{0\} \cup V}\right) .
$$

Indeed,

$$
\begin{aligned}
\widehat{\mathrm{TH}}(\mathbb{A}, \operatorname{Psd}(\mathbb{K})) & \subseteq \widehat{\mathrm{TH}}\left(\mathbb{S}^{V}, \operatorname{Psd}\left(\mathbb{S}_{+}^{V}\right)\right) & & \text { by }(5.31),(5.30 \mathrm{a}), \mathbb{A} \subseteq \mathbb{S}^{V}, \text { and } \mathbb{K} \subseteq \mathbb{S}_{+}^{V} \\
& =\widehat{\mathrm{TH}}\left(\mathbb{S}^{V}, \mathbb{S}_{+}^{\{0\} \cup V}\right) . & & \text { by }(5.25)
\end{aligned}
$$

We have that $\operatorname{diag}(\widehat{X}) \leq \mathbb{1}$ for every $\widehat{X} \in \widehat{\mathrm{TH}}\left(\mathbb{S}^{V}, \mathbb{S}_{+}^{\{0\} \cup V}\right.$ ) by $(5.22)$, so (5.20) implies that $\widehat{\mathrm{TH}}\left(\mathbb{S}^{V}, \mathbb{S}_{+}^{\{0\} \cup V}\right.$ ) is bounded. Hence (5.34) implies that $\widehat{\mathrm{TH}}(\mathbb{A}, \operatorname{Psd}(\mathbb{K}))$ is bounded.

Using (5.32), we have that

$$
\widehat{\mathrm{TH}}(\mathbb{A}, \operatorname{Psd}(\mathbb{K}))=\left\{\widehat{X} \in \operatorname{Psd}(\mathbb{K}) \mid \mathcal{B}_{V}(\widehat{X})=1 \oplus 0, \widehat{X}[V] \in \mathbb{A}\right\} .
$$

Equation (5.35) implies that $\widehat{\mathrm{TH}}(\mathbb{A}, \operatorname{Psd}(\mathbb{K}))$ is the intersection of three closed sets: $\operatorname{Psd}(\mathbb{K})$, which is closed by (5.24), and the preimages of the closed sets $\{1 \oplus 0\}$ and $\mathbb{A}$ under the linear functions $\mathcal{B}_{V}$ and $\widehat{X} \in \mathbb{S}\{0\} \cup V \mapsto \widehat{X}[V]$, respectively. Therefore $\widehat{\mathrm{TH}}(\mathbb{A}, \operatorname{Psd}(\mathbb{K}))$ is closed. 
The lifted theta body can be used to encode continuous optimization problems that are relaxations of combinatorial optimization problems. In the upcoming Section 5.3, we will exploit $\widehat{\mathrm{TH}}$ to obtain bounds related to the cuts of a graph. Its name and notation, however, are related to the stable set problem. For every $\mathbb{A} \subseteq \mathbb{S}^{V}$ and $\widehat{\mathbb{K}} \subseteq \mathbb{S}_{\{0\} \cup V}$, define

$$
\operatorname{TH}(\mathbb{A}, \widehat{\mathbb{K}}):=\{\operatorname{diag}(\widehat{X}[V]) \mid \widehat{X} \in \widehat{\mathrm{TH}}(\mathbb{A}, \widehat{\mathbb{K}})\} .
$$

Note that $\mathrm{TH}(\mathbb{A}, \widehat{\mathbb{K}}) \subseteq \mathbb{R}^{V}$. We may call sets of the form $\mathrm{TH}(\mathbb{A}, \widehat{\mathbb{K}})$ as theta bodies. Furthermore, for every $w \in \mathbb{R}_{+}^{V}$, define

$$
\vartheta(\mathbb{A}, \widehat{\mathbb{K}}, w):=\delta^{*}(w \mid \operatorname{TH}(\mathbb{A}, \widehat{\mathbb{K}})) .
$$

We warn the reader that this notation deviates from [28]. As expected, this definition captures the theta body of a graph and its most famous variants. Let $G:=(V, E)$ be a graph. Define

$$
\begin{aligned}
\operatorname{TH}(G) & :=\operatorname{TH}\left(\mathbb{A}_{E, E}, \operatorname{Psd}\left(\mathbb{S}_{+}^{V}\right)\right) . \\
\operatorname{TH}^{\prime}(G) & :=\operatorname{TH}\left(\mathbb{A}_{E \cup \bar{E}, E}, \operatorname{Psd}\left(\mathbb{S}_{+}^{V}\right)\right) . \\
\operatorname{TH}^{+}(G) & :=\operatorname{TH}\left(\mathbb{A}_{\varnothing, E}, \operatorname{Psd}\left(\mathbb{S}_{+}^{V}\right)\right) .
\end{aligned}
$$

Define

$$
\begin{aligned}
\vartheta(G, w) & :=\vartheta\left(\mathbb{A}_{E, E}, \operatorname{Psd}\left(\mathbb{S}_{+}^{V}\right), w\right)=\delta^{*}(w \mid \mathrm{TH}(G)), \\
\vartheta^{\prime}(G, w) & :=\vartheta\left(\mathbb{A}_{E \cup \bar{E}, E}, \operatorname{Psd}\left(\mathbb{S}_{+}^{V}\right), w\right)=\delta^{*}\left(w \mid \mathrm{TH}^{\prime}(G)\right), \text { and } \\
\vartheta^{+}(G, w) & :=\vartheta\left(\mathbb{A}_{\varnothing, E}, \operatorname{Psd}\left(\mathbb{S}_{+}^{V}\right), w\right)=\delta^{*}\left(w \mid \mathrm{TH}^{+}(G)\right)
\end{aligned}
$$

for every $w \in \mathbb{R}_{+}^{V}$ and recover $\vartheta$ as introduced by Lovász [77], $\vartheta^{\prime}$ as independently introduced by McEliece, Rodemich, and Rumsey [87] and Schrijver [108], and $\vartheta^{+}$as introduced by Szegedy [115]. Quite remarkably, [28, Proposition 16] proves that

$$
\operatorname{STAB}(G)=\operatorname{TH}\left(\mathbb{A}_{E, E}, \operatorname{Psd}\left(\mathbb{C P}^{V}\right)\right)
$$

and [28, Corollary 19] proves that

$$
\operatorname{QSTAB}(G)=\operatorname{TH}\left(\mathbb{A}_{E, E}, \operatorname{Schur}\left(\left(\mathbb{C P}^{V}\right)^{*}\right)\right) .
$$

Let $V$ be a finite set, and let $\mathbb{A} \subseteq \mathbb{S}^{V}$ and $\widehat{\mathbb{K}} \subseteq \mathbb{S}\{0\} \cup V$. Equations (5.38), (5.40), and (5.41) show that it is not uncommon for theta bodies to define convex corners. What properties should one impose on $\mathbb{A}$ and $\widehat{\mathbb{K}}$ in order to ensure that $\mathrm{TH}(\mathbb{A}, \widehat{\mathbb{K}})$ is a convex corner? This is one of the questions tackled by [28]. Their results are framed in terms of an interesting property. A set $\mathbb{K} \subseteq \mathbb{S}^{V}$ is diagonally scaling-invariant if

$$
\operatorname{Diag}(h) \mathbb{K} \operatorname{Diag}(h) \subseteq \mathbb{K}
$$

for every $h \in \mathbb{R}_{+}^{V}$. Every diagonally scaling-invariant set is a cone, since $\alpha X=\operatorname{Diag}(\sqrt{\alpha} \mathbb{1}) X \operatorname{Diag}(\sqrt{\alpha} \mathbb{1})$ for every $\alpha \in \mathbb{R}_{+}$. Proposition 5.3 and (5.11) imply that $\mathbb{S}_{+}^{V}, \mathbb{C P}^{V}$, and $\mathbb{C S}_{+}^{V}$ are diagonally scaling-invariant. One may also prove that diagonally scaling-invariant cones are closed under taking Schur and Psd liftings. Moreover, it also holds that

$$
\mathbb{A}_{E^{+}, E^{-}} \text {is diagonally scaling-invariant. }
$$

for every $E^{+}, E^{-} \subseteq\left(\begin{array}{c}V \\ 2\end{array}\right)$. The authors in [28] go even further and show that any polyhedral diagonally scaling-invariant cone which contains $\operatorname{Diag}\left(\mathbb{R}^{V}\right)$ is of the form $\mathbb{A}_{E^{+}, E^{-}}$for $E^{+}, E^{-} \subseteq\left(\begin{array}{c}V \\ 2\end{array}\right)$. In any case, their chosen hypotheses to ensure that $\mathrm{TH}(\mathbb{A}, \widehat{K})$ is a convex corner get reasonably technical. Whereas one may easily show that $\operatorname{TH}(\mathbb{A}, \operatorname{Psd}(\mathbb{K}))$ is a convex corner whenever $\mathbb{A}$ and $\mathbb{K}$ are diagonally scaling-invariant and $\mathbb{C P}^{V} \subseteq \mathbb{K} \subseteq \mathbb{S}_{+}^{V}$, this is not entirely satisfactory, as it excludes (5.41) and also

$$
\operatorname{FRAC}(G):=\left\{x \in[0,1]^{V} \mid x_{i}+x_{j} \leq 1 \text { for every } i j \in E\right\},
$$


which is a convex corner, and, by [28, Theorem 9],

$$
\operatorname{FRAC}(G)=\operatorname{TH}\left(\mathbb{A}_{E, E}, \operatorname{Schur}\left(\left\{X \in \mathbb{S}^{V} \mid X[\{i, j\}] \succeq 0 \text { for every } i, j \in V \text { with } i \neq j\right\}\right)\right) .
$$

Nevertheless, this discussion on convex corners is just a prerequisite for a deeper question: what is $\operatorname{abl}(\mathrm{TH}(\mathbb{A}, \widehat{\mathbb{K}}))$ ? Is it also a theta body? It turns out that under some mild extra hypotheses on $\mathbb{A}$ and $\widehat{\mathbb{K}}$, the answer is yes.

Theorem 5.7 ([28, Theorems 6 and 14]). Let $V$ be a finite set. Let $E^{+}, E^{-} \subseteq\left(\begin{array}{c}V \\ 2\end{array}\right)$, and set $\mathbb{A}:=\mathbb{A}_{E^{+}, E^{-}}$ and $\overline{\mathbb{A}}:=\mathbb{A}_{\overline{E^{+}}, \overline{E^{-}}}$. Let $\mathbb{K} \subseteq \mathbb{S}_{+}^{V}$ be a diagonally scaling-invariant closed convex cone such that $\operatorname{Diag}\left(\mathbb{R}_{+}^{V}\right) \subseteq \mathbb{K}$ and $\operatorname{int}(\mathbb{K}) \neq \varnothing$. Then

$$
\text { both } \operatorname{cl}\left(\operatorname{TH}\left(\overline{\mathbb{A}}, \operatorname{Schur}\left(\mathbb{K}^{*}\right)\right)\right) \text { and } \operatorname{TH}(\mathbb{A}, \operatorname{Psd}(\mathbb{K})) \text { are convex corners }
$$

and

$$
\operatorname{abl}\left(\operatorname{cl}\left(\operatorname{TH}\left(\overline{\mathbb{A}}, \operatorname{Schur}\left(\mathbb{K}^{*}\right)\right)\right)\right)=\operatorname{TH}(\mathbb{A}, \operatorname{Psd}(\mathbb{K})) .
$$

Let $V$ be a finite set. Let $E^{+}, E^{-} \subseteq\left(\begin{array}{c}V \\ 2\end{array}\right)$, and set $\mathbb{A}:=\mathbb{A}_{E^{+}, E^{-}}$and $\overline{\mathbb{A}}:=\mathbb{A}_{\overline{E^{+}}, \overline{E^{-}}}$. Let $\mathbb{K} \subseteq \mathbb{S}_{+}^{V}$ be a diagonally scaling-invariant closed convex cone such that $\operatorname{Diag}\left(\mathbb{R}_{+}^{V}\right) \subseteq \mathbb{K}$ and $\operatorname{int}(\mathbb{K}) \neq \varnothing$. We conclude that

$$
\begin{gathered}
\vartheta(\mathbb{A}, \operatorname{Psd}(\mathbb{K}), \cdot) \text { and } \vartheta\left(\overline{\mathbb{A}}, \operatorname{Schur}\left(\mathbb{K}^{*}\right), \cdot\right) \text { are real-valued positive definite monotone gauges, } \\
\text { dual to each other }
\end{gathered}
$$

since

$$
\begin{aligned}
\operatorname{unit}\left(\vartheta\left(\overline{\mathbb{A}}, \operatorname{Schur}\left(\mathbb{K}^{*}\right), \cdot\right)\right) & =\operatorname{unit}\left(\delta^{*}\left(\cdot \mid \operatorname{TH}\left(\overline{\mathbb{A}}, \operatorname{Schur}\left(\mathbb{K}^{*}\right)\right)\right)\right) & & \text { by }(5.37) \\
& =\operatorname{unit}\left(\delta^{*}\left(\cdot \mid \operatorname{cl}\left(\operatorname{TH}\left(\overline{\mathbb{A}}, \operatorname{Schur}\left(\mathbb{K}^{*}\right)\right)\right)\right)\right) & & \\
& =\operatorname{unit}\left(\gamma\left(\cdot \mid \operatorname{abl}\left(\operatorname{cl}\left(\operatorname{TH}\left(\overline{\mathbb{A}}, \operatorname{Schur}\left(\mathbb{K}^{*}\right)\right)\right)\right)\right)\right) & & \text { by item } 2 \text { of Theorem } 4.25 \text { and }(5.44) \\
& =\operatorname{unit}(\gamma(\cdot \mid \operatorname{TH}(\mathbb{A}, \operatorname{Psd}(\mathbb{K})))) & & \text { by }(5.45) \\
& =\operatorname{TH}(\mathbb{A}, \operatorname{Psd}(\mathbb{K})) . & & \text { by Proposition } 4.11 \text { and }(5.44)
\end{aligned}
$$

Recall the previous results implied by (5.46): (4.46) and (4.71).

Having proved the generic case, we recover known results. We have that

$$
\operatorname{abl}\left(\mathrm{TH}^{\prime}(G)\right)=\mathrm{TH}^{+}(\bar{G}) .
$$

This holds because

$$
\begin{aligned}
\mathrm{TH}^{+}(\bar{G}) & =\operatorname{TH}\left(\mathbb{A}_{\varnothing, \bar{E}}, \operatorname{Psd}\left(\mathbb{S}_{+}^{V}\right)\right) & & \text { by }(5.38 \mathrm{c}) \\
& =\operatorname{abl}\left(\operatorname{abl}\left(\operatorname{TH}\left(\mathbb{A}_{\varnothing, \bar{E}}, \operatorname{Psd}\left(\mathbb{S}_{+}^{V}\right)\right)\right)\right) & & \text { by }(5.44) \text { and item } 3 \text { of Theorem } 4.25 \\
& =\operatorname{abl}\left(\operatorname{cl}\left(\operatorname{TH}\left(\mathbb{A}_{E \cup \bar{E}, E}, \operatorname{Schur}\left(\mathbb{S}_{+}^{V^{*}}\right)\right)\right)\right) & & \text { by }(5.45) \\
& =\operatorname{abl}\left(\operatorname{cl}\left(\operatorname{TH}\left(\mathbb{A}_{E \cup \bar{E}, E}, \operatorname{Schur}\left(\mathbb{S}_{+}^{V}\right)\right)\right)\right) & & \text { since } \mathbb{S}_{+}^{V^{*}}=\mathbb{S}_{+}^{V} \\
& =\operatorname{abl}\left(\operatorname{TH}\left(\mathbb{A}_{E \cup \bar{E}, E}, \operatorname{Schur}\left(\mathbb{S}_{+}^{V}\right)\right)\right) & & \text { by }(4.28) \\
& =\operatorname{abl}\left(\operatorname{TH}\left(\mathbb{A}_{E \cup \bar{E}, E}, \operatorname{Psd}\left(\mathbb{S}_{+}^{V}\right)\right)\right) & & \text { by }(5.25) \text { and }(5.29) \\
& =\operatorname{abl}\left(\mathrm{TH}^{\prime}(G)\right) . & & \text { by }(5.38 \mathrm{~b})
\end{aligned}
$$

Similarly,

$$
\operatorname{abl}(\operatorname{TH}(G))=\operatorname{TH}(\bar{G}) .
$$


This holds because

$$
\begin{aligned}
\operatorname{TH}(\bar{G}) & =\operatorname{TH}\left(\mathbb{A}_{\bar{E}, \bar{E}}, \operatorname{Psd}\left(\mathbb{S}_{+}^{V}\right)\right) & & \text { by }(5.38 \mathrm{a}) \\
& =\operatorname{abl}\left(\operatorname{abl}\left(\operatorname{TH}\left(\mathbb{A}_{\bar{E}, \bar{E}}, \operatorname{Psd}\left(\mathbb{S}_{+}^{V}\right)\right)\right)\right. & & \text { by }(5.44) \text { and item } 3 \text { of Theorem } 4.25 \\
& =\operatorname{abl}\left(\operatorname{cl}\left(\operatorname{TH}\left(\mathbb{A}_{E, E}, \operatorname{Schur}\left(\mathbb{S}_{+}^{V^{*}}\right)\right)\right)\right) & & \text { by }(5.45) \\
& =\operatorname{abl}\left(\operatorname{cl}\left(\operatorname{TH}\left(\mathbb{A}_{E, E}, \operatorname{Schur}\left(\mathbb{S}_{+}^{V}\right)\right)\right)\right) & & \text { since } \mathbb{S}_{+}^{V *}=\mathbb{S}_{+}^{V} \\
& =\operatorname{abl}\left(\operatorname{TH}\left(\mathbb{A}_{E, E}, \operatorname{Schur}\left(\mathbb{S}_{+}^{V}\right)\right)\right) & & \text { by }(4.28) \\
& =\operatorname{abl}\left(\mathrm{TH}^{+}\left(\mathbb{A}_{E, E}, \operatorname{Psd}\left(\mathbb{S}_{+}^{V}\right)\right)\right) & & \text { by }(5.25) \text { and }(5.29) \\
& =\operatorname{abl}(\operatorname{TH}(G)) . & & \text { by }(5.38 \mathrm{a})
\end{aligned}
$$

Proposition 5.8. Let $G$ be a graph. Then

$$
\operatorname{STAB}(G) \subseteq \mathrm{TH}^{\prime}(G) \subseteq \mathrm{TH}(G) \subseteq \mathrm{TH}^{+}(G) \subseteq \operatorname{QSTAB}(G) .
$$

Proof. We first prove that

$$
\operatorname{STAB}(G) \subseteq \mathrm{TH}^{\prime}(G)
$$

Let $S \in \mathcal{S}(G)$. Since $S$ is stable, we have that

$$
\mathbb{1}_{S} \mathbb{1}_{S}^{\top} \in \mathbb{A}_{E \cup \bar{E}, E}
$$

Therefore

$$
\begin{aligned}
{\left[\begin{array}{cc}
1 & \mathbb{1}_{S}^{\mathrm{T}} \\
\mathbb{1}_{S} & \mathbb{1}_{S} \mathbb{1}_{S}^{\mathrm{T}}
\end{array}\right] } & \in \widehat{\mathrm{TH}}\left(\mathbb{A}_{E \cup \bar{E}, E}, \mathbb{C P}^{\{0\} \cup V}\right) & & \text { by }(5.21) \text { and }(5.51) \\
& \subseteq \widehat{\mathrm{TH}}\left(\mathbb{A}_{E \cup \bar{E}, E}, \operatorname{Psd}\left(\mathbb{C P}^{V}\right)\right) & & \text { by }(5.31) \text { and }(5.26) \\
& \subseteq \widehat{\mathrm{TH}}\left(\mathbb{A}_{E \cup \bar{E}, E}, \operatorname{Psd}\left(\mathbb{S}_{+}^{V}\right)\right) & & \text { by }(5.31),(5.30 \mathrm{a}), \text { and }(5.17)
\end{aligned}
$$

Both (5.38b) and (5.36) imply that $\mathbb{1}_{S} \in \mathrm{TH}^{\prime}(G)$. As this holds for every $S$, convexity of $\mathrm{TH}^{\prime}(G)$ implies (5.50).

We also have that

$$
\operatorname{TH}^{\prime}(G) \subseteq \operatorname{TH}(G)
$$

Indeed, note that

$$
\widehat{\mathrm{TH}}\left(\mathbb{A}_{E \cup \bar{E}, E}, \operatorname{Psd}\left(\mathbb{S}_{+}^{V}\right)\right) \subseteq \widehat{\mathrm{TH}}\left(\mathbb{A}_{E, E}, \operatorname{Psd}\left(\mathbb{S}_{+}^{V}\right)\right)
$$

by (5.31) and $\mathbb{A}_{E \cup \bar{E}, E} \subseteq \mathbb{A}_{E, E}$. Both (5.36) and (5.38) then imply (5.52).

Combining (5.50) and (5.52) with (4.26) we conclude that

$$
\operatorname{abl}(\operatorname{TH}(G)) \subseteq \operatorname{abl}\left(\operatorname{TH}^{\prime}(G)\right) \subseteq \operatorname{abl}(\operatorname{STAB}(G)) .
$$

Therefore, Proposition 4.27, (5.47), and (5.48) imply that

$$
\mathrm{TH}(\bar{G}) \subseteq \mathrm{TH}^{+}(\bar{G}) \subseteq \operatorname{QSTAB}(\bar{G}) .
$$

As this argument holds for every $G$, the statement follows.

Theorem 5.7 provides substance to our claim that the Psd and Schur liftings are dual to each other. The following proposition, which will be useful in Section 5.3, shows that this duality is actually even simpler.

Proposition 5.9 ([28, Proposition 7]). Let $V$ be a finite set. Let $\mathbb{K} \subseteq \mathbb{S}_{+}^{V}$ be a closed convex cone such that $\operatorname{Diag}\left(\mathbb{R}_{+}^{V}\right) \subseteq \mathbb{K}$ and $\operatorname{int}(\mathbb{K}) \neq \varnothing$. Then $[\operatorname{Psd}(\mathbb{K})]^{*}=\operatorname{Schur}\left(\mathbb{K}^{*}\right)$ and $\left[\operatorname{Schur}\left(\mathbb{K}^{*}\right)\right]^{*}=\operatorname{Psd}(\mathbb{K})$ 
Let $G$ and $H$ be graphs, and let $f \in \operatorname{Hom}(G, H)$. Proposition 5.3 and (5.11a) and (5.11c) imply that

$$
\begin{aligned}
P_{f}^{\top} \mathbb{C P}^{V(H)} P_{f} & \subseteq \mathbb{C P}^{V(G)}, \\
P_{f}^{\top} \mathbb{S}_{+}^{V(H)} P_{f} & \subseteq \mathbb{S}_{+}^{V(G)} .
\end{aligned}
$$

A similar property also holds for the polyhedral matrix cones that appear in (5.38). Using that

$$
\left(P_{f}^{\top} A P_{f}\right)_{i j}=A_{f(i) f(j)}
$$

for every $A \in \mathbb{S}^{V(H)}$, one may easily check that

$$
\begin{aligned}
P_{f}^{\top} \mathbb{A}_{E(H), E(H)} P_{f} & \subseteq \mathbb{A}_{E(G), E(G)}, \\
P_{f}^{\top} \mathbb{A}_{E(\bar{H}) \cup E(H), E(H)} P_{f} & \subseteq \mathbb{A}_{E(\bar{G}) \cup E(G), E(G)}, \\
P_{f}^{\top} \mathbb{A}_{\varnothing, E(H)} P_{f} & \subseteq \mathbb{A}_{\varnothing, E(G)} .
\end{aligned}
$$

These properties are interesting in that they relate distinct cones while exploiting the graph-theoretic information. Inspired by these examples, we may consider functions $\mathbb{K}$ on graphs associating a cone $\mathbb{K}_{G} \subseteq \mathbb{S}^{V(G)}$ for every graph $G$, and such that

$$
P_{f}^{\top} \mathbb{K}_{H} P_{f} \subseteq \mathbb{K}_{G}
$$

for every $G$ and $H$ graphs and $f \in \operatorname{Hom}(G, H)$. In our next result, we will use that, for every finite sets $S$ and $T$, for every function $f: S \rightarrow T$, and for every $x \in \mathbb{R}^{S}$,

$$
\operatorname{Diag}\left(P_{f} x\right)=P_{f} \operatorname{Diag}(x) P_{f}^{\top} .
$$

To see that (5.57), note that

$$
\begin{aligned}
\operatorname{Diag}\left(P_{f} x\right) & =\sum_{i \in S} x_{i} \operatorname{Diag}\left(P_{f} e_{i}\right) \\
& =\sum_{i \in S} x_{i} \operatorname{Diag}\left(e_{f(i)}\right) \\
& =\sum_{i \in S} x_{i} e_{f(i)} e_{f(i)}^{\top} \\
& =\sum_{i \in S} x_{i} P_{f} e_{i} e_{i}^{\top} P_{f}^{\top} \\
& =P_{f} \operatorname{Diag}(x) P_{f}^{\top} .
\end{aligned}
$$

Theorem 5.10. Let $\mathbb{A}$ and $\mathbb{K}$ be functions on graphs associating cones $\mathbb{A}_{F} \subseteq \mathbb{S}^{V(G)}$ and $\mathbb{K}_{F} \subseteq \mathbb{S} V(F)$ to every graph $F$, both satisfying (5.56). Then for every graphs $G$ and $H$ and $f \in \operatorname{Hom}(G, H)$,

$$
P_{f}^{\top} \operatorname{TH}\left(\mathbb{A}_{H}, \operatorname{Psd}\left(\mathbb{K}_{H}\right)\right) \subseteq \operatorname{TH}\left(\mathbb{A}_{G}, \operatorname{Psd}\left(\mathbb{K}_{G}\right)\right) .
$$

Proof. Let

$$
\left[\begin{array}{ll}
1 & x^{\top} \\
x & X
\end{array}\right]:=\widehat{X} \in \widehat{\mathrm{TH}}\left(\mathbb{A}_{H}, \operatorname{Psd}\left(\mathbb{K}_{H}\right)\right) .
$$

Since $\widehat{X} \in \mathbb{S}_{+}^{\{0\} \cup V(H)}$, we have that

$$
\begin{aligned}
\mathbb{S}_{+}^{\{0\} \cup V(G)} & \ni\left[\begin{array}{cc}
1 & 0^{\top} \\
0 & P_{f}^{\top}
\end{array}\right]\left[\begin{array}{cc}
1 & x^{\top} \\
x & X
\end{array}\right]\left[\begin{array}{cc}
1 & 0^{\top} \\
0 & P_{f}
\end{array}\right] \\
& =\left[\begin{array}{cc}
1 & x^{\top} \\
P_{f}^{\top} x & P_{f}^{\top} X
\end{array}\right]\left[\begin{array}{ll}
1 & 0^{\top} \\
0 & P_{f}
\end{array}\right] \\
& =\left[\begin{array}{cc}
1 & x^{\top} P_{f} \\
P_{f}^{\top} x & P_{f}^{\top} X P_{f}
\end{array}\right] .
\end{aligned}
$$

by Proposition 5.3 and (5.11c) 
Moreover, since both $\mathbb{A}$ and $\mathbb{K}$ satisfy (5.56), we have that $P_{f}^{\top} X P_{f} \in \mathbb{A}_{G} \cap \mathbb{K}_{G}$. Moreover, note that

$$
\operatorname{diag}\left(P_{f}^{\top} X P_{f}\right)=P_{f}^{\top} x .
$$

This is true as for every $y \in \mathbb{R}^{V(G)}$,

$$
\begin{aligned}
y^{\top} \operatorname{diag}\left(P_{f}^{\top} X P_{f}\right) & =\left\langle\operatorname{Diag}(y), P_{f}^{\top} X P_{f}\right\rangle & & \text { by }(2.22) \\
& =\operatorname{Tr}\left(\operatorname{Diag}(y) P_{f}^{\top} X P_{f}\right) & & \text { by }(5.1) \\
& =\operatorname{Tr}\left(P_{f} \operatorname{Diag}(y) P_{f}^{\top} X\right) & & \text { by }(2.18) \\
& =\operatorname{Tr}\left(\operatorname{Diag}\left(P_{f} y\right) X\right) & & \text { by }(5.57) \\
& =\left\langle\operatorname{Diag}\left(P_{f} y\right), X\right\rangle & & \text { by }(5.1) \\
& =y^{\top} P_{f}^{\top} \operatorname{diag}(X) . & &
\end{aligned}
$$

We conclude that

$$
\left[\begin{array}{cc}
1 & x^{\top} P_{f} \\
P_{f}^{\top} x & P_{f}^{\top} X P_{f}
\end{array}\right] \in \widehat{\mathrm{TH}}\left(\mathbb{A}_{G}, \operatorname{Psd}\left(\mathbb{K}_{G}\right)\right)
$$

which implies that $P_{f}^{\top} x \in \operatorname{TH}\left(\mathbb{A}_{G}, \operatorname{Psd}\left(\mathbb{K}_{G}\right)\right)$.

Theorem 5.11. Let $S$ and $T$ be finite sets. Let $f: S \rightarrow T$. Let $\beta_{S}: \mathbb{R}_{+}^{S} \rightarrow \mathbb{R}$ and $\beta_{T}: \mathbb{R}_{+}^{T} \rightarrow \mathbb{R}$ be positive definite monotone gauges. The following are equivalent:

1. $\beta_{S}\left(P_{f}^{\top} z\right) \leq \beta_{T}(z)$ for every $z \in \mathbb{R}_{+}^{T}$,

2. $\operatorname{unit}\left(\beta_{S}\right) \supseteq P_{f}^{\top} \operatorname{unit}\left(\beta_{T}\right)$,

3. $\beta_{S}^{\circ}(w) \geq \beta_{T}^{\circ}\left(P_{f} w\right)$ for every $w \in \mathbb{R}_{+}^{S}$, and

4. $P_{f} \operatorname{unit}\left(\beta_{S}^{\circ}\right) \subseteq \operatorname{unit}\left(\beta_{T}^{\circ}\right)$.

Proof. Item 1 implies Item 2 : let $z \in \operatorname{unit}\left(\beta_{T}\right)$. Item 1 implies $P_{f}^{\top} z \in \operatorname{unit}\left(\beta_{S}\right)$, since

$$
\beta_{S}\left(P_{f}^{\top} z\right) \leq \beta_{T}(z) \leq 1 .
$$

Item 2 implies Item 3: for every $w \in \mathbb{R}_{+}^{S}$,

$$
\begin{aligned}
\beta_{S}^{\circ}(w) & =\delta^{*}\left(w \mid \operatorname{unit}\left(\beta_{S}\right)\right) & & \text { by }(4.42) \\
& \geq \delta^{*}\left(w \mid P_{f}^{\top} \operatorname{unit}\left(\beta_{T}\right)\right) & & \text { by item } 2 \text { and Corollary } 4.16 \\
& =\delta^{*}\left(P_{f} w \mid \operatorname{unit}\left(\beta_{T}\right)\right) & & \text { by }(2.50) \\
& =\beta_{T}^{\circ}\left(P_{f} w\right) . & & \text { by }(4.42)
\end{aligned}
$$

Item 3 implies Item 4: let $w \in \operatorname{unit}\left(\beta_{S}^{\circ}\right)$. Item 3 implies $P_{f} w \in \operatorname{unit}\left(\beta_{T}^{\circ}\right)$, as

$$
\beta_{T}^{\circ}\left(P_{f} w\right) \leq \beta_{S}^{\circ}(w) \leq 1 .
$$

Item 4 implies Item 1: for every $z \in \mathbb{R}_{+}^{T}$,

$$
\begin{aligned}
\beta_{T}(z) & =\gamma\left(z \mid \operatorname{unit}\left(\beta_{T}\right)\right) & & \text { by Proposition } 4.10 \\
& =\gamma\left(z \mid \operatorname{abl}\left(\operatorname{abl}\left(\operatorname{unit}\left(\beta_{T}\right)\right)\right)\right) & & \text { by item } 3 \text { of Theorem } 4.25 \text { and Proposition } 4.10 \\
& =\gamma\left(z \mid \operatorname{abl}\left(\operatorname{unit}\left(\beta_{T}^{\circ}\right)\right)\right) & & \text { by item } 2 \text { of Theorem } 4.26 \\
& =\delta^{*}\left(z \mid \operatorname{unit}\left(\beta_{T}^{\circ}\right)\right) & & \text { by item } 2 \text { of Theorem } 4.25 \\
& \geq \delta^{*}\left(z \mid P_{f} \operatorname{unit}\left(\beta_{S}^{\circ}\right)\right) & & \text { by item } 4 \\
& =\delta^{*}\left(P_{f}^{\top} z \mid \operatorname{unit}\left(\beta_{S}^{\circ}\right)\right) & & \text { by }(2.50) \\
& =\gamma\left(P_{f}^{\top} z \mid \operatorname{abl}\left(\operatorname{unit}\left(\beta_{S}^{\circ}\right)\right)\right) & & \text { by item } 2 \text { of Theorem } 4.25 \\
& =\gamma\left(P_{f}^{\top} z \mid \operatorname{unit}\left(\beta_{S}^{\circ \circ}\right)\right) & & \text { by item } 2 \text { of Theorem } 4.26 \\
& =\gamma\left(P_{f}^{\top} z \mid \operatorname{unit}\left(\beta_{S}\right)\right) & & \text { by item } 3 \text { of Theorem } 4.26 \\
& =\beta_{S}\left(P_{f}^{\top} z\right) . & & \text { by Proposition } 4.10
\end{aligned}
$$


Proposition 5.12. Let $\mathbb{K}$ be a function on graphs associating cones $\mathbb{K}_{G} \subseteq \mathbb{S}^{V(G)}$ for every graph $G$ such that

1. for every graph $H:=(V, E)$, the set $\mathbb{K}_{H} \subseteq \mathbb{S}_{+}^{V}$ is a diagonally scaling-invariant closed convex cone such that $\operatorname{Diag}\left(\mathbb{R}_{+}^{V}\right) \subseteq \mathbb{K}_{H}$ and $\operatorname{int}\left(\mathbb{K}_{H}\right) \neq \varnothing$;

2. $\mathbb{K}$ satisfies (5.56).

Let $E^{+}, E^{-}$be function on graphs such that $E^{+}(H), E^{-}(H) \subseteq\left(\begin{array}{c}V(H) \\ 2\end{array}\right)$ for every graph $H$, and such that

$$
H \mapsto \mathbb{A}_{E^{+}(H), E^{-}(H)}
$$

satisfies (5.56). Then

$$
G \mapsto \vartheta\left(\mathbb{A} \overline{E^{+}(G)}, \overline{E^{-}(G)}, \operatorname{Schur}\left(\mathbb{K}_{G}^{*}\right), \mathbb{1}\right)
$$

is homomorphism monotone.

Proof. Let $G$ and $H$ be graphs, and let $f \in \operatorname{Hom}(G, H)$. For $F \in\{G, H\}$, set $\mathbb{A}_{F}:=\mathbb{A}_{E^{+}(F), E^{-}(F)}$, set $\overline{\mathbb{A}}_{F}:=\mathbb{A}_{\overline{E^{+}(F)}}, \overline{E^{-}(F)}$, let $\mathbb{1}_{F} \in \mathbb{R}^{V(F)}$ denote the all-ones vector, and set

$$
\beta_{F}:=\vartheta\left(\overline{\mathbb{A}}_{F}, \operatorname{Schur}\left(\mathbb{K}_{F}\right), \cdot\right) .
$$

We claim that for every $F \in\{G, H\}$.

$$
\operatorname{unit}\left(\beta_{F}\right)=\operatorname{TH}\left(\mathbb{A}_{F}, \operatorname{Psd}\left(\mathbb{K}_{F}\right)\right) .
$$

as

$$
\begin{aligned}
\operatorname{unit}\left(\beta_{F}\right) & =\operatorname{unit}\left(\vartheta\left(\overline{\mathbb{A}}_{F}, \operatorname{Schur}\left(\mathbb{K}_{F}^{*}\right), \cdot\right)\right) & & \text { by }(5.59) \\
& =\operatorname{unit}\left(\delta^{*}\left(\cdot \mid \operatorname{TH}\left(\overline{\mathbb{A}}_{F}, \operatorname{Schur}\left(\mathbb{K}_{F}^{*}\right)\right)\right)\right) & & \text { by }(5.37) \\
& =\operatorname{unit}\left(\delta^{*}\left(\cdot \mid \operatorname{cl}\left(\operatorname{TH}\left(\overline{\mathbb{A}}_{F}, \operatorname{Schur}\left(\mathbb{K}_{F}^{*}\right)\right)\right)\right)\right) & & \text { by }(2.46) \\
& =\operatorname{unit}\left(\gamma\left(\cdot \mid \operatorname{abl}\left(\operatorname{cl}\left(\operatorname{TH}\left(\overline{\mathbb{A}}_{F}, \operatorname{Schur}\left(\mathbb{K}_{F}^{*}\right)\right)\right)\right)\right)\right) & & \text { by }(5.44) \text { and item } 2 \text { of Theorem } 4.25 \\
& =\operatorname{unit}\left(\gamma\left(\cdot \mid \operatorname{TH}\left(\mathbb{A}_{F}, \operatorname{Psd}\left(\mathbb{K}_{F}\right)\right)\right)\right) & & \text { by }(5.45) \\
& =\operatorname{TH}\left(\mathbb{A}_{F}, \operatorname{Psd}\left(\mathbb{K}_{F}\right)\right) . & & \text { by }(5.44) \text { and Proposition } 4.11
\end{aligned}
$$

Then

$$
P_{f}^{\top} \operatorname{unit}\left(\beta_{H}\right) \subseteq \operatorname{unit}\left(\beta_{G}\right),
$$

since

$$
\begin{aligned}
\operatorname{unit}\left(\beta_{G}\right) & =\operatorname{TH}\left(\mathbb{A}_{G}, \operatorname{Psd}\left(\mathbb{K}_{G}\right)\right) & & \text { by }(5.60) \\
& \supseteq P_{f}^{\top} \operatorname{TH}\left(\mathbb{A}_{H}, \operatorname{Psd}\left(\mathbb{K}_{H}\right)\right) & & \text { by Theorem } 5.10 \\
& =P_{f}^{\top} \operatorname{unit}\left(\beta_{H}\right) . & & \text { by }(5.60)
\end{aligned}
$$

The statement follows:

$$
\begin{aligned}
\vartheta\left(\overline{\mathbb{A}}_{G}, \operatorname{Schur}\left(\mathbb{K}_{G}\right), \mathbb{1}_{G}\right) & =\beta_{G}\left(\mathbb{1}_{G}\right) & & \text { by }(5.59) \\
& =\beta_{G}\left(P_{f}^{\top} \mathbb{1}_{H}\right) & & \text { by }(2.15) \\
& \leq \beta_{H}\left(\mathbb{1}_{H}\right) & & \text { by Theorem } 5.11 \text { and }(5.61) \\
& =\vartheta\left(\overline{\mathbb{A}}_{G}, \operatorname{Schur}\left(\mathbb{K}_{G}\right), \mathbb{1}_{G}\right) . & & \text { by }(5.59)
\end{aligned}
$$

Proposition 5.12, (5.53b), and (5.55) provide a single proof that

$$
\overline{\vartheta^{\prime}}, \bar{\vartheta} \text {, and } \overline{\vartheta^{+}} \text {are homomorphism monotone }
$$

since these parameters are defined as in (5.39). Similarly, Proposition 5.12, (5.53a), and (5.55a) imply that

$$
\chi_{f} \text { is homomorphism monotone }
$$

since

$$
\chi_{f}(G, \cdot)=\delta^{*}(\cdot \mid \operatorname{QSTAB}(\bar{G}))=\delta^{*}\left(\cdot \mid \operatorname{TH}\left(\mathbb{A}_{E, E}, \operatorname{Schur}\left(\left(\mathbb{C P}^{V}\right)^{*}\right)\right)\right)
$$

by $(5.41)$. 


\subsection{Dual Conic-programming Bounds for the Maximum Cut and Cut-Covering Problems}

In this section we develop conic-programming bounds for the maximum cut and for the fractional cut-covering problems. These developments arise naturally from the concepts presented so far. Section 5.2 defined convex corners containing the stable set polytope, from suitable matrix cones. In Subsection 5.3.1 we translate such convex corners into convex corners containing the cut polytope, thus obtaining conic-programming approximations for the maximum cut problem. In Subsection 5.3.3 we use the geometric bound-conversion procedure from Section 4.3 to obtain bounds for the fractional cut-covering problem. Subsection 5.3.2 focuses on the special case of the semidefinite cone.

Before diving into our results, we recall some basic properties of the maximum cut and the fractional cut-covering numbers. Let $G:=(V, E)$ be a graph. Theorem 4.29 states that

$$
x(G, z)=\delta^{*}(z \mid \operatorname{abl}(\operatorname{CUT}(G)))
$$

for every $z \in \mathbb{R}_{+}^{E}$. Although $\operatorname{CUT}(G)$ is not itself a convex corner, Proposition 4.28 states that

both $\operatorname{lc}(\mathrm{CUT}(G))$ and $\operatorname{abl}(\mathrm{CUT}(G))$ are convex corners

and

$$
\operatorname{abl}(\operatorname{lc}(\operatorname{CUT}(G)))=\operatorname{abl}(\operatorname{CUT}(G)) .
$$

\subsubsection{Conic Approximations for the CUT Polytope}

In this subsection, we present conic programs which define real-valued positive definite monotone gauges that upper bound the value of the maximum cut of a graph. We make no authorship claims on the ideas presented here, as we mostly aim at introducing, in the language of this text, objects which are either well known or quite similar to well-known objects.

Let $G:=(V, E)$ be a graph. Define $\mathcal{A}_{G}: \mathbb{R}^{E} \rightarrow \mathbb{R}^{V \times V}$ as

$$
\mathcal{A}_{G}(w):=\sum_{i j \in E} w_{i j}\left(e_{i} e_{j}^{\top}+e_{j} e_{i}^{\top}\right)
$$

for every $w \in \mathbb{R}^{E}$. In words, $\mathcal{A}_{G}$ converts edge weights into weighted adjacency matrices, allowing us to conveniently move between $\mathbb{R}^{E}$ and $\mathbb{R}^{V \times V}$. Note that for every $w \in \mathbb{R}^{E}$,

$$
\mathcal{A}_{G}^{*}(X)=\sum_{i j \in E}\left(X_{i j}+X_{j i}\right) e_{i j},
$$

since for every $X \in \mathbb{R}^{V \times V}$ and $y \in \mathbb{R}^{E}$,

$$
\begin{aligned}
\left\langle\mathcal{A}_{G}^{*}(X), y\right\rangle & =\left\langle X, \mathcal{A}_{G}(y)\right\rangle \\
& =\sum_{i j \in E} y_{i j}\left\langle X, e_{i} e_{j}^{\top}+e_{j} e_{i}^{\top}\right\rangle \\
& =\sum_{i j \in E} y_{i j}\left(X_{i j}+X_{j i}\right) \\
& =\left\langle\sum_{i j \in E}\left(X_{i j}+X_{j i}\right) e_{i j}, y\right\rangle .
\end{aligned}
$$

We thus have that

$$
\mathcal{A}_{G}^{*}\left(\mathcal{A}_{G}(w)\right)=2 w
$$


for every $w \in \mathbb{R}^{E}$, since

$$
\begin{array}{rlrl}
\mathcal{A}_{G}^{*}\left(\mathcal{A}_{G}(w)\right) & =\mathcal{A}_{G}^{*}\left(\sum_{i j \in E} w_{i j}\left(e_{i} e_{j}^{\top}+e_{j} e_{i}^{\top}\right)\right) & & \text { by }(5.66) \\
& =\sum_{i j \in E} w_{i j} \mathcal{A}_{G}^{*}\left(e_{i} e_{j}^{\top}+e_{j} e_{i}^{\top}\right) & & \\
& =\sum_{i j \in E} w_{i j} 2 e_{i j} & & \text { by }(5.67) \\
& =2 w . &
\end{array}
$$

Let us do a simple calculation that implies that, for every $S \subseteq V(G)$,

$$
\mathcal{A}_{G}^{*}\left(\mathbb{1}_{\bar{S}} \mathbb{1}_{S}^{\top}\right)=\mathcal{A}_{G}^{*}\left(\mathbb{1}_{S} \mathbb{1} \frac{\mathrm{T}}{S}\right)=\mathbb{1}_{\delta(S)}
$$

where $\bar{S}:=V \backslash S$. Indeed,

$$
\begin{aligned}
\mathcal{A}_{G}^{*}\left(\mathbb{1}_{\bar{S}} \mathbb{1}_{S}^{\top}\right) & =\sum_{i j \in E}\left(\left(\mathbb{1}_{\bar{S}} \mathbb{1}_{S}^{\top}\right)_{i j}+\left(\mathbb{1}_{\bar{S}} \mathbb{1}_{S}^{\mathrm{\top}}\right)_{j i}\right) e_{i j} \\
& =\sum_{i j \in E}([i \in \bar{S}][j \in S]+[j \in \bar{S}][i \in S]) e_{i j} \\
& =\sum_{i j \in E}[i j \in \delta(S)] e_{i j} \\
& =\mathbb{1}_{\delta(S)} .
\end{aligned}
$$

It is obvious from (5.67) that $\mathcal{A}_{G}^{*}\left(X^{\top}\right)=\mathcal{A}_{G}^{*}(X)$ for every $X \in \mathbb{R}^{V \times V}$, so we conclude (5.69). We now use (5.69) to describe an object in $\mathbb{S}^{V}$ which encodes $\operatorname{CUT}(G)$. Define the cut polytope $\mathrm{CUT}_{U}^{ \pm 1}$ for every finite set $U$ as

$$
\operatorname{CUT}_{U}^{ \pm 1}:=\operatorname{conv}\left(\left\{x x^{\top} \mid x \in\{ \pm 1\}^{U}\right\}\right) .
$$

We recover $\operatorname{CUT}(G)$ from $\operatorname{CUT}_{V}^{ \pm 1}$ in a simple manner.

Proposition 5.13. For every graph $G:=(V, E)$,

$$
\operatorname{CUT}(G)=\frac{1}{4} \mathcal{A}_{G}^{*}\left(\mathbb{1} \mathbb{1}^{\top}-\mathrm{CUT}_{V}^{ \pm 1}\right) .
$$

Proof. Let $S \subseteq V$ and define $x_{S}:=\mathbb{1}_{S}-\mathbb{1}_{\bar{S}}$, where $\bar{S}:=V \backslash S$. We have that

$$
\mathcal{A}_{G}^{*}\left(\mathbb{1}^{\top}-x_{S} x_{S}^{\top}\right)=4 \mathbb{1}_{\delta(S)},
$$

since

$$
\begin{aligned}
\mathcal{A}_{G}^{*}\left(\mathbb{1}^{\top}-x_{S} x_{S}^{\top}\right) & =\mathcal{A}_{G}^{*}\left(\left(\mathbb{1}_{S}+\mathbb{1}_{\bar{S}}\right)\left(\mathbb{1}_{S}+\mathbb{1}_{\bar{S}}\right)^{\top}-\left(\mathbb{1}_{S}-\mathbb{1}_{\bar{S}}\right)\left(\mathbb{1}_{S}-\mathbb{1}_{\bar{S}}\right)^{\top}\right) \\
& =\mathcal{A}_{G}^{*}\left(2\left(\mathbb{1}_{S} \mathbb{1}_{\bar{S}}^{\mathrm{T}}+\mathbb{1}_{\bar{S}} \mathbb{1}_{S}^{\top}\right)\right) \\
& =2\left(\mathbb{1}_{\delta(S)}+\mathbb{1}_{\delta(S)}\right) \\
& =4 \mathbb{1}_{\delta(S)} .
\end{aligned}
$$

It is easy to see that

$$
\text { for every } x \in\{ \pm 1\}^{V} \text { there exists a unique } S \subseteq V \text { such that } x=x_{S} .
$$

Therefore

$$
\begin{aligned}
& \frac{1}{4} \mathcal{A}_{G}^{*}\left(\mathbb{1}^{\top}-\mathrm{CUT}_{V}^{ \pm 1}\right)=\left\{\frac{1}{4} \mathcal{A}_{G}^{*}\left(\mathbb{1} \mathbb{1}^{\top}-X\right) \mid X \in \mathrm{CUT}_{V}^{ \pm 1}\right\} \\
& =\operatorname{conv}\left(\left\{\frac{1}{4} \mathcal{A}_{G}^{*}\left(\mathbb{1}^{\top}-x x^{\top}\right) \mid x \in\{ \pm 1\}^{V}\right\}\right) \quad \text { by (5.70) and (2.49) } \\
& =\operatorname{conv}\left(\left\{\frac{1}{4} \mathcal{A}_{G}^{*}\left(\mathbb{1}^{\top}-x_{S} x_{S}^{\top}\right) \mid S \subseteq V\right\}\right) \quad \text { by }(5.72) \\
& =\operatorname{conv}\left(\left\{\mathbb{1}_{\delta(S)} \mid S \subseteq V\right\}\right) \quad \text { by }(5.71) \\
& =\operatorname{CUT}(G) \text {. }
\end{aligned}
$$


Let $T$ and $U$ be finite sets. Let $A \in \mathbb{R}^{U \times T}$. Define $\mathcal{C}_{A}: \mathbb{R}^{T \times T} \rightarrow \mathbb{R}^{U \times U}$ by

$$
\mathcal{C}_{A}(X):=A X A^{\top}
$$

for every $X \in \mathbb{R}^{T \times T}$. Note that $\mathcal{C}_{A}$ is a linear transformation, and if $A$ is invertible then $\mathcal{C}_{A}(X)$ is congruent to $X$ for every $X \in \mathbb{R}^{T \times T}$ — which explains the choice of notation.

Proposition 5.14. Let $T$ and $U$ be finite sets. Let $A \in \mathbb{R}^{U \times T}$. Then

$$
\mathcal{C}_{A}^{*}=\mathcal{C}_{A^{\top}}
$$

Proof. For every $X \in \mathbb{R}^{T \times T}$ and $Y \in \mathbb{R}^{U \times U}$,

$$
\begin{aligned}
\left\langle\mathcal{C}_{A}^{*}(Y), X\right\rangle & =\left\langle Y, \mathcal{C}_{A}(X)\right\rangle & & \\
& =\operatorname{Tr}\left(Y^{\top} \mathcal{C}_{A}(X)\right) & & \text { by }(5.1) \\
& =\operatorname{Tr}\left(Y^{\top} A X A^{\top}\right) & & \text { by }(5.73) \\
& =\operatorname{Tr}\left(A^{\top} Y^{\top} A X\right) & & \text { by }(2.18) \\
& =\left\langle A^{\top} Y A, X\right\rangle & & \text { by }(5.1) \\
& =\left\langle\mathcal{C}_{A^{\top}}(Y), X\right\rangle . & & \text { by }(5.73)
\end{aligned}
$$

Proposition 5.15. Let $T$ and $U$ be finite sets. Let $A \in \mathbb{R}^{U \times T}$. If $A$ is invertible, then so is $\mathcal{C}_{A}$, and

$$
\mathcal{C}_{A}^{-1}=\mathcal{C}_{A^{-1}}
$$

Proof. Note that

for every $X \in \mathbb{R}^{T \times T}$, and

$$
\mathcal{C}_{A}^{-1}\left(\mathcal{C}_{A}(X)\right)=A^{-1} A X A^{\top} A^{-\top}=X
$$

$$
\mathcal{C}_{A}\left(\mathcal{C}_{A^{-1}}(Y)\right)=A A^{-1} Y A^{-\top} A^{\top}=Y
$$

for every $Y \in \mathbb{R}^{U \times U}$.

One of Karp's original NP-complete problems [66] is to decide, given a graph $G$ and a natural number $k$, if there exists a cut in $G$ of cardinality at least $k$. For this reason, our goal is to exploit Proposition 5.13 to translate approximations for $\mathrm{CUT}_{V}^{ \pm}$into approximations for $\operatorname{CUT}(G)$, where $G$ is a graph and $V=V(G)$. If $V$ is a finite set and $0 \notin V$, we identify

$$
\mathbb{R} \oplus \mathbb{R}^{V}=\mathbb{R}^{\{0\} \cup V} .
$$

In particular, we have that $(1 \oplus x)(1 \oplus x)^{\top} \in \mathbb{S}^{\{0\} \cup V}$.

Proposition 5.16 ([28]). Let $V$ be a finite set. Let $\mathbb{K} \subseteq \mathbb{S}^{V}$ be a convex cone such that $\mathbb{C P}^{V} \subseteq \mathbb{K}$. Then

$$
\operatorname{conv}\left(\left\{(1 \oplus x)(1 \oplus x)^{\top} \mid x \in\{0,1\}^{V}\right\}\right) \subseteq \widehat{\mathrm{TH}}\left(\mathbb{S}^{V}, \operatorname{Psd}(\mathbb{K})\right) .
$$

Proof. Note that $\mathbb{C P}^{V} \subseteq \mathbb{K}$ implies that $\operatorname{Psd}\left(\mathbb{C P}^{V}\right) \subseteq \operatorname{Psd}(\mathbb{K})$ by (5.30a), which in turn implies that

$$
\widehat{\mathrm{TH}}\left(\mathbb{S}^{V}, \operatorname{Psd}\left(\mathbb{C P}^{V}\right)\right) \subseteq \widehat{\mathrm{TH}}\left(\mathbb{S}^{V}, \operatorname{Psd}(\mathbb{K})\right)
$$

by $(5.31)$.

Let $x \in\{0,1\}^{V}$. Then there exists $S \subseteq V$ such that $x=\mathbb{1}_{S}$, so

$$
\begin{aligned}
(1 \oplus x)(1 \oplus x)^{\top} & =\left[\begin{array}{cc}
1 & \mathbb{1}_{S}^{\top} \\
\mathbb{1}_{S} & \mathbb{1}_{S} \mathbb{1}_{S}^{\top}
\end{array}\right] & & \\
& \in \widehat{\mathrm{TH}}\left(\mathbb{S}^{V}, \mathbb{C P}^{\{0\} \cup V}\right) & & \text { by }(5.21) \\
& \left.\subseteq \widehat{\mathrm{TH}}\left(\mathbb{S}^{V}, \operatorname{Psd}\left(\mathbb{C P}^{V}\right)\right)\right) & & \text { by }(5.26) \text { and }(5.31) \\
& \subseteq \widehat{\mathrm{TH}}\left(\mathbb{S}^{V}, \operatorname{Psd}(\mathbb{K})\right) . & & \text { by }(5.30 \mathrm{a}),(5.31), \text { and } \mathbb{C P}^{V} \subseteq \mathbb{K}
\end{aligned}
$$

Since $\widehat{\mathrm{TH}}\left(\mathbb{S}^{V}, \operatorname{Psd}(\mathbb{K})\right)$ is convex, the proof is done. 
Let $V$ be a finite set. At a high level, the role of Proposition 5.16 is to provide an approximation for the boolean quadric polytope

$$
\operatorname{conv}\left(\left\{x x^{\top} \mid x \in\{0,1\}^{V}\right\}\right)
$$

using a convex cone contained in $\mathbb{S}^{V}$. Our next proposition combines Proposition 5.16 with a well-known linear function which maps the boolean quadric polytope to the cut polytope $\mathrm{CUT}_{V}^{ \pm 1}$. Following [29], define

$$
\text { Bool : }=\left[\begin{array}{cc}
1 & 0^{\top} \\
\frac{1}{2} \mathbb{1} & \frac{1}{2} I
\end{array}\right] \in \mathbb{S}^{\{0\} \cup V} .
$$

One may directly check that

$$
\mathrm{Bool}^{-1}=\left[\begin{array}{cc}
1 & 0^{\top} \\
-\mathbb{1} & 2 I
\end{array}\right] .
$$

Moreover, for every $S \subseteq V$

$$
\operatorname{Bool}^{-1}\left(1 \oplus \mathbb{1}_{S}\right)=1 \oplus\left(\mathbb{1}_{S}-\mathbb{1}_{\bar{S}}\right),
$$

where $\bar{S}:=V \backslash S$, since

$$
\operatorname{Bool}^{-1}\left(1 \oplus \mathbb{1}_{S}\right)=\left[\begin{array}{cc}
1 & 0^{\top} \\
-\mathbb{1} & 2 I
\end{array}\right]\left[\begin{array}{c}
1 \\
\mathbb{1}_{S}
\end{array}\right]=\left[\begin{array}{c}
1 \\
2 \mathbb{1}_{S}-\mathbb{1}
\end{array}\right]=1 \oplus\left(\mathbb{1}_{S}-\mathbb{1}_{\bar{S}}\right) .
$$

The reader may notice that we implicitly used Bool in the proof of Proposition 5.13.

Let $V$ be a finite set. Let $\mathbb{K} \subseteq \mathbb{S}^{V}$ be a convex cone such that $\mathbb{C P}^{V} \subseteq \mathbb{K}$. The generalized elliptope $\mathcal{E}_{\mathbb{K}}$ is defined by

$$
\mathcal{E}_{\mathbb{K}}:=\mathcal{C}_{\text {Bool }}^{-1}\left(\widehat{\mathrm{TH}}\left(\mathbb{S}^{V}, \operatorname{Psd}(\mathbb{K})\right)\right)[V] .
$$

We remark that in (5.78), one first applies $\mathcal{C}_{\text {Bool }}^{-1}$ and only then takes the principal submatrix; as this is the only order in which both operations can be applied, we omitted an additional pair of parenthesis. As the name suggests, $\mathcal{E}_{\mathbb{K}}$ is a generalization of the elliptope

$$
\mathcal{E}_{V}:=\left\{Y \in \mathbb{S}_{+}^{V} \mid \operatorname{diag}(Y)=\mathbb{1}\right\} .
$$

If $\mathbb{K}_{0}$ and $\mathbb{K}_{1}$ are convex cones in $\mathbb{S}^{V}$ which contain $\mathbb{C P}^{V}$, then

$$
\mathbb{K}_{0} \subseteq \mathbb{K}_{1} \Longrightarrow \mathcal{E}_{\mathbb{K}_{0}} \subseteq \mathcal{E}_{\mathbb{K}_{1}}
$$

This holds because $\mathbb{K}_{0} \subseteq \mathbb{K}_{1}$ and (5.30a) imply that $\operatorname{Psd}\left(\mathbb{K}_{0}\right) \subseteq \operatorname{Psd}\left(\mathbb{K}_{1}\right)$, which together (5.31) imply that

$$
\widehat{\mathrm{TH}}\left(\mathbb{S}^{V}, \operatorname{Psd}\left(\mathbb{K}_{0}\right)\right) \subseteq \widehat{\mathrm{TH}}\left(\mathbb{S}^{V}, \operatorname{Psd}\left(\mathbb{K}_{1}\right)\right),
$$

so (5.80) follows from (5.78).

Proposition 5.17. Let $V$ be a finite set. Let $\mathbb{K} \subseteq \mathbb{S}^{V}$ be a convex cone such that $\mathbb{C P}^{V} \subseteq \mathbb{K}$. Then

$$
\mathrm{CUT}_{V}^{ \pm 1} \subseteq \mathcal{E}_{\mathbb{K}}
$$

Proof. It holds that

$$
\begin{aligned}
& \operatorname{conv}\left(\left\{(1 \oplus x)(1 \oplus x)^{\top} \mid x \in\{ \pm 1\}^{V}\right\}\right) \\
& =\operatorname{conv}\left(\left\{\operatorname{Bool}^{-1}(1 \oplus y)(1 \oplus y)^{\top} \mathrm{Bool}^{-\top} \mid y \in\{0,1\}^{V}\right\}\right) \quad \text { by (5.77) } \\
& =\operatorname{conv}\left(\left\{\mathcal{C}_{\text {Bool }^{-1}}\left((1 \oplus y)(1 \oplus y)^{\top}\right) \mid y \in\{0,1\}^{V}\right\}\right) \quad \text { by (5.73) } \\
& =\mathcal{C}_{\text {Bool }^{-1}}\left(\operatorname{conv}\left(\left\{(1 \oplus y)(1 \oplus y)^{\top} \mid y \in\{0,1\}^{V}\right\}\right)\right) \quad \text { by }(2.49) \\
& \subseteq \mathcal{C}_{\mathrm{Bool}^{-1}}\left(\widehat{\mathrm{TH}}\left(\mathbb{S}^{V}, \operatorname{Psd}(\mathbb{K})\right)\right) \quad \text { by Proposition } 5.16 \\
& =\mathcal{C}_{\text {Bool }}^{-1}\left(\widehat{\mathrm{TH}}\left(\mathbb{S}^{V}, \operatorname{Psd}(\mathbb{K})\right)\right) \quad \text { by Proposition } 5.15
\end{aligned}
$$

The statement follows by applying $\widehat{X} \in \mathbb{S}\{0\} \cup V \mapsto \widehat{X}[V]$ to both sides of the inclusion and then using (5.78). 
Laurent, Poljak, and Rendl [75] use $\mathcal{C}_{\text {Bool }}^{-1}$ to relate known approximations for the maximum cut problem to known approximations for the stable set problem, and also cite older texts with the same observation [31, $63]$.

Proposition 5.18 ([75, Proposition 4]). Let $V$ be a finite set. Then

$$
\mathcal{C}_{\text {Bool }}^{-1}\left(\widehat{\mathrm{TH}}\left(\mathbb{S}^{V}, \mathbb{S}_{+}^{\{0\} \cup V}\right)\right)=\mathcal{E}_{\{0\} \cup V} .
$$

Therefore $\mathcal{E}_{V}=\mathcal{E}_{\mathbb{S}_{+}^{V}}$.

Proof. We first prove that

$$
\mathcal{C}_{\text {Bool }}\left(\mathcal{E}_{\{0\} \cup V}\right) \subseteq \widehat{\mathrm{TH}}\left(\mathbb{S}^{V}, \mathbb{S}_{+}^{\{0\} \cup V}\right)
$$

Let

$$
\widehat{Y}:=\left[\begin{array}{cc}
1 & y^{\top} \\
y & Y
\end{array}\right] \in \mathcal{E}_{\{0\} \cup V}
$$

where $y \in \mathbb{R}^{V}$ and $Y \in \mathbb{S}^{V}$. Proposition 5.3 and (5.11c) imply that Bool $\widehat{Y}$ Bool $^{\top} \succeq 0$. Furthermore,

$$
\begin{aligned}
\text { Bool } \widehat{Y} \text { Bool }^{\top} & =\left[\begin{array}{cc}
1 & 0^{\top} \\
\frac{1}{2} \mathbb{1} & \frac{1}{2} I
\end{array}\right]\left[\begin{array}{cc}
1 & y^{\top} \\
y & Y
\end{array}\right]\left[\begin{array}{cc}
1 & \frac{1}{2} \mathbb{1}^{\top} \\
0 & \frac{1}{2} I
\end{array}\right] \\
& =\left[\begin{array}{cc}
1 & y^{\top} \\
\frac{1}{2}(\mathbb{1}+y) & \frac{1}{2}\left(\mathbb{1} y^{\top}+Y\right)
\end{array}\right]\left[\begin{array}{cc}
1 & \frac{1}{2} \mathbb{1}^{\top} \\
0 & \frac{1}{2} I
\end{array}\right] \\
& =\left[\begin{array}{cc}
1 & \frac{1}{2}(\mathbb{1}+y)^{\top} \\
\frac{1}{2}(\mathbb{1}+y)^{\top} & \frac{1}{4}\left(\mathbb{1} \mathbb{1}^{\top}+y \mathbb{1}^{\top}+\mathbb{1} y^{\top}+Y\right)
\end{array}\right] .
\end{aligned}
$$

Since $\operatorname{diag}(Y)=\mathbb{1}$, we have that

$$
\operatorname{diag}\left(\frac{1}{4}\left(\mathbb{1} \mathbb{1}^{\top}+y \mathbb{1}^{\top}+\mathbb{1} y^{\top}+Y\right)\right)=\frac{1}{4}(\mathbb{1}+y+y+\mathbb{1})=\frac{1}{2}(\mathbb{1}+y) .
$$

Thus (5.82) holds. Therefore,

$$
\begin{aligned}
\mathcal{E}_{\{0\} \cup V} & =\mathcal{C}_{\text {Bool }}^{-1}\left(\mathcal{C}_{\text {Bool }}\left(\mathcal{E}_{\{0\} \cup V}\right)\right) \\
& \subseteq \mathcal{C}_{\text {Bool }}^{-1}\left(\widehat{\mathrm{TH}}\left(\mathbb{S}^{V}, \mathbb{S}_{+}^{\{0\} \cup V}\right)\right) .
\end{aligned}
$$

We now prove that

$$
\mathcal{C}_{\text {Bool }}^{-1}\left(\widehat{\mathrm{TH}}\left(\mathbb{S}^{V}, \mathbb{S}_{+}^{\{0\} \cup V}\right)\right) \subseteq \mathcal{E}_{\{0\} \cup V}
$$

Let

$$
\widehat{X}:=\left[\begin{array}{cc}
1 & x^{\top} \\
x & X
\end{array}\right] \in \widehat{\mathrm{TH}}\left(\mathbb{S}^{V}, \mathbb{S}_{+}^{\{0\} \cup V}\right),
$$

where $x \in \mathbb{R}^{V}$ and $X \in \mathbb{S}^{V}$. Proposition 5.3 and (5.11c) imply that $\mathrm{Bool}^{-1} \widehat{X} \mathrm{Bool}^{-\mathrm{T}} \succeq 0$. Furthermore

$$
\begin{aligned}
\text { Bool }^{-1} \widehat{X} \text { Bool }^{-\top} & =\left[\begin{array}{cc}
1 & 0 \\
-\mathbb{1} & 2 I
\end{array}\right]\left[\begin{array}{cc}
1 & x^{\top} \\
x & X
\end{array}\right]\left[\begin{array}{cc}
1 & -\mathbb{1}^{\top} \\
0 & 2 I
\end{array}\right] \\
& =\left[\begin{array}{cc}
1 & x^{\top} \\
2 x-\mathbb{1} & 2 X-\mathbb{1} x^{\top}
\end{array}\right]\left[\begin{array}{cc}
1 & -\mathbb{1}^{\top} \\
0 & 2 I
\end{array}\right] \\
& =\left[\begin{array}{cc}
1 & \left(2 x-\mathbb{1}^{\top}\right. \\
2 x-\mathbb{1} & 4 X-2 \mathbb{1} x^{\top}-2 x \mathbb{1}^{\top}+\mathbb{1}^{\top}
\end{array}\right] .
\end{aligned}
$$

Since $\operatorname{diag}(X)=x$, we have that

$$
\operatorname{diag}\left(4 X-2 \mathbb{1} x^{\top}-2 x \mathbb{1}^{\top}+\mathbb{1} \mathbb{1}^{\top}\right)=4 x-2 x-2 x+\mathbb{1}=\mathbb{1} .
$$


Using Proposition 5.15 we conclude that $\mathcal{C}_{\text {Bool }}^{-1}(\widehat{X})=\mathcal{C}_{\mathrm{Bool}^{-1}}(\widehat{X}) \in \mathcal{E}_{\{0\} \cup V}$, thus proving (5.83). Both (5.82) and (5.83) imply (5.81). Furthermore, the generalized elliptope generalizes the elliptope:

$$
\begin{aligned}
\mathcal{E}_{V} & =\left\{Y \in \mathbb{S}_{+}^{V} \mid \operatorname{diag}(Y)=\mathbb{1}\right\} & & \text { by }(5.79) \\
& =\left(\left\{\widehat{Y} \in \mathbb{S}_{+}^{\{0\} \cup V} \mid \operatorname{diag}(\widehat{Y})=\mathbb{1}\right\}\right)[V] & & \\
& =\left(\mathcal{E}_{\{0\} \cup V}\right)[V] & & \text { by }(5.79) \\
& =\left(\mathcal{C}_{\text {Bool }}^{-1}\left(\widehat{\operatorname{TH}}\left(\mathbb{S}^{V}, \mathbb{S}_{+}^{\{0\} \cup V}\right)\right)\right)[V] & & \text { by }(5.81) \\
& =\mathcal{E}_{\mathbb{S}_{+}^{V} .} & & \text { by }(5.78) \text { and }(5.25)
\end{aligned}
$$

Let $G:=(V, E)$ be a graph. Recall Proposition 5.13, and define

$$
\operatorname{CUT}_{G}(\mathbb{K}):=\frac{1}{4} \mathcal{A}_{G}^{*}\left(\mathbb{1}^{\top}-\mathcal{E}_{\mathbb{K}}\right)
$$

for every convex cone $\mathbb{K} \subseteq \mathbb{S}^{V}$ such that $\mathbb{C P}^{V} \subseteq \mathbb{K}$. It is obvious from (5.84) and (5.80) that

$$
\mathbb{K}_{0} \subseteq \mathbb{K}_{1} \Longrightarrow \operatorname{CUT}_{G}\left(\mathbb{K}_{0}\right) \subseteq \operatorname{CUT}_{G}\left(\mathbb{K}_{1}\right),
$$

for every closed convex cones $\mathbb{K}_{0}, \mathbb{K}_{1} \subseteq \mathbb{S}^{V}$ which contain $\mathbb{C P}^{V}$. The condition that the cones contain $\mathbb{C P}^{V}$ is motivated by Proposition 5.16: we want to use relaxations of $\mathrm{CUT}_{V}^{ \pm 1}$ to produce relaxations of CUT $(G)$.

Let $G:=(V, E)$ be a graph. The relaxation $\operatorname{CUT}_{G}\left(\mathbb{S}_{+}^{V}\right)$ is well known. Let $w \in \mathbb{R}_{+}^{V}$. Define

$$
\eta(G, w):=\max \left\{\frac{1}{4}\left\langle\mathcal{A}_{G}(w), \mathbb{1} \mathbb{1}^{\top}-Y\right\rangle \mid Y \in \mathbb{S}_{+}^{V}, \operatorname{diag}(Y)=\mathbb{1}\right\} .
$$

Goemans and Williamson [46] proved that

$$
\alpha_{\mathrm{GW}} \eta(G, w) \leq \delta^{*}(w \mid \mathrm{CUT}(G)) \leq \eta(G, w),
$$

where

$$
\alpha_{\mathrm{GW}}:=\inf _{0<\theta \leq \pi} \frac{2}{\pi} \frac{\theta}{1-\cos \theta} .
$$

The first ' $\leq$ ' in (5.87) is proved by rounding an optimal solution of the SDP (5.86) to produce a randomized cut with expected objective value at least $\alpha_{\mathrm{GW}} \eta(G, w)$. These results fit nicely into our discussion. First note that

$$
\operatorname{CUT}_{G}\left(\mathbb{S}_{+}^{V}\right)=\left\{\frac{1}{4} \mathcal{A}_{G}^{*}\left(\mathbb{1} \mathbb{1}^{\top}-Y\right) \mid Y \in \mathbb{S}_{+}^{V}, \operatorname{diag}(Y)=\mathbb{1}\right\}
$$

since

$$
\begin{aligned}
\operatorname{CUT}_{G}\left(\mathbb{S}_{+}^{V}\right) & =\frac{1}{4} \mathcal{A}_{G}^{*}\left(\mathbb{1}^{\top}-\mathcal{E}_{\mathbb{S}_{+}}\right) & & \text {by }(5.84) \\
& =\frac{1}{4} \mathcal{A}_{G}^{*}\left(\mathbb{1}^{\top}-\mathcal{E}_{V}\right) & & \text { by Proposition } 5.18 \\
& =\left\{\frac{1}{4} \mathcal{A}_{G}^{*}\left(\mathbb{1}^{\top}-Y\right) \mid Y \in \mathbb{S}_{+}^{V}, \operatorname{diag}(Y)=\mathbb{1}\right\} . & & \text { by }(5.79)
\end{aligned}
$$

This implies that

$$
\eta(G, w)=\delta^{*}\left(w \mid \mathrm{CUT}_{G}\left(\mathbb{S}_{+}^{V}\right)\right)
$$

as

$$
\begin{aligned}
\delta^{*}\left(w \mid \mathrm{CUT}_{G}\left(\mathbb{S}_{+}^{V}\right)\right) & =\sup \left\{w^{\top} x \mid x \in \mathrm{CUT}_{G}\left(\mathbb{S}_{+}^{V}\right)\right\} & & \text { by (2.45) } \\
& =\sup \left\{w^{\top}\left(\frac{1}{4} \mathcal{A}_{G}^{*}\left(\mathbb{1}^{\top}-Y\right)\right) \mid Y \in \mathbb{S}_{+}^{V}, \operatorname{diag}(Y)=\mathbb{1}\right\} & & \text { by (5.89) } \\
& =\sup \left\{\frac{1}{4}\left\langle\mathcal{A}_{G}(w), \mathbb{1}^{\top}-Y\right\rangle \mid Y \in \mathbb{S}_{+}^{V}, \operatorname{diag}(Y)=\mathbb{1}\right\} & & \\
& =\eta(G, w) . & & \text { by }(5.86)
\end{aligned}
$$


Theorem 5.19. Let $G:=(V, E)$ be a graph. Let $\mathbb{K} \subseteq \mathbb{S}^{V}$ be a closed convex cone such that $\mathbb{C P}^{V} \subseteq \mathbb{K} \subseteq \mathbb{S}_{+}^{V}$. Then

$$
\text { both } \operatorname{lc}\left(\mathrm{CUT}_{G}(\mathbb{K})\right) \text { and } \operatorname{abl}\left(\mathrm{CUT}_{G}(\mathbb{K})\right) \text { are convex corners }
$$

and

$$
\operatorname{abl}\left(\operatorname{lc}\left(\operatorname{CUT}_{G}(\mathbb{K})\right)\right)=\operatorname{abl}\left(\operatorname{CUT}_{G}(\mathbb{K})\right) .
$$

Furthermore,

$$
\operatorname{CUT}(G) \subseteq \operatorname{CUT}_{G}(\mathbb{K}) .
$$

Proof. Corollary 4.23 implies (5.92). We prove that

$$
\mathrm{CUT}_{G}\left(\mathbb{S}_{+}^{V}\right) \subseteq \mathbb{R}_{+}^{E}
$$

Both (5.67) and (5.89) reduce (5.94) to proving that $1-Y_{i j} \geq 0$ for every $Y \in \mathcal{E}_{V}$ and $i j \in E$. But this is immediate from $\operatorname{diag}(Y)=\mathbb{1}$ and (5.20). Therefore

$$
\begin{aligned}
\operatorname{CUT}(G) & =\frac{1}{4} \mathcal{A}_{G}^{*}\left(\mathbb{1}^{\top}-\mathrm{CUT}_{V}^{ \pm 1}\right) & & \text { by Proposition } 5.13 \\
& \subseteq \frac{1}{4} \mathcal{A}_{G}^{*}\left(\mathbb{1} \mathbb{1}^{\top}-\mathcal{E}_{\mathbb{K}}\right) & & \text { by Proposition } 5.17 \\
& =\mathrm{CUT}_{G}(\mathbb{K}) & & \text { by }(5.84) \\
& \subseteq \operatorname{CUT}_{G}\left(\mathbb{S}_{+}^{V}\right) & & \text { by }(5.85) \text { and } \mathbb{K} \subseteq \mathbb{S}_{+}^{V} \\
& \subseteq \mathbb{R}_{+}^{E} & & \text { by }(5.94)
\end{aligned}
$$

Thus (5.93) holds and $\operatorname{CUT}_{G}(\mathbb{K}) \subseteq \mathbb{R}_{+}^{E}$. The set $\mathrm{CUT}_{G}(\mathbb{K})$ has a strictly positive element, as

$$
\frac{2}{|V|} \mathbb{1}=\sum_{i \in V} \frac{1}{|V|} \mathbb{1}_{\delta(\{i\})} \in \operatorname{CUT}(G) \subseteq \operatorname{CUT}_{G}(\mathbb{K}) .
$$

Using Corollary 4.20, we may conclude that

$$
\operatorname{lc}\left(\mathrm{CUT}_{G}(\mathbb{K})\right) \text { is a convex corner }
$$

if we prove that $\mathrm{CUT}_{G}(\mathbb{K})$ is convex and compact.

The set $\widehat{\mathrm{TH}}\left(\mathbb{S}^{V}, \operatorname{Psd}(\mathbb{K})\right)$ is clearly convex. Proposition 5.6 implies that $\widehat{\mathrm{TH}}\left(\mathbb{S}^{V}, \operatorname{Psd}(\mathbb{K})\right)$ is compact when $\mathbb{K} \subseteq \mathbb{S}_{+}^{V}$ is closed. This in turn implies that $\mathcal{E}_{\mathbb{K}}$ is convex and compact, as it is the image of $\widehat{\mathrm{TH}}\left(\mathbb{S}^{V}, \operatorname{Psd}(\mathbb{K})\right)$ under the linear function $\widehat{X} \in \mathbb{S}^{\{0\} \cup V} \mapsto \mathcal{C}_{\text {Bool }}^{-1}(\widehat{X})[V]$ by (5.78). This in turn implies that $\mathrm{CUT}_{G}(\mathbb{K})$ is convex and compact, as it is the image of $\mathcal{E}_{\mathbb{K}}$ under the affine function $X \in \mathbb{S}^{V} \mapsto \frac{1}{4} \mathcal{A}_{G}^{*}\left(\mathbb{1} \mathbb{1}^{\top}-X\right)$ by (5.84). In short, $\mathrm{CUT}_{G}(\mathbb{K})$ is convex and compact because $\widehat{\mathrm{TH}}\left(\mathbb{S}^{V}, \operatorname{Psd}(\mathbb{K})\right)$ is convex and compact, and each function in the following diagram preserves such properties:

$$
\widehat{\mathrm{TH}}\left(\mathbb{S}^{V(G)}, \operatorname{Psd}(\mathbb{K})\right) \stackrel{\widehat{X} \mapsto \mathcal{C}_{\text {Bool }}^{-1}(\widehat{X})[V]}{\longrightarrow} \mathcal{E}_{\mathbb{K}} \stackrel{X \mapsto \frac{1}{4} \mathcal{A}_{G}^{*}\left(\mathbb{1} \mathbb{1}^{\top}-X\right)}{\longrightarrow} \operatorname{CUT}_{G}(\mathbb{K}) .
$$

We thus conclude (5.95).

The proof is done, as

$$
\begin{aligned}
\operatorname{abl}\left(\mathrm{CUT}_{G}(\mathbb{K})\right)=\operatorname{abl}\left(\operatorname{lc}\left(\mathrm{CUT}_{G}(\mathbb{K})\right)\right) & \text { by }(5.92) \\
\text { is a convex corner } & \text { by item } 1 \text { of Theorem } 4.25 \text { and (5.95). }
\end{aligned}
$$

Corollary 5.20. Let $G:=(V, E)$ be a graph. Then $w \in \mathbb{R}_{+}^{E} \mapsto \eta(G, w)$ is a real-valued positive definite monotone gauge, and

$$
\operatorname{unit}(\eta(G, \cdot))=\operatorname{abl}\left(\mathrm{CUT}_{G}\left(\mathbb{S}_{+}^{V}\right)\right)
$$


Proof. We have that

$$
\eta(G, w)=\gamma\left(w \mid \operatorname{abl}\left(\mathrm{CUT}_{G}\left(\mathbb{S}_{+}^{V}\right)\right)\right)
$$

for every $w \in \mathbb{R}_{+}^{E}$, as

$$
\begin{aligned}
\eta(G, w) & =\delta^{*}\left(w \mid \operatorname{CUT}_{G}\left(\mathbb{S}_{+}^{V}\right)\right) & & \text { by }(5.90) \\
& =\delta^{*}\left(w \mid \operatorname{lc}\left(\operatorname{CUT}_{G}\left(\mathbb{S}_{+}^{V}\right)\right)\right) & & \text { by Corollary } 4.22 \\
& =\gamma\left(w \mid \operatorname{abl}\left(\operatorname{lc}_{\left.\left.\left(\mathrm{CUT}_{G}\left(\mathbb{S}_{+}^{V}\right)\right)\right)\right)}\right.\right. & & \text { by }(5.91) \text { and item } 2 \text { of Theorem } 4.25 \\
& =\gamma\left(w \mid \operatorname{abl}\left(\operatorname{CUT}_{G}\left(\mathbb{S}_{+}^{V}\right)\right)\right) . & & \text { by }(5.92)
\end{aligned}
$$

The set $\operatorname{abl}\left(\mathrm{CUT}_{G}\left(\mathbb{S}_{+}^{V}\right)\right)$ is a convex corner by (5.91), so Proposition 4.11 and (5.97) finish the proof.

Corollary 5.21. Let $G:=(V, E)$ be a graph, and let $\mathbb{K} \subseteq \mathbb{S}^{V}$ be a closed convex cone such that $\mathbb{C P}^{V} \subseteq \mathbb{K} \subseteq \mathbb{S}_{+}^{V}$. Then

$$
\alpha_{\mathrm{GW}} \operatorname{lc}\left(\mathrm{CUT}_{G}\left(\mathbb{S}_{+}^{V}\right)\right) \subseteq \operatorname{lc}(\mathrm{CUT}(G)) \subseteq \operatorname{lc}\left(\mathrm{CUT}_{G}(\mathbb{K})\right) \subseteq \operatorname{lc}\left(\mathrm{CUT}_{G}\left(\mathbb{S}_{+}^{V}\right)\right)
$$

where $\alpha_{\mathrm{GW}}$ is defined as in (5.88).

Proof. Set $\eta:=\eta(G, \cdot)$. We first prove that for every $w \in \mathbb{R}_{+}^{E}$,

$$
\alpha_{\mathrm{GW}} \eta(w)=\delta^{*}\left(w \mid \alpha_{\mathrm{GW}} \operatorname{abl}(\operatorname{unit}(\eta))\right) .
$$

Let $w \in \mathbb{R}_{+}^{E}$. Corollary 5.20 and Proposition 4.10 imply that unit $(\eta)$ is a convex corner. Therefore

$$
\begin{aligned}
\alpha_{\mathrm{GW}} \eta(w) & =\alpha_{\mathrm{GW}} \gamma(w \mid \operatorname{unit}(\eta)) & & \text { by Proposition } 4.10 \text { and Co } \\
& =\alpha_{\mathrm{GW}} \gamma(w \mid \operatorname{abl}(\operatorname{abl}(\operatorname{unit}(\eta)))) & & \text { by item } 3 \text { of Theorem } 4.25 \\
& =\alpha_{\mathrm{GW}} \delta^{*}(\cdot \mid \operatorname{abl}(\operatorname{unit}(\eta))) & & \text { by item } 2 \text { of Theorem } 4.25 \\
& =\delta^{*}\left(w \mid \alpha_{\mathrm{GW}} \operatorname{abl}(\operatorname{unit}(\eta))\right) . & &
\end{aligned}
$$

Moreover, we claim that

$$
\alpha_{\mathrm{GW}} \operatorname{abl}(\operatorname{unit}(\eta)) \subseteq \operatorname{lc}(\mathrm{CUT}(G))
$$

Note that

$$
\begin{aligned}
\delta^{*}\left(w \mid \alpha_{\mathrm{GW}} \operatorname{abl}(\operatorname{unit}(\eta))\right) & =\alpha_{\mathrm{GW}} \eta(w) & & \text { by }(5.98) \\
& \leq \delta^{*}(w \mid \operatorname{CUT}(G)) & & \text { by }(5.87) \\
& =\delta^{*}(w \mid \operatorname{lc}(\operatorname{CUT}(G))) & & \text { by Corollary } 4.22
\end{aligned}
$$

for every $w \in \mathbb{R}_{+}^{V}$. Thus, by Corollary 4.16, we can conclude (5.99) once we prove that both $\alpha_{\mathrm{GW}} \operatorname{abl}(\operatorname{unit}(\eta))$ and $\operatorname{lc}(\operatorname{CUT}(G))$ are convex corners. The latter is true by (5.64). To see that $\alpha_{\mathrm{GW}} \operatorname{abl}($ unit $(\eta))$ is a convex corner, note that

$$
\begin{aligned}
& \eta \text { is a positive definite monotone gauge } \\
& \Longrightarrow \operatorname{unit}(\eta) \text { is a convex corner } \\
& \Longrightarrow \operatorname{abl}(\operatorname{unit}(\eta)) \text { is a convex corner } \\
& \Longrightarrow \alpha_{\mathrm{GW}} \operatorname{abl}(\operatorname{unit}(\eta)) \text { is a convex corner. }
\end{aligned}
$$

by Corollary 5.20

by Proposition 4.10

by item 1 of Theorem 4.25

by Proposition 4.9

This proves (5.99).

The statement follows:

$$
\begin{aligned}
\alpha_{\mathrm{GW}} \operatorname{lc}\left(\mathrm{CUT}_{G}\left(\mathbb{S}_{+}^{V}\right)\right) & =\alpha_{\mathrm{GW}} \operatorname{abl}\left(\operatorname{abl}\left(\operatorname{lc}\left(\mathrm{CUT}_{G}\left(\mathbb{S}_{+}^{V}\right)\right)\right)\right) & & \text { by }(5.91) \text { and item } 3 \text { of Theorem } 4.25 \\
& =\alpha_{\mathrm{GW}} \operatorname{abl}\left(\operatorname{abl}\left(\mathrm{CUT}_{G}\left(\mathbb{S}_{+}^{V}\right)\right)\right) & & \text { by }(5.92) \\
& =\alpha_{\mathrm{GW}} \operatorname{abl}(\operatorname{unit}(\eta)) & & \text { by }(5.96) \\
& \subseteq \operatorname{lc}(\operatorname{CUT}(G)) & & \text { by }(5.99) \\
& \subseteq \operatorname{lc}\left(\operatorname{CUT}_{G}(\mathbb{K})\right) & & \text { by }(5.93) \text { and }(4.13) \\
& \subseteq \operatorname{lc}\left(\operatorname{CUT}_{G}\left(\mathbb{S}_{+}^{V}\right)\right) . & & \text { by } \mathbb{K} \subseteq \mathbb{S}_{+}^{V}, \quad(5.85), \text { and }(4.13)
\end{aligned}
$$




\subsubsection{An SDP-based Approximation Algorithm for the Fractional Cut-covering Number}

This subsection and Subsection 5.3.3 study the antiblockers of the convex corners in Corollary 5.21. After presenting some basic facts about these antiblockers, we focus on $\operatorname{abl}\left(\mathrm{CUT}_{G}\left(\mathbb{S}_{+}^{V}\right)\right)$ and provide an SDP which approximates the fractional cut-covering number. As a by-product, we obtain a new proof of Proposition 3.81, which approximates the fractional cut-covering number using $\overline{\vartheta^{\prime}}$.

Let $V$ be a finite set. Recalling the definitions of the antiblocker (4.25) and of the polar (2.51) of a set, it is immediate that

$$
\operatorname{abl}(\mathcal{C})=\mathcal{C}^{\circ} \cap \mathbb{R}_{+}^{V}
$$

for every $\mathcal{C} \subseteq \mathbb{R}_{+}^{V}$.

Proposition 5.22. Let $S$ and $T$ be finite sets. Let $C \subseteq \mathbb{R}^{S}$ be a convex set, and let $\mathcal{A}: \mathbb{R}^{S} \rightarrow \mathbb{R}^{T}$ be a linear transformation. Then

$$
\operatorname{abl}(\mathcal{A} C)=\left\{y \in \mathbb{R}_{+}^{T} \mid \mathcal{A}^{*}(y) \in C^{\circ}\right\} .
$$

Proof. By (5.100), it is enough to prove that $(\mathcal{A C})^{\circ}=\left\{y \in \mathbb{R}^{T} \mid \mathcal{A}^{*}(y) \in C^{\circ}\right\}$. This standard result (see, for example, [102, Corollary 16.3.2]) is easy to prove, as for every $y \in \mathbb{R}^{T}$,

$$
\begin{aligned}
y \in(\mathcal{A} C)^{\circ} & \Longleftrightarrow\langle y, \mathcal{A}(x)\rangle \leq 1 \text { for every } x \in C \\
& \Longleftrightarrow\left\langle\mathcal{A}^{*}(y), x\right\rangle \leq 1 \text { for every } x \in C \\
& \Longleftrightarrow \mathcal{A}^{*}(y) \in C^{\circ} .
\end{aligned}
$$

Corollary 5.23. Let $G:=(V, E)$ be a graph, and let $\mathbb{K} \subseteq \mathbb{S}^{V}$ be a closed convex cone such that $\mathbb{C P}^{V} \subseteq \mathbb{K} \subseteq \mathbb{S}_{+}^{V}$. Then

$$
\operatorname{abl}\left(\mathrm{CUT}_{G}\left(\mathbb{S}_{+}^{V}\right)\right) \subseteq \operatorname{abl}\left(\mathrm{CUT}_{G}(\mathbb{K})\right) \subseteq \operatorname{abl}(\mathrm{CUT}(G)) \subseteq \frac{1}{\alpha_{\mathrm{GW}}} \operatorname{abl}\left(\mathrm{CUT}_{G}\left(\mathbb{S}_{+}^{V}\right)\right) .
$$

Proof. Note that

$$
\operatorname{abl}\left(\operatorname{lc}\left(\alpha_{\mathrm{GW}} \operatorname{CUT}_{G}\left(\mathbb{S}_{+}^{V}\right)\right)\right)=\frac{1}{\alpha_{\mathrm{GW}}} \operatorname{abl}\left(\mathrm{CUT}_{G}\left(\mathbb{S}_{+}^{V}\right)\right)
$$

since

$$
\begin{aligned}
\operatorname{abl}\left(\operatorname{lc}\left(\alpha_{\mathrm{GW}} \operatorname{CUT}_{G}\left(\mathbb{S}_{+}^{V}\right)\right)\right) & =\operatorname{abl}\left(\alpha_{\mathrm{GW}} \operatorname{CUT}_{G}\left(\mathbb{S}_{+}^{V}\right)\right) & & \text { by Corollary } 4.23 \\
& =\left\{y \in \mathbb{R}_{+}^{T} \mid \alpha_{\mathrm{GW}} y \in \mathrm{CUT}_{G}\left(\mathbb{S}_{+}^{V}\right)^{\circ}\right\} & & \text { by Proposition } 5.22 \\
& =\left\{\frac{1}{\alpha_{\mathrm{GW}}} y \in \mathbb{R}_{+}^{T} \mid y \in \mathrm{CUT}_{G}\left(\mathbb{S}_{+}^{V}\right)^{\circ}\right\} & & \\
& =\frac{1}{\alpha_{\mathrm{GW}}}\left\{y \in \mathbb{R}_{+}^{T} \mid y \in \mathrm{CUT}_{G}\left(\mathbb{S}_{+}^{V}\right)^{\circ}\right\} & & \\
& =\frac{1}{\alpha_{\mathrm{GW}}} \operatorname{abl}\left(\mathrm{CUT}_{G}\left(\mathbb{S}_{+}^{V}\right)\right) . & & \text { by }(5.100)
\end{aligned}
$$

Then Corollary 5.21 and (4.26) imply that

$$
\operatorname{abl}\left(\operatorname{lc}\left(\operatorname{CUT}_{G}\left(\mathbb{S}_{+}^{V}\right)\right)\right) \subseteq \operatorname{abl}\left(\operatorname{lc}\left(\operatorname{CUT}_{G}(\mathbb{K})\right)\right) \subseteq \operatorname{abl}(\operatorname{lc}(\operatorname{CUT}(G))) \subseteq \operatorname{abl}\left(\operatorname{lc}\left(\alpha_{\mathrm{GW}} \operatorname{CUT}_{G}\left(\mathbb{S}_{+}^{V}\right)\right)\right)
$$

so Corollary 4.23 (thrice) and (5.101) finish the proof.

Corollary 5.24. Let $G:=(V, E)$ be a graph, and let $\mathbb{K}$ be a convex cone such that $\mathbb{C P}^{V} \subseteq \mathbb{K} \subseteq \mathbb{S}_{+}^{V}$. Then

$$
\operatorname{abl}\left(\mathrm{CUT}_{G}(\mathbb{K})\right)=\left\{4 y \mid y \in \mathbb{R}_{+}^{E}, \mathcal{A}_{G}(y) \in\left(\mathbb{1}^{\top}-\mathcal{E}_{\mathbb{K}}\right)^{\circ}\right\} .
$$

Proof. The result follows from a simple computation:

$$
\begin{aligned}
\operatorname{abl}\left(\operatorname{CUT}_{G}(\mathbb{K})\right) & =\operatorname{abl}\left(\frac{1}{4} \mathcal{A}_{G}^{*}\left(\mathbb{1} \mathbb{1}^{\top}-\mathcal{E}_{\mathbb{K}}\right)\right) & & \text { by }(5.84) \\
& =\left\{w \in \mathbb{R}_{+}^{E} \mid \frac{1}{4} \mathcal{A}_{G}(w) \in\left(\mathbb{1}^{\top}-\mathcal{E}_{\mathbb{K}}\right)^{\circ}\right\} & & \text { by Proposition } 5.22 \\
& =\left\{4 w \mid w \in \mathbb{R}_{+}^{E}, \mathcal{A}_{G}(w) \in\left(\mathbb{1}^{\top}-\mathcal{E}_{\mathbb{K}}\right)^{\circ}\right\} . & &
\end{aligned}
$$


Let $G:=(V, E)$ be a graph, and let $\alpha_{\mathrm{GW}}$ be defined as in (5.88). Since semidefinite programming provides good approximations for the maximum cut problem - recall (5.87) - it is natural to attempt to determine a number $\beta \in \mathbb{R}$ such that

$$
\beta \leq x(G) \leq \frac{1}{\alpha_{\mathrm{GW}}} \beta
$$

using semidefinite programming. Neto and Ben-Ameur [94, Proposition 9] recently used a bisection argument involving solving several SDP's to find a number $\beta$ satisfying (5.102). However, we may obtain such number $\beta$ by solving a single SDP: as [94, Corollary 4] points out, a previous argument by Šámal [103, Theorem 5.2] implies that

$$
\beta=2\left(1-\frac{1}{\overline{\vartheta^{\prime}}(G)}\right)
$$

satisfies (5.102). Our upcoming results shall improve on this remark, and follow from the core observation that

for every $z \in \mathbb{R}_{+}^{E}$, as

$$
\eta^{\circ}(G, z)=\delta^{*}\left(z \mid \operatorname{abl}\left(\mathrm{CUT}_{G}\left(\mathbb{S}_{+}^{V}\right)\right)\right)
$$

$$
\begin{aligned}
\eta^{\circ}(G, z) & =\max \left\{z^{\top} x \mid x \in \operatorname{unit}(\eta(G, \cdot))\right\} & & \text { by }(4.42) \\
& =\max \left\{z^{\top} x \mid x \in \operatorname{abl}\left(\mathrm{CUT}_{G}\left(\mathbb{S}_{+}^{V}\right)\right)\right\} & & \text { by }(5.96) \\
& =\delta^{*}\left(z \mid \operatorname{abl}\left(\mathrm{CUT}_{G}\left(\mathbb{S}_{+}^{V}\right)\right)\right) . & & \text { by }(2.45)
\end{aligned}
$$

The following proposition captures the idea behind (5.102).

Proposition 5.25. For every graph $G:=(V, E)$ and for every $z \in \mathbb{R}_{+}^{E}$,

$$
\eta^{\circ}(G, z) \leq x(G, z) \leq \frac{1}{\alpha_{\mathrm{GW}}} \eta^{\circ}(G, z),
$$

where $\alpha_{\mathrm{GW}}$ is defined as in (5.88).

Proof. For every $z \in \mathbb{R}_{+}^{E}$,

$$
\begin{aligned}
\eta^{\circ}(G, z) & =\delta^{*}\left(z \mid \operatorname{abl}\left(\mathrm{CUT}_{G}\left(\mathbb{S}_{+}^{V}\right)\right)\right) & & \text { by }(5.103) \\
& \leq \delta^{*}(z \mid \operatorname{abl}(\operatorname{CUT}(G))) & & \text { by Corollary } 5.23 \text { and Corollary } 4.16 \\
& \leq \delta^{*}\left(z \mid \frac{1}{\alpha_{\mathrm{GW}}} \operatorname{abl}\left(\mathrm{CUT}_{G}\left(\mathbb{S}_{+}^{V}\right)\right)\right) & & \text { by Corollary } 5.23 \text { and Corollary } 4.16 \\
& =\frac{1}{\alpha_{\mathrm{GW}}} \delta^{*}\left(z \mid \operatorname{abl}\left(\mathrm{CUT}_{G}\left(\mathbb{S}_{+}^{V}\right)\right)\right) & & \\
& =\frac{1}{\alpha_{\mathrm{GW}}} \eta^{\circ}(G, z) & & \text { by }(5.103)
\end{aligned}
$$

which implies the statement, as $x(G, z)=\delta^{*}(z \mid \operatorname{abl}(\mathrm{CUT}(G)))$ by (5.63).

Let $G:=(V, E)$ be a graph. From a computational complexity standpoint, as soon as we proved (5.90), we could use that $\eta(G, w)$ is the optimal value of an SDP for every $w \in \mathbb{R}_{+}^{E}$ and invoke [50, Corollary 3.5] to conclude we can optimize over abl $\left(\mathrm{CUT}_{G}\left(\mathbb{S}_{+}^{V}\right)\right)$ in polynomial time. Therefore, Proposition 5.25 and (5.103) imply the existence of a polynomial-time computable $\beta \in \mathbb{R}$ satisfying (5.102). The remainder of this subsection will present an SDP whose optimal value is $\eta^{\circ}(G, z)$ for every $z \in \mathbb{R}_{+}^{V}$. Our developments are slightly unusual, in that we deliberately avoid the most straightforward argument to the desired results; rather, we prioritize making our exposition similar to its generalization in the upcoming Subsection 5.3.3.

Proposition 5.26. Let $V$ be a finite set. For every $A \in \mathbb{S}^{V}$,

$$
\delta^{*}\left(A \mid \mathcal{E}_{\mathbb{S}_{+}^{V}}\right)=\max \left\{\langle A, X\rangle \mid X \in \mathbb{S}_{+}^{V}, \operatorname{diag}(X)=\mathbb{1}\right\}=\min \left\{\mathbb{1}^{\top} y \mid y \in \mathbb{R}^{V}, \operatorname{Diag}(y) \succeq A\right\} .
$$

Proof. Note that

$$
\begin{aligned}
\delta^{*}\left(A \mid \mathcal{E}_{\mathbb{S}_{+}^{V}}\right) & =\delta^{*}\left(A \mid \mathcal{E}_{V}\right) \\
& =\delta^{*}\left(A \mid\left\{X \in \mathbb{S}_{+}^{V} \mid \operatorname{diag}(X)=\mathbb{1}\right\}\right) \\
& =\sup \left\{\langle A, X\rangle \mid X \in \mathbb{S}_{+}^{V}, \operatorname{diag}(X)=\mathbb{1}\right\} \\
& \leq \inf \left\{y^{\top} \mathbb{1} \mid y \in \mathbb{R}^{V}, \operatorname{Diag}(y) \succeq A\right\} .
\end{aligned}
$$

by Proposition 5.18

by $(5.79)$

by $(2.45)$

item 1 of Theorem 2.11 
Equality and attainment are implied by items 2 and 3 of Theorem 2.11 , as $\stackrel{\circ}{X}:=I$ and $\stackrel{\circ}{y}:=\left(\lambda_{\max }(A)+1\right) \mathbb{1}$ are Slater points of their respective SDPs.

Corollary 5.27 ([74, Proposition 2.1]). Let $V$ be a finite set. Then

$$
\left(\mathbb{1}^{\top}-\mathcal{E}_{\mathbb{S}_{+}^{V}}\right)^{\circ}=\left\{Y \in \mathbb{S}^{V} \mid \exists y \in \mathbb{R}^{V}, Y+\operatorname{Diag}(y) \succeq 0,\left\langle Y+\operatorname{Diag}(y), \mathbb{1}^{\top}\right\rangle \leq 1\right\} .
$$

Proof. Let $Y \in \mathbb{S}^{V}$. Note that $Y \in\left(\mathbb{1}^{\top}-\mathcal{E}_{\mathbb{S}_{+}^{V}}\right)^{\circ}$ if and only if $\left\langle\mathbb{1} \mathbb{1}^{\top}, Y\right\rangle+\langle X,-Y\rangle \leq 1$ for every $X \in \mathcal{E}_{\mathbb{S}_{+}^{V}}$. Thus

$$
Y \in\left(\mathbb{1}^{\top}-\mathcal{E}_{\mathbb{S}_{+}^{V}}\right)^{\circ} \text { if and only if }\left\langle\mathbb{1} \mathbb{1}^{\top}, Y\right\rangle+\delta^{*}\left(-Y \mid \mathcal{E}_{\mathbb{S}_{+}^{V}}\right) \leq 1
$$

On the other hand,

$$
\begin{aligned}
& \left\langle\mathbb{1} \mathbb{1}^{\top}, Y\right\rangle+\delta^{*}\left(-Y \mid \mathcal{E}_{\mathbb{K}}\right) \\
& =\left\langle\mathbb{1} \mathbb{1}^{\top}, Y\right\rangle+\min \left\{\mathbb{1}^{\top} y \mid y \in \mathbb{R}^{V}, \operatorname{Diag}(y) \succeq-Y\right\} \\
& =\min \left\{\left\langle\mathbb{1} \mathbb{1}^{\top}, Y\right\rangle+\mathbb{1}^{\top} y \mid y \in \mathbb{R}^{V}, \operatorname{Diag}(y) \succeq-Y\right\} \\
& =\min \left\{\left\langle\mathbb{1} \mathbb{1}^{\top}, Y\right\rangle+\mathbb{1}^{\top} y \mid y \in \mathbb{R}^{V}, Y+\operatorname{Diag}(y) \succeq 0\right\} \\
& =\min \left\{\left\langle\mathbb{1} \mathbb{1}^{\top}, Y+\operatorname{Diag}(y)\right\rangle \mid y \in \mathbb{R}^{V}, Y+\operatorname{Diag}(y) \succeq 0\right\},
\end{aligned}
$$

so (5.104) implies the statement.

Theorem 5.28. Let $G:=(V, E)$ be a graph. Then

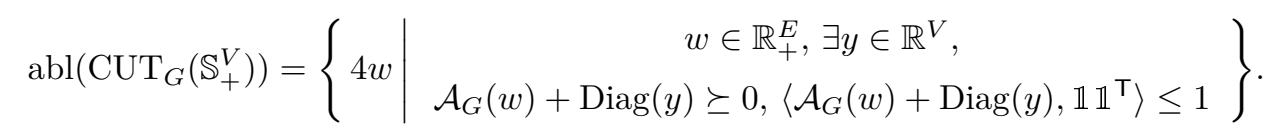

Consequently, for every $z \in \mathbb{R}_{+}^{E}$,

$$
\eta^{\circ}(G, z)=\max \left\{\begin{array}{l|c}
4 z^{\top} w & w \oplus y \in \mathbb{R}_{+}^{E} \oplus \mathbb{R}^{V}, \\
\mathcal{A}_{G}(w)+\operatorname{Diag}(y) \succeq 0,\left\langle\mathcal{A}_{G}(w)+\operatorname{Diag}(y), \mathbb{1} \mathbb{1}^{\top}\right\rangle \leq 1
\end{array}\right\} .
$$

Proof. We have that

$$
\begin{aligned}
\operatorname{abl}\left(\operatorname{CUT}_{G}\left(\mathbb{S}_{+}^{V}\right)\right) & =\left\{4 w \mid w \in \mathbb{R}_{+}^{E}, \mathcal{A}_{G}(w) \in\left(\mathbb{1}^{\top}-\mathcal{E}_{\mathbb{S}_{+}^{V}}\right)^{\circ}\right\} & \text { by Corollary } 5.24 \\
& =\left\{\begin{array}{l|l}
4 w \mid & \mathbb{R}_{+}^{E}, \exists y \in \mathbb{R}^{V}, \\
\mathcal{A}_{G}(w)+\operatorname{Diag}(y) \succeq 0,\left\langle\mathcal{A}_{G}(w)+\operatorname{Diag}(y), \mathbb{1}^{\top}\right\rangle \leq 1
\end{array}\right\} . & \text { by Corollary } 5.27
\end{aligned}
$$

Hence (5.105) holds. Furthermore

$$
\operatorname{abl}\left(\operatorname{CUT}_{G}\left(\mathbb{S}_{+}^{V}\right)\right) \text { is compact }
$$

by (5.91). Hence, for every $z \in \mathbb{R}_{+}^{E}$,

$$
\begin{aligned}
\eta^{\circ}(G, z) & =\delta^{*}\left(z \mid \operatorname{abl}\left(\mathrm{CUT}_{G}\left(\mathbb{S}_{+}^{V}\right)\right)\right) \\
& =\sup \left\{z^{\top} x \mid x \in \operatorname{abl}\left(\mathrm{CUT}_{G}\left(\mathbb{S}_{+}^{V}\right)\right)\right\} \\
& =\max \left\{z^{\top} x \mid x \in \operatorname{abl}\left(\mathrm{CUT}_{G}\left(\mathbb{S}_{+}^{V}\right)\right)\right\} \\
& =\max \left\{\begin{array}{c|c}
4 z^{\top} w \mid & w \oplus y \in \mathbb{R}_{+}^{E} \oplus \mathbb{R}^{V}, \\
& \mathcal{A}_{G}(w)+\operatorname{Diag}(y) \in \mathbb{S}_{+}^{V},\left\langle\mathcal{A}_{G}(w)+\operatorname{Diag}(y), \mathbb{1}^{\top}\right\rangle \leq 1
\end{array}\right\} .
\end{aligned}
$$

Corollary 5.29. Let $G:=(V, E)$ be a graph. Then for every $z \in \mathbb{R}_{+}^{E}$,

$$
\eta^{\circ}(G, z)=\max \left\{2\left\langle\mathcal{A}_{G}(z), X\right\rangle \mid X \in \mathbb{A}_{E \cup \bar{E}, \bar{E}} \cap \mathbb{S}_{+}^{V},\left\langle\mathbb{1} \mathbb{1}^{\top}, X\right\rangle \leq 1\right\},
$$

where $\bar{E}:=\left(\begin{array}{c}V \\ 2\end{array}\right) \backslash E$. 
Proof. The function $\mathcal{B}: \mathbb{R}_{+}^{E} \oplus \mathbb{R}^{V} \rightarrow \mathbb{A}_{E \cup \bar{E}, \bar{E}}$ defined by

$$
\mathcal{B}(w \oplus y):=\mathcal{A}_{G}(w)+\operatorname{Diag}(y)
$$

for every $w \in \mathbb{R}_{+}^{E}$ and $y \in \mathbb{R}^{V}$ is bijective. Furthermore, for every $w \in \mathbb{R}_{+}^{E}$ and $y \in \mathbb{R}^{V}$,

$$
2\left\langle\mathcal{B}(w \oplus y), \mathcal{A}_{G}(z)\right\rangle=4 w^{\top} z,
$$

as

$$
\begin{aligned}
2\left\langle\mathcal{B}(w \oplus y), \mathcal{A}_{G}(z)\right\rangle & =2\left\langle\mathcal{A}_{G}(w)+\operatorname{Diag}(y), \mathcal{A}_{G}(z)\right\rangle & & \text { by }(5.109) \\
& =2\left\langle\mathcal{A}_{G}(w), \mathcal{A}_{G}(z)\right\rangle & & \operatorname{since} \operatorname{diag}\left(\mathcal{A}_{G}(z)\right)=0 \\
& =2\left\langle w, \mathcal{A}_{G}^{*}\left(\mathcal{A}_{G}(z)\right)\right\rangle & & \\
& =4 w^{\top} z . & & \text { by }(5.68)
\end{aligned}
$$

It is immediate that $w \oplus y \mapsto \mathcal{B}(w \oplus y)$ is a homomorphism of optimization problems from (5.106) to (5.108), and that its inverse is a homomorphism of optimization problems from (5.108) to (5.106).

Specializing the SDP in Corollary 5.29 to the case $z=\mathbb{1}$, we shall recover the result from [94, 103] relating $\eta^{\circ}$ and $\overline{\vartheta^{\prime}}$. We will use that

$$
\text { if } E \neq \varnothing \text {, then } \overline{\vartheta^{\prime}}(G) \geq 2 .
$$

Propositions 3.29 and 4.41 imply that $\omega \leq \overline{\vartheta^{\prime}}$, which implies (5.110). We will also need another formulation of the $\vartheta^{\prime}$ function.

Theorem 5.30 ([28, Theorem 5]). Let $V$ be a finite set, and let $E^{+}, E^{-} \subseteq\left(\begin{array}{c}V \\ 2\end{array}\right)$. Let $\mathbb{K} \subseteq \mathbb{S}_{+}^{V}$ be a diagonally scaling-invariant closed convex cone such that $\operatorname{Diag}\left(\mathbb{R}_{+}^{V}\right) \subseteq \mathbb{K}$. Then

$$
\vartheta_{3}\left(\mathbb{A}_{E^{+}, E^{-}}, \operatorname{Psd}(\mathbb{K}), w\right)=\sup \left\{\left\langle\sqrt{w} \sqrt{w}^{\top}, X\right\rangle \mid \operatorname{Tr}(X)=1, X \in \mathbb{K}, X \in \mathbb{A}_{E^{+}, E^{-}}\right\} .
$$

Theorem 5.30 and $(5.39 \mathrm{~b})$ readily imply that

$$
\vartheta^{\prime}(G, w)=\sup \left\{\left\langle\sqrt{w} \sqrt{w}^{\top}, X\right\rangle \mid \operatorname{Tr}(X)=1, X \in \mathbb{S}_{+}^{V}, X \in \mathbb{A}_{E \cup \bar{E}, E}\right\}
$$

for every graph $G:=(V, E)$.

Corollary 5.31. Let $G$ be a graph. Then

$$
\eta^{\circ}(G, \mathbb{1})=2\left(1-\frac{1}{\overline{\vartheta^{\prime}}(G)}\right) .
$$

Proof. Let $(V, E):=G$, and set $\bar{E}:=\left(\begin{array}{c}V \\ 2\end{array}\right) \backslash E$. We claim that

$$
\frac{1}{2} \eta^{\circ}(G, \mathbb{1})=\max \left\{\left\langle A_{G}, X\right\rangle \mid X \in \mathbb{A}_{E \cup \bar{E}, \bar{E}} \cap \mathbb{S}_{+}^{V},\left\langle X, \mathbb{1}^{\top}\right\rangle \leq 1\right\},
$$

and that

$$
\overline{\vartheta^{\prime}}(G)=\max \left\{\left\langle\mathbb{1} \mathbb{1}^{\top}, X\right\rangle \mid X \in \mathbb{A}_{E \cup \bar{E}, \bar{E}} \cap \mathbb{S}_{+}^{V}, \operatorname{Tr}(X)=1\right\} .
$$

To see that (5.112) holds, note that

$$
\begin{array}{rlrl}
\frac{1}{2} \eta^{\circ}(G, \mathbb{1}) & =\frac{1}{2} \max \left\{2\left\langle\mathcal{A}_{G}(\mathbb{1}), X\right\rangle \mid X \in \mathbb{A}_{E \cup \bar{E}, \bar{E}} \cap \mathbb{S}_{+}^{V},\left\langle\mathbb{1} \mathbb{1}^{\top}, X\right\rangle \leq 1\right\} & & \text { by Corollary } 5.29 \\
& =\frac{1}{2} \max \left\{2\left\langle A_{G}, X\right\rangle \mid X \in \mathbb{A}_{E \cup \bar{E}, \bar{E}} \cap \mathbb{S}_{+}^{V},\left\langle\mathbb{1}^{\top}, X\right\rangle \leq 1\right\} & & \text { since } \mathcal{A}_{G}(\mathbb{1})=A_{G} \\
& =\max \left\{\left\langle A_{G}, X\right\rangle \mid X \in \mathbb{A}_{E \cup \bar{E}, \bar{E}} \cap \mathbb{S}_{+}^{V},\left\langle\mathbb{1}^{\top}, X\right\rangle \leq 1\right\} . &
\end{array}
$$

On the other hand, (5.113) simply restates (5.111) for $\bar{G}$. 
First note that if $E=\varnothing$, then $G \leftrightarrow K_{1}$, which implies that $\overline{\vartheta^{\prime}}(G)=1$. Therefore the RHS of the statement is zero. On the other hand, if $E=\varnothing$, then $A_{G}=0$, so (5.112) implies that $\eta^{\circ}(G, \mathbb{1})=0$. Hence, we assume that

$$
E \neq \varnothing \text {. }
$$

Let $X_{\eta}$ and $X_{\vartheta}$ be optimal solutions of the optimization problems in (5.112) and (5.113), respectively. Using (5.114) and (5.110) we conclude that $\left\langle\mathbb{1}^{\top}, X_{\vartheta}\right\rangle=\overline{\vartheta^{\prime}}(G)>0$, so $\left\langle\mathbb{1} \mathbb{1}^{\top}, X_{\vartheta}\right\rangle^{-1} X_{\vartheta}$ is defined and feasible in (5.112). Hence

$$
\begin{aligned}
\frac{1}{2} \eta^{\circ}(G, \mathbb{1}) & \geq\left\langle\left\langle X_{\vartheta}, \mathbb{1}^{\top}\right\rangle^{-1} X_{\vartheta}, A_{G}\right\rangle \\
& =\left\langle X_{\vartheta}, \mathbb{1}^{\top}\right\rangle^{-1}\left\langle X_{\vartheta}, \mathbb{1}^{\top}-I\right\rangle \\
& =1-\frac{1}{\left\langle X_{\vartheta}, \mathbb{1} \mathbb{1}^{\top}\right\rangle} \\
& =1-\frac{1}{\overline{\vartheta^{\prime}}(G)} .
\end{aligned}
$$

since $X_{\vartheta} \in \mathbb{A}_{E \cup \bar{E}, \bar{E}}$

since $\operatorname{Tr}\left(X_{\vartheta}\right)=1$

Thus ' $\geq$ ' holds in the statement. Hence, we can use (5.114) and (5.110) to conclude that

$$
\left\langle X_{\eta}, A_{G}\right\rangle=\frac{1}{2} \eta^{\circ}(G, \mathbb{1}) \geq 2\left(1-\frac{1}{\overline{\vartheta^{\prime}}(G)}\right)>0 .
$$

Thus $X_{\eta} \neq 0$, which together with $X_{\eta} \succeq 0$ implies that $\operatorname{Tr}\left(X_{\eta}\right)>0$. Consequently, $\operatorname{Tr}\left(X_{\eta}\right)^{-1} X_{\eta}$ is defined and feasible in (5.113). Therefore

$$
\begin{array}{rlrl}
\overline{\vartheta^{\prime}}(G) & \geq\left\langle\operatorname{Tr}\left(X_{\eta}\right)^{-1} X_{\eta}, \mathbb{1}^{\top}\right\rangle & \\
& =\operatorname{Tr}\left(X_{\eta}\right)^{-1}\left\langle X_{\eta}, I+A_{G}\right\rangle & & \text { since } X_{\eta} \in \mathbb{A}_{E \cup \bar{E}, \bar{E}} \\
& =1+\frac{\left\langle X_{\eta}, A_{G}\right\rangle}{\left\langle X_{\eta}, I\right\rangle} & & \\
& =1+\frac{\left\langle X_{\eta}, A_{G}\right\rangle}{\left\langle X_{\eta}, \mathbb{1} \mathbb{1}^{\top}\right\rangle-\left\langle X_{\eta}, A_{G}\right\rangle} & & \text { since } X_{\eta} \in \mathbb{A}_{E \cup \bar{E}, \bar{E}} \\
& \geq 1+\frac{\left\langle X_{\eta}, A_{G}\right\rangle}{1-\left\langle X_{\eta}, A_{G}\right\rangle} & & \text { by }(5.115) \text { and }\left\langle X_{\eta}, \mathbb{1} \mathbb{1}^{\top}\right\rangle \leq 1 \\
& =\frac{1}{1-\frac{1}{2} \eta^{\circ}(G, \mathbb{1})} . & & \text { since } \eta^{\circ}(G, \mathbb{1})=2\left\langle X_{\eta}, A_{G}\right\rangle
\end{array}
$$

Rearranging terms, we conclude that ' $\leq$ ' holds in the statement.

Freund [37] presents dual gauge programs. In his work, the gauge dual of a program is obtained from the Lagrangean dual by exchanging the constraint with the objective value, just as we have done in (5.112) and (5.113). In fact, once one proves the last step in

$$
\begin{aligned}
\frac{1}{2} \eta^{\circ}(G, \mathbb{1}) & =\max \left\{\left\langle A_{G}, X\right\rangle \mid X \in \mathbb{A}_{E \cup \bar{E}, \bar{E}} \cap \mathbb{S}_{+}^{V},\left\langle\mathbb{1} \mathbb{1}^{\top}, X\right\rangle \leq 1\right\} \\
& =\max \left\{\left\langle\mathbb{1} \mathbb{1}^{\top} X-I,\right\rangle \mid X \in \mathbb{A}_{E \cup \bar{E}, \bar{E}} \cap \mathbb{S}_{+}^{V},\left\langle\mathbb{1} \mathbb{1}^{\top}, X\right\rangle \leq 1\right\} \quad \text { since } A \in \mathbb{A}_{E \cup \bar{E}, \bar{E}} \\
& =1-\min \left\{\operatorname{Tr}(X) \mid X \in \mathbb{A}_{E \cup \bar{E}, \bar{E}} \cap \mathbb{S}_{+}^{V},\left\langle\mathbb{1}^{\top}, X\right\rangle \leq 1\right\},
\end{aligned}
$$

it is possible to directly use the result in [37]. Gauge duality has received a lot of attention in the optimization community recently; see [3, 38].

Recall that, in Subsection 3.4.8, we stated that the fractional cut-covering and the vector chromatic numbers are related because both parameters optimize "compatible" objective functions over representations of the graph in the unit sphere. Corollary 5.31 made such claims precise. Furthermore, the objective value in Corollary 5.29 makes it evident that this "compatibility" only holds when the weights in the edges are uniform. Let $G$ be a graph with nonempty edge set. We remark that

$$
2\left(1-\frac{1}{\overline{\vartheta^{\prime}}(G)}\right) \leq x(G)
$$


which follows directly from Proposition 5.25 and Corollary 5.31, was first proved in Corollary 3.80. We thus have yet another result which can be obtained using either homomorphism composition or antiblocking duality.

Corollary 5.32 ([94, 103]). For every graph $G$ with nonempty edge set,

$$
2\left(1-\frac{1}{\overline{\vartheta^{\prime}}(G)}\right) \leq x(G) \leq \frac{\pi}{\arccos \left(\left(1-\overline{\vartheta^{\prime}}(G)\right)^{-1}\right)} \leq \frac{2}{\alpha_{\mathrm{GW}}}\left(1-\frac{1}{\overline{\vartheta^{\prime}}(G)}\right)
$$

Proof. The first inequality is (5.116), whereas the second inequality comes from Proposition 3.81. Set

$$
\gamma:=-\frac{1}{\overline{\vartheta^{\prime}}(G)-1}
$$

so

$$
\begin{gathered}
-1 \leq \gamma<0 \text { and } \\
\frac{1}{1-\gamma}=1-\frac{1}{\overline{\vartheta^{\prime}}(G)} .
\end{gathered}
$$

We used (5.110) in (5.117b). By (5.117b), we may set $\theta:=\arccos \gamma$, so (5.88) implies that

$$
\alpha_{\mathrm{GW}} \leq \frac{2}{\pi} \frac{\theta}{1-\cos \theta}=\frac{2}{\pi} \frac{\arccos \gamma}{1-\gamma}
$$

Thus

$$
\begin{aligned}
\frac{\pi}{\arccos \left(\left(1-\overline{\vartheta^{\prime}}(G)\right)^{-1}\right)} & =\frac{\pi}{\arccos \gamma} & & \text { by }(5.117 \mathrm{a}) \\
& \leq \frac{2}{\alpha_{\mathrm{GW}}} \frac{1}{1-\gamma} & & \text { by }(5.118) \\
& =\frac{2}{\alpha_{\mathrm{GW}}}\left(1-\frac{1}{\overline{\vartheta^{\prime}}(G)}\right) . & & \text { by }(5.117 \mathrm{c})
\end{aligned}
$$

\subsubsection{Conic Approximations for the Fractional Cut-covering Number}

We now convert the upper bounds for the maximum cut problem presented in Subsection 5.3.1 into lower bounds for the fractional cut-covering problem, generalizing for other matrix cones what we achieved at Subsection 5.3.2 for the positive semidefinite cone. We exploit Proposition 5.9 to formulate the support function of the lifted theta body as a conic programming problem. With this formulation, we are able to calculate the polar of sets of the form $\mathbb{1}^{\top}-\mathcal{E}_{\mathbb{K}}$ for suitable cones $\mathbb{K}$, which is a key step in computing $\operatorname{abl}\left(\mathrm{CUT}_{G}(\mathbb{K})\right)$. This provides a family of conic programming lower bounds for the fractional cut-covering number.

Proposition 5.33. Let $V$ be a finite set. Let $\mathbb{K}$ be a closed convex cone such that $\mathbb{C P}^{V} \subseteq \mathbb{K} \subseteq \mathbb{S}_{+}^{V}$. Then $[\operatorname{Psd}(\mathbb{K})]^{*}=\operatorname{Schur}\left(\mathbb{K}^{*}\right)$ and $\left[\operatorname{Schur}\left(\mathbb{K}^{*}\right)\right]^{*}=\operatorname{Psd}(\mathbb{K})$.

Proof. Proposition 5.9 applies, as $\mathbb{C P}^{V}$ is full dimensional and contains $\operatorname{Diag}\left(\mathbb{R}_{+}^{V}\right)$.

Let $V$ be a finite, and recall the definition of $\mathcal{B}_{V}: \mathbb{S}\{0\} \cup V \rightarrow \mathbb{R} \oplus \mathbb{R}^{V}$ in (5.33). Note that for every $\alpha \in \mathbb{R}$ and $y \in \mathbb{R}^{V}$,

$$
\mathcal{B}_{V}^{*}(\alpha \oplus y)=\left[\begin{array}{cc}
\alpha & y^{\top} \\
y & -2 \operatorname{Diag}(y)
\end{array}\right],
$$


since for every $\widehat{X} \in \mathbb{S}\{0\} \cup V$,

$$
\begin{aligned}
\left\langle\mathcal{B}_{V}^{*}(\alpha \oplus y), \widehat{X}\right\rangle & =\left\langle\alpha \oplus y, \mathcal{B}_{V}(\widehat{X})\right\rangle \\
& =\alpha \widehat{X}_{00}+\sum_{i \in V} y_{i}\left(\widehat{X}_{0 i}+\widehat{X}_{i 0}-2 \widehat{X}_{i i}\right) \\
& =\alpha \widehat{X}_{00}+\sum_{i \in V} y_{i}\left\langle e_{0} e_{i}^{\top}+e_{i} e_{0}^{\top}-2 e_{i} e_{i}^{\top}, \widehat{X}\right\rangle \\
& =\left\langle\left[\begin{array}{cc}
\alpha & 0 \\
0 & 0
\end{array}\right], \widehat{X}\right\rangle+\left\langle\left[\begin{array}{cc}
0 & y^{\top} \\
y & -2 \operatorname{Diag}(y)
\end{array}\right], \widehat{X}\right\rangle \\
& =\left\langle\left[\begin{array}{cc}
\alpha & y^{\top} \\
y & -2 \operatorname{Diag}(y)
\end{array}\right], \widehat{X}\right\rangle .
\end{aligned}
$$

We now generalize Proposition 5.26.

Proposition 5.34. Let $V$ be a finite set, let 0 be an element not in $V$, and let $\mathbb{K}$ be a closed convex cone such that $\mathbb{C P}^{V} \subseteq \mathbb{K} \subseteq \mathbb{S}_{+}^{V}$. Then for every $Y \in \mathbb{S}^{V}$,

$$
\begin{aligned}
\delta^{*}\left(Y \mid \mathcal{E}_{\mathbb{K}}\right) & =\max \left\{\left\langle\mathcal{C}_{\text {Bool }}^{-*}(0 \oplus Y), \widehat{X}\right\rangle \mid \widehat{X} \in \operatorname{Psd}(\mathbb{K}), \mathcal{B}_{V}(\widehat{X})=1 \oplus 0\right\} \\
& =\inf \left\{\alpha \mid \alpha \oplus y \in \mathbb{R} \oplus \mathbb{R}^{V}, \mathcal{B}_{V}^{*}(\alpha \oplus y) \succeq_{\text {Schur }\left(\mathbb{K}^{*}\right)} \mathcal{C}_{\text {Bool }}^{-*}(0 \oplus Y)\right\} .
\end{aligned}
$$

Proof. We first prove that

$$
\mathbb{C P}^{\{0\} \cup V} \subseteq \operatorname{Psd}(\mathbb{K}) \subseteq \mathbb{S}_{+}^{\{0\} \cup V}
$$

Indeed,

$$
\begin{aligned}
\mathbb{C P}^{\{0\} \cup V} & \subseteq \operatorname{Psd}\left(\mathbb{C P}^{V}\right) & & \text { by }(5.26) \\
& \subseteq \operatorname{Psd}(\mathbb{K}) & & \text { by }(5.30 \mathrm{a}) \text { and } \mathbb{C P}^{V} \subseteq \mathbb{K} \\
& \subseteq \operatorname{Psd}\left(\mathbb{S}_{+}^{V}\right) & & \text { by }(5.30 \mathrm{a}) \text { and } \mathbb{K} \subseteq \mathbb{S}_{+}^{V} \\
& =\mathbb{S}_{+}^{\{0\} \cup V} . & & \text { by }(5.25)
\end{aligned}
$$

We conclude from (5.121) that $\operatorname{Psd}(\mathbb{K})$ is a regular cone - recall Section 2.6. The set Psd( $\mathbb{K})$ is a convex cone by construction, and it is clear from both (5.121) and (5.14) that $\operatorname{Psd}(\mathbb{K})$ has nonempty interior. We can also use (5.14) to conclude that $[\operatorname{Psd}(\mathbb{K})]^{*}$ has nonempty interior, since

$$
\begin{aligned}
\mathbb{C P}^{\{0\} \cup V} & \subseteq \mathbb{S}_{+}^{\{0\} \cup V} & & \text { by }(5.17) \\
& \subseteq[\operatorname{Psd}(\mathbb{K})]^{*} . & & \text { by }(5.121) \text { and }(5.4)
\end{aligned}
$$

Since $\operatorname{Psd}(\mathbb{K})$ is closed by $(5.24)$, we conclude that $\operatorname{Psd}(\mathbb{K})$ is regular.

We first prove that for every $\widehat{C} \in \mathbb{S}\{0\} \cup V$,

$$
\begin{aligned}
\delta^{*}\left(\widehat{C} \mid \widehat{\mathrm{TH}}\left(\mathbb{S}^{V}, \operatorname{Psd}(\mathbb{K})\right)\right) & =\sup \left\{\langle\widehat{C}, \widehat{X}\rangle \mid \widehat{X} \in \operatorname{Psd}(\mathbb{K}), \mathcal{B}_{V}(\widehat{X})=1 \oplus 0\right\} \\
& \leq \inf \left\{\alpha \mid \alpha \oplus y \in \mathbb{R} \oplus \mathbb{R}^{V}, \mathcal{B}_{V}^{*}(\alpha \oplus y) \succeq_{\operatorname{Schur}\left(\mathbb{K}^{*}\right)} \widehat{C}\right\}
\end{aligned}
$$

The first equality in (5.122) is a direct consequence of (5.32). Proposition 5.33 states that $[\operatorname{Psd}(\mathbb{K})]^{*}=$ $\operatorname{Schur}\left(\mathbb{K}^{*}\right)$. Item 1 of Theorem 2.11 implies (5.122).

As the linear transformations

$$
\begin{aligned}
\widehat{X} \in \mathbb{S}^{\{0\} \cup V} \mapsto \mathcal{C}_{\text {Bool }}^{-1}(\widehat{X})[V] \in \mathbb{S}^{V} \text { and } \\
X \in \mathbb{S}^{V} \mapsto \mathcal{C}_{\text {Bool }}^{-*}(0 \oplus X) \in \mathbb{S}^{\{0\} \cup V}
\end{aligned}
$$

are adjoint to each other,

$$
\begin{aligned}
\delta^{*}\left(Y \mid \mathcal{E}_{\mathbb{K}}\right) & =\delta^{*}\left(Y \mid \mathcal{C}_{\text {Bool }}^{-1}\left(\widehat{\mathrm{TH}}\left(\mathbb{S}^{V}, \operatorname{Psd}(\mathbb{K})\right)\right)[V]\right) & & \text { by }(5.78) \\
& =\delta^{*}\left(\mathcal{C}_{\text {Bool }}^{-*}(0 \oplus Y) \mid \widehat{\mathrm{TH}}\left(\mathbb{S}^{V}, \operatorname{Psd}(\mathbb{K})\right)\right) . & & \text { by }(2.50)
\end{aligned}
$$


Hence (5.122) proves (5.120a) - except attainment - and ' $\leq$ ' in (5.120b). Item 3 of Theorem 2.11 reduces proving the second equation in (5.120b) to exhibiting $\alpha \oplus y \in \mathbb{R} \oplus \mathbb{R}^{V}$ such that

$$
\mathcal{B}_{V}^{*}(\alpha \oplus y)-\mathcal{C}_{\text {Bool }}^{-*}(0 \oplus Y) \in \operatorname{int}\left(\operatorname{Schur}\left(\mathbb{K}^{*}\right)\right) .
$$

Note that $\mathbb{K} \subseteq \mathbb{S}_{+}^{V}$ and (5.4) imply $\mathbb{S}_{+}^{V} \subseteq \mathbb{K}^{*}$, so

$$
\mathbb{S}_{+}^{\{0\} \cup V}=\operatorname{Schur}\left(\mathbb{S}_{+}^{V}\right) \subseteq \operatorname{Schur}\left(\mathbb{K}^{*}\right) .
$$

From (5.123) we conclude that $\mathbb{S}_{++}^{\{0\} \cup V}=\operatorname{int}\left(\mathbb{S}_{+}^{\{0\} \cup V}\right) \subseteq \operatorname{int}\left(\operatorname{Schur}\left(\mathbb{K}^{*}\right)\right)$. Thus it suffices to find $\alpha \oplus y \in \mathbb{R} \oplus \mathbb{R}^{V}$ such that $\mathcal{B}_{V}^{*}(\alpha \oplus y) \succ \mathcal{C}_{\text {Bool }}^{-*}(0 \oplus Y)$. Define

$$
\begin{aligned}
y & :=-\frac{1}{2}\left(4 \lambda_{\max }(Y)+1\right) \mathbb{1}, \\
\gamma & :=\|y+2 Y \mathbb{1}\|_{2}^{2}+1, \\
\alpha & :=\mathbb{1}^{\top} Y \mathbb{1}+\gamma .
\end{aligned}
$$

Then (5.119) implies that

$$
\mathcal{B}_{V}^{*}(\alpha \oplus y)-\mathcal{C}_{\text {Bool }}^{-*}(0 \oplus Y)=\left[\begin{array}{cc}
\alpha-\mathbb{1}^{\top} Y \mathbb{1} & y^{\top}+2 \mathbb{1}^{\top} Y \\
y+2 Y \mathbb{1} & -2 \operatorname{Diag}(y)-4 Y
\end{array}\right],
$$

since

$$
\begin{aligned}
\mathcal{C}_{\text {Bool }}^{-*}(0 \oplus Y) & =\mathcal{C}_{\mathrm{Bool}^{-\top}}(0 \oplus Y) & & \text { by Propo } \\
& =\left[\begin{array}{cc}
1 & -\mathbb{1}^{\top} \\
0 & 2 I
\end{array}\right]\left[\begin{array}{cc}
0 & 0 \\
0 & Y
\end{array}\right]\left[\begin{array}{cc}
1 & 0^{\top} \\
-\mathbb{1} & 2 I
\end{array}\right] & & \text { by }(5.76) \\
& =\left[\begin{array}{cc}
\mathbb{1}^{\top} Y \mathbb{1} & -2 \mathbb{1}^{\top} Y \\
-2 Y \mathbb{1} & 4 Y
\end{array}\right] . & &
\end{aligned}
$$

We claim that

$$
-2 \operatorname{Diag}(y) \succ \frac{1}{\gamma}(y+2 Y \mathbb{1})(y+2 Y \mathbb{1})^{\top}+4 Y .
$$

This is true as, for every nonzero $x \in \mathbb{R}^{V}$,

$$
\begin{aligned}
x^{\top}\left(\frac{1}{\gamma}(y+2 Y \mathbb{1})(y+2 Y \mathbb{1})^{\top}+4 Y\right) x & =\frac{\left((y+2 Y \mathbb{1})^{\top} x\right)^{2}}{\gamma}+4 x^{\top} Y x & & \\
& \leq \frac{\|y+2 Y \mathbb{1}\|_{2}^{2}\|x\|_{2}^{2}}{\gamma}+4 x^{\top} Y x & & \text { by Cauchy-Schwarz } \\
& <\|x\|_{2}^{2}+4 x^{\top} Y x & & \text { by (5.124b) and } x \neq 0 \\
& \leq\|x\|_{2}^{2}+4 \lambda_{\max }(Y)\|x\|_{2}^{2} & & \\
& =x^{\top}\left(I+4 \lambda_{\max }(Y) I\right) x & & \\
& =x^{\top}(-2 \operatorname{Diag}(y)) x . & & \text { by }(5.124 \mathrm{a})
\end{aligned}
$$

We conclude that $\mathcal{B}_{V}^{*}(\alpha \oplus y) \succ \mathcal{C}_{\text {Bool }}^{-*}(0 \oplus Y)$ :

$$
\begin{array}{rlrl}
\mathcal{B}_{V}^{*}(\alpha \oplus y)-\mathcal{C}_{\text {Bool }}^{-*}(0 \oplus Y) & =\left[\begin{array}{cc}
\alpha-\mathbb{1}^{\top} Y \mathbb{1} & y^{\top}+2 \mathbb{1}^{\top} Y \\
y+2 Y \mathbb{1} & -2 \operatorname{Diag}(y)-4 Y
\end{array}\right] & \text { by }(5.125) \\
& \in \mathbb{S}_{++}^{\{0\} \cup V} & & \text { by }(5.28 \mathrm{~b})
\end{array}
$$

Note that the last step also used (5.126) and that $\alpha-\mathbb{1}^{\top} Y \mathbb{1}=\gamma>0$.

Let $V$ be a finite set, and let $\mathbb{K}$ be a closed convex cone such that $\mathbb{C P}^{V} \subseteq \mathbb{K} \subseteq \mathbb{S}_{+}^{V}$. By Theorem 2.11, we could attempt to prove attainment in $(5.120 \mathrm{~b})$ by exhibiting a matrix $\widehat{X} \in \operatorname{int}(\operatorname{Psd}(\mathbb{K}))$ such that $\mathcal{B}_{V}(\widehat{X})=1 \oplus 0$. However, as de Klerk and Pasechnik [30] point out, when $\mathbb{K}=\mathbb{C P}^{V}$ it may be the case that no such matrix $\widehat{X}$ exists. Luckily, this is not an issue for our developments, as we can generalize Corollary 5.27. 
Corollary 5.35. Let $V$ be a finite set not containing 0 , and let $\mathbb{K}$ be a closed convex cone such that $\mathbb{C P}^{V} \subseteq \mathbb{K} \subseteq \mathbb{S}_{+}^{V}$. Then $Y \in\left(\mathbb{1} \mathbb{1}^{\top}-\mathcal{E}_{\mathbb{K}}\right)^{\circ}$ if and only if

$$
\inf \left\{\left\langle\mathbb{1} \mathbb{1}^{\top}, Y\right\rangle+\alpha \mid \begin{array}{c}
\alpha \oplus y \in \mathbb{R} \oplus \mathbb{R}^{V}, \\
\mathcal{B}_{V}^{*}(\alpha \oplus y)+\mathcal{C}_{\text {Bool }}^{-*}(0 \oplus Y) \in \operatorname{Schur}\left(\mathbb{K}^{*}\right)
\end{array}\right\} \leq 1 .
$$

Proof. Let $Y \in \mathbb{S}^{V}$. Note that $Y \in\left(\mathbb{1}^{\top}-\mathcal{E}_{\mathbb{K}}\right)^{\circ}$ if and only if $\left\langle\mathbb{1} \mathbb{1}^{\top}, Y\right\rangle+\langle X,-Y\rangle \leq 1$ for every $X \in \mathcal{E}_{\mathbb{K}}$. Thus

$$
Y \in\left(\mathbb{1}^{\top}-\mathcal{E}_{\mathbb{K}}\right)^{\circ} \text { if and only if }\left\langle\mathbb{1} \mathbb{1}^{\top}, Y\right\rangle+\delta^{*}\left(-Y \mid \mathcal{E}_{\mathbb{K}}\right) \leq 1 \text {. }
$$

On the other hand,

$$
\begin{aligned}
& \left\langle\mathbb{1} \mathbb{1}^{\top}, Y\right\rangle+\delta^{*}\left(-Y \mid \mathcal{E}_{\mathbb{K}}\right) \\
& =\left\langle\mathbb{1} \mathbb{1}^{\top}, Y\right\rangle+\inf \left\{\begin{array}{l|c}
\alpha \mid \begin{array}{c}
\alpha \oplus y \in \mathbb{R} \oplus \mathbb{R}^{V}, \\
\mathcal{B}_{V}^{*}(\alpha \oplus y) \succeq_{\operatorname{Schur}\left(\mathbb{K}^{*}\right)} \mathcal{C}_{\mathrm{Bool}}^{-*}(0 \oplus(-Y))
\end{array}
\end{array}\right\} \quad \text { by Proposition } 5.34
\end{aligned}
$$

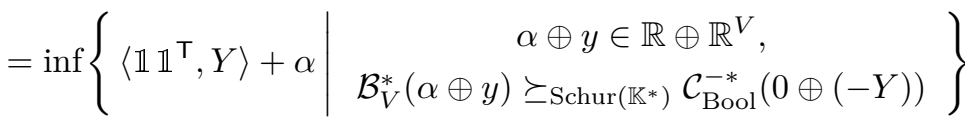

$$
\begin{aligned}
& =\inf \left\{\begin{array}{c|c}
\left\langle\mathbb{1} \mathbb{1}^{\top}, Y\right\rangle+\alpha \mid \begin{array}{c}
\alpha \oplus y \in \mathbb{R} \oplus \mathbb{R}^{V}, \\
\mathcal{B}_{V}^{*}(\alpha \oplus y)+\mathcal{C}_{\text {Bool }}^{-*}(0 \oplus Y) \in \operatorname{Schur}\left(\mathbb{K}^{*}\right)
\end{array}
\end{array}\right\},
\end{aligned}
$$

so (5.128) implies the statement.

We finish this section by obtaining a conic programming generalization of Theorem 5.28.

Theorem 5.36. Let $G:=(V, E)$, and let $\mathbb{K}$ be a closed convex cone such that $\mathbb{C P}^{V} \subseteq \mathbb{K} \subseteq \mathbb{S}_{+}^{V}$. Then for every $z \in \mathbb{R}_{+}^{E}$,

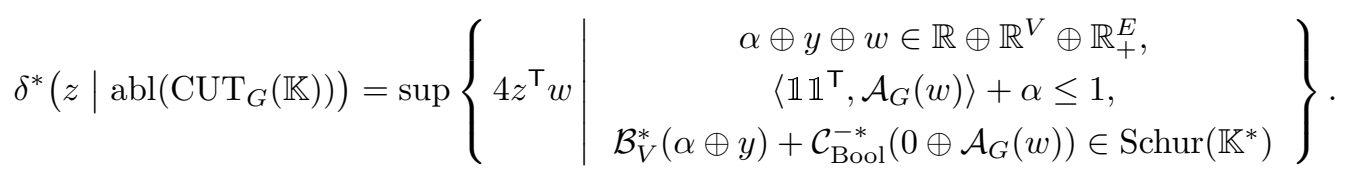

Proof. Denote by $\mathcal{F}$ the feasible region of the optimization problem in the RHS of the statement. Let $\alpha \oplus y \oplus w \in \mathcal{F}$. Corollary 5.35 implies that $\mathcal{A}_{G}(w) \in\left(\mathbb{1}^{\top}-\mathcal{E}_{\mathbb{K}}\right)^{\circ}$, so Corollary 5.24 implies that $4 w \in$ $\operatorname{abl}\left(\operatorname{CUT}_{G}(\mathbb{K})\right)$. Therefore ' $\geq$ ' holds in the statement.

Let $4 w \in \operatorname{abl}\left(\operatorname{CUT}_{G}(\mathbb{K})\right)$, and let $\varepsilon>0$. Corollary 5.24 implies that $\mathcal{A}_{G}(w) \in\left(\mathbb{1}^{\top}-\mathcal{E}_{\mathbb{K}}\right)^{\circ}$. By Corollary 5.35, there exists $\alpha \oplus y \in \mathbb{R} \oplus \mathbb{R}^{V}$ such that

$$
\begin{gathered}
\left\langle\mathbb{1} \mathbb{1}^{\top}, \mathcal{A}_{G}(w)\right\rangle+\alpha \leq 1+\varepsilon \text { and } \\
\mathcal{B}_{V}^{*}(\alpha \oplus y)+\mathcal{C}_{\text {Bool }}^{-*}\left(0 \oplus \mathcal{A}_{G}(w)\right) \in \operatorname{Schur}\left(\mathbb{K}^{*}\right) .
\end{gathered}
$$

From (5.129) it is easy to see that $(1+\varepsilon)^{-1}(\alpha \oplus y \oplus w) \in \mathcal{F}$, so

$$
\beta \geq 4\left((1+\varepsilon)^{-1} w\right)^{\top} z=(1+\varepsilon)^{-1} 4 w^{\top} z,
$$

where $\beta:=\sup \left\{4 w^{\top} z \mid \alpha \oplus y \oplus z \in \mathcal{F}\right\}$. As this holds for every $\varepsilon>0$, we conclude that $\beta \geq 4 w^{\top} z$. Since $4 w \in \operatorname{abl}\left(\mathrm{CUT}_{G}(\mathbb{K})\right)$ was arbitrary, the proof is done.

\subsection{Conic Formulations of Graph Homomorphisms}

Conic formulations of graph homomorphisms, as introduced by Roberson [100], uniformly capture graph homomorphisms, quantum graph homomorphisms, and other related notions. This section reformulates conic homomorphisms in a new language, highlighting its similarities to the well-studied notion of quantum channels [118, Chapter 2]: we define a conic homomorphism as a linear transformation between real square matrices, and then use the Choi representation [118, Subsection 2.2.2] of such transformations to encode conic restrictions. To the best of my knowledge, the connection between [100] and the Choi representation is new. 


\subsubsection{Kronecker Product of Matrices}

The current subsection exploits the Kronecker product of matrices to define the vectorization of matrices and the Choi representation of linear transformations. There are no new results in this subsection, and our exposition borrows heavily from [118], with a key difference: we work with the Kronecker product of real matrices, whereas [118] works with the abstract tensor product of linear transformations between complex Euclidean spaces. We start by introducing notation. Define

$$
\operatorname{Mat}(S, T):=\mathbb{R}^{S \times T}
$$

for every finite sets $S$ and $T$, and define

$$
\operatorname{Lin}(\operatorname{Mat}(S, T), \operatorname{Mat}(U, V)):=\{\Phi: \operatorname{Mat}(S, T) \rightarrow \operatorname{Mat}(U, V) \mid \Phi \text { is linear }\}
$$

for every $S, T, U$, and $V$ finite sets.

Let $S$ and $T$ be finite sets. Our main goal when defining the notation $\operatorname{Mat}(S, T)$ is to highlight the difference between sets such as

$$
\operatorname{Mat}(T \times T, S) \text { and } \operatorname{Mat}(T, T \times S) .
$$

Although both sets define isomorphic vector spaces, we must treat them as distinct objects as soon as we want to transpose or to multiply matrices. A similar distinction lies at the core of our development. Assume that $S=V(G)$ and $T=V(H)$ for graphs $G$ and $H$. As the title of the section implies, we are interested in conic formulations of graph homomorphisms. Roberson [100] defined conic homomorphisms as matrices in Mat $(T \times S, T \times S)$. Although this sensible definition is convenient when imposing conic restrictions (recall from Section 5.1 that we are interested in cones of symmetric matrices), it unfortunately makes the composition of conic homomorphisms cumbersome. We are thus interested in establishing a special isomorphism between

$$
\operatorname{Lin}(\operatorname{Mat}(S, S), \operatorname{Mat}(T, T)) \text { and } \operatorname{Mat}(T \times S, T \times S)
$$

in order to cast composition of conic homomorphisms as composition of linear transformations. This special isomorphism, its relationship to the vectorization of matrices, and some of its computational properties are the main subject of this subsection. Our interest in the relationship between the objects in (5.133) stems from our interest in composition and matrix cones. For convenience, define

$$
\operatorname{Mat}(V):=\operatorname{Mat}(V, V)
$$

and

$$
\operatorname{Mat}_{+}(V):=\left\{X \in \operatorname{Mat}(V) \mid \forall i, j \in V, X_{i j} \geq 0\right\}
$$

for every finite set $V$.

We now recall a notion defined in (2.6) and used in (3.190). Let $R, S, T$, and $U$ be finite sets. The Kronecker product is the function

$$
\otimes: \operatorname{Mat}(S, T) \times \operatorname{Mat}(U, V) \rightarrow \operatorname{Mat}(S \times U, T \times V)
$$

defined for every $A \in \operatorname{Mat}(S, T)$ and $B \in \operatorname{Mat}(U, V)$ by

$$
(A \otimes B)_{s u, t v}:=A_{s t} B_{u v}
$$

for every $s \in S, t \in T, u \in U$, and $v \in V$. Note from (5.135) that

$$
\alpha(A \otimes B)=(\alpha A) \otimes B=A \otimes(\alpha B),
$$

so we may denote this common value by $\alpha A \otimes B$ for every $A, B \in \operatorname{Mat}(S, T)$ and $\alpha \in \mathbb{R}$. One may easily prove from (5.135) that

$$
\begin{aligned}
& A \otimes(X+\alpha Y)=A \otimes X+\alpha A \otimes Y \text { and } \\
& (A+\alpha B) \otimes X=A \otimes X+\alpha B \otimes X,
\end{aligned}
$$


and that

$$
(A \otimes X)^{\top}=A^{\top} \otimes X^{\top}
$$

for every $A, B \in \operatorname{Mat}(S, T)$, for every $X, Y \in \operatorname{Mat}(U, V)$, and for every $\alpha \in \mathbb{R}$. The freedom of using arbitrary sets as row and column sets of matrices restricts our Kronecker product more than usual; in particular, our Kronecker product is not associative. Interestingly, even this "nuisance" fits nicely into our developments. The most important property of the Kronecker product of matrices is the mixed product property.

Proposition 5.37 (Mixed Product Property). Let $R, S, T, U, V$, and $W$ be finite sets. It holds that

$$
(A \otimes B)(C \otimes D)=A C \otimes B D
$$

for every $A \in \operatorname{Mat}(R, S), C \in \operatorname{Mat}(S, T), B \in \operatorname{Mat}(U, V)$, and $D \in \operatorname{Mat}(V, W)$.

Proof. It suffices to apply the definitions of the Kronecker and matrix products. For every $r \in R, u \in U$, $t \in T$, and $w \in W$,

$$
\begin{aligned}
((A \otimes B)(C \otimes D))_{(r, u),(t, w)} & =\sum_{s \in S} \sum_{v \in V}(A \otimes B)_{(r, u),(s, v)}(C \otimes D)_{(s, v),(t, w)} \\
& =\sum_{s \in S} \sum_{v \in V} A_{r, s} B_{u, v} C_{s, t} D_{v, w} \\
& =\left(\sum_{s \in S} A_{r, s} C_{s, t}\right)\left(\sum_{v \in V} B_{u, v} D_{v, w}\right) \\
& =(A C)_{r t}(B D)_{u w} \\
& =(A C \otimes B D)_{(r, u),(t, w)} .
\end{aligned}
$$

Proposition 5.38. Let $S$ and $T$ be finite sets. Then

$$
\operatorname{Tr}(A \otimes B)=\operatorname{Tr}(A) \operatorname{Tr}(B)
$$

for every $A \in \operatorname{Mat}(S)$ and $B \in \operatorname{Mat}(T)$.

Proof. This result follows directly from the definitions:

$$
\begin{aligned}
\operatorname{Tr}(A \otimes B) & =\sum_{s \in S} \sum_{t \in T}(A \otimes B)_{(s, t),(s, t)} \\
& =\sum_{s \in S} \sum_{t \in T} A_{s s} B_{t t} \\
& =\left(\sum_{s \in S} A_{s s}\right)\left(\sum_{t \in T} B_{t t}\right) \\
& =\operatorname{Tr}(A) \operatorname{Tr}(B) .
\end{aligned}
$$

Proposition 5.39. Let $S, T, U$, and $V$ be finite sets. It holds that

$$
\langle A \otimes X, B \otimes Y\rangle=\langle A, B\rangle\langle X, Y\rangle
$$

for every $A, B \in \operatorname{Mat}(S, T)$ and $X, Y \in \operatorname{Mat}(U, V)$.

Proof. We have that

$$
\begin{aligned}
\langle A \otimes X, B \otimes Y\rangle & =\operatorname{Tr}\left((A \otimes X)^{\top}(B \otimes Y)\right) & & \text { by }(5.1) \\
& =\operatorname{Tr}\left(\left(A^{\top} \otimes X^{\top}\right)(B \otimes Y)\right) & & \text { by }(5.137) \\
& =\operatorname{Tr}\left(A^{\top} B \otimes X^{\top} Y\right) & & \text { by Proposition } 5.37 \\
& =\operatorname{Tr}\left(A^{\top} B\right) \operatorname{Tr}\left(X^{\top} Y\right) & & \text { by Proposition } 5.38 \\
& =\langle A, B\rangle\langle X, Y\rangle . & & \text { by }(5.1)
\end{aligned}
$$


We can define the Kronecker product for vectors using the Kronecker product of matrices. Let $S$ and $T$ be finite sets. Whenever $|T|=1$, we will implicitly identify $\mathbb{R}^{S}$ with $\operatorname{Mat}(S, T)$ using $\iota_{S}: \mathbb{R}^{S} \rightarrow \operatorname{Mat}(S, T)$ defined by $\iota_{S}\left(e_{i}\right)=e_{(i, t)}$ for every $i \in S$, where $T=\{t\}$. In this manner, we identify

$$
\operatorname{Mat}(S, T) \equiv \begin{cases}\mathbb{R}, & \text { if }|S|=1 \text { and }|T|=1 \\ \mathbb{R}^{S}, & \text { if }|S|>1 \text { and }|T|=1\end{cases}
$$

Using (5.138) we obtain

$$
\otimes: \mathbb{R}^{S} \times \mathbb{R}^{T} \rightarrow \mathbb{R}^{S \times T}
$$

from

$$
\otimes: \operatorname{Mat}(S,\{0\}) \times \operatorname{Mat}(T,\{0\}) \rightarrow \operatorname{Mat}(S \times T,\{0\} \times\{0\}) .
$$

To appreciate (5.138), consider the expression

$$
x^{\top}(y \otimes z) \in \mathbb{R} \text { for } x \in \mathbb{R}^{S \times T}, y \in \mathbb{R}^{S} \text {, and } z \in \mathbb{R}^{T} .
$$

We use (5.138) to state that $x \in \operatorname{Mat}(S \times T,\{0\}), y \in \operatorname{Mat}(S,\{0\})$, and $z \in \operatorname{Mat}(T,\{0\})$. Matrix multiplication then implies that $x^{\top}(y \otimes z) \in \operatorname{Mat}(\{0\},\{0\} \times\{0\})$, so we use (5.138) again to obtain a scalar. The importance of scalars in linear-algebraic definitions motivates (5.138) recovering $\mathbb{R}$ whenever possible. For example, if $x^{\top}(y \otimes z)$ appears inside a trace, we can only apply linearity to move it outside the trace once we identify Mat $(\{0\},\{0\} \times\{0\})$ with $\mathbb{R}$. We also point out another commonly used identification closely related to (5.138). Matrix multiplication defines a linear transformation $\operatorname{Mat}(S,\{0\}) \rightarrow \operatorname{Mat}(T,\{0\})$ for every $A \in \operatorname{Mat}(T, S)$. Therefore (5.138) implies that

$$
(x \mapsto A x) \in \operatorname{Lin}\left(\mathbb{R}^{S}, \mathbb{R}^{T}\right) \text { for every } A \in \operatorname{Mat}(T, S) .
$$

This identifies Mat $(T, S)$ with $\operatorname{Lin}\left(\mathbb{R}^{S}, \mathbb{R}^{T}\right)$. Correspondences (5.138) and (5.141) are ubiquitous in matrix computations, and were used countless times in this text. For this reason, we do not introduce special notation for these correspondences, and we will only reference them to clarify exposition. As a rule of thumb, we use (5.138) to "promote" all of our vectors into matrices, and then perform our computations, possibly using (5.138) again to manipulate some scalars.

Let $S, T, U$, and $V$ be finite sets. Let $s \in S, t \in T, u \in U$, and $v \in V$. It is easy to see that

$$
e_{s} \otimes e_{t}=e_{(s, t)} \in \mathbb{R}^{S \times T} .
$$

We also have that

$$
e_{u} e_{v}^{\top} \otimes e_{s} e_{t}^{\top}=e_{(u, s)} e_{(v, t)}^{\top},
$$

since

$$
\begin{aligned}
e_{u} e_{v}^{\top} \otimes e_{s} e_{t}^{\top} & =\left(e_{u} \otimes e_{s}\right)\left(e_{v}^{\top} \otimes e_{t}^{\top}\right) & & \text { by Proposition } 5.37 \\
& =\left(e_{u} \otimes e_{s}\right)\left(e_{v} \otimes e_{t}\right)^{\top} & & \text { by }(5.137) \\
& =e_{(u, s)} e_{(v, t)}^{\top} . & & \text { by }(5.142)
\end{aligned}
$$

Furthermore, for every $A \in \operatorname{Mat}(U \times S, V \times T)$,

$$
A_{(u, s),(v, t)}=\left\langle e_{u} e_{v}^{\top} \otimes e_{s} e_{t}^{\top}, A\right\rangle,
$$

as

$$
\begin{aligned}
A_{(u, s),(v, t)} & =\left\langle e_{(u, s)} e_{(v, t)}^{\top}, A\right\rangle & \\
& =\left\langle e_{u} e_{v}^{\top} \otimes e_{s} e_{t}^{\top}, A\right\rangle . & \text { by }(5.143)
\end{aligned}
$$

Proposition 5.40. Let $S, T, U$, and $V$ be finite sets, and let $A \in \operatorname{Mat}(U \times S, V \times T)$. Then $A=0$ if and only if

$$
\langle Y \otimes X, A\rangle=0
$$

for every $X \in \operatorname{Mat}(S, T)$ and $Y \in \operatorname{Mat}(U, V)$. 
Proof. If $A \neq 0$, there exists $s \in S, t \in T, u \in U$, and $v \in V$ such that $A_{(u, s),(v, t)} \neq 0$. Then

$$
\begin{aligned}
0 & \neq A_{(u, s),(v, t)} \\
& =\left\langle e_{u} e_{v}^{\top} \otimes e_{s} e_{t}^{\top}, A\right\rangle
\end{aligned}
$$

If $A=0$, then it is clear that the inner product is always zero.

Corollary 5.41. Let $S, T, U$, and $V$ be finite sets, and let $A, B \in \operatorname{Mat}(U \times S, V \times T)$. Then $A=B$ if and only if

$$
\langle Y \otimes X, A\rangle=\langle Y \otimes X, B\rangle
$$

for every $X \in \operatorname{Mat}(S, T)$ and $Y \in \operatorname{Mat}(U, V)$.

Proof. We have that $A=B$ if and only if $A-B=0$. Proposition 5.40 and linearity of the tensor product finish the proof.

Proposition 5.42. Let $S, T, U$, and $V$ be finite sets. There exists a linear transformation

$$
\text { rep: } \operatorname{Lin}(\operatorname{Mat}(S, T), \operatorname{Mat}(U, V)) \rightarrow \operatorname{Mat}(U \times S, V \times T)
$$

such that

$$
\langle Y \otimes X, \operatorname{rep}(\Phi)\rangle=\langle Y, \Phi(X)\rangle
$$

for every $\Phi \in \operatorname{Lin}(\operatorname{Mat}(S, T), \operatorname{Mat}(U, V))$, for every $X \in \operatorname{Mat}(S, T)$, and for every $Y \in \operatorname{Mat}(U, V)$. Furthermore, rep is invertible and is the only linear transformation satisfying (5.145).

Proof. Let $\Phi \in \operatorname{Lin}(\operatorname{Mat}(S, T), \operatorname{Mat}(U, V))$. Define $\operatorname{rep}(\Phi) \in \operatorname{Mat}(U \times S, V \times T)$ by

$$
\operatorname{rep}(\Phi)_{(u, s),(v, t)}:=\left\langle e_{u} e_{v}^{\top}, \Phi\left(e_{s} e_{t}^{\top}\right)\right\rangle
$$

for every $s \in S, t \in T, u \in U$, and $v \in V$. For every $X \in \operatorname{Mat}(S, T)$ and $Y \in \operatorname{Mat}(U, V)$,

$$
\begin{array}{rlrl}
\langle Y \otimes X, \operatorname{rep}(\Phi)\rangle & =\sum_{u \in U} \sum_{v \in V} Y_{u v}\left\langle e_{u} e_{v}^{\top} \otimes X, \operatorname{rep}(\Phi)\right\rangle & & \text { by (5.136) } \\
& =\sum_{u \in U} \sum_{v \in V} \sum_{s \in S} \sum_{t \in T} Y_{u v} X_{s t}\left\langle e_{u} e_{v}^{\top} \otimes e_{s} e_{t}^{\top}, \operatorname{rep}(\Phi)\right\rangle & & \text { by (5.136) } \\
& =\sum_{u \in U} \sum_{v \in V} \sum_{s \in S} \sum_{t \in T} Y_{u v} X_{s t}\left\langle e_{(u, s)} e_{(v, t)}^{\top}, \operatorname{rep}(\Phi)\right\rangle & & \text { by (5.143) } \\
& =\sum_{u \in U} \sum_{v \in V} \sum_{s \in S} \sum_{t \in T} Y_{u v} X_{s t}\left\langle e_{u} e_{v}^{\top}, \Phi\left(e_{s} e_{t}^{\top}\right)\right\rangle & \text { by (5.146) } \\
& =\sum_{u \in U} \sum_{v \in V} Y_{u v}\left\langle e_{u} e_{v}^{\top}, \Phi(X)\right\rangle & & \\
& =\langle Y, \Phi(X)\rangle . &
\end{array}
$$

We conclude that rep satisfies (5.145). To see that rep is linear, note that for every $\Phi, \Psi \in \operatorname{Lin}(\operatorname{Mat}(S, T), \operatorname{Mat}(U, V))$ and for every $\alpha \in \mathbb{R}$,

$$
\begin{aligned}
\langle Y \otimes X, \operatorname{rep}(\Phi)+\alpha \operatorname{rep}(\Psi)\rangle & =\langle Y \otimes X, \operatorname{rep}(\Phi)\rangle+\alpha\langle Y \otimes X, \operatorname{rep}(\Psi)\rangle \\
& =\langle Y, \Phi(X)\rangle+\alpha\langle Y, \Psi(X)\rangle \\
& =\langle Y, \Phi(X)+\alpha \Psi(X)\rangle \\
& =\langle Y,(\Phi+\alpha \Psi)(X)\rangle \\
& =\langle Y \otimes X, \operatorname{rep}(\Phi+\alpha \Psi)\rangle .
\end{aligned}
$$

Corollary 5.41 implies that $\operatorname{rep}(\Phi+\alpha \Psi)=\operatorname{rep}(\Phi)+\alpha \operatorname{rep}(\Psi)$. It is clear from Corollary 5.41 that any other linear transformation satisfying (5.145) must be equal to rep. We finish the proof by showing that rep is 
invertible. Since rep is a linear transformation between vector spaces with the same finite dimension, it suffices to show that it has a right inverse. For every $A \in \operatorname{Mat}(U \times S, V \times T)$, define $\Phi_{A} \in \operatorname{Lin}(\operatorname{Mat}(S, T)$, $\operatorname{Mat}(U, V))$ by

$$
\left\langle e_{u} e_{v}^{\top}, \Phi_{A}\left(e_{s} e_{t}^{\top}\right)\right\rangle:=A_{(u, s),(v, t)}
$$

for every $s \in S, t \in T, u \in U$, and $v \in V$. We have that $\Phi_{A}$ is well defined and unique because $\left\{e_{s} e_{t}^{\top} \mid s \in S, t \in T\right\}$ and $\left\{e_{u} e_{v}^{\top} \mid u \in U, v \in V\right\}$ are bases of $\operatorname{Mat}(S, T)$ and $\operatorname{Mat}(U, V)$, respectively. Then (5.146) implies that $\operatorname{rep}\left(\Phi_{A}\right)=A$.

Let $S$ and $T$ be finite sets. Define $\operatorname{mat}_{T, S}: \operatorname{Lin}(\operatorname{Mat}(S), \operatorname{Mat}(T)) \rightarrow \operatorname{Mat}(T \times S)$ as the unique linear transformation such that

$$
\left\langle Y \otimes X, \operatorname{mat}_{T, S}(\Phi)\right\rangle=\langle Y, \Phi(X)\rangle
$$

for every $Y \in \operatorname{Mat}(T), X \in \operatorname{Mat}(S)$, and $\Phi \in \operatorname{Lin}(\operatorname{Mat}(S)$, Mat $(T))$. Proposition 5.42 implies that mat $_{T, S}$ is defined. The function mat ${ }_{T, S}$, which establishes the correspondence hinted at (5.133), has been studied in the context of quantum information theory. The Choi representation of a quantum channel, as presented in [118, Subsection 2.2.2], is not precisely equal to mat ${ }_{T, S}$ simply because we work with real matrices, whereas [118] works with complex matrices. Our notation highlights its similarity with another wellknown (see [61, Definition 4.2.9] or [118, Equation (1.127)]) correspondence: the vec operator. Define $\operatorname{vec}_{T, S}: \operatorname{Lin}\left(\mathbb{R}^{S}, \mathbb{R}^{T}\right) \rightarrow \mathbb{R}^{T \times S}$ as the unique linear transformation such that

$$
(y \otimes x)^{\top} \operatorname{vec}_{T, S}(A)=y^{\top} A x
$$

for every $y \in \mathbb{R}^{T}, x \in \mathbb{R}^{S}$, and $A \in \operatorname{Mat}(T, S)$ - note the use of correspondence (5.141) when we write $\operatorname{vec}_{T, S}(A)$ for $A \in \operatorname{Mat}(T, S)$. We have that $\operatorname{vec}_{T, S}$ is the unique linear transformation between

$$
\operatorname{Lin}(\operatorname{Mat}(S,\{0\}), \operatorname{Mat}(T,\{0\})) \rightarrow \operatorname{Mat}(T \times S,\{0\} \times\{0\})
$$

from Proposition 5.42, rewritten using the correspondence (5.138). We prove that

$$
\operatorname{vec}_{T, S}\left(y x^{\top}\right)=y \otimes x
$$

for every $y \in \mathbb{R}^{S}$ and $x \in \mathbb{R}^{T}$, which is usually given as the definition of $\operatorname{vec}_{T, S}$. For every $a, x \in \mathbb{R}^{S}$ and for every $b, y \in \mathbb{R}^{T}$,

$$
\begin{aligned}
(a \otimes b)^{\top} \operatorname{vec}_{T, S}\left(y x^{\top}\right) & =a^{\top} y x^{\top} b & & \text { by }(5.148) \\
& =(a \otimes b)^{\top}(y \otimes x) . & & \text { by Proposition } 5.39
\end{aligned}
$$

As this holds for every $a \in \mathbb{R}^{S}$ and $b \in \mathbb{R}^{T}$, Corollary 5.41 implies (5.149). We also have that

$$
\langle A, B\rangle=\operatorname{vec}_{T, S}(A)^{\top} \operatorname{vec}_{T, S}(B)
$$

for every $A, B \in \operatorname{Mat}(T, S)$. Indeed,

$$
\begin{array}{rlrl}
\operatorname{vec}_{T, S}(A)^{\top} \operatorname{vec}_{T, S}(B) & =\sum_{t \in T} \sum_{s \in S} A_{t, s} \operatorname{vec}_{T, S}\left(e_{t} e_{s}^{\top}\right)^{\top} \operatorname{vec}_{T, S}(B) & \\
& =\sum_{t \in T} \sum_{s \in S} A_{t, s}\left(e_{t} \otimes e_{s}\right)^{\top} \operatorname{vec}_{T, S}(B) & \text { by (5.149) } \\
& =\sum_{t \in T} \sum_{s \in S} A_{t, s} e_{t}^{\top} B e_{s} & \text { by (5.148) } \\
& =\sum_{t \in T} \sum_{s \in S} A_{t, s} B_{t, s} & \\
& =\langle A, B\rangle .
\end{array}
$$

Equation (5.150) states that $\operatorname{vec}_{T, S}$ is an isomorphism between Euclidean spaces, and not merely a linear isomorphism. 
Proposition 5.43. Let $S$ and $T$ be finite sets. Then

$$
(Y \otimes X) \operatorname{vec}_{T, S}(A)=\operatorname{vec}_{T, S}\left(Y A X^{\boldsymbol{\top}}\right)
$$

for every $Y \in \operatorname{Mat}(T)$, for every $X \in \operatorname{Mat}(S)$, and for every $A \in \operatorname{Mat}(T, S)$.

Proof. Set vec $:=\operatorname{vec}_{T, S}$. We first prove that

$$
\sum_{t \in T}\left(A_{t s}\left(Y e_{t}\right) \otimes\left(X e_{s}\right)\right)=\operatorname{vec}\left(Y A e_{s} e_{s}^{\top} X^{\top}\right)
$$

for every $s \in S$. Indeed,

$$
\begin{aligned}
\sum_{t \in T}\left(A_{t s}\left(Y e_{t}\right) \otimes\left(X e_{s}\right)\right) & =\left(\sum_{t \in T} Y e_{t} e_{t}^{\top} A e_{s}\right) \otimes\left(X e_{s}\right) \\
& =\left(Y A e_{s}\right) \otimes\left(X e_{s}\right) \\
& =\operatorname{vec}\left(Y A e_{s} e_{s}^{\top} X^{\top}\right) .
\end{aligned}
$$

This implies the statement, as

$$
\begin{aligned}
(Y \otimes X) \operatorname{vec}(A) & =\sum_{t \in T} \sum_{s \in S} A_{t s}(Y \otimes X) \operatorname{vec}\left(e_{t} e_{s}^{\top}\right) & & \\
& =\sum_{t \in T} \sum_{s \in S} A_{t s}(Y \otimes X)\left(e_{t} \otimes e_{s}\right) & & \text { by }(5.149) \\
& =\sum_{t \in T} \sum_{s \in S}\left(A_{t s}\left(Y e_{t}\right) \otimes\left(X e_{s}\right)\right) & & \text { by Proposition } 5.37 \\
& =\sum_{s \in S} \operatorname{vec}\left(Y A e_{s} e_{s}^{\top} X^{\top}\right) & & \text { by }(5.151) \\
& =\operatorname{vec}\left(Y A X^{\top}\right) . & & \text { since } \sum_{s \in S} e_{s} e_{s}^{\top}=I
\end{aligned}
$$

Let $S$ and $T$ be finite sets. Proposition 5.42 relates each element of $\operatorname{Mat}(T \times S)$ to a unique linear transformation from $\operatorname{Mat}(S)$ to $\operatorname{Mat}(T)$. It is convenient to make this relation more explicit. For every $A \in \operatorname{Mat}(T \times S)$ and for every $X \in \operatorname{Mat}(S)$, define $\operatorname{Tr}_{\otimes X}(A) \in \operatorname{Mat}(T)$ by

$$
\operatorname{Tr}_{\otimes X}(A)_{t t^{\prime}}:=\sum_{s, s^{\prime} \in S} A_{\left((t, s),\left(t^{\prime}, s^{\prime}\right)\right)} X_{s s^{\prime}}
$$

for every $t, t^{\prime} \in T$. For every $A \in \operatorname{Mat}(T \times S)$, for every $Y \in \operatorname{Mat}(T)$, and for every $X \in \operatorname{Mat}(S)$, we have that

$$
\left\langle\operatorname{Tr}_{\otimes X}(A), Y\right\rangle=\langle A, Y \otimes X\rangle,
$$

since

$$
\begin{aligned}
\langle A, Y \otimes X\rangle & =\sum_{t, t^{\prime} \in T} \sum_{s, s^{\prime} \in S} A_{\left((t, s),\left(t^{\prime}, s^{\prime}\right)\right)}(Y \otimes X)_{\left((t, s),\left(t^{\prime}, s^{\prime}\right)\right)} \\
& =\sum_{t, t^{\prime} \in T} \sum_{s, s^{\prime} \in S} A_{\left((t, s),\left(t^{\prime}, s^{\prime}\right)\right)} Y_{t t^{\prime}} X_{s s^{\prime}} \\
& =\sum_{t, t^{\prime} \in T} Y_{t t^{\prime}}\left(\sum_{s, s^{\prime} \in S} A_{\left((t, s),\left(t^{\prime}, s^{\prime}\right)\right)} X_{s s^{\prime}}\right) \\
& =\sum_{t, t^{\prime} \in T} Y_{t t^{\prime}} \operatorname{Tr}_{\otimes X}(A)_{t t^{\prime}} \\
& =\left\langle\operatorname{Tr}_{\otimes X}(A), Y\right\rangle .
\end{aligned}
$$


Similarly, for every $A \in \operatorname{Mat}(T \times S)$ and for every $Y \in \operatorname{Mat}(T)$, define $\operatorname{Tr}_{Y \otimes}(A) \in \operatorname{Mat}(S)$ by

$$
\operatorname{Tr}_{Y \otimes}(A)_{s s^{\prime}}:=\sum_{t, t^{\prime} \in T} A_{\left((t, s),\left(t^{\prime}, s^{\prime}\right)\right)} Y_{t t^{\prime}}
$$

for every $s, s^{\prime} \in S$. For every $A \in \operatorname{Mat}(T \times S)$, for every $Y \in \operatorname{Mat}(T)$, and for every $X \in \operatorname{Mat}(S)$, we have that

$$
\left\langle\operatorname{Tr}_{Y \otimes}(A), X\right\rangle=\langle A, Y \otimes X\rangle,
$$

since

$$
\begin{aligned}
\langle A, Y \otimes X\rangle & =\sum_{t, t^{\prime} \in T} \sum_{s, s^{\prime} \in S} A_{\left((t, s),\left(t^{\prime}, s^{\prime}\right)\right)}(Y \otimes X)_{\left((t, s),\left(t^{\prime}, s^{\prime}\right)\right)} \\
& =\sum_{t, t^{\prime} \in T} \sum_{s, s^{\prime} \in S} A_{\left((t, s),\left(t^{\prime}, s^{\prime}\right)\right)} Y_{t t^{\prime}} X_{s s^{\prime}} \\
& =\sum_{s, s^{\prime} \in S} X_{s s^{\prime}}\left(\sum_{t, t^{\prime} \in T} Y_{t t^{\prime}} A_{\left((t, s),\left(t^{\prime}, s^{\prime}\right)\right)}\right) \\
& =\sum_{s, s^{\prime} \in S} X_{s s^{\prime}} \operatorname{Tr}_{Y \otimes}(A)_{s s^{\prime}} \\
& =\left\langle\operatorname{Tr}_{Y \otimes}(A), X\right\rangle .
\end{aligned}
$$

Recall from (5.1) that we are working with the trace inner product, so (5.153) and (5.155) allow us to think of $\operatorname{Tr}_{\otimes X}(\cdot)$ and $\operatorname{Tr}_{Y \otimes}(\cdot)$ as partial traces for every $X \in \operatorname{Mat}(S)$ and for every $Y \in \operatorname{Mat}(T)$. One can prove that for every $A \in \operatorname{Mat}(T \times S)$, for every $X \in \operatorname{Mat}(S)$, and for every $Y \in \operatorname{Mat}(T)$, we have that

$$
\operatorname{Tr}_{\otimes X}(A)=\operatorname{Tr}_{\otimes I}((I \otimes X) A) \text { and } \operatorname{Tr}_{Y \otimes}(A)=\operatorname{Tr}_{I \otimes}((Y \otimes I) A) .
$$

This justifies a slightly different notation commonly used for the partial trace, which always assume that $X=I$ or $Y=I$; see [118, Section 1.1].

Let $S$ and $T$ be finite sets, and let $\Phi \in \operatorname{Lin}(\operatorname{Mat}(S), \operatorname{Mat}(T))$. We claim that

$$
\operatorname{Tr}_{\otimes X}\left(\operatorname{mat}_{T, S}(\Phi)\right)=\Phi(X)
$$

for every $X \in \operatorname{Mat}(S)$, and that

$$
\operatorname{Tr}_{Y \otimes}\left(\operatorname{mat}_{T, S}(\Phi)\right)=\Phi^{*}(Y)
$$

for every $Y \in \operatorname{Mat}(T)$. Indeed,

$$
\begin{aligned}
\left\langle\operatorname{Tr}_{\otimes X}\left(\operatorname{mat}_{T, S}(\Phi)\right), Y\right\rangle & =\left\langle\operatorname{mat}_{T, S}(\Phi), Y \otimes X\right\rangle \\
& =\left\langle Y \otimes X, \operatorname{mat}_{T, S}(\Phi)\right\rangle \\
& =\langle Y, \Phi(X)\rangle
\end{aligned}
$$

and

$$
\begin{aligned}
\left\langle\operatorname{Tr}_{Y \otimes}\left(\operatorname{mat}_{T, S}(\Phi)\right), X\right\rangle & =\left\langle\operatorname{mat}_{T, S}(\Phi), Y \otimes X\right\rangle \\
& =\left\langle Y \otimes X, \operatorname{mat}_{T, S}(\Phi)\right\rangle \\
& =\langle Y, \Phi(X)\rangle \\
& =\left\langle\Phi^{*}(Y), X\right\rangle
\end{aligned}
$$

for every $X \in \operatorname{Mat}(S)$ and $Y \in \operatorname{Mat}(T)$. Note that (5.156a) implies that

$$
\operatorname{mat}_{T, S}^{-1}(A)=\left(X \in \operatorname{Mat}(S) \mapsto \operatorname{Tr}_{\otimes X}(A) \in \operatorname{Mat}(T)\right)
$$

for every $A \in \operatorname{Mat}(T \times S)$.

Let $S$ and $T$ be finite sets, and let $A, B \in \operatorname{Mat}(T, S)$. We may define $\Phi(X):=A X B^{\top}$ for every $X \in \operatorname{Mat}(S)$, thus obtaining a linear transformation $\operatorname{Mat}(S) \rightarrow \operatorname{Mat}(T)$. The following proposition relates mat $_{T, S}$ and $\operatorname{vec}_{T, S}$ by proving that

$$
\operatorname{mat}_{T, S}^{-1}(\Phi)=\operatorname{vec}_{T, S}(A) \operatorname{vec}_{T, S}(B)^{\top} .
$$


Proposition 5.44. Let $S$ and $T$ be finite sets. Let $A, B \in \operatorname{Mat}(T, S)$. Then for every $X \in \operatorname{Mat}(S)$,

$$
\operatorname{Tr}_{\otimes X}\left(\operatorname{vec}_{T, S}(A) \operatorname{vec}_{T, S}(B)^{\top}\right)=A X B^{\top},
$$

and for every $Y \in \operatorname{Mat}(T)$,

$$
\operatorname{Tr}_{Y \otimes}\left(\operatorname{vec}_{T, S}(A) \operatorname{vec}_{T, S}(B)^{\top}\right)=A^{\top} Y B
$$

Proof. Set vec $:=\operatorname{vec}_{T, S}$. We first prove (5.157). Note that for every $Y \in \operatorname{Mat}(T)$,

$$
\begin{aligned}
\left\langle\operatorname{Tr}_{\otimes X}\left(\operatorname{vec}(A) \operatorname{vec}(B)^{\top}\right), Y\right\rangle & =\left\langle\operatorname{vec}(A) \operatorname{vec}(B)^{\top}, Y \otimes X\right\rangle & & \text { by }(5.153) \\
& =\operatorname{Tr}\left(\operatorname{vec}(B) \operatorname{vec}(A)^{\top}(Y \otimes X)\right) & & \text { by }(5.1) \\
& =\operatorname{vec}(A)^{\top}(Y \otimes X) \operatorname{vec}(B) & & \text { by }(2.18) \\
& =\operatorname{vec}(A)^{\top} \operatorname{vec}\left(Y B X^{\top}\right) & & \text { by Proposition } 5.43 \\
& =\left\langle A, Y B X^{\top}\right\rangle & & \text { by }(5.150) \\
& =\operatorname{Tr}\left(A^{\top} Y B X^{\top}\right) & & \text { by }(5.1) \\
& =\operatorname{Tr}\left(B X^{\top} A^{\top} Y\right) & & \text { by }(2.18) \\
& =\left\langle A X B^{\top}, Y\right\rangle . & & \text { by }(5.1)
\end{aligned}
$$

As this holds for every $Y \in \operatorname{Mat}(T)$, we conclude (5.157). The result follows, as for every $X \in \operatorname{Mat}(S)$,

$$
\begin{aligned}
\left\langle\operatorname{Tr}_{Y \otimes}\left(\operatorname{vec}(A) \operatorname{vec}(B)^{\top}\right), X\right\rangle & =\left\langle\operatorname{vec}(A) \operatorname{vec}(B)^{\top}, Y \otimes X\right\rangle & & \text { by }(5.155) \\
& =\left\langle\operatorname{Tr}_{\otimes X}\left(\operatorname{vec}(A) \operatorname{vec}(B)^{\top}\right), Y\right\rangle & & \text { by }(5.153) \\
& =\left\langle A X B^{\top}, Y\right\rangle & & \text { by }(5.157) \\
& =\operatorname{Tr}\left(B X^{\top} A^{\top} Y\right) & & \text { by }(5.1) \\
& =\operatorname{Tr}\left(X^{\top} A^{\top} Y B\right) & & \text { by }(2.18) \\
& =\left\langle X, A^{\top} Y B\right\rangle & & \text { by }(5.1) \\
& =\left\langle A^{\top} Y B, X\right\rangle . & &
\end{aligned}
$$

\subsubsection{Gram Matrices and Composition}

Any notion worth being called homomorphism must have an associated notion of composition. As we hinted at in (5.133), we want to work with linear maps between square matrices, and we want to have composition given by the usual composition of functions. On the other hand, we also want to use the cones from Chapter 5 to impose conic restrictions on such linear transformations. This motivates the topic of the current subsection: which matrix cones are "composition compatible"? To answer this question, we introduce the notion of Gram-like cones, present relevant examples, and exploit a key observation due to Roberson to show that such cones suit our needs.

An example may clarify our current task. Let $R, S$, and $T$ be finite sets. As we will later prove, it holds that

$$
\operatorname{mat}_{T, S}(\Phi) \in \mathbb{S}_{+}^{T \times S} \text { and } \operatorname{mat}_{S, R}(\Psi) \in \mathbb{S}_{+}^{S \times R} \text { imply that } \operatorname{mat}_{T, R}(\Phi \circ \Psi) \in \mathbb{S}_{+}^{T \times R}
$$

for every $\Phi \in \operatorname{Lin}(\operatorname{Mat}(S)$, Mat $(T))$ and $\Psi \in \operatorname{Lin}(\operatorname{Mat}(R)$, Mat $(S))$. Consequently, after using Proposition 5.44 to check that

$$
\operatorname{mat}_{S, S}(X \in \operatorname{Mat}(S) \mapsto X \in \operatorname{Mat}(S)) \in \mathbb{S}_{+}^{S},
$$

we can consider the sets

$$
\left\{\Phi \in \operatorname{Lin}(\operatorname{Mat}(S), \operatorname{Mat}(T)) \mid \operatorname{mat}_{T, S}(\Phi) \in \mathbb{S}_{+}^{T \times S}\right\}
$$

as defining the homomorphisms in a category whose composition is given by composition of linear maps. This subsection singles out a set of hypotheses on cones which are sufficient for implying (5.159). To the reader bothered by the absence of graphs in this discussion, we remark that graph homomorphisms are functions with extra conditions imposed; we are first obtaining a geometric encoding of functions, to later impose graph-theoretic conditions. 
Theorem 5.45. Let $S$ and $T$ be finite sets. Let $\mathbb{K}_{S} \subseteq \operatorname{Mat}(S)$ and $\mathbb{K}_{T} \subseteq \operatorname{Mat}(T)$ be convex cones. Let $\Phi \in \operatorname{Lin}(\operatorname{Mat}(S), \operatorname{Mat}(T))$. The following are equivalent:

1. $\operatorname{mat}_{T, S}(\Phi) \in\left(\mathbb{K}_{T}^{*} \otimes \mathbb{K}_{S}\right)^{*}$,

2. $\Phi\left(\mathbb{K}_{S}\right) \subseteq \operatorname{cl}\left(\mathbb{K}_{T}\right)$,

3. $\Phi^{*}\left(\mathbb{K}_{T}^{*}\right) \subseteq \mathbb{K}_{S}^{*}$.

Proof. Set mat $:=\operatorname{mat}_{T, S}$. Items 2 and 3 are equivalent by Proposition 5.1, so it suffices to prove that items 1 and 3 are equivalent. Assume item 3 holds, and let $Y \in \mathbb{K}_{T}^{*}$. Then for every $X \in \mathbb{K}_{S}$,

$$
\begin{aligned}
\langle Y \otimes X, \operatorname{mat}(\Phi)\rangle & =\langle Y, \Phi(X)\rangle & & \text { by }(5.147) \\
& =\left\langle\Phi^{*}(Y), X\right\rangle & & \\
& \geq 0 . & & \text { by item } 3
\end{aligned}
$$

Hence item 3 implies item 1.

Now assume that item 1 holds. Then for every $Y \in \mathbb{K}_{T}^{*}$ and $X \in \mathbb{K}_{S}$,

$$
\begin{aligned}
\left\langle\Phi^{*}(Y), X\right\rangle & =\langle Y, \Phi(X)\rangle & & \\
& =\langle Y \otimes X, \operatorname{mat}(\Phi)\rangle & & \text { by }(5.147) \\
& \geq 0 . & & \text { by item } 1
\end{aligned}
$$

Hence item 1 implies item 3.

Let $S$ and $T$ be finite sets, and let $\mathbb{K}_{S} \subseteq \operatorname{Mat}(S)$ and $\mathbb{K}_{T} \subseteq \operatorname{Mat}(T)$ be convex cones. Theorem 5.45 proves that

$$
\left\{\Phi \in \operatorname{Lin}(\operatorname{Mat}(S), \operatorname{Mat}(T)) \mid \Phi\left(\mathbb{K}_{S}\right) \subseteq \operatorname{cl}\left(\mathbb{K}_{T}\right)\right\}=\operatorname{mat}_{T, S}^{-1}\left(\left(\mathbb{K}_{T}^{*} \otimes \mathbb{K}_{S}\right)^{*}\right),
$$

making it immediate that the set in the LHS is a closed convex cone. Theorem 5.45 also can be used to prove that

$$
\text { for every } \Phi \in \operatorname{Lin}(\operatorname{Mat}(S), \operatorname{Mat}(T)) \text {, if } \operatorname{mat}_{T, S}(\Phi) \in \mathbb{S}_{+}^{T \times S} \text {, then } \Phi\left(\mathbb{S}_{+}^{S}\right) \subseteq \mathbb{S}_{+}^{T} \text {. }
$$

Similar results hold for the cones of symmetric, nonnegative, completely positive, and completely positive semidefinite matrices. We will not prove these now, as we obtain them as consequences of further developments.

The following definition, which is the main notion of this subsection, has a categorical interpretation we wish to emphasize. In order to do so, we define two categories. Denote by FinSet the category whose objects are finite sets, whose homomorphisms are functions, and whose composition is given by function composition. Denote by ConvCone the category whose objects are triplets $(S, T, \mathbb{K})$ where $\mathbb{K} \subseteq \operatorname{Mat}(T, S)$ is a convex cone. A homomorphism in ConvCone between the objects $\left(\mathbb{K}_{0}, S, T\right)$ and $\left(\mathbb{K}_{1}, U, V\right)$ is a linear transformation $\Phi: \operatorname{Mat}(S, T) \rightarrow \operatorname{Mat}(U, V)$ such that $\Phi\left(\mathbb{K}_{0}\right) \subseteq \mathbb{K}_{1}$. Composition of homomorphisms is given by function composition of linear transformations. For the next definition, we ask the reader to recall the notation from (2.12).

Definition 5.46. A Gram-like cone is function $\mathbb{K}$ on finite sets such that

1. for every finite set $V$, we have that $\mathbb{K}_{V}$ is a convex cone in $\operatorname{Mat}(V)$;

2. for every finite sets $U$ and $V$,

$$
\text { if } f \in \operatorname{Hom}_{\text {FinSet }}(U, V) \text {, then }\left(X \mapsto P_{f} X P_{f}^{\top}\right) \in \operatorname{Hom}_{\text {ConvCone }}\left(\mathbb{K}_{U}, \mathbb{K}_{V}\right) \text {; }
$$

3. for every finite set $V$ and for every $S \subseteq V$, if $X \in \mathbb{K}_{V}$, then $X[S] \in \mathbb{K}_{S}$;

4. for every finite sets $U$ and $V$, we have that $\mathbb{K}_{U} \otimes \mathbb{K}_{V} \subseteq \mathbb{K}_{U \times V}$.

We point out that items 1 and 2 of Definition 5.46 imply that a Gram-like cone $\mathbb{K}$ determines a functor FinSet $\rightarrow$ ConvCone. 
Proposition 5.47. The function on finite sets $V \mapsto \mathbb{S}^{V}$ is a Gram-like cone.

Proof. We directly prove each item from Definition 5.46. Items 1 and 3 are immediate. Let $U$ and $V$ be finite sets. Item 2 follows from the fact that

$$
\left(P_{f} X P_{f}^{\top}\right)^{\top}=P_{f} X^{\top} P_{f}^{\top}=P_{f} X P_{f}^{\top}
$$

for every $X \in \mathbb{S}^{U}$ and $f: U \rightarrow V$. Let $X \in \mathbb{S}^{U}$ and $Y \in \mathbb{S}^{V}$. Item 4 holds since

$$
\begin{aligned}
(X \otimes Y)_{(u, v),\left(u^{\prime}, v^{\prime}\right)} & =X_{u u^{\prime}} Y_{v v^{\prime}} & & \text { by }(5.135) \\
& =X_{u^{\prime} u} Y_{v^{\prime} v} & & \text { since } X \in \mathbb{S}^{U} \text { and } Y \in \mathbb{S}^{V} \\
& =(X \otimes Y)_{\left(u^{\prime}, v^{\prime}\right),(u, v)} & & \text { by }(5.135) \\
& =(X \otimes Y)_{(u, v),\left(u^{\prime}, v^{\prime}\right)}^{\top} & &
\end{aligned}
$$

for every $u, u^{\prime} \in U$ and $v, v^{\prime} \in V$.

Proposition 5.48. The function on finite sets $V \mapsto \operatorname{Mat}_{+}(V)$ is a Gram-like cone.

Proof. We directly prove each item from Definition 5.46. Items 1 and 3 are immediate. Let $U$ and $V$ be finite sets. Item 2 holds because for every $f: U \rightarrow V$,

$$
\begin{array}{rlrl}
\left(P_{f} X P_{f}^{\top}\right)_{i, j} & =e_{i}^{\top} P_{f} X P_{f}^{\top} e_{j} & \\
& =\mathbb{1}_{f^{-1}(\{i\})}^{\top} X \mathbb{1}_{f^{-1}(\{j\})} & \text { by }(2.13) \\
& \geq 0
\end{array}
$$

for every $X \in \operatorname{Mat}_{+}(U)$ and $i, j \in V$. Let $X \in \operatorname{Mat}_{+}(U)$ and $Y \in \operatorname{Mat}_{+}(V)$. Item 4 holds since

$$
\begin{aligned}
(X \otimes Y)_{(u, v),\left(u^{\prime}, v^{\prime}\right)} & =X_{u, u^{\prime}} Y_{v, v^{\prime}} & & \text { by }(5.135) \\
& \geq 0 & & \text { since } X \in \operatorname{Mat}_{+}(U) \text { and } Y \in \operatorname{Mat}_{+}(V)
\end{aligned}
$$

for every $u, u^{\prime} \in U$ and $v, v^{\prime} \in V$.

We now prove the cones in (5.11) to be Gram-like.

Proposition 5.49. Let $A, B, C, D, U$, and $V$ be finite sets. For every $f: U \rightarrow \operatorname{Mat}(A, B)$ and $g: V \rightarrow$ $\operatorname{Mat}(C, D)$,

$$
\operatorname{Gram}(f) \otimes \operatorname{Gram}(g)=\operatorname{Gram}(h)
$$

where $h: U \times V \rightarrow \operatorname{Mat}(A \times C, B \times D)$ is defined by

$$
h(u, v):=f(u) \otimes g(v)
$$

for every $u \in U$ and $v \in V$.

Proof. For every $u, u^{\prime} \in U$ and $v, v^{\prime} \in V$,

$$
\begin{aligned}
(\operatorname{Gram}(f) \otimes \operatorname{Gram}(g))_{\left((u, v),\left(u^{\prime}, v^{\prime}\right)\right)} & =\operatorname{Gram}(f)_{u u^{\prime}} \operatorname{Gram}(g)_{v v^{\prime}} & & \text { by }(5.135) \\
& =\left\langle f(u), f\left(u^{\prime}\right)\right\rangle\left\langle g(v), g\left(v^{\prime}\right)\right\rangle & & \text { by }(5.10) \\
& =\left\langle f(u) \otimes g(v), f\left(u^{\prime}\right) \otimes g\left(v^{\prime}\right)\right\rangle & & \text { by Proposition } 5.39 \\
& =\left\langle h(u, v), h\left(u^{\prime}, v^{\prime}\right)\right\rangle & & \\
& =\operatorname{Gram}(h)_{\left((u, v),\left(u^{\prime}, v^{\prime}\right)\right)} & & \text { by }(5.10)
\end{aligned}
$$

Theorem 5.50. Let $\mathbb{E}$ be a function on finite sets taking values in the objects of ConvCone. For every finite set $V$, define

$$
\operatorname{Gram}(\mathbb{E})_{V}:=\left\{\operatorname{Gram}(f) \mid f: V \rightarrow \mathbb{E}_{T} \text { for some finite set } T\right\} .
$$

Assume that 
1. $\operatorname{Gram}(\mathbb{E})_{V}$ is a convex cone for every finite set $V$,

2. that $\mathbb{E}_{S} \otimes \mathbb{E}_{T} \subseteq \mathbb{E}_{S \times T}$ for every finite sets $S$ and $T$.

Then the function on finite sets $V \mapsto \operatorname{Gram}(\mathbb{E})_{V}$ is a Gram-like functor.

Proof. We now prove each item in Definition 5.46.

Item 1 of Definition 5.46: as (5.10) implies that $\operatorname{Gram}(\mathbb{E})_{V} \subseteq \operatorname{Mat}(V)$ for every finite set $V$, we conclude this item directly from item 1.

Item 2 of Definition 5.46: let $U$ and $V$ be finite sets, let $\phi: U \rightarrow V$, and let $x: U \rightarrow \mathbb{E}_{S}$. Define $y: V \rightarrow \mathbb{E}_{S}$ by

$$
y(v):=\sum_{u \in U}\left(P_{\phi}\right)_{v u} x(u)
$$

for every $v \in V$. Note we have used that $\mathbb{E}_{S}$ is convex cone and that $P_{\phi} \geq 0$ to conclude that $y(v) \in \mathbb{E}_{S}$ for every $v \in V$. We have that $\operatorname{Gram}(y) \in \operatorname{Gram}(\mathbb{E})_{V}$, so

$$
\begin{array}{rlrl}
P_{\phi} \operatorname{Gram}(x) P_{\phi}^{\top} & =\operatorname{Gram}(y) & \text { by Proposition } 5.3 \\
& \in \operatorname{Gram}(\mathbb{E})_{V} .
\end{array}
$$

Item 3 of Definition 5.46: let $V$ be a finite set, and let $U \subseteq V$. Then for every finite set $S$ and $x: V \rightarrow \mathbb{E}_{S}$, we have that

$$
\begin{array}{rlrl}
\operatorname{Gram}(x)[S] & =\operatorname{Gram}\left(\left.x\right|_{S}\right) & \text { by Proposition } 5.2 \\
& \in \operatorname{Gram}(\mathbb{E})_{U} .
\end{array}
$$

Item 4 of Definition 5.46: let $U, V, S$, and $T$ be finite sets, and let $x: U \rightarrow \mathbb{E}_{S}$ and $y: V \rightarrow \mathbb{E}_{T}$. Define $z: U \times V \rightarrow \mathbb{E}_{S \times T}$ by

$$
z(u, v):=x(u) \otimes y(v)
$$

for every $(u, v) \in U \times V$. Note we have used Item 2 to conclude that $z(u, v) \in \mathbb{E}_{S \times T}$ for every $(u, v) \in U \times V$. Moreover, it is obvious that $S \times T$ is a finite set. Hence

$$
\begin{aligned}
\operatorname{Gram}(x) \otimes \operatorname{Gram}(y) & =\operatorname{Gram}(z) & \text { by Proposition } 5.49 \\
& \in \operatorname{Gram}(\mathbb{E})_{U \times V} . &
\end{aligned}
$$

Corollary 5.51. The function on finite sets $V \mapsto \mathbb{S}_{+}^{V}$ is a Gram-like cone.

Proof. For every finite set $S$, define $\mathbb{E}_{S}:=\mathbb{R}^{S}$. It is clear that

$$
\begin{aligned}
\mathbb{E}_{S} \otimes \mathbb{E}_{T} & =\mathbb{R}^{S} \otimes \mathbb{R}^{T} \\
& \subseteq \mathbb{R}^{S \times T} \\
& =\mathbb{E}_{S \times T}
\end{aligned}
$$

for every finite sets $S$ and $T$. Furthermore (5.11c) implies that $\operatorname{Gram}(\mathbb{E})_{V}=\mathbb{S}_{+}^{V}$ is a convex cone for every finite set $V$. Theorem 5.50 implies the statement.

Corollary 5.52. The function on finite sets $V \mapsto \mathbb{C S}_{+}^{V}$ is a Gram-like cone.

Proof. For every finite set $S$, define $\mathbb{E}_{S}:=\mathbb{S}_{+}^{S}$. We have that

$$
\begin{aligned}
\mathbb{E}_{S} \otimes \mathbb{E}_{T} & =\mathbb{S}_{+}^{S} \otimes \mathbb{S}_{+}^{T} \\
& \subseteq \mathbb{S}_{+}^{S \times T} \\
& =\mathbb{E}_{S \times T}
\end{aligned}
$$

by Corollary 5.51 and item 4 of Definition 5.46

Furthermore, (5.16) implies that $\operatorname{Gram}(\mathbb{E})_{V}=\mathbb{C S}_{+}^{V}$ is a convex cone for every finite set $V$. Theorem 5.50 implies the statement. 
Corollary 5.53. The function on finite sets $V \mapsto \mathbb{C P}^{V}$ is a Gram-like cone.

Proof. For every finite set $S$, define $\mathbb{E}_{S}:=\mathbb{R}_{+}^{S}$. We have that

$$
\begin{aligned}
\mathbb{E}_{S} \otimes \mathbb{E}_{T} & =\mathbb{R}_{+}^{S} \otimes \mathbb{R}_{+}^{T} \\
& \subseteq \mathbb{R}_{+}^{S \times T} \\
& =\mathbb{E}_{S \times T}
\end{aligned}
$$

Furthermore (5.14) implies that $\operatorname{Gram}(\mathbb{E})_{V}=\mathbb{C P}^{V}$ is a convex cone for every finite set $V$. Theorem 5.50 implies the statement.

Let $R, S$, and $T$ be finite sets. As we hinted at before, the work [100] does not mention spaces of the form $\operatorname{Lin}(\operatorname{Mat}(S), \operatorname{Mat}(T))$ : it develops its result entirely in terms of matrices in $\operatorname{Mat}(T \times S)$. A consequence of this approach is that expressing the composition of conic homomorphisms is much harder. Let $H_{1} \in \operatorname{Mat}(T \times S)$ and $H_{2} \in \operatorname{Mat}(S \times R)$. Define $H \in \operatorname{Mat}(T \times R)$ by

$$
H_{(t, r),\left(t^{\prime}, r^{\prime}\right)}:=\sum_{s, s^{\prime} \in S}\left(H_{1} \otimes H_{2}\right)\left(((t, s),(s, r)),\left(\left(t^{\prime}, s^{\prime}\right),\left(s^{\prime}, r^{\prime}\right)\right)\right)
$$

for every $t, t^{\prime} \in T$ and for every $r, r^{\prime} \in R$. Theorem 4.8 of [100] proves that conic graph homomorphisms are closed under composition by showing that $H$ determines a conic graph homomorphism whenever $H_{1}$ and $H_{2}$ determine conic graph homomorphisms. The language from Subsection 5.4.1 arose from an attempt to dissect (5.161) into meaningful constituents. The expression for mat ${ }_{T, R}(\Phi \circ \Psi)$ in the following theorem is the outcome of these efforts. The key argument used to show that Gram-like cones are "composition compatible" is present throughout [100], and is encapsulated by Theorem 5.54 .

Theorem 5.54. Let $R, S$, and $T$ be finite sets. Set

$$
U:=\left\{\left((t, s),\left(s^{\prime}, r\right)\right) \in(T \times S) \times(S \times R) \mid s=s^{\prime}\right\}
$$

and define $f: U \rightarrow T \times R$ by

$$
f((t, s),(s, r)):=(t, r)
$$

for every $((t, s),(s, r)) \in U$. Then for every $\Phi \in \operatorname{Lin}(\operatorname{Mat}(S), \operatorname{Mat}(T))$ and $\Psi \in \operatorname{Lin}(\operatorname{Mat}(R), \operatorname{Mat}(S))$,

$$
\operatorname{mat}_{T, R}(\Phi \circ \Psi)=P_{f}\left(\left(\operatorname{mat}_{T, S}(\Phi) \otimes \operatorname{mat}_{S, R}(\Psi)\right)[U]\right) P_{f}^{\top} .
$$

Proof. This proof is one big computation which we break down into smaller ones. Note that

$$
P_{f} \in \operatorname{Mat}(T \times R, U),
$$

and that for every $t \in T$ and $r \in R$, we have that

$$
P_{f}^{\top} e_{(t, r)}=\sum_{s \in S} e_{((t, s),(s, r))}
$$

by (2.13). Furthermore, it is clear that

$$
A=\sum_{s, s^{\prime} \in S}\left\langle A, e_{s} e_{s^{\prime}}^{\top}\right\rangle e_{s} e_{s^{\prime}}^{\top}
$$

for every $A \in \operatorname{Mat}(S)$.

Let $t, t^{\prime} \in T$ and $r, r^{\prime} \in R$. It holds that

$$
\begin{gathered}
\text { for every } s, s^{\prime} \in S, \\
\left(e_{t} e_{t^{\prime}}^{\top} \otimes e_{s} e_{s^{\prime}}^{\top}\right) \otimes\left(e_{s} e_{s^{\prime}}^{\top} \otimes e_{r} e_{r^{\prime}}^{\top}\right)=e_{((t, s),(s, r))} e_{\left(\left(t^{\prime}, s^{\prime}\right),\left(s^{\prime}, r^{\prime}\right)\right)}^{\top}
\end{gathered}
$$


since

$$
\begin{aligned}
\left(e_{t} e_{t^{\prime}}^{\top} \otimes e_{s} e_{s^{\prime}}^{\top}\right) \otimes\left(e_{s} e_{s^{\prime}}^{\top} \otimes e_{r} e_{r^{\prime}}^{\top}\right) & =e_{(t, s)} e_{\left(t^{\prime}, s^{\prime}\right)}^{\top} \otimes e_{(s, r)} e_{\left(s^{\prime}, r^{\prime}\right)}^{\top} & & \text { by (5.143) } \\
& =e_{((t, s),(s, r))} e_{\left(\left(t^{\prime}, s^{\prime}\right),\left(s^{\prime}, r^{\prime}\right)\right)}^{\top} & & \text { by }(5.143)
\end{aligned}
$$

Consequently,

$$
\begin{gathered}
\text { for every } s, s^{\prime} \in S, \\
\left\langle e_{t} e_{t^{\prime}}^{\top}, \Phi\left(e_{s} e_{s^{\prime}}^{\top}\right)\right\rangle\left\langle e_{s} e_{s^{\prime}}^{\top}, \Psi\left(e_{r} e_{r^{\prime}}^{\top}\right)\right\rangle=e_{((t, s),(s, r))}^{\top}\left(\operatorname{mat}_{T, S}(\Phi) \otimes \operatorname{mat}_{S, R}(\Psi)\right) e_{\left(\left(t^{\prime}, s^{\prime}\right),\left(s^{\prime}, r^{\prime}\right)\right)},
\end{gathered}
$$

as

$$
\begin{aligned}
& \left\langle e_{t} e_{t^{\prime}}^{\top}, \Phi\left(e_{s} e_{s^{\prime}}^{\top}\right)\right\rangle\left\langle e_{s} e_{s^{\prime}}^{\top}, \Psi\left(e_{r} e_{r^{\prime}}^{\top}\right)\right\rangle \\
& =\left\langle e_{t} e_{t^{\prime}}^{\top} \otimes e_{s} e_{s^{\prime}}^{\top}, \operatorname{mat}_{T, S}(\Phi)\right\rangle\left\langle e_{s} e_{s^{\prime}}^{\top} \otimes e_{r} e_{r^{\prime}}^{\top}, \operatorname{mat}_{S, R}(\Psi)\right\rangle \quad \text { by (5.147) } \\
& =\left\langle\left(e_{t} e_{t^{\prime}}^{\top} \otimes e_{s} e_{s^{\prime}}^{\top}\right) \otimes\left(e_{s} e_{s^{\prime}}^{\top} \otimes e_{r} e_{r^{\prime}}^{\top}\right), \operatorname{mat}_{T, S}(\Phi) \otimes \operatorname{mat}_{S, R}(\Psi)\right\rangle \quad \text { by Proposition } 5.39 \\
& =\left\langle e_{((t, s),(s, r))} e_{\left(\left(t^{\prime}, s^{\prime}\right),\left(s^{\prime}, r^{\prime}\right)\right)}^{\top}, \operatorname{mat}_{T, S}(\Phi) \otimes \operatorname{mat}_{S, R}(\Psi)\right\rangle \quad \text { by (5.164) } \\
& =\operatorname{Tr}\left(e_{\left(\left(t^{\prime}, s^{\prime}\right),\left(s^{\prime}, r^{\prime}\right)\right)} e_{((t, s),(s, r))}^{\top}\left(\operatorname{mat}_{T, S}(\Phi) \otimes \operatorname{mat}_{S, R}(\Psi)\right)\right) \quad \text { by (5.1) } \\
& =e_{((t, s),(s, r))}^{\top}\left(\operatorname{mat}_{T, S}(\Phi) \otimes \operatorname{mat}_{S, R}(\Psi)\right) e_{\left(\left(t^{\prime}, s^{\prime}\right),\left(s^{\prime}, r^{\prime}\right)\right) .} \quad \text { by }(2.18)
\end{aligned}
$$

We conclude that the matrices in the statement are equal:

$$
\begin{aligned}
& \operatorname{mat}_{T, R}(\Phi \circ \Psi)_{\left((t, r),\left(t^{\prime}, r^{\prime}\right)\right)} \\
& =\left\langle e_{(t, r)} e_{\left(t^{\prime}, r^{\prime}\right)}^{\top}, \operatorname{mat}_{T, R}(\Phi \circ \Psi)\right\rangle \\
& =\left\langle e_{t} e_{t^{\prime}}^{\top} \otimes e_{r} e_{r^{\prime}}^{\top}, \operatorname{mat}_{T, R}(\Phi \circ \Psi)\right\rangle \quad \text { by (5.143) } \\
& =\left\langle e_{t} e_{t^{\prime}}^{\top},(\Phi \circ \Psi)\left(e_{r} e_{r^{\prime}}^{\top}\right)\right\rangle \quad \text { by (5.147) } \\
& =\left\langle e_{t} e_{t^{\prime}}^{\top}, \Phi\left(\sum_{s, s^{\prime} \in S}\left\langle\Psi\left(e_{r} e_{r^{\prime}}^{\top}\right), e_{s} e_{s^{\prime}}^{\top}\right\rangle e_{s} e_{s^{\prime}}^{\top}\right)\right\rangle \quad \text { by (5.163) } \\
& =\sum_{s \in S} \sum_{s^{\prime} \in S}\left\langle e_{t} e_{t^{\prime}}^{\top}, \Phi\left(e_{s} e_{s^{\prime}}^{\top}\right)\right\rangle\left\langle\Psi\left(e_{r} e_{r^{\prime}}^{\top}\right), e_{s} e_{s^{\prime}}^{\top}\right\rangle \\
& =\sum_{s \in S} \sum_{s^{\prime} \in S} e_{((t, s),(s, r))}^{\top}\left(\operatorname{mat}_{T, S}(\Phi) \otimes \operatorname{mat}_{S, R}(\Psi)\right) e_{\left(\left(t^{\prime}, s^{\prime}\right),\left(s^{\prime}, r^{\prime}\right)\right)} \quad \text { by }(5.165) \\
& =\sum_{s \in S} \sum_{s^{\prime} \in S} e_{((t, s),(s, r))}^{\top}\left(\left(\operatorname{mat}_{T, S}(\Phi) \otimes \operatorname{mat}_{S, R}(\Psi)\right)[U]\right) e_{\left(\left(t^{\prime}, s^{\prime}\right),\left(s^{\prime}, r^{\prime}\right)\right)} \quad \text { by the definition of } U \\
& =\sum_{s \in S} e_{((t, s),(s, r))}^{\top}\left(\left(\operatorname{mat}_{T, S}(\Phi) \otimes \operatorname{mat}_{S, R}(\Psi)\right)[U]\right) P_{f}^{\top} e_{\left(t^{\prime}, r^{\prime}\right)} \quad \text { by }(5.162) \\
& =e_{(t, r)}^{\top} P_{f}\left(\left(\operatorname{mat}_{T, S}(\Phi) \otimes \operatorname{mat}_{S, R}(\Psi)\right)[U]\right) P_{f}^{\top} e_{\left(t^{\prime}, r^{\prime}\right)} \quad \text { by }(5.162) \\
& =\left(P_{f}\left(\left(\operatorname{mat}_{T, S}(\Phi) \otimes \operatorname{mat}_{S, R}(\Psi)\right)[U]\right) P_{f}^{\top}\right)_{\left((t, r),\left(t^{\prime}, r^{\prime}\right)\right)} .
\end{aligned}
$$

Corollary 5.55. Let $R, S$, and $T$ be finite sets, and let $\mathbb{K}$ be a Gram-like cone. If $\Phi \in \operatorname{Lin}(\operatorname{Mat}(S), \operatorname{Mat}(T))$ and $\Psi \in \operatorname{Lin}(\operatorname{Mat}(R), \operatorname{Mat}(S))$ are such that

$$
\operatorname{mat}_{T, S}(\Phi) \in \mathbb{K}_{T \times S} \text { and } \operatorname{mat}_{S, R}(\Psi) \in \mathbb{K}_{S \times R},
$$

then

$$
\operatorname{mat}_{T, R}(\Phi \circ \Psi) \in \mathbb{K}_{T \times R}
$$

Proof. Define $U \subseteq(T \times S) \times(S \times R)$ and $f: U \rightarrow T \times R$ as in Theorem 5.54. Then

$$
\begin{array}{lll}
\Longrightarrow & \operatorname{mat}_{T, S}(\Phi) \otimes \operatorname{mat}_{S, R}(\Psi) \in \mathbb{K}_{(T \times S) \times(S \times R)} & \text { by Definition } 5.46 \text { item } 4 \text { and (5.166) } \\
\Longrightarrow & \left(\operatorname{mat}_{T, S}(\Phi) \otimes \operatorname{mat}_{S, R}(\Psi)\right)[U] \in \mathbb{K}_{U} & \text { by Definition } 5.46 \text { item } 3 \\
\Longrightarrow & P_{f}\left(\left(\operatorname{mat}_{T, S}(\Phi) \otimes \operatorname{mat}_{S, R}(\Psi)\right)[U]\right) P_{f}^{\top} \in \mathbb{K}_{T \times R} & \text { by Definition } 5.46 \text { item } 2 \\
\Longrightarrow & \operatorname{mat}_{T, R}(\Phi \circ \Psi) \in \mathbb{K}_{T \times R} . & \text { by Theorem } 5.54 .
\end{array}
$$


Corollary 5.55 provides rigorous meaning to the idea that Gram-like cones are "composition compatible". Right after proving Theorem 5.45, we promised that (5.160) would follow from our developments. The following innocent-looking result is the key idea in making (5.160) fit in the result provided by Corollary 5.55.

Lemma 5.56. Let $S$ be a finite set. Define $\lambda: S \times\{0\} \rightarrow S$ by $\lambda(s, 0):=s$ for every $s \in S$. For every $\Phi \in \operatorname{Lin}(\operatorname{Mat}(\{0\}), \operatorname{Mat}(S))$ and for every $\alpha \in \mathbb{R}$,

$$
\Phi([\alpha])=\alpha P_{\lambda} \operatorname{mat}_{S,\{0\}}(\Phi) P_{\lambda}^{\top} .
$$

Proof. Since $\lambda: S \times\{0\} \rightarrow S$, we have that $P_{\lambda} \in \operatorname{Mat}(S, S \times\{0\})$. Moreover, as $\Phi \in \operatorname{Lin}(\operatorname{Mat}(\{0\})$, $\operatorname{Mat}(S))$, we have that $\operatorname{mat}_{S,\{0\}}(\Phi) \in \operatorname{Mat}(S \times\{0\})$. Therefore, the expression in the RHS of the statement is indeed a matrix in $\operatorname{Mat}(S)$.

Let $Y \in \operatorname{Mat}(S)$, and let $\alpha \in \mathbb{R}$. We claim that

$$
Y \otimes[\alpha]=\alpha P_{\lambda}^{\top} Y P_{\lambda} .
$$

This holds, as

$$
\begin{aligned}
(Y \otimes[\alpha])_{i 0, j 0} & =Y_{i j}[\alpha]_{00} \\
& =\alpha e_{i}^{\top} Y e_{j} \\
& =\alpha\left(P_{\lambda} e_{(i, 0)}\right)^{\top} Y P_{\lambda} e_{(j, 0)} \\
& =\left(\alpha P_{\lambda}^{\top} Y P_{\lambda}\right)_{i 0, j 0}
\end{aligned}
$$

for every $i, j \in S$. Hence

$$
\begin{aligned}
\langle Y, \Phi([\alpha])\rangle & =\left\langle Y \otimes[\alpha], \operatorname{mat}_{S,\{0\}}(\Phi)\right\rangle & & \text { by }(5.147) \\
& =\left\langle\alpha P_{\lambda}^{\top} Y P_{\lambda}, \operatorname{mat}_{S,\{0\}}(\Phi)\right\rangle & & \text { by }(5.167) \\
& =\alpha \operatorname{Tr}\left(P_{\lambda}^{\top} Y^{\top} P_{\lambda} \operatorname{mat}_{S,\{0\}}(\Phi)\right) & & \text { by }(5.1) \\
& =\alpha \operatorname{Tr}\left(Y^{\top} P_{\lambda} \operatorname{mat}_{S,\{0\}}(\Phi) P_{\lambda}^{\top}\right) & & \text { by }(2.18) \\
& =\alpha\left\langle Y, P_{\lambda} \operatorname{mat}_{S,\{0\}}(\Phi) P_{\lambda}^{\top}\right\rangle & & \text { by }(5.1) \\
& =\left\langle Y, \alpha P_{\lambda} \operatorname{mat}_{T,\{0\}}(\Phi) P_{\lambda}^{\top}\right\rangle . & &
\end{aligned}
$$

As this holds for every $Y \in \operatorname{Mat}(S)$, the statement follows.

Corollary 5.57. Let $S$ and $T$ be finite sets, and let $\mathbb{K}$ be a Gram-like cone. If $\Phi \in \operatorname{Lin}(\operatorname{Mat}(S), \operatorname{Mat}(T))$ is such that $\operatorname{mat}_{T, S}(\Phi) \in \mathbb{K}_{T \times S}$, then

$$
\Phi\left(\mathbb{K}_{S}\right) \subseteq \mathbb{K}_{T}
$$

Proof. Define

$$
\begin{gathered}
\lambda: S \times\{0\} \rightarrow S \text { by } \lambda(s, 0):=s \text { for every } s \in S, \\
\lambda^{\prime}: T \times\{0\} \rightarrow T \text { by } \lambda^{\prime}(t, 0):=t \text { for every } t \in T .
\end{gathered}
$$

Let $A \in \mathbb{K}_{S}$, and define $\Psi \in \operatorname{Lin}(\operatorname{Mat}(\{0\}), \operatorname{Mat}(S))$ by

$$
\Psi([\alpha]):=\alpha A
$$

for every $\alpha \in \mathbb{R}$. We have that

$$
\begin{aligned}
\operatorname{mat}_{S,\{0\}}(\Psi) & =P_{\lambda^{-1}} P_{\lambda} \operatorname{mat}_{S,\{0\}}(\Psi) P_{\lambda}^{\top} P_{\lambda^{-1}}^{\top} & & \text { by Proposition } 2.1 \\
& =P_{\lambda^{-1}} \Psi([1]) P_{\lambda^{-1}}^{\top} & & \text { by Lemma } 5.56 \\
& =P_{\lambda^{-1}} A P_{\lambda^{-1}}^{\top} & & \text { by }(5.168)
\end{aligned}
$$


Therefore Definition 5.46 item 2 and $A \in \mathbb{K}_{S}$ imply that

$$
\operatorname{mat}_{S,\{0\}}(\Psi)=P_{\lambda^{-1}} A P_{\lambda^{-1}}^{\top} \in \mathbb{K}_{S \times\{0\}},
$$

while $\operatorname{mat}_{T, S}(\Phi) \in \mathbb{K}_{T \times S}$ holds by hypothesis. Hence Corollary 5.55 applies, so

$$
\begin{array}{lll} 
& \operatorname{mat}_{T,\{0\}}(\Phi \circ \Psi) \in \mathbb{K}_{T \times\{0\}} & \text { by Corollary } 5.55 \\
\Longrightarrow & P_{\lambda^{\prime}} \operatorname{mat}_{T,\{0\}}(\Phi \circ \Psi) P_{\lambda^{\prime}}^{\top} \in \mathbb{K}_{T} & \text { by Definition } 5.46 \text { item } 2 \\
\Longrightarrow & \Phi(\Psi([1])) \in \mathbb{K}_{T} . & \text { by Lemma } 5.56 \\
\Longrightarrow & \Phi(A) \in \mathbb{K}_{T} . & \text { by }(5.168)
\end{array}
$$

As this holds for every $A \in \mathbb{K}_{S}$, the statement follows.

Corollary 5.58. Let $S$ and $T$ be finite sets, and let $\Phi \in \operatorname{Lin}(\operatorname{Mat}(S), \operatorname{Mat}(T))$. If $\operatorname{mat}_{T, S}(\Phi) \in \mathbb{S}^{T \times S}$, then $\Phi\left(\mathbb{S}^{S}\right) \subseteq \mathbb{S}^{T}$

Proof. Proposition 5.47 implies that $\mathbb{S}$ is a Gram-like cone, so Corollary 5.57 applies.

Corollary 5.59. Let $S$ and $T$ be finite sets. If $\Phi \in \operatorname{Lin}(\operatorname{Mat}(S), \operatorname{Mat}(T))$ is such that $\operatorname{mat}_{T, S}(\Phi) \in$ $\operatorname{Mat}_{+}(T \times S)$, then $\Phi\left(\operatorname{Mat}_{+}(S)\right) \subseteq \operatorname{Mat}_{+}(T)$.

Proof. Proposition 5.48 implies that Mat + is a Gram-like cone, so Corollary 5.57 applies.

Corollary 5.60. Let $S$ and $T$ be finite sets. If $\Phi \in \operatorname{Lin}(\operatorname{Mat}(S), \operatorname{Mat}(T))$ is such that $\operatorname{mat}_{T, S}(\Phi) \in \mathbb{S}_{+}^{T \times S}$, then $\Phi\left(\mathbb{S}_{+}^{S}\right) \subseteq \mathbb{S}_{+}^{T}$.

Proof. Corollary 5.51 implies that $\mathbb{S}_{+}$is a Gram-like cone, so Corollary 5.57 applies.

Corollary 5.61. Let $S$ and $T$ be finite sets, and let $\mathbb{K}$ be a Gram-like cone. Then

$$
\begin{aligned}
\mathbb{K}_{T \times S} & \subseteq\left(\mathbb{K}_{T}^{*} \otimes \mathbb{K}_{S}\right)^{*}, \text { and } \\
\mathbb{K}_{T}^{*} \otimes \mathbb{K}_{S} & \subseteq \mathbb{K}_{T \times S}^{*} .
\end{aligned}
$$

Proof. Let $H \in \mathbb{K}_{T \times S}$, and define $\Phi:=\operatorname{mat}_{T, S}^{-1}(H)$. Corollary 5.57 implies that

$$
\Phi\left(\mathbb{K}_{S}\right) \subseteq \mathbb{K}_{T} \subseteq \operatorname{cl}\left(\mathbb{K}_{T}\right),
$$

so Theorem 5.45 implies that

$$
H=\operatorname{mat}_{T, S}(\Phi) \in\left(\mathbb{K}_{T}^{*} \otimes \mathbb{K}_{S}\right)^{*} .
$$

Since $H \in \mathbb{K}_{T \times S}$ was arbitrary, we conclude (5.169a). The proof is done, as

$$
\begin{aligned}
\mathbb{K}_{T}^{*} \otimes \mathbb{K}_{S} & \subseteq \operatorname{cl}\left(\mathbb{K}_{T}^{*} \otimes \mathbb{K}_{S}\right) & & \\
& =\left(\mathbb{K}_{T}^{*} \otimes \mathbb{K}_{S}\right)^{* *} & & \text { by }(5.8) \\
& \subseteq \mathbb{K}_{T \times S}^{*} . & & \text { by }(5.4) \text { and }(5.169 \mathrm{a})
\end{aligned}
$$

\subsubsection{Conic Formulations of Graph Homomorphisms}

Let $\mathbb{K}_{0}$ and $\mathbb{K}_{1}$ be Gram-like cones. We denote by $\mathbb{K}_{0} \subseteq \mathbb{K}_{1}$ the fact that $\mathbb{K}_{0}(V) \subseteq \mathbb{K}_{1}(V)$ for every finite set $V$. In this notation, (5.17) becomes

$$
\mathbb{C P} \subseteq \mathbb{C S}_{+} \subseteq \mathbb{S}_{+} \subseteq \mathbb{S} .
$$

Definition 5.62. A Gram cone is a Gram-like cone $\mathbb{K}$ such that

$$
\mathbb{C P} \subseteq \mathbb{K} \subseteq \mathbb{S}_{+} .
$$


Definition 5.62 replaces the definition of "frabjous" cone in [100]. We now introduce notation which will be helpful when working with linear functions between square matrices. For every graph $G$, define

$$
\begin{aligned}
\operatorname{Diag}(G) & :=\operatorname{Diag}\left(\mathbb{R}^{V(G)}\right), \text { and } \\
\operatorname{Mat}(G) & :=\left\{A \in \operatorname{Mat}(V(G)) \mid A_{i j}=0, \forall i j \notin E(G)\right\} .
\end{aligned}
$$

Note that

$$
\begin{array}{r}
\left\{e_{i} e_{i}^{\top} \mid i \in V(G)\right\} \text { is a basis of } \operatorname{Diag}(G) \text { and } \\
\left\{e_{i} e_{j}^{\top} \mid i j \in E(G)\right\} \text { is a basis of } \operatorname{Mat}(G) .
\end{array}
$$

Finally, if $H$ is a graph, denote by

$$
\begin{aligned}
\operatorname{mat}_{H, G} & :=\operatorname{mat}_{V(H), V(G)}, \\
\operatorname{vec}_{H, G} & :=\operatorname{vec}_{V(H), V(G)} .
\end{aligned}
$$

Definition 5.63. Let $G$ and $H$ be graphs, and let $\mathbb{K}$ be a Gram cone. A $\mathbb{K}$-homomorphism from $G$ to $H$ is a linear transformation $\Phi \in \operatorname{Lin}(\operatorname{Mat}(V(G)), \operatorname{Mat}(V(H)))$ such that

1. $\Phi(\operatorname{Diag}(G)) \subseteq \operatorname{Diag}(H)$;

2. $\Phi(\operatorname{Mat}(G)) \subseteq \operatorname{Mat}(H)$;

3. $\Phi^{*}\left(\mathbb{1}^{\top}\right)=\mathbb{1}^{\top} ;$ and

4. $\operatorname{mat}_{H, G}(\Phi) \in \mathbb{K}_{V(H) \times V(G)}$.

We denote by $\operatorname{Hom}_{\mathbb{K}}(G, H)$ the set of $\mathbb{K}$-homomorphisms from $G$ to $H$.

Let $G$ and $H$ be graphs. If $\mathbb{K}_{0}$ and $\mathbb{K}_{1}$ are Gram cones, then it is obvious from Definition 5.63 that

$$
\mathbb{K}_{0} \subseteq \mathbb{K}_{1} \Longrightarrow \operatorname{Hom}_{\mathbb{K}_{0}}(G, H) \subseteq \operatorname{Hom}_{\mathbb{K}_{1}}(G, H)
$$

Together with (5.170), we conclude that

$$
\operatorname{Hom}_{\mathbb{C P}}(G, H) \subseteq \operatorname{Hom}_{\mathbb{C S}_{+}}(G, H) \subseteq \operatorname{Hom}_{\mathbb{S}_{+}}(G, H) .
$$

Let $G$ and $H$ be graphs, let $\mathbb{K}$ be a Gram-like cone, and let $\Phi \in \operatorname{Hom}_{\mathbb{K}}(G, H)$. The reader may find it odd that we are working with linear transformations between $\operatorname{Mat}(G)$ and $\operatorname{Mat}(H)$, which do not impose symmetry on the matrices manipulated. We remark that item 4 of Definition 5.63 and (5.171) imply that $\operatorname{mat}(\Phi) \in \mathbb{S}^{V(G) \times V(H)}$, so Corollary 5.58 implies that

$$
\Phi\left(\mathbb{S}^{V(G)}\right) \subseteq \mathbb{S}^{V(H)} .
$$

This, together with item 2 of Definition 5.62, implies that a conic homomorphisms maps the symmetric adjacency matrices of $G$ to the symmetric adjacency matrices of $H$.

As we have previously mentioned, Definition 5.63 attempts to define conic homomorphisms in a manner similar to quantum channels - see [118, Chapter 2]. If for a moment we pretend that our definitions were made taking complex numbers into account, one could define a quantum channel as a linear transformation $\Phi \in \operatorname{Lin}(\operatorname{Mat}(S), \operatorname{Mat}(T))$, for finite sets $S$ and $T$, such that

1. $\Phi^{*}(I)=I$ and

2. $\operatorname{mat}_{T, S}(\Phi) \in \mathbb{S}_{+}^{T \times S}$.

Quantum channels, which have been studied in quantum information theory, model discrete changes in the state of registers in a quantum computer. Note that quantum channels consist of linear transformations between square matrices whose Choi representation $\operatorname{mat}_{T, S}(\Phi)$ is constrained to be in a matrix cone, and whose adjoint must map a special matrix to a special matrix. In this manner, quantum channels are quite 
analogous to conic homomorphisms, and items 1 and 2 of Definition 5.63 stand out as encoding graph-theoretic information. We do, however, remark that this is nothing more than an analogy: we are working only with real matrices and not with complex ones. Furthermore, in our developments, quantum results are encoded by the completely positive semidefinite cone, not the positive semidefinite cone.

Let $G$ and $H$ be graphs, and let $\mathbb{K}$ be a Gram cone. One could use mat ${ }_{H, G}$ to obtain an equivalent definition of $\mathbb{K}$-homomorphism as a matrix in $\operatorname{Mat}(V(H) \times V(G))$ satisfying certain properties. This is precisely the approach in [100]. Our current goal is to recover this original definition. We start with an auxiliary result. Let $S$ and $T$ be finite sets. We claim that for every $A \in \operatorname{Mat}(T \times S)$,

$$
\operatorname{Tr}_{\otimes e_{i} e_{j}^{\top}}(A)_{k \ell}=A_{k i, \ell j}
$$

for every $i, j \in S$ and $k, \ell \in T$. Consequently, for every $\Phi \in \operatorname{Lin}(\operatorname{Mat}(S), \operatorname{Mat}(T))$,

$$
\Phi\left(e_{i} e_{j}^{\top}\right)_{k \ell}=\operatorname{mat}_{T, S}(\Phi)_{k i, \ell j},
$$

as $\Phi\left(e_{i} e_{j}^{\top}\right)=\operatorname{Tr}_{\otimes e_{i} e_{j}^{\top}}\left(\operatorname{mat}_{T, S}(\Phi)\right)$ by (5.156a). To see that (5.179) holds, note that

$$
\begin{aligned}
\operatorname{Tr}_{\otimes e_{i} e_{j}^{\top}}(A)_{k \ell} & =\left\langle\operatorname{Tr}_{\otimes e_{i} e_{j}^{\top}}(A), e_{k} e_{\ell}^{\top}\right\rangle \\
& =\left\langle A, e_{k} e_{\ell}^{\top} \otimes e_{i} e_{j}^{\top}\right\rangle \\
& =\left\langle A,\left(e_{k} \otimes e_{i}\right)\left(e_{\ell} \otimes e_{j}\right)^{\top}\right\rangle \\
& =\operatorname{Tr}\left(A^{\top}\left(e_{k} \otimes e_{i}\right)\left(e_{\ell} \otimes e_{j}\right)^{\top}\right) \\
& =\operatorname{Tr}\left(\left(e_{\ell} \otimes e_{j}\right)\left(e_{k} \otimes e_{i}\right)^{\top} A\right) \\
& =\left(e_{k} \otimes e_{i}\right)^{\top} A\left(e_{\ell} \otimes e_{j}\right) \\
& =e_{(k, i)}^{\top} A e_{(\ell, j)} \\
& =A_{k i, \ell j} .
\end{aligned}
$$

by Proposition 5.37

by $(5.1)$

by $(2.18)$

by $(5.142)$

Let $G$ and $H$ be graphs. In Subsection 3.4.7 we defined the homomorphic product $G \ltimes H$. Define $G \rtimes H$ as the graph on $V(H) \times V(G)$ with adjacency matrix

$$
A_{G \rtimes H}=\left(\mathbb{1}^{\top}-I\right) \otimes I+\left(\mathbb{1}^{\top}-A_{H}\right) \otimes A_{G} \in \mathbb{S}^{V(H) \times V(G)} .
$$

Compare with (3.190). It is easy to see that the graphs $G \ltimes H$ and $G \rtimes H$ are isomorphic, with the former having vertex set $V(G) \times V(H)$ and the latter having vertex set $V(H) \times V(G)$. When comparing (3.190) with (5.181), the difference between the vertex sets is related to the non-commutativity of the Kronecker product. Whereas $G \ltimes H$ fits nicely in Proposition 3.70, our current focus on matrices in Mat $(V(H) \times V(G))$ makes $G \rtimes H$ more convenient. Furthermore, note that (5.181) implies that

$$
\begin{aligned}
E(G \rtimes H)= & \{(k, i),(\ell, i)\} \mid i \in V(G), k, \ell \in V(H), k \neq \ell\} \\
& \cup\{\{(k, i),(\ell, j)\} \mid i, j \in V(G), k, \ell \in V(H), i j \in E(G), k \ell \notin E(H)\} .
\end{aligned}
$$

Proposition 5.64. Let $G$ and $H$ be graphs. If $A \in \operatorname{Mat}(V(H) \times V(G))$ is such that

$$
\begin{aligned}
& A \in \mathbb{S}^{V(H) \times V(G)}, \text { and } \\
A_{k i, \ell j}= & 0 \text { for every } k i, \ell j \in E(G \rtimes H),
\end{aligned}
$$

then $\Phi:=\operatorname{mat}_{H, G}^{-1}(A)$ satisfies

$$
\begin{aligned}
\Phi(\operatorname{Diag}(G)) & \subseteq \operatorname{Diag}(H), \\
\Phi(\operatorname{Mat}(G)) & \subseteq \operatorname{Mat}(H), \\
\operatorname{mat}_{H, G}(\Phi) & \in \mathbb{S}^{V(H) \times V(G)} .
\end{aligned}
$$

Conversely, if $\Phi \in \operatorname{Lin}(\operatorname{Mat}(V(G))$, Mat $(V(H)))$ satisfies (5.184), then $\operatorname{mat}_{H, G}(\Phi)$ satisfies (5.183). 
Proof. Assume $A \in \operatorname{Mat}(V(H) \times V(G))$ satisfies (5.183), and set $\Phi:=\operatorname{mat}_{H, G}^{-1}(A)$. For every $i, j \in V(G)$ and for every $k, \ell \in V(H)$,

$$
\Phi\left(e_{i} e_{j}^{\top}\right)_{k \ell}=\operatorname{Tr}_{\otimes e_{i} e_{j}^{\top}}(A)_{k \ell}=A_{k i, \ell j}
$$

by (5.179). We have that $\Phi\left(e_{i} e_{j}^{\top}\right) \in \operatorname{Diag}(H)$ for every $i \in V(G)$, as

$$
\begin{aligned}
\Phi\left(e_{i} e_{i}^{\top}\right)_{k \ell} & =A_{k i, \ell i} & & \text { by }(5.185) \\
& =0 . & & \text { by }(5.183 \mathrm{~b}) \text { and }(5.182 \mathrm{a})
\end{aligned}
$$

for every $k, \ell \in V(H)$ with $k \neq \ell$. We thus conclude (5.184a) from (5.174). We also have that $\Phi\left(e_{i} e_{j}^{\top}\right) \in \operatorname{Mat}(H)$ for every $i j \in E(G)$, as

$$
\begin{aligned}
\Phi\left(e_{i} e_{j}^{\top}\right)_{k \ell} & =A_{k i, \ell j} & & \text { by }(5.185) \\
& =0 . & & \text { by }(5.183 \mathrm{~b}) \text { and }(5.182 \mathrm{~b})
\end{aligned}
$$

for every $j k \notin E(H)$. We thus conclude (5.184b) from (5.175). Note that (5.184c) follows directly from (5.183a).

Assume $\Phi \in \operatorname{Lin}(\operatorname{Mat}(V(G)), \operatorname{Mat}(V(H)))$ satisfies (5.184), and set $A:=\operatorname{mat}_{H, G}(\Phi)$. For every $i, j \in V(G)$ and for every $k, \ell \in V(H)$,

$$
A_{k i, \ell j}=\operatorname{mat}_{H, G}(\Phi)_{k i, \ell j}=\Phi\left(e_{i} e_{j}^{\top}\right)_{k \ell}
$$

by (5.180). For every $i \in V(G)$ and $k, \ell \in V(H)$ with $k \neq \ell$ we have that

$$
\begin{aligned}
A_{k i, \ell i} & =\Phi\left(e_{i} e_{j}^{\top}\right)_{k \ell} & & \text { by }(5.186) \\
& =0 . & & \text { by }(5.184 \mathrm{a})
\end{aligned}
$$

Moreover, for every $i, j \in V(G)$ such that $i j \in E(G)$ and for every $k, \ell \in V(H)$ such that $k \ell \notin E(H)$, we have that

$$
\begin{aligned}
A_{k i, \ell j} & =\Phi\left(e_{i} e_{j}^{\top}\right)_{k \ell} & & \text { by }(5.186) \\
& =0 . & & \text { by }(5.184 \mathrm{~b})
\end{aligned}
$$

Using (5.182) we conclude that $A$ satisfies (5.183b). Moreover, (5.183a) follows directly from (5.184c).

Corollary 5.65. Let $G$ and $H$ be graphs, and let $\mathbb{K}$ be a Gram cone. Let $A \in \mathbb{K}_{V(H) \times V(G)}$. Then $\operatorname{mat}_{H, G}^{-1}(A) \in \operatorname{Hom}_{\mathbb{K}}(G, H)$ if and only if

1. for every $i, j \in V(G)$,

$$
\sum_{k, \ell \in V(H)} A_{k i, \ell j}=1
$$

2. for every $k i, \ell j \in E(G \rtimes H)$, it holds that $A_{k i, \ell j}=0$.

Proof. Set mat $:=\operatorname{mat}_{H, G}$. We claim that for every $\Phi \in \operatorname{Lin}(\operatorname{Mat}(V(G)), \operatorname{Mat}(V(H)))$ and $i, j \in V(G)$,

$$
\Phi^{*}\left(\mathbb{1}^{\top}\right)_{i j}=\sum_{k, \ell \in V(H)} \operatorname{mat}(\Phi)_{k i, \ell j} .
$$

Indeed,

$$
\begin{aligned}
\Phi^{*}\left(\mathbb{1}^{\top}\right)_{i j} & =\left\langle\Phi^{*}\left(\mathbb{1}^{\top}\right), e_{i} e_{j}^{\top}\right\rangle \\
& =\left\langle\mathbb{1} \mathbb{1}^{\top}, \Phi\left(e_{i} e_{j}^{\top}\right)\right\rangle \\
& =\left\langle\mathbb{1} \mathbb{1}^{\top} \otimes e_{i} e_{j}^{\top}, \operatorname{mat}(\Phi)\right\rangle \\
& =\left\langle\left(\sum_{k, \ell \in V(H)} e_{k} e_{\ell}^{\top}\right) \otimes e_{i} e_{j}^{\top}, \operatorname{mat}(\Phi)\right\rangle \\
& =\sum_{k, \ell \in V(H)}\left\langle e_{k} e_{\ell}^{\top} \otimes e_{i} e_{j}^{\top}, \operatorname{mat}(\Phi)\right\rangle \\
& =\sum_{k, \ell \in V(H)} \operatorname{mat}(\Phi)_{k i, \ell j} .
\end{aligned}
$$


Let $A \in \mathbb{K}_{V(H) \times V(G)}$. Set $\Phi:=$ mat $^{-1}(A)$. By (5.187), we have that $\Phi$ satisfies item 3 of Definition 5.63 if and only if item 1 holds. By Proposition 5.64, we have that $\Phi$ satisfies items 1 and 2 of Definition 5.63 if and only if item 2 holds. Since $\operatorname{mat}(\Phi)=A$, the proof is done.

Corollary 5.65 implies the equivalence of Definition 5.63 with the original definition in [100].

Proposition 5.66. Let $G, H$, and $K$ be graphs, and let $\mathbb{K}$ be a Gram cone. If $\Phi \in \operatorname{Hom}_{\mathbb{K}}(H, K)$ and $\Psi \in \operatorname{Hom}_{\mathbb{K}}(G, H)$, then $\Phi \circ \Psi \in \operatorname{Hom}_{\mathbb{K}}(G, K)$.

Proof. It is clear that $\Phi \circ \Psi$ satisfies items 1 and 2 of Definition 5.63. Moreover, note that

$$
\begin{array}{rlrl}
(\Phi \circ \Psi)^{*}\left(\mathbb{1}^{\top}\right) & =\left(\Psi^{*} \circ \Phi^{*}\right)\left(\mathbb{1} \mathbb{1}^{\top}\right) \\
& =\Psi^{*}\left(\mathbb{1}^{\top}\right) & \text { by Definition } 5.63 \text { item } 3
\end{array}
$$$$
=\mathbb{1} \mathbb{1}^{\top} \text {. } \quad \text { by Definition } 5.63 \text { item } 3
$$

Finally, Corollary 5.55 and both $\operatorname{mat}_{K, H}(\Phi) \in \mathbb{K}_{V(K) \times V(H)}$ and $\operatorname{mat}_{H, G}(\Psi) \in \mathbb{K}_{V(H) \times V(G)}$ imply that

$$
\operatorname{mat}_{K, G}(\Phi \circ \Psi) \in \mathbb{K}_{V(K) \times V(G)} .
$$

Let $\mathbb{K}$ be a Gram cone. To prove that Hom $_{\mathbb{K}}$ are the homomorphisms of a category, it remains to exhibit identity homomorphisms. We do slightly more here, by proving a characterization of Hom $_{\mathbb{C P}}$ in terms of graph homomorphisms, and then exploiting (5.178).

Proposition 5.67. Let $G$ and $H$ be graphs, and let $f: V(G) \rightarrow V(H)$. Then $f \in \operatorname{Hom}(G, H)$ if and only if

$$
\operatorname{vec}_{H, G}\left(P_{f}\right) \operatorname{vec}_{H, G}\left(P_{f}\right)^{\top} \in \operatorname{mat}_{H, G}\left(\operatorname{Hom}_{\mathbb{C P}}(G, H)\right) .
$$

Proof. Set vec $:=\operatorname{vec}_{H, G}$, mat $:=\operatorname{mat}_{H, G}$, and $\Phi:=\operatorname{mat}^{-1}\left(\operatorname{vec}\left(P_{f}\right) \operatorname{vec}\left(P_{f}\right)^{\top}\right)$. For every $X \in \operatorname{Mat}(V(G))$ we have that

$$
\Phi(X)=P_{f} X P_{f}^{\top},
$$

since

$$
\begin{aligned}
\Phi(X) & =\operatorname{Tr}_{\otimes X}\left(\operatorname{vec}\left(P_{f}\right) \operatorname{vec}\left(P_{f}\right)^{\top}\right) & & \text { by }(5.156 \mathrm{a}) \\
& =P_{f} X P_{f}^{\top} . & & \text { by }(5.157)
\end{aligned}
$$

For every $i \in V(G)$, we have that

$$
\begin{aligned}
\Phi\left(e_{i} e_{i}^{\top}\right) & =P_{f} e_{i} e_{i}^{\top} P_{f}^{\top} & & \text { by }(5.188) \\
& =e_{f(i)} e_{f(i)}^{\top} & & \text { by }(2.12) \\
& \in \operatorname{Diag}(H) . & &
\end{aligned}
$$

Hence (5.174) implies $\Phi$ satisfies item 1 of Definition 5.63.

We also have that $\Phi$ satisfies item 3 of Definition 5.63 , as

$$
\begin{aligned}
\Phi^{*}\left(\mathbb{1} \mathbb{1}^{\top}\right) & =\operatorname{Tr}_{\mathbb{1} \mathbb{1}^{\top} \otimes}\left(\operatorname{vec}\left(P_{f}\right) \operatorname{vec}\left(P_{f}\right)^{\top}\right) & & \text { by }(5.156 \mathrm{~b}) \\
& =P_{f}^{\top} \mathbb{1}^{\top} P_{f} & & \text { by }(5.158) \\
& =\mathbb{1}^{\top} . & & \text { by }(2.15)
\end{aligned}
$$

Item 4 of Definition 5.63 follows from $\operatorname{vec}\left(P_{f}\right) \geq 0$, since

$$
\operatorname{mat}(\Phi)=\operatorname{vec}\left(P_{f}\right) \operatorname{vec}\left(P_{f}\right)^{\top} \in \mathbb{C P}^{V(H) \times V(G)} .
$$

by Proposition 5.4.

We finish the proof by showing that $\Phi$ satisfies item 2 of Definition 5.63 if and only if $f \in \operatorname{Hom}(G, H)$. 
Assume $f \in \operatorname{Hom}(G, H)$. Then $\Phi \in \operatorname{Hom}_{\mathbb{K}}(G, H)$, as for every $A \in \operatorname{Mat}(G)$,

$$
\begin{array}{rlrl}
\Phi(A) & =\sum_{i, j \in V(G)} A_{i j} \Phi\left(e_{i} e_{j}^{\top}\right) & \\
& =\sum_{i, j \in V(G)} A_{i j} P_{f} e_{i} e_{j}^{\top} P_{f}^{\top} & & \text { by (5.188) } \\
& =\sum_{i, j \in V(G)} A_{i j} e_{f(i)} e_{f(j)}^{\top} & & \text { by }(2.12) \\
& \in \operatorname{Mat}(G) . & & \text { by (5.175) and } f(i) f(j) \in E(H)
\end{array}
$$

Conversely, assume $\Phi \in \operatorname{Hom}_{\mathbb{K}}(G, H)$. Then $f(i) f(j) \in E(H)$ for every $i j \in E(G)$, since

$$
\begin{aligned}
e_{f(i)} e_{f(j)}^{\top} & =P_{f} e_{i} e_{j}^{\top} P_{f}^{\top} & & \text { by }(2.12) \\
& =\Phi\left(e_{i} e_{j}^{\top}\right) & & \text { by }(5.188) \\
& \in \operatorname{Mat}(H) . & & \text { by item } 2 \text { of Definition } 5.63
\end{aligned}
$$

Theorem 5.68. Let $S$ and $T$ be finite sets. Then

$$
\left\{A \in \operatorname{Mat}(T, S) \mid A^{\top} \mathbb{1}=\mathbb{1}, A \geq 0\right\}=\operatorname{conv}\left(\left\{P_{f} \mid f: S \rightarrow T\right\}\right) .
$$

Theorem 5.68 is a straightforward generalization of the Birkhoff-von Neumann Theorem (see [60, Theorem 8.7.1] or [109, Corollary 18.1]).

Proposition 5.69. Let $G$ and $H$ be graphs, and let $\Phi \in \operatorname{Lin}(\operatorname{Mat}(V(G))$, Mat $(V(H)))$. Then

$$
\Phi \in \operatorname{Hom}_{\mathbb{C P}}(G, H) \text { and } \operatorname{rank}\left(\operatorname{mat}_{H, G}(\Phi)\right)=1
$$

if and only if

$$
\text { there exists } f \in \operatorname{Hom}(G, H) \text { such that } \operatorname{mat}_{H, G}(\Phi)=\operatorname{vec}_{H, G}\left(P_{f}\right) \operatorname{vec}_{H, G}\left(P_{f}\right)^{\top} .
$$

Proof. Set mat $:=\operatorname{mat}_{H, G}$ and vec $:=\operatorname{vec}_{H, G}$.

If $f \in \operatorname{Hom}(G, H)$, then Proposition 5.67 implies that $\operatorname{vec}\left(P_{f}\right) \operatorname{vec}\left(P_{f}\right)^{\top}$ is a rank-one element of $\operatorname{mat}\left(\operatorname{Hom}_{\mathbb{C P}}(G, H)\right)$.

Let $\Phi \in \operatorname{Hom}_{\mathbb{C P}}(G, H)$ be such that $\operatorname{rank}(\operatorname{mat}(\Phi))=1$. Proposition 5.4 implies there exists $x \in$ $\mathbb{R}_{+}^{V(H) \times V(G)}$ such that mat $(\Phi)=x x^{\top}$. Since vec is invertible by Proposition 5.42, there exists $A \in \operatorname{Mat}(T, S)$ such that $x=\operatorname{vec}(A)$. Combining both results, we have that

$$
\operatorname{mat}(\Phi)=\operatorname{vec}(A) \operatorname{vec}(A)^{\top} .
$$

For every $i \in V(G)$,

$$
\begin{array}{rlrl}
1 & =\left\langle\mathbb{1} \mathbb{1}^{\top}, e_{i} e_{i}^{\top}\right\rangle & & \\
& =\left\langle\Phi^{*}\left(\mathbb{1}^{\top}\right), e_{i} e_{i}^{\top}\right\rangle & & \text { by Definition } 5.63 \text { item } 3 \\
& =\left\langle\operatorname{Tr}_{\mathbb{1}} \mathbb{1}^{\top} \otimes\right. \\
& =\left\langle A^{\top} \mathbb{1} \mathbb{1}^{\top} A, e_{i} e_{i}^{\top}\right\rangle & & \text { by }(5.156 \mathrm{~b}) \text { and }(5.189) \\
& =\operatorname{Tr}\left(A^{\top} \mathbb{1} \mathbb{1}^{\top} A e_{i} e_{i}^{\top}\right) & & \text { by }(5.158) \\
& =\left(e_{i}^{\top} A^{\top} \mathbb{1}\right)^{2} . & & \text { by }(5.1) \\
& & \text { by }(2.18)
\end{array}
$$

We also have that $A \geq 0$, since

$$
\begin{aligned}
A_{t s} & =\left\langle A, e_{t} e_{s}^{\top}\right\rangle & & \\
& =\operatorname{vec}(A)^{\top} \operatorname{vec}\left(e_{t} e_{s}^{\top}\right) & & \text { by }(5.150) \\
& =\operatorname{vec}(A)^{\top}\left(e_{t} \otimes e_{s}\right) & & \text { by }(5.149) \\
& =x^{\top}\left(e_{t} \otimes e_{s}\right) & & \\
& \geq 0 & & \text { since } x \geq 0
\end{aligned}
$$


for every $t \in T$ and for every $s \in S$. Therefore, we conclude $A \geq 0$ and that $A^{\top} \mathbb{1}=\mathbb{1}$. Theorem 5.68 implies there exists $F \subseteq V(H)^{V(G)}$ such that $A=\sum_{f \in F} \lambda_{f} P_{f}$, with $\lambda \in \mathbb{R}_{++}^{F}$ such that $\mathbb{1}^{\top} \lambda=1$. Hence

$$
x x^{\top}=\operatorname{vec}(A) \operatorname{vec}(A)^{\top}=\sum_{f, g \in F} \lambda_{f} \lambda_{g} \operatorname{vec}\left(P_{f}\right) \operatorname{vec}\left(P_{g}\right)^{\top} .
$$

Let $i \in V(G)$. Then

$$
\begin{aligned}
\Phi\left(e_{i} e_{i}^{\top}\right) & =\operatorname{Tr}_{\otimes e_{i} e_{i}^{\top}}\left(x x^{\top}\right) & & \text { by }(5.156 a) \text { and }(5.189) \\
& =\sum_{f, g \in F} \lambda_{f} \lambda_{g} \operatorname{Tr}_{\otimes e_{i} e_{i}^{\top}\left(\operatorname{vec}\left(P_{f}\right) \operatorname{vec}\left(P_{g}\right)^{\top}\right)} & & \text { by }(5.190) \\
& =\sum_{f, g \in F} \lambda_{f} \lambda_{g} P_{f} e_{i} e_{i}^{\top} P_{g}^{\top} & & \text { by }(5.157) \\
& =\sum_{f, g \in F} \lambda_{f} \lambda_{g} e_{f(i)} e_{g(i)}^{\top} . & & \text { by }(2.12)
\end{aligned}
$$

Definition 5.63 item 1 states that $\Phi\left(e_{i} e_{i}^{\top}\right)$ is a diagonal matrix, so we use $\lambda \in \mathbb{R}_{++}^{F}$ to conclude that $f(i)=g(i)$ for every $f, g \in F$. As this holds for every $i \in V(G)$, it follows that $F=\{f\}$, for some $f: V(G) \rightarrow V(H)$. Proposition 5.67 then implies that $f \in \operatorname{Hom}(G, H)$.

Theorem 5.70 ([100]). Let $G$ and $H$ be graphs. Then

$$
\operatorname{mat}_{H, G}\left(\operatorname{Hom}_{\mathbb{C P}}(G, H)\right)=\operatorname{conv}\left(\left\{\operatorname{vec}_{H, G}\left(P_{f}\right) \operatorname{vec}_{H, G}\left(P_{f}\right)^{\top} \mid f \in \operatorname{Hom}(G, H)\right\}\right) .
$$

Proof. Set vec $:=\operatorname{vec}_{H, G}$ and mat $:=\operatorname{mat}_{H, G}$. Proposition 5.67 and convexity of $\operatorname{Hom}_{\mathbb{C P}}(G, H)$ imply that

$$
\operatorname{conv}\left(\left\{\operatorname{vec}\left(P_{f}\right) \operatorname{vec}\left(P_{f}\right)^{\top} \mid f \in \operatorname{Hom}(G, H)\right\}\right) \subseteq \operatorname{mat}\left(\operatorname{Hom}_{\mathbb{C P}}(G, H)\right) .
$$

Let $\Phi \in \operatorname{Hom}_{\mathbb{C P}}(G, H)$. Then mat $(\Phi) \in \mathbb{C P}^{V(H) \times V(G)}$ by Definition 5.63 item 4, so item 3 of Proposition 5.4 implies there exists a finite set $S$ and $a: S \rightarrow \mathbb{R}_{+}^{V(H) \times V(G)}$ such that

$$
\begin{aligned}
& \operatorname{mat}(\Phi)=\sum_{s \in S} a_{s} a_{s}^{\top}, \text { and } \\
& a_{s} \neq 0 \text { for every } s \in S .
\end{aligned}
$$

Let $k \in S$, and define $\Phi_{k}:=\operatorname{mat}^{-1}\left(a_{k} a_{k}^{\top}\right)$. We claim that

$$
\alpha_{k} \Phi_{k} \in \operatorname{Hom}_{\mathbb{C P}}(G, H) \text { for some } \alpha_{k} \in \mathbb{R}_{++} .
$$

Note that for every $i, j \in V(G)$,

$$
\begin{aligned}
\Phi\left(e_{i} e_{j}^{\top}\right) & =\operatorname{Tr}_{\otimes e_{i} e_{j}^{\top}}(\operatorname{mat}(\Phi)) & & \text { by (5.156a) } \\
& =\sum_{s \in S} \operatorname{Tr}_{\otimes e_{i} e_{j}^{\top}\left(a_{s} a_{s}^{\top}\right)} & & \text { by (5.191a) } \\
& \geq \operatorname{Tr}_{\otimes e_{i} e_{j}^{\top}\left(a_{s} a_{s}^{\top}\right)} & & \text { by Corollary } 5.59 \text { and } a_{s} a_{s}^{\top} \geq 0 \text { for every } s \in S \\
& =\Phi_{k}\left(e_{i} e_{j}^{\top}\right) & & \\
& \geq 0 . & & \text { by Corollary } 5.59 \text { and } a_{s} a_{S}^{\top} \geq 0
\end{aligned}
$$

In short, $\Phi\left(e_{i} e_{j}^{\top}\right) \geq \Phi_{k}\left(e_{i} e_{j}^{\top}\right) \geq 0$. Consequently, if $\Phi_{k}\left(e_{i} e_{j}^{\top}\right)$ is not a diagonal matrix, then neither is $\Phi\left(e_{i} e_{j}^{\top}\right)$. Using (5.174) we conclude that $\Phi_{k}$ satisfies Definition 5.63 item 1. Similarly, $\Phi\left(e_{i} e_{j}^{\top}\right) \geq \Phi_{k}\left(e_{i} e_{j}^{\top}\right) \geq 0$ 
also implies that $\Phi_{k}\left(e_{i} e_{j}^{\top}\right) \in \operatorname{Mat}(H)$ for every $i j \in E(G)$. Using (5.175) we conclude that $\Phi_{k}$ satisfies Definition 5.63 item 2. Moreover,

$$
\begin{aligned}
\mathbb{1} \mathbb{1}^{\top} & =\Phi^{*}\left(\mathbb{1}^{\top}\right) & & \text { by Definition } 5.63 \text { item } 3 \\
& =\operatorname{Tr}_{\mathbb{1} \mathbb{1}^{\top} \otimes}(\operatorname{mat}(\Phi)) & & \text { by }(5.156 \mathrm{~b}) \\
& =\sum_{s \in S} \operatorname{Tr}_{\mathbb{1}^{\top} \otimes}\left(a_{s} a_{s}^{\top}\right) & & \text { by }(5.191 \mathrm{a}) \\
& \succeq \operatorname{Tr}_{\mathbb{1} \mathbb{1}^{\top} \otimes}\left(a_{k} a_{k}^{\top}\right) & & \text { by Corollary } 5.60 \text { and } a_{s} a_{s}^{\top} \succeq 0 \text { for every } s \in S \\
& =\Phi_{k}^{*}\left(\mathbb{1} \mathbb{1}^{\top}\right) & & \\
& \succeq 0 . & & \text { by Corollary } 5.60 \text { and } a_{s} a_{s}^{\top} \succeq 0
\end{aligned}
$$

In short, $\mathbb{1} \mathbb{1}^{\top} \succeq \Phi_{k}^{*}\left(\right.$ ones $\left.\mathbb{1}^{\top}\right) \succeq 0$, so we conclude that $\Phi_{k}^{*}\left(\mathbb{1}^{\top}\right)$ is a scalar multiple of $\mathbb{1}^{\top}$. We prove (5.192) by showing that $\Phi_{k}^{*}\left(\mathbb{1}^{\top}\right) \neq 0$, which holds because

$$
\begin{aligned}
& a_{k} a_{k}^{\top} \neq 0 \quad \text { by (5.191b) } \\
& \Longrightarrow \Phi_{k} \neq 0 \quad \text { as mat }\left(\Phi_{k}\right)=a_{k} a_{k}^{\top} \text { and mat injective } \\
& \Longrightarrow \Phi_{k}\left(e_{i} e_{j}^{\top}\right) \neq 0 \text { for some } i, j \in V(G) \\
& \Longrightarrow\left\langle\Phi_{k}\left(e_{i} e_{j}^{\top}\right), \mathbb{1}^{\top}\right\rangle>0 \text { for some } i, j \in V(G) \quad \text { since } \Phi_{k}\left(e_{i} e_{j}^{\top}\right) \geq 0 \text { by Corollary } 5.59 \\
& \Longrightarrow \Phi_{k}^{*}\left(\mathbb{1}^{\top}\right) \neq 0 \text {. }
\end{aligned}
$$

For every $s \in S$, define $\Psi_{s}:=\alpha_{s} \Phi_{s}$, where $\alpha_{s}$ is a nonnegative number whose existence is implied by (5.192). Clearly,

$$
\begin{aligned}
\Phi & =\sum_{s \in S} \Phi_{s} & & \text { by }(5.191 \mathrm{a}) \\
& =\sum_{s \in S} \alpha_{s}^{-1} \Psi_{s} . & & \text { by definition of } \Psi_{s}
\end{aligned}
$$

Moreover, we have that $\sum_{s \in S} \alpha_{s}^{-1}=1$, as

$$
\mathbb{1}^{\top}=\Phi^{*}\left(\mathbb{1}^{\top}\right)=\sum_{s \in S} \alpha_{s}^{-1} \Psi_{s}^{*}\left(\mathbb{1} \mathbb{1}^{\top}\right)=\left(\sum_{s \in S} \alpha_{s}^{-1}\right) \mathbb{1}^{\top} .
$$

In short, $\Phi$ is a convex combination of $\left\{\Psi_{s} \mid s \in S\right\}$. As for each $s \in S$, we have that $\Psi_{s} \in \operatorname{Hom}_{\mathbb{C P}}(G, H)$ and $\operatorname{rank}\left(\operatorname{mat}\left(\Psi_{s}\right)\right)=1$, Proposition 5.69 concludes the proof.

Corollary 5.71 ([100]). Let $G$ and $H$ be graphs. Then

$$
\operatorname{Hom}(G, H) \neq \varnothing \Longleftrightarrow \operatorname{Hom}_{\mathbb{C P}}(G, H) \neq \varnothing .
$$

Proof. Immediate from Theorem 5.70.

Theorem 5.72. For every Gram cone $\mathbb{K}$, there exists a category $\mathbb{K}$-Graph whose objects are graphs and whose homomorphisms between graphs $G$ and $H$ are given by $\operatorname{Hom}_{\mathbb{K}}(G, H)$.

Proof. Proposition 5.66 implies that conic homomorphisms compose. To finish the proof, we must establish that every graph has an identity homomorphism. Let $G$ be a graph, set vec $:=\operatorname{vec}_{G, G}$ and mat $:=\operatorname{mat}_{G, G}$, and let id: $V(G) \rightarrow V(G)$ denote the identity function. Then

$$
\begin{aligned}
\operatorname{vec}(I) \operatorname{vec}(I)^{\top} & =\operatorname{vec}\left(P_{\mathrm{id}}\right) \operatorname{vec}\left(P_{\mathrm{id}}\right)^{\top} & & \text { since } P_{\mathrm{id}}=I \text { by Proposition } 2.1 \\
& \in \operatorname{mat}\left(\operatorname{Hom}_{\mathbb{C P}}(G, G)\right) & & \text { by Proposition } 5.67 \text { and id } \in \operatorname{Hom}(G, G) \\
& \subseteq \operatorname{mat}\left(\operatorname{Hom}_{\mathbb{K}}(G, G)\right) . & & \text { by }(5.178) \text { and } \mathbb{C P} \subseteq \mathbb{K}
\end{aligned}
$$

Define $\Phi:=$ mat $^{-1}\left(\operatorname{vec}(I) \operatorname{vec}(I)^{\top}\right)$. Then (5.156a) and Proposition 5.44 imply that

$$
\Phi(X)=\operatorname{Tr}_{\otimes X}\left(\operatorname{vec}(I) \operatorname{vec}(I)^{\top}\right)=X
$$

for every $X \in \operatorname{Mat}(V(G))$, which implies that $\Phi$ is the identity homomorphism in $\operatorname{Hom}_{\mathbb{K}}(G, G)$. 
Some of the ideas captured by conic homomorphisms have been around for a few decades. Feige and Lovász [36], in 1992, optimized over objects similar to $\operatorname{mat}_{H, G}\left(\operatorname{Hom}_{\mathbb{C P}}(G, H)\right)$ and $\operatorname{mat}_{H, G}\left(\operatorname{Hom}_{\mathbb{S}_{+}}(G, H)\right)$ for graphs $G$ and $H$ when studying two-prover one-round protocols. Not only that, [36] mentions the homomorphism game, and its [36, Lemma 5.22] states that $\mathbb{S}_{+}$-homomorphisms compose. We will prove that $\mathbb{S}_{+}$-homomorphisms define the same preorder on graphs as the $\rightarrow^{\vartheta}$ relation, introduced in Subsection 3.4.7; this result comes from a paper from 1995 by Bačík and Mahajan [9]. In other words, results regarding $\mathbb{C P}_{-}$, $\mathbb{C S}_{+^{-}}$, and $\mathbb{S}_{+}$-homomorphisms appeared before the definition of conic homomorphisms, similar to how graph homomorphism optimization problems are usually not introduced as graph homomorphism optimization problems. However, the definition of quantum homomorphisms (recall Subsection 3.4.4) [85] and the definition of the completely positive semidefinite cone [73] are likely what sparked the work in [100]. Recall the measurement graph, defined in (3.164), and also recall our discussion following (3.168).

Theorem 5.73 ([100]). Let $G$ and $H$ be graphs. There exists a one to one correspondence between

$$
\bigcup\{\operatorname{Hom}(G, \mathcal{M}(H, d)) \mid d \in \mathbb{N}\} \text { and } \operatorname{Hom}_{\mathbb{C S}_{+}}(G, H) \text {. }
$$

Let $\mathbb{K}$ be a Gram cone. Theorem 5.72 allows us to discuss some categorical notions. We say that $\Phi \in \operatorname{Hom}_{\mathbb{K}}(G, H)$ is a $\mathbb{K}$-isomorphism from graph $G$ to graph $H$ if $\Phi^{-1}$ exists and belongs to $\operatorname{Hom}_{\mathbb{K}}(H, G)$. In other words, a $\mathbb{K}$-isomorphism is an isomorphism in the category $\mathbb{K}$-Graph. If there exist a $\mathbb{K}$-isomorphism from $G$ to $H$, we say that $G$ and $H$ are $\mathbb{K}$-isomorphic. Perhaps Definition 5.63 improves our understanding of $\mathbb{K}$-isomorphisms, as it casts such objects as invertible linear transformations.

Proposition 5.74. Let $G$ and $H$ be graphs, and let $\mathbb{K}$ be a Gram cone. If $G$ and $H$ are $\mathbb{K}$-isomorphic, then

$$
|V(G)|=|V(H)| \text { and }|E(G)|=|E(H)| .
$$

Proof. Let $\Phi \in \operatorname{Hom}_{\mathbb{K}}(G, H)$ be an isomorphism in $\mathbb{K}$-Graph. We have that

$$
\begin{aligned}
\Phi(\operatorname{Diag}(G)) & =\operatorname{Diag}(H) \text { and } \\
\Phi(\operatorname{Mat}(G)) & =\operatorname{Mat}(H),
\end{aligned}
$$

as

$$
\begin{aligned}
\operatorname{Diag}(H) & =\left(\Phi \circ \Phi^{-1}\right)(\operatorname{Diag}(H)) \\
& \subseteq \Phi(\operatorname{Diag}(G)) \\
& \subseteq \operatorname{Diag}(H) .
\end{aligned}
$$

by Definition 5.63 item 1

by Definition 5.63 item 1

and

$$
\begin{aligned}
\operatorname{Mat}(H) & =\left(\Phi \circ \Phi^{-1}\right)(\operatorname{Mat}(H)) \\
& \subseteq \Phi(\operatorname{Mat}(G)) \\
& \subseteq \operatorname{Mat}(H) .
\end{aligned}
$$

by Definition 5.63 item 2

by Definition 5.63 item 2

Therefore

$$
\begin{aligned}
|V(G)| & =\operatorname{dim}(\operatorname{Diag}(G)) & & \text { by }(5.174) \\
& =\operatorname{dim}(\operatorname{Diag}(H)) & & \text { by }(5.194 \mathrm{a}) \\
& =|V(H)| . & & \text { by }(5.174)
\end{aligned}
$$

and

$$
\begin{aligned}
|E(G)| & =\operatorname{dim}(\operatorname{Mat}(G)) & & \text { by }(5.175) \\
& =\operatorname{dim}(\operatorname{Mat}(H)) & & \text { by }(5.194 \mathrm{~b}) \\
& =|E(H)| . & & \text { by }(5.175)
\end{aligned}
$$


Although Proposition 5.74 is not too impressive, it is related to an interesting question when we consider the $\mathbb{S}_{+}$and $\mathbb{C S}_{+}$cones - note that Theorem 5.70 implies that $\mathbb{C P}$-isomorphic graphs are simply isomorphic graphs. Atserias et al. [4] introduced a notion of quantum isomorphism of graphs based on a nonlocal game. Quantum isomorphisms were recently related to the notion of quantum permutation groups [79], and also to homomorphism counting and planar graphs [86]. We do not know the relation between quantum isomorphisms and $\mathbb{C S}_{+}$-isomorphisms, and this predicament is correlated to a lack of understanding of $\mathbb{C S}_{+}$-isomorphisms. Hence, it is reasonable to believe that Definition 5.63 may shed light on this open question, and Proposition 5.74 may be a first sign of the effectiveness of this approach. We now relate the $\rightarrow^{\vartheta}$ order on graphs to $\mathbb{S}_{+}$-homomorphisms.

Theorem 5.75. Let $\mathbb{K}$ be a Gram cone. Let $G$ and $H$ be graphs. Then

$$
\operatorname{mat}_{H, G}\left(\operatorname{Hom}_{\mathbb{K}}(G, H)\right)=\left\{\begin{array}{l|c}
\operatorname{Tr}(X)=|V(G)|, \\
X \in \mathbb{K}_{V(H) \times V(G)} & \begin{array}{c}
X_{k i, \ell j}=0 \text { for every } k i, \ell j \in E(G \rtimes H), \\
\left\langle\mathbb{1} \mathbb{1}^{\top}, X\right\rangle=|V(G)|^{2} .
\end{array}
\end{array} .\right.
$$

Proof. Set mat $:=\operatorname{mat}_{H, G}$, set $J_{G}:=\mathbb{1}^{\top} \in \operatorname{Mat}(V(G))$, and set $J_{H}:=\mathbb{1}^{\top} \in \operatorname{Mat}(V(H))$. Define

$$
\mathcal{C}:=\left\{X \in \mathbb{K}_{V(H) \times V(G)} \mid X_{k i, \ell j}=0 \text { for every } k i, \ell j \in E(G \rtimes H)\right\} .
$$

By Proposition 5.64, it suffices to show that for every $\Phi \in$ mat $^{-1}(\mathcal{C})$,

$$
\begin{aligned}
\left\langle J_{H} \otimes J_{G}, \operatorname{mat}(\Phi)\right\rangle= & |V(G)|^{2} \text { and } \operatorname{Tr}(\operatorname{mat}(\Phi))=|V(G)| \\
& \text { if and only if } \\
& \Phi^{*}\left(J_{H}\right)=J_{G} .
\end{aligned}
$$

We claim that

$$
\operatorname{Tr}(\operatorname{mat}(\Phi))=\operatorname{Tr}\left(\Phi^{*}\left(J_{H}\right)\right)
$$

and that

$$
\begin{gathered}
\left\langle J_{H} \otimes J_{G}, \operatorname{mat}(\Phi)\right\rangle=\operatorname{Tr}(\operatorname{mat}(\Phi))|V(G)| \\
\text { if and only if }
\end{gathered}
$$

there exists $\alpha \in \mathbb{R}_{+}$such that $\Phi^{*}\left(J_{H}\right)=\alpha J_{G}$

for every $\Phi \in$ mat $^{-1}(\mathcal{C})$. Note that both (5.197) and (5.198) imply (5.196). Indeed, if $\Phi^{*}\left(J_{H}\right)=J_{G}$, then (5.197) implies that

$$
\operatorname{Tr}(\operatorname{mat}(\Phi))=\operatorname{Tr}\left(\Phi^{*}\left(J_{H}\right)\right)=\operatorname{Tr}\left(J_{G}\right)=|V(G)|,
$$

so (5.198) implies that $\left\langle J_{H} \otimes J_{G}\right.$, mat $\left.(\Phi)\right\rangle=|V(G)|^{2}$. Conversely, if $\Phi \in$ mat $^{-1}(\mathcal{C})$ is such that $\operatorname{Tr}(\operatorname{mat}(\Phi))=$ $|V(G)|$ and $\left\langle J_{H} \otimes J_{G}\right.$, mat $\left.(\Phi)\right\rangle=|V(G)|^{2}$, we can use (5.198) to conclude that there exists $\alpha \in \mathbb{R}_{+}$such that $\Phi^{*}\left(J_{H}\right)=\alpha J_{G}$, and then use (5.197) to show that $\alpha=1$, since

$$
|V(G)|=\operatorname{Tr}(\operatorname{mat}(\Phi))=\operatorname{Tr}\left(\Phi^{*}\left(J_{H}\right)\right)=\alpha \operatorname{Tr}\left(J_{G}\right)=\alpha|V(G)| .
$$

We first prove (5.197). Let $\Phi \in$ mat $^{-1}(\mathcal{C})$. Proposition 5.64 implies $\Phi$ maps diagonal matrices to diagonal matrices, so

$$
\begin{aligned}
\operatorname{Tr}\left(\Phi^{*}\left(J_{H}\right)\right) & =\left\langle\Phi^{*}\left(J_{H}\right), I\right\rangle & & \text { by }(5.1) \\
& =\left\langle J_{H}, \Phi(I)\right\rangle & & \\
& =\langle I, \Phi(I)\rangle & & \text { since } \Phi(I) \text { is a diagonal matrix } \\
& =\langle I \otimes I, \operatorname{mat}(\Phi)\rangle & & \text { by }(5.147) \\
& =\operatorname{Tr}(\operatorname{mat}(\Phi)) . & & \text { by }(5.1)
\end{aligned}
$$

We now prove (5.198). Let $\Phi \in$ mat $^{-1}(\mathcal{C})$. We have that mat $(\Phi) \succeq 0$, since $\operatorname{mat}(\Phi) \in \mathbb{K}_{V(H) \times V(G)} \subseteq$ $\mathbb{S}_{+}^{V(H) \times V(G)}$. Corollary 5.51 implies that $\mathbb{S}_{+}^{V(H) \times V(G)}$ is Gram-like, so we can combine Corollary 5.61 and 
Theorem 5.45 to obtain that $\Phi^{*}\left(J_{H}\right) \succeq 0$. Consequently, we may bound the largest eigenvalue of $\Phi^{*}\left(J_{H}\right)$ by the sum of its eigenvalues, so

$$
\left\|\Phi^{*}\left(J_{H}\right)\right\|_{2}=\lambda_{\max }\left(\Phi^{*}\left(J_{H}\right)\right) \leq \operatorname{Tr}\left(\Phi^{*}\left(J_{H}\right)\right)=\operatorname{Tr}(\operatorname{mat}(\Phi)) .
$$

Therefore

$$
\begin{aligned}
\left\langle J_{H} \otimes J_{G}, \operatorname{mat}(\Phi)\right\rangle & =\left\langle J_{H}, \Phi\left(J_{G}\right)\right\rangle & & \text { by }(5.147) \\
& =\left\langle\Phi^{*}\left(J_{H}\right), J_{G}\right\rangle & & \\
& \leq\left\|\Phi^{*}\left(J_{H}\right)\right\|_{2}\left\|J_{G}\right\|_{2} & & \text { by Cauchy-Schwarz } \\
& \leq \operatorname{Tr}(\operatorname{mat}(\Phi))\left\|J_{G}\right\|_{2} & & \text { by }(5.199) \\
& =\operatorname{Tr}(\operatorname{mat}(\Phi))|V(G)| . & &
\end{aligned}
$$

Equality holds in the Cauchy-Schwarz inequality if and only if $\Phi^{*}\left(J_{H}\right)=\alpha J_{G}$ for some $\alpha \in \mathbb{R}$, so taking (5.199) into account, we prove (5.198).

Recall from Subsection 3.4.7 that we defined

$$
G \rightarrow^{\vartheta} H \text { if and only if } \vartheta(G \ltimes H)=|V(G)| .
$$

Corollary 5.76 ([9]). Let $G$ and $H$ be graphs. Then there exists a $\mathbb{S}_{+}$-homomorphism from $G$ to $H$ if and only if $G \rightarrow^{\vartheta} H$.

Proof. Define $\mathbb{K}:=\mathbb{S}_{+}^{V(G \rtimes H)}$ and $\mathbb{A}:=\mathbb{A}_{E(G \rtimes H), E(G \rtimes H)}$. Then

$$
\begin{array}{rlrl}
\vartheta(G \ltimes H) & =\vartheta(G \rtimes H) & & \text { since } \vartheta \text { is a graph parameter } \\
& =\vartheta(\mathbb{A}, \operatorname{Psd}(\mathbb{K}), \mathbb{1}) & \text { by }(5.39 \mathrm{a}) \\
& =\sup \left\{\left\langle\mathbb{1} \mathbb{1}^{\top}, X\right\rangle \mid \begin{array}{c}
\operatorname{Tr}(X)=1, \\
X_{k i, l j}=0, \forall k i, l j \in E(G \rtimes H), \\
X \succeq 0 .
\end{array}\right.
\end{array} \quad \begin{array}{ll}
\text { by Theorem } 5.30
\end{array}
$$

Since $\mathbb{S}_{+}^{V(H) \times V(G)}$ is closed, the supremum above is attained. If $X$ is a feasible solution with objective value $|V(G)|$, Theorem 5.75 implies that $|V(G)| \operatorname{mat}_{H, G}^{-1}(X) \in \operatorname{Hom}_{\mathbb{S}_{+}}(G, H)$. Conversely, if $\Phi \in \operatorname{Hom}_{\mathbb{S}_{+}}(G, H)$, Theorem 5.75 implies that $|V(G)|^{-1} \operatorname{mat}_{H, G}(\Phi)$ is a feasible solution to the optimization problem above with objective value $|V(G)|$.

For every Gram cone $\mathbb{K}$, denote by $\mathcal{P}_{\mathbb{K}}$ the preordered class on graphs obtained from $\mathbb{K}$-Graph via Proposition 3.22. Recall Section 3.3. We point out that some of generalized clique and coloring numbers defined using these conic preorders have already been studied. Recall the notations that $\mathcal{Q}_{K}=\left\{K_{n} \mid n \in \mathbb{N}\right\}$ and that $g_{K}\left(K_{n}\right)=n$ for every $n \in \mathbb{N}$. Corollary 5.71 implies that

$\chi$ is the largest $\left(\mathcal{P}_{\mathbb{C P}},[-\infty,+\infty]\right)$-monotone function which is at most $g_{K}$ on $\mathcal{Q}_{K}$,

while Corollary 5.76 and Theorem 3.74 states that

$$
\lceil\bar{\vartheta}\rceil \text { is the largest }\left(\mathcal{P}_{\mathbb{S}_{+}},[-\infty,+\infty]\right) \text {-monotone function which is at most } g_{K} \text { on } \mathcal{Q}_{K},
$$

and Theorem 5.73 implies that

$\chi_{q}$ is the largest $\left(\mathcal{P}_{\mathbb{C S}_{+}},[-\infty,+\infty]\right)$-monotone function which is at most $g_{k}$ on $\mathcal{Q}_{K}$.

We finish the subsection by relating the conic graph parameters from Section 5.2 with conic homomorphisms.

There is a small computation which will be useful in our next result. Let $V$ be a finite set, let $S \subseteq V$, and let $A \in \operatorname{Mat}(S)$. Define $\iota: S \rightarrow V$ by $\iota(s):=s$ for every $s \in S$. We first show that

$$
P_{\iota} P_{\iota}^{\top}=\sum_{s \in S} e_{s} e_{s}^{\top} \in \operatorname{Mat}(V)
$$


This holds as

$$
\begin{aligned}
P_{\iota} P_{\iota}^{\top} & =\sum_{s \in S} P_{\iota}\left(e_{\iota(s)} e_{s}^{\top}\right)^{\top} \\
& =\sum_{s \in S} P_{\iota} e_{s} e_{\iota(s)}^{\top} \\
& =\sum_{s \in S} e_{\iota(s)} e_{\iota(s)}^{\top} \\
& =\sum_{s \in S} e_{s} e_{s}^{\top}
\end{aligned}
$$

Moreover

$$
P_{\iota}^{\top} A P_{\iota}=A[S]
$$

since for every $j, k \in S$

$$
\begin{aligned}
\left(P_{\iota}^{\top} A P_{\iota}\right)_{j k} & =e_{j}^{\top} P_{\iota}^{\top} A P_{\iota} e_{k} \\
& =e_{\iota(j)}^{\top} A P_{\iota(k)} \\
& =e_{j}^{\top} A e_{k} \\
& =A[S]_{j k} .
\end{aligned}
$$

since $j, k \in S$

Combining the previous results, we have that

$$
P_{\iota} A[S] P_{\iota}^{\top}=\left(\sum_{s \in S} e_{s} e_{s}^{\top}\right) A\left(\sum_{s \in S} e_{s} e_{s}^{\top}\right)
$$

since

$$
\begin{aligned}
P_{\iota} A[S] P_{\iota}^{\top} & =P_{\iota} P_{\iota}^{\top} A P_{\iota} P_{\iota}^{\top} \\
& =\left(\sum_{s \in S} e_{s} e_{s}^{\top}\right) A\left(\sum_{s \in S} e_{s} e_{s}^{\top}\right) .
\end{aligned}
$$

Theorem 5.77. Let $G:=(V, E)$ be a graph, and let $\mathbb{K}$ be a Gram cone. Then

$$
\operatorname{TH}\left(\mathbb{A}_{E, E}, \operatorname{Psd}\left(\mathbb{K}_{V}\right)\right)
$$

is a convex corner. Consequently, $w \in \mathbb{R}_{+}^{V} \mapsto \vartheta\left(\mathbb{A}_{E, E}, \operatorname{Psd}\left(\mathbb{K}_{V}\right), w\right)$ is a real-valued positive definite monotone gauge.

Proof. We first prove that for every $i \in V$,

$$
\text { if } X \in \mathbb{K}_{V} \text { then } \operatorname{Diag}\left(\mathbb{1}-e_{i}\right) X \operatorname{Diag}\left(\mathbb{1}-e_{i}\right) \in \mathbb{K}_{V} \text {. }
$$

Let $X \in \mathbb{K}_{V}$, let $i \in V$, set $S:=V \backslash\{i\}$, and let $\iota: S \rightarrow V$ be defined by $\iota(s):=s$ for every $s \in S$. Then

$$
\begin{aligned}
\operatorname{Diag}\left(\mathbb{1}-e_{i}\right) X \operatorname{Diag}\left(\mathbb{1}-e_{i}\right) & =\left(\sum_{s \in S} e_{s} e_{s}^{\top}\right) X\left(\sum_{s \in S} e_{s} e_{s}^{\top}\right) \\
& =P_{\iota} X[S] P_{\iota}^{\top}
\end{aligned}
$$

by (5.203).

Since $X \in \mathbb{K}_{V}$, item 3 of Definition 5.46 implies that $X[S] \in \mathbb{K}_{S}$, so item 2 of Definition 5.46 implies that $P_{\iota} X[S] P_{\iota}^{\top} \in \mathbb{K}_{V}$. In short, (5.204) holds.

Set $\mathbb{A}:=\mathbb{A}_{E, E}$. It is clear that $\widehat{\mathrm{TH}}\left(\mathbb{A}, \operatorname{Psd}\left(\mathbb{K}_{V}\right)\right)$ is convex. We have that $\mathbb{K}_{V} \subseteq \mathbb{S}_{+}^{V}$ by (5.171). Proposition 5.6 then applies, so $\widehat{\mathrm{TH}}\left(\mathbb{A}, \operatorname{Psd}\left(\mathbb{K}_{V}\right)\right)$ is compact. Hence $\mathrm{TH}\left(\mathbb{A}, \operatorname{Psd}\left(\mathbb{K}_{V}\right)\right)$ is compact and convex, 
as it is the image of $\widehat{T H}\left(\mathbb{A}, \operatorname{Psd}\left(\mathbb{K}_{V}\right)\right)$ under the linear function $\widehat{X} \in \mathbb{S}\{0\} \cup V \mapsto \operatorname{diag}(\widehat{X}[V])$. Furthermore (5.36) implies that $e_{i} \in \mathrm{TH}\left(\mathbb{A}, \operatorname{Psd}\left(\mathbb{K}_{V}\right)\right)$ for every $i \in V$, as

$$
\begin{aligned}
{\left[\begin{array}{cc}
1 & e_{i}^{\top} \\
e_{i} & e_{i} e_{i}^{\top}
\end{array}\right] } & \in \widehat{\mathrm{TH}}\left(\mathbb{A}, \mathbb{C P}^{\{0\} \cup V}\right) & & \text { by }(5.21) \text { and } e_{i} e_{i}^{\top} \in \mathbb{A} \\
& \left.\subseteq \widehat{\mathrm{TH}}\left(\mathbb{A}, \operatorname{Psd}\left(\mathbb{C P}^{V}\right)\right)\right) & & \text { by }(5.26) \text { and }(5.31) \\
& \subseteq \widehat{\mathrm{TH}}\left(\mathbb{A}, \operatorname{Psd}\left(\mathbb{K}_{V}\right)\right) . & & \text { by }(5.171),(5.31), \text { and }(5.30 \mathrm{a})
\end{aligned}
$$

It follows that $\frac{1}{|V|} \mathbb{1} \in \mathrm{TH}\left(\mathbb{A}, \operatorname{Psd}\left(\mathbb{K}_{V}\right)\right)$. On the other hand,

$$
\begin{aligned}
\widehat{\mathrm{TH}}\left(\mathbb{A}, \operatorname{Psd}\left(\mathbb{K}_{V}\right)\right) & \subseteq \widehat{\mathrm{TH}}\left(\mathbb{S}^{V}, \operatorname{Psd}\left(\mathbb{S}_{+}^{V}\right)\right) & & \text { by }(5.171) \text { and }(5.31) \\
& =\widehat{\mathrm{TH}}\left(\mathbb{S}^{V}, \mathbb{S}_{+}^{\{0\} \cup V}\right) & & \text { by }(5.25)
\end{aligned}
$$

so (5.22) and (5.36) imply that $\operatorname{TH}\left(\mathbb{A}, \operatorname{Psd}\left(\mathbb{K}_{V}\right)\right) \subseteq \mathbb{R}_{+}^{V}$. By Corollary 4.20,

$$
\operatorname{lc}\left(\operatorname{TH}\left(\mathbb{A}, \operatorname{Psd}\left(\mathbb{K}_{V}\right)\right)\right) \text { is a convex corner. }
$$

Thus, to finish the proof, it suffices to show that $\mathrm{TH}\left(\mathbb{A}, \operatorname{Psd}\left(\mathbb{K}_{V}\right)\right)$ is lower-comprehensive. Proposition 4.2 reduces our argument to proving that

$$
x-x_{i} e_{i} \in \mathrm{TH}\left(\mathbb{A}, \operatorname{Psd}\left(\mathbb{K}_{V}\right)\right) \text { for every } x \in \operatorname{TH}\left(\mathbb{A}, \operatorname{Psd}\left(\mathbb{K}_{V}\right)\right) \text { and } i \in V .
$$

Let $i \in V$, and let

$$
\left[\begin{array}{ll}
1 & x^{\top} \\
x & X
\end{array}\right]:=\widehat{X} \in \widehat{\mathrm{TH}}\left(\mathbb{A}, \operatorname{Psd}\left(\mathbb{K}_{V}\right)\right)
$$

Set $h:=\mathbb{1}-e_{i}$. As

$$
\operatorname{Diag}(h) x=\operatorname{Diag}\left(\mathbb{1}-e_{i}\right) x=\left(I-e_{i} e_{i}^{\top}\right) x=x-x_{i} e_{i},
$$

we conclude (5.206) once we prove that

$$
\left[\begin{array}{cc}
1 & x^{\top} \operatorname{Diag}(h) \\
\operatorname{Diag}(h) x & \operatorname{Diag}(h) X \operatorname{Diag}(h)
\end{array}\right] \in \widehat{\mathrm{TH}}\left(\mathbb{A}, \operatorname{Psd}\left(\mathbb{K}_{V}\right)\right) .
$$

Since $\widehat{X} \in \mathbb{S}_{+}^{\{0\} \cup V}$, Proposition 5.3 and (5.11c) imply that

$$
\begin{aligned}
0 & \preceq\left[\begin{array}{cc}
1 & 0^{\top} \\
0 & \operatorname{Diag}(h)
\end{array}\right]\left[\begin{array}{cc}
1 & x^{\top} \\
x & X
\end{array}\right]\left[\begin{array}{cc}
1 & 0^{\top} \\
0 & \operatorname{Diag}(h)
\end{array}\right] \\
& =\left[\begin{array}{cc}
1 & x^{\top} \\
\operatorname{Diag}(h) x & \operatorname{Diag}(h) X
\end{array}\right]\left[\begin{array}{cc}
1 & 0^{\top} \\
0 & \operatorname{Diag}(h)
\end{array}\right] \\
& =\left[\begin{array}{cc}
1 & x^{\top} \operatorname{Diag}(h) \\
\operatorname{Diag}(h) x & \operatorname{Diag}(h) X \operatorname{Diag}(h)
\end{array}\right] .
\end{aligned}
$$

Moreover, we have that

$$
\operatorname{Diag}(h) x=\operatorname{diag}(\operatorname{Diag}(h) X \operatorname{Diag}(h))
$$

since for every $k \in V$,

$$
\begin{aligned}
\operatorname{diag}(\operatorname{Diag}(h) X \operatorname{Diag}(h))_{k} & =(\operatorname{Diag}(h) X \operatorname{Diag}(h))_{k k} & & \text { by }(2.21) \\
& =e_{k}^{\top} \operatorname{Diag}(h) X \operatorname{Diag}(h) e_{k} & & \\
& =[k \neq i]^{2} X_{k k} & & \text { by definition of } h \\
& =[k \neq i] X_{k k} & & \\
& =[k \neq i] \operatorname{diag}(X)_{k} . & & \text { by }(2.21)
\end{aligned}
$$

The matrix $\operatorname{Diag}(h) X \operatorname{Diag}(h)$ belongs to $\mathbb{A}$ by (5.42) and to $\mathbb{K}_{V}$ by (5.204). This concludes the proof of (5.207), and thus, of (5.206). 
All the arguments in the proof of Theorem 5.77 that come after (5.204) can be found in [28, Proposition 2]. Theorem 5.78 ([100]). Let $G$ and $H$ be graphs, and let $\mathbb{K}$ be a Gram cone. If $\operatorname{Hom}_{\mathbb{K}}(G, H) \neq \varnothing$, then

$$
\vartheta\left(\mathbb{A}_{G}, \operatorname{Psd}\left(\mathbb{K}_{G}\right), \mathbb{1}\right) \leq \vartheta\left(\mathbb{A}_{H}, \operatorname{Psd}\left(\mathbb{K}_{H}\right), \mathbb{1}\right),
$$

where $\mathbb{A}_{K}:=\mathbb{A}_{E(\bar{K}), E(\bar{K})}$ and $\mathbb{K}_{K}:=\mathbb{K}_{V(K)}$ for $K \in\{G, H\}$.

Proof. Set mat $:=\operatorname{mat}_{H, G}$. Let $\Phi \in \operatorname{Hom}_{\mathbb{K}}(G, H)$. We prove that $\Phi$ is a homomorphism of optimization problems between the formulations from Theorem 5.30. In other words, we prove that

$$
\begin{gathered}
\left\langle\Phi(X), \mathbb{1}^{\top}\right\rangle=\left\langle X, \mathbb{1}^{\top}\right\rangle \text { for every } X \in \mathbb{K}_{G} \cap \mathbb{A}_{G} \text { with } \operatorname{Tr}(X)=1, \\
\Phi\left(\left\{X \in \mathbb{K}_{G} \mid X \in \mathbb{A}_{G}, \operatorname{Tr}(X)=1\right\}\right) \subseteq\left\{X \in \mathbb{K}_{H} \mid X \in \mathbb{A}_{H}, \operatorname{Tr}(X)=1\right\} .
\end{gathered}
$$

Definition 5.63 item 3 states that $\Phi^{*}\left(\mathbb{1} \mathbb{1}^{\top}\right)=\mathbb{1}^{\top}$, so (5.208) follows.

Note that

$$
\mathbb{A}_{K}=\mathbb{S}^{V(K)} \cap \operatorname{Mat}(K)
$$

for every graph $K \in\{G, H\}$. Since mat $(\Phi) \in \mathbb{S}^{V(H) \times V(G)}$, Corollary 5.58 implies that $\Phi\left(\mathbb{S}^{V(G)}\right) \subseteq \mathbb{S}^{V(H)}$. Definition 5.63 item 2 states that $\Phi(\operatorname{Mat}(G)) \subseteq \operatorname{Mat}(H)$, so we can use (5.210) to conclude that $\Phi\left(\mathbb{A}_{G}\right) \subseteq \mathbb{A}_{H}$. We have that $\Phi\left(\mathbb{K}_{G}\right) \subseteq \mathbb{K}_{H}$ by Definition 5.63 item 4 and Corollary 5.57. To conclude (5.209), and thus the proof, we show that $\operatorname{Tr}(\Phi(X))=\operatorname{Tr}(X)$ for every $X \in \mathbb{A}_{G}$. Let $X \in \mathbb{A}_{G}$, and set

$$
x:=\operatorname{diag}(X) \text { and } A:=X-\operatorname{Diag}(x) .
$$

Since $X \in \mathbb{A}_{G}$, we have that $A \in \operatorname{Mat}(G)$, so Definition 5.63 item 2 implies that $\Phi(A) \in \operatorname{Mat}(H)$. Therefore $\langle I, \Phi(A)\rangle=0$. The result follows:

$$
\begin{aligned}
\operatorname{Tr}(\Phi(X)) & =\langle I, \Phi(X)\rangle & & \text { by }(5.1) \\
& =\langle I, \Phi(\operatorname{Diag}(x))\rangle+\langle I, \Phi(A)\rangle & & \\
& =\langle I, \Phi(\operatorname{Diag}(x))\rangle & & \\
& =\left\langle\mathbb{1} \mathbb{1}^{\top}, \Phi(\operatorname{Diag}(x))\right\rangle & & \text { by Definition 5.63 item } 1 \\
& =\left\langle\Phi^{*}\left(\mathbb{1} \mathbb{1}^{\top}\right), \operatorname{Diag}(x)\right\rangle & & \\
& =\left\langle\mathbb{1} \mathbb{1}^{\top}, \operatorname{Diag}(x)\right\rangle & & \\
& =\left\langle\mathbb{1} \mathbb{1}^{\top}, \operatorname{Diag}(\operatorname{diag}(X))\right\rangle & & \\
& =\langle I, X\rangle & & \\
& =\operatorname{Tr}(X) . & & \text { by }(5.1)
\end{aligned}
$$




\section{Chapter 6}

\section{Conclusions}

The first research task my supervisor gave me when I started my MSc. was to read Rockafellar's book [102]. I suspect he would have given me this task even sooner, if it was acceptable for a professor to walk around telling undergraduate students to read a specific book. As its title implies, this book is concerned with convex analysis, and presents a myriad of results on convex sets and functions. I diligently read the book and tried my best to grasp its knowledge. Yet, years later, I still find myself asking whether it is the intersection of the closures that is always contained in the closure of the intersection, or the other way around. I even know that if the intersection of the relative interiors of the sets in question is nonempty, then this distinction does not matter as both sets are equal [102, Theorem 6.5], but I always forget which side of the inclusion holds in general. In this specific case, I usually write a single line in some draft paper and conclude that the closure of the intersection is the smaller set. But this instance that can be quickly solved is the exception, rather than the rule: if left to myself, I must think for a while before stating and proving a technical result like Corollary 4.24. In any case, let us call this scenario in which one has to stop and work some tangential technical details the closure-of-the-intersection situation, and call the actual tangential technical details the closure-of-the-intersection result.

I consider the closure-of-the-intersection situation to be a serious predicament. What aggravates it is that I am usually really busy when it comes up: what requires me to use some closure-of-the-intersection result is always a problem I am trying to solve, sometimes an open research problem. These situations force me to halt my work in the problem I actually care about and load into my head lots of basic definitions from another area. Then, after proving some result that is likely some decades old, I must load back into my head all the details of the original problem. To make things worse, the very style of mathematics I strive for makes closure-of-the-intersection situations common. I believe it is epistemologically better to solve a problem using an older theory, leveraging years of research, than it is to come up with ad-hoc techniques. Of course, one does what it takes to solve a problem, but it is reasonable to look for better grounded approaches. In short, my highest-standard problem-solving approach is based on closure-of-the-intersection results.

Luckily, there are two simple weapons one can wield against closure-of-the-intersection situations: reading and writing. Nowadays this is my biggest use of [102]: I swiftly get by several closure-of-the-intersection situations by locating the appropriate result in the book. Obviously, there is only so much in a single book, so there are closure-of-the-intersection results I must figure out by myself. And this is hard: it turns out it is not always simple to wield simple weapons. The core issue is that it takes some amount of work to obtain a closure-of-the-intersection result, but it takes considerably more to make it into a drawer-friendly theorem. The phrase drawer friendly is supposed to communicate something stronger than re-usability: the idea is that you can safely forget the proof of the theorem, perhaps after spending some months working on unrelated matters, and then come back to the theorem, fetch it from its drawer and effortlessly use it. The set of drawer-friendly theorems is a proper subset of the set of theorems, as a theorem is reusable by definition, but not all of them are drawer friendly. A theorem stated in terms of highly specific objects which pertain only to its domain is not drawer friendly. Or perhaps its statement is really simple, so simple as to forbid its use in relevant cases. Needless to say, a theorem that forces you to copy its proof almost verbatim could not possibly be happy about being in the drawer. Granted, this definition sets an extremely high bar for calling a theorem drawer friendly: it may be the case that the research community as a whole is unable to 
make some argument easy to use.

Hence, as a researcher pestered by closure-of-the-intersection situations, it is only reasonable to cherish the few drawer-friendly theorems I stumble upon. As a computer scientist by formation, it is only natural to reach for programming in an attempt to produce drawer-friendly theorems. The inhumane nature of a computer is actually a feature: obviously, "drawer friendliness" is in the eye of the beholder, but striving to make an idea digestible to a machine (or a child) may as well make it more digestible to fellow humans.

The search for drawer-friendly theorems is the only reasonable explanation for several style decision throughout this text. Chapter 5 has at least 41 occurrences of "be finite sets" and 33 of "be a finite set". Why keep telling the reader that $V$ is a finite set, over and over again? Because the reader may be in a closure-of-the-intersection situation and does not want to skim over the whole chapter looking for a remark specifying whether the theorem in question applies to infinite sets as well. Furthermore, as the only way to check if a result is easy to use is to actually use it, the dialogues with Lamport's child [69] can be seen as a technique for reaching drawer friendliness. This disciplined approach to proving, aside from reducing errors, makes evident the arguments that need to be encapsulated, and how they tend to be used. Moreover, even though you will never need to see the proof of a platonic drawer-friendly theorem, a drawer-friendly proof can be really helpful in real life.

Making some theorems fit in the drawer requires more than form. $\mathrm{LAT}_{\mathrm{E} X} \mathrm{X}$ tells me the only results from Chapter 4 used outside of Chapter 4 are: Theorems 4.25, 4.26, and 4.29, Corollaries 4.16, 4.20, 4.22, and 4.23, and Propositions 4.2, 4.10, 4.11, 4.27, 4.28, 4.40, and 4.41. In other words, if we ignore the equations for the sake of simplicity, we have 14 results, out of 36 in the chapter, which are used outside of it. Sure, many of those results are from the new results in Sections 4.4 and 4.5, but there are still many statements like Proposition 4.7, which could easily be substituted for more direct arguments without having any impact whatsoever in the remainder of this work. It is not only possible, but it is actually much easier to write Chapter 4 without Theorem 4.17. Yet, Theorem 4.17 seems to fit comfortably in a drawer: it is a powerful result (it implies everything nontrivial in Subsection 4.1.2) stated using easy-to-grasp objects. A reader who, in a closure-of-the-intersection situation, feels puzzled by the lower-comprehensive hull, can easily reach for Propositions 4.1, 4.5, and 4.7. From a personal perspective, there is a nontrivial use of a hyperplane separation argument in the proof of Theorem 4.17 which will never confuse me again as long as I can apply the theorem as stated. All of its corollaries, including Theorems 4.25 and 4.26, show that one can get a good mileage out of Theorem 4.17 without tampering with its proof.

Section 4.3 is a drawer with theorems actually used to obtain new developments: the ideas in my coauthored paper [13], as well as the unpublished new results in Section 5.3. Remarkably, this is done by exploiting decade old ideas [39, 40, 49, 50]. The revised manuscript for [39] was received more than 50 years ago, and it has been almost 37 years since the Journal of Combinatorial Theory Series B received a manuscript which considered the antiblocker of a non-polyhedral set [49]. With academia growing by the day, it becomes imperative for a researcher to find processes for handling the ever increasing information stream. To read an influential paper, even if it is decades old, and then to properly accommodate its core ideas in your personal drawer may be a sensible approach.

Chapter 3 demarcates the objects studied in this text, specializing from graph homomorphism optimization problems into the notions of generalized clique and coloring numbers. To reason about the duality present in Definitions 3.24 and 3.25, it is enlightening to compare it with linear programming duality. Let $S$ and $T$ be finite sets, let $A \in \mathbb{R}^{T \times S}$, let $b \in \mathbb{R}^{S}$, and let $c \in \mathbb{R}^{T}$. If confronted with the question of whether

$$
\begin{gathered}
\inf \left\{y^{\top} b \mid y \in \mathbb{R}_{+}^{S}, A y=c\right\} \text { and } \\
\sup \left\{c^{\top} x \mid A^{\top} x \leq b, x \in \mathbb{R}^{T}\right\}
\end{gathered}
$$

are dual linear programs, any reader familiar with linear programming will instinctively check if the constraints conspire to ensure weak duality, i.e., check if

$$
c^{\top} x \leq y^{\top} b
$$

for every $x \in \mathbb{R}^{T}$ feasible in (6.1b), and for every $y \in \mathbb{R}^{S}$ feasible in (6.1a). This shows that it is not enough that both objects in (6.1) are of the same kind - linear programs - and are built in a clearly related manner using the same information - the triplet $(A, b, c)$. To put it briefly, we must specify what is meant by duality 
before checking if something is a dual pair. Now consider the following optimization problems:

$$
\begin{aligned}
& \inf \left\{n \in \mathbb{N} \mid G \rightarrow K_{n}\right\}, \text { and } \\
& \sup \left\{n \in \mathbb{N} \mid K_{n} \rightarrow G\right\} .
\end{aligned}
$$

Both objects are of the same kind - graph homomorphism optimization problems — and are built in a clearly related manner using the same information - the set $\mathcal{Q}_{K}:=\left\{K_{n} \mid n \in \mathbb{N}\right\}$ and the function $g_{k}: \mathcal{Q}_{K} \rightarrow[-\infty,+\infty]$ given by $g_{K}\left(K_{n}\right):=n$ for every $n \in \mathbb{N}$. To support the claim that there exists a duality in (6.3), Chapter 3 attempted to find a proper analogue of (6.2). In other words, Chapter 3 defined a duality which captures (6.3). The sizeable Section 3.4 surveys the literature for graph homomorphism optimization problems, providing examples which a suitable definition must capture. In this sense, Section 3.4 is the battery of tests - or perhaps, the test suite - used for the definitions made in Section 3.3, and consequently, for the developments of Section 3.1. Figure 3.1 stands as strong evidence in favor of our definitions in general, and of Theorem 3.28 in particular.

Perhaps the most important drawer-friendly part of Section 3.1 is the language provided for computing order-theoretic duals. Order duality is a wonderful intuition that tends to be presented in an unusable manner. If humble dual results like (3.26) are not properly stated, a reader who stumbles upon a phrase of the type " $S$ follows from duality" has only two options: to blindly trust the writer, or to rewrite the argument while changing $\preceq$ for $\succeq$. You see, a theorem that forces you to copy its proof almost verbatim. Textbook drawer aversion. Propositions 3.6 and 3.19 came from a deliberate effort to provide building blocks for computing order-theoretic duals, and are the crucial steps in the proof of (3.90), the most important duality result of Chapter 3. Note that we are actually talking about three dualities right now: linear programming duality, graph homomorphism optimization problem duality, and order duality. With the three examples in mind, it becomes evident that the duality in Definitions 3.24 and 3.25 is much closer to linear programming duality than to order duality. The preordered set $P^{*}$ is the dual of the preordered set $P$. On the other hand, Definitions 3.24 and 3.25 provide a dual pair of graph homomorphism optimization problems for every triplet $(P, Q, g)$, where $P$ is a preordered set, $Q \subseteq V(P)$, and $g: Q \rightarrow[-\infty,+\infty]$. Similarly, (6.1) provides a dual pair of linear programs for every triplet $(A, b, c)$. An unexpected ramification is that (6.3) does not really says that $\omega$ is the dual of $\chi$, as (6.3a) is not the formulation of $\chi$ as a graph homomorphism optimization problem, but simply a formulation of $\chi$ as a graph homomorphism optimization problem. We have only defined the duality between the graph homomorphism optimization problems in (6.3), and so far, we cannot extend this duality to the graph parameters they define. To make things concrete, recall how the graphs defined in (3.181) provide another formulation of $\chi$ as a graph homomorphism optimization problem. Once again, this is analogous to linear programming duality: the linear program in (3.109) provides a formulation of $\chi_{f}$ as a linear program, not the formulation.

Given the interest of this text on matrix cones and graph homomorphisms, the results by Roberson [100] on conic formulations of graph homomorphisms were compulsory. In a work that employs geometric techniques to study graph homomorphisms, it is imperative to dive into a paper that geometrically encodes graph homomorphisms. However, it really took some time to make [100] fit into my drawer. This effort culminated in Section 5.4, which completely reformulates conic homomorphisms. Subsection 5.4.1 is quite remarkable, in that it implements the operations used in the remainder of Section 5.4 completely in terms of matrix manipulations. The fundamental result is Proposition 5.42, which captures both the Choi representation of a linear transformation and the vectorization of matrices, and establishes a crucial correspondence between $\operatorname{Lin}(\operatorname{Mat}(S), \operatorname{Mat}(T))$ and $\operatorname{Mat}(T \times S)$, for $S$ and $T$ finite sets. As far as I know, the relationship between [100] and the Choi representation of linear transformations is new. This correspondence completely changes the interpretation of [100], and leads into the deep question addressed in Subsection 5.4.2: what kind of conic restrictions on the Choi representation define categories? In other words, what properties of the positive semidefinite cone ensure that (5.159) holds? Gram-like cones, as defined in Definition 5.46, are the abstraction we provide to answer this question. Gram-like cones reformulate the "frabjous" cones from [100]. Once again, our approach provides new meaning to what was present in [100]: when Roberson presents the hypotheses required by his cones, he singles out one of them saying it "requires a little explanation". This hypothesis is equivalent to item 2 of Definition 5.46, which we understand as the core assumption of functoriality. Perhaps the best example in this work of a complex argument encapsulated for clean use is Theorem 5.54, which implies that Gram-like cones define categories in the sense defined in Subsection 5.4.2. If the examples in Section 3.4 were the test for Definitions 3.24 and 3.25, the proof of Theorem 5.54 and its corollaries were the 
test for Subsection 5.4.1. As we point out right before its proof, Theorem 5.54 encapsulates an argument which Roberson admits the reader may find "mysterious". Subsection 5.4.3 then simply reaps the benefits of its previous subsections, defining a conic homomorphism to be a linear transformation and proving some of its basic properties.

We are finally in a good position to make the best case for the technical cohesion of this work, as we can enumerate important results which appear throughout the text. The Lovász $\vartheta$ function and its variants, as well as the fractional chromatic number $\chi_{f}$ make important appearances in each major chapter of this text: as generalized colorings in Chapter 3, as real-valued positive definite monotone gauges in Chapter 4, and as real-valued positive definite monotone gauges obtained from matrix cones in Chapter 5 . Furthermore, homomorphism monotonicity of these graph parameters is deeply connected with central results of this work. Focusing on $\chi_{f}$ for simplicity, we have that it is homomorphism monotone

1. by Definition 3.25 and its formulation as a graph homomorphism optimization problem in (3.102),

2. by Proposition 5.12 and its formulation in terms of matrix cones implied by (5.41) and (3.112), and

3. by Theorem 5.78 and its formulation in terms of matrix cones implied by (5.41) and (3.112).

Furthermore, Section 5.3 hints that the relationship between matrix cones, convex corners, and composition is robust in some sense. The fractional cut-covering number was introduced to study cut-continuous mappings [103]. Cut-continuous mappings are also closed under composition, and thus, define an alternative category on graphs. Subsection 4.1.3 formulates the fractional cut-covering number as a real-valued positive definite monotone gauge. In short, another category, another gauge. Theorem 5.77 shows that the technical hypotheses on matrix cones from the work by de Carli Silva and Tunçel [28] may be related to Gram-like cones, which derive from the work by Roberson [100]; this is quite remarkable considering the results each paper strives to capture. Finally, Theorem 5.75 shows how to recover the conic homomorphisms between two graphs $G$ and $H$ using the optimal solutions of one of the formulations of $\vartheta\left(\mathbb{A}_{E, E}, \operatorname{Psd}\left(\mathbb{K}_{V}\right), \mathbb{1}\right)$, where $(V, E):=G \rtimes H$, thus further strengthening the connection between $\vartheta$ and conic homomorphisms.

Theorems are the tools of our craft, and it should not be controversial to take good care of our tools. Beyond its direct uses, like allowing one to more competently employ what one reads, we believe that the methodological approach we vindicate for leads directly to new results, as it forces us to dissect our ideas and repackage them over and over again. It is a mechanical discipline that complements and enables human creativity. We hope to have provided the reader with a comprehensive and cohesive work that serves as an opportunity to appreciate the value of such methodology. 


\section{Bibliography}

[1] J. Adámek, H. Herrlich, and G. E. Strecker. Abstract and concrete categories: the joy of cats. Dover Publications, 2009. 528 pages (cited on page 13).

[2] M. O. Albertson and K. L. Collins. "Homomorphisms of 3-chromatic graphs". In: Discrete Mathematics 54.2 (1985), pages 127-132. DOI: 10.1016/0012-365X (85)90073-1 (cited on page 96).

[3] A. Y. Aravkin, J. V. Burke, D. Drusvyatskiy, M. P. Friedlander, and K. J. MacPhee. "Foundations of gauge and perspective duality". In: SIAM Journal on Optimization 28.3 (2018), pages 2406-2434. DOI: 10.1137/17M1119020 (cited on pages 81, 129).

[4] A. Atserias, L. Mančinska, D. E. Roberson, R. Šámal, S. Severini, and A. Varvitsiotis. "Quantum and non-signalling graph isomorphisms". In: Journal of Combinatorial Theory. Series B 136 (May 1, 2019), pages 289-328. DOI: 10.1016/j.jctb.2018.11.002 (cited on page 157).

[5] D. Avis, J. Hasegawa, Y. Kikuchi, and Y. Sasaki. "A Quantum Protocol to Win the Graph Colouring Game on All Hadamard Graphs". In: IEICE Transactions on Fundamentals of Electronics Communications and Computer Sciences E89A (2005). DOI: 10.1093/ietfec/e89-a.5.1378 (cited on page 63).

[6] C. Bachoc, A. Gundert, and A. Passuello. "The theta number of simplicial complexes". In: Israel Journal of Mathematics 232.1 (August 1, 2019), pages 443-481. DOI: 10.1007/s11856-019-1880-8 (cited on page 57).

[7] C. Bachoc, A. Pêcher, and A. Thiéry. "On the theta number of powers of cycle graphs". In: Combinatorica 33.3 (2013), pages 297-317. DOI: 10.1007/s00493-013-2950-x (cited on page 70).

[8] R. Bačík. "Structure of graph homomorphisms". Ph.D. Simon Fraser University, 1997. 103 pages (cited on pages 75,76$)$.

[9] R. Bačík and S. Mahajan. "Semidefinite programming and its applications to NP problems". In: Computing and combinatorics (Xi'an, 1995). Volume 959. Lecture Notes in Comput. Sci. Berlin: Springer, 1995, pages 566-575. DOI: 10.1007/BFb0030878 (cited on pages 76, 156, 158).

[10] I. Balla, S. Letzter, and B. Sudakov. "Orthonormal Representations of $H$-Free Graphs". In: Discrete $\mathcal{G}^{3}$ Computational Geometry 64.3 (October 1, 2020), pages 654-670. DOI: 10.1007/s00454-020-00185-0 (cited on page 65).

[11] M. Ben-Or, S. Goldwasser, J. Kilian, and A. Wigderson. "Multi-prover interactive proofs: how to remove intractability assumptions". In: Proceedings of the twentieth annual ACM symposium on Theory of computing. STOC '88. Chicago, Illinois, USA: Association for Computing Machinery, January 1, 1988, pages 113-131. DOI: 10.1145/62212.62223 (cited on page 62).

[12] A. Ben-Tal and A. Nemirovski. Lectures on modern convex optimization. MPS/SIAM Series on Optimization. Society for Industrial and Applied Mathematics (SIAM), Philadelphia, PA; Mathematical Programming Society (MPS), Philadelphia, PA, 2001, pages xvi+488. DOI: 10.1137/1.9780898718829 (cited on page 102).

[13] N. Benedetto Proença, M. K. de Carli Silva, and G. Coutinho. Dual Hoffman Bounds for the Stability and Chromatic Numbers Based on SDP. To appear in SIAM Journal on Discrete Mathematics. 2019. arXiv: 1910.05586 [math.CO] (cited on pages 97, 98, 100, 101, 103, 164). 
[14] A. Berman and N. Shaked-Monderer. Completely positive matrices. World Scientific Publishing Co., Inc., River Edge, NJ, 2003. x+206. DOI: 10.1142/9789812795212 (cited on page 108).

[15] N. Biggs. Algebraic graph theory. 2nd edition. Cambridge mathematical library. Cambridge University Press, Cambridge, 1993. viii+205 (cited on page 100).

[16] I. M. Bomze, M. Dür, E. de Klerk, C. Roos, A. J. Quist, and T. Terlaky. "On Copositive Programming and Standard Quadratic Optimization Problems". In: Journal of Global Optimization 18.4 (December 1, 2000), pages 301-320. DOI: 10.1023/A:1026583532263 (cited on page 107).

[17] J. A. Bondy and P. Hell. "A note on the star chromatic number". In: Journal of Graph Theory 14.4 (1990), pages 479-482. DOI: 10.1002/jgt.3190140412 (cited on page 54).

[18] G. Brassard, R. Cleve, and A. Tapp. "Cost of Exactly Simulating Quantum Entanglement with Classical Communication". In: Physical Review Letters 83.9 (August 30, 1999), pages 1874-1877. DOI: 10.1103/PhysRevLett.83.1874 (cited on page 63).

[19] J. Briët and J. Zuiddam. "On the orthogonal rank of Cayley graphs and impossibility of quantum round elimination". In: Quantum Inf. Comput. 17.1-2 (2017), pages 106-116 (cited on page 65).

[20] S. Burer. "On the copositive representation of binary and continuous nonconvex quadratic programs". In: Mathematical Programming 120.2 (2009), pages 479-495. DOI: 10.1007/s10107-008-0223-z (cited on pages 107, 109).

[21] A. Cabello, S. Severini, and A. Winter. "(Non-)Contextuality of Physical Theories as an Axiom". In: arXiv:1010.2163 [quant-ph] (October 11, 2010). arXiv: 1010.2163 (cited on page 57).

[22] P. J. Cameron, A. Montanaro, M. W. Newman, S. Severini, and A. Winter. "On the quantum chromatic number of a graph". In: Electronic Journal of Combinatorics 14.1 (2007), Research Paper 81, 15 (cited on pages 62,63$)$.

[23] V. Chvátal. "Edmonds polytopes and a hierarchy of combinatorial problems". In: Discrete Mathematics 4 (1973), pages 305-337. DOI: 10.1016/0012-365X (73)90167-2 (cited on pages 72, 74).

[24] V. Chvátal. Linear Programming. New York, NY: Bedford Books, June 14, 2016. 494 pages (cited on page 21).

[25] R. Cleve, P. Høyer, B. Toner, and J. Watrous. "Consequences and limits of nonlocal strategies". In: Proceedings. 19th IEEE Annual Conference on Computational Complexity, 2004. (2004), pages 236-249 (cited on pages 62,63).

[26] A. Coja-Oghlan. "The Lovász number of random graphs". In: Combinatorics, Probability and Computing 14.4 (2005), pages 439-465. DOI: 10.1017/S0963548305006826 (cited on page 104).

[27] M. K. de Carli Silva and L. Tunçel. "A Notion of Total Dual Integrality for Convex, Semidefinite, and Extended Formulations". In: SIAM Journal on Discrete Mathematics 34.1 (January 1, 2020), pages 470-496. DOI: 10.1137/18M1169710 (cited on page 109).

[28] M. K. de Carli Silva and L. Tunçel. "An axiomatic duality framework for the theta body and related convex corners". In: Mathematical Programming 162.1 (2017), pages 283-323. DOI: 10.1007/s10107016-1041-3 (cited on pages 6, 57, 59, 101, 108, 111-113, 119, 128, 161, 166).

[29] M. K. de Carli Silva and L. Tunçel. "Vertices of spectrahedra arising from the elliptope, the theta body, and their relatives". In: SIAM Journal on Optimization 25.1 (2015), pages 295-316. DOI: 10.1137/130945818 (cited on page 120).

[30] E. de Klerk and D. V. Pasechnik. "Approximation of the stability number of a graph via copositive programming". In: SIAM Journal on Optimization 12.4 (2002), pages 875-892. DOI: 10.1137 / S1052623401383248 (cited on page 132).

[31] C. De Simone. "The cut polytope and the Boolean quadric polytope". In: Discrete Mathematics 79.1 (1989), pages 71-75. DOI: 10.1016/0012-365X (90) 90056-N (cited on pages 95, 121).

[32] W. Deuber and X. Zhu. "Circular Coloring of Weighted Graphs". In: Journal of Graph Theory 23 (1994), pages 365-376 (cited on page 72). 
[33] M. DeVos, J. Nešetřil, and A. Raspaud. "On edge-maps whose inverse preserves flows or tensions". In: Graph theory in Paris. Trends Math. Birkhäuser, Basel, 2007, pages 109-138. DOI: 10.1007/978-37643-7400-6_10 (cited on page 76).

[34] S. Eilenberg and S. MacLane. "General Theory of Natural Equivalences". In: Transactions of the American Mathematical Society 58.2 (1945), pages 231-294. DOI: 10.2307/1990284 (cited on page 13).

[35] S. Fallat and L. Hogben. "Variants on the minimum rank problem: A survey II". In: arXiv:1102.5142 [math] (October 8, 2014). arXiv: 1102.5142 (cited on page 65).

[36] U. Feige and L. Lovász. "Two-prover one-round proof systems: their power and their problems (extended abstract)". In: Proceedings of the twenty-fourth annual ACM symposium on Theory of Computing. STOC '92. Victoria, British Columbia, Canada: Association for Computing Machinery, July 1, 1992, pages 733-744. DOI: 10.1145/129712.129783 (cited on pages 62, 76, 156).

[37] R. M. Freund. "Dual gauge programs, with applications to quadratic programming and the minimumnorm problem". In: Mathematical Programming 38.1 (1987), pages 47-67. DOI: 10.1007/BF02591851 (cited on pages 81, 129).

[38] M. P. Friedlander, I. Macêdo, and T. K. Pong. "Gauge optimization and duality". In: SIAM Journal on Optimization 24.4 (2014), pages 1999-2022. DOI: 10.1137/130940785 (cited on pages 81, 129).

[39] D. R. Fulkerson. "Anti-blocking polyhedra". In: Journal of Combinatorial Theory. Series B 12 (1972), pages 50-71. DOI: 10.1016/0095-8956 (72)90032-9 (cited on pages 3, 4, 88, 164).

[40] D. R. Fulkerson. "Blocking and anti-blocking pairs of polyhedra". In: Mathematical Programming 1 (1971), pages 168-194. DOI: 10.1007/BF01584085 (cited on pages 3, 88, 164).

[41] C. Godsil, D. E. Roberson, B. Rooney, R. Šámal, and A. Varvitsiotis. "Graph homomorphisms via vector colorings". In: European Journal of Combinatorics 79 (2019), pages 244-261. DOI: 10.1016/j . ejc.2019.04.001 (cited on page 57).

[42] C. Godsil, D. E. Roberson, B. Rooney, R. Šámal, and A. Varvitsiotis. "Vector coloring the categorical product of graphs". In: Mathematical Programming 182.1 (July 1, 2020), pages 275-314. DOI: 10 . 1007/s10107-019-01393-0 (cited on pages 57, 72).

[43] C. Godsil, D. E. Roberson, R. Šámal, and S. Severini. "Sabidussi versus Hedetniemi for three variations of the chromatic number". In: Combinatorica 36.4 (2016), pages 395-415. DOI: 10.1007/s00493-0143132-1 (cited on pages 57, 72).

[44] C. Godsil and G. Royle. Algebraic graph theory. Volume 207. Graduate Texts in Mathematics. SpringerVerlag, New York, 2001. xx+439 (cited on pages 4, 12, 49, 75, 100).

[45] C. Godsil and K. Meager. Erdös-Ko-Rado Theorems: Algebraic Approaches. Cambridge University Press, November 2015 (cited on page 103).

[46] M. X. Goemans and D. P. Williamson. "Improved approximation algorithms for maximum cut and satisfiability problems using semidefinite programming". In: Journal of the Association for Computing Machinery 42.6 (1995), pages 1115-1145. DOI: 10.1145/227683.227684 (cited on pages 80, 95, 122).

[47] J. Gouveia, P. A. Parrilo, and R. R. Thomas. "Theta bodies for polynomial ideals". In: SIAM Journal on Optimization 20.4 (2010), pages 2097-2118. DOI: 10.1137/090746525 (cited on page 57).

[48] S. Gribling, D. de Laat, and M. Laurent. "Bounds on entanglement dimensions and quantum graph parameters via noncommutative polynomial optimization". In: Mathematical Programming 170.1 (2018), pages 5-42. DOI: 10.1007/s10107-018-1287-z (cited on page 62).

[49] M. Grötschel, L. Lovász, and A. Schrijver. "Relaxations of vertex packing". In: Journal of Combinatorial Theory. Series B 40.3 (1986), pages 330-343. DOI: 10.1016/0095-8956(86)90087-0 (cited on pages 4, 101, 164).

[50] M. Grötschel, L. Lovász, and A. Schrijver. "The ellipsoid method and its consequences in combinatorial optimization". In: Combinatorica 1.2 (1981), pages 169-197. DOI: 10.1007/BF02579273 (cited on pages 126, 164).

[51] N. Gvozdenović and M. Laurent. "The operator $\Psi$ for the chromatic number of a graph". In: SIAM Journal on Optimization 19.2 (2008), pages 572-591. DOI: 10.1137/050648237 (cited on page 76). 
[52] G. Hahn and C. Tardif. "Graph homomorphisms: structure and symmetry". In: Graph symmetry (Montreal, PQ, 1996). Volume 497. NATO Adv. Sci. Inst. Ser. C Math. Phys. Sci. Kluwer Acad. Publ., Dordrecht, 1997, pages 107-166 (cited on pages 1, 12, 13, 42, 96).

[53] R. Hammack, W. Imrich, and S. Klavžar. Handbook of product graphs. 2nd edition. Discrete Mathematics and its Applications (Boca Raton). CRC, 2011. xviii+518 (cited on page 72).

[54] J. Håstad. "Clique is hard to approximate within $n^{1-\varepsilon " . ~ I n: ~ A c t a ~ M a t h e m a t i c a ~} 182.1$ (1999), pages 105142. DOI: $10.1007 / \mathrm{BF} 02392825$ (cited on page 3).

[55] S. T. Hedetniemi. "Homomorphisms of Graphs and Automata". Ph.D. University of Michigan, 1966. 92 pages (cited on page 72 ).

[56] P. Hell. "An introduction to the category of graphs". In: Topics in graph theory (New York, 1977). Volume 328. Ann. New York Acad. Sci. New York Acad. Sci., New York, 1979, pages 120-136 (cited on pages $4,72-74)$.

[57] P. Hell and J. Nešetřil. Graphs and homomorphisms. Volume 28. Oxford Lecture Series in Mathematics and its Applications. Oxford University Press, Oxford, 2004. xii+244. DOI: 10.1093/acprof : oso/ 9780198528173.001.0001 (cited on pages 1, 42, 49, 96).

[58] A. J. Hoffman. "On eigenvalues and colorings of graphs". In: Graph Theory and its Applications (Proc. Advanced Sem., Math. Research Center, Univ. of Wisconsin, Madison, Wis., 1969). Academic Press, New York, 1970, pages 79-91 (cited on pages 98, 100).

[59] L. Hogben, K. F. Palmowski, D. E. Roberson, and S. Severini. "Orthogonal representations, projective rank, and fractional minimum positive semidefinite rank: connections and new directions". In: Electronic Journal of Linear Algebra 32 (2017), pages 98-115. DOI: 10.13001/1081-3810.3102 (cited on page 66).

[60] R. A. Horn and C. R. Johnson. Matrix analysis. Corrected reprint of the 1985 original. Cambridge: Cambridge University Press, 1990, pages xiv+561 (cited on pages 99, 103, 108, 110, 153).

[61] R. A. Horn and C. R. Johnson. Topics in matrix analysis. Cambridge University Press, Cambridge, 1991. viii+607. DOI: 10.1017/CB09780511840371 (cited on pages 9, 138).

[62] P. Hudak, J. Hughes, S. P. Jones, and P. Wadler. "A History of Haskell: being lazy with class". In: (June 9, 2007) (cited on page 13).

[63] P. L. Ivănescu. "Some Network Flow Problems Solved with Pseudo-Boolean Programming". In: Operations Research 13.3 (June 1, 1965), pages 388-399. DOI: 10.1287/opre.13.3.388 (cited on page 121).

[64] K. E. Iverson. "Notation as a tool of thought". In: Communications of the ACM 23.8 (August 1, 1980), pages 444-465. DOI: 10.1145/358896.358899 (cited on page 5).

[65] D. Karger, R. Motwani, and M. Sudan. "Approximate graph coloring by semidefinite programming". In: Journal of the Association for Computing Machinery 45.2 (1998), pages 246-265. DOI: 10.1145/ 274787.274791 (cited on pages 2, 57, 59).

[66] R. M. Karp. "Reducibility among combinatorial problems". In: Complexity of computer computations (Proc. Sympos., IBM Thomas J. Watson Res. Center, Yorktown Heights, N.Y., 1972). 1972, pages 85103 (cited on page 119).

[67] D. E. Knuth. "The sandwich theorem". In: Electronic Journal of Combinatorics 1 (1994), Article 1, approx. 48 (cited on pages 4, 69, 100, 103).

[68] O. Kuryatnikova, R. Sotirov, and J. Vera. "The maximum $k$-colorable subgraph problem and related problems". In: arXiv:2001.09644 [math] (January 27, 2020). arXiv: 2001.09644 (cited on page 75).

[69] L. Lamport. "How to write a 21st century proof". In: Journal of Fixed Point Theory and Applications 11.1 (2012), pages 43-63. DOI: 10.1007/s11784-012-0071-6 (cited on pages 5, 164).

[70] M. Larsen, J. Propp, and D. Ullman. "The fractional chromatic number of Mycielski's graphs". In: Journal of Graph Theory 19.3 (1995), pages 411-416. DOI: 10.1002/jgt. 3190190313 (cited on page 75$)$. 
[71] M. Laurent. "A Comparison of the Sherali-Adams, Lovász-Schrijver, and Lasserre Relaxations for 0-1 Programming". In: Mathematics of Operations Research 28.3 (August 1, 2003), pages 470-496. DOI: 10.1287/moor.28.3.470.16391 (cited on page 109).

[72] M. Laurent. "Sums of squares, moment matrices and optimization over polynomials". In: Emerging applications of algebraic geometry. Volume 149. IMA Vol. Math. Appl. Springer, New York, 2009, pages 157-270. DOI: 10.1007/978-0-387-09686-5_7 (cited on page 57).

[73] M. Laurent and T. Piovesan. "Conic approach to quantum graph parameters using linear optimization over the completely positive semidefinite cone". In: SIAM Journal on Optimization 25.4 (2015), pages 2461-2493 (cited on pages 63, 108, 156).

[74] M. Laurent and S. Poljak. "On a positive semidefinite relaxation of the cut polytope". In: Linear Algebra and its Applications. Honoring Miroslav Fiedler and Vlastimil Ptak 223-224 (July 1, 1995), pages 439-461. DOI: 10.1016/0024-3795(95)00271-R (cited on page 127).

[75] M. Laurent, S. Poljak, and F. Rendl. "Connections between semidefinite relaxations of the max-cut and stable set problems". In: Mathematical Programming 77 (1997), pages 225-246. DOI: 10.1016/S00255610 (96) 00084-6 (cited on pages 95, 121).

[76] A. Lévy. "The Role of Classes in Set Theory". In: Studies in Logic and the Foundations of Mathematics. Edited by G. H. Müller. Volume 84. Sets and Classes on The Work by Paul Bernays. Elsevier, January 1, 1976, pages 173-215. DOI: 10.1016/S0049-237X(08) 70894-2 (cited on pages 7, 39).

[77] L. Lovász. "On the Shannon capacity of a graph". In: IEEE Transactions on Information Theory 25.1 (1979), pages 1-7. DOI: 10.1109/TIT.1979.1055985 (cited on pages 2, 57, 59, 65, 69, 100, 103, 111).

[78] C. Lund and M. Yannakakis. "On the hardness of approximating minimization problems". In: Journal of the Association for Computing Machinery 41.5 (1994), pages 960-981. DOI: 10.1145/185675. 306789 (cited on page 3).

[79] M. Lupini, L. Mančinska, and D. E. Roberson. "Nonlocal games and quantum permutation groups". In: Journal of Functional Analysis 279.5 (September 15, 2020), page 108592. DoI: 10.1016/j.jfa. 2020.108592 (cited on page 157).

[80] C. J. Luz and A. Schrijver. "A convex quadratic characterization of the Lovász theta number". In: SIAM Journal on Discrete Mathematics 19.2 (2005), 382-387 (electronic). DOI: 10.1137/S0895480104429181 (cited on page 101).

[81] C. J. Luz. "A characterization of the weighted Lovász number based on convex quadratic programming". In: Optimization Letters 10.1 (2016), pages 19-31. DOI: 10.1007/s11590-015-0911-6 (cited on page 101).

[82] C. J. Luz. "An upper bound on the independence number of a graph computable in polynomial-time". In: Operations Research Letters 18.3 (1995), pages 139-145. DOI: 10.1016/0167-6377 (95) 00042-9 (cited on page 101).

[83] C. J. Luz and D. M. Cardoso. "A quadratic programming approach to the determination of an upper bound on the weighted stability number". In: European Journal of Operational Research 132.3 (2001), pages 569-581. DOI: 10.1016/S0377-2217(00)00162-4 (cited on page 101).

[84] S. Mac Lane. Categories for the working mathematician. 2nd edition. Graduate Texts in Mathematics. Springer-Verlag, New York, 1998. xii+314 (cited on pages 13, 14).

[85] L. Mančinska and D. E. Roberson. "Quantum homomorphisms". In: Journal of Combinatorial Theory. Series B 118 (2016), pages 228-267 (cited on pages 62, 63, 65, 66, 69, 70, 96, 156).

[86] L. Mančinska and D. E. Roberson. "Quantum isomorphism is equivalent to equality of homomorphism counts from planar graphs". In: arXiv:1910.06958 [quant-ph] (October 18, 2019). arXiv: 1910.06958 (cited on page 157).

[87] R. J. McEliece, E. R. Rodemich, and H. C. Rumsey Jr. "The Lovász bound and some generalizations". In: Journal of Combinatorics, Information \& System Sciences 3.3 (1978), pages 134-152 (cited on pages 57,111$)$. 
[88] E. Moggi. "Notions of computation and monads". In: Information and Computation. Volume 93. 1. 1991, pages 55-92. DOI: 10.1016/0890-5401(91)90052-4 (cited on page 13).

[89] B. Mohar and H. Wu. "Dichromatic Number and Fractional Chromatic Number". In: Forum of Mathematics, Sigma 4 (2016). DOI: 10.1017/fms.2016.28 (cited on page 48).

[90] B. Mohar and H. Wu. "Fractional chromatic number of a random subgraph". In: Journal of Graph Theory 95.3 (2020), pages 467-472. DOI: 10.1002/jgt.22571 (cited on page 48).

[91] J. Mycielski. "Sur le coloriage des graphs". In: Colloquium Mathematicum 3 (1955), pages 161-162. DOI: $10.4064 / \mathrm{cm}-3-2-161-162$ (cited on page 75 ).

[92] G. Narasimhan. The Maximum K-Colorable Subgraph Problem. Technical Report. University of Wisconsin-Madison Department of Computer Sciences, 1989 (cited on page 75).

[93] A. Nemirovski. Introduction to Linear Optimization. 2012. URL: https://www2.isye.gatech.edu/ $\sim$ nemirovs/OPTI_LectureNotes.pdf (visited on 10/10/2019) (cited on page 21).

[94] J. Neto and W. Ben-Ameur. "On fractional cut covers". In: Discrete Applied Mathematics. The Journal of Combinatorial Algorithms, Informatics and Computational Sciences 265 (2019), pages 168-181. DOI: 10.1016/j.dam.2019.03.020 (cited on pages 76, 80, 126, 128, 130).

[95] M. W. Newman. "Independent Sets and Eigenspaces". PhD thesis. Department of Combinatorics and Optimization, University of Waterloo, 2004 (cited on page 100).

[96] V. I. Paulsen, S. Severini, D. Stahlke, I. G. Todorov, and A. Winter. "Estimating quantum chromatic numbers". In: Journal of Functional Analysis 270.6 (March 15, 2016), pages 2188-2222. DOI: 10.1016/ j.jfa.2016.01.010 (cited on page 63).

[97] A. Pultr and V. Trnková. Combinatorial, algebraic and topological representations of groups, semigroups and categories. Volume 22. North-Holland Mathematical Library. North-Holland Publishing Co., Amsterdam-New York, 1980, pages $\mathrm{x}+372$ (cited on page 13).

[98] R. Rabelo, C. Duarte, A. J. López-Tarrida, M. T. Cunha, and A. Cabello. "Multigraph approach to quantum non-locality". In: Journal of Physics A: Mathematical and Theoretical 47.42 (October 2014), page 424021. DOI: 10.1088/1751-8113/47/42/424021 (cited on page 57).

[99] E. Riehl. Category Theory in Context. 2016 (cited on pages 13, 18).

[100] D. E. Roberson. "Conic formulations of graph homomorphisms". In: Journal of Algebraic Combinatorics 43.4 (2016), pages 877-913 (cited on pages 6, 65, 133, 134, 141, 145, 149, 150, 152, 154-156, 161, 165, 166).

[101] D. E. Roberson. "Variations on a Theme: Graph Homomorphisms". PhD thesis. Department of Combinatorics and Optimization, University of Waterloo, 2013 (cited on page 57).

[102] R. T. Rockafellar. Convex analysis. Princeton Landmarks in Mathematics. Reprint of the 1970 original, Princeton Paperbacks. Princeton, NJ: Princeton University Press, 1997, pages xviii +451 (cited on pages 19, 20, 81, 89, 106, 125, 163).

[103] R. Śámal. "Cubical coloring - fractional covering by cuts and semidefinite programming". In: Discrete Math. Theor. Comput. Sci. 17.2 (2015), pages 251-266 (cited on pages 76, 77, 80, 95, 126, 128, 130, 166).

[104] R. Šámal. "Cycle-continuous mappings - order structure". In: Journal of Graph Theory 85.1 (2017), pages 56-73. DOI: 10.1002/jgt.22047 (cited on page 76).

[105] R. Šámal. "On XY mappings". PhD thesis. Charles University in Prague, 2006. 152 pages (cited on pages $76,77,95)$.

[106] E. R. Scheinerman and D. H. Ullman. Fractional graph theory. A rational approach to the theory of graphs, With a foreword by Claude Berge, Reprint of the 1997 original. Dover Publications, Inc., Mineola, NY, 2011, pages xviii+211 (cited on pages 4, 5, 48, 49).

[107] A. Schrijver. Theory of Linear and Integer Programming. Wiley-Interscience Series in Discrete Mathematics. Chichester: John Wiley \& Sons Ltd., 1986. xii+471 (cited on page 21). 
[108] A. Schrijver. "A comparison of the Delsarte and Lovász bounds". In: IEEE Transactions on Information Theory 25.4 (1979), pages 425-429. DOI: 10.1109/TIT.1979.1056072 (cited on pages 57, 111).

[109] A. Schrijver. Combinatorial optimization. Polyhedra and efficiency. Vol. A. Volume 24. Algorithms and combinatorics. Springer-Verlag, Berlin, 2003 (cited on page 153).

[110] Y. Shitov. "Counterexamples to Hedetniemi's conjecture". In: Annals of Mathematics. Second Series 190.2 (2019), pages 663-667. DOI: 10.4007/annals.2019.190.2.6 (cited on pages 48, 72).

[111] M. A. Shulman. "Set theory for category theory". In: arXiv:0810.1279 [math] (October 7, 2008). arXiv: 0810.1279 (cited on pages 7, 14).

[112] J. Sikora and A. Varvitsiotis. "Linear conic formulations for two-party correlations and values of nonlocal games". In: Mathematical Programming 162.1 (2017), pages 431-463. DOI: 10.1007/s10107016-1049-8 (cited on page 62).

[113] W. Slofstra. "The Set of Quantum Correlations is not Closed". In: Forum of Mathematics, Pi 7 (2019). Publisher: Cambridge University Press. DOI: 10.1017/fmp.2018.3 (cited on pages 62, 108).

[114] R. Smullyan and P. Fitting. Set theory and the continuum problem. Oxford Logic Guides. Oxford University Press, Oxford, 1996 (cited on page 7).

[115] M. Szegedy. "A note on the theta number of Lovász and the generalized Delsarte bound". In: Proceedings of the 35th Annual IEEE Symposium on Foundations of Computer Science. 1994, pages 36-39 (cited on pages $57,59,111)$.

[116] A. Vince. "Star chromatic number". In: Journal of Graph Theory 12.4 (1988), pages 551-559. DoI: 10.1002/jgt.3190120411 (cited on pages 2, 53, 56).

[117] P. Wadler. "Propositions as types". In: Communications of the ACM 58.12 (November 23, 2015), pages 75-84. DOI: 10.1145/2699407 (cited on page 5).

[118] J. Watrous. The Theory of Quantum Information. Cambridge University Press, 2018. 598 pages (cited on pages $63,133,134,138,140,149)$.

[119] P. Wocjan, C. Elphick, and A. Abiad. "Spectral upper bound on the quantum $k$-independence number of a graph". In: arXiv:1910.07339 [math] (October 16, 2019). arXiv: 1910.07339 (cited on page 63).

[120] X. Zhu. "Circular chromatic number: a survey". In: Discrete Mathematics 229.1-3 (2001). Combinatorics, graph theory, algorithms and applications, pages 371-410 (cited on pages 53, 71).

[121] X. Zhu. "Circular perfect graphs". In: Journal of Graph Theory 48.3 (2005), pages 186-209. DOI: 10.1002/jgt. 20050 (cited on pages 2, 53, 54, 56, 70).

[122] X. Zhu. "The fractional version of Hedetniemi's conjecture is true". In: European Journal of Combinatorics 32.7 (2011), pages 1168-1175. DOI: 10.1016/j.ejc.2011.03.004 (cited on pages 48, $72)$. 